

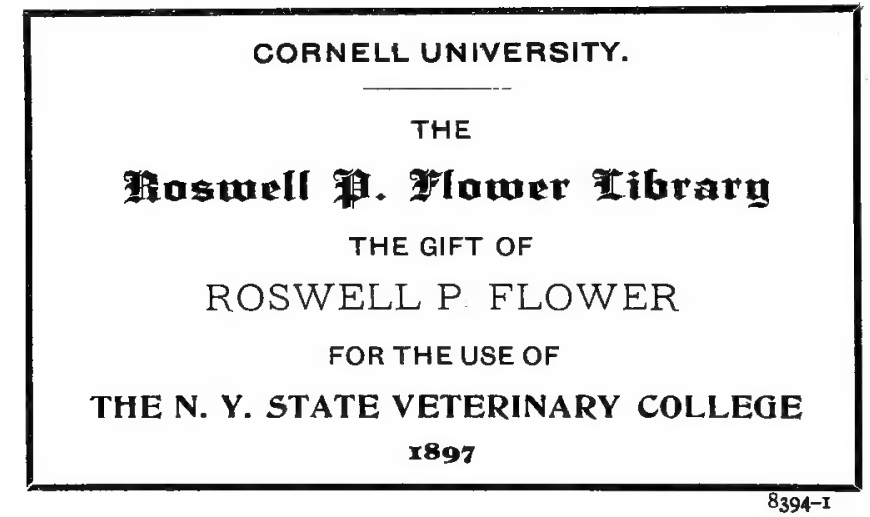




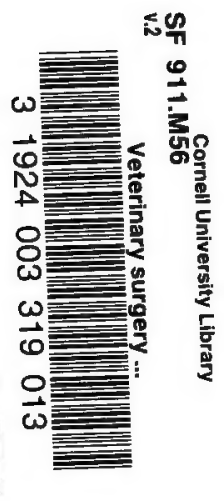




\section{Cornell University Library}

The original of this book is in the Cornell University Library.

There are no known copyright restrictions in the United States on the use of the text.

http://www.archive.org/details/cu31924003319013 




\title{
VETERINARY SURGERY
}

By Louis A. Merillat, V. S.

\author{
Volume II
}

THE PRINCIPLES OF VETERINARY SURGERY. 


\section{VETERINARY SURGERY}

By LOUIS A. MERILLAT, V. S.

VOLUME I

ANIMAL DENTISTRY AND DISEASES OF THE MOUTH

VOLUME II

THE PRINCIPLES OF VETERINARY SURGERY

Volume III

VETERINARY SURGICAL OPERATIONS 




\title{
Veterinary Surgery
}

\author{
Volume II
}

\section{THE PRINCIPLES}

\section{OF \\ VETERINARY SURGERY \\ BY \\ Louis A. Merillat, V. S.}

AUTHOR OF ANIMAL DENTISTRY AND DISEASES OF THE MOUTH; COLLABORATOR AMERICAN,VETERINARY REVIEW; PROFESSOR OF VETERINARY SURGERY IN THE CHICAGO VETERINARY COLLEgE; LATE SECRETARY AND PROFESSOR OF DENTISTRY

IN THE M'KILLIP VETERINARY COLLEGE; LATE PRESIDENT CHICAgo VETERINARY SOCIETY; LATE SECKETARY OF THE ASSOCIATION OF VETERINARY FACULTIES AND EXAMINING BOARDS OF NORTH AMERICA, ETC., ETC., ETC.

Including an Authorized Translation, Enlargement and Rearrangement of

\section{GENERAL VETERINARY SURGICAL PATHOLOGY}

BY

Profs. C. CADEAC, P. LigBlanC and C. CAROUGeAU of the Veterinary High School at Lyons, France.

CHICAGO

ALEXANDER EGER

1907 
Entered according to Act of Congress in the year 1808 by ALEXANDER EGER

In the office of the Librarian of Congress at Washington.

\section{Daniels Company Press} Chicago 


\section{PREFACE.}

In the three-year course of American veterinary colleges the subject of surgery is taught during the last two years. The junior student is taught the essentials of surgical science and the principles of operative technique while the senior, continuing along the same lines, must in addition complete the full quota of surgical diseases with regard to their causes, symptoms, pathologic lesions and their surgical treatment. At the opening of each college year, when the new student of veterinary surgery is given an outline of this vast scope before him, an inquiry about books covering the ground is invariably forthcoming. "What books shall I procure?" is the universal cry of our new students of veterinary surgery, and owing to the fact that the pressing need of complete books on veterinary surgery has not been met by English-writing authors, the teacher is forced to admit that no books exist that will 'satisfactorily fill the requirement. We have books on surgical technique, books on surgical diseases and books on special surgical subjects, original works and translations, but books on the pathological subjects and the other essentials which form the foundation of surgical knowledge are wanting. As a consequence the student must be directed to glean here and there through works written for the benefit of students of human pathology only. This condition, little less then a calamity in the present-day veterinary college, will only be improved when we have a series of surgical books written by and for the veterinarian. It is deplorable that we should have been so long content with foundations laid by men who admittedly have no knowledge of the superstructure of our science. 
While this volume is intended to meet the requirement of the junior student studying the principles upon which sound surgical knowledge is based, it; details will indoubtedly be of service as a reference to both the senior student and practitioner.

The promulgation of the many intricate subjects involved would have been impossible through my own efforts. It was only through the consent to translate "Pathologie Chirurgical Generale," by LeBlanc, Cadeac et Carougeau, that the publication of these details become possible. Their book, which is the second part of this volume translated from the original French edition, commended itself to me as a most valuable work for the English-reading veterinarian and veterinary student. For two years I urged its translation and publication, but meeting with no encouragement, I undertook the task alone. I desire to thank their publishers for their liberal arrangement in permitting me to translate the work into English, and to apologize to the authors for any errors the translation may contain. To meet the demands of a certain classification of the diseases it was found necessary to rearrange the chapters.

I am also deeply indebted to Prof. G. E. Amadon, Director of the Pathological Laboratories of the Chicago Veterinary College, for assistance in preparing several of the chapters, whereby the usefulness and value of the volume have been greatly enhanced.

L. A. Merillat. 


\section{CONTENTS.}

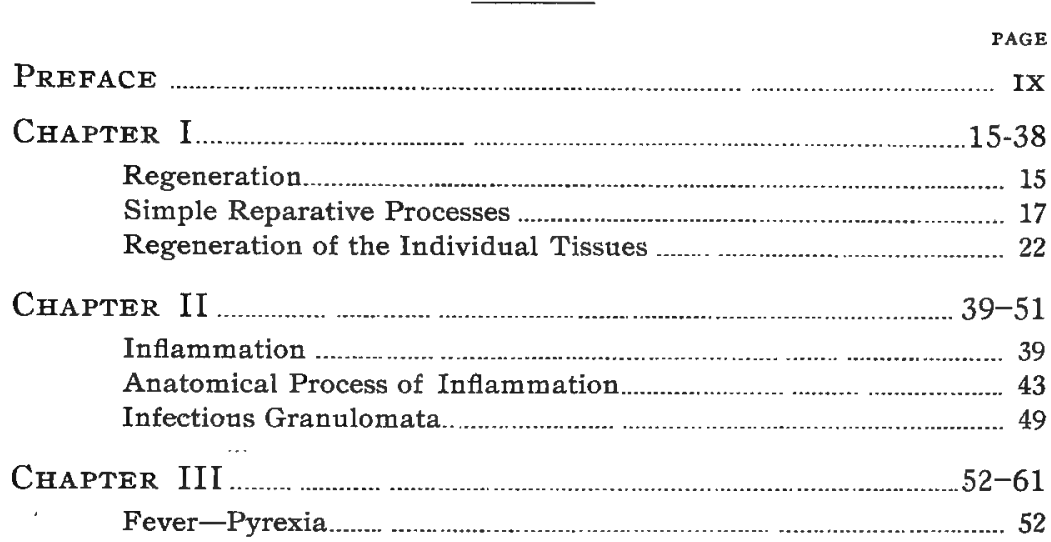

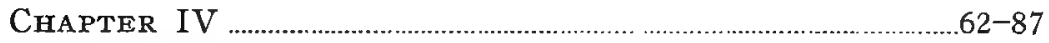

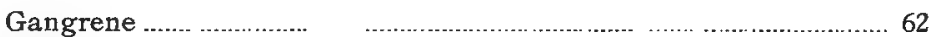

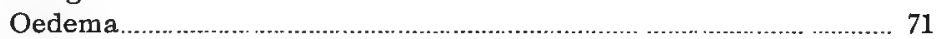

Thrombosis and Embolism .......................................................... 76

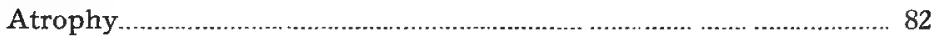

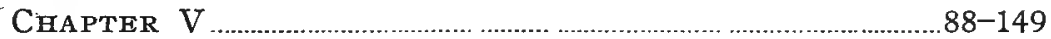

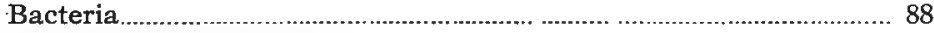

Microscopic Examination of Bacteria

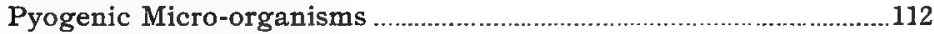

Specific Surgical Micro-organisms.....................................................126

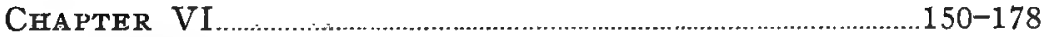

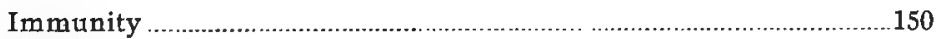

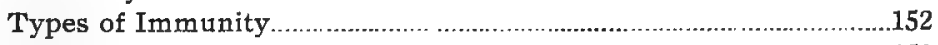

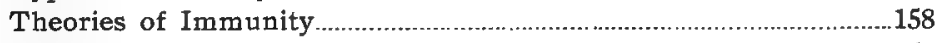

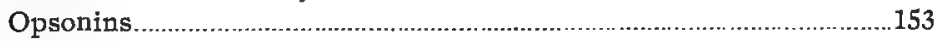

Serums-Diagnosis, Preparation, etc ............................................. 176

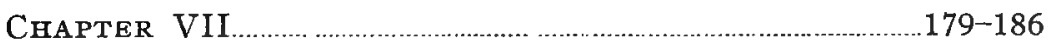

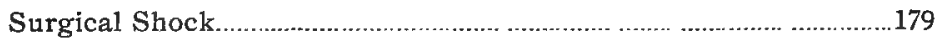

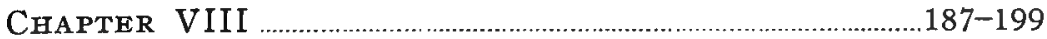

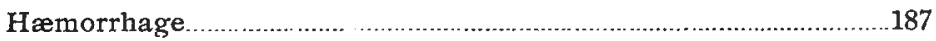

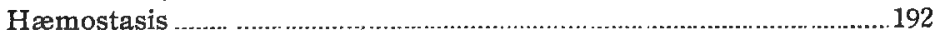

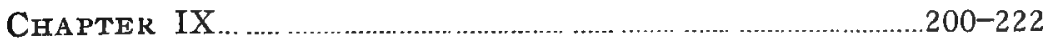

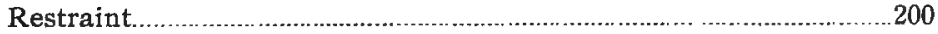




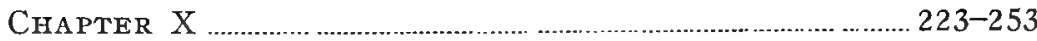

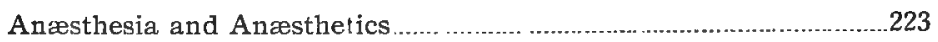

Local Anæsthesia and Local Anæsthetics.......................................... 247

Chapter XI

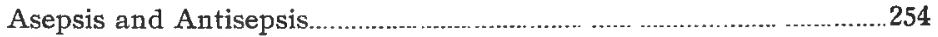

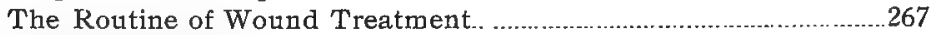

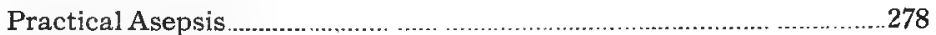

Recommendations for an Aseptical Operation..................................281

\section{PART II}

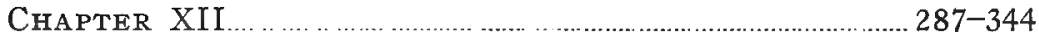

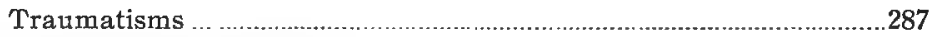

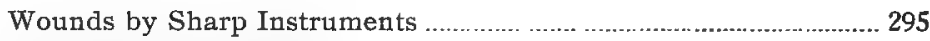

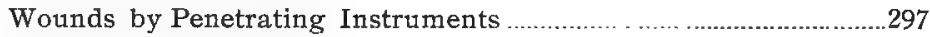

Wounds by Tearing ...........................................................................299

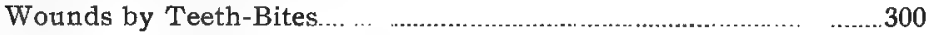

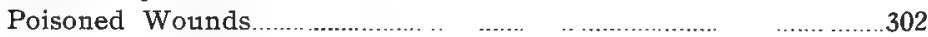

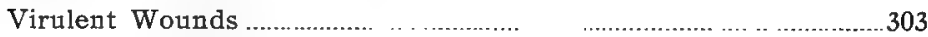

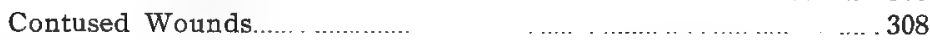

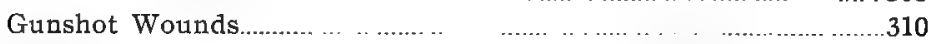

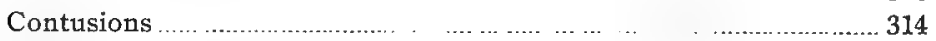

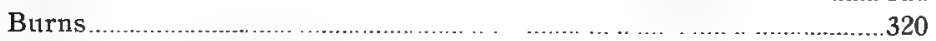

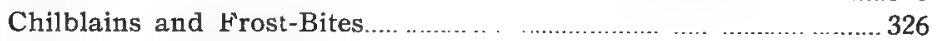

Traumatic Hæmorrhage and Anæmia........................................... 330

'Traumatic Emphysema................................................................. 332

Cicatrices of the Skin-Cutaneous Scars........................................ 333

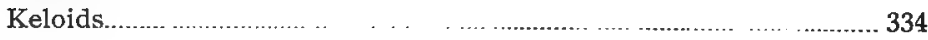

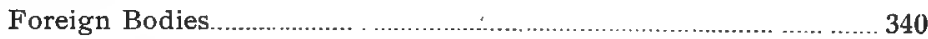

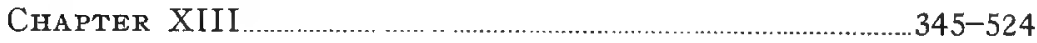

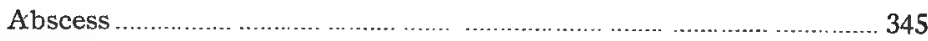

Hot or Acute Abscess...................... . . .................................. 350

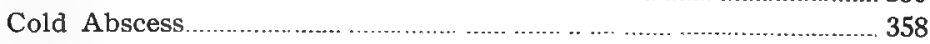

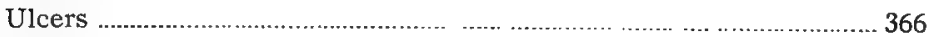

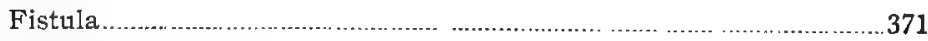

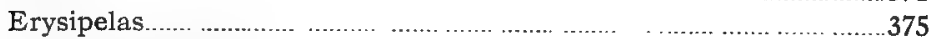

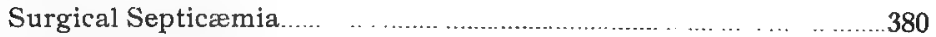

Malignant Edema-Gangrenous Septicæmia .... ..........................386

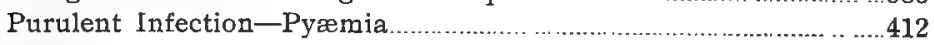

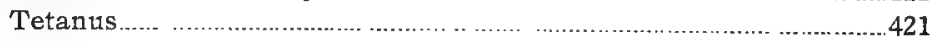

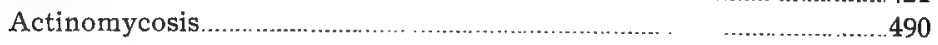

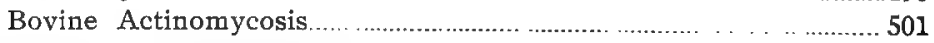

Porcine Actinomycosis........................ . . . . . . . . ...................... 514

Equine Actinomycosis ................ ........................................ 517

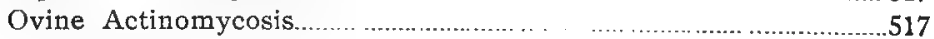

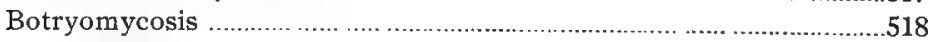

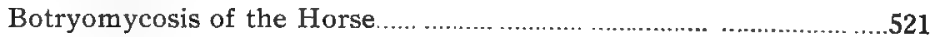




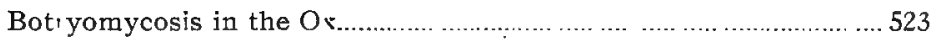

Botryomycosis in the Hog ....................................................524

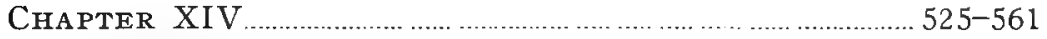

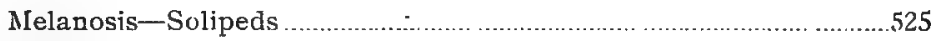

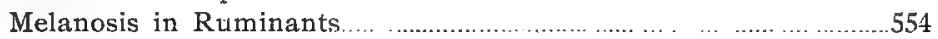

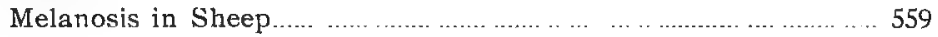

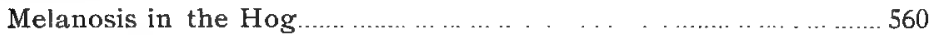

Melanosis in the Dog .................................. . . ....................5 561

Chapter XV $\quad$.

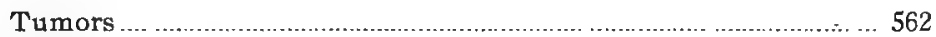

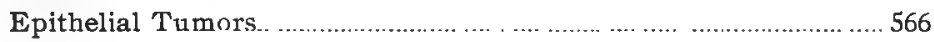

Connective Tissue Tumors ........................................... 570

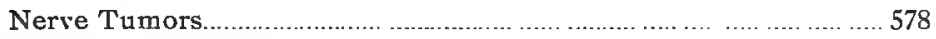

Tumors of Muscular 'Type ..................................................580

Complex Tumors .................................................................... 580

Osseous Tissues-Greneral Affections-Rachrtis ......................... 583

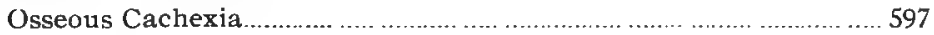

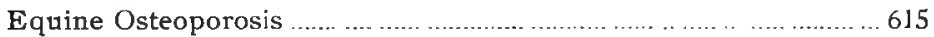

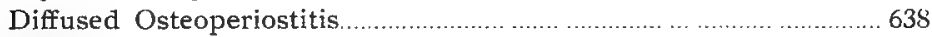

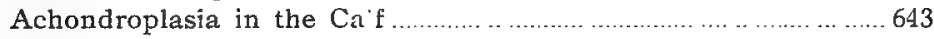

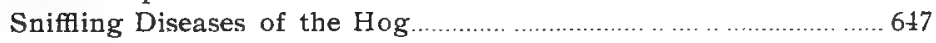





\section{CHAPTER I.}

\section{REGENERATION.}

DEFINITION.-Regeneration is the process which leads to the replacement of lost tissue. It takes place under normal and physiological conditions and in all pathological processes. In both cases the reactive phenomena are the same.

The annual moulting of the feathers of the bird, the periodic loss and growth of the horns of the stag, and the breaking down of cells in different parts of the body after they have been active for a time, and their replacement by new cells are examples of the phenomena of physiological regeneration. As a general rule, the higher the tissue stands in the scale of differentiation, the smaller the tendency of its cells to proliferate. The power of regeneration in mammals is limited as compared with that of many of the lower animals. The upper part of the hydra, if separated, will reproduce the rest of the body; if the lower part is cut off, it will add the rest. Certain worms may be cut into several pieces, and each part will regain what is needed to complete the mangled organism. The starfish can reproduce its arms; the holothurian, its stomach; the snail, its tentacles; the lobster, its claws; the spider, its legs; the fish, its fins; the lizard, its tail.

In the domestic animals, nature facilitates the struggle for existence in another way. If a leg is cut off, an animal can make three do; if one kidney is destroyed, the other will enlarge, and together with the skin make it possible for the animal to live and work in comfort; if the spleen is removed, the lymphatic glands will perform its functions almost as 
well as the organ itself. In man, the upper part of the duodenum has been trained to do the work of the stomach, and even one hemisphere of the brain can be removed, and the other made to take its place. Almost the entire thyroid gland can be taken out and the remaining part will perform the physiological duties of the whole organ. If one ovary or tes. ticle be removed, it will not interfere with the generative functions of the animal. The bladder can be dispensed with by transplanting the ureters into the intestines; the lower bowel, by attaching the remaining portion of intestines to the external abdominal wall; the osophagus, by inserting a tube into the stomach; and furthermore, the various organs have been transplanted from one animal to another, such as the ovary, kidney, thyroid gland, etc. This is still in the experimental stage.

In pathological regeneration, the new tissue is formed by the proliferation of cells in the immediate neighborhood of the area from which the cells have been cast off or destroyed. The new tissue is derived from tissue of like kind; epithelium from epithelium, muscle from muscle and connective tissue from connective tissue. There are two methods of reproducing cells,-direct division or amitosis, and indirect division or karyokinesis. In amitosis, the mother-cell simply increases in size and finally divicles directly into two daughtercells after a division of the nucleus. In karyokinesis, the process is more complicated, consisting in a cycle of definite changes involving first the nucleus and then the cell-body. The latter mode of division is the most common.

The faculty of reproduction is not possessed by all the tissues in the same degree. Ganglion-cells are rarely reproduced, if at all, in the adult, and many glandular epithelial cells have a limited power of reproducing themselves; whereas the surface epithelium, the epithelial cells in the lining of ducts, and particularly the connective-tissue cells, have great power 
of regeneration. Connective tissue is prone to fill the spaces not occupied by other cells, and this is a marked picture in pathological processes.

In pathological repair, the new tissue takes its origin from the adjacent tissues of the same kind if the lesion is slight; but in chronic abscesses and cases of severe wounds and burns, etc., with extensive loss of substance, granulations form which are ultimately replaced by cicatricial or connective tissue. This imperfect partial regeneration forms a scar.

When the normal processes of repair are interfered with by mechanical or chemical agents, or particularly by the invasion of micro-organisms, an inflammatory process sets up. The granular tissue by which repair was being effected at once shows marked changes. If infiltrated with a serous or fibrinous fluid, then an exudative inflammation is the result; if leucocytes predominate, then it is a purulent inflammation. In all cases the pure healing process is stopped until the disturbing cause is removed.

DIRECT AND INDIRECT HEALING.-Every wound heals by the formation of new tissue. If this is microscopic in amount, as in a perfectly coaptated wound, direct or primary union is effected, and there remains no visible scar. This is also known as healing by "first intention." Indirect or secondary healing occurs in wounds which have suffered an extensive loss of substance and therefore require the formation of a large amount of new tissue. This can readily be seen with the naked eye. In some cases it may be necessary to hold in contact two granulating surfaces until they fuse together, sometimes called healing by "third intention."

\section{SIMPLE REPARATIVE PROCESSES.}

The pathological conditions to be repaired usually consist of wounds, infections, or some organic lesion; and the repar- 
ative process involves not only the making of new tissue cells, but also the removal and absorption of the old. If the body has been invaded by foreign substances, organic or chemical, these also must be removed.

The body possesses two principal means of disposing of the tissue-debris and foreign invaders; the leucocytes of the blood and certain fixed cells have the power of ingesting foreign substances, while the serous fluids of the blood and tissues can wash them away through the lymphatics into the general circulation. To this end the invasion of the body by any foreign agent excites the circulatory system to summon its means of defense to the injured part.

The changes ushered in by the processes of repair are usually included among inflammatory processes. Instances of simple destruction on the one hand, and simple regeneration on the other are difficult to obtain. The injury to the tissue is usually so great as to incite marked vascular changes and these accompany every reparative process. They are prompted by the irritating factor in the cause of the injury, whether mechanical, chemical, thermal or bacterial, and vary in degree according to the amount of irritation present. The muscle-cells and endothelium of the vessel-walls, and the corpuscles of the blood-stream are stimulated by the products of irritation to which they respond somewhat as follows in the process of healing:

I. Dilatation of the Vessels.-This is most marked in the arterioles and is also present in veins and capillaries. The vessels may increase one-fourth larger than normal.

2. Retardation of the Blood Current.-The axial current of the stream becomes broader, leucocytes fall out into the marginal stream, rolling slowly along. Stopping here and there they finally come to a standstill, lodging in the wall of the vessel. 
3. Oscillation.-Contents of the vessels cease to move, and sway back and forth.

4. Stasis.-There is now no movement of any kind; thrombosis may occur.

5. Emigration.-The leucocytes work their way through the vessel wall, by means of their active amœboid processes. Red cells in fewer numbers escape through spaces between the endothelial cells of the capillaries.

6. Exudation.-Owing to the increased permeability of the vessel wall, variable quantities of the serous fluids escape ; !ater a plasma-like fluid may accumulate, and fibrin may be precipitated. The greater the damage to the vessels the more nearly the exudation approximates that of the blood, and the greater the number of corpuscles it contains.

The emigrated cells and exuded fluids at once begin their work of repair. Both red and white corpuscles at first remain near the vessels from which they have escaped, but they are soon pushed away by other corpuscles or washed on by the escaping fluid. The white corpuscles have in addition their own peculiar power of locomotion, stimulated and directed by the irritating substance which caused the injury to the tissue. Hence ultimately, they may be found far from their point of egress from the vessels. The leucocytes and also certain fixed tissue cells take up into their own bodies whatever foreign substances they find in the area of irritation. In this way the bacteria, chemical irritants and tissue debris are disposed of while the extded fluids aid in washing all away into the lymphatics. Any unremoved blood-corpuscles or fibrin later undergo fatty degeneration, and thus complete removal of the irritating products is eventually brought about.

The tissue cells which have been destroyed are replaced by multiplication of the surviving cells, epithelium by epithelium, muscle by muscle, nerve by nerve, etc. The stasis 
in the blood-vessels gives place to a renewed circulation, the corpuscles of the stagnant blood one by one moving off wntil a slow stream is established through the inflamed area. The stream quickens, and the normal contraction of the vessels is restored. Thus the heat and swelling of the injured part are reduced, and finally complete repair takes place.

The foregoing processes are characteristic changes which accompany the healing of slight wounds only; in case of severe wounds or deep cuts with the surgeon's knife, much tissue is destroyed and there is usually an abundant hemorrhage into the part, which forms a clot temporarily filling up the wound. The repair which follows is in essence the same as takes place in slight injuries, only much more tissue has been destroyed and therefore much more must be regenerated.

HEALING OF AN INCISED WOUND.-In case a large amount of tissue has been destroyed, as would occur in an incised wound, or in an extensive surgical operation, the gap in the tissue fills up with clotted blood, which forms a temporary bridge between the divided parts and makes a path for the regenerating tissue cells as soon as they begin to multiply. The endothelial cells of the vessels, and the old connective tissue cells in the parts adjacent to the edges of the wound throw out protoplasmic processes and invade the blood-clot. New connective tissue cells are formed from the old, which possess independent locomotion or ameboid movement, and they move about in the fibrinous meshes of the clot, throwing out a fibrous stroma until finally a firmer bridge of young connective tissue is formed. Simultaneously new capillaries sprout from the old, and nourish the newly formed connective tissue, or granulation tissue so-called. This granulation tissue is characterized by many different varieties of cells. The proliferating connective tissue cell is present in all 
stages of growth. The youngest forms are small, round cells with a large, deeply staining nucleus and a small rim of protoplasm. As the cell increases in size it takes on more body, its nucleus becomes vesicular, and we have the socalled epithelioidal cell. These throw out protoplasmic processes and become typical fibroblasts, the immediate antecedents of the connective tissue fibres. There are many other cells present in granulation tissue, which may be summarized as follows :

I. Giant-cells.-These may be formed by repeated division of nuclei in the same cell, or by the union of several cells. Giant-cells usually attend any new formation of tissue. They are oval in shape, from 50 to Ioo $\mu$ in diameter, and contain many nuclei scattered throughout their protoplasm.

2. Plasma cells. - The protoplasm of these cells is usually distinctly granular, and sometimes consists wholly of cosinophile granules or of fine granules taking a dark stain. The nuclei are dark, circular, and often multiple. Origin not definitely known.

3. Leucocytes.-Lymphoid cells and polymorphonuclear leucocytes are usually present in large numbers.

4. Mast-cells.-These cells are characterized by small spheric granules which stain deep blue.

Eventually the granulation-tissue cells are replaced by connective tissue fibres which form more or less of a scar or cicatrix. This is the result of the new tissue becoming more and more dense, and undergoing shrinkage until finally the blood vessels are crowded out, the cellular elements are decreased in number and a firm mass of contracted fibrous tissue makes up the cicatricial scar.

Sometimes the granulation tissue forming is of such a low type that it never becomes adult tissue. This is true of the tubercles of tuberculosis and glanders, which are 
largely composed of small round cells, the youngest form of connective tissue cells.

\section{REGENERATION OF THE INDIVIDUAL TISSUES.}

One law holds good for the regeneration of every kind of tissue. "Omnis cellula e cellula ejusdem generis." This law was first formulated by Bard and is universally applicable. Muscle is formed from pre-existing muscle-cells, but it cannot produce nerve. In the same way connective tissue will produce any of its own varieties, but it never becomes epithelium, and vice versa.

The blood-vessels are almost the first to regenerate. for if the new tissue forming is not nourished, it will soon degenerate. The endothelial cells of the old capillaries send out protoplasmic processes into the surrounding tissues. These processes meet and unite into endothelial tubes which eventually become hollowed out as the blood begins to circulate and push its way through the capillary system. The vessels probably acquire their adventitia from the adjacent connective tissues, but the origin of their muscle fibres is not definitely known.

CONNECTIVE TISSUE.-The regeneration of connective tissue is by far the most frequent in the body, for it enters into the organization of every kind of tissue that has become useless or destroyed. Its proliferation occurs not only in replacing connective tissue, but in replacing tissues and organs which have not the power of reproducing their own specific cells. It occurs also as a secondary process after a number of inflammatory conditions. It is characteristic of old age. If the parenchymatous organs of old animals, the liver, kidney or spleen, and also the heart are examined microscopically, they will be seen to contain a large amount of connective tissue. In animals that have been worked in 
harness, especially horses, the places that have been rubbed by the harness, the traces, the collar, etc., show a marked increase of connective tissue in the skin.

Connective tissue can be formed only from pre-existing connective-tissue cells and from the closely related endothelial cells of the vascular and lymphatic systems. The newly-formed connective-tissue cells possess the power of moving about. They work their way into fibrinous networks, into thrombi, tumors, and minute cavities of foreign bodies. They must not be confounded with the leucocytes which also have amoboid movement. They are larger than the latter and have a round or oval, vesicular nucleus instead of the dark polymorphous nucleus of the leucocyte. Aside from the amoboid properties of these formative cells, they also possess the faculty of taking up corpuscles and foreign bodies into their protoplasm. They are phagocytes. Metchnikoff, the discoverer of phagocytosis, called them "macrophages" on account of their size. The equally phagocytic leucocytes he designated "microphages." Corpuscles and foreign bodies of every description, both such as enter the organism from without, and such as are formed by dead cells within, may be taken up by phagocytes.

The first changes observed in proliferating connective tissue consist in the enlargement of the pre-existing adult cells. Larger, shorter and thicker forms develop, which have polyhedral protoplasmic bodies with several processes. The nuclei are oval, or round, and vesicular. These are the socalled formative cells or "fibroblasts." They are connectivetissue builders. On account of their amœboid movement, their forms are often very bizarre. Some are large, round, or oval masses of protoplasm like epithelioid cells; others roughly star-shaped; and others irregular with long processes or bundles of fibres at the ends as if the protoplasm had been frayed out. The epithelioidal forms are probably 
younger cells and direct antecedents of the more matured fibroblasts. These formative cells have received various names depending upon the kind of mature tissue they are intended to form-if fibrous tissue, fibroblasts; if cartilage, chondroblasts; and if bone, osteoblasts. As they continue proliferating, the old fibrous intercellular substance disappears and the new tissue becomes soft and yielding like embryonal tissue. The cells often overlie one another and may exhibit an "epithelioid" arrangement. As soon as the defect in the tissue has been made good, the fibroblasts begin secreting and storing a stroma-forming substance and their protoplasmic processes break up into small fibrils. Ultimately their cell bodies do likewise, forming small bundles of fibrils arranged in parallel rows. They thus lose the greater part of their protoplasm and finally return to the adult form of connective tissue cell, the small narrow cell with a dark spindle-shaped nucleus. The intercellular substance becomes more fibrous and the new tissue gradually approximates that of normal tendon, fascia, skin or whatever the form of connective tissue may be.

The method of repair varies somewhat according to whether a blood clot is or is not present in the wound. After division of a tendon, if no blood-clot be present, the sheath simply collapses, becomes adherent to the tendon stump and new tissue immediately is formed by the cells of the sheath and cut tendon. When a blood-clot is present, the method of repair is not so simple. Large numbers of leucocytes first infiltrate the clot and the ends of the cut tendon degenerate, becoming soft and succulent. But in a few days, the cells of the sheath and tendon begin to proliferate, new capillaries are formed and a mass of granulation tissue replaces the clot, extending some distance into the ends of the tendon. Eventually the new vessels in the granulations join those of the tendon, the embryonal cells develop into the adult form, 
and the tendinous tissue becomes completely regenerated. The sheath usually remains slightly adherent at the point of section.

ELASTIC FIBRES.-The formation of elastic tissue is a slow process. In wounds of the skin, the regeneration of elastic elements does not begin for four or five weeks. They are always formed from the old elastic fibres which begin sending out little delicate twigs from their sides, not from their ends. These gradually grow toward one another and finally unite to form an interlacing network of small elastic fibrils. It sometimes takes years before they are of normal size. The fact that elastic tissue is so slow in healing has a marked influence in the repair of some abscesses. In pollevil, the ligamentum nuchæ takes so long to granulate that often the abscess is slow to heal; a necrosis of the surrounding tissues and a purulent inflammation of the bursa may baffle the best treatment.

FAT.-Formative tissue, fibrous connective tissue or medullary tissue of bone may give origin to fat cells. The nucleated protoplasmic remains of former fat cells divide ind multiply into large, round, mono- or multi-nuclear cells - the fibro-blasts. These develop within the membrane surrounding the original fat-cells (Dürck) and there manufacture the globules of fat.

The mature connective tissues possess different degrees of regenerative power. It is most marked in ordinary fibrous connective tissue, in periosteum and in the medullary tissue of bone; whereas cartilage and bone substance proper possess but little ability to form new tissue. If they. are repaired, it is by proliferation of the cells of the periosteum or of fibrous connective-tissue; otherwise the defects are filled in with scar tissue. The connective tissues are able to change from the one to the other by a change of the intercellular substance only. This is called metaplasia. Cartilage may 
change into bone, fibrous tissue into cartilage, etc., by a transformation of the matrix in each case. (Hektoen.)

CARTILAGE.-The formative cells or chondroblasts generally originate in the periosteum or perichondrium, the medulla of bone, and sometimes in fibrous connective-tissue. The cells secrete a homogeneous intercellular substance which gradually encroaches upon them and they become compressed into small spaces. This substance may remain hyaline, or it may develop white or elastic fibres in its matrix. Cartilage does not easily regenerate. Some deny that it does at all. More often wounds of cartilage are repaired through the formation of fibrous connective tissue which forms a scar.

BONE.-Osseous tissue undergoes as perfect a regeneration as any tissue of the body through the calcification and subsequent ossification of an embryonal tissue known as callus. The formative cells or osteoblasts arise from the periosteum, perichondrium and bone-marrow. These grow into the blood-clot which forms in a fractured or amputated bone, and produce a large mass of granulation tissue, the callus. This is purely connective tissue at first, but later changes into cartilage and then into bone, by metaplastic processes similar to those in the original formation of bone. The callus forms a temporary bridge to hold the broken fragments together until the process of cicatrization in bonytissue takes place.

As early as the second day after a wound in osseous tissue, the lacerated periosteum, muscles and blood-clot are infiltrated with leucocytes. At some distance from the immediate neighborhood of the fracture, the cells of the periosteum, especially of the osteogenic layer, swell up and multiply. Numerous angular and stellate cells, the osteoblasts, appear and at the same time an active proliferation of connective tissue cells begins in the mequl- 
lary cavity. The blood-vessels also take part in the process and a granulation tissue very rich in cells is formed at the ends of the bony fragments and gradually replaces the bloodclot; in this way a provisional union takes place. This soft tissue eventually becomes a fibrous mass poorly supplied with nuclei and extends some distance above and below the seat of fracture. It is the callus. It does not have a well-defined outline and may involve some of the surrounding tissues. In a few days the inflamed mass begins to take on much firmer consistency and if examined during the second week of repair, there is found a thick layer of cells embedded in a finely striated intercellular substance somewhat like cartilage. This is the so-called osteoid tissue, especially common in dogs near the ends of the bones and also in the center of the callus. The intercellular substance has a hyaline appearance and in the early period of repair consists largely of cartilage.

The cartilage cells now form a kind of hard secretion impregnated with calcium salts which gradually surrounds them and incloses their bodies in the ground substance. Presently it is found that trabecula of bone have formed, and the cartilage cells, now shrunken and spindle shaped, have become typical bone-cells, sending out wavy processes into the bone substance. Between the trabeculæ are spaces containing blood vessels. This new spongy bone begins forming some distance from the seat of the fracture, gradually growing thicker as the ends of the bones are approached. As it grows out from each end of the bone, it invades the cartilaginous callus and finally the two developing ends grow together making a bony bridge which unites the fracture. At first the whole mass of callus fills up the medullary cavity and the space between the fractured ends of the bones, and can be differentiated into its periosteal and myelogenic layers. It produces a marked thickening of the bone from an exces- 
sive deposition of tissue, but that is absorbed later through the action of the osteoclasts. These are multinuclear giant cells situated along the bone-trabeculæ. They perform the same function in the repair of bone as in its development, viz: the removal of superfluous tissue both in the periosteum and medullary cavity.

The periosteal callus may ossify directly through the action of the osteoblasts, the preliminary cartilaginous stage being dispensed with. They excrete the calcareous salts which surround them and ultimately convert them into bone corpuscles.

In from fourteen to twenty-one days, callus-formation has fully begun; twelve or fourteen days later, i. e. during the fourth or fifth week, ossification occurs; and at the end of one or two months, a mass of spongy bone occupies the seat of fracture. The medullary cavity sometimes takes years to become patulous. The amount of callus varies greatly in different cases. In animals that are allowed to run about during the process of repair, the two ends of the bone are imbedded in a luxuriant callus which involves a considerable portion of the shaft and this is sometimes impossible to suppress.

Sometimes there appears to be an inability on the part of the bone-producing structures to form new bone. The granulation-tissue is either absorbed or becomes fibrous; no new bone is thrown out, and as a result of this we get a non-bony union. The two ends of the bone are united by a ligamentous band. In some cases nature attempts to form a new joint; the fractured ends become covered with hyaline cartilage, and are held together by a capsule, which contains a small amount of clear serum. This condition is known as pseudoarthrosis.

The regeneration of fractured and diseased bones varies greatly among the domestic animals. This may be due to 
differences in the anatomy of their various bones and joints, but in all probability the specific use to which an animal is put, plays the most important part. The process is always more or less hindered by imperfect immobilization of the part.

MUSCLE.-Of the three kinds of muscle tissue, striated voluntary muscle has the most marked power of regeneration. It is formed from the old muscle fibres only, and never from connective tissue. It can take place independently of any nerve supply. After an injury of striated muscle, the broken ends necrose and become homogeneous irregular clumps. The nuclei in the adjacent portions begin to multiply and form chains, which accumulate in the swollen ends of the old fibres presenting an appearance closely resembling giant-cells. These are the "muscle-buds" of Neumann. They are not always single; in some places several buds may be seen coming from one fibre. The new muscle fibres develop from these multinucleated masses, which increase in size and length, gradually presenting a longitudinal striation and then a transverse. As the fibres grow, they lose their parallel arrangement and become entangled with one another. The nuclei which at first occupy the middle of the fibre later on take up a position nearer the surface and that at further intervals apart. At this stage a new envelop or sarcolemma is formed, probably from the surrounding connective tissue. This has developed simultaneously with the muscle, and if the wound be large, has formed a connective tissue cicatrix, which the rewly formed fibres gradually invade from opposite sides of the wound, and, if possible, meet and interlace with one another. In this way the connectivetissue scar disappears, unless the wound be too large for the new muscle to span. In time the great irregularity in the direction of the fibres changes, and they become parallel as in normal muscle. 
The regeneration of heart muscle is very limited; wounds practically always heal by means of connective-tissue scars, and the continuity of the contractile substance is never restored. Involuntary muscle may be reproduced to some degree after traumatic and other necroses; but wounds in the alimentary and urinary tracts are largely healed by scartissue. In the muscular layer of the uterus, where the organ increases in size from ten to twenty times during pregnancy, there are very few new muscle-fibres formed, but the individual fibres undergo a marked increase in size,-hypertrophy. These are normally about I-IOOO to I-50O of an inch in length, while during pregnancy their length increases from $I-25$ to $\mathrm{I}-75$ of an inch. There is also a corresponding increase in the width. The blood vessels also show an increase in the size of their muscle fibres.

Some animals possess a more marked degree of muscle regeneration than others. In man this occurs only after. a slight injury of the muscle. In the horse, complete and very extensive atrophies may manifest a marked tendency to regenerate and resume their normal constitution and function. The process is generally slow but it is certain. Examples are found in the crural atrophy of azoturia and the shouldder atrophy of "shoulder-slip" so-called.

NERVOUS TISSUE.-The peripheral nerves are capable of extensive regeneration. When a nerve is divided, it is invariably followed at once by degeneration of the entire peripheral segment and of a variable portion of the central segment, sometimes as far as the sixth node of Ranvier. Not infrequently single fibres degenerate for long distances toward the centre, even though surrounded by intact fibres. This degeneration is characterized by disintegration of the axis-cylinder and medullary sheath. The axis-cylinder first swells up, presenting small villous dilations along its sides, and these break up into small fragments. Secondarily, the 
myelin sheath also breaks down into small fragments and particles, and the nuclei of the sheath of Schwann begin an active cell proliferation. They swell up and seek the centre of the nerve fibre among the fragments of myelin, forming small cellular tubules at various intervals apart. Regeneration begins long before the retrogressive changes are complete, and is accomplished by an outgrowth of the axiscylinder of the central end, which splits up into several small fibrils, often spiral, and sometimes one coiled within the other. These early receive a covering of delicate medullary sheaths, and may enter the old sheaths of the peripheral portion of the nerve and there complete their structure. However, they may also extend into the adjacent tissue. This is more likely to occur if much scar-tissue has formed in the healing of the wound, the cicatrix preventing the downward growth of the fibres.

The cells of the sheath of Schwann are connective-tissue cells and their functions in the regeneration of nerve seem to be phagocytic, rather than forming a part of the neurones proper. (Hektoen).

When a section of a nerve is removed or when a limb is amputated, the axis-cylinders may permeate the scar tissue of the wound in every direction and form a very sensitive scar which has been erroneously included among the true tumors. In human surgery it is designated as "amputation. neuroma." The so-called neuroma following neurotomy of the plantars is an example of this regenerative process. It is an unsuccessful effort to restore the function of the cut. nerve. The axis-cylinders, in their effort to grow into the unnerved area, become lost in the intervening cicatrix.

The regeneration of nerves has been the subject of numerous clinical and experimental observations which have yielded a fairly accurate knowledge of the process. It is now. well known that a divided nerve trunk will slowly re-estab- 
lish the innervation of the part by the outward growth of its axis-cylinders, providing, however, the growth is not hindered by loss of substance or obstructed by cicatricial tissue. That is to say, if a nerve trunk is divided and the ends are immediately brought into perfect apposition, the axis-cylinders will rather rapidly grow down the old channel. It is even claimed that the new axis-cylinders appropriate some of the elements of the old ones as they advance toward the periphery. Functional restoration under these most favorable conditions may occur after several weeks or several months, but the function is not perfect even after several years. The sensation is often blunted for life and the muscular activity, if not assisted by compensatory augmentation of other muscles, will remain rather imperfect for a long time, if not forever.

When a part of a nerve trunk, e. g. one-half to one inch, is removed,perfect anatomical union and physiological restoration do not usually occur. The descending axis-cylinders are blocked by the scar or are lost while wandering into the surrounding tissues; a few of them may find the old channel but they will establish only a very imperfect innervation of the unnerved area. It has been shown by experiments that if the lost piece is replaced by a similar piece, even though taken from another animal, it will serve as a guiding channel for the growing axones, and thus direct them into the old trunk. Pieces of tubing, bone, rubber, etc., have been used for the same purpose but with less favorable results. Thus Cluck implanted a short piece of the sciatic nerve of a dog into a corresponding breach of the same nerve in a rabbit and found that the unnerved part responded to chemical and mechanical stimulants after eleven days. Unfortunately the degree of the re-established sensibility and motion is not mentioned. Tillman succeeded in restoring the function of a grafted nerve. In all of the experimental observa- 
tions in which the function of the nerve was restored the conditions for growth were always the best. No part of the nerve trunk was destroyed. The solution of continuity was artificially restored at once.

Quinn and Desperes claim to have restored the function of the median nerve of an animal by grafting it to the ulnar. After making this experiment they report that the "use of the foot was recovered." This experiment becomes somewhat inconsistent when it is known that the median nerve of animals at any available point of operation is purely sensory, and that veterinarians repeatedly divide this nerve surgically to relieve pain in the articulations below. The median nerve of animals has motor fibres but not at any point where it could be successfully grafted to the ulnar. It can be cut with impunity far above the elbow, without impairing the function of a single muscle. The fact is, these learned experimentalists grafted two sensory nerves to each other, both of which might be cut without disturbing the use of the leg.

In a word, cut nerves appropriately approximated will promptly unite anatomically and will slowly resume their function by the downward growth of their axis-cylinders. while cut nerves not appropriately approximated resume their function very imperfectly or not at all, according to whether or not the axis-cylinders meet with any obstacle in finding the old channel, which is indispensable to the re-innervation of an area unnerved by the division of a nerve trunk.

EPITHELIUM.-New epithelium must spring from pre-existing epithelial cells and the regeneration is usually quite complete. The phenomena occurring immediately after a wound are very similar to those which take place in the reaction of other tissues to injury. The epithelial cells immediately bordering the wound, die and degenerate, while the cells in adjacent parts swell up and multiply (both by direct 
division and also by karyokinesis), and ultimately push their way over the gap in the tissue which has been filled up with granulations. Comparatively few epithelial cells are requisite to cover defects that originally were very large, for the contraction of the granulation-tissue lessens the superficial area of the wound.

The multiplication of cells takes takes place chiefly from the deep layers of the rete Malpighii, but if the whole thickness of the skin has not been destroyed, the epithelial cells of remnants of glands and hair-follicles may give rise to the new epithelium, little islets of new epithelium being scattered over the granulating surface, a so-called "insular" epidermization.,

Cylindrical epithelium is usually accompanied by a change of form when regenerating. The new cells are lower and more squamous in form, but later assume their normal shape. In the intestine, the surface cells are replaced by proliferation of the cells in Lieberkühn's glands.

The appendages of the skin, the hoofs and hair, are restored if sufficient portions of the matrix or root remain from which the new tissues may grow. In large wounds of the skin which heal with a cicatrix, the hair and glands are not restored.

As regards the regenerative power of the glandular epithelium, opposite conclusions have been drawn by various investigators, some claiming that glands will readily repair their parenchyma, while others directly deny this. Experiments with lower animals seem to confirm the first conclusion, while practical experience suggests the opposite as the more usual result. According to Dürck, true regeneration of glandular substance capable of functionating probably never takes place. The reparative process consists in simple scar-formation when a liver, kidney, ovary or testicle is to be healed. The cicatricial tissue varies slightly in the different 
glands. An old liver-scar may contain cyst-like cavities surrounded with epithelium somewhat like liver cells, while a scar forming in the kidney may show tubular formations.

Certain compensatory changes may occur if a large portion of a gland is removed or if one of the kidneys is removed: the remaining portion or gland undergoes hypertrophy, the cells increase in number, showing marked karyokinetic division of nuclei, so that a loss of volume may be completely made good in a comparatively short time.

HOOF.-Hoof, horn and claws are products of epithelium. They proceed from a matrix that is rich in cells having a special function to perform-that of secreting the insensitive hoof, horn or claw fibres. This matrix is analogous to the hair follicle which performs the function of secreting the hair. If a part of a hair follicle remains intact the remaining cells will immediately proceed to reproduce a new hair, and if a part of the hoof, horn or claw matrix is left in any given part the fibers for a new insensitive organ (hoof, horn or claw) will soon be reproduced by the same genetic process. That is to say, hoof, horn or claw tissue will promptly regenerate by the outward growth of new fibers from the genetic matrix, so long as the latter is not destroyed. When there is loss of matrix in whole or in part, it, too, is capable of regenerating, but the quality of fiber thereafter produced may be defective. Thus, if the coronary cushion of a horse or ox is partially destroyed, the wall of the hoof proceeding from the regenerated matrix will be abnormal. If a portion of the sensitive laminæe is destroyed by disease or injury, the wall will never again be perfectly united to the subjacent regenerated laminæ.

The hoof of the horse is divided into wall, sole and frog, all of which are secreted by a highly organized matrix-the keratogenous membrane,-which occupies the confined position between the hoof and the bones. This genetic structure 
is divided anatomically into the coronary cushion, which forms the wall, the sensitive laminæ, which forms the insensitive laminæ, and the velvety tissue, which forms the sole and the frog. The coronary cushion which occupies the superior border of the hoof is studded with papillae, each one of which produces an individual wall-fibre. If all of the wall is removed these papilla will reproduce it in its entirety in about eight months. At the heels the distance between coronet and sole being short the reproduction is complete in eight weeks to three months. When the coronary cushion is divided transversely from disease or injury and the wound heals by second intention the scar tissue which connects the separated ends will contain a dearth of genetic cells and the wall fibers proceeding from them will be so defective in quality and often in quantity as to produce a seam in the wall of the hoof that will vary in character according to the amount of scar tissue that was required to heal the breach. This scarcity of hoof-producing cells in the scar may however not be permanent, as the regenerating process in the scar tissue may continue month after month, and even for several years with the result of again bringing the wall back to its normal condition. Such seams of abnormal hoof in young horses may entirely disappear after several years through the gradual resumption of the normal function of the coronary cushion. More often, however, after severe injuries, an indelible blemish remains.

When the sensitive laminæ are destroyed over a consid. able area, their function of uniting the wall to the bone is never entirely restored although the defect may not be noticeable from without. No matter how trivial is the loss of tissue in this structure, the accordion-pleat arrangement is never restored. An irregular arrangement of the pleating always results and often the surface remains quite smooth and a considerable space intervenes between it and the 
wall. The condition known as "seedy toe" although usually due to pre-existing inflammations of obscure origin, is a fair sample of the condition produced from traumatic lesions that destroy large areas of the sensitive laminæ.

The scar tissue replacing the lost laminæ is, however, always replete with hoof-producing cells that are even more active in hoof-production than the original ones. The hoof producing quality of new laminal tissue is a striking example of augmented cellular activity. The amount of hoof produced is often exuberant, especially when the membrane is not destroyed as deep as the periosteum. And even when the bone is laid bare over a considerable surface the regenerative propensities of the region are always strikingly exemplified. When a large portion of the wall is destroyed this membrane repairs the breach by coating it over with a rather firm, thick, horny substance, pending the growth of the new wall from the coronary cushion, which permanently covers the defect in time.

In a word, the regenerative powers of the sensitive lamina are great, but the anatomical arrangement of the pleats is never perfectly restored.

When the velvety tissue is destroyed it is much more prone to resume its normal anatomical and physiological condition than the coronary cushion and sensitive laminæ. In fact, most any breach will be replaced by a very active horn-producing tissue that will almost immediately secrete a normal sole or frog. Sometimes the new sole is somewhat spongy, but this only for a very brief period. Within several weeks it can not be distinguished from the original organ. The entire anterior two-thirds of the frog and its matrix may be surgically removed and then be entirely restored to its normal condition, and that within the brief period of two or three months, or even less.

Hoof-producing cells always abound in the granulation 
tissue of any wound adjacent to the hoof of solipeds. During sclerogenesis of wounds about the feet a considerable amount of modified horn tissue is always produced and the result is the "horn cicatrix" so frequently seen after the barbed wire wounds of horses' pasterns. This example is a striking illustration of the hoof-producing powers of the region. 


\section{CHAPTER II.}

\section{INFLAMMATION.}

DEFINITION.-Inflammation is not a simple process. Many pathologists have ceased to define it. It is now associated with such a complex series of symptoms that it is no longer an entity. According to Stengel, inflammation is a series of changes which occur in living tissue, which are designated the vascular, exudative, degenerative, and regenerative as a result of irritation by chemical, mechanical, or thermal agents, - the most common being toxic, due to the products of pathogenic bacteria. In other words, inflammation is the reaction of tissue to injury. Clinically, the phenomena of inflammation, as expressed by Galen, consist of tumor (swelling), rubor (redness), calor (heat), and dolor (pain), to which has been added functio læsa (impaired function). Its study not only includes the acute processes caused by bacteria, other fungi and protozoa, but the remote changes which occur as secondary reactions. Hence it will be seen that a large part of special pathology is included under this heading. Even some pathologists believe that tumors are of this origin. Malformations can hardly be excluded, for most of them are due to injurious agents acting upon the embryo and influencing or preventing development. In this chapter we shall attempt to give only a general description, principally microscopical, of the most common conditions found in domestic animals which are produced by either pathogenic bacteria or other injurious agents.

ETIOLOGY.-Bacteria are by far the most common cause of inflammation. The mere presence of these small vegetable cells in the tissue produces little or no change; but 
the injury to the tissue is brought about by their poisonous products-toxins. These toxic substances are definite chemical bodies which differ greatly in the different species of bacteria. They consist of toxalbumins. It is not always necessary for the bacteria to be present in order to produce their lesions or diseases. They can be grown upon artificial culture media and their toxins can be separated out by a process of filtration and injected into an animal, and the same symptomalogy and changes in the tissue will occur as if the bacteria were allowed to multiply and produce their toxins in the tissue themselves.

Many bacteria have a selective action, i. e., they have an affinity for certain tissues of the body. For example, the toxin of tetanus bacilli have an affinity for brain tissue and no matter where they gain entrance into the body, their toxins must reach the nerve cells before they can do any harm. Bacillus coli communis has an affinity for the gastrointestinal tract; anthrax, for the blood; the tubercle bacillus, for the lymph-glands; the virus of hydrophobia, for nervous tissue; while other bacteria may act principally on the parenchymatous organs - the liver, spleen and kidney. In the human, the gonococcus has a great affinity for the urethra, the diphtheria bacillus for the throat; while on the other hand, such bacteria as streptococcus and staphylococcus will produce their lesions in any part of the body. Staphylococci will make an abscess in the withers as readily as in the kidney. The streptococci may produce a skin-lesion, or an inflammation of the uterus, or a general infection of the blood, -septicremia. There are also certain non-pathogenic bacteria, whose products-ptomaines-may set up indirectly a slight inflammatory process.

There are other factors which influence a bacterial inflammation besides the bacteria themselves. These we call predisposing causes. First is susceptibility; an animal's tis- 
sues must have the power to combine with toxin before the inflammation can be produced. Many animals possess a marked resistance to bacterial invasion. This must be lowered before infection can take place. Second, virulence of bacteria. Bacteria cannot produce disease unless they are in a very active and virulent condition, and usually a large number of them gain entrance to the body at the same time. Third, a previous or concurrent injury. The tetanus bacilli are usually carried in with a nail, the staphylococci enter through some abrasion, etc. As soon as there is an abrasion of the skin or injury of some tissue, the way is opened for bacteria to enter the body and set up an inflammatory process.

Inflammations due to traumatic, thermal and chemical injuries are completely dealt with in chapter on Traumatism.

CLASSIFICATION.-The classifications of inflammation are various. In general we may classify them as acute and chronic. The acute form is rapid in onset, of short duration, and has a tendency to produce pus in the tissue. Streptococcus, staphylococcus and bacillus pyocyaneus enter largely into this form. Chronic inflammation is insidious in onset, protracted in duration, and characterized by progressive lesions in the tissue; ; it shows little tendency to recover. Fistulæ and ulcers are examples of chronic inflammations. They heal with the formation of cicatricial tissue, or a scar.

Inflammations may be further classified according to their location in the tissue, and the kind of irritation produced during the reaction:

I. Parenchymatous, involving largely the essential functionating cells of an organ, as in anthrax.

2. Interstitial, affecting the supporting connective-tissue frame work, or stroma, of an organ; found in chronic conditions.

3. Catarrhal, affecting the mucous membranes with a 
superficial exudation of leucocytes: it is found in bronchitis.

4. Desquamated, characterized by a desquamation, or casting off of the epithelium, as in dermatitis.

5. Vesicular or herpetic, characterized by the formation of small vesicles or blisters filled with serous fluid: it is found in herpes.

6. Papular, forming little hard elevations or nodules, as in equine syphilis.

7. Pustular, having small elevations filled with pus; found in cow-pox.

8. Diphtheritic or croupous, the surface of mucous membrane covered with a pseudo-membrane, made up of fibrin, leucocytes, and degenerated cells. The specific cause may be the diphtheria bacillus or the streptococcus. It is found in fowl diphtheria.

9. Ulcerative, characterized by ulcers with a prolonged suppuration, necrosis, or gangrene.

Io. Degenerative, accompanied by an unusual amount of disintegration of tissue, as in tuberculosis with mixed infection.

I I. Hemorrhagic, associated with hemorrhages into the tissues; found in purpura hemorrhagica.

12. Gangrenous, associated with gangrene.

I3. Suppurative, when the process culminates in suppuration, as in abscess.

I4. Phlegmonous, when cellular tissue becomes infiltrated with pus; found in some forms of cellulitis.

I5. Specific, one caused by definite known bacteria such as symptomatic anthrax.

The foregoing classification may be simplified by considering all the various forms under four divisions: (I) Exudative, characterized by serous, fibrinous and hemorrhagic discharges; (2) emigrative or purulent, associated with emigration of certain corpuscular elements of the blood into the 
tissues-the polymorphonuclear leucocyte, the lymphocyte, the eosinophile and the mast-cell (the phagocytes) (by the older authorities including Cohnheim and Metchnikoff, emigration is considered the principal phenomenon of all infiammations); (3) productive, a form of inflammation in which the proliferation of new cells is the main characteristic; (4) specific or infectious granulomata, special inflammatory growths due to specific micro-organisms, not only bacteria, as the glanders bacillus, but other fungi also, the ray-fungus, botryomyces, etc. In nearly all these infectious granulomata, the lesions are focal, the course is sub-acute or chronic, resulting in circumscribed collections of granulationtissue. Granulomatous tumors usually continue to enlarge as long as the micro-organisms continue to vegetate, and when they die, secondary retrogressive changes take place in the granulomata. Another marked characteristic of these tumors is that the change produced in the tissue is so specified that in most cases it is possible to make a diagnosis by a microscopical examination of the tissues without isolating the parasites. Under this form of inflammation may be included tuberculosis, actinomycosis, botryomycosis, glanders, and farcin du boeuf.

\section{ANATOMICAL PROCESSES OF INFLAM- MATION.}

These consist of four distinct phenomena on the part of the tissues, viz: the changes which take place in the vessels, the emigration of the leucocytes, the inflammatory exudates and the changes in the fixed cells. These phenomena are the same in essence as occur in every reparative process.

VASCULAR CHANGES.-Marked changes in the blood-vessels take place at the beginning of an inflammation in vascular tissue. This is largely due to the stimulating action of the irritant upon the vaso-motor nerves. However, 
we may have all the vascular changes in an acute inflammation without the intervention of the nervous system. When the irritant is sufficiently toxic to affect the blood-vessels, a momentary spasm of the vessels is noted, together with an acceleration of the blood current. This is immediately followed by a dilatation of the capillaries and arterioles accompanied by a marked retardation of the current of blood. In severe inflammations, degenerations occur in the wall of the blood-vessels which increase their porosity. The endothelial cells lining the blood-vessels and lymphatics are not only contractile, but according to Metchnikoff, are phagocytic and mobile. As the current of blood slows down, the corpuscles pass into the periphery of the stream and the axial character of the current is lost. Normally the corpuscles are carried in the central current of the circulating blood, while the marginal zone consists wholly of plasma. As the leucocytes reach the periphery of the vessel, they attach themselves to its wall and finally force their way through it. This is emigration, designated by some authors, diapedesis and transmigration. Diapedesis, however, is a term usually applied to the red blood corpuscles. The leucocytes represent an active process on account of their ameboid movement. The red blood cells which have collected to form dense masses also escape through the vessel wall, being passively forced through by increased pressure. They generally succeed the passage of the leucocytes except in those cases where there is a severe injury to the vessels, thus causing a hemorrhage. A small amount of serous fluid usually escapes at the same time.

EMIGRATION.-Normally, a few leucocytes are continually passing from the capillaries into the tissues; they are wandering cells of the body; but in inflammation, their emigration is increased very markedly when the irritant consists of pyogenic bacteria, and slightly in such cases as tetanus. 
Chemotaxis is the principal cause of the emigration. This is the phenomenon shown by living cells (phagocytes) of moving toward (positive) or away from (negative) certain other cells or substances. The positive chemotaxis exerted by the inflammatory irritant attracts the leucocyte, which is ever looking for food-stuff and debris. The exact explanation of chemotaxis is not definitely given. Metchnikoff suggests that this phenomenon is similar to the action of the plant reaching upward, the root penetrating downward or the leaf turning toward the light. It is known that certain leucocytes are attracted toward bacteria which they pick up into their protoplasm, and attempt to destroy by a ferment which they possess, called "cytase." This is phagocytosis. There are two kinds of phagocytes-the polymorphonuclear leucocytes, or "microphages," with a ferment called microcytase, and the lymphocytes, endothelial cells, and fibroblasts, or "macrophages" whose ferment is called "macrocytase." This cytase is the alexin of Buchner, or complement of Ehrlich. It has been given several names by other authors. Cytase enters largely into the action of anti-bodies, which play such an important part in immunity.

The kind of leucocyte which emigrates in inflammation depends upon the irritant. The most common cell is the polymorphonuclear leucocyte. In acute inflammations, especially pyogenic, these cells are attracted into the tissue in large numbers; and, together with degenerative changes in the tissue, viz., liquefaction necrosis, form pus. In catarrhal inflammation, mucous membrane is the seat of the reaction. Here the leucocytes collect in fairly large numbers and the degeneration of the epithelial cells is slight. In every inflammation, more or less of these leucocytes are present. They will be found especially in the neighborhood of the bloodvessels. They have the same characteristic appearance, even when degenerated, as in the blood. A few eosinophilic leu- 
cocytes appear in acute inflammations, and are sometimes found in pus. They are also present in tissues infected with trichina and animal parasites. Lymphocytes, both small and large, are present in some inflamed tissues, the large predominating. Chronic inflammations, infectious granulomata, such as tuberculosis, actinomycosis, etc., attract lymphocytes. Mast-cells are not always found among the emigrating cells, but appear in some inflammations of the mucous membrane and skin. Myelocytes and nucleated red blood corpuscles most often appear in the neighborhood of bone marrow, from whence they originally come.

Red blood-corpuscles take part in every acute inflammation, distending the blood vessels and surrounding them; in some cases they will be found over the entire inflammatory area. Their passage from the blood vessels is purely a passive one, due especially to the increased pressure within the vesse1. This is called diapedesis.

EXUDATES.-Inflammatory exudates vary in quality and quantity according to the tissue concerned, and the nature and intensity of the inflammatory agent. Dense tissue allows but little exudation to take place, while vascular structures, cutaneous and serous surfaces readily permit exudation. Some exudates are found in acute inflammations while others are characteristic of chronic. Serous exudates occur chiefly in a mild inflammation, but also in certain virulent cases such as anthrax, and are present to some extent in every inflammation. In pleuritis and pericarditis, there may be a large amount of serous exudate, one or two quarts in the pleural or pericardial cavity. The fluid contains little or no fibrin and few or no cells. A fibrinous exudate is characterized by the formation of fibrin; it does not differ materially from a coagulated serous exudate. When the exudate consists of a mixture of fibrin and serum, it is known as a sero-fibrinous exudate. Fibrinous exudates are com. 
mon in pneumonia and fowl-diphtheria. Fibrinous pneumonia is called croupous pneumonia. These exudates may form in the tissue spaces, in serous cavities, or in mucous membranes. They sometimes give rise to adhesions, such as are found in pleurisy and pneumonia, called fibrinous adhesions. Under the microscope, fibrin appears as delicate fibrils interlacing among the cells, and is of a blue color with ordinary tissue staining. 'There are also special fibrin stains which can be used in differentiation. The fibrinous exudate may be the forerunner of newly formed connective tissue. The hemorrhagic exudate has already been described under emigration as consisting of red blood corpuscles.

If resolution occurs before there are marked changes in the fixed cells, the exudate is conveyed to the circulation by way of lymphatics; and the leucocytes having performed their phagocytic functions, likewise return to the circulating blood. Otherwise the exudates may terminate in suppuration, cicatrization, or repair.

\section{CHANGES IN THE FIXED CELLS OF THE TIS-} SUE.-The changes in the fixed tissue in inflammation are partly retrogressive, and partly progressive. These two changes are often closely associated. In many inflammatory areas, the retrogressive changes are found near the centre, while the proliferation occurs near the periphery. The former are more often found in the beginning of an inflammation, while the latter predominate when actual healing begins. In chronic inflammations, the proliferative changes are the most marked.

The inflammatory irritant always produces certain lesions in the fixed tissue cells. Cloudy swelling and fatty degeneration will be found in most all the parenchymatous tissues in inflammations associated with toxæmia or septicæmia. All kinds of degenerative changes occur when the serous exudate infiltrates the tissue; the fluid may soak into 
various histological structures such as nerve-fibres, musclefibres, and cells, and cause dropsical vacuoles. Mucoid degeneration is frequent especially in catarrhal inflammations; hyalin and amyloid, common in chronic. When the acute degenerative changes of inflammation subside, leaving an ulcer or abscess, the development of formative cells from pre-existing cells occurs for regenerative purposes. If a limited amount of tissue has been destroyed, the ordinary processes of repair occur, each new tissue arising from old cells of the same kind. On the other hand, if there has been an extensive loss of substance, granulations form, which are ultimately converted into cicatricial tissue or scar-formation.

Microscopical Picture of an Acute and Chronic Inflammation.-Histologically, the acute inflammation is characterized by a cloudy swelling and parenchymatous degeneration of the epithelial cells, which are enlarged, granular, and vacuolated, and also poorly stained, especially the nuclei. Marked infiltration of leucocytes occurs in the tissues irritated, a round cell-infiltration. Any of the exudates described above may be seen in the inflamed areas and can be differentiated by special stains. If the irritant be microparasites, they can many times be demonstrated in the tissues.

A chronic inflammation is characterized by the formation of connective tissue, which as regards its cells, can be seen in all stages of development-small round cells, epithelioidal cells, fibroblasts, matured connective-tissue cells, giant and plasma cells. The younger the tissue, the more cellular it is, while in old chronic tissues, fibres predominate, together with marked forms of degeneration. Exudates may or may not be present. The irritant can more often be demonstrated than in the acute inflammation.

As will be seen from the foregoing, there is nothing in the histological findings of an ordinary inflammation that would indicate the exact cause of the irritation unless the ir- 
ritant be demonstrated. The changes above described may be due to any one of a large number of micro-parasites and to many other causes. A marked emigration of leucocytes, for instance, occurs in streptococcus infection, staphylococcus infection, or with bacillus pyocyaneus; the changes in the fixed cells are much the same in tissue irritated by chemicals as in tissue irritated by toxins; the hyperamia caused by a lash of a whip upon the skin is similar to the hyperæmia beginning a bacterial inflammation. And furthermore, in order to prove the etiology of such inflammations as those just mentioned, it is necessary to find the exciting factor. With limitations this does not apply to the infectious granulomata.

\section{INFECTIOUS GRANULOMATA.}

These form a distinct group of productive inflammations and appear practically always in the form of a sharply circumscribed new formation of tissue, which is due to the invasion of the body by certain definite infectious micro-organisms. If these parasitic germs are transported by the blood or lymph to some distant portions of the body, they set up an inflammation precisely similar to that which takes place at the site of the primary invasion. Thus an infectious granuloma may form metastases, and this is very common among the domestic animals. But these must not be regarded as true tumors, the etiology of which has not as yet been demonstrated. Generally speaking, it is possible to infer the species of infectious invader from the histologic appearance of the tissue proliferation. The microscopical findings are pathognomonic. This can be illustrated by the description of any one of the infectious granulomata, and as examples, tuberculosis and glanders will be compared. Actinomycosis and botryomycosis are taken up in detail in chapters.

The tubercle of glanders can easily be differentiated from that of tuberculosis. While both are characterized by a pro- 
liferation of embryonal connective-tissue cells, in tuberculosis, different forms appear than in glanders. In the former, the tubercle is made up of a central giant cell surrounded by several rows of epithe!ioidal cells, with a mass of lymphoid cells, encircling the whole. The giant cell has a dense row of nuclei massed together on one border. This characteristic appearance has classified it as the giant cell of tuberculosis, other giant cells having their nuclei scattered throughout the protoplasm. By a special staining process the tubercle bacilli can usually be demonstrated within the giant cells, and also elsewhere in the tissue.

Large areas of caseation can be seen scattered here and there. They are usually adjacent to the lymphoid cell masses, of which they are a degeneration. The lymphoid tissue is the youngest form of embryonic connective tissue and having almost no blood supply, easily degenerates into caseated areas. The epithelioiclal cells are probably an advanced stage of the lymphoid cells, antecedent to fibroblasts, the characteristic cell in most regenerative tissue, but in tuberculosis and glanders, the proliferating tissue is of such a low type that the matured forms of connective tissue cells seldom appear.

The tubercle of glanders is made up almost wholly of lymphoid cells, and no such regular formation occurs as in that of tuberculosis. Areas of caseation are present and a few epithelioidal cells may be found, but no characteristic giant cells. If the cells have degenerated so that a liquefaction necrosis of the gland occurs, the typical glanders bacilli can be demonstrated by staining a little of the pus. A young tubercle is necessary for the demonstration: in old tubercles, the germs have usually degenerated along with the cells.

The infectious venereal tumor is a pathological lesion practically limited to dogs, although a similar condition is found in the human. It is most common in the bull dog and 
terrier breeds. It is very contagious and capable of being communicated from dog to bitch, and vice versa, during copulation. Macroscopically, these tumors have a cauliflower appearance similar to a soft papilloma. They begin as small yellowish white raised patches, about the size of a mustardseed, growing into clusters of friable masses, which bleed readily when touched. Microscopically all these tumors possess several distinct characteristics, which, however, are by no means pathognomonic, being found also in sarcomata. When seen under the low power of the microscope, these tumors present the appearance of a round-celled sarcoma, or an endothelioma, but they have a thin covering of stratified squamous epithelium. Under the higher power, the tumorcells have the appearance of embryonic connective tissue cells in every stage of growth. Many are small round-cells with a vesicular nucleus. Mitosis can be seen in many of the cells. Some of these cells are spindle-shaped, and others fusiform, while many typical branched cells-the fibroblasts -are present. There is usually a leucocytic infiltration. In some of Dr. C. A. White's cases, a characteristic endothelioma could be seen. The endothelial cells can be seen springing from the endothelial lining of the blood-vessels, and occasionally epithelioidal cells are present.

Some pathologists classify these tumors as true sarcomata, others as infectious granulomata, but recent investigations by Reitman and Amadon would suggest that these are true mycotic tumors of an infectious nature. 


\section{CHAPTER III.}

\section{FEVER-PYREXIA.}

DEFINITION.-Fever may be defined as a general, systemic disease or symptom of disease, manifested by elevation of the temperature, and characterized by increased destructive metamorphosis, accelerated circulation, and disturbed secretion. The latter is but a symptom of the former. The word applies particularly to a more or less persisting rise of temperature.

ETIOLOGY.-The real cause of fever is an aberration of the vital function that maintains the equilibrium between heat-generation and heat-dissipation. This vital function of heat regulation, which Warren named thermotaxis, maintains the temperature of all warm blooded animals at a certain point in spite of external influences, so long as the organism is in a state of health. Heat-generation is due to oxidation or combustion in the tissues. The blood dumps oxygen into the tissues and thus produces a chemical action that ends with the casting off of carbonic acid. The chemical change occurring between the introduction of oxygen and the elimination of carbonic acid produces the heat. This chemical change is, however, not the simple combination of oxygen with carbon and hydrogen. It is a complicated process in which complex chemical molecules are disintegrated. Heat generation is, therefore, the result of complex chemical changes in the tissues produced by oxygen being thrown into them by the blood.

Heat-dissipation, or loss of heat, is caused by the exposure of the blood to external cold in the superficial capillaries; by exposure of the blood to the inspired air in the 
lungs; and by evaporation. The maintenance of an equal temperature under all circumstances in health, must therefore depend not only upon the production of a given amount of heat, but also upon its continuous loss. If the production exceeds the loss the temperature will immediately rise, but if the reverse occurs the body temperature will fall below normal. There being an internal furnace and an external refrigerator, the mechanism maintaining the equilibrium would appear to depend simply upon the cooling of the blood brought to the surface, the amount being regulated by the vaso-motor nerves. But this explanation is not entirely correct because the evolution of heat in any given muscle does not vary in obedience to the amount of blood flowing through it, which fact, being amply proven, demonstrates that heat generation is augmented and depressed under the direction of cerebro-spinal nerves that have no influence upon the blood vessels.

Whether or not there is a thermic center in the brain that presides over the heating apparatus of the body, does not specially interest the pathologist, who will be content to know that heat (fever) is governed by the nervous system, independently of its influence on the arteries, as well as by external exposure of the blood and evaporation.

Whether fever is due more to the repressed dissipation than the increased production, is a question that naturally arises in the study of its etiology. Fever might easily be explained by the anæmic condition of the periphery during the the first stage of diseases. It would be only logical to conclude that the lack of blood coming to the surface to be cooled would produce increased internal heat, but experiments have shown that depressed dissipation is not the sole cause, but that there is also a marked increased generation. Proof of this is shown by the rise of fever in a perspiring animal, and also by the fact that a feverish animal will heat 
more water after its temperature has been reduced to normal by cold baths, than a healthy animal. This experiment alone is ample proof that fever depends largely upon increased generation of heat, in fact that it is due to augmented oxidation of the blood and the tissues. The increased elimination of urea and other products, even when nitrogenized food is withheld, still further confirms the hypothesis. And furthermore there is always an increased elimination of carbonic acid-the end product of combustion-in a fever patient.

Fever being due to both diminished loss and increased production of heat, the real cause must be found in a disturbance of the regulating apparatus, - the nervous system. It is well known that the animal body is capable of, and in fact does, generate enough heat in twenty-four hours to coagulate the living protoplasm; that is to say, the body would be burned up in less than twenty-four hours were it not for the inhibition to which the mechanism is subjected. Exceptionally high temperatures are often supposed to be caused by imperfect elimination of heat, which statement cannot be entirely contradicted, but the fact that fever does not run riot and burn up the body when the eliminating mechanism is completely suspended points to the existence of an internal governor, the disturbance of which is the actual cause of elevations of temperature.

High temperature must not, however, be confused with increased combustion. A very high temperature may be caused by only a nominal degree of increased combustion, when the elimination is impaired, while on the other hand a nominal elevation of the temperature may in reality enanate from a markedly increased combustion because of the normal state of elimination.

Heat-production being the chief factor in fever, the question of its cause naturally arises. In this connection various theories have been advanced. The oldest one ascribes fever 
to the heat of inflammation. It was supposed that the inflammatory process generated heat and that the heat diffused through the entire organism. This hypothesis was not long entertained because the amount of heat in a local inflammatory condition is always insufficient to elevate the temperature to any considerable extent, and especially, because there is no regular relation between the degree of fever and the intensity or extent of the local inflammations, which do not in themselves, produce heat. It has also been supposed that fever is an inflammation of the blood,-a hæmitis. The theory was evidently based upon the perceptible changes in the blood in fever cases, which are now known to be due to micro-organisms and combustion products. Woods states that "There are nerve centers which are directly concerned in the thermogenic function and which affect the production of animal heat independently of the circulation, by direct action upon the tissues." Besides the theories above mentioned there is the bacterial theory of the modern pathologists, which will account well enough for many febrile states but which can not be accepted as the universal cause in the face of the fact that many febrile conditions are known to be provoked by conditions in which bacteria play no part. Fever can be experimentally produced in animals by inoculation with pus, purulent products and certain microbian cultures, but it can also be produced by chemical substances. The fever caused experimentally by microbian inoculation, does not, however, like that of chemical agents, depend upon the amount injected. The virulence of the inoculated virus is a more potent factor than the amount.

The part played by bacteria in the production of fever is still not satisfactorily explained. Whether they or their toxins simply disturb the nervous system, or produce changes in the blood and tissues which directly augment 
heat generation, is not known. It is, however, positively known that fever produced by chemical substances, by fear, by shock, or any external cause is not lasting, and that the only really persisting febrile states found in animals, are perpetuated if not caused by the influence of micro-organisms. In this connection Warren says: "In general it may be said that fever is due to the presence in the blood of a pyrogenous substance of an organic nature, that may have been produced by bacteria; or to the presence of bacteria; or finally to some ferment-like substance which has resulted from cell-clisintegration." When such eminent writers as Warren will not take a positive stand as to the exact role of micro-organisms in the causation of fever, it may be safely concluded that the matter is not definitely settled. A few years ago writers on surgery referred to all obscure surgical fevers as the result of impressions on the nervous system, and even today the term "aseptic fever" is by no means obsolete. The fever that follows in the wake of an apparently aseptic operation is conveniently attributed to nervous disturbances, or to the absorption of entirely harmless products in small quantities, proceeding from the wounded tissues. It is, however, interesting to note that these so-called "aseptic febrile states" are becoming fewer and fewer as surgical complications are becoming better understood. It is pretty safe for the present-clay veterinarian to act under the quite logical deduction that fever in surgery is synonymous with microbian infection. In a word, fever in veterinary pathology is caused by micro-organisms, by their toxins, or by both.

Fever has also been described as nature's protection against microbian invasion, the supposition being that either the high temperature or the morbid condition responsible for it were inimical to the welfare of the intruding micro-organisms. This theory is entirely without any foundation to support it, except the fact that attempts to lower the body tem- 
perature in certain diseases often prove harmful to the feverish subject.

The surgical fevers, so-called, are aseptic fever, surgical septicæmia, and purulent infection or pyæmia, the latter two being special morbid processes described in subsequent chapters.

Aseptic träumatic fever refers to the febrile state that is supposed to follow strictly aseptic operations. It is recognized from the septic febrile states by its strictly benign form. There is a nominal elevation of temperature, but no perceptible systemic derangement. The pulse is almost normal, or but slightly accelerated, the mucous membranes are of a natural color and the appetite is not impaired. The wound shows no sign of septic complications. The cause of these febrile conditions is usually explained as being due to nervous disturbances as before stated, but the present knowledge of wound diseases points more to the microbian origin of even these simple aberrations. That there are indeed few strictly aseptic surgical operations in animals is no longer denied. The deeper layers of the skin are known to contain micro-organisms at all times, that are ever ready to elaborate poisons at the proper opportunity. In comparatively clean operations, when the micro-organisms are few and the tissues healthy, it is only reasonable to suppose that a transient fever may result from a brief fight between the animal and the micro-organisms, in which the former is victorious before any perceptible alterations in the wound can appear. Experimental proof or disproof is, however, wanting.

SYMPTOMS.-The symptoms of fever are elevation of the temperature, determined by the clinical thermometer or by the tactile sense; more or less acceleration of the respirations; pronounced increase in the pulse rate; loss of spirits, dejected countenance and torpidity; injection of the visible mucous membranes; and more or less anorexia. Frequently 
these symptoms are preceded by chills of variable severity. The chill may be described as the first stage of fever or the period of invasion. During this phase of the disease, or soon after, the temperature reaches its highest mark. At this point the heat regulating apparatus seems to gain partial control of the situation and prevents impending death from hyperpyrexia. When the period of invasion is prolonged there may be alternate exacerbations and remissions, and the body will, during the entire time, be warmed with the greatest difficulty. The coldness of the surface will persist as stubbornly as it does during the chill, which is manifested by shaking of the body. The period of invasion or stage of chill is then supplanted by the second stage of fever or period of dominance. During this phase of the febrile state the temperature fluctuates about a given point. It may be high in the evening and lower during the morning hours. Sometimes the reverse is seen but more often there is no regularity nor certainty in the variation. The same temperature is, however, never maintained for a long time. Oscillation of the temperaiure is a characteristic feature of this stage. The period of dominance varies, as to symptoms, according to its cause. In organic inflammations the temperature must, of course, vary largely in obedience to the trend of the inflammatory process. When due to the continuous entry of toxins into the organism the variation and the gravity will vary according to the amount and the character of the poison absorbed. The fact that most febrile states in animals are due to either local inflammations or continuous morbid absorption renders this stage of fever very atypical. Death may occur during this period from the exhausting influence of a very high temperature, or from the onward march of the organic disease responsible for it. Dejection, inappetance and high pulse are markedly in evidence.

Next comes the period of defervescence, or third stage of 
fever. Sometimes, especially when invasion was rapid, this period is very short and amounts to nothing more than a sudden drop of the temperature. More frequently, the decline is gradual during two or three days. When it reaches a point near to the normal there is always a marked improvement in the patient's general condition. The spirits return, the eyes brighten, the mucous membranes become pink, the pulse slow, and the appetite improves. Often the latter becomes ravenous. The termination of fevers by crises is seldom ever seen in animals, or at least the crisis passes unobserved. When the period of defervescence is unusually prolonged there is reason to suspect grave internal abnormalities that the organism has failed to throw off. In septic infections, it points to secondary abscess. In pleurisy it indicates hydrothorax. In pneumonia, influenza, bronchitis, strangles, etc., it indicates gangrene, abscess, purpura hæmorrhagica, etc.

TREATMENT.-The treatment of fever always depends upon its cause. From the very earliest days the lowering of the temperature of sick patients was considered the physician's most patent mission. The modern therapist has, however, upset the old tradition and now only makes special effort to combat fever that is extremely high and which, as a consequence of its high mark, threatens early exhaustion of the vital forces. In domestic animals temperatures of $106^{\circ}$, $107^{\circ}$ or $107.5^{\circ}$ Fahr. demand somewhat special attention, especially when this high degree is maintained for some hours. But even here, the lowering of the temperature must not be purchased at the expense of the patient's strength. In the surgical fevers the temperature is combated chiefly by destroying the infective hot-beds. The infected wound.is opened, drained, curetted, irrigated, etc., often with the result of causing an immediate fall of the temperature, and prompt return of the general health. When such efforts fail or for 
some reason can not be executed, other means of treatment may be necessary. Diaphoresis, induced by diffusible stimulants and warm clothing, is one of the most valuable expedients in veterinary patients, especially in the horse. The perspiration, by causing the dissipation of heat, is generally followed by a drop in the temperature. This recourse is specially valuable during the period of invasion. Often it actually will permanently "break up" an approaching febrile state. In the later stages perspiration is less effectual, but it is always admissible. In fact, fever in animals can not be very successfully combated when the skin is kept cold. Clothing that will keep the body in a constant state of warmth, verging on perspiration, constitutes no small part in the treatment of any fever. Cold baths or tepid baths will lower the temperature to any point desired, and under all circumstances. Hence if the lowering of the temperature were the only essential in the treatment of fever a universal remedy is at hand. But since it has been shown by experience that such marked decrease of temperature as is produced by baths is more harmful than beneficial to the fever patient, these expedients are now only utilized to keep the fever within due bounds. In the treatment of heat stroke, a disease due to heat, one without internal lesions, the cold bath is a specific. In these cases when the temperature is lowered by a point nearing the normal the patient rises perfectly cured. In symptomatic fevers the case is entirely different. Chilling the periphery, or even gradually cooling it, seems always to lower the temperature at the expense of the internal strength. Internal medicines, aconite, acetanilid, antipyrin, quinine, and many others, may often be given to advantage, but they must never be depended upon alone to accomplish much, nor must they be expected to accomplish wonders. Aconite lowers the temperature by depressing the heart, acetanilid by diminishing combustion, quinine by pre- 
venting putrefaction, etc. The relative value of the various so-called antipyretics can not easily be determined. Some are valuable here, others there, while still others are probably always ineffectual if not harmful to most patients. Quinine is undoubtedly the most potent of the antipyretics for the fevers of domestic animals. It should be given in somewhat large doses and not repeated more than once or twice daily. In the temperature that runs riot, a good liberal dose (and only one) of acetanilid followed by the application of exceptionally warm clothing to induce a profuse sweat, will often result in a drop of several degrees. The permanence of the temperature thus lowered varies with the disease. In some cases it will rise again with increased intensity, while in others, it may remain lower throughout the entire course of the period of dominance. Subcutaneous injections of normal salt solution given copiously and frequently give perceptible results in persistent septic fevers, by stimulating the emunctories to greater activity. 


\section{CHAPTER IV.}

\section{GANGRENE.}

\section{SYNONYMS.-Necrosis, mortification, sphacelus.}

DEFINITION.-Gangrene is the death of a considerable area of tissue due to arrested nutrition. The word "gangrene" in modern surgery is used largely to designate the death of superficial, soft parts of the body, in distinction to "necrosis" which is applied to death in bone tissue or in the internal organs. The arbitrary use of these words is, however, admissible so long as they are applied to the death of tissues en masse. The word "ulceration" must be applied to molecular disintegration, and "sphacelus" to death of unusually large areas, such as an entire limb.

Death of circumscribed areas of tissue is a very common condition in domestic animals. It is encountered at every turn, in many phases, and in all the various degrees of extent and seriousness. No domestic animal is exempt from this morbid process, but those used for working purposes are particularly predisposed to both the superficial and the organic forms, owing to the greater liability of sustaining the injuries and diseases upon which gangrene so largely depends in animals.

ETIOLOGY.-Gangrene, or necrosis, in a word, is arrested nutrition due to obturation of the nutrient vessels of a given part. The causes of gangrene are largely physical causes-vascular obstructions-but it may also result from inflammation of extreme intensity (mycotic gangrene). In animal surgery, the causes are chiefly determining causes, although gangrene may be predisposed by both local and systemic debility. A local area affected with a pre-existing dis- 
ease or injury will more readily become necrotic than an absolutely healthy tissue; and an animal weakened from disease, over-work or bad hygiene is much more liable to develop gangrene from lesions that would otherwise prove less serious.

Etiologically, gangrene may be divided into primary, or traumatic and secondary or spontaneous.

I. Primary or traumatic gangrene is caused by direct injury to, or disease of, a given tissue. The injuries may be mechanical, chemical or thermic. These influences either kill the tissues at a single blow, injure them beyond repair, or else reduce their vitality so as to make them easy prey for intruding micro-organisms. Various forms of mechanical violence are capable of producing injury severe enough to cause immediate or subsequent death of the wounded part; in fact, all contusions of more than nominal severity, cause the death of a portion of the bruised tissue. These include kicks, blows, treads, lacerations, etc., which cause death by crushing, disorganizing and separating the anatomical elements, and by creating favorable food for microbian growth. Chemical substances,-caustics,-coagulate the protoplasm and like the contusion, either entirely kill the tissue at once or else injure it beyond repair. Burns and scalds affect the tissues in much the same manner, while freezing produces gangrene by arresting the flow of blood and causing general systemic enfeeblement.

Gangrenous dermatitis (mud-fever) affecting the legs of horses is a striking example of gangrene resulting from a trivial injury, becoming infected with virulent micro-organisms. It is a true mycotic gangrene. The skin becomes literally choked with micro-organisms and dies as the result of the strangulation of its capillaries and the dissolving influence of microbian products. A trivial injury badly infected is capable of producing a gangrene even more serious than if 
a serious injury had been inflicted. In this disease the trauma is usually a simple flexion crevice or trivial abrasion of the skin that admits micro-organisms into the subcutaneous connective tissue. Operative gangrene occurs from various causes after veterinary operations. The ligation of a large artery or even a large vein is, however, the most common cause. Whenever such recourses are necessary in a surgical operation or in the treatment of an accidental wound the area supplied by the ligated vessels often becomes the seat of an extensive gangrene. Pressure gangrene, or pressure necrosis, as it is often conveniently called, is also a common form of the disease. This form is caused from the application of taut bandages (bandage necrosis); from the suturing of wounds (stitch necrosis); from prolonged decumbency (decubitus); from pressure of the harness, saddle or collar (sit-fast); from pulling heavily upon the bit (bit gnathitis); or from any severe but especially prolonged pressure upon any given part of the body. An example of pressure gangrene is seen in strangulated hernia, when the constricted hernial orifice compressed the incarcerated bowel. In all of these cases the primary injury is followed by the invasion of pathogenic microbes which provoke intense inflammatory action and thus become largely instrumental in determining the seriousness of the condition. They always cause the putrefaction which the dead tissues undergo, and without them the gangrene is always subordinate to the force of the initial injury. The so-called aseptic inflammations, simple inflammations, or non-microbian inflammations which in reality are no more than pure physiological regenerations, are incapable of producing a gangrene beyond the area actually killed at the initial blow. All spreading gangrenes of the primary, traumatic types are microbian. That is to say if the prick of a nail or the kick of a shoe results in the sloughing of a considerable area, it is the invasion of micro- 
organisms that must be blamed. The exact role of microorganisms in the causation of traumatic gangrene must, however, not be misconstrued. They enter into the gangrenous process after some other action has interfered with the nutrition of the affected area. Etiologically, they are secondary; the bruise, the pressure, the ligated artery, etc., are the primary causes.

Necrosis of the internal organs of animals occurs from various causes. The presence of a foreign body in the alimentary tract will often produce necrosis of a part of the organ in which it lodges. In this manner the pharynx, the osophagus, the stomach, the rumen, the intestines, the rectum, etc., often become the seat of serious fatal gangrenes. Pharyngeal and œsophageal obstructions always, when not removed, terminate in fatal necrosis at the seat of obstruction from the continued pressure of the foreign body. Jones of Illinois, found an extensive gangrene of the rumen, caused from the accumulation of a large amount of binder-twine, and analogous conditions are frequently reported of other ingested foreign bodies. The pressure of tumors on blood vessels or directly against organs may cause necrosis, and severe infective inflammations are always liable to terminate in softening from gangrene, abscess, or both. In the lungs, hæmorrhage, apoplexy, and pneumonia often terminate in the death of a large area of the invaded tissue. A whole lobe or even an entire lung may be found to be the seat of a widespread gangrene. Here the condition is due both to strangulated circulation and to putrefaction of the large quantity of exudates exposed to the micro-organisms of inspired air. The gangrenous lung follows, either a very intense morbid process (apoplexy, pneumonia, etc.) or else is predisposed by the general systemic enfeeblement of the patient.

2. Secondary or spontaneous gangrene refers to all of 
those gangrenous conditions that occur in the absence of any direct injury. They are called secondary becautse their actual cause is always an obturation of blood vessels, more or less remote from the original seat of the gangrene. This form is not as common in animals as the traumatic. Widespread thrombosis and arterial sclerosis, the two common causes of occlusion of blood vessels, are seldom seen in animals. The former is, however, encountered occasionally in the large arteries of both the anterior and the posterior limbs of horses. Thrombosis of the iliac arteries is occasionally found, and is capable at any time of producing an obstruction sufficiently complete to cause sphacelus of the entire limb and death of the patient. The same phenomena are also seen in the anterior limbs. They simulate attacks of lymphangitis in the first stages. Another example of spontaneous gangrene is the necrosis of molar teeth from atheromatous degenerations of the radicals of the dental arteries. In these cases the teeth lose their principal blood supply and die as a consequence.

PATHOLOGICAL ANATOMY.-In every wound caused by bruising there are three kinds of tissues; the dead, the dying, and the viable. In the center of the traumatic focus the dead elements are found attached to, and surrounded by, the dying elements which soon become prey for microorganisms. The viable elements surrounding the former two, thanks to the reaction of the organism, revive from their weakened state and promptly separate themselves from the dead tissues they encircle. The point of separation soon becomes distinctly visible and constitutes the "line of demarcation" which in due time forms a crevice in the tissues and thus separates the living from the dead. In certain tissues (tendons, ligaments, cartilage, etc.) the dead elements remain attached to the living, and although the process may be old, no distinct line of demarcațion is formẹd: Necrotic portions of 
ligaments, tendons and aponeuroses will remain attached to the living portions indefinitely without material change in color, strength or texture. They, however, separate from their bony attachments and surrounding tissues, and may thus be recognized from the living tendinous tissues. The "line of demarcation" readily' forms in the skin, in bone, in muscle and in the parenchymatous organs. Slowly developing gangrenes of the skin caused by moderate but constant pressure often become dry and remain attached to the subjacent tissues (sit-fast), but the line of demarcation is never wanting as in the case of white, fibrous organs, such as tendons.

The line of demarcation is serrated and irregular in all of its outlines. It may be deep at some points and shallow at others. When the slough or sequestrum is removed it uncovers typical, rosy granulations which proceed to cicatrize in the usual manner.

Gangrene is often described as moist gangrene and dry gangrene. This classification is not without fault. It is apt to be misleading for both of these varieties may be due to the same cause, and the reputed cause of one may determine the other. For example, an inflammatory condition may cause dry gangrene as is observed in sit-fast and decubitus of animals, while the occlusion of a large artery which is supposed to cause only the dry form may, in reality, produce the moist variety. The kind of gangrene is determined by the amount of fluids the tissues contain at the time the nutrition was arrested, and upon their capability of preventing its evaporation, and not upon the cause.

The gangrenous tissues may also contain gas as in the case of malignant œdema and symptomatic anthrax. Gangrenous processes even of small areas always cause some systemic disturbances. When the area is great grave disorders always supervene and death may ensue from septic 
absorption. When located in vital organs the systemic disturbances will vary with the location. They may be fatal, grave or trivial.

Necrosis of bone in animals is seen only in the traumatic form. It is never spontaneous nor diffused. The necrosis of bone encountered in veterinary subjects is always of a circumscribed and rather trivial character. Injuries that cestroy the periosteum, or circumscribed inflammations that impair its osteogenic function are always the exciting causes. Lesions of sufficient magnitude to cause an extensive necrosis in the bones of animals usually prove fatal from other causes before the necrotic process has had time to evolve. The special necroses of animals are:.(I) necrosis of the superficial bones from accidental wounds that implicate the periosteum; (2) necrosis of the retrossal and basilar apophyses of the os pedis from suppurating corns; (3) necrosis of the solar surface of the os pedis from nail pricks; (4) necrosis of the inferior maxilla in the interdental space from bit pressure; (5) necrosis of the superior extremities of the superior spinous apophyses of the dorsal vertebræ from fistulæ of the withers; (6) and necrosis of the anterior angle of the ilium from prolonged decumbency or violence. It will be seen that all of the conditions are traumatic, rather trivial, and limited in extent. In this form of local death a given section of bone is deprived of its nutrition by the loss of the fibrous covering-the periosteum-upon which its life depends. The extent is always subordinate to the area stripped of its periosteum by disease or injury, and owing to the fact the bone tissue receives nutrition from within, the necrosis is never deep. Like gangrene of soft tissues deprived of their nutrient blood supply, the bone tissue stripped of its periosteum, separates from the contiguous, living bone after the formation of a distinct line of demarcation, and then is çast off in the form of a somewhat irregular sequestrum, 
leaving a depression that is not always entirely refilled with bone tissue.

The separation of necrotic bone from its contiguous living bone tissue occurs through the active proliferation of the cells of those portions of the Haversian canals, medulla and periosteum that immediately surround the diseased area. The cells of these structures build a bed of new tissue between the dead and the living tissues that is analogous to the granulations which underlie gangrenous soft structures. The process of separation is hurried somewhat, in infected cases, by the dissolving influence of bacterial ferments.

SYMPTOMS.-Traumatic gangrene is always manifested by intense pain at the seat of the injury: When located in the limbs there is severe lameness of several days' duration before the true nature of the disturbance can be determined. The skin then becomes cold, less sensitive, moist, as if perspiring, and finally sheds its hairs. If the hair surrounding the diseased area is shaved the line of demarcation can be scen, and the tissues within its boundary are found to emit an offensive smell. After several more days the slough falls off and leaves the granulating surface exposed. At this period, if the gangrene has not transgressed upon the neighboring synovials, the lameness ceases and the wound heals without further event, but leaves a hairless scar as an indelible blemish.

Extensive gangrene progresses in much the same manner, with the exception that the systemic disturbances are grave and usually terminate the patient's existence before the gangrenous area has separated from the surrounding living tissues. A whole limb, the whole withers, the entire poll or the buttocks may become the seat of an extensive gangrene,-sphacelus,-that rapidly terminates fatally. In these cases the pulse is fast, the temperatire high, the move- 
ments extremely painful or impossible, the respirations accelerated and the appetite nil. The affected area is first hot, but soon becomes cold and moist. The skin will be found oozing a thin serum. If, by chance, the patient's life is spared, the gangrenous area sloughs off in great chunks, leaving sections of living tissues here and there, that have escaped the entire force of the morbid phenomenon. In the limbs the skin and subjacent tissues may slough off at numerous points and in large sections as from the elbow to the foot. Cicatrization is slow and imperfect and usually its constricting tendency leaves the subject a hopelessly blemished cripple for life.

TREATMENT.-In the treatment of the various gangrenous conditions of animals, it must be remembered that the damage is already done at the time treatment usually begins, and as a consequence there is no possibility of preventing the impending death of the affected area. In surgical operations gangrene must be prevented by preserving large vessels instead of sacrificing them to the knife. Bandage necrosis, stitch necrosis and decubitus are to be prevented rather than cured. When once the circulation of a given part is occluded no form of treatment will revive it, and hence treatment aiming to limit the extent of the dying area will always fail. Hot fomentations may occasionally serve to augment the collateral circulation and thus enlarge the limits of the surrounding viable tissues. The dying area must, however, be constantly immersed in antiseptics to prevent putrefaction. With this precaution the absorption of septic products is materially lessened and as a consequence the affected part may be given ample time to separate completely from the living tissues before it is removed. It is only when the systemic symptoms are grave and threatening that premature extirpation of the gangrenous area is beneficial. That 
is to say, surgical intervention is intended for systemic and not local amelioration. In certain cases free incision and even ablation of the part may be necessary to prevent the burrowing of purulent products into the neighboring structures. Prompt operation upon the fistula of the withers, the poll evil, the quittor, etc., may prevent the formation of complicated fistulous tracts, but even here, and always in the superficial gangrenes, the "waiting" treatment is by far the most appropriate.

Amputation, which constitutes the essential treatment for extensive spreading gangrenous conditions in the human being is not applicable to veterinary subjects, except, possibly here and there, in the dog or cat, and occasionally for gangrene of the tails of any of the domestic animals.

The palpable duty of the veterinarian in the treatment of gangrene is: (I) to apply hot fomentations to encourage the collateral circulation; (2) to incise or ablate when purulent products may penetrate into important neighboring structures; (3) to remove the sloughs or sequestra when they are well separated; and (4) to encourage cicatrization in the usual manner.

\section{OEDEMA.}

DEFINITION-Edema is simply the transudation of serum from the blood channels, due to obstruction or retardation of the flow of venous blood toward the heart. When the fluid accumulates in the subcutaneous tissue over a circumscribed area, it is usually referred to as "œdematous swelling" or "simple cdema." If the accumulation is in a cavity it is called "dropsy." In the peritoneal cavity the name "ascites" is given, in the cerebral ventricles, "hydrocephalus," and in the scrotum, "hydrocele." If spread throughout the subcutaneous and intermuscular areolar tissues it is called "anasarca." 
PATHOLOGY AND ETIOLOGY.-The pathology and etiology of œdema are comparatively simple. The return circulation from any given part, when obstructed, either partially or completely, causes an engorgement of the blood carried to it by the arteries. The tension thus produced leads to the straining of the watery constituents of the blood through the vessel wall into the areolar spaces of large cavities.

The intercellular spaces of normal tissues are filled with a clear, watery serum (lymph) that is filtered from the blood serum through the walls of the capillaries (and probably partly secreted by the cells). When the production of this serum exceeds the absorption, the tissues become saturated with fluid, the anatomical elements are crowded apart to make room for the accumulation and the result is an œdematous swelling. Both the lymphatics and the blood vessels are concerned in absorbing lymph and carrying it back to the general circulation. If the former is obstructed the latter will generally perform the function of absorbing the excess of lymph, but if a blood vessel is obstructed, lymphatics seem entirely incapable of offering equal compensation. That is to say, if a lymphatic vessel is obstructed the veins will probably absorb the excess of serum in the intercellular spaces and no permanent œdema will result, while, on the other hand, if there is even a trivial interference with the free flow of blood from any given part to the heart more or less œdema is certain to supervene and to remain until the obstruction is removed.

The accumulation of transudates (œdema) may be favored by pathologic conditions of the blood vessels and of the composition of the blood as in purpura hæmorrhagica, but by far the most common cedemas of animals are those die directly to passive hyperæmia. Veins become obstructed in one way or other and the part drained by them becomes 
the seat of a copious transudation of serum into the tissuespaces. The passive hyperæmia may be local or general. In local odema only a small vein is obstructed and the swelling remains limited to the tissues directly concerned. But often a circumscribed œdema may spread from the pressure it itself exerts upon neighboring veins. Thus a trivial swelling of the abdomen may become an enormous cedema of the entire ventral surface of the body. The local odemas observed in animals are caused from inflammatory swellings which press upon veins, solution in the continuity of veins in accidental or surgical wounds, ligation of veins, pressure of tumors, venous emboli, pressure of bandages, and in fact any condition that is capable of retarding the free backward flow of venous blood from any given tissue. Thus a bruise to the forearm, by interfering with the upward movement of blood in the radical veins, will promptly cause a pronounced cedema of the entire limb below. In the same manner a disease of the liver will cause ascites by hindering the flow of intestinal blood through it.

General œdema is due to tricuspid insufficiency, obstruction of the venæ cavæ, or general depression of the circulatory apparatus. When the tricuspid fails to effectually close the right auriculo-ventricular opening with each contraction of the right ventricle the inward flow of venous blood into the right auricle is retarded. The entire venous current from the remotest capillaries feels the effect of the added obstacle to its already difficult passage from the periphery to the heart, and the result is a transudation of serum at different parts of the body. A general dropsy may thus be provoked. When the obstruction is but partial the total effect may be an œdema of the legs, where gravity greatly hinders the return current. If the circulation is depressed from disease or overwork, gravity alone may be sufficient to determine an obstinate œdema of the hind legs. The arterial blood may 
flow freely into the legs, but often there is not sufficient force to again lift it back through its upward, tortuous course to the heart. Under such circumstances odema is the inevitable result.

Pendant parts of the body (the legs, the sheath, the abdominal walls, the pectoral region) are the favorable locations for œdematous swellings. Here the return current of blood is at a disadvantage while the direct current,- - the arterial, - is favored by gravity. The blood flows freely into such parts, but as it cannot as easily return, the most trivial interference with the current will favor transudation from the peripheral capillaries, and œdema is the result. CEdema is also favored in such tissues by the rather loose arrangement of the subcutaneous connective tissue and the spacious cavities it affords the transuded fluid.

The common œdemas seen in animals,are: ( I) œdema of the sheath and prepuce, caused from bruises, filth, idleness, tumors, castration, etc.; (2) œdema of the extremities, caused by bruises, wounds, debility, scratches, febrile diseases, etc.; (3) œdema of the abdomen, caused by contusions, wounds, pregnancy, febrile diseases, general debility, erythema, etc.; (4) ascites of the dog, due to pathologic conditions of the liver, kidneys or tricuspid valves, tumors compressing the vena cava, etc.; (5) hydrocephalus of the horse, due to obstruction of the cephalic veins, lesions of the heart, tumors, etc.

Besides these special examples œdema is found in connection with almost every inflammatory swelling of traumatic origin, with every abscess of any considerable dimension, and with every wound that divides veins. In these examples chere is no plain line of demarcation between the cdema and the inflammatory swelling. The former surrounds the latter and is especially prominent in the tissues farthest from 
the center of the circulation and beneath where the fluid precipitates by gravity.

The lesions of odema consist of: (I) increased permeability of the capillary walls; (2) separation and saturation of the anatomical elements of the part; (3) slight increase in the size of the cells from absorption of fluid; (4) accumulation of fluid in the tissue spaces.

SYMPTOMS AND TREATMENT.-Subcutaneous œdema is easily recognized by its abrupt boundary, its doughy consistency, the absence of pain on palpation, and the absence of proportionate systemic disturbances. When scarified or punctured the fluid will freely drip from the wounds in the form of a clear, yellowish serum. The inflammatory swelling accompanying a contusion or abscess is firm and painful, while the surrounding œdema exhibits only the above symptoms.

Edema on the surface of the body is in itself a very bcnign affection that disappears when the cause is removed or after the collateral circulation begins to perform the function of the obturated vein or veins. The traumatic œedemas quite readily yield to this reaction, but when the cause persists grave results may follow. In the limbs, long-standing œdema is always certain to stimulate the production of new connective tissue that remains thereafter as a permanent tumefaction of the affected members. In other parts necrosis of no small proportions may supervene from pressure of the infiltrated fluid upon the nutrient vessels of the œdematous tissues. This complication is particularly frequent in odemas of the sheath and prepuce.

The abdominal odema of pregnancy disappears with parturition, and that of the limbs and sheath often yields to exercise. Baths, massage, kneading, friction, support, slight pressure, scarification, puncture, and exercise are so many 
remedial expedients, the value of which depends upon the location and the cause.

The removal of the cause would promptly terminate most any ordinary œdema of animals, but this can seldom ever-be immediately effected. The divided vein, the ligated vein, the obturated vein, the inflammatory swelling, the defective heart, the depressed circulation, etc., which cause œdema can not be dispatched at one stroke: The treatment niust, however, be directed at the cause as well as at the effect whenever possible.

Tonics, healthful exercise, better hygiene, good food and regular dieting must not be omitted in the treatment of œdemas due to, or augmented by, general depression of the circulatory apparatus.

Ascites of the dog, being due to incurable affections of the liver or other organs, can never be permanently remedied. The aspiration of the fluid is always followed by refilling of the cavity, and the medicines given for the sclerotic liver are generally ineffectual.

\section{THROMBOSIS AND EMBOLISM.}

DEFINITION.-Thrombosis is the coagulation of blood within the blood vessels of the living body. It is death of a portion of the blood. The coagulated mass is called a thrombus. The coagulation of blood after death even if outside of the blood vessels is called a coagulum or a clot. The word "thrombus" is still more properly defined as a fixed coagulum within the blood vessels of a living body. Embolism is the obturation of blood vessels from the lodgment of a solid object that has floated in the blood current from other parts of the body. The floating particle is known as an embolus. The thrombus is always composed of clotted blood while the embolus may be any floating solid substance, such as detached particles of thrombi, clusters of bacteria; clusters of 
endothelial cells, parasites, foreign bodies, shreds of tissues, etc.

ETIOLOGY AND PATHOLOGY.-In the living healthy blood flowing in healthy vessels the solid constituents are held in suspension or in solution. As long a's the normal blood flows at a certain rate of speed and meets no other object within the smooth endothelium of the intima, it retains its liquid state, but when the current is stopped or retarded, or the lining of the vessels becomes wounded or diseased, a certain amount of coagulation will promptly result.

Thrombosis may be caused by slowing or arrest of the blood current, by pathological changes in the walls of the blood vessels, or by changes in the blood itself.

If the blood current in the heart, in the large vessels, or in any local part becomes very slow, the leucocytes adhere to the intima and fibrin becomes deposited upon them. More leucocytes impregnate the mass and more fibrin forms, until the lumen of the vessels becomes small and even entirely obliterated with a firm clot that varies in color from yellow to dark red, according to the number of leucocytes it contains.

These thrombi often form very rapidly just before death, and may be found to entirely fill up such large vessels as the posterior aorta, the pulmonary arteries, the coronary arteries, the brachial arteries, the carotids and the large veins. The firmness of their organization would often suggest a much slower process of formation. In the cavities of the heart they are found with great frequency post-mortem, in the form of free, elongated masses of whitish coagula that tear with some difficulty. The facts anent these thrombi are that they form during the few moments, or at least the few hours, before death. The real ante-mortem thrombi that have existed in the heart or blood vessels for some time betore death are found adherent to the endothelial layer of the 
vesset or cavity containing them. In the heart they adhere to the valves. The free ones are all of rapid formation. The socalled "chicken-fat" thrombus abounds with leucocytes and contains few red blood corpuscles, while in the "currentjelly" variefy the opposite condition exists. These two varieties may co-exist in any given thrombus of rapid formation, the first layer around the intima being whitish and the remainder jelly-colored. The clot that forms just before death or immediately after may be either whitish or dark, but it is always stringy, quite firm and elastic, while the slow forming ante-mortem clot is always whitish and possessed of a characteristic friability.

The changes in the blood vessels that favor thrombosis are inflammations of bacterial or traumatic origin. These cause an erosion or wrinkling of the intima and thus lead to the accumulation of leucocytes and then fibrin upon the altered area. A trivial lesion may produce a clot of large dimensions by supplying the nucleus upon which more and more blood coagulates until the vessel is obturated a considerable distance from the initial lesion. Aneurisms in the same manner are very prone to cause the formation of thrombi. The ligation of blood vessels in surgical operations always causes the formation of a clot in the ligated stump, which may extend into the main trunk and thus lead to a very extensive coagulum.

The blood itself may be altered so as to provoke the formation of thrombi by the accidental introduction of foreign matter into the blood current. Medicaments administered intravenously, air accidentally admitted into a vein, pus absorbed from a suppurating focus, and micro-organisnıs and their products, may readily cause the blood to coagulate within the vessels. And finally one thrombus may lead to the formation of others. Particles become detached (em- 
boli) and then float to other parts of the body and there form new thrombic foci.

\section{TERMINATIONS AND EFFECTS-Thrombi may} become organized, that is, they may be replaced with connective tissue through the activity of the endothelial cells which perform the function of fibroblasts. In this event the - vessel becomes permanently obliterated. Its lumen is filled with a firm fibroconnective tisste that will henceforth permanently obstruct the channel. Striking examples of this termination are found in the iliac arteries of horses, because of which seizures of very transient but of very acute lameness may occur in one or both hind-legs. Again, thrombi may liquefy and restore the vessel to its normal condition, they may undergo septic disintegration with results varying with the nature of the infection, they may calcify or they may become detached and float awray as emboli and then create new thromboses. Finally, they may produce gangrene by obstructing the blood supply to any given part, or abscess by becoming infected at the initial seat or by the floating of infected emboli to remote regions. Thus embolic pneumonia may readily result by the flow of a septic embolus from a peripheral trauma through the veins to the capillaries of the lungs.

The thrombus retains its original condition for a very brief period only. It is no sooner formed than changes begin to occur. . At first soft as any ordinary clot of blood it soon becomes firm, fibrous, softened, granular, calcified or purulent.

The effect of thrombi and emboli depends upon the importance of the vessels concerned and the nature of the clot. If infected they are always more serious than when microorganisms do not enter into the process of their formation. Wide-spread cedema of an entire extremity, circumscribed or diffused gangrene, multiple abscesses, embolic pneumonia, 
purulent infection, and septicæmia are among the conditions that may result from them.

SYMPTOMS.-The clinical pictures produced by thrombosis will of course vary according to the location, the extent of the obstruction, and the character of the infection if any exists. The choking up of the blood vessels of the skin of the heels of horses following virulent infections and resulting in a circumscribed gangrene of the integument is an example of the effects of thrombosis of strictly bacterial origin.

Thrombosis in animals always produces severe pain in the affected region. When a radial, brachial, tibial, femioral or iliac vessel is the seat of the obstruction, the leg becomes œdematous and extremely painful to the touch; and very grave systemic disturbances immediately follow. If the obstruction is wide-spread death will often result from pain and exhaustion before the co-lateral circulation is sufficiently augmented to offer adequate compensation. When the acute stage is survived and the clot is replaced with connective tissue, the pain may only appear during exercise, and then promptly subside with rest. Smaller arteries of the legs may become the seat of thromboses and cause very excruciating pain without producing any swelling. If buried deeply in the muscles palpation may fail to locate the seat of the lesion in spite of the excruciating pain. In the acute case, and also during seizure in the chronic case the leg will support little or no weight, the respirations will be augmented, and the body bathed with perspiration.

A certain well known race-horse began to show signs of distress in one hind leg after each work-out of one-half to one mile. During rest and during short runs nothing abnormal was noticeable, but with each long run a narked lameness developed with great regularity. With each seizure there was trembling. perspiration, distress and severe lameness in the left hind leg. The horse was treated in different joints of the legs during two years, at the end of which time an effort to speed him provoked the very same symptoms, unpalliated and unaltered. An examination of the iliacs through the rectum revealed a hard formation at the aortic quadrification, and absence of perceptible pulsations in the left external iliac. The left internal iliac 
and both right iliacs were apparently normal on palpation in the living animal. Dissection of the parts post-mortem disclosed an ovid aneurism of the aorta dt its quadrification, measuring four inches long, two and a half inches wide and one and one-half inches thick. The left external iliac artery was completely obstructed with a firm connective tissue thrombus extending downward fourteen inches into the femoral. The artery itself was degenerated to less than one-half its normal diameter, and the thrombus was firmly adhered to its walls. In fact it had become a part of the artery itself. The connective tissue thrombus also occupied a part of the aneurism in the aorta, and extended with small branches into all of the other iliacs, without entirely occluding them.

Hill reports the history of a cavalry horse that suffered from an acute fatal thrombosis of the popliteal artery. The attack was sudden and terminated fatally after five days of excruciating suffering. The horse was found suffering pain and unable to support weight on one hind leg, on the morning inspection. Careful and repeated inspection and palpation of the leg failed to disclose either the nature or the location of the affection, although the lameness and systemic symptoms continued to increase in severity. On the fifth day the patient, being in a dying condition. was killed and the examination post-mortem disclosed a thrombus of the popliteal artery. The report, unfortunately, does not include a pathological nor anatomical description of the abnormality.

Analogous conditions are occasionally encountered in the fore extremities of horses, but here the disease is generally acute, and resembles attacks of lymphangitis until the increasing gravity of the process reveals its true nature. In the acute cases affecting the extremities, death supervenes on the eighth to the tenth day, from pain, exhaustion, absorption of morbid products, fever, anorexia, etc., or else the process terminates in an extensive gangrene that will prove fatal at a later date. It is only the rare cases that terminate in chronic fibro-connective-tissue thrombosis, but when this event does not occur it is through the influence of the collateral vessels which undergo compensatory augmentation soon after the principal one is obturated.

Thromboses in other parts of the body, notably the parenchymatous organs, produce symptoms according to the particular organ affected and the extent of the obstruction.

TREATMENT.-The disease resulting from thrombosis must be treated according to its nature and location. In the limbs. baths, friction, massage and moderate exercise are the 
best to recommend for the acute case. Internal medicines, reputed to possess the property of defibrinating the blood and of dissolving clots, may be administered. Ammonium inuriate and potassium nitrate are said to be of some value in that connection. In the internal organs, the treatment is limited to the administration. of appropriate systemic remedies.

\section{ATROPHY.}

DEFINITION-In its etymological sense, the word "atrophy" signifies want of nutrition. In pathology it is defined as the wasting of a part or all of the body, or in other words the diminution of the normal volume of the body, of any organ or of any tissue. General atrophy, that is, the uniform wasting of the whole apparatus of the body, is however usually referred to as "emaciation" when due to disease or privation, and as "senile changes" or "senile degeneration" when due to old age.

CLASSIFICATION.-Atrophy may advantageously be divided into several classes: viz., (I) Pathologic atrophy or wasting due to disease; (2) physiologic atrophy or wasting due to inactivity; (3) local or circumscribed atrophy when limited to a small area or single structure; (4) diffused atrophy, when affecting a whole group of muscles or a whole apparatus; and (5) general atrophy when the whole organism is involved. As to cause, there is (I) nervous atrophy or wasting due to impaired trophic innervation; (2) ischemic atrophy or shrinking of a part due to inadequate blood supply; (3) pressure atroplyy or wasting from prolonged compression; and (4) senile atrophy or wasting from old age.

ETIOLOGY AND PATHOLOGY.-Atrophy of any given part results in the diminution of the size or of the number of cells or both, and not in any change in their chemical constitution. Degenerations and disintegrations 
are not atrophies. The use of the word "atrophy" must be restricted to the wasting process in which the normal constitution of the part is fairly well maintained. When a degenerative process predominates the phenomenon is not an atrophy.

In the simple atrophy commonly encountered in domestic animals the affected part exhibits a dearth of cells and a manifest diminution in their size, while the intercellular substance remains unchanged. The loss of volume is due strictly to this behavior of the cellular elements.

The causes of local atrophies are: ( I) Peripheral paralysis, (2) inactivity or disuse, (3) pressure, and (4) centric paralysis.

(I) Peripheral Paralysis.-When a nerve-trunk supplying the motor innervation of a part is bruised, compressed, violently stretched, or divided, a wasting process will supervene. In the case of muscles where the normal volume depends upon their activity, the wasting process may be exceptionally rapid, if the motor innervation is wanting. A musc'e made perfectly vapid by impairment of its motor nerve will shrink much more rapidly than inactive muscles normally supplied with nerves. Whether this difference is due to the absence of trophic influences or not, cannot easily be determined because absolute inactivity of normal muscles seldom ever exists. The very rapid wasting of certain muscles of the horse after their motor nerve is injured would, however, indicate that inactivity is not alone responsible, but that some other causative factor enters into the process. Whether this subtile influence is failure of the trophic innervation, thrombosis, or local vaso-motor disturbance, is diffi. cult to decide.

There are three special examples of atrophy from peripheral paralysis in the horse, that beggar description from this standpoint. These are: ( I) Atrophy of the external 
scapula muscles from injury to the supra-scapular nerve, (2) atrophy of the crural muscles following azoturia, (3) atrophy of the caput and adjacent muscles following injuries to the brachial plexus.

In each of these examples the wasting process is a much more rapid one than inactivity alone could produce. The nutrition as well as the motion of the vapid muscle is undoubtedly impaired. The almost total disappearance of a muscle within the short space of ten days could hardly be attributed solely to motor paralysis in the face of so many instances in which muscles remain dormant for weeks with only nominal atrophy. As the result, in each of these atrophies the cause is peripheral nerve paralysis. Two of them are of traumatic and the other of unknown origin, and in each instance the wasting process is extremely rapid in spite of the fact that the blood supply of the paralyzed muscles is fairly well maintained by the activity of the surrounding muscles. Furthermore the wasting process is not prevented by attempts to excite the circulation of the parts with irritants, massage, etc., before the wasting process has begun. From these facts it becomes evident that muscular atrophy is due both to motor and trophic paralysis.

(2) Inactivity or Disuse.-Unless an organ is in active use it will diminish in volume and even entirely disappear. Organs that are specially concerned in fotal development waste away after birth when they have no further function to perform; muscles not in active use shrink until only their stroma remains, and in fact every tissue and every organ of the body will enlarge and shrink in strict obedience to its state of activity. This is physiologic atrophy. It is the most common of the wasting processes of animals. Lameness due to diseased processes in the supporting structures of the extremities will cause atrophy of the muscles fal above the seat of dis- 
ease. The atrophy may be slow but it is always certain and will vary according to the severity and the duration of the lameness. If a limb remains absolutely inactive for a long time the muscles may almost completely disappear, leaving only their fibrous stroma and a few starved cells as a trace of their former existence. With renewed activity regeneration will occur, slowly perhaps, but always with certainty. Atrophy of the gluteals from subacute and chronic affections of the hind legs, of the shoulder and pectoral muscles from lamenesses in the fore extremities, of the masseter: firom lesions in the inferior maxillary, are so many examples of this form of atrophy in the large domestic animals.

(3) Pressure.-If any part of the organism is submitted to constant pressure not sufficiently severe to provoke necrosis, atrophy of the compressed part will result. The pressure may be mechanical upon the surface of the body or that of encroaching tumors upon certain internal organs.

(4) Centric Paralysis.-The atrophies caused from centric paralysis are not common in animals, because they seldom survive the primary affection. The stroke of apoplexy that is followed by a general unilateral atrophy is se dom seen in animals because death occurs during the original trouble or soon after, from complications. The muscles of mastication and deglutition sometimes waste rather rapidly from bulbar paralysis in the horse, and those of the hind extremities from a paraplegia of spinal origin, in the dog and hog. Otherwise central lesions are of little import in the study of atrophy of domestic animals.

SYMPTOMS.-Atrophy is recognized by the small size of the affected organ when compared with the normal. In the muscles it appears as an excavation in the affected part, exposing the skeletal apophyses. In the hips it gives the animal the appearance of being "lop-sided," and exposes prominently the angles of the ilium, the great trochanter, the 
ischial tuberosity, and the sacral spines. In the shoulders, the spine of the scapula, the coracoid process, the external tuberosity of the humerus are prominent. In the head, the bony outlines of the jaws are markedly visible; and in addition there is the impaired function due to the weakened state of the shrunken muscles.

In atrophy of internal organs, the physiologic activity is correspondingly reduced, and as a consequence gives symptoms according to the particular organ affected. In paired organs, the normal one will undergo compensatory augmentation in size and function.

TREATMENT.-Atrophy due to peripheral paralysis requires the restoration of the nerve's function before there will be any perceptible amelioration of the condition. If the function of the nerve has been destroyed beyond repair, the atrophy will be permanent. No form of treatment is capable of making the least impression upon the wasted organ that would not also restore the function of the nerve, and since the progress of nerve regeneration is one that is but little inflienced by either external applications or internal medicines, it is very evident that there is no very effectual treatment for this form of atrophy. If the nerve is only bruised, if only a part of its axones are injured, or if the trunk is not injured or diseased beyond repair, the function of the nerve will sooner or later be restored, and with that event the muscle will promptly resume its normal size. Friction, massage, electricity, setons, blisters, liniments, internal administration of strychnia and potassium iodide are useful only where policy dominates the situation. Exercise that will encourage general good health while the nerve regenerates, and then friction, massage, etc., to stimulate the growth of the muscle tissue thereafter is the appropriate line of treatment to pursue. It is often noted that the atrophied process remains tenaciously obstinate for several months or more, and then 
rather suddenly the muscle regeneration will begin and then continue until completely restored to the normal size. In crural paralysis of azoturia it does not matter how much local or systemic treatment is given, the atrophy will persist until a certain time (which is often as long as seven to nine months) and then, (the nerve function being restored) the muscles will show a decided tendency to "fill up" vacant excavation.

In atrophy from inactivity of local muscles due to lameness, the cause must first be removed before there is any possible help for the shrunken muscles. Exercise is the best remedy. In very lame animals the paddock, the pasture and the loose box are preventives of no small value. External applications to muscles wasted from this cause are ineffectual, and only add more torture to the suffering animal.

Pressure atrophy is corrected by removing the pressing object, tumor, collar or whatever it may be. 


\section{CHAPFER V.}

\section{BACTERIA.}

HISTORICAL.-By unveiling the mysteries of fermentation, and upsetting the theories of spontaneous generation that had gained root during the last half of the nineteenth century, Pasteur earned the welldeserved title of "father of bacteriology." As early as $185^{8}$ he established the facts that have proven to be the foundation of a new science, although prior to that date various investigators had demonstrated the existence of the minute forms of life and their supposed connection with disease, decomposition, fermentation and putrefaction. As early as 1675, Leeuwenhoek, discovered infusoria in saliva and feces with the aid of a crude magnifying instrument. In the early part of the nineteenth century Gay-Lussac experimented with fermentation, and in I837, Schwann found that alcoholic fermentation occurred in the presence of the yeast plant. These facts were, however, very slowly accepted by the scientists of the succeeding years, and it remained for Pasteur to prove their exactness, and to show the relations of micro-organisms to disease. About the middle of the nineteenth century Davaine found the bacillus anthracis in the carcasses of animals that died of anthrax. This was prior to Pasteur's discovery, but Davaine failed to disprove the fast-gaining theory of spontaneous generation. In fact, his discovery of the bacillus anthracis seemed more to awaken new interest in the erroneous supposition, until Pasteur's demonstrations unveiled the mystery of disease-producing bacteria. But the crowning event in the history of bacteriology,-especially surgical bacter- 
iology,-was the demonstrations of Sir Joseph Lister in the treatment, or rather the prevention, of wound diseases. These investigations began in 1865 and were perfected by investigations from time to time, until the present day. Lister proved that the miserable behavior of wounds was due to irttruding microbes, and that by excluding them the healing occurred by the simple, uncomplicated process that is today known as "reparative hyperæmia," or simple inflammation.

While the evolution of bacteriology cannot be discussed at length here, it is only fair to recognize the valuable work of Guerin, Tyndal, Kircher, Klebs, Koch, Behring, Weigert, Gram, Kitasato, Nicolaier, Shultz, Rosenbach, Arloing, Chaveau, Moore, Bollinger and many others, all of whom have added much to the store-house of modern bacteriological knowledge.

At the present day, however, many questions remain to be solved, and although a great number of specific bacteria have been discovered, much remains to be learned as to the therapentic management of the diseases they produce. And besides, there are still many diseases known to be of bacterial origin, in which the specific agent defies discovery in spite of the modern methods of investigation and the many instructive precedents to follow. Although bacteriology has given us but few cures, it has furnished many an impressive lesson in prophylaxis. Diphtheria antitoxin is really the only specific curative weapon it has furnished to date. Mallein is credited with marked curative properties in equine glanders, and tuberculin is said by many to possess analogous effects in human and bovine tuberculosis, but additional data seems necessary to establish these facts beyond doubt. Anthrax, rabies, glanders, symptomatic anthrax, tick fever and tetanus are prevented by artificial immunization, and disinfertion, isolation, quarantine and hygiene, owing to a better knowledge of etiology, can be more intelligently utilized. 
In surgery, bacteriology has accomplished wonders, by the one simple discovery of the cause of suppuration and its allies. Septicæmia, pyæmia, sapræmia, etc., are universally prevented by the simple process of excluding the causative bacteria from intruding into the surgical trauma, and by rendering them innocuous in the wound accidentally sustained. These valuable data owe their existence to the rapid evolution of the science of bacteriology, under the directorship of many earnest and faithful martyrs to the cause.

In diagnosis much has been accomplished by the science of bacteriology. Tuberculin and mallein are fully recognized as valuable agents for the diagnosis of tuberculosis and glanders in their most obscure phases. Their exactness for this purpose is no longer questioned. Besides these two examples, there are now many standard methods of diagnosing mycotic diseases by bacteriological investigations. In the human being diphtheria is promptly recognized by the examination of bacterial cultures obtained from the throats of the infected patients, and pulmonary tuberculosis is readily rliagnosed by a simple method of examining the sputum expectorated by the patient. In animals, while the bacteriological investigation cannot be said to have come into general use for the diagnosis of any of the mycoses, except in the experiment stations and colleges, there is a broad field open for such methods of diagnosis. By the intelligent application of the present knowledge about bacteria, many fruitful investigations are possible in diseases that remain a mystery to the perplexed practitioner. In dealing with the wellknown diseases the practitioner may usually accomplish satisfactory results from the clinical symptoms or from the post mortem examination, but too frequently, outbreaks of rare endemic and very mysterious diseases, present themselves for solution, in which neither the clinical aspects nor the post mortem will disclose their true nature. Here the 
bacteriological investigation becomes the essential and often the only means of arriving at any definite conclusions.

DEFINITION.-A bacterium is a minute, unicellular thallophyte (a vegetable without a distinct root, stem or leaf). Gould defines bacterium as "a genus of schizomycetous fungi (fission fungi) characterized by short, linear, inflexible, rod-like forms. The word 'bacteria,' as now employed, is practically synonymous with schizomycetes, micro-organisms, and microbes." More recently the exactness of including the bacteria among the fungi has been questioned. owing to the fact that some of them contain chlorophyl, and are therefore neither algæe nor fungi, but belong to a group by themselves. The classification of bacteria with fungi is regarded by botanists as purely an arbitrary one.

DISTRIBUTION.-Bacteria have an exceedingly wide distribution throughout the world. They are almost everywhere. They are found in food, water, dust, clothing, soil, tools, utensils, instruments, animal feeds, vegetables, dust of dwellings and stables, street dirt, the surface and the open cavities of the animal body, diseased organs and tissues within the body, the excrement of man and animals, etc., etc. In fact, everything external harbors them and very frequently they find their way into the remotest recesses of things animate and inanimate.

On the living healthy body of domestic animals microorganisms are found in abandance, on and within the recesses of the skin, in the mouth, pharynx, stomach and intestines. These organs are capable of harboring pathogenic microorganisms that are ever ready to produce serious derangement when the proper opportunity presents itself. The respiratory tract always contains micro-organisms that under ordinary conditions of health are harmless, but which are capable of generating a serious morbid phenomenon if the tissues harboring them become weakened from any cause. 
The vagina, the uterus, the teat ducts, the conjunctiva, and the external ear contain them.

Bacteria, however, are not found in the living, healthy tissues and humors of the body. The tissues other than the skin and mucous membranes are free from micro-organisms except in disease. The blood, the synovia, the serum of the great cavities, and the cerebro-spinal fluid do not contain micro-organisms in health, and even in mycotic diseases these liquids, including the blood, do not often abound with them. During the progress of disease the micro-organisms entering the blood stream are promptly disposed of by the resisting forces and emunctories of the body. It is only as death approaches, when the resisting forces are depressed and exhausted, that microbian flora flourish within the body. After death when the resistance is entirely removed, all the tissues abound with them.

Air itself is free from micro-organisms, but it is kept more or less constantly contaminated by the dust and flying particles it is capable of containing at all tines in closely inhabited districts, in buildings, streets, etc. The air of the distant prairies, of the pine forest, the mountain, and in sparsely inhabited regions in general is comparatively free from pathogenic microbian agents. "Wherever man, animals or even plants live, die and decompose, bacteria are sure to be present" (McFarland). Decomposing organic matter, cesspools, manure-heaps, dead animal-bodies, seethe with them. The upper layers of well-tilled soil abound with all kinds of microbian flora, but the lower layers are relatively free from them owing to the absence of the nitrogenous food necessary for their support. Water of the city hydrant, the dug well, rivers, creeks, ponds, lakes, etc., is seldom ever free from bacteria. They are, however, not found in the water of deep, drilled wells and springs that are not contaminated from the surface. 
CLASSIFICATION.-Morphologically, bacteria are divided into Cocci or spheres; Bacilli or rods; and Spirilla or screws.

Cocci.-The coccus, or micrococcus, has a round or ovoid contour, and presents several different forms of growth which are subdivided into: diplococcus, when arranged in pairs, streptococcus, when arranged in chains, and staphylococcus, when arranged in clusters. When a second division of the diplococcus occurs, thus making a group of four, the tetracoccus is formed, and when the development continues until a bale-like mass is formed the micro-organism is called sarcina. Cocci are also divided into flagellated and unflagellated, the former being a rather rare variety.

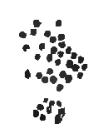

1

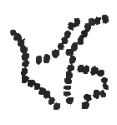

2

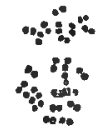

3

FIG. 1.

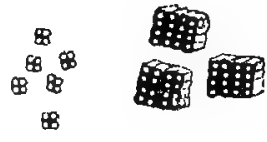

4

Various Forms of Cocci. 1, Staphylococci ; 2, Streptococçi ; 3, Diplococci ; 4, Tetracocci; 5, Sarcinae.

Bacilli.-The bacilli (rods) are staff-shaped or rodshaped, and consist of elongated cells. They vary greatly in shape, from elliptical forms to long slender threads, and in size, from very small to very large bacteria. Some have rounded extremities and others square ones. They divide by transverse fission. There are both flagellated and unflaggellated varieties.
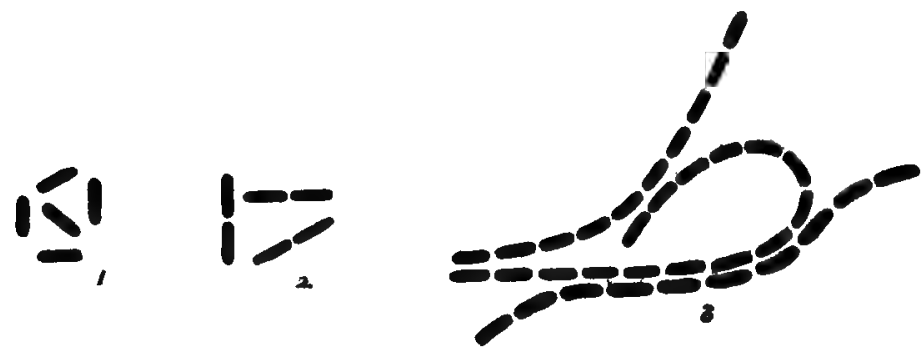

FIG. 2.

Forms of Bacilli. 1, Single Bacilli ; 2, Bacilli in Pairs ; 3 Bacilli in Threads. 
Spirilla.-The spirillum is a bacterium shaped like a corkscrew. Some are short, like bent bacilli, while others are long and slender. They appear in both the flagellated and unflagellated forms.
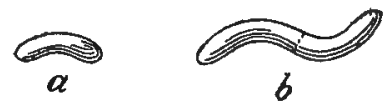

FIG. 3.

a, Vibrio; b, Spirillum.

According to their function bacteria may be divided into the following important classes.
a. Saprogenic.
f. Photogenic.
b. Zymogenic.
g. Pathogenic.
c. Aerogenic.
h. Alkali-forming.
d. Acid-forming.
i. Nitrifying.
e. Chromogenic.

a. Saprogenic bacteria are those which produce putrefaction. These bacteria decompose the complex nitrogenous compounds into simpler chemical combinatiohs. They transform albumen into peptones and then split up the latter into salts, acids and gases, and sometimes into very poisonous alkaloidal compounds called ptomains. Example, bacillus tremulus.

b. Zymogenic bacteria are those which produce fermentation. These micro-organisms possess the power of reducing the complex molecules of non-nitrogenous matter (e. g. carbohydrates) into simple compounds. Examples, bacillus aceticus, bacillus butyricus, etc. These bacteria are of much more importance in the study of chemistry, physiology, and physiological chemistry than in pathology.

c. Aerogenic bacteria are gas producing bacteria. During putrefaction and fermentation various gases are elaborated, among which are carbonic acid, hydrogen, hydrogen sulphide, ammonia, etc. Gas production is the property of many bacteria, but the process may continue unnoticed unless the decomposition is occurring in a confined place-the 
depth of a solid culture media, a covered vessel, or deeply in the recesses of the living tissues. Examples, bacillus aerogenes capsulatus, bacillus œdematous maligni, bacillus anthracis symptomatici, etc.

d. The formation of acids is the property of many microorganisms. Acetic, lactic and butyric acid fermentation is created in the culture media by bacteria bearing the names of these acid products. In most instances the generation of acids continues until the media becomes untenable for further growth of the microbes, which then die in their own products.

e. Chromogenic bacteria are color producing bacteria. These micro-organisms are largely non-pathogenic, yet some are capable of producing disease. The various pigmentforming bacteria are capable of producing practically all of the known colors, red, yellow, black, green, blue, lemon, etc. Examples, staphylococcus pyogenes aureus, bacillus pyocyaneus, bacillus prodigiosus, etc.

f. Photogenic and Other Bacteria.-Besides the roles played by the preceding classes of bacteria, photogenesis and other functions might be enumerated, but these are of but minor import in the special study of surgery. The production of phosphorescence-light-is the property of numerous micro-organisms, notably the bacillus phosphorescens. The light-producing property is often so active as to dimly illuminate the interior of the incubator.

g. Pathogenic bacteria, or disease producing bacteria, are the most important of the micro-organisms to the pathologist. All other forms are included in the great group called non-pathogenic bacteria. A distinct line of demarcation cannot, however, be drawn between these two groups. Some bacteria are pathogenic to all warm blooded animals and yet incapable of producing any derangement in the cold blooded. Some will kill in a certain genus and prove per- 
fectly innocuous in others. Some will kill the weak and prove harmless to the strong, etc. However, any bacterium that is capable of producing derangement of the living cells deserves to be classed among the pathogenic micro-organisms, whether the lesion produced is a simple local phenomenon in a particular species, or a grave or fatal malady in another. Besides the variable disease-producing quality seen in different species, and under different conditions in the same species, no two given samples of the same micro-organism possess the same pathogenic capacity. The virulence of microbian cultures is variable. No two inoculations, experimental or accidental, will produce precisely the same degree of disease. Various influences are capable of attenuating micro-organisms to the point of enfeebling and even destroying their pathogenic powers. Unnatural environment, heat, cold, desiccation, prolonged exposure, their number, the point of entrance, etc., are among the conditions which modify their virulence. Furthermore, the pathogenicity of many micro-organisms is materially increased by inoculating them with other bacteria-favoring bacteria. The tetanus bacillus is harmless unless associated with other microbes; the bacillus of malignant œdema may be increased in virulence by inoculating it with the bacillus prodigiosus, etc.

The disease-producing power of bacteria depends upon various properties which differ with the different forms. The pathogenicity, however, largely depends upon their ability to elaborate poisonous products-toxins, toxalbumins or bacterio-proteins, which are absorbed in sufficient quantities to intoxicate the organism. "The intoxication is, however, but a part of infection which consists of the sum of all the vital phenomena manifested by the bacterium in its parasitic life." (McFarland.) The toxins are not all thoroughly understood. They are unstable substances, with few exceptions. that are readily destroyed by exposure to light, air. 
heat, cold, etc. Some are highly poisonous, as tetanic toxin, while others may be tolerated in relatively large quantities, or be successfully eliminated by the emunctories, as in animals recovering from septicæmia.

Bacteria may also produce disease by mechanically obstructing the capillaries, by absorbing the oxygen essential to the construction of tissues, by impairing the function of vital organs, and by kindling exhausting fevers; all of which might occur independently of the absorption and dissemination of the toxins which most micro-organisms elaborate. Some bacteria, in fact many, possess a combinedi action of provoking organic disturbance, intoxicating the system, producing fever, and blocking the construction of tissues, all of which in their totality determine grave disorders. Others have strictly a local action, or at least a local effect, which predominates over the slight systemic disturbance. In animals the pyogenic cocci are capable of producing local lesions of considerable magnitude without causing much systemic disorder, and only produce death through the constant and exhausting drain of prolonged suppuration. Tetanus bacilli, on the other hand, produce scarcely perceptible local lesions, and yet may inject the system with a fatal dose of poison.

The number of micro-organisms inoculated in any given infection has a patent influence upon the pathogenicity of the microbe. While it is true that a single bacterium by multiplying may reproduce numbers in the course of several days that would seem improbable on mathematical calculation, it is, nevertheless, proven over and over that unless a sufficient number of them are injected the results are usually negative. It seems that they fall an easy prey to the englobing phagocytes, and besides, in numerous instances it has been shown that specific micro-organisms, unassisted with other associated (favoring) microbes (which seem to 
play the role of battling with the phagocytes while the specific agent elaborates its toxin) are incapable of exhibiting any pathogenic powers sufficient to overcome the resisting forces of the body. Rogers has shown that it requires a certain given number of tuberculous bacilli to kill a cavy and analogous facts with otber bacteria are legion.

The channel of infection also has much to do with determining the pathogenic quality of many bacteria. Microbes that will produce pneumonia when introduced into the lungs may cause but a local suppurating process when inoculated elsewhere in the body. The bacillus mallei when ingested or inhaled will produce an entirely different clinical tableau if inoculated into the skin. The bacillus tuberculosis may cause consumption when inhaled. When ingested it is capable of provoking entirely different localizations and again, if inoculated directly into a trauma a still different picture is produced. Pyogenic cocci entering the umbilicus provoke a grave polyarthritis and when injected subcutaneously a simple and even trivial abscess is the only result. These examples might be enumerated in considerable numbers, but the foregoing are sufficient to establish the fact that the avenue of entrance is a marked determinating factor in regard to the pathologic behavior of the disease producing bacteria.

Bacteria seem entirely incapable of traversing the epithelial lining of the skin or mucous membranes, in the absence of any breach of continuity. The skin or mucosa must first be injured or diseased before the bacteria can pass their protecting epithelial coating. A mere scratch of a pin, a disturbed hair follicle, a slight erosion of a mucous membrane, trivial irritation, congestion or inflammation, or a morbid process of whatever character is sufficient to admit them. In a large percentage of instances the bacteria remain quite persistently localized at the seat of infection or its im- 
mediate environs, spreading out, by multiplication, directly through the tissues. In their local habitat they elaborate toxins which are absorbed by the usual absorbing channels of the tissues, and then diffused throughout the system. But bacteria themselves are capable of entering the circulation and of localizing at points of the body quite remote from the seat of initial infection. The exact manner by which they gain entrance into the blood stream is not definitely settled. They may be taken up by the lymph vessels from the lymph spaces of the infected focus; they may enter the capillaries when the pressure of the inflammatory exudates exceeds the blood pressure; or they may enter the veins directly by first producing phlebitis and then traversing through the three consecutive layers which are more or less dissolved, separated and weakened by the morbid process; and finally they may be englobed by leucocytes which carry them into the current by their amcboid movements.

h. The formation of alkalies, notably ammonium, occurs in many microbic cultures. The gas either escapes into the surrounding atmosphere or else combines to form salts of ammonia. Like acid fermentation the production of abundant alkalies inhibits the growth of the microbes that produce it.

Many bacteria are capable of producing the well known odors of hydrogen sulphide and ammonium, while most of them emit odors peculiar to themselves, some of which are pungent and unpleasant while others are aromatic and agreeable. The production of odors is characteristic of most all bacteria.

i. Nitrifying bacteria.-Many micro-organisms possess the property of reducing nitrogenous compounds into sim1. ler combinations. In the cultivated soil they perform the important function of breaking up nitrogen compounds into combinations assimilable by plant life, and of gathering 
nitrogen from the air and combining it into compounds useful for both vegetable and animal life.

Many micro-organisms have the property of peptonizing the casein of milk. Some digest casein without changing the appearance of the milk, some coagulate it, some gelatinize it, while still others reduce it to a watery fluid. (More extensive works on bacteriology must be consulted for a more comprehensive study of these functions.)

The bacteria belonging to each of these classes may or may not produce disease, which fact, in the study of pathology, necessitates the division of all bacterial forms into two great classes, viz., (I) Pathogenic bacteria, or those which produce disease, and (2) non-pathogenic bacteria, or those which do not produce disease.

According to the oxygen required for their growth, micro-organisms are divided into three important groups: aerobic bacteria, anaerobic bacteria, and facultative anaerobic bacteria. It must be remembered that all micro-organisms require oxygen for their propagation. Oxygen is indispensable to all living things, and the bacterial forms are no exception. This classification refers to the requirement of free oxygen only.

Aerobic bacteria inclucle all those forms that will thrive in the presence of free, uncombined oxygen atoms. Examples, bacillus tuberculosis, bacillus diphtherix, bacillus sul)tilis, etc.

Anaerobic bacteria include all those forms which cannot thrive or even grow in the presence of free oxygen. To these micro-organisms free oxygen is a deadly poison, and will either destroy them or prevent their successful reproduction. Examples, bacillus of Nicolaier, septic vibrion, bacillus butyricus, etc.

Facultative bacteria, facultative-anaerobic bacteria or 
optiona!-anaerobic bacteria are equally appropriate desigilations for this variety, which possess the property of growing either with or without free oxygen. These forms are capable of thriving when exposed to the air or when hidden in the recesses of a sheltered environment. Examples, the pyogenic cocci, the typhoid bacillus, cholera spirillum, etc.

According to their place of abode micro-organisms may be classified into: saprophytic bacteria and parasitic bacteria. They may even be designated by the simple names of saprophytes and parasites.

I. Saprophytic bacteria are those which cannot live in the living tissues, but depend upon decaying organic matter for their existence. The purely saprophytic micro-organism is incapable of existing in the living body of animals and is devoid of harmful action, while others may, under certain conditions, elaborate a poison and, intoxicate the system. The fact that certain bacteria are only encountered in the lesions of disease, and that others are only found in extraneous abodes, does not conclusively show that a distinct line of demarcation can be drawn between these two classes. It is well known that many parasites may thrive as saprophytes, which fact necessitates the grouping of nany important bacteria into a third class which might appropriately be called optional bacteria. This group would then include such highly important micro-organisms as the bacillus of Nicolaier and the septic vibrion actinomyces bovis and probably the bacillus of glanders, which is known to live about the stable watering trough indefinitely.

2. Parasitic bacteria include those micro-organisms found only upon or within the living body of animals. There are many microbes that up to the present time have not been found except in the lesions which they have produced. Nothing is known of their exogenous existence, 
and hence they deserve to be classed as strictly parasitic bacteria. This class includes all of the micro-organisms that remain closely associated to the living body of animals and only produce disease when a favorable opportunity presents itself. For example, the staphylococcus pyogenes aureus will live upon the skin without harm until a favorable wound is sustained, under which circumstance it will promptly and quite certainly provoke suppuration and even more grave conditions. The pneumococcus may be harmless in a healthy bronchiole, but is capable of causing fatal pneumonia if the environment is made suitable by exposure or debility.

MEASUREMENT OF MICRO-ORGANISMS.-Bacteria are too minute to be conveniently measured by the ordinary commercial scale of dimensions. A special unit has been adopted for this purpose. The unit applied to the measurement of bacteria, is the micro-millimeter or micron, which is the equivalent of one twenty-five thousandth of an inch, or one-thousandth of a millimeter. Its symbol is $\mu$.

In mental calculations of the size of bacteria during microscopic examinations the red blood cell is largely used as the unit of measurement by practical microscopists.

S*me bacteria are but a fraction of a micro-millimeter in length, while others reach a length of forty or more. The measurement is obtained by the eyepiece micrometer, the manipulations of which must vary according to the construction of the instrument. The operator should follow the dealer's instructions which accompany such instruments.

REPRODUCTION.-Bacteria propagate by fission (binary division). 'That is to say, each bacterium divides into two separate beings, by which process an appalling number may be reproduced in a relatively short time. If the process of fission continues unrestricted the number of bacteria resulting, even from a single germ, could scarcely be 
estimated, but the growth is usually arrested either by the exhaustion of the nutrition or by the repressing effect of metabolic products they elaborate in the environment.

In addition to reproduction by fission some bacteria form spores which ripen, split open and liberate the young organisms. The spore develops within (endospore) the bacterium, which degenerates as the spore enlarges toward maturity. The process is called sporulation, and the microorganism reproducing itself by this process is referred to as a sporogenic bacterium. Sporulation is one of the processes by which bacteria preserve their existence when threatened with destruction. It is not their usual mode of reproduction. The newly-hatched bacterium liberated from the spore develops into its characteristic form and then proceeds at once to reproduce itself by fission. Sporulation is a mode of self-preservation, while fission is the real mode of reproduction.

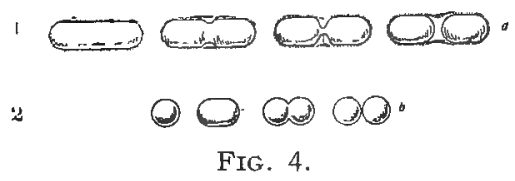

Bacilli (1) and Cocci (2) Undergoing Binary Division.

The formation of arthrospores, which consists of the transformation of the entire bacterium into a spore, is a mode of self-preservation that is known to exist in some few species of cocci and small bacilli. It is a transformation forced upon a bacterium through the influence of an environment unfavorable for its preservation.

CULTIVATION OF BACTERIA.-In the study of any given bacterium it is absolutely essential that it be separated from its associates and a pure culture obtained; otherwise its special characteristics cannot be -accurately determined. One organism may destroy the products of another, will change the reaction of the media, and will in various ways so inhibit the propagation of the miçrobe under surveil- 
lance as to render accurate deductions impossible. The microscopic examination alone would accomplish but little in the study of bacteria. It is the study of the living germs, their mode of life, their behavior in the media, the poisons they elaborate, and the experimental inoculation of the artificially cultivated bacteria that unfold their true pathogenic powers and differentiate one from another. The study of bacteria artificially grown in vitro, followed by their experimental inoculation in vivo, has brought the science of pathologic bacteriology to its present status. Numerous mixtures have been used for the cultivation of bacteria, a few of which have become standard preparations. For the pathogenic micro-organisms it seems essential that the composition of the media approximate the juices of the body. They should contain about 80 per cent of water and be of neutral or feebly alkaline reaction. Transparency is a desirable quality as it gives a better opportunity of observing the various changes which the media undergo under the influence of bacterial growth. Solid media are preferable to liquid under many circumstances, especially when isolating the different varieties of bacteria and transplanting them to fresh media until the bacterium has been passed through several successive generations. The bouillon culture fluid is the artifice of Pasteur, while the idea of solid media originated in the laboratory of Koch.

All culture media must be absolutely sterilized as a pre-requisite to the successful cultivation and isolation of, bacteria. The materials from which they are made contain bacteria that must first be eliminated. The feat is accomplished by hoiling, steaming, chemicals, fil tion, cold, and by dry heat with the aid of specially improvised apparatus. When thus prepared the media is transferred into specially prepared, sterilized vessels and inoculated with the infectious matter to be cultivated by means of the 
sterilized platinum needle, pipette or other instrument. When the infectious matter is inoculated into a solid or semi-solid medium, the name stab-culture is applied, and when spread over the surface the process is designated as smear-culture.

The mixture thus inoculated is then submitted to a temperature which must vary according to the particular requirements of the micro-organisms-under investigation, which is usually $37^{\circ} \mathrm{C}$. The incubator is usually necessary for this purpose, as by its use the temperature can be changed or maintained at a certain degree of heat at will. Often cultures will grow well in ordinary temperatures of a room, but high temperatures,--blood heat,-are more favorable to the exuberant growth of bacteria in artificial media.

At a given time, varying with each particular bacterium, the macroscopic and microscopic developments furnish important revelations for differentiation. The medium may be peculiarly changed, may emit a special odor, may be liquefied or not, may be cloudy or clear, may contain a precipitate, or finally it may have developed a characteristic color. An accurate knowledge of these macroscopic changes is no small part of the successful investigator's attainments. A subsequent microscopic examination of the microbian flora oi the culture, the inoculation of fresh cultures to obtain new generations of the microbe, and finally inoculation experiments upon living animals completes the investigation

The anaerobic bacteria must be cultivated in the absence of free oxygen. This is accomplished by excluding air, by means of various mechanical devices, by cultivating them in the presence of inert gases-hydrogen-or by creating a vacuum in the container.

The more common culture media are bouillon, potato, agar-agar, and blood-serum. Gelatin, gelose, milk 
ancl various alkaline and saccharine mixtures are also used for special purposes.

In addition to these media, all of which must be prepared with due respect to certain rules, there are others which are required to meet the peculiar demands of certain bacteria. A detailed description of the preparation of culture media cannot be undertaken here. The reader is referred to works on bacteriology, which cover the essential details.

\section{MICROSCOPIC EXAMINATION OF BACTERIA.}

When it is possible the bacteria should be examined in the living state, which then reveals their actual shape, size, motility and grouping. When a complicated process of preparation is found necessary, as is often the case, the bacterium may present a totally unnatural appearance. Drying, fixing, boiling, steaming and staining, although absolutely necessary processes of preparation in many instances are capable of giving an entirely wrong impression of the real appearance of any given bacterium. When these preparatory steps are used, due allowance must always be made for the changes they are certain to bring about.

The simplest microscopic examination of bacteria is accomplished by placing a drop of the culture on the slide, covering it with the corer-glass and then focusing the instrument upon it. This method answers only for immediate examination; the evaporation of the solution destroys it for further use. The one chief objection to this simple process is that the movement of the fluid under the cover-glass might be mistaken for the motility of bacteria.

The hanging-drop is by far the best method of microscopical examination of bacteria. By this method they are actually observed in their natural abode. They can be examined as regards shape, size, motility, grouping, fission, sporulation with the greatest accuracy, and for several days 
until the nutrition is exhausted. The process requires a hollow-ground slide, a large cover-glass and a bit of vaseline. The vaseline is penciled around the concavity of the slide, and a drop of the culture placed upon the center of the cover-glass, which is then inverted over the concavity of the slicle. The drop should hang into the concavity without touching the bottom. It is advisable to examine the edges of the drop rather than the center, which is usually too thick. Cultures grown in solid media may be examined in this manner by first mixing a small portion of the culture with some sterilized fluid.

STAINING.-The necessity of coloring bacteria to identify them from other particles on the slide was recognized during the earliest days of bacteriology. Carmin and hematoxylin were used until Weigert demonstrated the virtue of the aniline dyes and their remarkable affinity for bacteria. The number of these dyes is very large, but only a few are in common use; in fact, two or three simple stains will demonstrate all the bacteria ordinarily encountered, and aid in their diagnosis.

Most of the bacteria have an affinity for basic dyes such as methylene-blue, gentian-violet, fuchsin, etc.; a very few stain better in an acid solution, e. g., the tubercle bacillus. They vary in their capacity for taking up the stain. The glanders bacillus stains slowly, and therefore requires an intensive dye like gentian-violet; in case the bacteria are associated with blood, pus, milk, etc., methylene-blue is better to use, as it does not readily over-stain. For all general staining methylene-blue is the best to begin with; if necessary, certain differential stains can be applied later.

It is of the greatest importance that the stains used should be good. Grubler's are the best. These should be obtained in powder form and made up into saturated alcoholic solutions from which small amounts of the aqueous 
solutions can be made from time to time. The aqueous solutions have a tendency to decompose, especially the gentian-violet; the alcoholic keep indefinitely.

If the smears have been made from blood or pus, where histological elements also claim attention, first stain the cellular protoplasm with a $\mathrm{I} \%$ aqueous solution of eosin. then use the methylene-blue as above described. This method not only demonstrates the bacteria, but also differentiates the various kinds of tissue-cells which may be present.

In case the urine is to be stained, some difficulty is usually experienced in making the smear adhere to the slide, on account of the numerous salts and small amount of albumin present. Let the urine settle first, or centrifuge it; then wash the sediment with distilled water several times. The sediment then forming is fairly free from the salts of the urine and can usually be fixed to the glass with heat. If not, a little albumin-fixative first applied to the glass will ensure good fixation.

Staining with gentian-violet is the same as with methylene-blue, except that the time of staining is shorter. This dye is so penetrating that its tendency is to over-stain and hence should be applied for two or three minutes only. A fresh aqueous solution should be made up at time of using. The old solutions in water are apt to be decomposed. All the staphylococci and the glanders bacillus are better stained with gentian-violet.

Of the various staining-methods, three principal ones will be described: the common method, the Ziehl-carbolfuchsin stain for tubercle bacilli, and Gram's method.

The ordinary method of staining necessitates the use of either meth vlene-blue or gentian-violet. Take one part of the saturated alcoholic solution and nine parts of water. 
This is the regular staining solution, and a simple technique is as follows:

I. With a platinum loop spread a thin layer of the specimen on a clean cover-glass. Dilute specimen with distilled water if too thick.

2. Air-dry by allowing the cover-glass to remain in the air until absolutely dry.

3. Fix by passing cover-glass three times through a Hame, either a Bunsen burner or alcohol lamp.

4. Apply stain to the cover-glass and allow to remain from 3 to 5 minutes.

5. Pour off stain and wash cover-glass in water to remove surplus stain.

6. Dry cover-glass with filter paper; add a drop of Canada balsam to the center of the stained side of the covergiass.

7. Mount cover-glass on slide, turning the loaded slide down.

8. Label, add a drop of cedar oil, and examine under the one-twelfth lens.

The foregoing method can be used in the staining of bacteria from culture media, or in the staining of pus, excretions or secretions, or in the examination of any organ or tissue for bacteria. Not only are the bacteria stained, but the blood-cells and epithelial cells also.

Examination of pus by this method would not only reveal the character of the bacteria present, but also the nature of the pus cells. In cold abscesses these would consist largely of lymphocytes together with broken down tissue-cells; while in acute abscesses, the pus cells are mainly of the polymorphonuclear variety. This method will stain all bacteria except the tubercle bacillus.

Tubercle bacilli do not take the ordinary stains, but when once they do take a stain, they give it up with great 
difficulty. Ziehl's carbol-fuchsin is the stain generally used for tubercle bacilli and its formula is as follows:

Fuchsin Powder ............. gm.

Absolute Alcohol .............. Io c.c.

Carbolic Acid c. p. ........... . . c.c.

Distilled Water q. s. ........... roo c.c.

Filter clear. This solution will keep for years. The technique of using it in staining is as follows:

I. Spread a thin layer of the naterial on a cover-glass.

2. Air-dry.

3. Fix in a flame.

4. Apply carbol-fuchsin to the cover-glass, and hold over a flame until steam arises.

5. Pour off the stain.

6. Dip rapidly into a $10 \%$ solution of nitric acid.

7. Wash freely in $95 \%$ alcohol until the specimen on the cover-glass is colorless.

8. Stain again with a watery solution of methylene-blue for 3 minutes.

9. Wash in water, dry with filter paper, and mount in Canada balsam.

Io. Add a drop of cedar oil and examine under the onetwelfth lens.

By this method of staining, the tubercle bacilli, if present, will appear as short red rods. All other bacteria and tisstue-cells will be stained blue.

There are a few other bacteria that retain the carbolfuchsin stain after being treated with acid and alcohol, but these are not met with in general work. They are the leprosy, smegma, and Lustgarten bacilli.

Gram's method of staining has the advantage that by its use, certain bacteria are stained a violet color, and other bacteria are not stained at all. Two solutions are necessary in using this stain: (I) Lugol's solution and (2) aniline oil gentian-violet. 
I. Lugol's Solution or Gram's Solution:

Iodine ............ gram

Potassium Iodide......2 grams

Water ........ . IOO c.c.

2. Aniline oil gentian-violet:

Aniline water ( 2 c.c. of aniline oil to 50 c.c. of water-filter clear) .............. ro c.c.

Gentian-violet (saturated alcoholic solution).. I c.c. .

The aniline oil gentian-violet should be prepared just before using, as the solution does not keep. The various steps in using Gram's method are as follows:

I. Spread a thin layer on the cover-glass or slide.

2. Air-dry.

3. Fix in a flame.

4. Stain with aniline oil gentian-violet 5 minutes.

5. Pour off stain and wash in watèr.

6. Apply Lugol's solution 2 minutes.

7. Wash freely in alcohol until no more color is given off.

8. Wash in water, dry, and mount in Canada ba!sam.

9. Examine with the oil-immersion lens.

Eosin or Bismark-brown can be used as contrast stains for the bacteria which are decolorized by this process of staining. After washing in water (step 8), apply either eosin or Bismark-brown for one minute, wash in water again, and mount, etc.

The bacteria "taking" the Gram stain are of a deep purple color; those "refusing," are colored pink or brown, according to the counterstain used-eosin or Bismark-brown.

SURGICAL BACTERIA.-Nearly all of the pathogenic micro-organisms might, on some pretext or other, be classified with the so-called "surgical bacteria." Most all bacteria are capable of entering the body through the avenue opened up by the accidental or the surgical wound, which fact alone greatly enlarges the scope of surgical bacteriology. But 
besides the strictly "traumatic micro-organisms" the surgeon must also respect all the bacteria that might be encountered, or which might need consideration, in one way or another, in the course of surgical treatment of diseases. The surgeon must cope with all bacterial forms that are capable of producing local or systemic derangement by directly invading the trauma and all of those that produce diseases requiring surgical intervention. 'This leaves the domain of surgical bacteriology practically analogous to that of pathogenic bacteriology. The pathogenic bacteria are, however, not of equal interest to each branch of veterinary medicine. The sanitarian, the physician and the surgeon each find that certain micro-organisms are of more importance than others, in the pursuit of their respective missions. The surgeon is particularly interested in the more common wound infections, the physician in the fevers and internal diseases, and the sanitarian in the contagions.

The following list of bacteria are those of special interest to the student of veterinary surgery.

\section{A. PYOGENIC MICRO-ORGANISMS.}

I. STAPHYLOCOCCUS PYOGENES AUREUS.This bacterium, either alone or associated with other similar forms, is the most common cause of suppurative processes in the wounds of clomestic animals. It is the arch-enemy of the veterinary surgeon. Found universally as a parasite on the skin of both the human hands and the skin of animals, it seldom fails to invade every solution in the continuity of the latter. It is a parasite, and from its parasitic abode, it is readily disseminated over every article, around and about a thickly inhabited environment. The skin, the clothing, the working tools, the blankets, the dust of the stables, the surgical instruments, the air of the veterinary hospital, and in fact every exposed article, abounds with this creature. The 
careful surgeon suspects its existence everywhere. It is the common micro-organism of suppuration and is found in the pus of many abscesses. Although suppuration is not a specific disease, the staphylococcus pyogenes aureus, might, with at least some propriety, be regarded as the specific microbe of acute abscess in domestic animals, so frequent is it found in this connection. It was discovered by Ogston, but was first cultivated by Rosenbach, who gave it the name it has retained.

Description.-Non-motile. Non-flagellated. Liquefying.

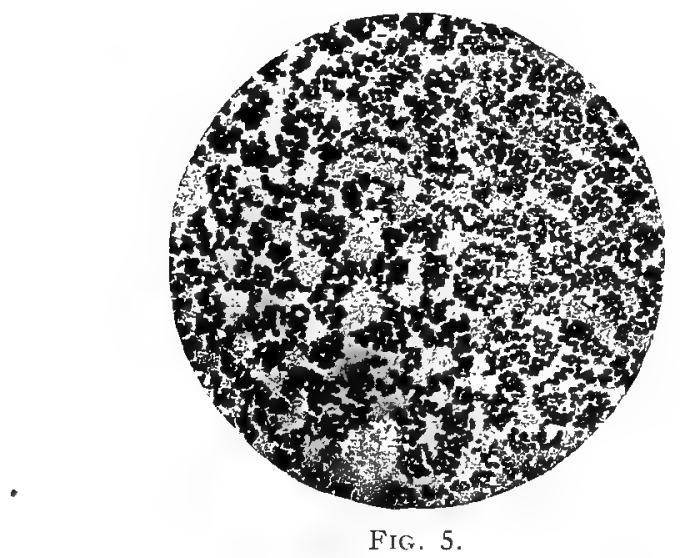

Staphylococcus Pyogenes Aureus from Artificial Culture.

Chromogenic. Aerobic or facultative anaerobic. Nonsporogenous. Size, $0.7 \mu$. Aggregated in masses.

Cultivation.-It can be grown upon gelatin, agar-agar, coagulated blood-serum and potato and in boutlion and milk. Grows readily at ordinary temperatures but more rapidly at about $85^{\circ} \mathrm{Fahr}$. and the normal temperature of the body. It peptonizes albumin, liquefies gelatin and coagulates milk. In solid media, it grows along the tract of the needle and upon the surface, and it imparts a gold-yellow color to the culture by the formation of pigment. It will grow either in the presence or in the absence of oxygen.

Isolation.-The organism may easily be isolated by 
smearing a drop of pus over gelatin or agar-agar. The subsequent cultivation will bring out colonies that are recognized from other forms by their orange color. Other cocci may produce every intermediate color between white and yellow, and thus prove confusing. This feature of the cultures has often led to the belief that the staphylococcus pyogenes albus is but a non-chromogenic form of the same species.

Staining.-The organism stains brilliantly with aniline solutions and by Gram's method.

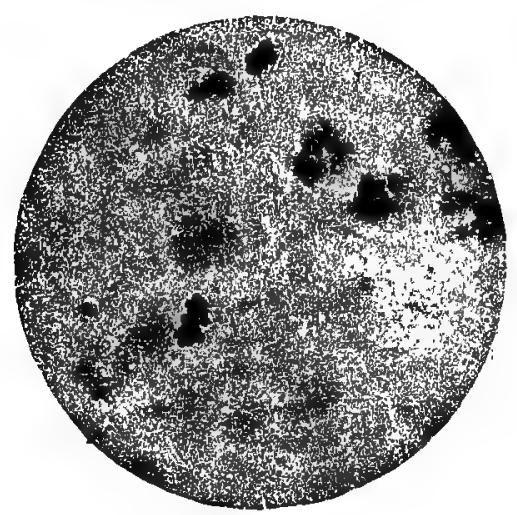

FIG. 6.

Staphylococcus Pyogenes Aureus from Pus.

Pathogenicity.-The staphylococcus pyogenes aureus may be appropriately designated as a deadly organism, although at times it may only cause a rather trivial suppuration. When injected subcutaneously it will promptly cause a collection of pus, and not infrequently it generalizes and causes fatal septicæmia. If the resisting powers of the body are sufficient a sub-acute purulent infection-pyæmia-may result. The golden coccus is more deadly to animals than to man. In the former, especially in the debilitated, fatal generalization is quite certain from any prolonged and active suppuration. The most common cause of death is embolic pneumonia. Coagula containing them, loosen from 
the walls of the veins in the infected area and lodge in the rapillaries of the lungs, at which point new, and often fatal abscesses evolve.

It is also found in superficial inflammations, deep abscesses, furuncles, and ordinary suppurating wounds.

Virulence and Resistance.-Mercuric chloride in solution of $\mathrm{I}$ to $8 \mathrm{I}, 400$ will arrest its growth in the culture media. It is incapable of living without moisture, drying for one hour often being sufficient to reduce its pathogenic properties, but it is not entirely killed after ten days desiccation. It requires boiling of several minutes duration to entirely arrest its growth. The staphylococcus pyogenes aureus must be looked upon as a very durable form of micro-organism, as it is difficult to destroy in the tissues. The ordinary antiseptic solutions have but little effect in permanently preventing its multiplication in a diseased tissue. Its virulence is, however, variable. The variability in virulence seems greater than that of the other well studied micro-organisms. In one instance it provokes a trivial suppuration, -a mere pustule,-while in other cases fatal septicæmia is the result. Even with due consideration for the variability of the resisting powers of the body, it is very evident that the golden coccus is found in many different degrees of virulence. Its pathogenic properties are greatest when taken from the first culture made directly from infected tissues. The cultivation of successive generations greatly attenuates its powers, which may, however, be revived by passing it through the animal body.

Toxic Products.-Leber describes the toxin of pus cocci under the name of phlogosin, a crystalline substance soluble in alcohol and ether. Other investigators have repeatedly shown that sterilized pus is toxic. Mannatti has shown that injections of pus deprived of its living microbes by heat will cause chronic intoxication that simulates the systemic de- 
rangements accompanying chronic suppuration. The toxic product of pus cocci has also been described under other names. Van de Velde called it leukocidin and claimed for it the power of destroying the leucocytes. Wechsberg named it staphylolysin. Ribbert produced lesions of the liver, spleen, heart and kidneys by the injection of sterilized cultures of pus cocci. Experiments along this line all show analogous results from sterilized and unsterilized cultures. It is, however, evident that the staphylococcus elaborates but little, if any, free, extracellular toxin, because filtered cultures, of high virulence, seem incapable of causing any marked reaction.

Antitoxin.-Several laboratories in both America and Europe, widely advertise, and claim extravagant results from, serum derived from streptococci, but the therapists of the present day have not as yet been supplied with an "antistaphylococcus serum." The streptococcus antitoxin is claimed to be potent against suppuration.

\section{STAPHYLOCOCCUS PYOGENES ALBUS.-} This bacterium occurs only as an associate of the aureus, with which it is so frequently found that the two are frequently described under the name of Staphylococci Pyogenes Aureus et Albus. Morphologically they are identical, and they can only be recognized, one from another, by the orange color the auretts imparts to the culture.

Albus is non-chromogenic and but feebly pathogenic, and when isolated, cultivated and then inoculated into animals it is found incapable of provoking any of the grave systemic disorders produced by the aureus. Only a trivial, local phlegmon results from subcutaneous injection of the most highly virulent cultures obtainable. Its extraneous abode is not known. It is found only in pus and then only when associated with the aureus. The fact that the chromogenic function of pus cocci can be changed by various mani- 
pulations would indicate that this bacterium is but a modified form of the golden coccus. It is of no special importance in veterinary surgery.

\section{STAPHYLOCOCCUS EPIDERMIS ALBUS.-} This coccus is described by Welch as an habitual parasite of the skin, in which structure it is often found as deep as the Malpighian layer. Welch believes it to be an attenuated form of the albus. Moore found it impossible to entirely rid the skin of animals of pyogenic cocci and Robb refers to the development of stitch abscesses from catgut inserted into a thoroughly disinfected skin. It is but feebly pathogenic and only provokes disturbances under such potent predisposing influences as stitch pressure. Its general characteristics are similar to those of the aureus and albus, and it is cultivated and stained by the same methods. The impossibility of obtaining a universal immunity from stitch suppuration in veterinary operations, points as forcibly as these experiments to the existence of pus microbes in the deep layers of the skin of animals.

\section{STAPHYLOCOCCUS PYOGENES CITREUS.-}

This bacterium answers to the same general morphologic description as the preceding forms. It differs from the aureus in that it forms a sulphur-colored pigment on the surface of the agar-agar culture that does not, like the latter, become orange-yellow. It is only feebly pathogenic, but will produce abscess when inoculated subcutaneously into animals. It is much less important than the preceding.

5. STREPTOCOCCUS PYOGENES.- "The confusion which exists concerning streptococci and the variety of antistreptococcic serums on the market, renders a summary of the present knowledge concerning this group of bacteria somewhat desirable."-Moore. It is very evident that there is no satisfactory classification of the many species of streptococci that are known to exist, and that the exact role of 
this genus of the coccacere in the production of disease is largely a matter for future investigation. That streptococci produce various diseases in which different forms of the genus are the specific causes, is not questioned. From time to time, as investigations proceed, different forms are recognized as the specific causative agents of certain diseases. For example the diplococcus of pneumonia (once described as a streptococcus) is now recognized as having a potent infuence in provoking inflammations in the lungs; the streptococcus erysipelatis, is no longer doubted as the specific microbe of human erysipelas; and the streptacoccus equi (Shultz) is believed to be the cause of equine strangles The forms described under the single name of streptococcus pyogenes include all the varieties that are capable of producing suppuration. Pus taken from different lesions does not always exhibit the same arrangement of the chains, which feature is much depended upon for differentiation. It is therefore evident that various forms of streptococci are pyogenic. It is even shown the streptococcus erysipelatis can not be differentiated from the streptococcus found in abscesses. Speaking along this line Moore says: "The study of streptococci from various sources, more especially from the tissues of diseased animals, suggests the desirability of delaying a further classification until more definite data are obtained concerning the natural history, not only of these, but also of the species normally present on the, mucous membranes of animals, and in nature generally." The specific name is, pathologically or even biologically speaking, of little moment unless we can attach a certain definite meaning to it concerning the morphologic characters, culture manifestations and the degree of disease-producing power possessed by the organism designated."

The streptococcus pyogenes, of Rosenbach, which name we shall use for the purpose of describing the pus-producing 
streptococcus of interest to the veterinary surgeon, is found abundantly in discharges from mucous membranes, on the skin rather deeply imbedded in the sebaceous ducts and hair follicles, in the mouth, throat, intestines and bronchial tubes. It is very frequently found in the pus of such well known lesions as fistula of the withers, poll-evil, infective mastitis and cold abscess of the mastoido-humeralis. In these suppurative processes the staphylococcus pyogenes aureus et albus is very frequently associated with it.

Cultivation.-The pyogenic streptococci can be grown

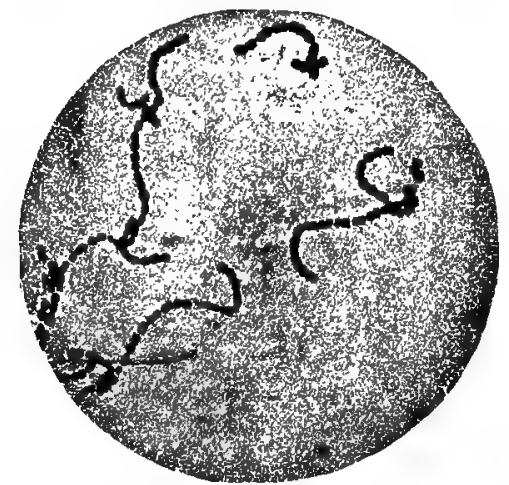

FIG. 7.

Streptococcus Pyogenes from Culture.

on gelatin, blood-serum, potato, agar-agar, or in bouillon and in milk. It grows at ordinary house temperature, but its most rapid growth occurs at the temperature of the body. It grows best on blood serum.

Isolation.-It may be isolated from smear cultures, but the best method is to inoculate a rabbit or mouse and then search for the micro-organism in the blood, in which it can usually be found.

Staining.-It stains by Gram's method or with the aqueous anilins. Methylene blue stains it.

Pathogenicity.-The streptococcus pyogenes has been found in a variety of lesions, varying in gravity from fatal human erysipelas to a simple dermal pustule of a mouse. It 
is a common inhabitant of pus from most any source. In the more grave suppurations of the domestic animals it is usually associated with other pyogenic micro-organisms. The poll-evil, the quittor, the fistula of the withers, etc., contain them either alone or mixed with the staphylococci. In these cases they are the determining causes. When the resisting forces of the body are depressed or the infections extremely virulent and abundant, fatal septicæmia or pyæmia are the certain terminations. At autopsies on animals having died from generalization of septic infections, the strepto-

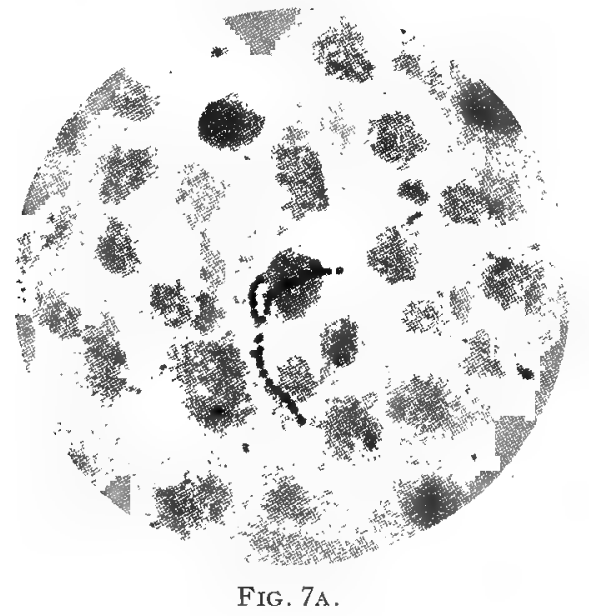

Streptococcus Pyogenes from Pus.

coccus is found in the organs in a larger percentage of cases than any of the other pyogenic agents. It is often associated with the septic vibrion, the bacillus of Nicolaier, the bacillus coli communis, the bacillus anthracis, etc., in the lesions of the diseases each of these specific micro-organisms is capable of producing. Inoculative experiments with virulent cultures usually prove fatal to cavies, rabbits, mice, etc.

Resistance and Virulence.-It is probably more virulent in man than in animals. Animals suffering from puerperal infection caused by streptococcus often get well, while in the 
human, the case is far different. Like the staphylococcus it has a variable virulency. The cultures are most virulent to the species of animals from which they are taken. Often a culture from one species will prove rather innocuous to another. Marmorek secured the highest virulence by passing the coccus through rabbits and then cultivating it in media composed of human blood serum and bouillon. Holst reports a virulent artificial culture that was kept, without special precautions, for eight years. They possess a remarkable tolerance to desiccation.

Toxic Products.-It has not been shown that the streptococcus pyogenes elaborates any considerable amount of free toxin. Cultures sterilized by heat are, however, capable of causing a fatal intoxication. . The toxic products of the streptococcus pyogenes is said to exert a specific curative action on certain malignant tumors, notably on sarcomatous growths. It has been observed that such tumors occasionally sloughed out and cicatrized when accidentally infected with streptococci; this circumstance has led to the experimental inoculation of tumors with sterilized cultures. Numerous reports of favorable results from prominent investigators would indicate that the expedient is not without merit.

Antitoxin.-The value of the antitoxins used against streptococcic infections is a matter for future determination. While flattering results are reported here and there, the antistreptococcic serums have not come into general use in either human or veterinary medicine.

The antitoxin is prepared by securing a high degree of immunity in a horse, through the repeated injection of virulent cultures of the micro-organism. The process is not unlike the preparation of antitetanic serum.

6. BACILLUS COLI COMMUNIS.-Next to the staphylococci and the streptococcus pyogenes, the bacillus coli 
communis is the most important pyogenic bacterium in veterinary surgery. It is also described under the name of bacillus pyogenes fœtidus on account of the very offensive odor of the pus it produces. It is often referred to as the colon bacillus. It is a constant and habitual inhabitant of the large intestines, small intestines and bile ducts of domestic animals and can usually be found in the feces of most all mammals. It establishes its abode in the intestinal tract soon after birth and remains there as a parasite throughout the life of the animal. Its constant presence in the intestines has led to

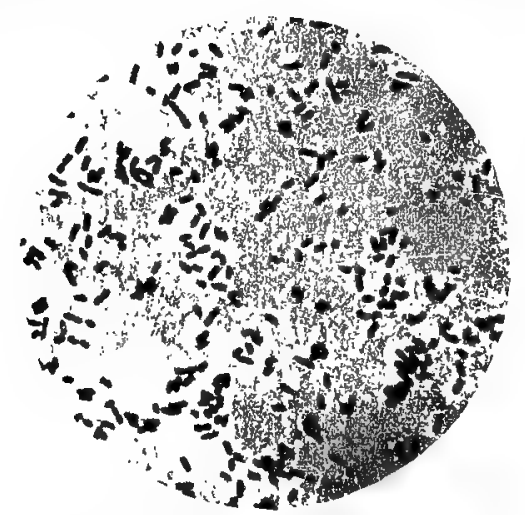

FIG. 8.

Colon Bacillus without Flagella.

many erroneous impressions as to its pathogenicity. It has been wrongfully accused at different times of producing dysentery, diarrhœa, cholera, typhoid fever, and hog cholera, besides various other cliseases. At the autopsy, it is one of the common micro-organisms found in the tissues of the carcass, owing to its remarkable penetrating and contaminating powers after death. It has the property of spreading directly through the tissues after death. Soon after death it passes directly through the intestinal walls into the surrounding tissues. Its multiplication in dead tissues is very rapid. The colon bacillus is frequently found in rivers, streams, drinking water and sewage. 
Description.-A short, thick bacillt1s. Motile. Flagellated. Non-sporogenous. Non-chromogenic. Nonliquefying. Aerogenic. Aerobic and facultative anaerobic. Saprophytic and occasionally pathogenic. Size $\mathrm{I} \mu$ to $3^{\mu}$ long by $0.4^{\mu}$ to $0.7 \mu$ thick. The bacilli are actively motile even in cultures that are quite old. On the slide they are found in various sizes. Some appear like cocci while others are considerably elongated and present from four to twelve flagella. They are quite distinctly separated and may be seen in pairs here and there at different points of the specimen.

Cultivation.-The colon bacillus may be grown on any of the ordinary media-gelatin, potato, agar-agar, milk, and houillon-preferably at the temperature of the body.

Staining.-The bacillus will not take Gram's stain, but can be stained with the aqueous solutions of the anilin dyes. and methylene blue.

Resistance.-It is killed by exposure to a heat of $140^{\circ}$ Fahr. for ten minutes, but is very resistant to chemical antiseptics, especially carbolic acid.

Pathogenicity.-Although a harmless inhabitant of the bowels under usual conditions, it is ever ready to enter into lesions of the intestine and provoke suppurations. It was once thought to be the specific micro-organism of appendicitis of the human being, but its pathogenicity in this connection seems to have been greatly over-estimated. In the veterinary subjects, it is found to provoke suppuration in the rectum, perineum and intestines. In the abscesses following proctitis, periproctitis and traumatic vaginitis, the colon bacillus may often be found in large numbers. Abscesses of the abdominal walls caused by paracentesis abdominis are very frequently found to contain this pyogenic agent in large numbers, having been inoculated into the abdominal muscles by the withdrawal of the canula. In 
every case, the pus emits a characteristic fetor and some gas, and often holds in suspension numerous connective tissue sloughs, indicating the intensity, and gangrenous characteristics, of the inflammation the micro-organism is capable of developing. When the colon bacillus is found in various lesions at the post mortem, its faculty of penetrating the tissues after death must always be taken into account. This feature of the coli communis, its pathogenic power under favorable conditions and its constant presence in the bowels, have in the past led to erroneous conclusions and

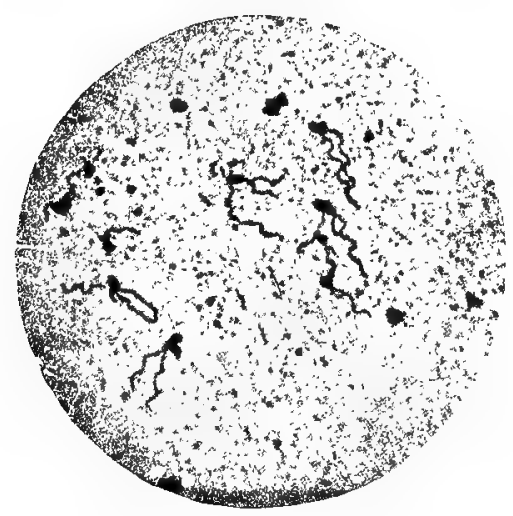

FiG. 9.

Colon Bacillus with Flagella.

some wild speculations as to the place where it actually belongs in the category of pathogenic bacteria.

Toxins.-It does not liberate free toxin, in which con. nection it simulates the pyogenic cocci. An intracellular poison has been shown to exist, by the injection of sterilized cultures into animals.

7. BOTRYOMYCES EQUI.-This bacterium has been described under several different names, among which are: dyscomyces equi, botryococcus, micrococcus ascoformans, and micrococcus botryogenes. Its role as a disease-producing bacterium, and even its very existence as a special being, has been questioned. Moore, after investigating the 
microbian flora of several botryomycostic lesions, says that the disease known as botryomycosis of the horse is "a wound infection, and that several species of micro-organisms are capable of producing it." The more recent experiments of De Jong, Kitt and Rabe seem, however, to entirely disprove the exactness of this deduction. Rabe has shown that the botryococcus found in lesions of horses will, by inoculation, produce analogous lesions in other horses. He succeeded in reproducing botryomycostic growths in the horse, but found that the same agent caused entirely different lesions,--necrotic inflammations, - in other species, which fact confirms the conclusions made from our own clinical observations as to the receptivity of the horse to this special bacterium. De Jong found that botryococci and staphylococci could not be made to show any analogous cultural manifestations, and that when the former were infected into the subcutem of the horse it always produced a mycofibroma while the latter only caused its characteristic phenomenon,abscess.

Description.-Coccus. Non-motile. Non-flagellated. Chromogenic. Aerobic. Size, I $\mu$. Arranged in groups somewhat smaller than the staphylococcus.

Cultivation.-Gelatinized peptone, glycerinated gelose, gelatin agar-agar and potato are the most suitable media for its cultivation.

Isolation.-The botryococcus may be isolated by its rather characteristic behavior on agar. (Its colonies promptly produce an orange pigment, which, unlike the staphylococcus pyogenes aureus, when inoculated into fresh media and heated to body heat, becomes white in twenty. four hours, and then orange-yellow if the temperature is lowered to $60^{\circ}$ Fahr.) It is also differentiated from the aureus by the variable colors it produces. The chromogenic function of the aureus is quite uniform. 
Pathogenicity.-From the experiments already mentioned it is evident that the botryococcus produces a neoplasm when injected into horses. It is also found in harness and collar lesions that tend to encroach upon the surrounding tissues. It is found very frequently in the scirrhous cord following castration. It is probably the specific bacterium of this disease in the horse, although it cannot be discovered in the recent case. All of the old scirrhous cord examined at the Chicago Veterinary College during four years were found to contain them. The pathogenic role of the botryococcus in this affection, is evidently that of perpetuating rather than causing the morbid process. The pre-existing funiculitis caused by improper methods of castration is primary and gives the botryococcus an avenue of entrance and a suitable environment for growth. Besides provoking the production of new tissue, it is pyogenic (See chapter on Botryomycosis, Part II).

\section{B. SPECIFIC SURGICAL MICRO-ORGANISMS.}

In studying the surgical bacteria, it must be remembered that the so-called pyogenic forms are not numerous, but that many other micro-organisms may cause pus formation. Pyogenesis is not a specific disease. It is simply the result of the reaction of the organism against the micro-organism, and may therefore occur in the presence of most any pathogenic bacterium that attempts, but fails, to successfully invade the living body. The first seven forms described above might be appropriately referred to as the microbian agents most likely to provoke suppuration in animals. They might also be appropriately accused of being rather unsuccessful invaders. At most every attempt they make to enter the organism they are successfully repulsed by the leucocytes, and suppuration is the result. Occasionally, however, their attack is successful. They surmount the barrier put up 
against them, invade the body and cause such grave and fatal conditions as septicamia and pyrmia. The botryococcus and the actinomyces, are undeniably pyogenic, but the pyogenesis is but a minor feature of the lesions for which they are responsible, viz, the neoformations. The following bacteria together with the last two are specific. They are known to produce definite lesions, from which they can be isolated, then cultivated, and then, when inoculated into susceptible animals they reproduce lesions similar to the original.

I. BACILLUS MALLEI.-This specific bacterium was.

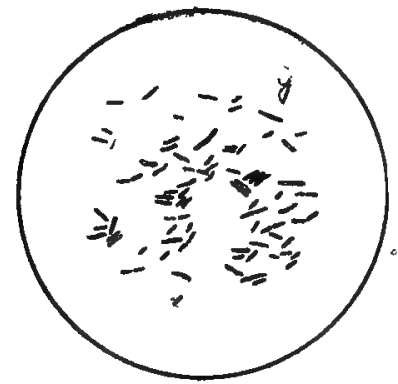

Fitc. 10.

Bacillus Mallei. (Pure Culture.)

discovered by Shultz and Loeffler in I882. They found it in the lesions and discharges of glandered horses. The bacillus mallei is probably purely parasitic. It has never been found except associated with the disease and its discharges, which become desiccated upon watering troughs, mangers, harness, wagons, neck-yokes, hitching-posts, trees in the pasture, fences, etc., and may, even after the lapse of considerable time, prove highly virulent to animals accidentally inoculating themselves from these objects.

Description.-Bacillus. Non-motile. Non-flagellated. Non-chromogenic. Non-liquefying. Non-sporogenous. Aerobic and facultative anaerobic. Size, I.5 $\mu$ to $3 \mu$ long and $0.25 \mu$ to $0.4 \mu$ thick. They are slightly bent and may exist in the form of spheres (coccoid) or branched forms. 
Cultivation.-It can be grown in bouillon, milk, and upon the usual solid media. It grows particularly well upon glycerin agar-agar and upon blood serum. It grows best between $75^{\circ}$ and I $10^{\circ}$ Fahr.

Isolation.-The most satisfactory and effectual method of obtaining the bacillus unassociated, is by inoculating a cavy with pus from a gland or nodule and then securing the bacillus from its tissues. Attempt to isolate it by the usual smear-culture method generally fails owing to the presence of the associated bacteria which usually grow more rapidly than the specific microbe. When animals are inoculated for this purpose the appearance of secondary lesions is awaited and then the culture is obtained from the soft pustule before it ruptures and has thus been exposed to external micro-organisms.

Staining.-The bacillus mallei will not take Gram's, but can be stained with the aqueous solutions of the anilin dyes.

Virulence and Resistance.-The cultivation through several successive generations reduces the virulence markedly. Attenuation by the use of heat has not been successful, the micro-organism being promptly killed by an exposure of five minutes to a heat of $130^{\circ}$ Fahr. It yields to the ordinary disinfectants, but is capable of resisting desiccation for several months and, very probably, much longer. The marked resistance of the bacillus mallei to desiccation would indicate that it is a sporogenous microbe, which is however not the case. It is simply very resistant to the various extraneous influences to which it might be subjected in the stable and out-of-door environments.

Pathogenicity.-When injected into the tissues of receptive animals, the bacillus mallei will cause the wellknown disease glanders, the lesions of which will always be found to contain the bacillus. It is capable of producing both an acute and a chronic glanders and of localizing in a 
great variety of different points of the body, but shows a special affinity for the lungs, skin, lymphatic glands and the liver. Any part may however, become the seat of a glander lesion.

It is capable of producing the disease in the horse, ass, mule, goat, dog, cat, hog, rabibit, cavy, field-mouse and in man. The ox, white mice and rats are immune. The horse, ass and mule are the most receptive of all the domestic species. The dog and cat by inhabiting stables badly infected with the disease, sometimes become inoculated with the virus and develop typical cases. The hog is likewise slightly susceptible but much less so than the other species. Cattle never have the disease. The bacillus will not grow in their serum, which Chenot claims will cure the disease in experimentally-infected cavies.

The lesions consist of nodules containing the bacillus, leucocytes and some epithelioid cells. The nodules may become necrotic, discharge their contents on the surface and leave a ragged ulcer which may cicatrize rather slowly. They may be numerous, few, acute or chronic.

Avenue of Entrance.-The channel through which the bacillus mallei enters the animal has a bearing on the clinical phases of the resulting phenomena. When the admission is through an abrasion of the skin the result may be a threatening, phlegmonous swelling that soon suppurates at different points; or a mere pustule, with the formation of secondary foci in remote regions. The neighboring lymphatics are first to become involved, whence it diffuses to other parts. The conjunctiva has been found to admit a fatal infection, that first destroyed the globe of the eye. The Schneiderian membrane, by direct inhalation of stable dust containing the specified germ, is often the very first structure to become involved. Halter abrasions at the poll, collar sores on the neck, saddle galls on the withers, often become 
contaminated with germs harbored on the harness. The chief source of entrance is the digestive tract. The microorganisms are ingested with the food, pass unimpaired through the digestive and absorbent mechanism and lodge in the lungs, where they produce the first lesion. This deduction has been proven over and over by numerous experiments and observations. Nocard's feeding experiments have proven it beyond question. The watering trough and the feed box are found to be the principal habitats of the bacillus mallei in the stab?e. It is deposited there by the nasal discharge, which in many and many instances may be trivial in quantity, and may entirely escape notice, and yet be capable of depositing an abundance of virulent microbes. The direct inoculation of the lungs through the respiratory tract is not doubted, but it is known to be rare as compared to the alimentary route.

Immunity.-Mallein, under certain conclitions and by repeated inoculation, will confer an immunity against the disease, but as Law says, "Altogether, attempts to immunize the equine population are not hopeful where it is dense, where they must be kept stabled, where the climate is moist and the glanders is deadly or tends to persist in the chronic form for years in the same animal." The value of the immunizing agents at our command is strikingly e!ucidated in these few words. The writer's own experience with the disease in a large city fully confirms them. Mallein is an immunizing agent of some potency when used repeatedly and when suitable hygienic and working conditions are accorded the infected and exposed anima!s. It seems to require the assistance of the resisting forces of the body. Rabe has asserted that single injections wi1l immunize anjmals. If this is true it applies only to transient passing immunity, that can only be prolonged by subsequent repetitions of the mallein inoculations. 
Diagnosis of Glanders.-Glanders can be positively diagnosed by either of the following methods: (I) By animal inoculation: and (2) by the use of mallein. The former. usually referred to as the Straus test, is carried out by inoculating scrapings or discharges of glander lesions subcutaneously or intraperitoneally into male caries, and then making deductions from the resulting phenomena. The seat of inoculation swells and in about two or three days after the testicles and inguinal glands become markedly tender and swollen from a secondary inflammation. A post mortem examination of the experimental cavy at this time will reveal the presence of the specific agent in the secondary lesions in the testicles and lymphatic glands. Other organs may also be found invaded. For all practical purposes the clinical phenomena presented by the experimental cavy may be regarded as sufficient. That is, if the testicles swell and become painful the case is one of glanders, while on the other hand, if no such evidence appears, glanders may be safely excluded. The microscopic examination shou'cr, however, be made when possible.

The latter, is now universally designated in reterinary medicine as the Mallein test. It consists of administering subcutaneous injections of from $2.5 \mathrm{cc}$ to $5 \mathrm{cc}$ of mallein solution into the suspected subject, which is first submitted to a preliminary examination, especially as regards the temperature curve. Other symptoms should, however, be taken into account, among which are the general spirits, appetite and local lesions. Eight hours after the injection, which is generally made in the lateral cervical region, the temperature curve is taken for twelve to sixteen hours, during which time observations are also made as to the behavior of the seat of inoculation and the general spirits of the patient. An elevation of $1.5^{\circ}$ Fahr., continuing through the entire period of observation, a painful cedema around the 
seat of inoculation, together with some loss of appetite and spirits, may be regarded as a chain of condemning circumstances. A mere swelling withont fever is negative. A sudden burst of fever, even to $104^{\circ}$ Fahr., that drops as suddenly within several hours, is likewise negative, or at least is never a positive indication of glanders. And again, a high elevation of the temperature without any marked local swelling at the seat of inoculation, must always be cautiously judged. It may have been provoked by other causes. The mallein test for equine glanders is valued highest by those who have used it the most, and is condemned only by the inexperienced or unobserving diagnosticians, largely because of the fact that only the febrile feature of the test is taken into account. Post mortems, exceeding four hundred in number, made by Wright, on tested horses, have proven conclusively that mallein will "ferret out" the minutest glander lesion. Failure to find them may often be traceable to the crudeness of the autopsy and the minuteness of the lesions. It is nolonger the custom to kill all the reacting subjects. Only those having external symptoms are regarded as dangerous infection-carriers. Although this plan, which is now quite universally adopted in the veterinary profession, is not without fault, it is, from the economic standpoint, the only practical one. The best sanitarians in America-Rutherford, Pearson, Gill, Moore, Wright, Tiffany, Reynolds, et al., have adopted this plan in dealing with the disease in large cities, but all of them are known to recognize the danger of the occult case disseminating the disease without ever developing into a clinical case.

Mallein.-Mallein is the metabolic product (toxin). of the bacillus mallei in artificial media. It is prepared from artificial cultures of the micro-organism in glycerin boullion. 
The culture is allowed to grow for several weeks, then the micro-organisms are killed by heat and filtered out with a porcelain filter. The remaining liquid is then reduced to one-tenth its volume by evaporation. The manufacture of mallein from potato cultures is said to yield a much stronger product. There are also other, more complex, methods of preparing the substance. Bonome prepares it directly from the blood, or viscera of artificially inoculated animals.

Mallein does not affect healthy animals, nor animals not affected with glanders. When inoculated into the body of an animal having no lesions of glanders the results are negative. It causes no perceptible signs of intoxication, even in very large doses; but when inoculated into glandered subjects, although the disease may be limited to very trivial internal lesions, it will cause a characteristic disturbance manifested chiefly by fever, dejection, anorexia and œdema at the seat of inoculation, all of which vary greatly in severity. The disease is made temporarily worse and in some instances it may cause the death of the glandered patient; after several days of illness. This fatal termination is by no means rare. The mechanism by which it causes these phenomena is not well understood, but the certainty of their appearance in the infected subjects, renders mallein an invaluable agent for the purpose of diagnosing glanders in the soliped. Its curative properties have been recognized for some years. Nocard, first attracted attention to this action after a series of experiments that leave no room for doubt. In this country it is found no less valuable in the hands of experienced veterinarians. For this purpose mallein is injected at intervals of six weeks to two months, in addition to improving the hygiene of the stable. The sick are thus discovered, benefited or found dangerous enough to destroy; and the healthy ones are rendered at least tempor- 
arily inmune against infection. By thus revealing the exact extent to which a given stable of horses is infected, other rules of sanitary medicine, too complicated to discuss here, can be inangurated and carried ont, with the result of entirely eliminating the disease.

2. BACILLUS OF NICOLAIER (bacillus tetani).The specific micro-organism of tetanus, is anaerobic, flagellated, sporogenous, motile, non-chromogenic, pathogenic and toxic bacillus. It is a microbe of well-tilled soil and is found abundant'y in street dirt, stable dust, dung of herbivora, in-

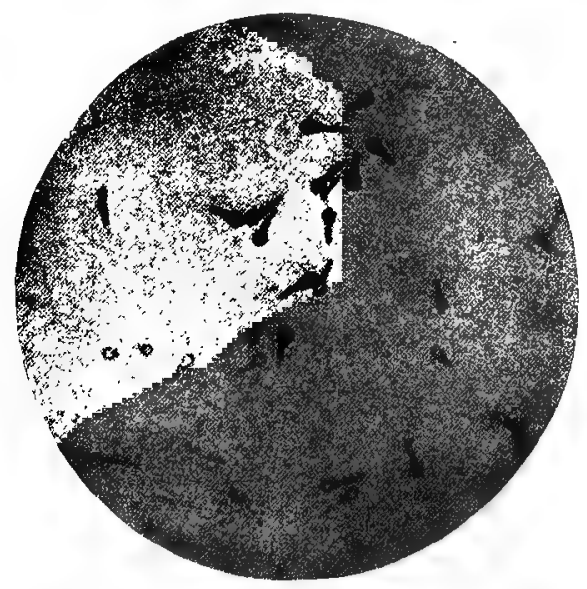

FIG. 11.

Tetanus Bacillus from a Lesion.

testines of herbivora, the unclean skin of man and animals. It is very evident that it is ingested with food containing particles of earth and that it rapidly multiplies in the intestines owing to the sheltered environment (anaerobic) these organs furnish for its growth. The inert gases, minus oxygen, in the bowels furnishes a very suitalsle medium for its rapid multiplication. It is 2 to 4 micromillimeters long by 0.3 to 0.5 thick. It was discovered by Nico'aier in 1884. (For detailed description see chapter on Tetanus, Part II).

3. ACTINOMYCES BOVIS (ray fungus), the specific 
micro-organism of actinomycosis, is a pathogenic parasite, aerobic and facultative anaerobic, rion-motile, non-flagellated, and non-sporogenous, pyogenic microbe belonging to the higher bacteria. The ray fungus is a parasite of vegetables, particularly the beards of cereals. Johne and Brazzola found it on barley and it is known to produce disease only through abrasions made by or contaminated with such substances. It was first observed by Langenbeck in 1845 , but was only definitely proven to be the specific microbe of actinomycosis by Bollinger, in 1878 . (For detailed description see chapter on Actinomycosis, Part TI).

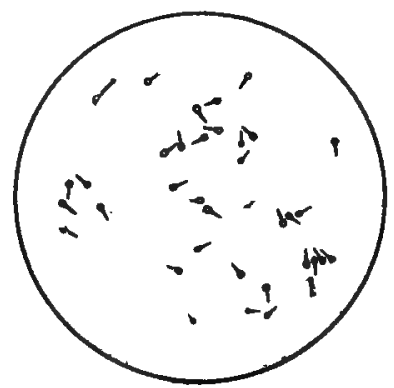

FIG. 12.

Tetanus Bacillus from Pure Culture.

4. BACILLUS TUBERCULOSIS is the specific microorganism producing the well-known disease-tuberculosis, which in the words of McFarland "is no respecter of persons but affects alike, the young and the old, the male and the female, the rich and the poor, the enlightened and the savag $\epsilon$ the human being and the lower animals." Tuberculosis no only affects mammals, but is found also in fishes, birds, rep tiles and batrachians. Wild animals in captivity are ver. susceptible, but seem to escape it in the wild state. It wa discovered by Koch in 1882 .

Description.-Non-motile. Non-flagellated. Non-sporo genous. Non-chromogenic. Non-liquefying. Distinctl aerobic. Highly pathogenic Purely parasitic. It occs. 
in the form of slender, slightly-curved rods with round ends, I. $5 \mu$ to $3.5 \mu$ long by $0.2 \mu$ to $0.5 \mu$ thick.

Cultivation.-The best method of obtaining a specimen of the bacillus is to inoculate an experimental animal with the pus of a lesion or human sputum, and then after the disease has developed, destroy it and make cultures from some of the tuberculous glands. It is cultivated with blood serum, dogs' blood serum, potato, and egg. The tubercle bacillus can be accustomed to grow on very simple media containing no albumen. It can be grown on glycerin containing carbonate of ammonia, phosphate of potash, and sulphate of

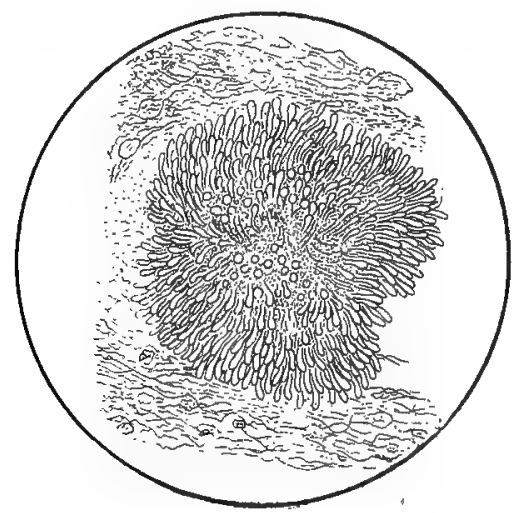

FIG. 13.

Ray Fungus.

magnesium, in considerable quantities. It is in a mixture of this character that Koch produced tuberculin.

It grows best in a temperature between $85^{\circ}$ to $105^{\circ} \mathrm{Fahr}$. and is exceptionally sensitive to extremes. It is killed at I6 $5^{\circ} \mathrm{F}$. It requires oxygen, but no light. Sunlight kills it promptly.

Staining.-The bacillus tuberculosis is difficult to stain, and when stained is as difficult to decolorize, which characteristic, once confusing, is now used as a valuable means of differentiating it from other forms that take stains promptly, and are easily decolorized with mineral acids. The 
difficulty of staining, and then the subsequent difficulty of decolorizing, is a property belonging to the tubercle bacillus alone. It can be stained with watery or alcoholic solutions of anilin dyes, but special and more effectual methods are now in general use. Koch stained it with an aqueous solution of a basic anilin dye, - a little potassium hydrate as a mordant,-then after washing with water, counterstained with vesuvin. Ehrlich improved the method by using pure anilin instead of potassium hydrate, and then decolorized all the other tissues except the bacilli with a strong minera! acid. This latter method is said to be the best one for stain-

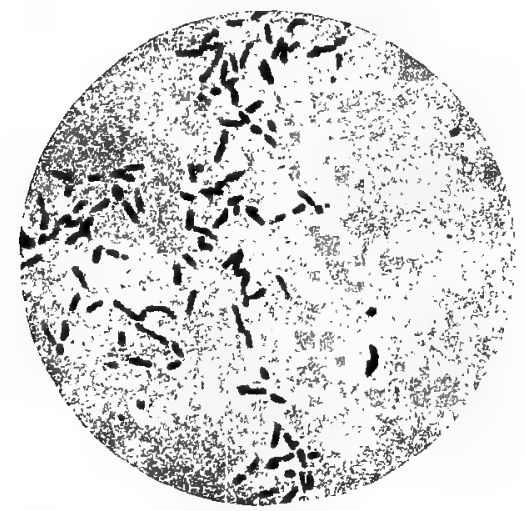

FIG. 14.

Bacillus Tuberculosis. (Pure Culture.)

ing the tubercle bacillus. This micro-organism has been so well studied that many special manipulations are used in dealing with it under the different conditions with which it is encountered. For these special processes the reader is respectfully referred to more elaborate works on general pathogenic bacteriology.

Diagnosis.-Tuberculosis in animals is diagnosed by three special effectual methods;-(I) By microscopic examination of the elements in the lesions; (2) By animal inoculation; and (3) By the tuberculin test.

I. Microscopic examinations are made by mounting 
and staining according to well laid-down rules, which differ somewhat with the particular tissue from which the specimen is taken. In this method the peculiar behavior of the bacillus in regard to staining and the tenacity of its stain is regarded as sufficient evidence that the microbe at hand is the tubercle bacillus. The fact that relatively large quantities of tissue may contain but few bacilli must always be taken into account. They may escape notice. When several attempts give negative results, and when considerable importance is attached to the diagnosis, the second recourse must supplement the microscopic examination.

2. Animal Inoculation.-The suspected tissues are emulsified and inoculated into a cavy which three weeks later is killed and examined for the tubercle bacillus. The lesions in the center of the lymphatic glands is the most likely place to find them. These specimens are mounted and stained in the usual manner prescribed.

3. The Tuberculin Test.-This test is the most practical and the most effectual in reterinary subjects. It is used more particularly in the bovine species. It is the only method by which the occult form can be recognized, and through which the number of affected animals of a given herd can be determined. The preceding tests are not available except when the lesions can be reached, or at the postmortem examination.

In this test the temperature curve alone is depended upon. The preliminary and the final examination, after the inoculation with tuberculin, consists exclusively of taking the temperature. The tuberculin is injected into the shoulder after the temperature has been observed at least three times during the twenty-four hours preceding the date of injection, "yet in busy field work one night temperature taken just before injecting rarely fails to give a satisfactory normal as to the standard for the animal." (Law). The 
injection is macle preferably at ten P. M. and at six A. M. the final observations begin. Temperatures are taken every two hours during the next sixteen hours. A gradual rise to a certain point, no less than two degrees above the highest preliminary temperature, followed by a gradual fall, is the typical reaction. Sudden elevations are of no significance, owing to the many external infuences that may temporarily disturb the temperature of an excitable bovine.

As in the case of glanders, the tuberculin test is valued highest by those having the most experience, and is quite universally condemned by the careless and inexperienced as being unreliable and even harmful.

Tuberculin.-The well known substance now universally designated as "tuberculin" is the metabolic product (toxin) of the bacillus tuberculosis, elaborated in artificial media. It was first prepared by Koch in I890, and is now manufactured by various well known firms, each of which probably uses a special process of preparation. The principles are, however, the same. A pure culture of the bacillus is allowed to grow for a certain time, until the media is saturated with the poison (toxin). The living bacteria are killed with heat and then filtered out by passing the mixture through porcelain. It is then evaporated to a certain strength and preserved in sterilized vials with carbolic acid. Tuberculin is an albuminous derivative that is not soluble in alcohol. It seems to be a bacterio-proteid, that differs from the toxalbumins by resisting excessive heat. Tuberculin is not destroyed by boiling for hours. When injected into healthy animals it is quite harmless but it proves very poisonous to tuberculosis patients.

Immunity.-Numerous investigators have experimented with antitubercle sertums, hoping to apply the principles of serum therapy to tuberculosis, but nothing yet achieved has been recognized by either the medical or veterinary profes- 
sions, although rather extravagent claims have been coming "thick and fast" from every corner of the world.

The problem of artificially immunizing cattle against tuberculosis is now occupying the attention of some leading veterinarians of the present day, but the methods recommended are still in the experimental stage and can therefore neither be approved nor condemned. Judging from the discussion on the subject at the meeting of the American Veterinary Medical Association of 1905 , it is very evident that there are no convincing data to submit to the veterinary

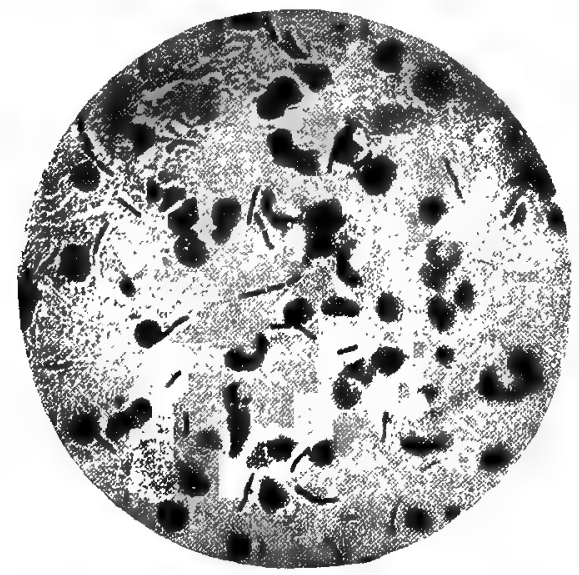

FIG. 15.

Bacillus Tuberculosis from Sputum.

student at the present time, and that the processes recommended are tedions, uncertain and impractical.

Certain vaccines consisting of attenuated tubercle bacilli are actually dangerous in that they produce the disease in its generalized form in at least a small percentage of the vaccinated animals. The International Veterinary Congress (1905) held at Budapest, by resolution advised in the following words that "until the field of usefulness of vaccination against tuberculosis is established, the continued employment of the sanitary measures known to be effectual is essential." 
Virulence.-The bacillus tuberculosis has a variable virulence dependent iargely upon its source. It matters considerably whether the source is a particular lesion or a particular species of animals. Those from miliary tuberculosis are very virulent, while those from pulmonary lesions with ulcerations are generally feeble. The bovine bacillus, generally speaking, is a highly virulent form, and is much more active in the cavy than specimens from human sputum.

Avenue of Entrance.-The tubercle bacillus enters the body chiefly through the respiratory tract, although its admission via the alimentary canal is now known to be much more common than was once supposed. There is now an overwhelming amount of evidence to prove that ingested milk frequently causes the disease in the human being, as well as in the sucking animal. Wounds on the surface of the body may become the seat of tuberculous processes, although this channel cannot be said to be a common one in animals, which fact rather diminishes the importance of tuberculosis in veterinary surgery. The tuberculous, surgical lesion is a rare one in veterinary practice, and is only encountered, here and there, and under such conditions as preclude surgical intervention.

5. BACILLUS ANTHRACIS.-This bacterium, which was the first specific micro-organism to be recognized as such, causes the disease now universally designated as anthrax, a fatal infective condition affecting man, oxen, sheep, horses, swine, goats, and the small, laboratory animals. Dogs and cats are generally referred to as immune species, but the observations of several investigators have shown that they may contract the disease by eating rather freely of the carcasses of animals that have died of the disease. The bacillus anthracis was discovered by Davaine in $185^{\circ}$, but it remained for Pasteur and Koch to definitely confirm his discovery by artificial cultivation and inoculation, some years later. 
Description.-Non-motile. Non-flagellated. Sporogenous. Non-chromogenic. Liquefying. Aerobic. Pathogenic. Size, $5 \mu$ to $20 \mu$ long by $\mathrm{I} \mu$ to $\mathrm{r} .25 \mu$ thick. It is a very large bacterium, which fact facilitated its early discovery. It has a very pronounced tendency to grow into long threads which distinctly show the separate bacilli, each of which is rectangular and somewhat rounded at the ends. The sportulation is always prolific, each spore forming at or very near the center of the rod, without increasing its diameter at the point of formation. The spore increases in length only, and finally ruptures to release the new bacillus.

Cultivation.-The bacillus may be grown on agar-agar, gelatin, potato and blood serum and in milk and bouillon. The growth is most active at the temperature of the body, but is not arrested in an environment as low as $68^{\circ}$ Fahr., nor as high as II $2^{\circ}$ Fahr.

Staining.-The bacillus takes Gram's and Weigert's and can also be colored with the ordinary aqueous anilin solutions. The spores are stained with carlool-fuchsin, after which the remaining parts of the bacilli are clecolorized with a weak acid and then re-stained with methyl blue.

Isolation.-The bacillus anthracis is an excellent specimen for studying the behavior of micro-organisn's, and their resistance to antiseptics. Other bacteria are constantly compared with it as regards size, sporulation, thread-formation and resistance to chemical and thermic actions $I t$ is easily isolated on account of its size, appearance of the colonies, and the receptivity of the experimental animals, by making smear-cultures or by inoculation of an animal and obtaining it from the blood.

Pathogenicity.-Infection occurs through the alimentary tract or through wounds in the surface of the body. The respiratory tract is always referred to as a channel of entrance, owing to the "wool-sorter's disease" it produces 
when admitted through this arenue. Insect bites are known to produce a suitable abrasion for the admission of the microbe into the skin, and surgical wounds made with unclean instruments are not uncommon channe!s of infection.

Dependent upon the avenue of entrance the bacillus anthracis produces two distinct forms of the disease,-internal anthrax and external anthrax. When ingested they pass into the bowels and multiply in the form of bacillary networks around the villi and enter the blood through the absorbent vessels. The resulting phenomena proves fatal in four to twenty-four hours. If the channel of admission is an

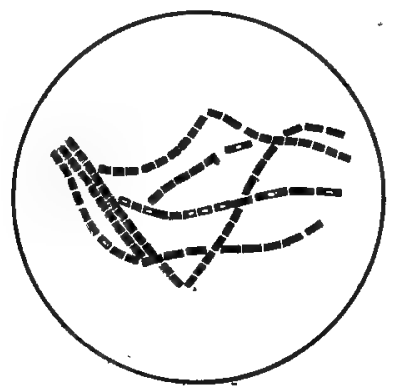

FIG. 16.

Bacillus Anthracis.

external breach, the grave symptoms are preceded by the appearance of a large, threatening, doughy swelling that does not crepitate nor manifest any tendency toward suppuration, by which it is differentiated from malignant cedema, symptomatic anthrax and phlegmons. In the horse these lesions often appear on the thigh, neck, withers and. shoulders.

Resistance.-The bacillus is noted for its resistant propensities. Dried spores retain their vitality for years, and it requires no less than five minutes boiling to kill them. They can be immersed in a five per cent solution of carbolic solution for twenty-four hours, and then germinate, but are killed in a short time with mercuric chloride $I$ to $I, 000$. 
It was once supposed that they lived for a long time when cleeply buried with the carcasses of animals having died of the disease and that they subsequently were brought to the surface by moisture currents or earth worms. But this has been proven erroneous. It is now known that, buried, they die like any other living cell. It is the desiccated spore that is so viable, and not the buried one. Pasteur has always maintained that it was the living, diseased animal, that disseminated the infectious matter, and not the dead; and subsequent investigation bears out his statements. The living animal, by its excrement, spreads the germs over the surface where they are capable of living for long periods, and whence they can be blown to remote places by wind-carried dust.

Toxin.-The metabolic product of the bacillus anthracis is a curling ferment that will digest both casein and fibrin. It is, properly speaking, not a toxin, and it is indeed doubtful whether the bacillus elaborates any poison at all. Several experimenters have isolated various toxic products, but they were found to be fatal only in very large doses, which clearly indicates that they are not the death dealing elements of the germ. The anthrax poison is an intra-cellular poison, and not an extra-cellular toxin.

Immunity.-Vaccination. Pasteur has provided a practical means of immunization, which consists of inoculating exposed animals with attenuated bacilli, now sold by the Pasteur Vaccine Company under the name of "anthrax vaccine." The attenuation of the bacillus is accomplished by various means, but the Pasteur Company alone seem to have the secret of reducing its virulence to a uniformly safe degree of attenuation. Attempts to produce a similar product by other manufacturers of biological products have thus far proved disastrous, in that vaccinated animals often developed fatal forms of the disease. The scientific world is in- 
debted to Pasteur, but not for the secret he withheld in regard to the manufacture of anthrax vaccine. The vaccination, as carried out under their direction, consists of two separate inoculations. First with a weak virus, and then, some days after, with a more virulent one. This is known as the double vaccine. A single vaccine is also furnished which is said to impart equal protection against infection. The claims of Koch and other Germans that Pasteur's vaccine did not protect animals against intestinal infection is probably not true, as years of use seem only to prove the universal benefit of the vaccination.

Serum Therapy.-The antitoxic serum recommended by Ogata in I 890 has not been adopted, although he proved that such a substance could be produced. The greater practicability of the protective inoculation of Pasteur has apparently stopped all researches along that line.

Field Diagnosis.-In the field anthrax must be differentiated from other acute disease having a galloping course and termination.. These are malignant odema, symptomatic anthrax, tick fever, corn-stalk diseases, etc. The differentiation is made by the clinical symptoms, by the postmortem appearances of the blood and organs, and by a bacteriological investigation. The anthrax carcass has a characteristic engorgement of the spleen and an infiltration of the internal organs, and a characteristic darkness of the blood. These are seldom combined in other diseases. When a bacteriological diagnosis is desired, a piece of an ear is taken to the laboratory in a closed jar. An ear will not readily decompose, and contains sufficient blood for the purpose. Putrefaction of the specimen greatly interferes with the investigation.

6. BACILLUS ANTHRACIS SYMPTOMATICI.This bacterium is the specific causative agent of the disease known under the various names of anthrax bacteridian, 
symptomatic anthrax, black leg, quarter evil, and black quarter. The bacillus is also known by the names of bacillus anthracis emphysematosa, Rauschbrand bacillus, and bacillus Chauvai. It was discovered by Arloing, Cornevin and Thomas, between I880-I883.

Description.-Motile. Flagellated. Sporogenous. Anaerobic. Aerogenic. Non-chromogenic. Liquefying. Pathogenic. Size, $3 \mu$ to $5 \mu$ long by $0.5 \mu$ to $0.6 \mu$ thick. It has rounded ends and is never arranged in chains. It forms oval spores which distend the bacillus at the point of formation.

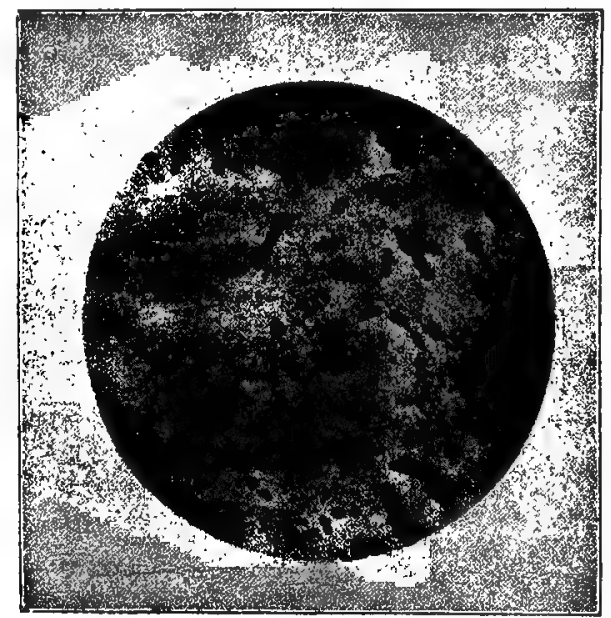

FIG. 17.

Bacillus Anthracis Symptomatici from Infected Tissues.

When examined in the hanging drop the motility is readily observed in the middle of the specimen, while those at the edges die from their exposure to oxygen.

Cultivation.-It is strictly anaerobic and therefore can only be cultivated in sheltered environments. Deep stabs in gelatin or agar-agar are the appropriate methods of growing black leg bacillus. I_iquid media-milk or bouillon-in a vacuum or in hydrogen are also suitable.

Staining.-It will not take Gram's, but can be colored by 
the watery anilins. Loeffler's solution will stain it in the tissues.

Pathogenicity. - The bacillus will produce the disease in young cattle, between the ages of six months and four years. Older animals are seldom attacked. It may also affect sheep and goats, but horses, dogs and hogs are immune. The disease is strictly a wound disease. The microbe is introduced into the subcutem, where it produces an advancing odematous and emphysematous swelling at the seat of entrance, together with grave systemic disturbances that prove fatal in several days. The bacillus gains admission through insect bites, punctures, abrasions, etc.

Resistance.-The spores may be preserved indefinitely in a dry environment, and cold as low as $98^{\circ}$ Fahr. below zero has failed to kill it (Law). It is killed by boiling for twenty minutes, but the spores resist boiling for six hours. It is killed quite readily with the ordinary chemical antiseptics, mercuric chloride, carbolic acid, sulphate of copper, and boracic acid. In the soil, if sheltered from the atmosphere, it will live indefinitely.

Immunity.-Immunity may be acquired by vaccination. The blackleg vaccine is prepared from the dried and pulverized muscles of calves that have been artifically infected. When the calf dies the infected muscles are taken out, cut into pieces and dried slowly at about the heat of the body. When dried it is pulverized and the living bacteria are attenuated with heat of about $2 \mathrm{I} 2^{\iota} \mathrm{Fahr}$. or slightly higher for six or seven hours. The resulting product is the first vaccine. The second vaccine is submitted to but $185^{\circ} \mathrm{Fahr}$. These two vaccines are injected subcutaneously at an interval of one week. More recently, however, it has been found that a safe immunity may be obtained from a single inoculation with a virus submitted to $185^{\circ}$ for six or seven hours. The vaccination is not entirely without the danger of produc- 
ing the disease itself, and thus causing out-breaks that would otherwise not have occurred.

It is very evident that the immunity against black-leg is strictly one of degree. It is very slight in the young ox, but increases with age, and is never entirely complete. It has been shown that non-receptive animals can be artificially infected by artificially increasing the virulence of the bacteria or by artificially diminishing the resisting forces of the tissues at the seat of inoculation. Absolutely refractory animals, artificially immunized animals and naturally immune animals, can be infected by injecting the tissues with cultures associated with certain harmless bacteria, notably the

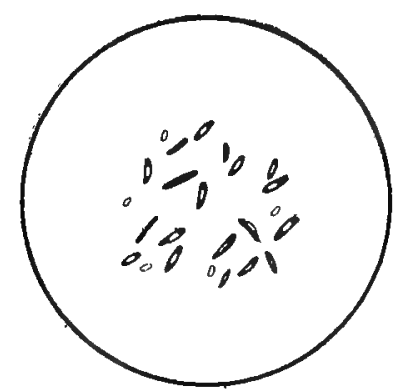

FIg. 18.

Sporulation of B. Oedematis Maligni.

bacillus prodigiosus. And finally, lactic acid added to the culture renders successful infection of non-receptive subjects, possible.

Diagnosis.-The emphysematous symptom and the rapid course of black-leg brings out a clinical tableau that bears considerable resemblance to malignant œdema, from which disease it must always be differentiated. The fact, however, that the former disease attacks chiefly young cattle, and usually a number of them simultaneously, seldom leaves any room for doubt. Malignant odema cases are always single cases. It would indeed be a rare coincidence to see two animals attacked at the same time, considering: 
the relative rarity of the disease among the bovine species. And besides, malignant œdema seldom ever affects the ox except in the region of the pudendum, and that only after parturition.

It is recognized from external anthrax by the absence of emphysema in the latter, although on inspection the swellings may appear similar. When there is any confusion a bacteriological examination will remove all doubt. (See description of each bacterium).

8. BACILLUS EDEMATIS MALIGNI.-This microbe is the specific causative bacterium of malignant odema, a disease affecting all the domestic animals and man. It is also known by the names of gangrenous septicæmia, gaseous gangrene and emphysematous gangrene. The bacterium is known as the septic vibrion in France, and is often referred to as the bacillus septicus by English writers. It was found by Pasteur in 1875 .

It is a motile, flagellated, sporogenous, anaerobic, aern genic, liquefying, non-chromogenic and pathogenic bacillus. It is found in well-tilled soil, and in the dust derived from that source. Hay, stable and house dust is rich in this bacterium. It measures from $2 \mu$ to Io $\mu$ long by. $0.8 \mu$ to I $\mu$ thick. (For detailed description see chapter on Gangrenous Septicamia, Part II). 


\section{CHAPTER VI.}

\section{IMMUNITY.}

DEFINITION AND SCOPE.-Immunity is that phenomenon by which an animal or whole class of animals exhibits such resistance to an infectious disease as to withstand it, while under the same conditions another anirnal or class of animals will yield. In its broad sense immunity is exemption from disease, but it is usually limited to diseases of parasitic origin, either animal or vegetable. There is no such thing as immunity from accident or death. When a pathogenic and parasitic microbe invades the body of an animal, complicated phenomena ensue, the whole process being conventionally looked upon in the nature of a conflict. On the one hand, offensive weapons brought into action by the microbe enable it to pass a phase of its existence in the body before it makes exit. On the other hand, the host, by various defensive mechanisms, seeks to protect its cellular units from the advancing microbe. From the character of the vital processes of both the invader and the invaded, one can easily comprehend that the phenomena of infection and immunity are at once complex.

Immunity is not a theory. It is a science, although not perfectly understood; but relatively speaking, we have a better knowledge of the action of the protective substances in the blood,- - antitoxins, etc.,- - than of the physiological action of quinine or aconite. The only real theory concerning immunity, and this will be taken up in detail later, is in regard to the manner in which these substances are formed and in regard to their mode of action. That the antitoxins and allied bodies are of great therapeutic, diagnostic and prophy- 
lactic value, is conceded by the entire scientific world. "The controversy relates wholly to the manner of their development and action, and these theories are far from speculative.

The study of immunity has revolutionized therapeutics to such an extent that more than one-half of the indicated treatment in infectious diseases is with immune substances. In prophylaxis, some idea can be had of the extensive usage of antitoxin by the fact that during the last ten years over $50,000,000$ doses of blacklegoids have been given. In many diseases, the diagnosis is wholly dependent upon the manifestation of substances formed in the body during the process of infection.

As will be seen from the foregoing, the study of immunity includes etiology; the preparation and administration of serums and vaccines; serums and toxins in the diagnosis of disease; the agglutinins, precipitins, lysins and opsonins; and the theories of the manner by which these bodies act, namely, Metchnikoff's theory of phagocytosis and Ehrlich's side-chain theory. The older hypotheses have either proven to be erroneous or else have been modified to harmonize with those of Ehrlich and Metchnikoff, and hence will not be considered here. And, as will be seen later, both the theories of Ehrlich and Metchnikoff, with a few possible exceptions, are but different parts of the same hypothesis.

HISTORY.-Immunity is not a recent discovery, having been known for many centuries. It has been known that certain animals are not susceptible to some diseases, such as a horse to symptomatic anthrax, cattle to glanders, etc.; and that an attack of a certain infectious disease brings about such a change in the animal's tissue as to protect it from further attacks of the same disease. It has also been well known that certain diseases which attack man cannot be very easily inoculated into the lower animals. Not only 
have these facts been known concerning infectious diseases, but similar facts have been demonstrated of snake-venom, protozoan diseases, and a few vegetable poisons, abrin, ricin, etc. However, it is only during the last few years that immunity has made such wonderful strides, and has now reached the stage of so great practicability. This is due largely to the work of such men as Pasteur, Buchner, Metchnikoff, Behring, Koch, Chaveau, Bordet, Wasserman, Ehrlich, Nuttall, Wright, and many others. Due credit must also be given to the various pharmaceutical houses, Parke Davis, Frederick Sterns, Mulford and the Pasteur Vaccine Company. These commercial plants have greatly simplifies the practical application of immune sera. Probably no other branch of science is being investigated at this time in different parts of the world with such enthusiasm as the various aspects of immunity. In all the government laboratories, this work is being pushed, and many philanthropists have made large gifts to further this work. Finally, immunity in its different phases has more to offer to the investigator than any other branch of veterinary medicine. We are looking forward to the time that for every infectious disease we shall have an antitoxin, and, better still, a preventive.

\section{TYPES OF IMMUNITY.}

The following outline will aid in classifying the various forms of immunity which may occur:

Immunity-Antitoxic or Antibacterial.

I. Natural-Inherited by species, families, or individuals.

2. Acquired

a. Artificial

Active, as in vaccination.

Passive, as in antitoxin injection. 
b. Natural-i. e., as the result of an infectious disease. Active only.

Two principal types of immunity occur, natural and acquired. Immunity may be acquired naturally as the result of an infection, but this is a very different thing from natural immunity. Immunity which is acquired artificially may be active, as in vaccination; or passive, as in antitoxin injection. Furthermore, depending upon the nature of the immune serum, whether its immunizing substance neutralizes toxin or kills bacteria, immunity is said to be antitoxic or antibacterial.

Natural immunity is one which for the most part is inherited. This is usually the case where a whole class of animals is involved. On the other hand, the immunity may run in certain breeds or families. The Poland China hog, for example, is more susceptible to cholera than the "razor back." Even in a given family there may be marked variations. An infection of distemper may attack six puppies, while four may escape, and all these animals living under the same conditions. In immunity, as in everything else, the "personal equation" is an important factor.

Old animals are by nature less liable to infectious diseases than young. Young rats are markedly susceptible to anthrax, while under ordinary conditions, old rats will not succumb. In the human, measles, whooping-cough and scarlet fever are practically limited to children; adults seldom become infected.

Hogs which overcome an attack of cholera may transmit immunity to their offspring; on the other hand, the offspring of tubercular cattle may be immune or markedly susceptible to tuberculosis. Thus, susceptibility to infection as well as immunity, may be inherited by a whole species of animals. 
Natural immunity is not absolute and complete; for example, the horse is immune to anthrax as a rule, but under conditions whereby his vitality is lowered, either by overwork, by some other disease, or by malnutrition, he can be infected with anthrax. Until within the last year or two, it was thought that animals were immune to venereal diseases; but recently Metchnikoff and Roux have succeeded in inoculating the chimpanzee with syphilis, and before the medical society of Berlin a horse was recently shown to have been inoculated with syphilis from the human. Moreover, an aninal immune to a certain disease by nature, may acquire it if inoculated with bacteria of unusual virulence. Pathogenic bacteria, after passing through a series of susceptible animals, finally becomes so virulent that, together with a lowered resistance on the part of the animal, they can overcome one which naturally has a considerable degree of immunity. Absolute immunity, however, can be acquired either by vaccination or as a result of an attack of the disease. As a rule an animal having been thus protected cannot acquire the disease, no matter how lowered his vitality, or how virulent the bacteria. For example, it is possible for an animal protected against tetanus to withstand a dose of toxin that is sufficient to kill a thousand unprotected animals. 'Physical exhaustion, hunger, exposure to cold, pestilence, and famine are factors which may reduce the natural resistance of an animal.

Acquired Immunity.-This may be artifical (active and passive) or natural (active only). If the immunizing substance is developed in the body of the immune animal, we call it active immunity; if it has been elaborated in the body of another animal and then inoculated into the animal to be immunized, we call it passive immunity. Active immunity may also be produced naturally as the result of 
recovery from an attack of disease. Artificial active immunity may be produced in three ways:

Ist. By vaccination.

2nd. By injection of toxins.

3rd. By recovering from an infectious disease.

Vaccination is a form of preventive inoculation. It was the knowledge that mild and severe attacks of an infectious disease are equally efficacious in producing immunity, which has given rise to our present methods of preventive inoculation. Jenner discovered vaccination: Pasteur was the first to place it on a practical basis. He demonstrated that the virulence of bacteria can be varied by experimental procedures. By these means, the bacteria can be very much weakened or attenuated and still produce immunity against subsequent attacks, if inoculated into an animal. They may be attenuated in several ways: By cultivation at temperatures above their optimum; by successive inoculation into non-susceptible animals; by prolonged artificial cultivation in the presence of oxygen; by exposure to certain inorganic chemical substances, as diphtheria to trichlorid of iodine, anthrax to bichromate of potash, and tetanus to a weak solution of carbolic acid; by exposure of cultures to organic extracts, such as that of thymus gland; by drying, as is done in making the virus of hydrophobia; by exposure to sunlight, electricity, X-ray, radium, etc. By these methods the bacteria are not killed; their capacity for producing disease is simply lowered.

After they have been attenuated by any of the foregoing methods, they may be inoculated into the animal with but a slight reaction on its part. Later, gradually increasing doses may be injected until the animal is immune.

No single method of attenuation is suitable for all bacteria. Anthrax bacilli may be attenuated by cultivating at $42^{\circ} \mathrm{C}$; the bacillus of chicken cholera, by exposing to light 
and air; the virus of hydrophobia, by desiccating the spinal cords of affected rabbits.

The second method of producing artificial active immunity is by the injection into an animal of gradually increasing doses of the products of bacteria-their toxinseither with or without the dead bodies of the bacteria themselves; the toxin of tetanus, for example. The virulence of the toxin may be diminished by heating it; by mixing it with organic extracts or with certain chemical substances; by greatly diluting it; by adding small doses of already prepared antitoxin, or by adding blood serum.

The third method is by recovering from an attack of an infectious disease. If an ox recovers from anthrax infection, thereafter he is immune. This active immunity may be permanent or temporary. In case of anthrax, the immunity will last for a lifetime, whereas a horse that has recovered from tetanus is immune for only a few weeks. In the majority of cases, the active immunity conferred upon an animal by an attack of clisease is permanent; it may be temporary, however, and on the other hand, it may make the animal more susceptible, as in pneumonia. A horse having had pneumonia once is more apt to have it again. Many times one attack of disease renders future attacks very mild; but they may prove fatal.

Acquired passive immunity differs only from the active in that the immunizing substance is developed first in the body of another animal and then injected into the animal we wish to immunize. This immunizing substance is antitoxin, and it is in the blood-serum where it is developed. Hence the blood-serum of an animal immune to tetanus injected into another animal will make it immune also. This is passive immunity. We immunize an animal with a substance which has been developed in the serum of another animal. 
Whatever the type of immunity occurring, it is either antitoxic or antibacterial. The serum of an animal immune to tetanus is antitoxic; for it is the extracellular toxin of the tetanus bacillus which does the harm, and not the mere body of the bacillus itself, which never comes in contact with the tissue it destroys. Its toxin, however, goes into solution and is carried from the wound to the nervous system,- the bacteria remaining at their place of entrance,-and there destroys the tissue. Hence it is the toxin which must be neutralized by a serum immune to tetanus, and the immunity is in a peculiar sense antitoxic. On the other hand, immunity to the cholera vibrio is antibacterial. The toxin of the cholera vibrio is intracellular, and is never diffused in a soluble form, so that the bacteria themselves must come in contact with the tissues they are to destroy. Experiments seem to show that the serum of an animal immune to cholera, is able to kill the bacteria. but apparently is not able to neutralize their intracellular toxins which are liberated only after the bacteria are disintegrated. Such immunity is antibacterial. It is obvious, therefore, that immunity, whether natural or acquired, may be in one case antitoxic and in another antibacterial.

Antibacterial immunity does not always imply a bactericidal serum. For example, the dog and rat both are immune to anthrax, yet the serum of the dog has no bactericidal effect on the anthrax bacillus, while that of the rat is strongly bactericidal. This would indicate that the serum is not the only factor governing an immunity. In the same way immunity to toxins is not in all cases antitoxic in the sense that the serum contains demonstrable antitoxin which will neutralize and render innocuous its corresponding toxin. 


\section{THEORIES OF IMMUNITY.}

Before discussing the theories of immunity, we shall take up the natural defenses of the body.

Every time bacteria come in contact with a susceptible animal, infection does not necessarily follow, otherwise the animal race would soon be destroyed. Most all pathogenic bacteria can be isolated from the hide and hoof of animals - streptococcus, staphylococcus, tetanus, etc.-while in the mouth are normally found such germs as pneumococcus and influenza bacillus; in the intestinal tract, the colon bacillus, or the bacillus dysenteria. The body has natural defenses which prevent the development of these bacteria. First, the

Skin constitutes a covering of great importance as a means of protection against microbial invasion. In all the domestic animals it is a prolific seat of bacterial flora, which seize every favorable opportunity to attack the animal, producing abcesses, phlegmons, or even general infection of blood and tissue, as in septicæmia and pyæmia. Normally, the skin can resist these attacks by the constant production and expulsion of its corneal cells. This continued exfoliation of the cells of the deeper layers of the epidermis results in a simultaneous eviction of such micro-organisms as are living on them. The connective-tissues of the skin, also the hair, horns, and hoof, are normally able to protect themselves against bacteria by reason of their impenetrability. All these tissues have the service of the phagocytes, which wander about everywhere, ever ready to pick up foreign invaders.

Mucous membranes are able to resist bacteria by the throwing-oft of their cells, which take the germs with them, or by the antiseptic protection of the mucus and saliva with which the germs come in contact. These secretions 
may carry the bacteria from the pharyngeal cavities into the stomach, or out of the body by way of the mouth and nose. The food stuffs also may carry the bacteria into the stomach. There are numerous saprophytic or non-pathogenic bacteria all along the alimentary tract which may antagonize and destroy the pathogenic bacteria.

The conjunctiva rids itself of bacteria chiefly by removing them mechanically and washing them down through the lachrymal duct into the nasal cavity. The mucous membrane of the nasal cavity throws off the bacteria by its secretions, aided by the ciliary action of the epithelium. The stomach destroys bacteria through the action of the hydrochloric acid. Few bacteria can live in an acid medium. The vagina is normally free from pathogenic bacteria, owing to the fact that there are constantly present in the vagina saprophytic bacteria which have an acid secretionthe bacillus Doederlein, for example.

One of the most important means of defense which the body possesses is the blood. Normal blood-serum, even when free from leucocytes, is toxic for bacteria; and furthermore, it not only destroys these vegetable cells, but animal cells also. Whether the power exists primarily in the serum or is one conferred on it by the leucocytes, leads to the discussion of the two great theories of immunity.

METCHNIKOFF THEORY : - Phagocytosis. - Eli Metchnikoff, the distinguished biologist and bacteriologist, who is now one of the heads of the Pasteur Institute in Paris, and successor to Pasteur, first gave his theory of phagocytosis to the world in an address given at Odessa, before the congress of naturalists and medical men in 1883 ; and since that time by many ingenious experiments and much painstaking work has been constantly adding new proof to his primary hypothesis, until now many bacteriologists believe that his theory satisfactorily explains im- 
munity, and the entire scientific world admits it to be at least a link in the solution of a most complex and interesting problem, one which deals with the vital welfare of all the domestic animals and man.

If a few anthrax bacilli are introduced into the body of an animal, it will be soon noticed that some of the leucocytes are attracted by the chemotactic influence of the bacteria. The cells pick up the bacteria, incorporate them into their protoplasm and digest them by means of a ferment which they possess called cytase. All cells which pick .up and digest bacteria are called phagocytes. Metchnikoff describes two kinds, the microphages and macrophages. The microphage is the polymorphonuclear leucocyte, with its irregular lobulated nucleus, which is regarded as an adaptation to enable the cell to pass as rapidly as possible through the walls of the capillaries and venules. This active process is called amœboid movement. The macrophages consist of the mononuclear lencocytes or the so-called large lymphocytes. Other cells have phagocytic properties also, such as fibroblasts and endothelial cells, but they have little or no power of movement.

Phagocytosis Metchnikoff states to be one of the principal means of defence possessed by an animal. As soon as bacteria have penetrated the body, a whole army of leucocytes proceeds toward the point of invasion, and there enters upon a struggle with the micro-organisms. The macrophages follow the microphages and become mixed in greater or less numbers with the exudation. The leucocytes having arrived at the spot where the bacteria are found, seize them within their bodies and subject them to intercellular digestion. This digestion takes place in the vacuoles, which usually contain a weakly acid fluid containing ferments. Micro-organisms attract not only leucocytes, but also other bodies, inert particles of dust, 
aseptic fluicls, chemicals, croton oil, turpentine, cells from other animals, such as blood corpuscles and spermatozoa; and in fact, most all foreign sulbstances, when introduced into an animal possess chemotactic power, both positive and negative.

The phagocytes choose borlies best suited for their use. The macrophages seize by preference animal cells such as blood corpuscles, sperniatozoa and the animal parasites, etc, and those micro-organisms which set up chronic diseases like glanders, tuberculosis, actinomycosis, etc. The macrophages can also ingest bacteria of acute diseases, but with the exception of a few cases, their intervention is of little moment. The microphages appear to play their part especially in acute infection, such as one' caused by streptococci, hog cholera bacilli, etc. Their intervention against animal cells is nil, or almost so. They appear to be repelled by parasites of animal origin and by certain bacteria which set up chronic processes.

The morphological and physiological differences between the two great categories of mobile phagocytes correspond to the differences in their soluble ferments or cytases. These cytases, (alexins of Buchner, or complements of Ehrlich) can be extracted from the leucocytes, and under certain conditions are found free in the blood. The macrocytase is capable of dissolving the red bloodcorpuscles and other cells, which the microcytase will destroy and digest many micro-organisms. Thus Metchnikoff states that there are two kincls of cytase, while Ehrlich holds that there are many kincls. These cytases are not thrown off by the phagocytes so long as they remain intact. When these cells are injured, however, they allow a part of their cytase to escape, which gives to the blood its antitoxic, hemolytic, and bactericiclal properties. The destruction of the phagocytes by which the cytase is set free into 
the blood is called phagolysis. Metchnikoff states that besides the cytase, another substance exists in the blood which attaches itself to the bacterial cell and renders it susceptible to the action of the cytase. This he designates by the name of fixative. The fixative and cytase are both soluble ferments, but can easily be differentiated. The cytase is clestroyed by heating at $55^{\circ} \mathrm{C}$, while the fixative is destroyed by heating $60^{\circ}$ or $65^{\circ} \mathrm{C}$. The fixatives are differentiated by a high specificity never observed in cytase, in that the majority of them are not capable of attaching them. selves to more than one species of bacteria or to one class of animal cells. The microcytase, on the other hand, can attack all kinds of micro-organisms and the macrocytase all kinds of animal cells. As to the origin of this fixative (the amboceptor of Ehrlich) Metchnikoff believes that it comes from the phagocytes. It is involved in antibacterial immunity.

The fixatives and cytases are not the only soluble ferments which appear in the fluids of immunized animals. The serum is often characterized by another distinct property which Metchnikoff designates the protective substance. This substance resists higher heating than any of the other ferments, even to $70^{\circ} \mathrm{C}$. Under this temperature a protective serum loses its bactericidal power, if this is present, but will prevent infection when injected into another animal,-Asiatic cholera, for example. Similarly, normal serums contain no fixatives, but they are protective. On the other hand the fixative may be present in serums which are not protective, and still further, not every immune serum will protect other animals when injected into them. Very often there are found agglutinative substances in immune serums. These resist the same temperatures as the fixatives, and are characterized by the same specificity, but a slight agglutinative property may be associated with a 
very marked protective or bactericidal (fixative) property and therefore the part it plays in immunity is not sufficiently constant to base any theory upon its presence.

A number of facts appear paradoxical. If cavies are immunized against the vibrio Metchnikovi, the blood of the cavy is very bactericidal as regards the vibrio. If some of these vibrios are placed into a test-tube containing blood serum from one of these pigs, they will be destroyed within less than an hour. Nevertheless, if a small dose of this culture is injected subcutaneously into these hyper-vaccinated cavies, the vibrio will remain alive for several days up until the time that they are ingested and destroyed by the leucocytes. This apparent contradiction Metchnikoff explains by the fact that in the test-tube the vibrios encounter the microcytase which has escaped from the microphages at the time of the formation of the clot and the separation of the serum; while in the subcutaneous tissue of the immunized pig the vibrios live on until the leucocytes collect in sufficient numbers to destroy them by the cytase which they set free. Then again, the blood of animals which are immune to a certain disease, is not always bactericidal against the germ causing the disease. This Metchnikoff explains from the fact that cytase escapes from the leucocytes with great difficulty and hence an animal can be immune whose serum is not bactericidal because it does not contain the free cytase.

'There must exist, then, something other than the powers of the fluids of the body, that is to say some other factor which plays the predominant part in immunity, and according to Metchnikoff, phagocytosis is the most constant and general phenomenon. He finds it in cases where the humoral properties are the most marked, as well as in those in which they are only slightly developed or entirely absent.

With these factors at hand-the phagocytes, and their 
soluble ferments, the cytases and fixatives, Metchnikoff exp.ains the various types of immunity as follows:

In natural immunity the microphages stand out as the defensive cells par excellence against micro-organisms. The macrophages may act in some degree, but there is no help on the part of the body fluids. The phagocytes seize and ingest the living micro-organisms which they dissolve completely by means of their intracellular ferments, the cytases. The agglutinative power of the normal fluids plays no important part in natural immunity.

In acquired immunity, there exist as a fairly genera! rule, other substances whose role in the defensive action offered by the animal against micro-organisms is very important. These are the fixatives, which permeate the bacteria and render them more susceptible to the bactericidal action of the microcytase. These fixatives, however, although found in the serum, are undoubtedly of cellular origin, probably the phagocytes. Having absorbed the micro-organisms, they set to work to elaborate large quantities of fixatives, which they secrete and pass into the blood plasma and other fuids. This excretion, however, is not an indispensable act for the functioning of the fixatives. In the cases of acquired immunity where no fixatives are found in the body fluids, they remain lodged in the phagocytes, where they act in conjunction with the cytases.

As, in the majority of cases of acquired immunity, the blood-serum contains fixatives in considerable proportion which aid the action of the cytases, we can understand that the injection of such a serum into other animals would increase their resistance also. The fixatives injected with the serum, fix themselves with avidity upon the micro-organisms and make them a more ready prey to the phagocytes. Thus Metchnikoff explains passive immunity.

Immunity is much more readily acquired against micro- 
organisms than against their toxins. Antitoxic immunity Metchnikoff presents as far more difficult of solution than is that of acquired immunity against micro-organisms. He says that it is impossible to look to the body fluids for a satisfactory explanation, but claims an interceptive power on the part of the cells of the organs less vital than those which the organisms aim to attack. In an animal immune to tetanus for example, the liver cells may fix the toxin before it reaches the nervous centres, and prevent it from doing harm.

\section{EHRLICH'S THEORY-SIDE-CHAIN THEORY.-} The author of this theory, Dr. Panl Ehrlich, A. M., the CELL
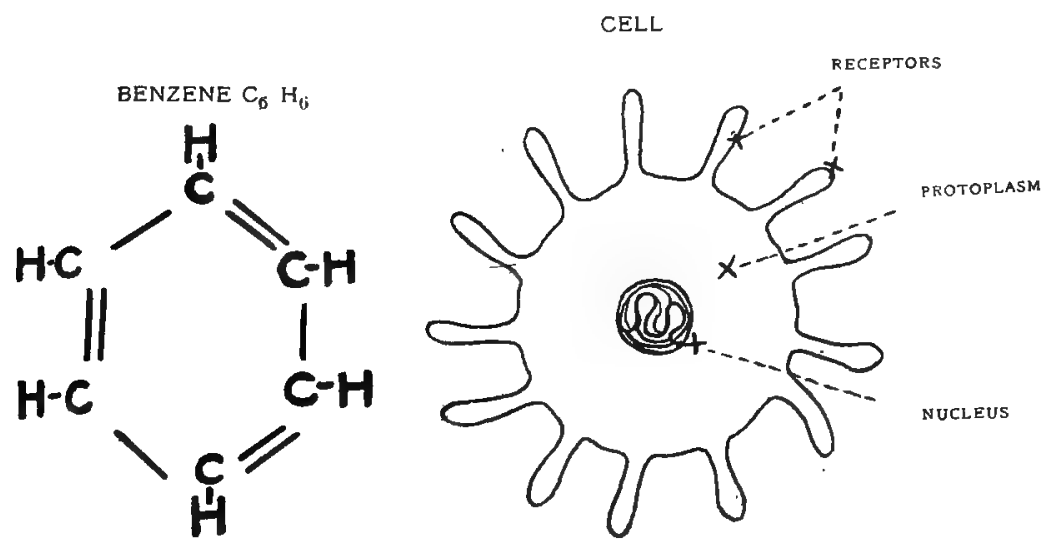

FIG. 18A.

director of the Royal Institute for Experimental Medicine in Frankfort, first announced his brilliant discovery in I885. $\mathrm{He}$ is not only the author of the most probable theory of immunity, but he is the most distinguished living hamatologist and the author of our method of standardization of antitoxin.

Side-Chain Theory.-According to Ehrlich, every cell of the body contains, besides its nucleus, a number of sidechains, or receptors, arranged around it much the same as the atoms in the benzene ring, in which six atoms of hy- 
drogen unite to six atoms of carbon. The chemical formula of benzene with its various side-chains may be graphically compared with a cell and its receptors.

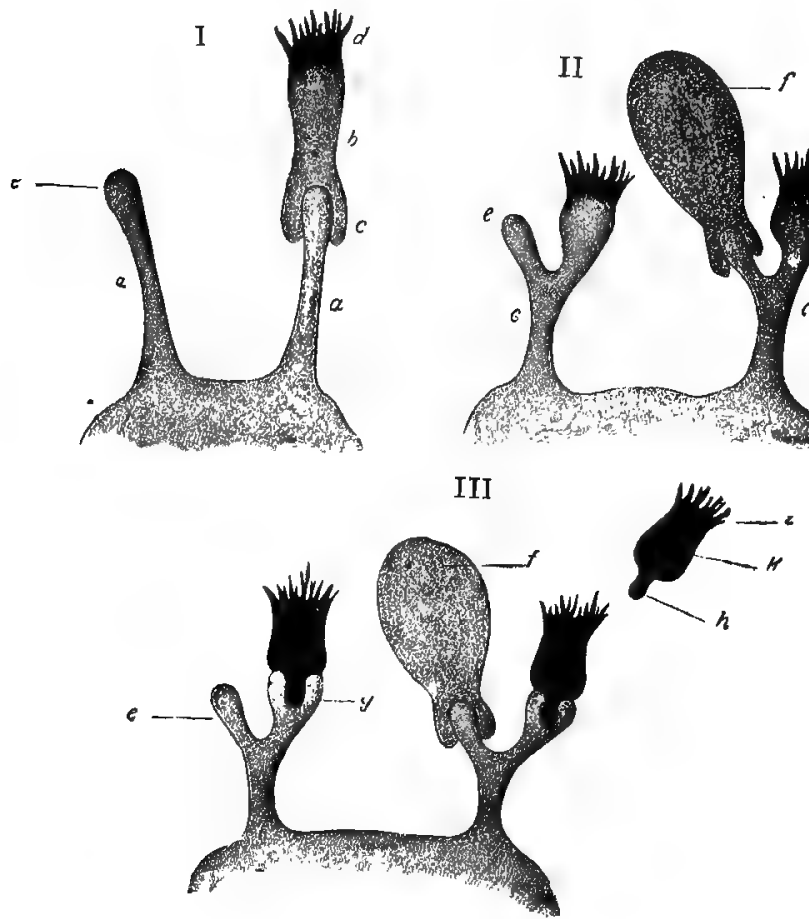

FIG. 19.

Receptors of the First Order, are represented in Fig. 19, I., at $a$, being attached to the cell, molecule beneath. The portion $e$ represents the heptophorous complex, whilst $b$ represents a toxin molecule, which possesses a haptophorous $c$ and toxophorous $d$ group: This represents the union of toxin and antitoxin, or ferment and antiferment, the latter possessing but one haptophorous group, the union between antibody and the toxin or ferment being direct.

Receptors of the Second Order, are represented at $\sigma$, in Fig. 19, II., in which $e$ represents the haptophorous, and $d$ the zymophorous group of the complex, $f$ being the food-molecule with which it enters into combination. Receptors of this order are possessed by agglutinins and precipitins. The digestive cell, or the antibody present in the serum in this case, possesses one haptophorous and one zymophorous group.

Receptors of the Third Order, are represented in Fig. 19, III, $e$ being the baptaphorous group, $g$ the complementophile group of the complex, $k$ tho complement with its haptophorous $h$ and $z$ ymotoxic $z$ group ; whilst $f$ represents the food-molecule which has become linked to the receptor. Such receptors are found in hemolysins, bacteriolysins and other cytolysins, the union with these cellular elements being effected by means of the immune-body, this permitting the complement to act as a digestive ferment. - Nuttall.

The sicle-chains, or receptors, fulfil various accessory functions, but serve especially for the nutrition of the cell. A molecule of assimilable substance, coming in contact with the cell protoplasm, is absorbed by combining with one of 
the sicle-chains much the same as a key fits into a lock. In this way it enters the cell itself and becomes a chemical part of it. A side-chain combining with a simple substance such as toxin molecule, Ehrlich calls a uniceptor; one combining with a complex substance he calls an amboceptor. These receptors have a great affinity for the various substances maintaining the life of the cell. Under normal conditions these receptors seize nutritive molecules which serve as food; while under special conditions these receptors lay hold of complex molecules of albuminoid substances like toxins, and instead of combining with a molecule which nourishes the cell, unite with one which poisons the cell.

According to Ehrlich's theory, the toxin molecule contains two anatomic groups,-one which combines with a receptor of the cell, the haptophore, and one which poisons the cell, or the toxophore. This is illustrated as follows:

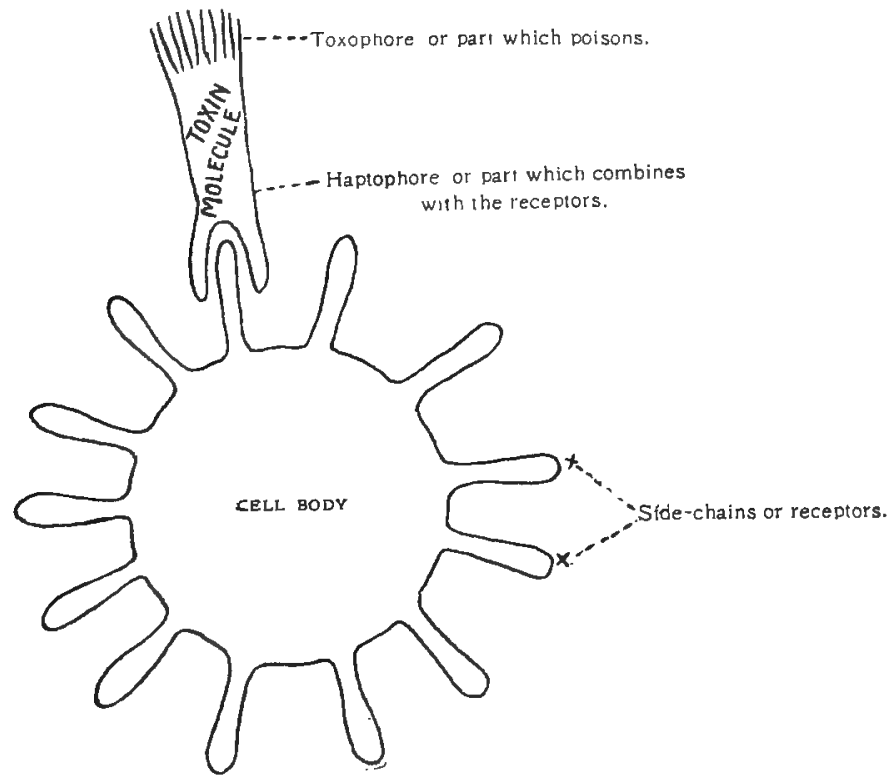

FIG. 19A.

Unless a cell has receptors that fit the haptophore group of the ioxin molecule, they will not combine. They will lie 
side by side without fixing each other. On the other hand, when the toxin molecule unites with the cell, it not only diverts the receptors from their normal functions of nutrition, but it actually poisons the cell so that the more toxin introduced, the more receptors abound, and the greater the injury to the cell. This injury the cell makes up by a process of regeneration resulting in the manufacture of new receptors which are reproduced in excess of the number destroyed by the toxin: This excess may be so great that the cell is over-filled with them-so over-filled that many are cast off into the circulation and exist independently of the cell. These cast-off receptors can still unite with toxin, and thus become a means of protection to the cells. Let us suppose that one thousand molecules of toxin get into the body and one thousand free receptors unite with the toxin. Then no toxin molecules reach the cells and no cells are poisoned. This is immunity.

These free receptors constitute our antitoxin and thus become a means of protection to the cells. If we stimulate a horse to produce free receptors by injecting him with several non-fatal doses of tetanus toxin, we produce a state of active immunity in his body. If we then bleed him and inject his sertum containing free receptors of antitoxin into a horse stricken with tetanus, we find that the molecules of tetanus toxin in the stricken horse are pulled away from his tissue cells and become fixed to the free receptors of the injected serum. We thus produce a passive immunity.

As regards the structure of the antitoxin or cast-off receptor, it remains the same as when attached to the cell, i.e., it consists of one combining group only, the haptophore. Ehrlich designates it as uniceptor.

The foregoing explains only one type of immunity, namely, antitoxic immunity. Another type remains, antibacterial immunity, in the explanation of which Ehrlich 
takes up the phenomena of "lysis." These phenomena invo:ve not only the dissolution (lysis) of bacteria, but also of other cells. Three general lysins are named:

I. Bacteriolysins, - those which dissolve bacteria.

2. Hemolysins,-those which dissolve red blood corpuscles.

3. Cytolysins,-those which dissolve other cells, such as spermatozoa, etc.

The practical application of all the lysins is discussed under a special heading. We shall now take up the constitution and action of those which are involved in antibacterial immunity. They are the same in structure as the hamoiysins and cytolysins, and the action of all is similar.

It was Pfeiffer who discovered that a cavy which has been immunized to cholera vibrios will dissolve them if they be injected into the peritoneal carity. The freslily drawn serum of the animal also possesses the same power, but loses it if heated to $55^{\circ} \mathrm{C}$., or if it stands for some eight or ten days. But if this heated or old serum be injected into the peritoneal cavity, or blood of another guineapig, or of any animal, or if any fresh serum or blood be added to it in a test-tube, its bactericidal property is restored, and it will again destroy the bacilli. From these observations the fact has become graclually apparent that there are two vital principles in a bactericidal serum: one which is resistant to heat and is produced by the immunization of an animal to cholera bacilli, and one which is sensitive to heat and is present in the healthy normal serum of every animal. Ehrlich designates these two substances as immune body and complement. The immune body he considers to originate the same as the antitoxin from the receptors of the cells. Inasmuch as it cannot destroy the bacteria alone, but can when the complement is present, he represents its 
body as having two combining groups,- - one which unites with the bacteria, the "cytophilic haptophore," and one which unites with the complement or "complementophilic haptophore." He therefore calls the immune body, the amboceptor. It attaches itself to the bacteria and renders them vulnerable to the complement, which alone could not dissolve them. The complement evidently has a combining group by which it can unite with the amboceptor, and a lytic group by which it destroys the bacterial cell if united to it. It is, therefore, in structure, similar to the toxin molecule. The action of the inmune body and complement upon the bacteria may be graphically represented as follows:

As we have already stated, the lysins not only dissolve bacteria, but they also dissolve other cells. If we inject the red blood-corpuscles of a horse into a rabbit, the rabbit's blood will dissolve the red blood-corpuscles of the horse. This takes place in a test-tube as well as in the body. The serum of one animal will dissolve and destroy the red blood cells of another animal of different species. The practical application of this experiment is that it prevents us from inoculating the blood of one animal into another for therapeutic purposes. For example, we cannot replace the blood of a calf which has lost a great deal of blood with that of a horse. However, we could inject him with blood from a cow or another calf with benefit.

Ehrlich's theory also explains another kind of cell receptor, which governs the phenomena of agglutination and the action of precipitins. The Widal Test for hog cholera depends upon the action of the agglutinins. The effect of the cholera poisoning, after a few days, is an increase in a number of receptors on the cells of the boly. These receptors are stimulated into existence by the cholera toxin, and in consequence they fit nothing except the cholera bacilli. As the cell becomes overloaded with these recep- 
tors, they are shed into the plasma, where they unite with the cholera bacilli for which they are specific, and so affect them that the bacteria become non-motile, and stick together, forming clumps, i. e., they are agglutinated. The receptors thus have two characteristic groups; one, a haptophore which combines with the bacterial cell, and another the zymophore, or agglutinating property, which agglutinates the cells.

Similar to the action of the agglutinins is that of the precipitins. If a rabbit is repeatedly injected with ox-blood, his tissue-cells are stimulated to form receptors which fit ox-blood only. After a few days, the cells shed receptors into the serum. Now, when this rabbit's serum is mixed with ox-blood, the receptors, through their haptophores. unite with certain chemical substances in the ox-blood, which they precipitate by means of their zymophores. This zymophore portion of the receptor corresponds to the toxophore portion of the toxin molecule, and the complementophile portion of the complement. The zymophore group consists of a ferment which has the peculiar property of causing certain cells to agglutinate, or to form precipitate in other cases.

AGGLUTININS:-These have a large practical value in veterinary science, in that they are of valuable aid ir: diagnosis. The agglutination test has taken its place among the routine methods of diagnosis, and has the advantage of being pathognomonic, in that it will diagnose certain bacteria, - the glanders bacillus, hog cholera, tubercle bacillus, bacillus dysenterix, streptococcuis and many others. The test is as follows:

Cover-Glass Method:-Let us take, for example, the hog cholera bacillus. Obtain a drop of blood from the suspected animal, and let it dry on a slide or piece of paper. Then get a culture of known hog cholera bacilli, preferably, 
a young culture about is hours old. O:d cultures, or even dead bacteria, will do. Take a platinum-loopful of these known cholera bacilli and place upon the centre of a coverglass. Dilute the suspected blood with distilled water from 50 to roo times. This can be done by mixing the blood and water on a slide until the desired dilution is obtained. Then add a loopful of this diluted blood to the bacteria on the cover-glass. Take this cover-glass and turn it, loaded side down, on a concave slide which has first been rimmed with vaseline around its concavity, thus making an airtight chamber. Care must be taken not to mix the drop with the vaseline or allow it to dry. Place the slide under the microscope, using the oil-immersion lens. The procedure is the same as that of the ordinary hanging-drop method. Upon examining the slide, the bacilli will be seen to be motile and moving about if a live culture has been used; if a dead culture has been taken, the bacilli will be scattered over the field as single bacteria. If the blood is that of hog cholera, the bacilli will be seen to lose their motility, become shortened, rounded, and to stick to each other, forming clumps. They have been agglutinated. The reaction takes place in from five to thirty minutes; it is always well to wait at least an hour. A negative test shows no clumps, i. e., no agglutination, and the bacteria retain their motility.

Test-Tube Method:-This method of making the agglutination test has the advantage of not requiring a microscope. Let us take the bacillus dysenteria for example. Obtain a culture of the bacilli in a test-tube of bouillon (I8-hour-old culture is best; old culture will do) and also place some blood from the suspected animal in another test-tube. After the blood has clotted, take some of the serum, about I-5O of the bouillon, and add it to the testtube containing the given bacilli. If the boutllon measures 
tcn c.c., then I-5 c.c., or about three drops, would be the amount. The next step is to place the test-tube in the incubator at $37^{\circ} \mathrm{C}$ or in room temperature for eighteen hours: then examine: If the test is positive, i. e., if the animal is infected with bacillus dysenterice, the boutllon has lost its opacity; the bacteria will have agglutinated, and will form clumps at the sides and bottom of the test-tube which are visible to the naked eye.

Care must be taken to dilute the blood, as normal serum? undiluted will also cause agglutination. The amount of dilution differs with different species. With some bacteria the reaction is positive with a dilution of one-thousand.

The agglutination not only takes place with bacteria, but also with red blood corpuscles and other animal cells. Pathologists are beginning to explain thrombosis and embolism by this phenomenon.

PRECIPITINS:-The precipitin test is being used in biology to classify the different species of animals. Its practical application in veterinary science lies in the differentiation of bloods, meats, and albumins. In the chapter on Hrematology, the test is applied to the blood It may become necessary sometime to decide whether a piece of meat is that of beef, of a horse, or of some other animal. Neither anatomical, nor histological, nor chemical examination can decide. It is necessary to resort to the precipitin test.

If we wish to determine whether a given sample of meat is beef or not, we inject a little ox serum into a rabbit, (about 5 to ro c.c.) twice a week for a month. Then take some of the rabbit's serum and place in a test-tube; make an aqueous extract of the sample of meat and acld about $\mathrm{I} c$. $\mathrm{c}$. of it to the ox-serum. Put in the incubator for a few hours. If the meat in question be beef, we shall get a precipitate, a marked, flocculent, clouding. But, if the meat be from 
some other animal, the serum will remain clear. If we wish to determine the actual source of the meat, we inoculate rabbits with blood from all the probable animals from which the suspected meat might come (using only one kind of serum for each rabbit). After a sufficient number of inoculations, usually about eight or ten, place a little serum from each into as many test-tubes, and also into each testtube a little of the aqueous extract of the nnknown meat. The test-tube giving a precipitate indicates the source of the meat.

The precipitin test can be used also for spermatozoa, and all tissues of the bodies, even the urine.

\section{OPSONINS.}

Certain bodies called opsonins represent a property of serum which renders bacteria more susceptible to the phagocytes and are especially associated with infections caused by streptococci, staphylococci, pneumococci, etc. The study of immunity against these last-named microbes has led to the greatest diversity of opinion, some claiming antitoxic properties, others lytic, and others phagocytic properties for the immune sera. A very remarkable advance toward the definite solution of this controversy has been made by Wright and Douglas (1903), who have shown that the serum of the blood forms a definite and independent role in connection with phagocytosis and is brought about by the action of certain substances which have been designated opsonins, whose function in some way is to so change the microbe that it will fall an easy prey to the leucocyte. If the opsonins are removed from the blood-serum, the leucocytes will not manifest any phagocytic action. Opsonins are very susceptible to heat and also, like complement, disappear spontaneously from drawn serum in a short time. They seem to have a structure like that of toxin. 
The "opsonic power or index" represents the relation between the phagocytic power of an animal and that of a normal animal of the same species. It is obtained by mixing a little of the animal's serum with washed leucocytes and a few bacteria, and determining from a stained specimen of the mixture after it has been incubated at $37^{\circ} \mathrm{C}$ for I 5 minutes, the average number of bacteria per leucocyte ingested, which may be ten, twenty, etc. Staphylococci per leucocyte in the presence of a normal serum for man equals twenty. If that of a given serum say is fifteen, then the opsonic power would be $15-20$ or $3 / 4$ of normal. The normal index varies in clifferent animals.

The pathological value of a low and high index has not as yet been worked out. A low index has been observed, however, at the beginning of pneumonia, while at the crisis it may rise as high as 1.6.

There are many other interesting bodies which have entered into the explanation of immunity, such as anti-antibodies, cytotoxins, auto-antibodies, iso-antibodies, toxids, toxons, etc. For an understanding of these, the reader must be referred to a more complete work on the subject of immunity.

From the foregoing, it will be seen that the explanation of inmunity while yet a theory, has too many practical features for the veterinarian to ignore it.

As to the structure and action of toxin and antitoxin, they have proved to be chemical bodies of definite action. Antitoxin can be introduced into an animal without producing any harm. Also, if portions of toxin and antitoxin are mixed together in a test-tube, and inoculated into the body, no harm results. Thus it will be seen that antitoxin combines with toxin in a test-tube as well as in the body. 


\section{SERUMS-DIAGNOSIS, PREPARATION, ETC.}

THE TOXINS IN DIAGNOSIS:-It has been found that if we inject an animal suffering from tuberculosis with some toxin of the tubercle bacilli, or tuberculin, we shall notice an elevation of temperature. This is called the $\mathrm{Tu}^{-1}$ berculin Test. Similarly, if we inject some toxin of the glanders bacilli into an animal suspected of glanders infection, a reaction occurs in the form of a marked swelling at the site of injection, and an increased temperature. This is the Mallein Test. Similar reactions are sometimes seen when other infections are tested with the toxins of the corresponding bacteria, but as yet have not found universal acceptance.

\section{THE MANUFACTURE AND STANDARDIZATION OF SERUMS - VACCINES : - First - Antitoxic Ser-} ums: These are usually prepared by injecting an animal, preferably a horse, with gradually increasing doses of the toxin until the animal is able to withstand a very large dose, which if injected at first, would have proved fatal. This process we call "Immunizing the Animal." The toxins used for the injections are obtained from the living bacteria. These organisms are first grown in various culture media. The cultures are then run through a Pasteur Chamberlain filter, or similar one, to remove the bodies of the bacteria. The filtrate contains the toxin only, and is used for immunizing the horse.

After the horse has been thoroinghly immunized, he is bled, the blood allowed to coagulate, and the serum is separated from the clot. This serum contains the antitoxin and is ready for use.

Antibactericidal serums are produced similarly to the antitoxic serums, but the actual bacteria themselves are injected instead of the toxins: We begin by inoculating 
deacl or attenuated cultures into the animal, gradually increasing the virulence or dose of the bacteria, until the animal is able to stand a close which in the beginning would have killed him.

In the manufacture of anti-streptococcus serum, many varieties of streptococcus are used. Such a serum is called a "polyvalent" serum.

The manner of measuring the amount of serum to be injected into an animal is as follows:

We first determine the amount of toxin necessary to kill a guinea-pig of 250 grams weight. This is called the "minimum lethal dose". The amount of antitoxin necessary to neutralize Ioo lethal doses is called a unit. A cubic centimeter of diphtheria antitoxin contains 200 or 300 units. A cubic centimeter of tetanus antitoxin may contain a rooo units.

Antibactericidal serums are not standardized. They are either diluted or are mixed with some mild antiseptic, or heated in order to attenuate the bacteria.

By the method of vaccination we usually inoculate a small dose of the bacteria themselves or a fairly large dose of toxin into the animal, thereby giving the animal a mild attack of the disease. He thus produces his own antitoxins.

PRACTICAL VALUE OF THE VARIOUS SERUMS.-As curative, prophylactic and diagnostic means, the serums are valuable in the following diseases: Anthrax, glanders, tuberculosis, cattle plague, Texas fever, symptomatic anthrax, swine erysipelas, swine plague, hog cholera, sheep and cow-pox, foot and mouth disease, distemper, gangrenous septicamia, streptococcus and staphylococcus infections and many others. These serums are also used in malignant diseases caused by cancers and o!lıt' tumors. 
The experience of some veterinarians would tend to prove that the antitoxins are of little therapeutic value; but serum-therapy is comparatively a new science, and we are learning more about the nature of these bodies, how to manufacture them and administer them, and the future is full of splendid possibilities. We believe that the medicine case of the future veterinarian will contain a large stock of antitoxin. 


\section{CHAPTER VII.}

\section{SURGICAL SHOCK.}

DEFINITION.-The word "shock" is now pretty generally applied in various forms of collapse that follow traumatisms, either accidental or surgical. It includes "true shock," "secondary or delayed shock," "traumatic syncope," "fainting" and other various forms of more or less mysterious collapsing states which leave no identifying lesions behind. In a word, true shock is still a mystery. Different authors, from the earliest history of surgery until the present day, have interpreted it differently. By some it is attributed to an exhaustion of the nervous system; others asscribe the entire phenomena to a disturbance in the equilibrium of the circulatory apparatus; while still others refer to it as a reflex inhibition. Whatever is the real feature of the condition, shock, so far as it concerns animal surgery, must be defined as a sudden collapse of the functions of the whole body due to physical injury, prolonged pain, loss of blood, or circulatory disturbance. It is, in fact, a depression of the whole body, without reaction.

More recently shock has been attributed to the accumulation in the nerve centers of the products of destructive metamorphosis in excess of their elimination. That is to say, it is an autointoxication. The nerve centers and the nerves poison themselves by not being capable of eliminating wastes, and thus causing the general depression of all the functions, which in turn accounts for the unusual symptoms seen in typical shock. The flow or stagnation of blood in the splanchnics is explained by the fact that while all of the blood vessels are dilated the former offer a "point of 
least resistance" that is ever ready and always capable of taking an excess of blood. Reaction, from shock is said to occur from the gradual resumption of elimination of these wastes.

ETIOLOGY.-Shock is not as common in animals as in man, in whom, mental emotion and a greater sensitiveness of the nerres, seem to provoke attacks that animals escape, owing to their relatively slight sensibility. In animals shock is induced by three principal causes: (I) Physical injury; (2) prolonged pain or suffering that is suddenly relieved; (3) painful and bloody operations.

I. Physical injury is one of the chief causes of shock. When death supervenes after an injury that has not materially interfered with any of the vital organs, for example, an extensive mutilation of a limb, shock is its cause. In animals only very serious injuries are capable of provoking the state under consideration. Mental impression, apprehension of death or terror, which in man both cause and augment shock, play but a minor role in animals. Serious injiries are capable of abruptly arresting action of the heart and respirations, of producing loss of consciousness, or of depressing all of the functions for several hours. The seriousness of shock in animals is subordinate to the character of the lesion, the degree of pain, and the amount of blood lost. It may rapidly terminate fatally or gradually yield to the reaction of the organism. The trivial lesion is never followed by shock, in the domestic animals; it requires such serious traumata as crushing of a foot, wrenching off of.a hoof, extensive mutilation of the muscles of the hip, etc., to bring on the state. The writer has seen a horse die in six hours after the accidental wrenching of a hoof from wedging the shoe-calk on the rail of a street car track, and a dog succumb to the crushing of a fore leg at the carpus after suffering from shock for fourteen hours. Although a care- 
ful post-mortem was made in both of these subjects, no internal injuries were found.

2. Prolonged Pain or Suffering.-This cause is the important one to the veterinarian. Animals may suffer from a severe pain or some condition of discomfort for a long time and maintain a fair condition of general strength, but if by some intervention sudden relief is given, shock may promptly supervene. Shock from this cause follows lancing of large abscesses; evacuation of intestinal gases, tapping the dropsical thorax or abdomen; ablation or reposition of an inverted uterus, etc., etc. Any surgical operation that affords instant relief of a long-standing suffering may cause fatal shock. A fatal shock was observed in a gelding from the simple operation of relieving an over distended bladder by lancing a stricture that had resulted from amputation of the penis some two weeks previously. The amputated stump cicatrized over the urethra and gradually obstructed the flow of urine. When examined the bladder was found enormously distended, but the patient was in fair condition of health. The appetite was normal, there was no fever and the countenance was normal. The only abnormality apparent was the frequent unsuccessful attempts to void urine. The urethra behind the seat of amputation, distended and fluctuating, was lanced with the scalpel. The bladder rapidly evacuated its contents, but instead of affording the expected relief the patient almost immediately began to tremble as if in a chill. Soon a slight perspiration appeared about the forehead and ears and then over the entire body. The countenance became anxious, the eyes staring, the respirations accelerated, the pulse small, fast and weak, and the mucous membranes pale and anæmic, and finally cyanotic. This chain of symptoms continued incessantly for two hours, at which time death terminated its 
existence. The post-mortem disclosed no perceptible internal lesions.

Similar phenomena are often seen to follow amputations of the uterus in the cow, or even the simple reposition of a uterus that has been inverted for some hours. The sudden evacuation of intestinal gases of enormously bloated horses or oxen frequently gives the most striking examples of shock from this special cause. Soon after the sudden relief from such grave conditions the above symptoms rapidly follow and continue until death.

This special form of surgical shock, which is ever liable to occur in veterinary manipulations that are capable of disturbing the equilibrium of the circulation is very easily explained. The whole disturbance is due to an overflow of blood into the splanchnic vessels. In the case of the bloated horse or ox, practically all of the blood is driven out of the abdominal viscera by the pressure of the gas. In addition the small vessels of the viscera are weakened, in fact, paralyzed by the severe tension, when suddenly the pressure is removed by evacuation of the gas through the trocar, the blood pent up in other parts of the body suddenly overflows and over-fills the visceral blood vessels. The amount of blood that the weakened vessels are capable of holding within them is sufficient to deprive the peripheral vessels of their necessary supply. The skin becomes cold and clammy because its blood is within the abdomen, the brain becomes anæmic and hence incapable of governing the functions because its necessary blood is elsewhere, and the heart, owing to the deficiency of the blood volume, weakens as if an external hæmorrhage had occurred. Shock of this variety is, in fact, analogous to hamorrhage, from which it varies only in the fact that the blood, instead of being outside the body, is stagnated in the splanchnic vessels. Similar phenomena may occur in the uterus, the 
lungs, the pleura, the peritoneum, etc., but the shock from these causes is less severe than that occurring from engorgement of the abdominal viscera. The post-mortem in these cases always confirms the exactness of the theory; the splanchnic circulatory apparatus is found over-full of stagnated blood.

3. Painful and Bloody Operations.-The shock that follows long, painful, sanguineous surgical operations on animals is similar to that following the accidental wound. Loss of blood, associated with the pain and the injury of a surgical operation, augments the degree of shock. But surgical shock may occur mysteriously in the absence of both pain and hæmorrhage. In man it often follows the bloodless operation performed under the influence of profound anæsthesia, and in these instances it is not unusual for the symptoms to make their appearance before the patient has revived from the anasthetic. In animals this particular phase of shock is seldom, if ever, observed. The collapse of an animal occurring during the procedure of an operation is generally traceable to some internal rupture or chloroform syncope. It is after the long operation, the painful operation, and the sanguinary operation, but especially a combination of all three, that shock is so frequently observed in veterinary surgical operations. Some minutes, and sometimes several hours elapse before the grave symptoms appear. The patient is already in the standing posture and returned to the stall before any unusual symptoms are observed. Sometimes after a long operation performed under the restraint of casting ropes, the shock may prevent the patient from arising immediately. Some assistance may be necessary to return the patient to the standing attitude.

The influence of pain in the production of surgical shock in animals is shown by the fact that it seldom follows an operation performed under anæsthesia, and especially from 
the fact that in human surgery it has greatly diminished since the introduction of anæsthetics, although it can not be denied that occasionally the combined influence of shock znd the anxsthetic may prove the undoing of the surgical patient. But these cases are exceptional in animal surgery; the anresthetic prevents shock instead of causing it.

Shock is also produced in its most typical form by the rupture of the abdominal viscera, the invagination of a bowel or the strangulation of a hernia. Often during the course of an acute intestinal disease the patient will suddenly become quiet, the pain will entirely cease, the respirations will become fast, the pulse will become weak and even imperceptible, and a profuse perspiration will break o11t over the whole body, beginning at the head. The symptoms 'will continue until death, and the post-mortem will disclose a ruptured stomach or bowel, with dissemination of the contents throughout the peritoneal cavity. This is shock.

Operations upon bones are more liable to result seriously than other operations. The relative rarity of bone oper. ations in animals is in a measure responsible for the relative rarity of shock in domestic animal surgery. Operations on nerves, castrations, ovariotomy, and amputations of the tail in horses are often followed by slight, but never by severe shock.

PATHOLOGY.- It is quite safe to assert that all cases of shock in animals are caused by the engorgement of the splanchnic blood vessels, which feature of the disease is always found at the post-mortem. The only exception is found after bloody operations where tlie state depends chiefly upon the loss of blood instead of its flow to the viscera. As already stated, other vascular organs may also become the receptacies for blood in sufficient quantities to produce anæmia of the periphery, the brain, etc. In the 
shock that follows the return of the inverted uterus to its normal position, that organ, in addition to the bowels, will be found engorged with blood. When the operation does not directly influence the abdominal vessels, as in the case of ablations in the anterior parts of the body or operations on the extremities, the same condition is found in the visceral blood vessels. In these cases, MacCormac explains the phenomenon as being attributed to the loss of inhibitory influence exerted upon the splanchnics.

SYMPTOMS.-Shock of animals is manifested by symptoms which vary according to the degree of the condition. Mild cases are passed unnoticed, or revive without special treatment. Cataleptic states and syncope must not be in$c^{\prime}$ uded in this category. The real shock seen in animals appears immediately after a wound or operation, or else several hours later. Secondary, or delayed shock, so frequently seen in the human patient, is never observed in animals. The secondary shock of animals-if it may be so regarded -occurs several hours after the infliction of the initial cause. Tremor of the large muscles of the shoulders and thighs is the first symptom usually observed. It is almost always in evidence. If the shock is slight, reaction may set in and no further notice is given to the matter, while if the state is severe, other symptoms will soon make their appearance. The patient, whether horse, ox or dog will lie down as if desiring to rest after the ordeal of the accident or operation. Presently the respirations will be seen to accelerate and beads of sweat will appear at different parts of the body, but especially about the head. The pulse, at first somewhat full, will soon become weak, thready and even imperceptille. The temperature will stand at $98^{\circ}$ or $99^{\circ}$ Fahr. Is these various symptoms become more pronounced the temperature may fall as low as $97^{\circ}$, and death will soon supervene without a struggle. Between the mild form man- 
ifested only by tremor and this grave fatal form, come all the intermediate states, some cases of which die and others recover. In the dog shock is manifested by lassitude and a sinking state not unlike the fatal cases of man.

PROGNOSIS.-Shock is always grave. Its first, mild manifestation may develop into grave symptoms with alarming rapidity. When the disease develops to the point of producing marked alterations in the character of the pulse, respirations and temperature, death is the usual termination. It is only by the application of the most energetic treatment that any of the more serious cases can be saved.

TREATMENT.-Friction to the surface, hot blanket wraps over the entire body, and the internal administration of vascular stimulants (aromatic spirits of ammonia) are the appropriate treatment at the beginning. Coffee, digitalis, caffein, strychnin and nitro-glycerin are also highly recommended. As soon as this first course of treatment is applied, subcutaneous and intravaneous injections of normal salt solution must be administered in large quantities and often repeated until the state is markedly ameliorated. 


\section{CHAPTER VIII.}

\section{HÆMORRHAGE.}

HEMORRHAGE is the flow of blood from its natural channels, resulting from a breach or other defect in an artery, a vein, or the capillaries. It occurs in two distinct forms :(I) Hæmorrhage by rhexis or division of the vessel wall and (2) hæmorrhage by diapedesis, or oozing of the blood from the vessels, without any perceptible breach in their continuity.

I. Hæmorrhage by rhexis (diabrosis) occurs from breaches in the walls of arteries, veins or capillaries, or in the heart itself. It may be caused by any disease, any injury or any increase in the blood pressure that is capable of perforating the blood vessels, or capillaries. The degenerative changes in the walls of blood vessels most likely to cause hæmorrhage are aneurisms, atheromata, arterial sclerosis, abscesses, malignant neoplasms and fatty degeneration. These morbid conditions often act as predisposing causes when the blood pressure is markedly increased, or when the diseased part is subjected to some form of indirect violence, such as strain, traction, etc. The most common cause of hamorrhage, however, is traumatism, which produces a direct mechanical solution of continuity in the blood channel.

2. Hæmorrhage by diapedesis is synonymous with hæmorrhagic exudation. It consists of a transudation of somewhat limited quantities of blood into the tissues of a circumscribed area. It is called petechial when the blnod gathers in a number of small spots under epithelial tissues. The spots are called petechiæ. It is designated as ecchy- 
motic when the spots are larger and fuse together, and thus discolor a considerable surface. When a very large surface is involved the word extravasation is usually applied. And finally it is called infarction when tissues become saturated with more or less blood from obstruction of capillaries or veins. Hæmatoma is used to designate the cullection of a considerable amount of blood in the tissues.

Hæmophilia is that condition in which there is a tendency to bleed profusely on slight provocation. The disease exists without any perceptible abnormality of the organism. The patient bleeds for hours and often for several days from a trivial abrasion and every effort to arrest the flow may fail. It is saicl to be hereditary. In the human being the female bleeder transmits the condition to her male offspring. In animals hæmophilia is occasionally encountered in asses, and mules, and in certain individual horses. The most rational explanation of the phenomenon, and the one most generally accepted, is that the condition depends upon an aberration of blood coagulation. There is no perceptible a!teration in the blood vessels, or in the general welfare or health of the patient. The true nature of the condition is, however, little understood.

Certain special names are applied to hamorrhages from different organs, as follows:- Hæmaturia is bleeding from the urinary channel, hrmoptosis, from the lungs, epistaxis from the nasal cavities, enterorrhagia, from the bowers, hæmatomesis, from the stomach, and menorrhagia, from the uterus.

Hæmorrhage is also classified into primary when it occurs immediately after infliction of the injury; and secondary when it occurs some time after the injury from sloughing of tissues or ligatures.

ARTERIAL HÆMORRHAGE.-Himorrhage from arteries is more serious than that from veins of the same 
size. The flow of blood is always more copious. It is distinguished by the scarlet color of the blood and the spurting of the jet with each beat of the heart. Its most common cause is traumatisn, but serious and fatal arterial hæmorrhages may result from rupture of diseased vessel walls. The most common causes of dissolution of arteries are encroaching neoplasms and abscesses. Chronic diseases of the arteries are somewhat rare in animals. Rupture of the coronary artery may occur from fatty degeneration of its coats, and aneurisms of parasitic origin sometimes cause fatal hæmorrhages from the mesenteric arteries, but even these events are not of very common occurrence.

The arterial hæmorrhages of accidental origin may be divided into three classes: The fatal, the serious and the trivial.

Fatal Hæmorrhages supervene after solutions in the continuity of the aortas, the iliacs, the femorals, the carotids, the external thoracics, the external carotids, the occipitals and the brachials. The radials and the tibials also give fatal hæmorrhages, although occasionally these may be controlled before fatal bleeding ensues. As regards the others, a division of their walls is generally fatal, owing to the difficulty of coping with the situation in time to arrest the flow.

Serious Hæmorrhages occur from the metatarsals, the metacarpals, the glosso-facials, the palatines, the sphenopalatines, the superior cervicals, the inferior cervicals, the spermatics, the mammaries, the external pudics, the interna! pudics, the middle coccygeal, and the intercostals. Often unnamed arteries that serve as the nutrient vessels of tumors give very serious hæmorrhages. Such vessels enlarge to accommodate the growing neoplasm, and being anatomically defective, they lead to more copious bleeding of the normal vessels of the same size.

All of these arteries, although placed second in the scale 
of gravity as to the hrmorrhage they yield, may cause fatal bleeding, but here the flow can more readily be controlled before sufficient blood is lost to endanger life. From this standpoint these vessels deserve to be placed in this separate category in the study of surgery.

Trivial Hæmorrhages occur from the digitals, the arteries of the toe, the plantars, the palato-labial, the nasals. the dentals, and the chief radicles of the arteries classed in the second group.

These vessels under normal conditions will very seldom bleed sufficiently to endanger life, and the flow is easily controlled, hence their classification as third in the scale of gravity. All of the other superficial arteries may be included in this group, owing to the comparatively trivial bleeding they are capable of provoking.

VENOUS HEMORRHAGE is relatively less copious than arterial, and is recognized by the dark color of the blood and the continuous flow. In venous hamorrhage the blood flows from the wound like a fountain, and runs sheetlike over the surrounding parts in marked contrast to the spurting of a wounded artery.

These hæmorrhages are manifestly less serious than arterial ones, but are by no means trivial under all circumstances. Very serious and even fatal hæmorrhages may occur from veins. The jugulars, the femorals, brachials, and even the tibials and radials will bleed sufficiently to endanger life. The subcutaneous abdominals, the saphenas, the superficial radials, the cervicals, the occipitals, the thyroids, and the mammaries give serious hæmorrhages, while the other superficial ones may be classified among the less important vessels from the standpoint of surgical hæmorthage.

CAPILLARY HÆMORRHAGE.-The blood of capillary hæmorrhage has a color intermediate between the 
dark blood of venous, and the scarlet red blood of arterial hæmorrhage. The flow is trivial, constant, oozing and comes from all parts of the trauma. It is relatively short in duration in a strictly normal tissue. It is only in formative tissue, in neoplasms or in degenerated areas that capillary hæmorrhage persists for more than a few minutes.

RESULTS OF HÆMORRHAGE.-The seriousness of hamorrhage varies chiefly according to the amount of blood lost, although it cannot be denied that sudden loss is much more detrimental than the gradual loss of the same quantity. If a large artery, such as the carotid or femoral, is opened, the sudden outpouring will rapidly exhaust the vital forces by disturbing the equilibrium of the cardiac movements. When the flow is gradual the heart will only yield to the depressing effect when the aortic pressure is markedly diminished, which event occurs after about $2 \frac{\mathrm{T}}{2}$ per cent of the entire weight of the body is lost in blood. Thus a horse weighing I,Ooo pounds may lose 25 pounds of blood before there is any manifest influence upon the heart. Two per cent of the body weight may be drained out with impunity, three per cent will cause a transient depression of the vital forces, four per cent will endanger the heart's action and larger quantities will threaten life according to the amount withdrawn and the regenerative vitality of the subject. Healthy horses may lose from I to I $/ 2$ per cent of their entire weights in blood, weekly, and maintain a good condition of health, as has frequently been shown on animals used for the production of antitoxins.

The bleeding animal is protected against death by the contractility of the arteries, which accommodate themselves 11 the diminishing volume until the quantity lost reaches a certain amount. And besides, when the flow is gradual, the capillaries replenish the supply by absorbing liquids in the tissues, which fact accounts for the greater 
seriousness of the sudden hæmorrhage, where there is no time for this absorption to take place.

Death occurs from cardiac arrest, which in turn is caused by the diminished volume of blood in its cavities, and the starvation of its nerves. With a diminished volume of blood to stimulate its contractions, and an impoverished, anæmic state of the nerve centers which control its movements, the heart falters and finally stops entirely. Internal hæmorrhage may cause death even when the loss is of secondary importance, according as its location directly or indirectly affects vital organs.

TREATMENT.-The loss of large quantities of blood is always to be avoided in surgical operations, on account of the clepressing effect it always produces. It augments shock, impedes regeneration, and favors complications. The modern surgeon respects every drop of his patient's blood, and makes every possible effort to preserve it. Durnng the operation even the small bleeding vessels are carefully twisted as soon as they are met, the larger ones are promptly ligated, and the yery large ones are avoided when possible or ligated before they are divided. When the operation is over, the flow of blood is very carefully controlled before the dressings are applied.

The treatment of the patient that has lost large quantities of blood consists of the free and repeated injections of normal salt solution. These are given intravenously and subcutaneously every few hours until the blood pressure and the heart's action are revived. In addition, ammoniacal stimulants and warm clothing are not to be omitted. Alcoholic stimulants are harmful.

\section{HÆMOSTASIS.}

\section{HEMOSTASIS,-ARREST OF HÆMORRHAGE,} depends upon two influences, which may be described as, 


\section{Ist, Natural Hæmostasis and 2nd, Artificial Hæmostasis.}

Ist. NATURAL HÆMOSTASIS.-If the blood channel is perforated, even at a dependent part of the body, all of: the blood within will not escape. It will not flow out in obedience to the law of gravity as in the case of the liquid contents of a perforated barrel. When the breach is confined to capillaries, small veins or small arteries, the outward flow of blood, under normal conditions, is trivial. Only a small quantity will escape. If the breach, on the other hand, is located in a vein or artery of considerable size, the flow may be copious, but ordinarily, not in sufficient quantity to cause death. And lastly, if a large vessel, such as the caroticl artery or jugular vein, is severed, the flow will cause death, but in spite of the large breach, an abundance, of blood will still persist in remaining in the organism. Nature's forces which protect the organism against the loss of all of its blood from every trivial breach in the contintuity of the b.oor channel, are three in number:-a, The coagulability of the blood; b, the contractility of the blood vessels; $c$, the diminution in the force of the heart beats as the volume of blood diminishes.

a. Coagulability of the Blood.-Blood that is no longer in motion, that comes in contact with foreign matter, or that is exposed to external influences, promptly coagulates. In the bleeding wound it gathers around the severed blood vessels, adheres to the ragged fibers of the wounded tissues and accumulates so as to fill the traumatic cavity, and thus, more or less effectually, blocks the outward flow. of blood. This force alone is sufficient to arrest the flow of trivial wounds, but it is often subordinate to such external influences as motion, friction, etc., and to the character of the tissues in which a wound is located. The wound of a large vessel traversing between a bone and the skin. 
could not retain a clot as effectually as one located in the recesses of muscular tissue, and if the part were kept in motion by walking, moving about the stall, etc., the accumulating clot would be repeatedly cast off and thus prevented from exerting its plugging propensities. A wound of the palatine artery, which is located between the bony and the fibrous palate, both of which are unyielding structures, always bleeds profusely. There is no suitable cavity to gather an effectual coagulum, and the motion of the tongue constantly wipes away the clot as fast as it forms.

In the large wound this influence is no small part of the natural hrmostasis. The coagulum fills every recess of the traumatic cavity and even heaps up above the level of the skin. When several hours old it is quite effectually organized and is not as easily dislodged as during the first stage of the bleeding. In a favorable wound not subjected to mucli motion, especially jerking, sudden motion or friction, the coagulability of the blood is nature's greatest protection against death from hæmorrhage. It is also of equal value, in fact, it is indispensable, in permanently obturating the lumen of ligated vessels after the ligature has sloughed away and before the blind channel is obliterated.

b. The Contractility of the Vessels.-The blood vessels, especially the arteries, are very elastic. When cut they retract into the walls of the traumatic cavity, diminish in caliber, and the tunica intima recedes. By retracting into the tissues, the formation of a formidable plug of coagulated blood is facilitated; by diminishing in caliber, the bloodcurrent is narrowed; and by receding of the tunica intima, its rough subjacent stroma is exposed to the out-flowing blood and thus gathers particles of coagulated blood, which still further diminish the size of the out-pouring current and often entirely obturate the lumen. The contractility of the blood vessels, acting in conjunction with the coagulability 
of the blood, is the essential protective which prevents death from hæmorrhages.

c. Diminution in the Force of the Heart Beats comes into evidence as a protective influence only as a last resort. It is only active after a large amount of blood has escaped. The heart, weakened from the loss of blood, and through the diminution in the volume of blood essential to stimulate its contractions, no longer drives the current so forcibly through the open blood vessels, and thus arrests the flow, either from sheer lack of force or from permitting the coagulum to organize in the traumatic cavity.

These three mechanisms may always be depended upon to act in behalf of the organism, in a precise, uniform and effectual manner when the trauma is located in a normal tissue and when the blood vessels are not affected with degenerative changes. The vessels of a diseased structure often, in fact, generally, share in the morbid process and may, as a consequence of their altered anatomical and physiological constitution, fail in the essential behavior to block the flow of their blood currents. Certain diseases of the arteries themselves, either general or local, may likewise deprive them of their protective properties. Diseases of the blood, by diminishing its coagulability, may also serve to augment and perpetuate a hæmorrhage that would have been triviàl under normal conditions. And finally certain individuals, from entirely obscure causes, are more prone to bleed profusely than others. This latter fact is manifest in many surgical operations upon animals.

2nd. ARTIFICIAL HEMOSTASIS.-The artificial arrest of hæmorrhage is accomplished by. different processes which must vary according to the gravity of the hæmorrhage and the location of the trauma.

Ist. By Direct Compression.-Direct compression is probably the most common recourse in veterinary surgery. 
The bleeding leg or foot is promptly met by the simple application of a taut bandage, that is allowed to remain undisturbed for several hours, or even more. Three to six hours of bandage compression is usually sufficient to control even a serious hæmorrhage of the metacarpals, digitals, plantars or their radicles. A firm wad of cotton or oakum previously soaked in Monsel's solution, placed directly upon the wound before applying the bandage, is serviceable. In locations where bandages cannot be applied, compression may be advantageously applied by wadding the traumatic cavity with cotton, gauze, oakum, etc., and then retaining by suturing the wound tightly over it. This method is frequently used in dealing with the bleeding fistula, quittor, poll-evil, or tumor just operated upon. It always effectually arrests the outward flow of blood, but may prove rather disastrous by forcing the flow inward and in every direction irom the wound, beneath the skin and between the layers of muscle, often carrying along septic matter that disseminates the disease over a greater area than before the operation. Abscesses near the sternum have resulted from packing a bleeding fistulæ of the withers, and paralysis has followed pressure of the cord by blood flowing into a closed poll-evil wound.

2nd. By Indirect Compression (the Tourniquet.)Pressure above the bleeding wound often constitutes a convenient method of coping with hæmorrhage. It is especially valuable as a temporary expedient to withhold the flow of blood during surgical manipulations. In amputations it is indispensable. The rubber tourniquet applied sufficiently taut to prevent the flow of blood, may, if allowed to remain several hours, permanently prevent hæemorrhage in an amputated stump, but the method is never certain unless, at least the principal vessels have been searched out and ligated. In the amputation of the tails of horses by the dove-tail 
method, a cord tourniquet will seldom fail to prevent secondary hæmorrhage if left on for three hours. Compression of the metacarpals for two hours will usually permanently arrest a hæmorrhage of the foot.

3rd. By Ligation.-Ligation is the ideal hemostasis. It seldom fails, even in the arrest of the copious flow that occurs from such large vessels as the carotid arteries, the jugular veins, the nutrient arteries of large tumors, etc. In surgical operations large vessels should always be ligated before they are cut, instead of depending upon the possibility of accomplishing the feat afterwards. The chief obstacle of ligation is that of grasping the vessel after it has retracted into the tissues, and its exact location is masked by the rapid flow of blood into the cavity.

The ligation should be done with a thread that varies according to the size of the vessel. A large vessel requires a large thread, one that will not cut through the vessel wall before the coagulum has firmly organized. Often, several threads should be applied to the large vessel and these may even be made still more safe by the application of a hemostatic forcep, for twenty-four hours, between the last thread and the main trunk. Ligated large vessels must not be disturbed until the thread has safely sloughed away from the stump. Traction upon the thread earlier may provoke a serious secondary hæmorrhage.

4th. By Forcep Torsion.-Forcep torsion is the expedient made use of during the surgical operation for bleeding vessels of nominal size. The small vein or artery is grasped, crushed or twisted, chiefly to cope with the blood that masks the surgical wound, and to eliminate the flow prior to closure of the wound with sutures. This method is not always effectual. Vessels are often difficult to grasp in certain tissues, and others persist in bleeding even after repeated twistings. The most effectual results are obtained 
by allowing the forceps to hang to the bleeding spot for several minutes as the surgical work proceeds.

5th. By Bailing.-Bailing or dipping the blood from wounds during the surgical operation is the universal method of dealing with the capillary bleeding and that of small vessels. It is carried out more to facilitate the surgical work than to arrest the bleeding. Absorbent cotton or sponges are used for the purpose. They are wrung out of the bailing dish as dry as possible and then firmly pressed into the wound just long enough to absorb the blood. The wound must not be wiped with the cotton or sponge, as wiping provokes rather than arrests bleeding. By firmly pressing the sponge into the surgical wound the blood in the region is momentarily pressed back, and thus does not immediately refill the cavity.

6th. By Cauterization.-Cauterization, especially with the hot iron, is a very useful expedient in veterinary surgery. It serves purposes that could hardly be accomplished otherwise. For caudal amputations the actual cautery is particularly satisfactory. In arresting the bleeding from the resection of exuberant granulations, especially old ones growing about the fetlocks, pastern and coronets, the actual cautery promptly seals the oozing area and promotes cicatrization. Spurting vessels of the skin following the lancing of abscesses, sanguineous tumors, cysts, etc., although more annoying than dangerous, are promptly closed with a touch of the hot iron. In performing sanguineous ablations, such as large tumors, the numerous though not large vessels frequently yield a dangerous amount of blood, as well as hinder accurate work. These may be met effectually, as fast as they are cut, by merely touching them with the thermo-cautery.

7th. By Application of Styptics.-Monsel's solution, tannin solutions, zinc sulphate, copper sulphate, etc.; may 
occasionally be employed to advantage in assisting in the arrest of slight bleeding from capillaries and small veins. They should, however, be avoided as much as possible, as they retard regeneration and are always inimical to the general welfare of the injured tissue. Adrenalin has a powerful hemostatic action in preventing as well as in arresting bleeding. Applied to the conjunctiva, it is of actual service in carrying out trivial operations. Subcutaneously it renders the connective tissue anæmic, but has no effect upon the cutaneous vessels.

The other expedients used to control hxmorrhage are cold packs, hot packs, elevation of the bleeding part, and the internal administration of styptics. The latter, however, are of but little value. They are always found wanting, when in critical situations they are depended upon to help the surgeon save the life of a bleeding patient. 


\section{?. \\ CHAPTER IX.}

\section{RESTRAINT.}

While it might rightly be claimed that "restraint" belongs to the domain of operative surgery, it cannot be entirely ignored as a principle of veterinary surgery. Its details may well be left to the operative strgery, but its principles belong here. There are certain elements which enter into this feature of veterinary surgical operations which demand the greatest attention from the student. No clescription of restraint can be too thorough, and its details must be built upon a well-laid foundation. It is a conspiculous part of veterinary surgery from three stand points; (I) The personal safety of the surgeon; (2) the protection of the patient against injury; and (3) the thoroughness and exactness of the surgical technique.

Every operation, no matter how brief or trivial, must be preceded by some form of restraint from either or all of these standpoints; and each species, as well as each operation in each species, requires some special method of restraint to acquire control of the situation. The horse, the ox, the dog, the cat, the bird and the wild animal are encountered in the course of a surgical career, and each of them may forcibly resent the infliction of the least pain by effectually using their particular organs of clefense. The horse will kick and strike, the ox will kick and hook, the dog will bite, the cat will scratch and bite, and the bird will peck with its beak. Among the wild animals the danger is much greater. Their terrible claws and canines are capable of inflicting fatal injuries. Even such apparently harmless animals as reindeer and deer can disembowel a human 
being with a single blow with the fore foot. These special propensities of the different species must be known and must be taken into account in veterinary surgery.

Among the domestic species, the temper of individuals is varied. Some may be submitted to torturing pain without offering any great amount of resistance, while others of the same species may forcibly resent the slightest interference with their comfort. What will irritate one animal may provoke no resistance in another. A horse may strike out viciously from the simple attempt to trim the long hairs from its ears, or from the slightest handling of its neck at the seat of a previous collar gall. The simple operation of paracentesis of the intestines for flatulence, may provoke an unexpected wicked side-sweep of the hind leg. The administration of a rectal enema is accepted complacently by most horses, but occasionally it will be met with a vicious kick with both hind legs. Manual explorations of the generative organs of the mare during obstetrical operations are not always safe, although most mares will offer no resistance. The vicious horse is seldom dangerous, because special preparation is made to prevent the expected resentment. It is the apparently harmless animal, the one from which personal injury is not expected, that usually proves the undoing of a thoughtless veterinarian. Furthermore. personal injury may be sustained when there is no intention on the part of the patient to inflict it. A tread of the foot, a sudden fall to the floor, or the swing of an accidentally released leg, may often prove disastrous. In the wild species, there is less danger, because they are seldom handled without first taking due precautions to prevent them from inflicting injury. The dog, even though assured to be perfectly harmless, seldom ever deceives a trained yeterinarian, because many dogs are known to bite on the least provocation, and all of them are known to resent the 
infliction of the least pain. The cat is fairly complacent unless forcibly handled or injured, and because of its known propensities to claw, the surgeon is seldom caught unawares.

Personal injury sustained from surgical patients cannot be looked upon in the light of misfortune. It is more an exemplification of unskillfulness, clumsiness, carelessness or inexperience. To know the habits of our patients, to understand how to approach them, to devise ingenious and effectual methods of controlling them, and to be ever on the alert for personal injury, are attainments of no small importance. They are essential. Although the inborn faculty which enables one to effectually command a situation, whether encountered in the pig-sty, stable or parlor, is not possessed by all, thoughtfulness and a little experience will soon develop the essential accomplishments.

The patient must also be protected against injury, especially the horse. The other animals rarely ever sustain bodily injury from surgical restraint because they are relatively less powerful. The small animals, and even the ox, are incapable of directing the same enormous amount of force against the restraining apparatus, and are hence more easily confined and less susceptible to injury. The powerful muscular system, the large size of the animal and the natural inclination to fight restraint and resent the infliction of pain, all contribute to the susceptibility of the horse to bodily injury when forcibly secured with mechanical appliances. These injuries may be trivial or grave, according to their location and cause. They vary from slight excoriations of the cuticle to fractures of the vertebral column. Trivial or grave, they must be provided against. The humiliation of fracturing the spine of an old horse may be no greater than of bruising the orbit of a valuable animal. Injuries are usually caused by the display of rather poor 
judgment in selecting the particular kind of restraint most appropriate for the particular subject or particular operation. A horse that violently resents the application of the firing iron in the standing position from the very beginning of the operation must be promptly cast and effectually secured. Early in such operation the surgeon should carefully judge the temperament of such subjects. The old spavined horse may also have an anchylosis of the vertebræ which will render recumbent restraint rather hazardous. Such animals are operated upon by other methods and are thus protected against injury. In fact, every operation and every single patient must be carefully judged with the view of preventing even the more trivial accidents, which is accomplished only by thoroughly understanding the nature of the patient and the technique of the restraining method.

From the standpoint of thoroughness, perfect restraint is especially necessary. There are some operations that can only be performed in certain particular positions. The seat of operation, whether it be a leg or a region of the trunk, neck or head, must be exposed and immobilized to the best advantage, so that the steps of the operation can be consecutively executed with the least possible interference from the struggles of the patient. A median neurotomy would be impossible if the internal aspect of the humeroradial articulation were not well exposed and forcibly kept in a certain stretched position. The castration of the cryptorchid, the ablation of a scirrhous cord, or the operation for inguinal hernia necessitate the forcible parting and flexing of the hind legs. These are only a few of the many surgical operations that are best performed with a particular form of restraint. All of them require attention in this direction. Perfect surgery is impossible without perfect restraint. They go hand in hand. The restraint is more 
than half the battle in very many of the operations, which fact gives decided advaintage to the ingenious veterinarian over the less skillful in the art of surgical restraint. The young surgeon, who is none too familiar with the technique of the operation, may err seriously, or even abandon an operation, because the patient is not in the proper position or because the struggles were not preconsidered as an adverse element.

The recommenclations here are to adopt certain wellknown methods (standard methods) and then never deviate from them, but instead improve in their application, and never to begin an operation of any kind or character without first selecting the most appropriate means of restraint. Whether the operation is that of simply lancing a subglossal abscess or the removal of an abdominal testicle, the restraint must be given some consideration. In the one the mere application of the twitch will suffice, while in the other a complicated method of exposing the inguinal region is absolutely necessary. From the standpoint of accuracy both are important. Each reveals the surgeon's attainments.

The equipment necessary to meet every demand consists of: ( I) An operating table; (2) a casting harness; (3) a side-line and stocks for "standing" operations; (4) a small table for operations on small animals; (5) a hoodwink; (6) a twitch.

THE OPERATING TABLE.-The large operating table for the horse is a necessary apparatus for the veterinary hospital. There is no intention here to discuss the merits of the different kinds. It will be admitted that each one has its special points of superiority and its special demerits. It will suffice to say that great strength and simplicity must be its predominating features. The materials from which it is constructed, the wood, the iron, the hop- 
ples, the chains, etc., etc., must be strong to a fault. A single weak point in a horse operating table will soon give way, and often with disastrous results. The struggles of a large horse as he feels the belts lifting him from his footing will often test the strength of the best table, and the struggles with the uppermost hind foot when the patient is down will test the temper of the strongest hopple and chain.

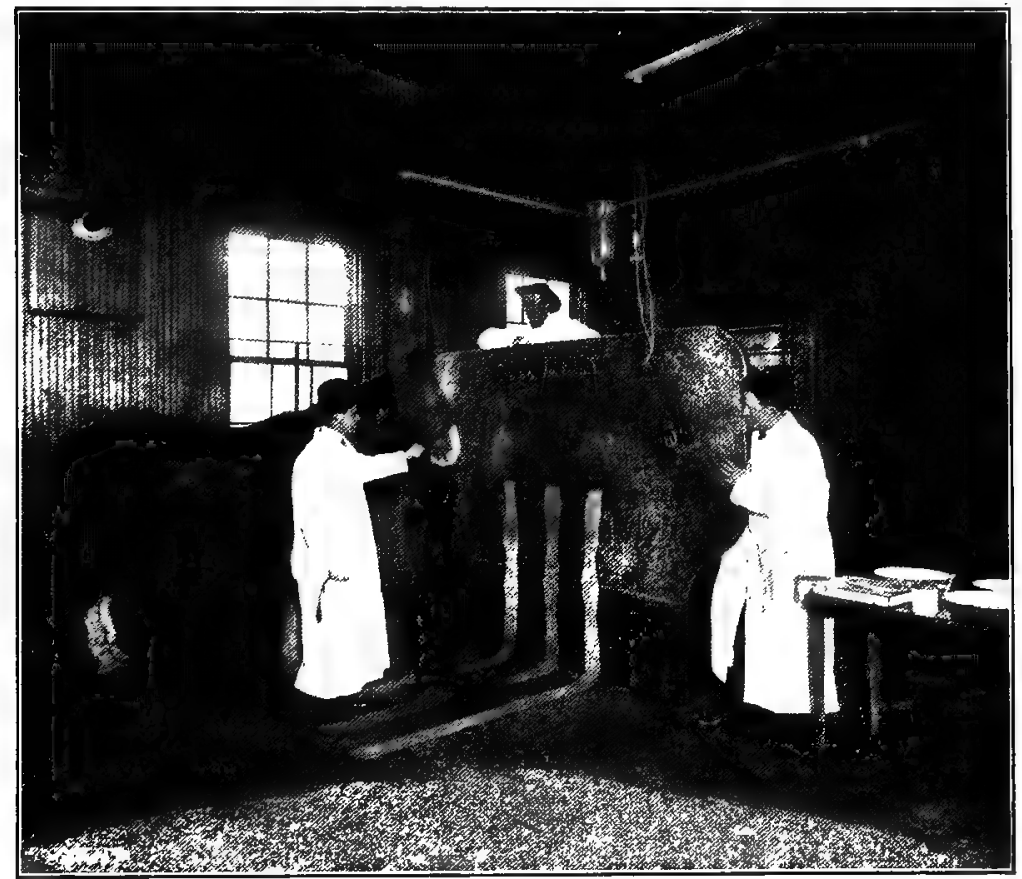

FIG. 20.

Operating Table. Bringing the Horse into Position; Assistants Properly Placed.

As to its simplicity, the table must be absolutely free from projecting objects capable of wounding the animal"s body, and it should require no more than two or three men to operate it. It must be geared so that both power and speed are respected. A table that will rapiclly turn down a large horse may require the strength of two or three men to return the horse to the standing position. The floor 
around the table should be wooden, to give good footing to the horse while being secured and released.

The simplest form of operating table is shown in Fig. 20. It consists of a simple wooden frame, a simple wooden table, moderately padded with oakum and painted canvas, and a simple gearing that turns the table from horizontal to perpendicular in fifteen turns of the crank. The three body belts are of five-inch, five-ply cloth belting. The four foot hopples are of three-ply harness leather, of good quality, hooked to the frame with strong chains, which automatically tighten the legs by the shifting of the table from the perpendicular to the horizontal position.

From the standpoint of the surgeon's safety the operating table compares favorably with the casting harness. The only danger connected with the method is that of being struck with the legs when they are released preparatory to returning the horse to the standing position, or when a hopple should acciclentally break during the operation.

In regard to the patient's safety against accident, there is much to be recommended. Horses are very seldom ever injured. A trivial abrasion of the orbit from improper attention of the head, or insufficient padding of the hoodwink and friction abrasions from struggling in the hopples, especially the uppermost hind foot, are about the only possible accidents. A horse that will persist in fighting constantly through a long operation may also bruise the hip, shoulder and stifle, but these subjects are rare and when met they should be promptly anæsthetized. Animals reviving from anæsthesia may sustain injuries of the limbs if returned to the standing position too soon. In the state of partial unconsciousness they may hang with the full weight in the belts, and when released one or more of the legs may become folded in an unnatural position under the body as it falls heavily to the floor. 
The greatest advantage of the operating table in this connection is the leisurely manner with which an operation may proceed without discomfort to the confined horse, and the decided freshness of the patient after the operation is over. The operating table does not often provoke exhausting struggles. With harness restraint the patient is uncomfortable throughout, and it is essential to hurry the operation in order to shorten the duration of the confinement. The patient always arises more or less exhausted, even from short operations, while with the table two; or even three hours' confinement will have no exhausting effect. In short, a horse is much safer on the operating table than upon the floor secured with the casting harness.

From the standpoint of thoroughness and exactness of the surgical technique, the table has both good and bad points. The operations are much cleaner. There are no flying particles from the litter, and less dust in the operating place, and as the surgical field can be more securely tied there is much less danger of soiling the surgical wound, and a much better opportunity of accurately executing the various steps of the operation. The dissection, the hæmostasis, the suturing and the dressing are greatly facilitated by the fixed state of the operating field.

On the other hand, there are operations which cannot be properly performed on the operating table, notably those in the inguinal region. Operation for cryptorchids, scrotal hernias; scirrhous cords, mammary tumors, etc. are performed with greater ease if the patient is properly tied with the harness. The operating table is strictly deficient in these special operations. Operations upon the throat are more easily performed with the harness method. In comparing the two methods,-table and harness, - the former is often condemned for bilateral operations because the horse must be twice secured. That is, when one side 
is operated upon, the patient must be returned to its feet and then replaced on the table on the opposite side. This is an objection only in as much as it requires slightly more time than to simply roll the horse over on the floor. This objection is, however, a trivial one.

The Manipulation of the Horse Operating Table seems

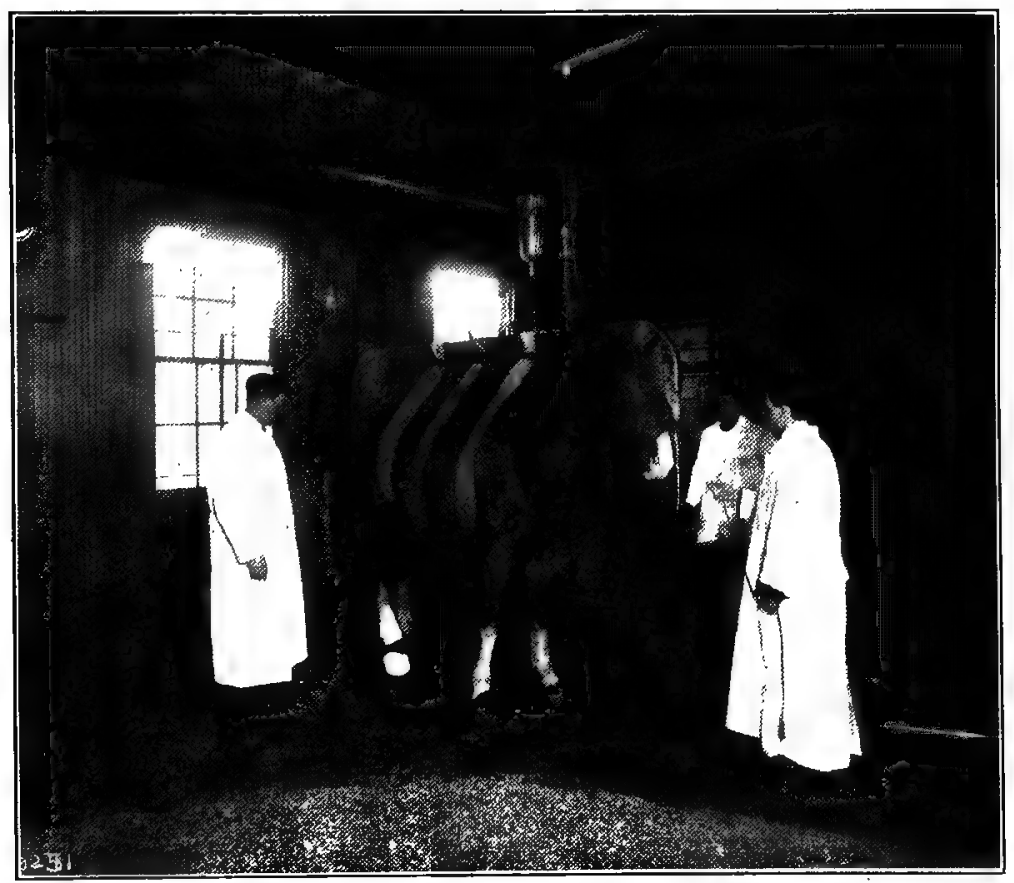

FIG. 21.

Operating Table. Horse Ready to be Turned Down; Assistants in Their Proper Stations.

very simple so far as its general principles are concerned. It consists of belting the body to the table while in the perpendicular position, strapping the legs to the hopples and then turning the table down with the crank. These are, however, "easier said than done." The securing of a horse on a table, like all of the other methods of restraint, can only be satisfactorily clone when certain essential rules are care- 
fully observed. It is accomplished in three separate steps: (I) The first step or feat is to induce the horse to approach near enough to the table to strap the body to it. Hoodwinked, there is comparatively little trouble in leading the average horse to the proper position, but many horses will obstinately persist in turning the hind quarters from the table after the head is brought into contact with it, and in spite of the aid of two or three strong assistants will cause some annoyance. The difficulty is quite readily overcome by patiently bringing the body parallel to the table without touching it and then rapidly securing the foremost belt, the halter shank and the neck strap before the patient is aware of what is being done. (2) The next step is to fasten the body firmly against the table and to secure the feet in the hopples. The foremost belt, the halter shank and the neck strap having already been fixed loosely, the next feat is to push the hind quarters bodily against the table as the assistant above takes up the slack of the hindermost belt: Here the horse may struggle some, or may hang heavily in the fixed belts, especially in the hindermost one. This is generally met by delivering a few gentle raps to the buttocks with a strap, or by applying a twitch to the nose, either of which will usually promptly bring the horse to an upright position. It is essential here to induce the horse to stand upright before the remainder of the appliances are adjusted, even though some difficulty is encountered in doing so. When the standing position is satisfactorily attained, or maintained, the middle belt is adjusted and the slack from the others is taken up. The hopples may then be buckled to the pasterns. The hindermost hopples must be buckled to the outer legs, on the fore legs as well as the hind ones in order to prevent the innermost leg from stepping over the chain attached to the outer one. The coronet, pastern and fetlock of the outer hind leg (which will be the 
uppermost when the table is down) must be well wrapped with a thick cloth or bandage to prevent injury from the struggles. The other legs are comparatively immune against injury. (3) The third step is to turn the table down as fast as possible while an assistant supervises the behavior of the chains and hopples. A chain may kink in the

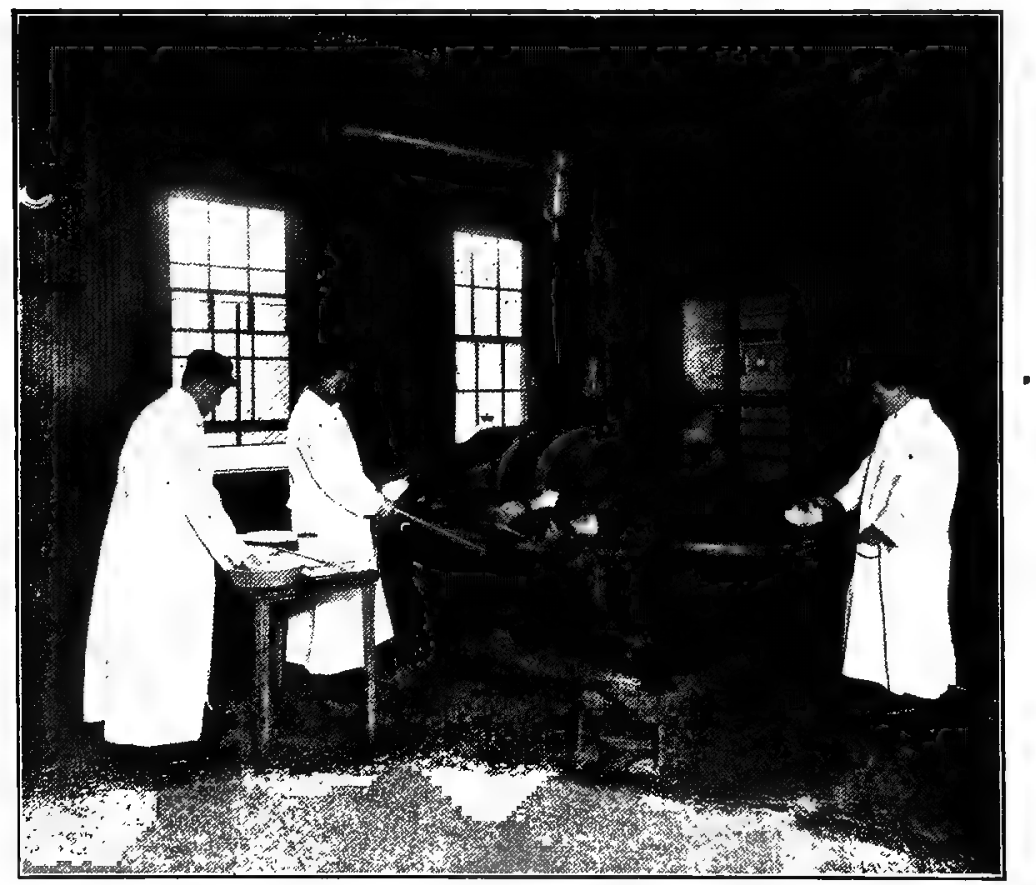

FIG.22.

Operating Table. Horse Ready for Operation; Assistants at Their Proper Stations.

loop-holes of the table, or a shoe-calk may catch in a link of the chains or in the hopple of another foot. These accidents must be recognized immediately to prevent injury to the horse or table.

When the recumbent position is reached the twitch is applied to the nose and the seat of operation exposed and secured according to the particular demands of each case. 
It is the habit of some surgeons to fasten the hind legs to each other with a leather strap wound around them in the form of a figure 8 , with the object of diminishing the force of the struggles. This expedient is, however, of questionable merit, as it often provokes rather than limits the struggling:

When the operation is completed the legs are released from the hopples, the twitch is removed from the nose, the neck strap is unbuckled and the belts are loosened several inches. These are done in the order named. The table is then rapidly turned to the perpendicular position. The assistant at the head holds it firmly against the table with the halter-shank and unbuckled neckstrap, and as soon as the feet approach the floor the hoodwink is removed. The removal of the hoodwink at this particular time will cause the horse to promptly assume the upright position instead of hanging heavily into the belts. When the footing is regained the belts, the unbuckled neck-strap and the haltershank are simultaneously released, and the horse is thus entirely liberated. If a horse hangs into the belts, instead of standing upright, some care must be taken not to throw its body heavily to the floor by releasing all of the belts. A gentle tap with a strap, or slowly releasing the foremost belt, will usually induce the horse to stand on its feet. The manipulation of the neck-strap must always be cautiously supervised with the aim of preventing a horse from hanging by the neck should it not be released before the belts.

THE CASTING HARNESS.-The casting harness (or casting ropes) is named as one of the essential apparatus because it is portable and also on account of the fact that certain operations are more easily performed with the rope method of restraint. For castrations, especially of cryptorchids, and for all other operations about the inguinal region, the ropes are par excellence the best restraint. The 
hind legs can be well flexed upon the pelvis and well parted to perfectly expose the region.

From the standpoint of the surgeon's personal safety, the ropes compare favorably with the table. There is some danger of a kick while buckling the hopples, and some slight danger while they are being released, but otherwise the method cannot be regarded as a hazardous one, especially

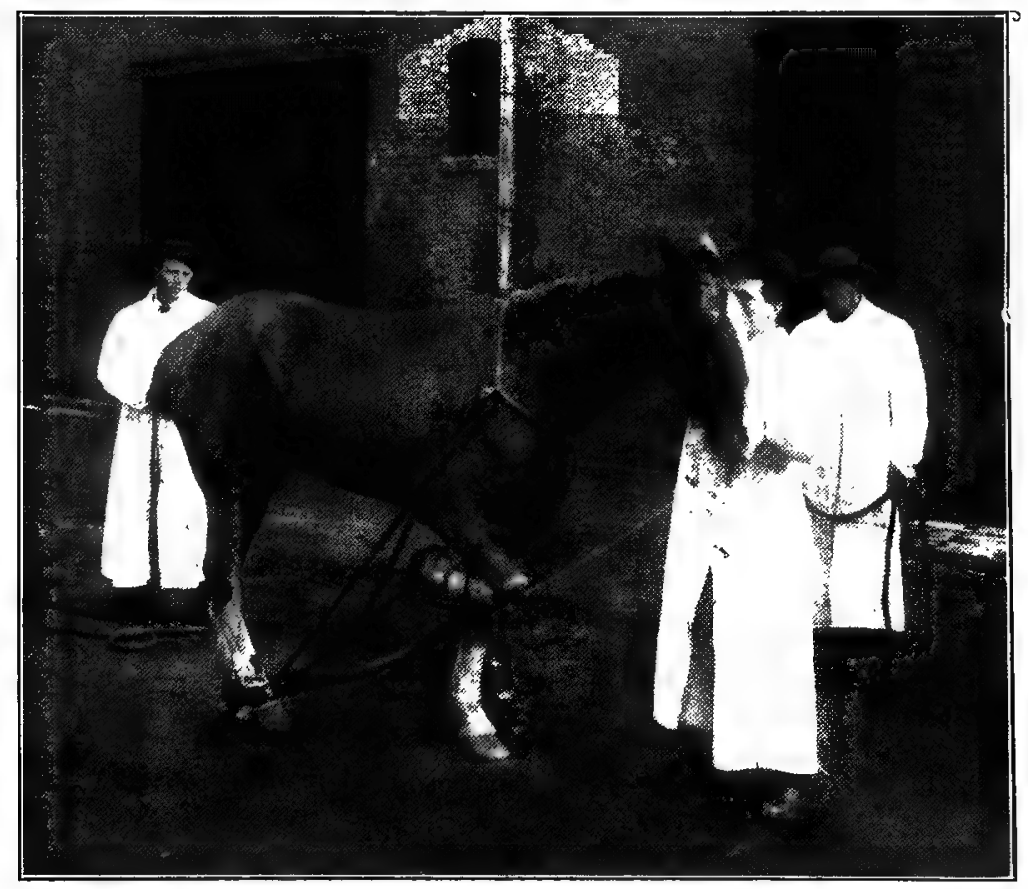

FIG. 23.

Miles' Casting Ropes. Horse Ready to Cast.

when the usual precautions are taken against the kicks and strikes of a vicious horse.

As regards the patient's safety and comfort, the ropes cannot compare with the operating table. The accidents of surgical restraint are ten to one in favor of the table, or even more. Such terrible accidents as fractures of the vertebrae of old horses, or fracture of the femur of the young, rickety 
subject, never occur in table restraint. It seems that accidents with the ropes are inevitable. They will occur in some form or other, no matter what precautions are taken. With the surgeon who has had a wide experience they are somewhat less frequent than with the new, inexperienced practitioner, but none have an absolutely clean record in this connection. Iractures of the vertebra, the pelvic arch, the femur, the humerus and even the tibia, facial paralysis, radial paralysis and bruises, abrasions and excoriations at the prominent points of the body, are the more common accidents of this method. Another feature of the method that cannot be overlooked is the exhaustion induced by keeping a horse securely tied during an operation of long duration. The cramped position of the legs is especiaily uncomfortable to the middle aged or the aged, owing to the diminished flexibility of the articulations. In animals suffering from spavin, diffused tarsitis or gonitis, the pain of forced flexion is often so severe that the horse rises with some difficulty after the operation is over.

For the young horse, for the brief operation, for certain special operations already enumerated and for out-of-door practice the ropes are useful and in fact indispensable.

From the standpoint of accurate surgery, both pro and con, premises are legion. The clean aseptic operations on the limbs are best performed upon the table, while the inguinal operations are greatly facilitated by the rope method.

The usefulness of the ropes for inguinal operations, and the fact that they are easily carried about from place to place, are the very best arguments in their favor.

The Miles casting ropes, which are as practical and as simple as any other, consist of sixty-five feet of hemp rope seven-eighths of an inch thick, looped in the middle to form a collar and wrapped at the cut ends to prevent unraveling of the strands; two leather hopples made of two plies of 
good strong harness leather and equipped with iron D's for passage of the ropes; several pieces of common sash cords to fasten the legs and to secure the limbs in different positions for operations. In the middle of each side of the collarloop an iron thimble is fastened between the strands to receive the ropes. (Fig. 23.) The hopples are buckled to each hind pastern and the collar slipped over the head and adjusted to the base of the neck. The ropes proceeding from the collar are passed backward between the forelegs to the hopples on the hind legs. Each rope is passed from within

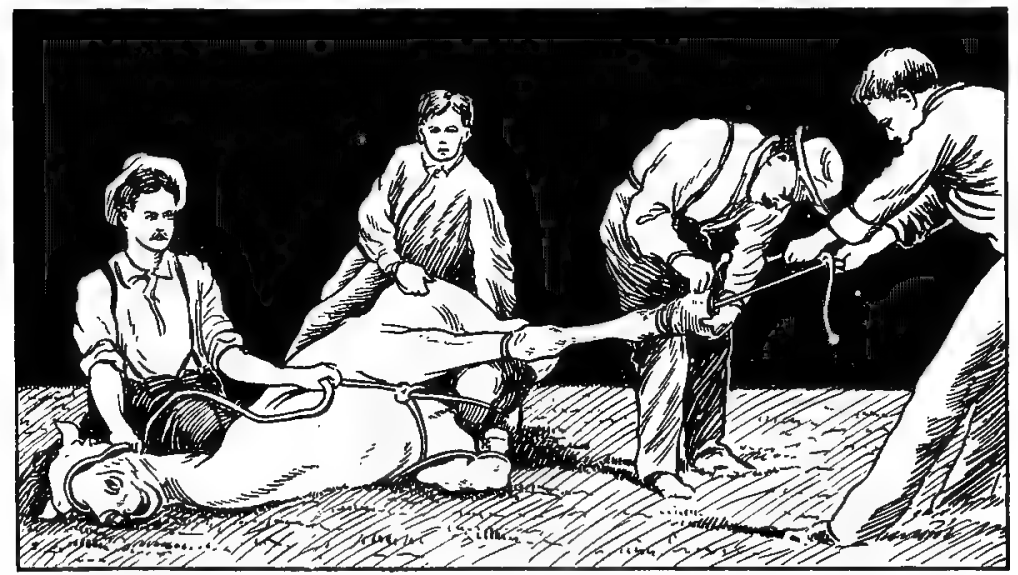

FIG. 24.

Horse Secured with Miles' Ropes, in Position for Foot Operation.

outward through the $\mathrm{D}$ of each hopple, and then through the thimbles at the collar. The rope on the side the horse is to be thrown is passed backward again through the hopple (not through the D) from above downward and then over to the opposite side to the hands of an assistant. This rope will subsequently be used to pull the hind leg from under the horse at the proper time. The rope on the opposite side is placed in the hands of a second assistant, stationed behind the horse. The front leg on the side the horse is to be cast is now tied up and a firm hold taken of the head at 
the halter. At the word, the assistant at the head starts the horse backwards and the other two pull firmly at the ropes. (Fig. 23.) The horse having but one foreleg to stand upon falls first upon the shoulder, then the hind quarters.

When down, the assistant at the head places one knee on the poll and with the hands to the halter lifts the nose from the floor. In this position the head is easily kept under control, and protected from injury. The hoodwink previously acljusted may also be used to prevent contusions of the orbit and to protect the eyes against foreign bodies.

The tying of the legs is the most important part of the wethod, as it is here the patient is protected against serious injury. The rope on the uppermost side is hitched over. the hind feet, passed around the loins and then hitched twice to the same foot after drawing the leg tightly into extreme flexion. This requires two assistants, one at the dorsal side to draw the leg to the flexed position and one on the ventral sicle to take up the slack. The front leg is then secured in the flexed position with the sash cord, looped first around the pastern and then passed around the two ropes that pass from the collar to the hind foot. The horse is then rolled over and the other side tied in the same fashion.

The seat of operation must then be exposed and securely immobilized according to its location. For an operation upon a fore foot or leg, a sash cord is looped around the metacarpus and then anchored to the hind hopple and a second one is looped around the foot and given into the hands of an assistant or two. For a hind foot the hopple and the hitches must be transferred to the tibial region. For inguinal operations the ropes which now cross each other over the region so as to obstruct free access to it, must be transferred each to the opposite foot. Most all parts of the body may be satisfactorily exposed by this method, except the neck at the collar seat, the shoulders 
and the pectoral region. But since most operations in these regions can be performed in the standing position, no great objection can be raised on this account. The casting harnesses such as Zeigler's are preferable for such operations. The Zeigler harness and other similar contrivances encircle the body at the girth instead of at the neck, but are otherwise similar to the above described ropes.

THE STOCKS.-(Fig: 25.) This apparatus is highly recommended by many veterinarians, especially where only one assistant is available. With moșt any other form of

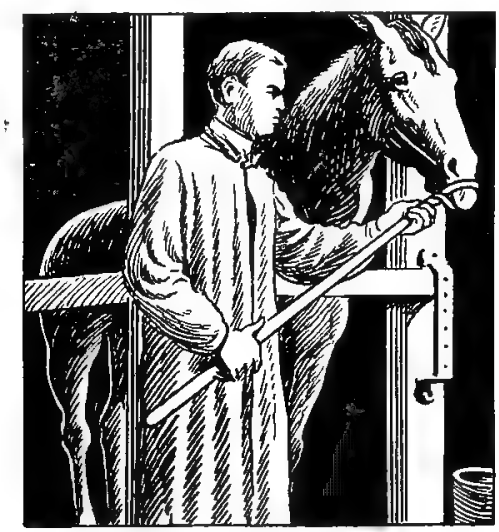

Fig. 25.

Horse in the Stocks.

restraint two or three assistants are absolutely necessary to assure the safety of the patient and the comfort of the surgeon. With the stocks one assistant is sufficient. They are quite satisfactory for operations on the withers, the acnestis, the shoulders, the buttocks, and the tail. For the legs they are much less effectual as a means of restraint, and are none too safe for the patient.

They are constructed of four perpendicular beams set in the form of a rectangle two and a half feet wide and six feet long. The sides are connected with a single strong stationary plank about two and a half by twelve inches 
while the ends are closed with strong removable timbers. The plank is equipped with four belts, two anteriorly and two posteriorly. The horse is led into the apparatus and locked into it with the removable timbers as the belts are fastened around the body, two above and two below, to prevent either rearing or falling. The application of the twitch completes the restraint.

This method is not without its faults for promiscuous operations on all kinds of horses, owing to the dangers of injuring the animal that may persist in violently resenting the confinement: Such a horse must either be perfectly secured beyondl the possibility of offering any resistance at all oryelse it 1aust be given considerable freedom. The partial confinement, such as the stocks provide, is dangerous when the animal is a fighter.

For ovariectomy either in the mare or the cow, the stocks are very commendable. They are, in fact, essential. For flank operations in the cow, especially when a number of them are performed in a herd, such a restraint apparatus is highly recommended. It is constructed with a hinged door on one side which can be dropped to expose the flank, or on one end if the vaginal operation is to be performed.

In a certain establishment where upwards of two thousand horses are confined annually for tail operations,-pricking and docking, - the stocks were abandoned after a trial of four years, on account of the accidents that occurred. These, while not specially numerous, were too frequent to warrant a continuation of their use. This is practically the final conclusion of all experienced operators. One hundred horses may be confined successfully without accident, but the one hundred and first one sustains a serious injury to the limbs or body while kicking or plunging. The stocks are credited with two fatal accidents to the writer's personal knowledge, and these after several years of experience 
with them. One case sustained a rupture of the colon and the other, showing no perceptible lesions on post-mortem, was attributed to traumatic syncope.

THE SINGLE SIDE LINE.-(Fig. 26.) For operations in the standing position the single side line is indispensable. It consists of a hopple, a rope twenty feet long, and a special

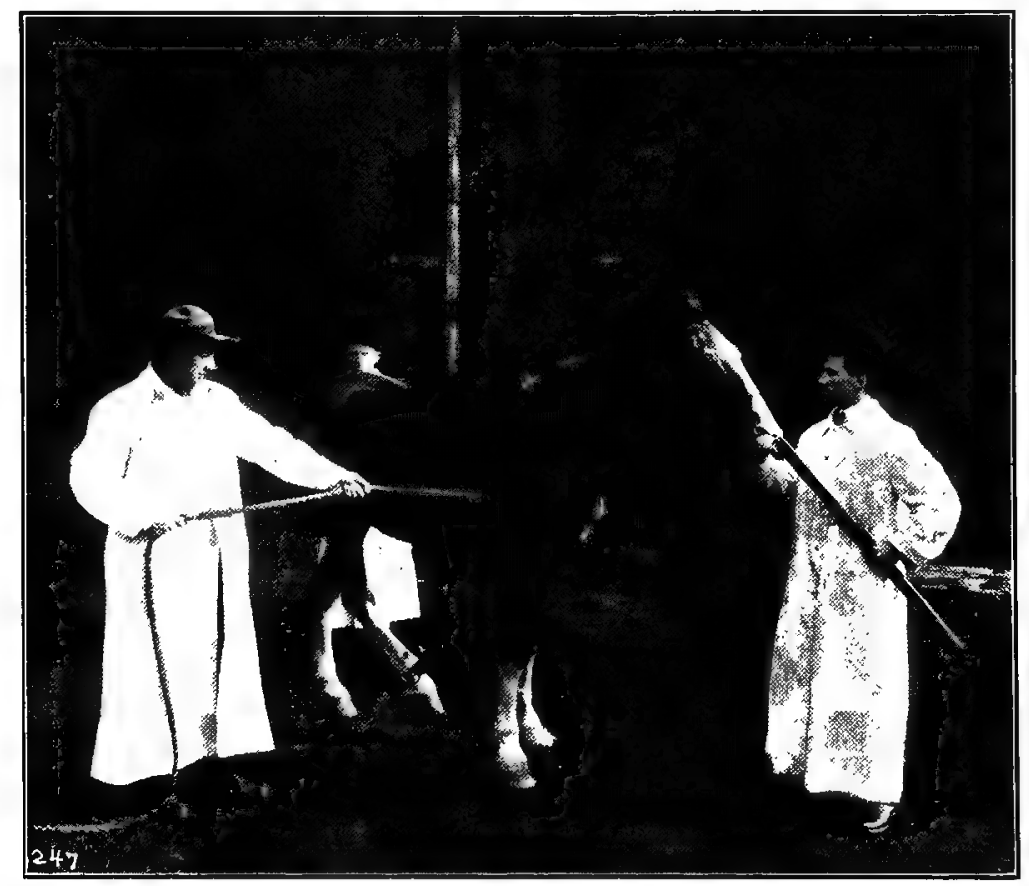

Fig. 26.

The Side Lines, for Operations in the Standing Position.

collar, and is used principally to lift one of the hind legs from the floor, which position leaves a horse quite helpless. The rope and hopple alone may be used to lift a fore leg for the purpose of operating upon the opposite one. By lifting a hind leg many of the operations can be safely performed. Firing, tail operations, operations upon the abdominal walls, hips and buttocks, vaginal and rectal explorations are ren- 
dered perfectly safe, and there is but little danger of injuring the horse if the operating place is large and some freedom is given the lifted leg if the animal lunges forward. Twitching the nose is a necessary adjunct to the method. Without the twitch few horses will remain satisfactorily quiet with a hind leg elevated from the floor. Strains of the leg thus forced to carry all of the weight of the posterior quarters are possible accidents from this expedient, but these are only liable to occur when the lifted leg is given no freedom as the patient hops forward. The leg must never be fixed; it must be held by an assistant instructed to relax the hold when the patient lunges. Sprain of the flexor metatarsi, curb, and thoroughpin are the possible accidents.

\section{OPERATING TABLE FOR SMALL ANIMALS.-} (Fig. 27). Where operations on the canine and feline species are numerous a specially designed operating table is an essential equipment, owing to the delicacy of many of the operations performed upon these animals. The operations which expose the viscera demand the adoption of methods which cannot be effectually carried out except in an absolutely clean environment, which in turn is rendered possible only by the use of strictly aseptic restraining appliance. The artifice of White (Fig.27) in every way meets all the requirements. It is made of enameled metal. The top is studded with a series of key-holes by means of which the hopple keys may be placed at any point to suit any size of animal or any particular position. The holes also serve the purpose of draining fluids into the pan 10cated six inches beneath. It is also supplied with two swinging basins and a glass irrigator.

Various other operating tables may be easily made to meet the requirements of ordinary veterinary hospitals. A polished and varnished plank two feet wide and five feet 
long fastened to the wall with hinges whereby it can be turned against it when not in use is a very convenient appliance as well as a very practical one for this purpose. Its edges are supplied with a series of screw-eyes for the securing cords, and there are two hinged legs at the free end, which fall automatically to the perpendicular position as the table is brought down from the wall.

THE TWITCH.-(See Fig. 25). The twitch, a valuable restraining appliance in equine surgery, consists of a

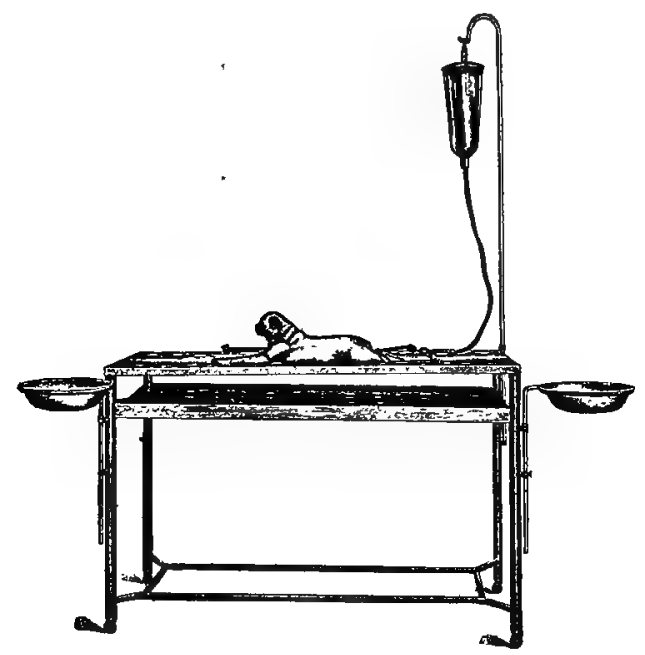

Fig. 27.

White's Operating Table.

plain round stick of wood with a loop of cord fastened through a hole at one end. It is used to inflict pain to the upper lip to distract the patient's attention from the pain inflicted by the operation. Although brutal in principle its use is absolutely necessary in many, in fact, most surgical operations, performed without general anæsthesia. Lancing an abscess on the anterior parts of the body, suturing accidental wounds of the head, ears, neck or shoulders, paring of a corn, removal of a shoe, clipping the hair of a limb, etc., etc., are always materially facili- 
tated by its use. The application of setons to the shoulder, the lancing of a cold abscess of the mastoido-humeralis and even castration, can often be performed with no other form of restraint. In most of the operations its application should precele all other methods of restraint. Before the single side line is applied preparatory to an operation in the standing position, or while the casting harness is being arranged, the twitch should already have been adjusted to the nose in every case.

The twitch is particularly effectual in preventing resistance of trivial manipulations, such as paring a hoof, clip-

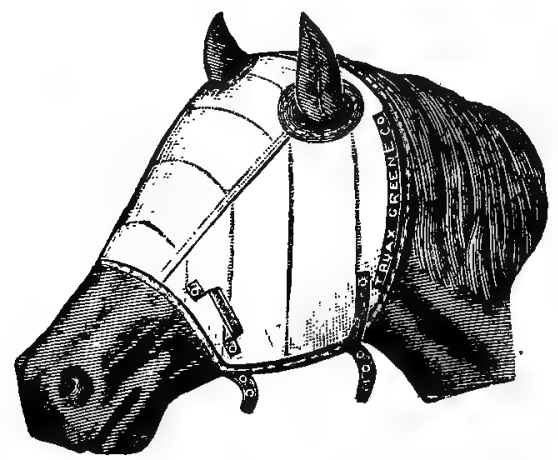

FIG. 28.

Hoodwink.

ping the hair, washing a wound, arranging the casting harness, etc.; but it is also effectual against the infliction of severe pain, especially if not of long duration.

The twitch should be no less than three feet long, and the loop no more than four to five inches in diameter, so that one or twe twists will be sufficient to tighten it, and it should be used with discretion, so as not to inflict unnecessary pain.

THE HOODWINK.-The hoodwink is made use of by veterinarians for the purpose of protecting the head from injury in operations in the recumbent position, and as a blind-fold for operations in the standing posture. In 
the former it is essential in every case, while in the latter it may even be harmful. Some horses will fight much more when blind-folded than when their view of the surroundings is not obstructed. It consists of a padded hood that covers the face, eyes, forehead, mastoid region and poll. (Fig. 28.) 


\section{CHAPTER X.}

\section{ANÆSTHESIA AND ANÆSTHETICS.}

\section{Divinum opus est sedare dolorem.-Hippocrates.}

DEFINITION.-Anæsthesia is defined as the relief of surgical pain by artificial means. Literally, the word signifies want of feeling or sensibility, but today it is seldom applied to diminution or loss of sensibility caused by pathological states. Anæesthesia is now synonymous with surgical anæsthesia.

HISTORY.-From the remotest antiquity surgeons recognized the absolute necessity of attenuating the pain of surgical operations, as an essential prerequisite to the successful progress of the art, and as a consequence various attempts were made, continuously, during all epochs, to accomplish some patent results in that connection. $\mathrm{Pa}$ tients were intoxicated with alcohol or stupefied with other narcotics; the vessels of the neck were compressed to lower the sensibility of the brain; nerve trunks and blood vessels were compressed above the seat of operation; and parts of the body were chilled or even frozen; all in vain efforts to diminish the inevitable pain that rendered surgery quite incapable of advancing. The practice of surgery in those days was restricted to the absolutely necessary operations, such as.amputations, reposition of fractures and luxations, treatment of accidental wounds, etc. There was no effort to add to the category of surgical operations, because surgery was no more inviting to the surgeon than to the suffering patient. The major surgical operation was a veritable horror, and even minor procedures were always approached with the greatest reluctance. 
The history of "scientific inhalation anæsthesia" begins September 3oth, I846, on which date, Morton, a Boston dentist, performed the first surgical operation under profound anæsthesia. During the four or five years preceding this date, Morton, Wells and Jackson had been conducting experiments with nitrous oxide, and finally at the suggestion of the latter rather accidentally proved that the vapor of ether possessed analogous properties that were more regular, more certain and much more effectual. The discovery was soon heralded over the entire civilized worlcl. Within a single year it had already been tested in every European country, with results that soon bespoke its future position among the great discoveries of the age. About one year later, Simpson of Edinburgh, discovered and announced to the world the value of chloroform in the same capacity. Thus within two years (I846-I847) the two great inhalation anæsthetics-chloroform and ether-were laid down as the first step toward the high place occupied by surgery at the beginning of the twentieth century.

In veterinary surgery, anæesthesia has no history. It is used in a kind of desultory fashion that reflects no great creclit to the present generation of veterinarians. At the clinics of the great colleges the general anæsthetic is quite generally used in the experimental operations on the large and small animals, and among the leading practitioners of the large cities and some country districts it is occasionally employed; but that general inhalation anæsthesia is in general use in the veterinary profession of this country would be a claim that is far from the truth. Many veterinarians of rather wide experience have never in a whole lifetime administered a general anæsthetic in performing their operations. It reflects greatly to the credit of the canine specialist, however, that he alone has adopted anæsthesia to any considerable extent. The veterinarian engaged 
largely in the treatment of small animals seems to recognize that the delicate surgical operations to which these animals must be submitted can only be appropriately carried out successfully with the aid of anæsthesia, and that the sentiment against the infliction of pain to the pet animal is considerably greater than with the working animal, whose value alone is respected alike by the owner and the practitioner.

Anæsthesia in veterinary surgery today is a means of restraint and not an expedient to relieve pain. So long as an operation can be performed by forcible restraint without imminent danger to the technique, the operator or the animal, the thought of anæsthesia does not enter into the proposition. But, on the other hand, when a certain technique requires perfect repose of the surgical field, or when there is danger of personal injury or injury to the animal, it is sometimes administered, minority reports to the contrary notwithstanding.

There is, however, some evidence of a change in the proper direction. A more lively interest is being taken in the matter as veterinary surgery is advancing, and at the present there is some indication that the practitioner of the near future will take advantage of the expedient that made the rapid advancement of human surgery possible.

\section{PHYSIOLOGICAL ACTION OF CHLOROFORM} AND ETHER.-For all practical purposes and intent the actions of these two agents are identical. The slight difference in the anæsthesia they each produce is trivial and immaterial, and is limited strictly to one of degree.

From the earliest history of inhalation-anæesthesia various theories were propounded as to the remote action of these agents on the organism, and the subject has since been thoroughly studied by niany carefully executed experiments. At first it was thought that the insensibility 
supervening the inhalation of the vapor was due to arrested hæmatosis. That is, the anæsthetic state was supposed to depend upon the intoxication of the blood with carbonic acid. This theory was, however, very short-lived, as the phenomena attending the accumulation of $\mathrm{CO}_{2}$ in the blood and that accompanying the inhalation of an anæsthetic possèss nothing in common. The action of narcotics generally cannot be appiied to anæsthetics, because of the markedly transient effect of the latter. If a body is narcotized with alcohol, opium, belladonna, etc., the diminished sensibility is lasting and it is never so regular, rapid nor effectual as the state of anæsthesia. The total suspension of all consciousness, all sensibility and the diminution of all the vital forces to the point of almost destroying life, accomplished in a few moments, and then as promptly banished by a few breaths of air, is a phenomenon that is as difficult to comprehend as to explain.

The theory most generally accepted today, and which seems to have been substantiated, in part, by experiments, is that inhaled anæsthetics produce a transient alteration of the protoplasm of the living cells. Claude Bernard and Dubois go still farther by insisting that the alteration consists of a dehydration of the protoplasm, which temporarily suspends its activity. They compare their action on cells with that of water applied to certain infusoria, which remain dormant when dried but promptly become active in the presence of moisture. In these low forms the dormant state and the active state can be produced at will by alternate desiccation and hydration. The effect is prompt and regular and simulates the effect of inhaled anxsthetics. It has been shown that all living cells are susceptible to the action of anrsthetics, and that the sensibility to them varies with their sensibility in general. That is, the higher the order the more sensible is the cell to the influence. It 
is shown that while all protoplasm may be anæsthetized, the brain cell is more sensible than the nerve, and the nerve more sensible than the muscle, and the muscle more than the tendon, etc., down the scale. In this same manner it is found that man is more sensible than the horse, the horse more sensible than the ox, the ox more sensible than birds, birds more sensible than the frog, and finally the frog is more sensible than the vegetable. These facts explain, beyond reasonable doubt, the phenomena following the inhalation of the highly volatile anæsthetic, that can be rapidly introduced into the blood and finally as rapidly eliminated or destroyed. The nerve cells of the brain and spinal cord being very highly sensitive to its action are promptly dehydrated (altered, disorganized, etc.) as the molecules of inhaled vapor are carried to them, the result being the state known as anresthesia. Some authors have insisted that these theories cannot be accepted entirely unchallenged until an explanation is forthcoming as to why the nerve cells governing the vital functions do not also yield as promptly to this subtile action. These are certainly highly sensitive elements. Why should not the cells governing the cardiac and respiratory functions become promptly altered, modified or dehydrated and then suspend their activity as readily as the others? A careful study of the brain and spinal cord, together with the phenomena observed in the subject sinking to the influence of an inhaled anæsthetic, answers the question to a nicety. It will be admitted that the cerebral hemispheres are the most highly sensitive structures in the body, and that the very first real narcosis from chloroform or ether, is hypnosis and then total loss of consciousness, which of course is due to their influence upon these organs, i. e., the cerebral hemispheres. The loss of consciousness in anæsthesia always precedes the loss of the reflexes. If an attempt is made to 
begin an operation too soon, even after the patient has lost all consciousness, there will be a marked resistance to the pain inflicted by the knife. The cerebrum,-- the most sensitive organism, -is therefore first to yield to the influence of the molecules of inhaled vapor that are carried to the cranial cavity by the blood. If the administration is discontinued at this moment a few breaths of air immediately terminates the phenomenon and the subject promptly revives, none the worse from the ordeal; but if the inhalation of the vapor is continued, then the less sensitive cells of the body begin to fall before the influence, one after another. The structure next in the scale of sensitiveness is the excito-motor apparatus, which being attacked by the molecules of the anæsthetic cause first a partial and then a total suspension of all voluntary motion. When the latter occurs the vital functions are already yielding, and unless the supply is curtailed at this moment they too suspend activity, and death supervenes from toxic apnœa, from overdosing.

The chain of events produced by anæsthetics from the very beginning to the end (death) are usually divided into three stages known as the stages of anæsthesia. These are:

I. The Stage of Excitement.-So far as the domestic animals are concerned this period of anæsthesia is nothing more than the natural resentment of the subject to the irritation of the anæsthetic vapor to the nasal mucosa and skin, and a sense of suffocation produced by obstructing the free admission of air into the air passages. It is but a fight against the anæsthetist, the apparatus and the anæsthetic. During this period the animal struggles violently,-with all its might,-to escape the impending discomfort. The horse will struggle against the restraining apparatus with the legs, strain every muscle of the body and will attempt to release the head from its confinement. 
The inspirations will at first be avoided, then several short respirations will be taken as if attempting to avoid the passage of the vapor deeply into the respiratory tract, and finally, when actually forced by threatened asphyxiation, the first deep breath will be inhaled, which may be referred to as the first important event of the phenomenon. It marks the passing of the stage of excitement and the beginning of the next period. The pulse will be found full and rapid, the respirations accelerated and now deep, the reflexes of the cornea not altered, the pupil somewhat contracted and the eyeball rolling around in its socket. The horse generally whinnies or even screams, the ox bellows and the dog bakks or whines.

2. The Stage of Narcosis.-This stage is often confounded with the last phases of the preceding and the opening events of the one succeeding. It is, nevertheless, to the observing and experienced anæsthetist, a distinct period intervening between the above mentioned first deep breath and the subsequent first shallow and soft breath, which event isolates it distinctly as a so-called stage of anæsthesia. It is marked by deep and rather harsh inspirations, from seven to fifteen in number, depending upon the dilution of the anæsthetic with air. If too much air is not admitted, seven to eight inspirations terminate the stage. The eyes roll in the orbit, the reflexes of the cornea are less acute, the sensibility, if the knife is applied, is but little modified and the consciousness is blunted and fast disappearing. With the latter event the first real event of anæsthesia proper begins. At this phase, according to Wilheme, the cells of the cerebrum are yielding to the blunting influence of the molecules of the vapor that have been carried to them by the blood vessels. They are being temporarily dehydrated, and the subject passes into a sleep as a consequence of their suspended activity. An examina- 
tion of the cornea now reveals its insensitiveness, the muscles are limp and immobile, the knife can be applied with impunity, the respirations rather suddenly become soft and shallow, the pulse slow and somewhat weak, although not markedly so, in fact;

3. The Stage of Anæsthesia has begun. This period is recognized from the preceding stage by the symptoms just enumerated. It is the surgical stage. The subject is ready for the waiting surgeon. The cornea no longer responds to the touch, the respirations are slow, soft, shallow and sometimes almost imperceptible, especially the exhalations. The heart beats are regular but rather weak and the arteries compressible. The entire body can be cut to pieces without provoking the least sign of pain or reflex action on part of either the voluntary or involuntary muscles. The cerebrum, the pons, and the excito-motor apparatus have yielded to the so-called dehydration of their cellular elements. The medulla and the sympathetic system, upon which the few remaining movements of the body are depending, are exhibiting evidences of yielding to the same modifications. If the administration continues they, too, suspend their activity, and death ensues.

Chloroform and ether are irritants. They blister the integument, and irritate the mucous membranes to no small degree. As a result of this action the bronchial secretion is always greatly augmented. Expectoration is profuse. The mucus can be heard in the bronchial tubes by auscultation, and it frequently produces a clicking in the throat. In the dog and in the ox there is also a marked increased flow of saliva, due evidently to increased secretion from irritation and to arrested deglutition. This feature is generally absent in the horse, but is not always wanting.

DURATION OF AN ESTHESIA.-Anæsthesia can be prolonged at will by continuing the administration. The 
domestic animals, by careful administration, may be kept in the anasthetic state for seven or eight hours before fatal intoxication occurs, but prolonged anæsthesia is always dangerous. The subject is revived from the state by cessation of the administration. If the anesthetic state was brief, and especially if the amount of vapor inhaled was small, the revival is rapid. The reflexes of the cornea return, the eyes roll about in the orbit, the subject begins to move the limbs and to raise the head, and in from ten to thirty minutes will stagger to the standing position. The gait will be groggy for a few minutes, but soon the normal equilibrium returns completely. The body is usually cold; often a cold perspiration will break out as the anæsthetic stage is disappearing.

Posology.-The dose of any anæsthetic for any animal is quantity sufficient. The aim should be to secure the greatest amount of anæsthesia with the smallest possible quantity of the anæsthetic. It is better to err in the direction of giving too little, than too much. And it must never be forgotten that a small quantity undiluted with air is much more dangerous than a large amount of well diluted vapor. Air is the antidote of the anasthetic; without it the patient must soon succumb; with it the anæsthesia may be prolonged indefinitely.

In the horse it requires about two ounces of chloroform to successfully carry the subject to the stage of anæsthesia, which amount should be placed upon the sponge as the administration begins. For the ox about three ounces of chloroform, and for the dog about two to six drams of ether. For the cat two drams of ether is usually sufficient. The quantity to be given subsequently to keep the surgical subject in the state of anæsthesia must depend entirely upon the condition of the patient. It must never be governed by measurement. As fast as the patient revives from 
the state, just so fast must the anæsthetic be given. The aim is to keep the subject at a perfectly safe distance from syncope, and yet prevent the reflexes from returning.

MODE OF ADMINISTRATION.-There are various apparatus recommended for the administration of anæsthetics to animals, but no one is more practical and satisfactory than the plain rubber or oil-cloth sheet and sponge. The adjustable nose-bags are rather treacherous and always somewhat difficult to manage.

Horse.-The subject must be securely tied in the recumbent position to meet the requirements of the violent

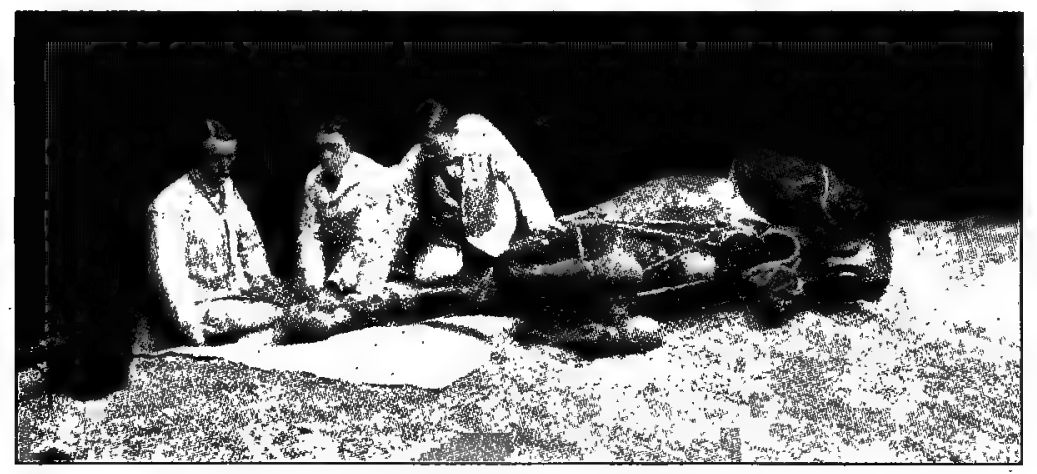

FIG. 29.

Administration of Chloroform.

struggles that are certain to ensue. The rubber sheet, which should be about one yard square, is spread out under the head with about one-half of its surface extending in front of the muzzle. The sponge, which should be of medium coarseness and large enough to hold two or three ounces of the anæsthetic without dripping, is sprinkled with the chloroform, placed in front of the nostrils and the sheet gathered up over it. The anæsthetist lies flat upon the horse's head and holds the sheet rather loosely around the nose, behind the nostrils. On the operating table the anæsthetist leans over the poll from behind forward, rest- . 
ing the chest heavily upon it as the sheet is gathered up and held. Instantly the struggles begin, but being expected they are met with forcible efforts to prevent disturbing the apparatus. The anæsthetist keeps "on the offensive" as long as the struggling continues, and until the subject is forced to take the first deep breath, which is usually about two minutes. Sometimes it is four minutes and sometime six minutes, but seldom longer. The first deep breath is accompanied with a somewhat marked relaxation of the muscular system. The struggles are less violent. From this point the hold is released, and more air is allowed to accompany the anæsthetic vapor, during seven to fifteen inspirations, which consume about another minute. When the respirations become soft, and shallow, the sheet is spread out and the sponge is placed on but one nostril throughout the remainder of the process. The soft respirations mark the beginning of the surgical stage. The subsequent duty of the anxesthetist is to administer the anæsthetic in limited quantities through the uppermost nostril, allowing the undermost one to freely admit air. A very satisfactory method of administration after the patient is once anæsthetized is by dropping the anæsthetic through a muslin cloth laid over the uppermost nostril. It is given drop by drop continuously, and is only withheld when there is evidence of a too profound state. This is preferable to the use of the large sponge, which is necessary only to hold the large quantity of the anæsthetic required to produce rapid anæsthesia at the beginning of the administration. The anæsthesia is produced rapidly by rapid administration with a large sponge and is continued by the "drop by drop" method through a muslin cloth.

The anæsthetist must never divert his attention from his work, and must take no part in the operation, if the killing of a large percentage of anæsthetized patients is to be 
avoided. He must direct his attention incessantly upon:(I) The condition of the respirations; (2) the condition of the reflexes as indicated by the cornea; and (3) the condition of the circulatory apparatus.

I. The respirations, although slow, shallow and soft, with the exha'ations often imperceptible, should be regular at all times in normal anæsthesia. The respiratory movements should be well divided between thoracic and abdominal. Pronounced abdominal breathing is unfavorab ${ }^{1} e$, especially when the act is jerky and somewhat irregular. The movements of the nostrils, although slight in profound anæsthesia must never be absent. The constant inspection of the respiratory movements is the anæsthetist's most serious duty, for it is here that the first sign of a fatal termination will appear in great majority of cases. Chloroform or ether poisoning-the administration of too much undiluted vapor-reflects first upon the respiration, and if the very first sign of apnoa is noticed, there is generally ample time to successfully revive the patient; in fact, the simple act of removing the anæsthetic apparatus is often sufficient to resuscitate the arrested function, but if in a moment the movements do not begin artificial respirations will gradually prove successful. There is very little danger of fatal chloroform intoxication if the state of the respirations is carefully guarded.

2. The condition of the reflexes as indicated by the cornea. The state of the sensibility (reflexes) is always well indicated, both by the touch to the cornea and by the resistance provoked by the surgical pain. If the patient is struggling against the surgeon's knife, the cornea will be found sensitive, while on the other hand, if the cornea does not respond to the touch, the operator may proceed without molestation. The cornea indicates the state of anæsthesia perfectly, but it is no indicator of the condition 
of the vital functions. The patient may be dying and still respond to the touch to the cornea, which fact the anæsthetist must always bear in mind.

3. The condition of the circulation as indicated by the pulse should not be entirely overlooked in anæsthetizing animals, as it may foretell an approaching syncope. The pulse should be rapid, full and regular, in the stages of excitement and narcosis, and slow, wavy and compressible during the anæsthetic stage. The quick, isolated heartbeat is a certain sign of impending danger. The anæsthetist should occasionally examine into the condition of the circulation with the aim of recognizing the faltering heart at any early period. When the subject is difficult to anæsthetize, or appears to succumb too readily to the influence, this precaution is especially indicated.

Ox.-The ox is anæsthetized with chloroform in about the same manner as the horse, after being well secured in the recumbent position. In this animal the anæsthetist must, however, take into consideration the fact that the mouth freely admits air into the air-passages, and that the sensibility to inhaled anæsthetics is somewhat less than in the horse. About three ounces of chloroform is placed upon the sponge, which is applied to the muzzle with the same sheet as the one used for the horse. When the anæsthesia is complete, it is continued throughout the operation by replenishing the supply to the sponge, instead of resorting to the "drop by drop" method recommended for the horse.

Dog and Cat.-Ether is by far the safest anæsthetic for these animals, although chloroform is successfully used by some cynologists. The writer's experience with chloroform in carnivora has been disastrous. White (Chicago) recommends the alcohol-chloroform-and-ether mixture, while Pertus (Paris) a mixture of ether, two parts and 
chloroform one part. In the large medical colleges, where a large number of dogs are anæsthetized for experimental operations, chloroform is in quite general use. The fatalities are, however, too numerous, while with ether they are quite rare. Ether in the dog and cat very seldom cause cardiac syncope, while chloroform always seems to kill by that method. Ether may provoke arrest of the respirations, but in the great majority of cases if the accident is observed promptly, resuscitation is generally successfully accomplished by artificial respirations.

In these animals a specially improvised apparatus is rec-

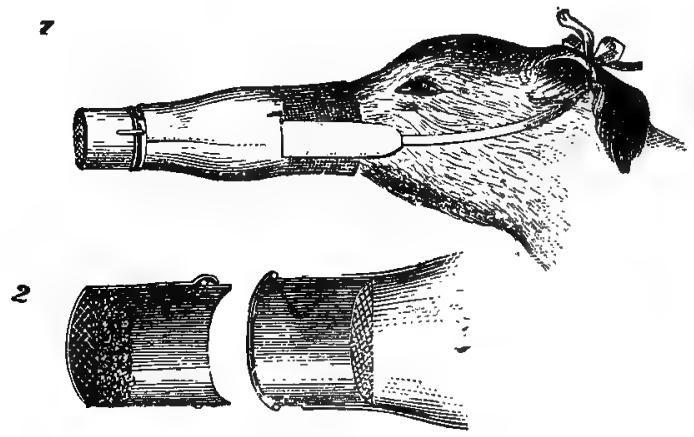

FIG. 30.

1. Perten's Method of Administering Anæsthetics to Dogs. 2. The Apparatus Taken Apart.

ommended. A leather cone to fit the nose, supplied with a strap to fasten it around the poll, a wire screen within to prevent the sponge from touching the nose and a removable and perforated end to hold the sponge, is the most practical apparatus (Fig. 30). The operation begins with two to five drams of ether (according to the size of the animal) applied to the sponge, which is then placed into the removable end of the cone just before the latter is adjusted to the apparatus, which has previously been attached to the subject's.head. The patient is held or tied firmly until the anæsthetic stage is reached; then a relaxed state of the 
body is preferable. It is quite difficult to kill a dog with ether if the respiratory movements are not restricted by forcible. or uncomfortable restraint, while, on the other hand, the least pressure to the thorax or tension on the abdominal muscles may "snuff out" the patient's existence without a moment's warning.

The anæsthetic state is maintained by replenishing the supply to the sponge from time to time as required, and as in the horse, the respirations are kept constantly under surveillance.

Small Laboratory Animals.-These subjects are best anesthetized by placing them under an inverted vessel containing a sponge saturated with a limited amount of ether or chloroform. As soon as they become unconscious the vessel is removed and the anæsthesia prolonged by dropping the anæsthetic through a cloth wrapped about the head.

HYPODERMIC ANÆSTHETICS are highly recommended in canine practice in lieu of those administered by inhalation. They are depended upon alone or as auxiliaries to the inhalation-anæsthetics. For' the latter purpose they are administered about twenty minutes before the ether or chloroform, with the purpose of producing a narcosis that will assist the action of the inhaled anæsthetic. The claim, however, that an animal previously narcotized can be anæsthetized with less ether or chloroform can not be proven by any series of comparative experimental tests. They accept them more complacently however, and thus facilitate the administration.

The hypodermic injection of from two to even six grains of morphia sulphate, often produces a very satisfactory state of narcosis in the dog and cat, without any subsequent inhaled anæsthetic.

THE ANESTHETICS.-Chloroform. for the horse 
and the ox and ether for the dog and the cat, will give results that leave but little room for improvement. The mixture of anæsthetics is absolutely unsatisfactory, in that the effects are uncertain, irregular and often treacherous. The administration of hypodermic narcotics previous to the inhalation anæsthetic is unnecessary and often harmful in the horse and the dog, but the use of morphia alone in the dog is quite effectual. Ethyl chloride, ethyl bromide, and the alcohol-ether-chloroform mixture cannot reclaim their places among the anæsthetics for animals. Chloral hydrate administered internally in doses of one to one and a half ounces dissolved in a quart of hot water, given on an empty stomach, is an effectual anæsthetic. It produces a partial anæsthesia suitable for certain minor operations. For clipping nervous horses, for shoeing the unbroken horse, for the tenesmus of difficult parturition in the mare and the cow, or for any manipulation that requires considerable time and that is aggravating but not very painful. this expedient is quite satisfactory. The anæsthesia begins about thirty minutes after the drench is administered if the stomach is not full of food. When full, two to three hours may elapse before the effect is observed.

Chloretone produces the most perfect anæsthesia for experimental purposes. From a single dose it causes a lasting and profound cataleptic state continuing without cessation for three days to one week, at the end of which time death supervenes. Given internally the dose is one grain for every two pounds of weight. In the horse, one grain for every three to four pounds of weight, injected intravenously in the form of an alcoholic solution, produces an immediate effect which will terminate fatally after about two to three days of profound anæsthesia. Chloretone is certain in its effects. It can always be depended upon to cause profound anæsthesia if the proper amount be given and 
absorbed by the blood. Some individual animals require more than others, per given weight, to produce the complete dòrmant stàte.

\section{FATALITIES FROM INHALATION ANÆSTHE-} SIA.-Inhaled anæsthetics may cause death by (I) toxic asphyxia; (2), reflex syncope; (3) direct syncope; or by (4) post-anæsthesia complications. The fatalities in ariæsthetized animals are more numerous than in man. largely because the veterinarian is less experienced in the administration than the trained human anæsthetist. Veterinarians of wider experience lose fewer than the one only occasionally resorting to the general anæsthetic. And besides, the veterinarian is seldom properly assisted in his operation. He must generally divide his attention between the anæsthetic and the operation, or else depend upon an inexperienced assistant to administer the latter. Furthermore, domestic animals are more difficult to manage and hence offer more obstacles to the administration of the anæsthetic in its proper dilution with air. That is to say, the amount of inhaled vapor is much more difficult to regulate. The mortality with chloroform in the horse from all causes is about I per 800, and with ether in the dog about I per 400 . In the human it is I per I 6000 with ether and I per 3 I 50 with chloroform. It is much greater in the sick patient than in the surgical subject in good general health. The rate of mortality in very sick horses,- say from septic infections, -is really very high, especially from complitions. Chloroform is very dangerous to the fat, idle horse, and ether is equally dangerous in the old, fat dog that has had insufficient exercise. Heaving horses are hazardous risks, and the roarer is none too safe. Animals that have been narcotized with drugs, especially with cocaine, are very susceptible to chloroform syncope.

I. Toxic Asphyxia.-Over-dosing with inhaled chlo- 
roform or ether may cause death, like any other chemical poison. Whenever the blood is impregnated with too liberal quantities of the drug, its toxicity produces death. The death from this cause is the one chiefly to be avoided by the anæsthetist. It is caused by arrested respirations, in almost every case, and is followed closely by cardiac syncope. This order is seldom reversed.

It is prevented by diluting the vapor with sufficient air, and by not prolonging the anæsthetic state unnecessarily. The rapid inhalation of large quantities of pure chloroform vapor or its continuous administration for a long period must be avoided in animals. When the accident is observed the anæsthetic must immediately be taken away from the nostrils and artificial respirations resorted to. In the horse, the best method to force the respirations is by pushing the foot firmly and deeply into the abdominal walls at intervals of about five seconds until the movements are spontaneously affected. The restraining ropes and all objects that might restrict the free action of the movements must be simultaneously removed. The tongue may be pulled forward to widen the pharyngeal lumen. No time must be lost in executing these manipulations. The internal or the hypodermic administrations of stimulants must never precede them because the time lost in preparing such medicaments is too valuable. A patient that once sinks into this state should not be re-anæsthetized. The subsequent steps of the operation must proceed without the aid of anæsthesia.

In the dog and cat, toxic asphyxia is treated by artificial respirations, as in the horse. The most effectual method is to expand the thorax by grasping the fore limbs and pumping them outward and inward at the rate of about one movement for every two seconds until the respiratory act returns. Satisfactory results can also be obtained by grasp- 
ing a hind leg in each hand and swinging the body like a pendulum for a few moments.

2. Reflex Syncope-This accident occurs during the stage of excitement, often before any great amount of the anæsthetic has been inhaled. It is cardiac syncope pure and simple, due to reflex action. It is undoubtedly caused by the combined action of fear, irritation of the anæsthetic on the sensory nerves and the sense of suffocation, predisposed, probably, by some functional or organic derangement of the heart, or the unusual sensitiveness of the individual. The writer has observed three such cases:-(I) A trotting bred stallion six years old that had been idle and fattened for several months was cast for the operation of cryptorchidectomy. About two ounces of chloroform was placed on the sponge, which was held to the muzzle in the usual manner, covered over with an oil cloth sheet. Instantly the struggles began, but before a single deep" inspiration was taken the patient was noticed to be dead. The respirations were stopped and the pulse imperceptible. Auscultation proved that the heart was arrested. All efforts to resuscitate were futile. Less than one minute elapsed between the time the sponge was placed to the muzzle and the time of death. (2) A coach horse nine yeyrs old; that was cast with the side-lines for a neurotomy of the peroneal nerve, died in about thirty seconds after the sponge containing two ounces of chloroform was applied to the muzzle. This patient had been cocainized over the plantar nerves with about six grains of cocaine hydrochlorate twenty hours previously. (3) A grade horse; weighing less than one thousand pounds was cocainized over the carpus to attenuate the pain of firing. The resistance being too annoying to the operator, the subject was cast with the side-lines. The struggling in this new attitude was continued so incessantly by the animal that it was found nec- 
essary to administer a general anæsthetic. The subject survived less than one minute after the sponge was applied. Williams (N. Y.) reports three similar cases in draft horses.

That the trifacial nerve is capable of conducting impressions which may arrest the heart's action is demonstrated in a case reported by Wright. A horse in apparent good health was brought out of the stall for the examination of a trivial nail puncture, and as he resented the doctor's manipulations a twitch was applied to the upper lip. It had scarcely been tightened firmly when the horse elevated the head, reeled backward and fell dead upon the floor. Prompt examination proved the heart to be stopped. Other analogous cases have been reported.

There is absolutely no prevention nor remedy for this unfortunate accident, unless the cocaine previously administered in two of these cases is really a causative factor. The writer is of the opinion that chloroformization following the subcutaneous injections of cocaine is a dangerous procedure.

Two cases of cardiac syncope in the human subject, were recently revived by direct massage of the heart, one through a thoracic wound which was under treatment, and the other through a laparotomy in the epigastrium, made through the diaphragm. The same expedient may be tried in animals, but its success remains to be determined.

3. Direct Syncope-Unlike the preceding, this accident occurs during the profound stage of anæsthesia, as the operation is proceeding. In most cases it is easily traced to either some organic disease of the heart, or to the seriousness of the operation being performed. It may follow immediately the execution of some salient part of the procedure. Prolonged anæsthesia when the alimentary canal is over-full, prolonged anæsthesia in fat animals, and pro- 
longed anæsthesia generally, may cause this accident. The latter, however, usually causes arrest of the respirations.

The prevention depends upon a careful selection of the patients, and their thorough preparation for the ordeal. Dieting, purgation and exercise for some days prior to the operation is often essential. Animals suspected of having some cardiac lesion, dilatations, hypertrophies, valvular lesions, etc., should be regarded as undesirable subjects. The administration of stimulants prior to the operation, although theoretically logical, seems to be useless.

4. Post-anæsthesia Complications.-The most common complications are bronchitis and broncho-pneumonia. The former may be rather trivial, but it is a very common sequel, especially after prolonged anæsthesia of the horse. It is manifested by fever, inappetance, and cough that either runs a short and favorable course or else terminates in a grave and generally fatal pneumonia. Chloroform pneumonia in the horse, and ether pneumonia in the dog, make their appearance on the third day by the usual symptoms of that disease. If the disease is wide-spread the respirations are greatly accelerated (heaving), the pulse rate is fast, and all food is refused. Death often supervenes after a very short illness of twenty-four hours. In milder cases several days terminate the life of the patient. It is caused by prolonged anæsthesia in the debilitated or seriously diseased animal, by exposure to cold after the operation, by pre-existing or approaching infectious diseases of the air passages, or from being predisposed by embolic pneumonia emanating from a suppurating focus at some other point of the body.

The prevention consists of limiting the duration of the anæesthetic in all cases, by careful attention to the warmth of the patient after the operation, and by avoiding anæsthe- 
sia in the debilitated and diseased. The treatment is that of pneumonia in general.

The other principal sequel of general anæsthesia in animals is "chloroform dementia," a condition which follows imimediately after the operation. Instead of reviving promptly as usual, the unconscious subject remains semicomatose, is incapable of regaining the standing posture, and struggles violently and incessantly with alt-fours for a period of from forty minutes to one hour or more. When finally the standing posture is again resumed, the subject will stagger, fall or plunge blindly against the wall. The majority gradually recover after six hours, but rare cases die, either from shock or from the congestion of the brain upon which the condition undoubtedly depends.

Blistering of the muzzle is a trivial complication, but is nevertheless one that should be avoided by anointing the skin about the nose with vaseline before administering the anasthetic.

INDICATIONS FOR GENERAL AN ESTHESIA.-

It cannot be denied that general anæsthesia is unnecessary in a large majority of the veterinary surgical operations. The veterinary-surgical patient must be submitted to the discomfort (and pain) of forcible restraint before the anæsthetic can be administered. Often this part of the procedure is quite as uncomfortable as the brief operation itself. If discomfort of administering the anæsthetic is added to that of restraining the patient, the pain of operation itself is often found to be a rather insignificant part of the entire procedure. The brief pain of dividing a nerve, a ligament, a tendon, of amputating a tail, of enucleating a small tumor, of inserting a seton, of removing the testicles, of suturing a lacerated wound, etc., etc., cannot be compared with the discomfort and misery' of administering chloroform and reviving from its influence, and with the 
danger of death from syncope, asphyxia or the post-anæsthesia complications.

These facts account somewhat for the apathy for the general anæsthetic among veterinarians. There are, however, many operations which cannot be properly performed without its aid, and as. many others that should not, on account of the severe and prolonged pain inflicted to the patient. The ablation of large tumors is both painful and dangerous from hæmorrhage and shock without the anzsthetic. The castration of a cryptorchid is an operation that is difficult to perform and painful, and hence is an indication for general anæsthesia. Ovariotomy of the bitch, and cat, herniotomies, abdominal operations that expose the viscera, laryngeal operations for roaring in the horse, radical operations for poll-evil, fistula of the withers, and quittor, neurotomies of the large nerve trunks, ablation of the eye-ball, and many others, are all procedures that are too painful and too tedious to carry out accurately without its use.

All of these major operations can be more successfully performed with the seat of operation in the perfect state of repose induced by general anæsthesia. The dissection is more accurate, the bleeding vessel is more easily located and ligated, the diseased area is brought into better view, the sutures can be more accurately adjusted, the protective dressing can be securely and effectually applied, and finally the danger of shock is materially lessened. Furthermore the pitiful shrieks and plaintive moans of the dumb brute should not be entirely ignored.

CONTRA-INDICATIONS.-General anæsthesia is harmful in some operations and strictly contra-indicated in others. It is particularly dangerous in animals carrying excessive, internal fat. The animal that has been fat for years, especially the one that has had insufficient exercise 
prior to the operation, is to be avoided. Debilitated subjects, either from over-work, bad hygiene or from disease, are never welcome surgical cases. Structural alterations of the heart,--although these can seldom be diagnosed,are generally considered as contra-indications. It is, however, doubtful whether there is more danger with than without, the anæsthetic in these cases. The writer has had a death from rupture of the coronary artery caused by severe straining during a quittor operation, that would certainly have been avoided had an anæsthetic been administered. Operations on the upper air passages should generally be performed without general anæsthesia, on account of the greater danger of aspirating blood, saliva and putrid matter into the lungs. Among these are trephining the skull for nasal catarrh, ablation of nasal tumors, operations on the guttural pouches and the reposition of comminuted fractures of the facial bones.

General anæsthesia is contra-indicated, also, when the alimentary canal is overloaded, or even normally full of aliment. In this instance the respirations are mechanically obstructed, and hence rather susceptible to arrest from the depressing influence of the anæsthetic.

Disease of the lungs (heaves), diseases of the pleura (pleural adhesions) and diseases of the large blood-vessels (aneurism) are ever prone to prove the undoing of an anæsthetized patient. Like the disease of the heart the latter are found at the post-mortem only.

PARTIAL GENERAL AN ESTHESIA.-There are certain conditions encountered in veterinary surgery in which complete general anæsthesia is neither advisable nor practical, and which can be coped with by blunting the sensibility with narcotics. In the dog, the hypodermic injettions of morphia, already referred to, produce a state of partial anasthesia which renders possible many of the sur- 
gical operations. Spaying of bitches, herniotomies, ablation of mammary tumors, trimming the ears, and many other minor and even major procedures can be quite well executed by thus blunting the sensibility. In the horse, and in the ox, chloral hydrate administered internally has many indications. For clipping the restive horse or for shoeing the vicious, unbroken colt, a state of partial insensibility can be thus produced that will greatly facilitate the work. In obstetrics, to arrest the forcible straining which interferes with the manipulation of the mal-presented fœtus, chloral is often helpful.

In the horse, chloral hydrate should be given only upon an empty stomach if prompt and uniform results are desired. One ounce to one ounce and a half, dissolved in a quart of hot water and administered as a drench, will bring about the desired state of blunted sensibility in about twenty minutes. If the stomach is full the results are less certain and the full effect of the drug may not appear for several hours. For clipping, trimming and shoeing, this method is very satisfactory.

\section{LOCAL ANÆSTHESIA AND LOCAL ANÆSTHETICS.}

HISTORY.-The scientific use of local anæsthesia dates back no earlier than I884, although ice, snow, cold water and other various expedients were used ineffectively since time immemorial. Halstead in 1884 , Corning in 1885 , and Reclus and Wall in 1886 , were the first to describe subdermal applications of chemical anæsthetics in medical literature. Some years later (1890) Schleich in his "Schmerzlose Operationen" gave the first detailed description of the effects of cocaine in various dilutions and from various modes of application. Schleich, however, gave preference to his "infiltration method" which con- 
sists of forcing the tissues apart with weak solutions of chemical analgesics. His solutions, consisting chiefly of cocaine, morphine and sodium chloride were forced in considerable quantities between the two layers of the skin or between the subjacent tissue layers. The claim was made that parting the anatomical elements of the tissues had in itself a marked anæsthetic effect; but later developments. have shown that the good results were due more to the action of the chemical analgesics than to the separation of the tissues, and hence the method, as such, became obsolete.

Cocaine was the first chemical anæsthetic to attract attention and it has held its place at the head of the list until the present day in spite of the frequent introduction of other substances claimed to possess equal anæsthetic action and less toxicity:

In veterinary surgery local anæsthesia did not come into general use until the late 9o's, although there is some evidence that it was used here and there by some veterinarians as early as I888. During the last few years it has come into very general use wherever scientific veterinary surgery is practiced. Today it has numerous indications for which it is used effectually. By its judicious use certain operations otherwise requiring recumbent restraint are performed with perfect precision in the standing position without the least discomfort to the patient. It has come into quite getreral use for very painful operations that are rather too brief to warrant the administration of a general anæsthetic. In surgery of the eye it has become indispensable. In the diagnosis of lameness it is invaluable. The lameness of unknown origin is located by the cocainization of the nerve trunk supplying the sensory innervation of the suspected region. The great value of local anrsthesia in these several connections is no longer questioned, in spite of the 
fact that the toxicity of the chemical anæsthetics renders their too free and too promiscuous application quite hazardous.

DEFINITION.-The term "local anæsthesia" refers to the relief of surgical pain in a circumscribed area. It is accomplished by the use of drugs called local anæsthetics. The area, although generally circumscribed, may by their application to a large nerve trunk, extend over a considerable surface. Thus, by injecting cocaine upon the plantar nerve the entire foot may be rendered insensible.

The local anæsthetics in general use in veterinary practice are cocaine hydrochlorate, in a $4 \%$ to a $10 \%$ solution; eucaine hydrochlorate in a $5 \%$ to a Io $\%$ solution; ethyl chloride; morphine sulphate in a Io\% solution with cocaine hydrochlorate $5 \%$; adrenalin chloride $0.0 \%$ with chloretone, I ; mixtures of cocaine, morphia, carbolic acid and sodium chloride in proper strengths; and stovane.

The chief one is cocaine hydrochlorate in a solution varying from $4 \%$ to $10 \%$, according to the surface to be anæsthetized. From the standpoint of efficacy it cannot be easily supplanted, although excellent effects are obtained when it is mixed with morphia, carbolic acid and sodium chloride. Eucaine is somewhat less toxic, but also less effectual as an anæsthetic. Ethyl chloride is admirable as a spray to the surface after cocaine has been injected subcutaneously, and to spray upon the tissues as the operation proceeds. It is practically non-toxic.

ACTION OF COCAINE.-When cocaine at $5 \%$ is applied to a mucous membrane or to the skin deprived of its epithelium, it rapidly deprives the seat of application of all its sensibility. After a few minutes it produces an anzmic condition of the part by constricting the vessels. The sensibility begins to return in about twenty minutes. The insensibility is very superficial, for cocaine does not 
penetrate when applied to a part. When applied directly to a nerve trunk it almost completely destroys the sensibility of the tissues supplied by it. But the action in this connection is somewhat uncertain because the liquid may not come into contact with all of the fibers of the trunk. A nerve trunk may, however, be entirely rendered insensible by bringing the drug into contact with all of its anatomical elements. Cocaine affects both the nerve ending and the nerve trunk, it need only come into contact with them.

Cocaine, when absorbed in sufficient quantities, produces constitutional disturbances of serious import. Instead of anæsthesia, it provokes a state of excitability and sensitiveness that is entirely opposite to the action desired. The patient becomes excited, refuses to remain quiet, breaks out with a slight perspiration, assumes a wild-looking appearance, and will violently resent the infliction of the slightest pain. If trotted out to the halter the head is held high, the action reckless, the eyes staring, and the animal is found difficult to control. When large doses are administered these symptoms are followed by a delirious, semi-comatose state, during which the animal will push forward in the stall or fall down and fight wildly with all four legs. Death occurs from arrest of the heart.

Five grains of cocaine hydrochlorate is sufficient to cause the state of excitement in a sensitive animal, but it often requires upwards of fifty grains to produce death in any horse. A horse that received twenty-four grains at a single dose (hypodermically) became delirious and semicomatose, but exhibited no signs of the ordeal after fortyeight hours. Another horse (a four-year-old thoroughbred) received forty grains, in two doses one-half hour apart, and showed no unusual inconvenience after two days. McEvers reports the death of a coach horse that received 
about one dram of a I0\% solution in the tail preparatory to the operation of caudal myotomy. But this horse plunged violently in the stocks and resisted every attempt to confine him, and probably died from traumatic syncope or reflex syncope. The post-mortem revealed no lesion to which the death could be traced.

Posology and Administration.-The toxicity of cocaine or any other potent local anæesthetic must always be kept prominently in mind. The reckless injection of these medicaments is often disastrous, although the lower animals are much more difficult to kill with them than the human family, certain individuals of which seem to possess manifest sensibility to their systemic actions. These idiosyncrasies do not exist in animals to any appreciable extent. Deaths from cocaine are rare in the horse, and they occur in the dog only when the dose has been excessive. Five grains should be the maximum dose for the horse and one-eighth to one-fourth grain for the dog. In the latter animal there is little demand for its use, owing to the facility with which operations can bé executed without anæsthesia, as well as the facility with which the general anæsthetic can be administered.

The five per cent aqueous solution of cocaine hydrochlorate is the most appropriate local anæsthetic for general uses in the veterinary subjects. When only very small areas are to be anæsthetized, the ten per cent solution may be used, but for general use this is too concentrated, and is positively harmful in any considerable quantity. With the five per cent concentration one dram of the solution (three grains) can be injected with impunity. This quantity of the solution is sufficient for the large majority of indications. When a larger surface is to be anæsthetized, an additional half dram can be safely given, but the better method is to dilute the solution to $4 \%, 3 \%$ or $2 \%$ when larger quantities are required. 
Local anæsthetics are used hypodermically in veterinary subjects. It is seldom used on the mucous membranes except the conjunctiva; it is seldom applied into the deep structures; and it cannot be injected intradermically. The skin of animals will not separate to receive an injected liquid.

An effectual method of anæsthetizing an operating field is to (I) shave and disinfect the part; (2) inject a dram of the five per cent solution of cocaine, here and there, hypodermically; and (3) spray the surface thoroughly with ethyl chloride. As the operation proceeds the latter may be sprayed directly into the tissues to carry the anæsthetic state beyond its original extent.

It is needless to state that injected anæsthetics must be aseptic, and the instruments used for its administration absolutely immaculate. Septic liquids and septic syringes cannot be used with impunity in the subcutaneous tissue. The anæsthetic solution must not be exposed to the atmosphere of the veterinary hospital, the horse stable, or the pharmacy, and each time before the needle of the syringe is inserted into the bottle, it must first be passed through the flame of a match or lamp. The syringe itself requires special care. The all-glass, or the all-steel syringes are preferable, owing to the difficulty of sterilizing the leather plunger of the ordinary hypodermic syringe.

A mixture of local anæsthetics containing the following ingredients is also highly recommended for veterinary use :

R Cocaine hydrochlorate

Morphia sulphate

Carbolic acid, 95\%

Sodium chloride

Aqua q. s. ad $3 i$

Misce et signa. One dose. 
SPINAL COCAINIZATION.-The posterior extremities, the pelvic organs and some of the abdominal viscera can be perfectly anæsthetized by the injection of a $2 \%$ solution of cocaine into the subarachnoidean space of the lumbar division of the spinal cord. The experiment was tried in human surgery for the past several years, with some success, but owing to the danger of complications it has been losing ground instead of being generally adopted. The uncertainties of asepsis, the difficulties met in delivering the solution to the proper place, and the gravity of the complications, seem to have rendered the method somewhat obsolete. In surgery of domestic animals it was never used, although a number of experiments made upon the dog have demonstrated the possibility of its application in animals. In the large animals it must not be thought of. The distance from the cord to the surface alone would prevent its adoption, without taking into account the dangers of sepsis and the difficulty of managing a half-paralyzed horse while the sensibility was gradually returning. 


\section{CHAPTER XI.}

\section{ASEPSIS AND ANTISEPSIS.}

DEFINITION.-The terms "asepsis and antisepsis," "aseptic surgery" and "antiseptic surgery" are now quite commonly used to designate the standard manipulations which have emanated from the teachings of Lister as to the cause of wound diseases. Aseptic surgery may be defined as the essential precaution to prevent infection of the surgical wound, while antiseptic surgery refers both to the method by which the former is accomplished and to the treatment of unavoidable infections. Thus, the absolute sterilization of everything more or less intimately connected with a surgical operation, is asepsis, and the operation performed under such conditions is "an aseptic su'gical operation." The process of sterilization requires the use of antiseptics,- - heat or chemicals, - hence the "antiseptic precaution" precedes the "aseptic technique." The former renders the latter possible. The whole aim in this particular connection is toward asepsis. The surgical wound is kept aseptic, while the already infected one is made as nearly aseptic as possible. To prevent a trauma from becoming infected and to disinfect the infected one is the surgeon's most serious duty.

From this standpoint, the veterinary surgical operations are often open to adverse criticism, in that they are not always performed in strict obedience to the laws which now govern surgical manipulations, viz., the laws which emanate from the common knowledge about the etiology of wound diseases. All mankind now knows that wound diseases are caused by the intrusion of bacteria; that the 
surgeon should prevent such intrusion; that the bacteria already lodged in the recesses of a wound may be difficult to dislodge; and that cleanliness in the surgical sense is nothing less than absolute sterilization. The human surgeon performs his operations in the seclusion of his operating, room, the veterinarian before a criticising public, which fact, particularly, necessitates the adoption of surgical manipulations that will bear investigation. Furthermore, aseptic wounds heal promptly and without complications, while the septic ones undergo varieties of serious complications that require a whole chapter to describe.

Asepsis and antisepsis in surgery are synonymous with cleanliness in the surgical sense of the word. They signify, chiefly the prevention of infection, but also include the clestruction of micro-organisms that have already invaded the surgical or accidental wound. These modern surgical manipulations are based upon well known facts:

Ist. The phenomena which exposed wounds undergo in the process of healing are abnormal. They are pathological instead of physiological. They are due to the invasion of pathogenic bacteria into the wound-the invasion of bacteria from without. They are carried into wounds by objects which touch them, such as dusty air, foreign bodies, surgical instruments, the surgeon's hands, etc.

2nd. Pathogenic bacteria are everywhere, the surgeon pre-supposes. The skin of the patient, the skin of the surgeon, the surgical instruments, the antiseptic solutions and their containers, the surgical dressings, the dusty air of the environment, the clothing, etc., etc., are known to contain them. Some of these objects which must come into contact with wounds may be aseptic, but the surgeon has no practical method of determining their purity, and hence treats everything as dangerous. 


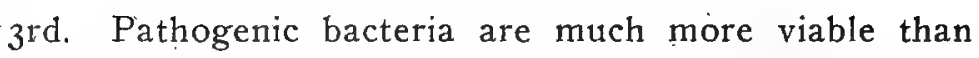
the cells of the body, and hence cannot be destroyed with chemical substances that will not also destroy the cells of the body. This fact necessitates the prevention of infection, because when a bacterium has once invaded the tissues it cannot easily be destroyed or removed.

4th. Wound diseases are prevented by protecting the wound against microbian invasion. This fact was Lister's hypothesis, and it has not been changed to the present day. Although Lister was not as familiar with the nature of the intruding agent as the present-day surgeon, his deductions were correct. He observed and even emphatically recommended the execution of the minutest details in preventing the unknown enemy from invading the open trauma. This then unknown enemy was washed from the seat of operation with soap and water and then with solutions of carbolic acid, the instruments were sterilized, the hands were cleaned, the sutures were immersed for several days in carbolized oil, the air was sprayed with carbolic acid solution and finally, after the sutures were applied, the wound was protected against subsequent infection by covering it with impervious antiseptic dressings. These few words embody the principles of antiseptic surgery of today: It has changed only in the details which have since emanated from a better understanding of bacteria. Lister paid too much attention to the air and too little to the instruments and hands, but otherwise there is little to criticise in the technique he recommended.

5th. Wounds occasionally become infected by way of the circulation, but that this form of localization is rather rare is now well"known. In veterinary subjects it is indeed rare. Animals affected: with tuberculosis, glanders, pyæinia, or septicamia, and debilitated subjects generally, may occasionally sustain wound infections from within but since 
the accident is extremely rare, this cause of infection may be ignored entirely. Bacteria in health, or even in the earlier stages of disease, are never found floating abundantly in the circulation, as was once supposed. The healthy body destroys them. They are found in the diseased faci, in the blood of the animal dying from certain infectious diseases, and in the carcass, but not in the circulating blood of the comparatively healthy animal.

The technique of an aseptic operation is based upon the foregoing five well-known and well proven facts.' The pathogenic powers of bacteria, their wide diffusion, and their great viability, necessitate the adoption of a rather complicated technique to prevent their invasion into the surgical wound. If the pathogenic powers were trivial, if the bacteria were few and if they were easily killed, aseptic precautions would hardly be necessary. But owing to the fact that very serious, very fatal or very harmful complications result from bacteria, and that almost everything around a surgical operation harbors them, and finally that they are very tenacious agents to destroy, the aseptic technique becomes an absolute necessity. - It is standard, it is expected, it is malpractice to ignore it,

The aseptic surgical operation begins, therefore, by sterilizing or rendering harmless, all objects that will come into direct contact with the wound. The conveyors of iniection are: (I) Air; (2) the surgical instruments; (3) the hands of the surgeon; (4) the environs of the wound; (5) the sutures; (6) the antiseptics and the containers of the antiseptics; (7) the surgical dressings; (8) the patient's habitat-the stall, kennel or sty; and (9) the litter.

I. The Air.-As stated above, Lister placed entirely too much importance on the air as a conveyor of infection. He supposed that myriads of "unknown poisonous bodies" fell upon the wound as the operation proceeded, and recom- 
mended that the air be sprayed with carbolic acid during the entire procedure. This supposition has since been proven erroneous. The air itself contains no microbes, but the dust it suspends, or the particles that may fly along with its currents are known to be the real conveyors. If the air is quiet and its dust is permitted to precipitate to the floor, it is incapable of contaminating a wound with microorganisms. Furthermore, the dust of the air of a room, while known to usually contain bacteria, may not contain pathogenic bacteria. They may be harmless, and may be left to invade the wound with impunity. The dust of a veterinary hospital harboring all kinds of patients, the dust of hay, the dust of straw, and the dust of an ordinary stable, must however, never be taken as entirely harmless. Numerous infections are traceable to this source, especially in the city practice.

In dealing with the air as a conveyor of wound infections. the veterinarian adopts practical methods. The operating room is kept clean, its floor is dampened and the air is never agitated prior to the hour for operations. The patients are brushed of superfluous hairs and wiped with dampened cloths before being admitted. If the patients are secured with the casting harness, the litter is dampened so that the minimum amount of dust will be raised by the struggling. The operating table, and the floor mattress are excellent expedients to prevent infections from this source, while the straw or hay litters are rather treacherous. Tanbark, peat, shavings or saw-dust can be rendered almost dust-proof, but particles are always liable to be whirled through the air and into the wound by the patient's struggles.

The country practitioner must avoid shaking up a fresh. litter of straw or hay, just before the operation. Castrations, especially of cryptorchids, often prove fatal from 
this cause. The out-of-door operation, on a grass plot, is the ideal one. But when it is necessary to perform the operation within the stable the litter should be laid and dampened some hours previous.

2. The Surgical Instruments.-For the sterilization of the instruments the writer recommends their immersion in 95 per cent carbolic acid for a few minutes, as the most practical method. The acid is contained in a wide saltmouth jar carpeted at the bottom with felt, cork or cotton to prevent the points of the knives from striking against the glass. While the preparations for the operation are proceeding, all of the instruments required are placed in the jar. In a few minutes they are safely sterilized, and can be removed with a forcep and rinsed in a three-percent solution of carbolic to render them safe to handle. They are either left in this weak solution, or else spread out on the instrument table ready for use. In out-of-door practice the resting place of the instruments must be somewhat carefully selected. The instrument tray containing a three-per-cent carbolic solution is the safest, although a clean towel or well cleaned, smooth board will answer. With a few sensible precautions, absolutely sterilized instruments may thus be obtained in almost any environment and without much inconvenience.

The boiling of instruments is, however, the safest form of sterilization. In the hospital, where some pretense is made to perform operations properly, heat sterilization should be adopted. It is easily and rapidly effected where a gas stove is available, but is entirely impractical under any other conditions. The lighting of a coal or wood stove to boil instruments for each operation would soon lead to the abandonment of the effort entirely.

Instruments should be boiled for fifteen to twenty minutes in a weak solution of sodium carbonate, and then 
laid out on the instrument table to cool or else placed in the three-per-cent carbolic solution. Only all-steel instruments can be thus treated. The catheter, the rubber syringe, the syringe with a leather plunger, the rubberhandled knife, the rubber-handled trephine, etc., must be placed in the 95 per cent carbolic acid.

3. The Surgeon's Hands.-As conveyors of infection into wounds, the hands of the veterinary surgeon stand first. They are always dangerous. If the cleanest hands of the cleanest human surgeon are never absolutely safe, what must be said of the hands of the veterinarian, who, just a moment before beginning the operation, must handle all kinds of infected objects-the halter, the patient, the casting ropes, etc.? In dealing with this item the veterinarian must first cope with the fact that the human skin is never entirely aseptic and that the very dirty hands cannot be rapidly rendered safely clean. The hands are capable of carrying dangerous infective microbes for several days after being contaminated. If we cope with a putrid fœtus or placenta, no washing will make the hands safe enough to operate upon a cryptorchid within twenty-four hours. If a putrid fistula is handled one moment and a horse castrated the next, although the hands have been cleansed, serioús results may follow. These facts are well established by experience.

The most important precaution to take in this connection is to avoid manual manipulations of surgical wounds as much as possible. It is remarkable how little one really needs to touch the surgical wound, if appropriate use is made of the dissecting forceps, the tenaculum, the retractors, the rat-tooth forceps, and the needle-holder. While there are operations in which the fingers must be used, there are indeed many in which the handling of the tissues is entirely unnecessary. To learn to avoid the direct 
use of the fingers is a valuable accomplishment in veterinary surgery.

Besides the above precaution, the hands should be washed with soap and water and then rinsed out in a solution of mercuric chloride, I-500.. The nails are kept short and free from dirt. Scrubbing with a stiff brush is thought to be harmful rather than beneficial, in that it brings the microbian flora of the deeper layers to the surface (Murphy). It is also advisable to repeatedly immerse the hands in the mercuric chloride solution during the operation.

Rubber gloves, although more cumbersome than the bare hands can be perfectly sterilized, and may therefore be adopted when absolute asepsis is desired.

Cleanliness of the hands is important, also, from the standpoint of the surgeon's personal safety against dangerous infections. Fatal septicæmia, glanders, anthrax, tuberculosis, tetanus, malignant œedema or actinomycosis may be acquired by ignoring these possibilities. Trivial wounds of the hands are particularly dangerous in obstetrical operations, in dental operations, and in autopsies. Before undertaking such work the veterinarian should protect abrasions with collodion, iodine or iron, and during the procedure frequently immerse the hands in strong antiseptics. Small, invisible abrasions may be located by rinsing the hands in vinegar and then exposing them to the air for a few moments. The pricking sensation discloses their exact locations.

4. The Environs of the Wound. The Surgical Field.Before a surgical wound is made the skin must be rendered as nearly aseptic as possible, not only along the line of incision but also over a liberal surface around it. In the accidental wound the surrounding skin requires cleansing. Here the same obstacles are encountered as with the surgeon's hands, with the addition that all veterinary wounds 
are "scalp-wounds," that is they are in a hairy environment. If the human hands are difficult to clean what is to be said of the skin of a horse, an ox $_{\text {m }}$ or a pig? The skin of these animals are veritable hot-beds for bacteria, and its constitution is such as to render its sterilization impossible. Experiments have shown that the skin of animals is seldom made aseptic, the latest being those of Pendergast and Linch at the New York State Veterinary College, who have shown by tests of skin taken from ten horses that disinfection cannot be absolutely depended upon to destroy all of the bacteria harbored within it.

Cleansing of the surgical field or the environs of an accidental wound is accomplished by (I) clipping and then shaving the hair; (2) washing with soap and water; (3) washing with mercuric chloride, I-500 or even I-200; and (4) washing with pure alcohol. These processes may be carried out twenty-four hours before the operation, and then the field may be covered with a pack soaked in a weaker mercuric chloride solution. As the operation begins a second immersion with alcohol may be applied. Ordinarily, however, the execution of these steps just before the operation is satisfactory.

In the accidental wound, washing, shaving and irrigating the surroundings may prove harmful by washing dirt and hairs into the abrasion. Often the hairs may be left alone for this reason.

After the wound is sutured the environs are kept dry to prevent microbian growth. If the wound secretions are left to flow over the surroundings, beneath a protective bandage, the bacteria which might have remained harmless upon or within the skin, may grow rapidly and thus gain admission to a wound that was otherwise properly treated. Dryness of the environs is no small part of aseptic treatment in veterinary subjects. 
5. The Sutures.-Sutures come into such close contact with the wounded tissues, being imbedded into them, and they remain there so long, that they, above all other conveyors of infection, require particular attention. Lister recognized this from the beginning, and immersed his suturing materials in carbolized oil. Absolute sterilization of sutures is as possible as it is necessary. It should never be omitted, nor be only half accomplished. A whole ball of cheap suturing material, such as Irish Linen Thread, can be safely sterilized by keeping it immersed in alcohol. A sait mouth jar with a small perforation in the lid to give exit to the thread is a good container for keeping an abundance of cheap suturing material in a state of perfect asepsis. For more particular work sterilized silk in hermetically sealed tubes is, however, advisable. For buried sutures, cat-gut in sealed tubes, is essential.

In preparing sutures for an operation they must be handled only after cleaning the hands, and a needle should be threaded for each stitch required. It is wrong to arm a needle with a long thread in a veterinary operation because it will surely be dragged over unclean surfaces. The threaded needles are placed in a separate tray or alone on the instrument table, so as not to become contaminated or tangled up while handling the other instruments. They should be picked up with the dissecting forceps: and then taken in the jaws of the needle-holder with which they are. inserted. In treating accidental wounds, the environs of which have not been shaved, or even in any wound.where absolute asepsis is desired, the needle should be passed from within outward to prevent carrying infection (or hairs) into the wound.

6. The Antiseptics and Their Containers.-Antisepties may not always be what their name indicates. Some of them are actually harmful from the great number of micro- 
organisms they carry into a trauma. The veterinary surgeon can make no greater mistake in surgical operations or wound treatment than that of placing explicit confidence in the microbicide action of antiseptics. The fact is, instead of killing microbes, the antiseptic powder or antiseptic solution that is not submitted to the same careful sterilization as the other infection conveyors, is much more harmful than useful. If an antiseptic is made strong enough to promptly kill micro-organisms or arrest their growth, it will also injure the living cells with which it comes into contact; if it is made weak enough not to injure the living cells of the wound, unless it is sterilized, it will carry new infection into the wound.

The powdered antiseptic, by virtue of its dryness, hinders microbian growth by absorbing their nutrition, which fact renders them less liable to add infection to the wound than the liquids. The precaution to take in this connection is to keep the dry antiseptics,-iodoform, boric acid, etc.,-in clean and protected packages; to make the solutions only with water that has not been contaminated or that has been sterilized by boiling; and finally to place them only in sterilized containers. The only really clean water is that coming from a deep drilled well or a spring. All other waters should be boiled. The containers should be of metal or glass; so as to facilitate cleansing. A well washed porcelain, tin, granite or glass vessel is practically safe, while the wooden bucket is always dangerous.

In the hospital the water and containers can readily be made and kept safely aseptic. It is only in the out-of-door practice that real obstacles are encountered in keeping these rules of asepsis inviolate. A kettle of boiled water and a well rinsed tin pail to contain it offers the best solution of the problem. Water from a stream, from a well that is contaminated with surface water, from the city hydrant, or from the 
rain-water cistern may contain very harmful micro-organisms, and should therefore never be used except for making exceptionally strong antiseptic solutions.

7. The Surgical Dressings.-These include, woundpackings, bandages, drainage tubes, plastic dressings, the bailing sponge, and often the dry antiseptic. Lister paid a great deal of attention to this item. He covered up the wound with layer after layer of antiseptic fabrics, and then with sheets of impervious material, with the purpose of excluding the microbes of the air, but it has since been shown that rather unnecessary efforts were made in this direction during the first years of antiseptic surgery. The packings placed into the wounds of animals to arrest hæmorrhage or to absorb wound secretions are either absorbent cotton, gauze, oakum, lint or waste. These substances may be purchased in various forms,-antiseptic or aseptic,- - packed in sealed jars or metal packages, but they are too expensive for veterinary operations generally. It is necessary to use the crude product and to make it safe by economical methods. The ordinary absorbent cotton purchased in pound packages is clean, and it can be made quite safe by immersing in mercuric chloride or carbolic solutions for some minutes before inserting it into the wound. Dry, it can not be recommended as absolutely aseptic until it has been passed through some form of disinfection. Waste, oakum and lint must be immersed for some time before they are absolutely safe.

A very economical and safe wound packing is made from cheese-cloth. It is torn into strips four inches wide and several yards long, wound up into loose bandages and kept immersed continually in a strong solution of mercuric chloride. A large, two-gallon, salt mouth bottle may be kept full of such packing ready for use at all times. The same recommendation is made for the protecting bandages, with the 
exception that these are made from a stronger muslin. A two-gallon bott.e containing strong muslin bandages and one containing cheese-cloth bandages, each filled with a I-IOOO mercuric chloride solution, are two very valuable and economical items for the veterinary operating room.

A similar recommendation is made regarding the "bailing sponges" used to wipe blood from the wound as the operation proceeds. These objects are capable of infecting every surgical wound, as they generally come inco contact with every part at frequent interva's cluring the operation. The sponge, from the fact that it is durable and is used over and over, is a very dangerous infection conveyor. No greater mistake can be made than that of using the same sponges for a number of consecutive operations, or to use the same sponges day after day, and week after week, with no greater precaution than that of immersing them in the antiseptic solutions just before the operation. Their physical constitution renders them capable of harboring infectious matter, which, of course, can not be made harmless in a moment. Small pledgets of cotton wrung out dry from I-IOoO mercuric chloride solution is always the safer bailing material. When soaked with blood these are cast aside and new, fresh and clean pledgets substituted. The sponge must be frequently wrung out in the antiseptic solution and this greatly diminishes its potency, as well as infects the solution.

When sponges must be used, they must be kept like the cheese-cloth and muslin bandage, in a salt-mouth jar containing mercuric chloride solution $1-500$ or I-IOOO ready for use. Small, fine, bleached sponges are selected for this purpose, and a quart salt-mouth jar to contain them.

8. The Patient's Habitat.-The surgical patient, more than any other, requires a clean place of abode, to prevent wound contamination, from lying upon or leaning against 
infected objects. It should in addition be kept comparatively free from dust or currents of air capable of conveying infectious matter to the wound. The soil of the pasture or the floor of the stable may convey tetanic infection into an unprotected wound. Whole herds of recently spayed hiefers, and whole droves of recently castrated pigs have sustained serious wound infections from having been placed into germridden habitats. The sheep-pen often infects a large percentage of castrated lambs, and the stall litter may readily cause a fatal septic peritonitis in the castrated horse. Not only the wound of castration, but any wound, may thus be contaminated. The sensible recommendation in this connection must emanate from an accurate knowledge of bacteria, especially in regard to their extraneous behavior. The greatest danger is found in the crowded environment, kept none too clean, the grassless paddock containing dust and animal excrement, the manure heap, the crowded pasture in dry seasons, and pastures harboring such micro-organisms as the bacillus tetanus, the bacillus anthracis, the bacillus anthracis symptomatici, etc. There is little fear in the clean stable or in the veterinary hospital where the value of intelligent disinfection is constantly respected.

\section{THE ROUTINE OF WOUND TREATMENT.}

A special, regular and methodical curriculum must be followed in the treatment of every wound. There must be no variation, except in the detailed execution of each step. The order is as follows :-

I. The examination.

2. The surgical treatment proper.

(a) The dissection, resection, etc.

(b) The hæmostasis.

(c) The sterilization.

3. Closure and drainage.

4. Protection and after-care. 
I. THE EXAMINATION.-A very careful examination of a wound must precede all other steps of treatment. In no case must this step be omitted, and in no case must it be carelessly or ignorantly performed. A wound that might on casual inspection appear to be a slight dermal laceration, might subsequently prove to be a serious penetrating wound of the thorax or abdomen. A wound may contain a foreign body, a spicula of bone, a necrotic cartilage, slough, or a recess to harbor wound secretions, which, if not discovered, would materially accentuate its seriousness and prevent its cicatrization in the shortest possible time.

The examination of a wound should include its history, which in turn reveals: (I) Its duration; (2) its previous symptoms; (3) its previous treatment; (4) the nature of the object with which it was inflicted; (5) the nature and possibly the virulence of the infectious matter conveyed into it.

I. The Duration.-The date of the accident is always taken into account first of all in making an inquiry about any given trauma, for here several important deductions are made. Certain phenomena may be serious at one stage and quite trivial at another. For example, severe lameness following the infliction of a wound may be a trivial matter, while the same degree of lameness on the second, third or fourth day would indicate the existence of serious trouble. Again, a certain amount of œdema at different stages of the process of repair points to entirely different conditions. An encroaching, painful œdema appearing twenty-four hours after a wound is inflicted would be regarded as threatening, while the same amount on the third or fourth day or at the end of a week might be expected as the natural result of any infected wound. The signs of infection that follow soon after the wound is inflicted are gen- 
erally an indication of a higher virulence than those occurring later.

2. The Previous Symptoms.-An inquiry into the symptoms presented prior to the date of examination may often be made to some advantage, although this feature is less important in the diagnosis of wounds than in that of internal diseases. When systemic disturbances accompany the trauma, this part of the history should not be omitted. Previous dejection, previous inappetence, previous pyrexia, previous lameness, previous discharges of such or such character, and previous swellings, may all alter the final conclusions. It is here that one learns the duration of certain phenomena that might have a bearing in both the diagnosis and the prognosis of the condition.

3. The Previous Treatment.-Wounds are so frequently submitted to empirical treatments that not only change their physiognomy but also render them more serious, that it is absolutely necessary to take into account every phase of the treatment'administered. Often aggravated, threatening and even painful swellings are traceable to the previous application of an irritating liniment. These might readily be mistaken for serious infective inflammations if the exact cause were not determined. Again, the treatment might have been of such a character as to have conveyed dangerous infections into the wound. Cob-webs to arrest hæmorrhage, and animal excrements as poultices are two of the many empirical remedies which demand special counter-remedies when the more intelligent wound treatment begins. And finally, caustic substances change wounds into conditions that would be confusing if the cause were not known. Diarrhœa might be caused from septicæmia, or it might be the result of a purgative medicament previously administered.

4. The Nature of the Object Inflicting the Wound.- 
'The object that penetrates into the body requires more than ordinary consideration in every case. The surgeon shoula examine it, if possible, with the aim of determining whether any part of it has remained in the depth of the wound. A broken nail, a piece of glass, a splintered piece of wood, a broken tine, etc., always leads to the examination of the wound for the remaining section. The character of the wound will also vary with the character of the woundingbody. Contusions, contused wound, lacerations and incisions all depend upon the nature of the object producing them.

5. The Nature and the Virulence of the Infection.The history of a wound should, if possible, reveal this feature. The street-nail is known to frequently cause tetanus, and the dog-bite, rabies. Both of these diseases are easily foreseen, and hence call for special treatment to prevent them. Other infections, especially the so-called bloodpoisonings cannot be prognosticated as to their possible seriousness; nevertheless, the wound made with a very dirty object coming from a very dirty environment is always given more attention than the one inflicted with a comparatively clean instrument. The infection is generally more virulent. Furthermore, wounds on debilitated subjects are more liable to become seriously infected than those of vigorous subjects.

II. THE SURGICAL TREATMENT PROPERThe second step of wound treatment is surgical. It includes three distinct manipulations. (I) The dissection, resection, etc.; (2) the hæmostasis; and (3) the sterilization.

I. The dissection, resection, amputation, curettage, avulsion, enucleation, torsion and ablation, whichever becomes necessary, begins the real treatment in most instances. In some instances these may be preceded by arresting the hæmorrhage, for example if the flow of blood is 
profuse. More often the two are simultaneously executed. In the accidental wound this step of wound treatment consists of removing foreign bodies, dissecting away shreds of unviable and dead tissues, dividing tissues to establish drainage and cutting away or curetting badly infected tissues. It is the trimming up or smoothing up of the anfractuous trauma, a part of wound treatment that, more than any other, determines its future behavior. It not only mechanically disinfects the wound, but also takes away available nutriment from the micro-organisms that still remain. In the surgical wound this step is the operation proper. The tumor is ablated; the necrotic cartilage, the necrotic bone, the necrotic ligament, is removed; the nerve is resected; the tendon is divided; the tooth is repulsed; etc.; etc. It is here that one surgeon demonstrates his skill over another. One lays bare a whole hot-bed of disease and removes it completely at one stroke, while the other makes a half-hearted incision and leaves morbid tissues behind to perpetuate the condition, until a second or a third operation becomes necessary.

2. The Hæmostasis.-Before, at the time, or immediately after the preceding step, the flow of blood is controlled. Sometimes it is so profuse that all other steps of wound treatment must be abandoned for the time being. It may be found necessary to immediately apply a compressing bandage, or a compressing pack without further ceremony. Ordinarily, however, the flow is nominal and may be leisurely attended to as the treatment proceeds. In no case must this step be neglected. The serious hæmorrhage is arrested to save life, and the trivial one to favor the healing process. Blood clots confined beneath the sutured skin furnish a favorable field for microbian growth, and otherwise mechanically interfere with the process of repair. The accumulation of blood within a wound should be 
prevented, but its outward flow during the surgical treatment of a wound may, in fact, be beneficial in washing out infectious matter. (See Hæmostasis, page 192).

3. The Sterilization.-The surgical wound made in a clean field requires no form of sterilization because it is not permitted to become infected. All surgical wounds made in an aseptic field are aseptic, and all accidental wounds, no matter how they are made or where they are located, must be regarded as septic and must as a consequence be submitted to the process of sterilization. As far as possible the accidental wound is mechanically disinfected, that is the infected tissues are cut away. The newly inflicted wound is thus disinfected by dissecting its mutilated shreds, and the older one is scraped of its infected granulation by curettage or other mechanical means.

The principles of chemical disinfection consist of rendering the micro-organisms as innocuous as possible by prolonged irrigation with weak antiseptics that will not injure the tissues, and then create a condition unfavorable for their growth by depriving them of their nutrition. Powerful antiseptics, while destructive to the micro-organisms, in one sense, may actually prove harmful by weakening the re. active powers of the living tissues. A half-killed cell falls an easy prey to the wound-bacterium, while on the other hand a bacterium partially blunted with a weak antiseptic is readily englobed by the active leucocytes. It is on this principle that wound disinfection must proceed, whenever prompt cicatrization is essential. On the other hand when the infectious matter threatens life, as in the case of tetanus or rabies, the strongest kind of germicides are demanded. Here the caustic is indicated.

After the wound is mechanically disinfected, and then chemically disinfected with the weak antiseptic, the next step is to rob the remaining bacteria of food necessary for 
their sustenance. Bacteria require moisture, the nitrogenous secretions of the wound, the dead or the half-dead elements, in order to successfully propagate and elaborate their poisonous products. If a wound is kept dry by absorbing the secretions or by allowing them to flow out by gravity drainage, and if the tissues are not seriously injured, the remaining bacteria may well be left to the mercy of the reacting forces of the body,- the leucocytes.

III. THE CLOSURE AND DRAINAGE.-An infected wound must never be entirely closed, while the aseptic (surgical) one, may or may not be closed, according to the amount of products it will secrete. As no wound is ever hermetically sealed against infection it must never be permitted to harbor quantities of food favorable for microbian growth. Wound cavities of animals containing serum will sooner or later become infected, unless the quantity is limited and is rapidly absorbed. The large, lacerated contusions should be left open and their surface made unińhabitable with absorbent antiseptic powders, instead of closing them to the benefit of the microbian flora. Punctured wounds that cannot be drained by gravity are irrigated to wash out the microbes and render them less virulent, and then packed with an absorbent antiseptic fabric as a drainage expedient to take away their food. In any case where free gravity drainage can be provided, combined closure and drainage is the appropriate treatment. Here the wound is closed with sutures except at a pendent part, which is left open to allow the secretions to flow freely over the surface of the body. Incised surgical wounds and well disinfected incised accidental wounds may be completely closed without any provision being made for drainage. But these must always be carefully watched for evidences of sepsis in order to forestall serious complications.

The closure of wounds with sutures is seldom successful 
in animals where the part cannot be perfectly immobilized. On the forehead, on the anterior surface of the face, on the back or on the croup or in certain parts of the legs, sutured wounds generally heal without separating, but in all other parts of the body the sutures often fail to accomplish the intended purpose, on account of the unavoidable friction between the edges caused by the animal's movements. The movements may be trivial, but they are generally sufficient to disturb the first agglutination that must precede the formation of the more stable reuniting tissue. In order to prevent this friction as much as possible it is necessary to apply two separate sets of sutures; a set of immobilizing sutures inserted some distance from each edge, and a set of uniting sutures applied to the edges of the wound. The first set may be of the interrupted button variety, and the second common interrupted stitches. The first creates an immobile area; that is, the whole area moves as a single body, and thus prevents the friction. In other instances (on the legs) the immobilization can be accomplished by bandaging, and in still others (on the body) by the application of a large and thick plastic dressing.

The drainage is accomplished by gravity, by irrigation, and by absorption. Gravity drainage is always provided when possible, and it may also be facilitated by irrigation.

Drainage tubes are very seldom used in veterinary surgery. They are replaced by wadding the opening with cotton or gauze for a few days until a wide orifice is established. Irrigation and absorption are used simultaneously in all wounds that harbor secretion by reason of their physical constitution. Antiseptic solutions are used for the irrigation and antiseptic powders or antiseptic fabrics for the packing. The ideal condition to obtain and to maintain in any wound is dryness. As the secretions diminish from the 
gradual destruction of the micro-organisms, they should be left to dry on the surface into the form of a scab.

IV. PROTECTION AND AFTER-CARE-Wounds of animals must be protected, not only against infection, but also against mechanical violence and motion. The protection against infection is no more important than the others. As mentioned in a preceding paragraph, Lister emphasized too strongly the necessity of post-operative protection. It was then thought that an absolutely impervious wrap was necessary to prevent invasion of poisons into the wound. Strictly air-tight wraps and layer after layer of antiseptic fabrics were placed over the trauma. Now, that the behavior of micro-organisms is better understood, the protective covering is known to be effectual if it will prevent dirt and dust from permeating through it. When a wound is secreting freely the bandage may even be harmful by spreading discharges over the surrounding surface and by preventing their free outward flow. Protecting wraps should be aseptic, or better still antiseptic, and they should neither obstruct drainage nor impede the free circulation of the blood to the part.

For incised, aseptic, surgical wounds a liberal dusting with boric acid covered over, first with absorbent cotton, and then with a muslin bandage, constitutes a perfectly safe dressing. Such a dressing may be left in place for six or seven days without renewal, or it may be renewed every day providing the wound itself is not disturbed. The daily dressing of such wounds should consist simply of renewing the boric acid and the re-adjusting the bandage, which is very liable to become uncomfortable by pressing too taut at different points. This recourse is advisable, because the first signs of sepsis are then noticed. The recommendation to allow such wounds in animals to remain under cover until the union is complete is not an absolutely safe one, because 
sepsis may supervene unobserved. The swelling and pain that accompany sepsis may be trivial, and thus remain unnoticed beneath the bandage. At the end of six to eight days the sutures are removed and the dressing renewed for three to four days longer, when cicatrization will be found to be complete. Wounds of this same character, located where bandages can not be applied, are protected with plastic dressings,--clay or collodion.

If in the course of the treatment signs of sepsis appear, antiseptic baths, more potent dry antiseptics, removal of the sutures, or irrigations may, each or all, become necessary according to the seriousness of the infection. The signs which give warning as to complications of this character are unaccountable swelling, pain out of proportion to the character of the wound, too copious secretion, and systemic disturbances.

For sutured wounds requiring drainage an entirely different course is pursued. Wraps that will gather the secretions, unless changed very frequently, are harmful. They are unnecessary. The sutures themselves may be covered with clay or collodion, but the drainage orifice must be left unobstructed after the second or third day, when the discharge becomes copious. Irrigations carefully injected are admissible, but they must never disturb the formation of the new uniting tissue along the edges of the sutured portion. The plastic dressing on the sutures is repaired as required. If signs of suppuration appear between the sutures, the dressing is substituted with a coating of idoform or some other active antiseptic powder.

The sutures are removed after six to seven days, at which time the stitches will be found to be of no further service. Either there is a safe union, or else a separation of the edges from stitch necrosis. If the skin has united the traumatic cavity will soon fill and terminate the healing 
process, but if the edges part the process of cicatrization will be rather slow in bringing them together again. Open-wound treatment will then be necessary. Irrigations with strong antiseptics followed with the application of dry powders is the most effectual until the granulations have completed their mission of bringing the cavity to the level of the surface. When the cavity is full of granulation tissue, drugs other than antiseptics are indicated for the first time. Astringents should then be applied to control the granulations. If too exuberant, caustics or even the actual cautery are indicated, but such complications are rare when the treatment has been correct throughout.

Open wound treatment is not unlike that recommended in the preceding paragraph. The wound is irrigated with potent antiseptic solutions and dusted with. powders several times daily until the granulations are level with the surrounding skin, at which time the astringent remedies are indicated until the scar is formed.

The healing of a wound depends upon the formation of the granulations, a process which in turn depends upon the vitality of the tissues and never upon the application of any medicaments. The formation of granulation tissue cannot be easily augmented by the application of any chemical substance. Powerful drugs are more apt to retard their growth than to augment it. The surgeon engages himself with strenuous efforts to kill the micro-organisms, to wash them out and to deprive them of their nutrition, while the vital forces repair the breach by first forming the succulent granulations and then transforming them into scar tissue. During the transformation (sclerogenesis) their growth must be arrested in order to limit the size of the scar.

The protection of wounds against mechanical violence is a very important part of wound treatment in animals. By intentionally or accidentally rubbing the wound against 
the side of the stall, by lying upon it, by flexing a wounded limb, or by moving about, the large animals always more or less mechanically disturb their traumas. In the same manner they accumulate dirt upon them. The protection against such accidents is never perfect. Animals cannot be placed in permanent decumbency, and their wounded arms cannot be placed in slings. The best expedient here is to keep the horse or ox in the standing position. The horse may be tied short, tied on the pillar reins or even placed in slings. In the standing position a wounded limb may be immobilized with bandages, wounds of the body can be kept from coming into contact with the stall, and the floor, and wounds of the head can be prevented from touching the manger. In the small animals wounds on the body or limbs can be more perfectly protected by wraps which encircle the body or which immobilize a limb.

Wounds in the course of healing are less liable to become infected if left alone than when their care is left to the hostler, muleteer, shepherd or cow man. Wound treatment requires the adoption of special manipulation that the ignorant layman cannot comprehend and will not enforce. The stable syringe in the hands of the stable man is sure to convey infection. Allowing the hostler to wash a wound, to irrigate it or to dress it in any way, is an error in veterinary wound treatment.

\section{PRACTICAL ASEPSIS.}

The term "practical asepsis" is appropriate in veterinary surgery because of the existence of certain inimical conditions which cannot be ignored. Everything around a veterinary surgical operation is abominably filthy,--germ ridden. The veterinary surgeon must actually "wade through germs" in his surgical work, and the magnitude of the precautions necessary to effectually cope with the situa- 
tion is often out of proportion to the importance of the operation. The relatively small economic importance of each surgical procedure necessitates the adoption of a practical technique that will create the best possible conditions under the circumstances. To create an aseptic condition in a badly septic environment is often possible, but the success so universally obtained in human surgery cannot be expected in veterinary surgery.

Asepsis depends not only upon obeying the standard details, but also upon the general cleanliness of the surroundings. Some years ago a certain eccentric human surgeon, probably in a spirit of contrariety, refuted the germ-theory of wound diseases and still had success that compared very favorably with those of his colleagues. An investigation proved that he was scrupulously clean in his: work. His operating room was immaculate. He washed everything thoroughly, - his hands, his patient and his instruments. Soap and water were his sole weapons. When everything is dirty around a surgical operation or around a surgical convalescent, micro-organisms will often creep into the wound in spite of all the precautions.

Operations performed in the operating room or out-ofdoors, on patients afterwards placed in reasonably clean surroundings yield the highest percentage of so-called aseptic results. Country operations are more successful in this connection than those performed in crowded cities, although abominable conditions may also exist in certain country stables. On the whole, howwever, pyogenic microorganisms are less abundant in country districts. Where pathogenic germs abound surgical asepsis is less certain; where they are partially eliminated by cleanliness, normal success may be expected, even in veterinary surgery.

Furthermore, the skin of the hairy domestic mammals may badly defeat the most painstaken technique. Exper- 
ience has shown, time after time, that operations consisting of the simplest incisions, performed in strict obedience to all of the standard regulations, will yield pus in about twenty-five per cent of the cases. Often it amounts to nothing more than a trivial stitch suppuration, but there it is, nevertheless. If four interrupted sutures are carefully inserted into the skin of the horse at different points of the body under the strictest aseptic precautions, it is quite certain that at least one of them will be found purulent on the fourth day. The infection may be limited to one point of entrance of the needle, it may be found at both, or finally in other cases the entire traumatic cavity may be purulent. These experiments prove the existence of pyogenic micro-organisms in the skin, that are capable of causing a reaction even in a simple incised wound where there are no mutilated elements or exudates to create a favorable medium for their growth. The behavior of these same micro-organisms in an accidental wound where the injured elements, blood and serum furnish abundant nutrition, can be imagined.

Lastly, the constant state of motion to which the wounds of animals are constantly subjected favors the spreading of infections which would have remained local in a perfectly immobilized trauma. Thus a trivial stitch suppuration that might subside and desiccate in a quiet wound, will spread into the surroundings from the movements of the parts. It is not a mere speculation to assert that a very trivial infection may be transformed into a very grave and even fatal disease from no other cause than the motion to which the wound is constantly subjected.

The best results are obtained in veterinary operations, as in human surgery, by the sterilization of everything more or less intimately connected with the procedure; but as it is stated above, this is not always practical. 


\section{RECOMMENDATIONS FOR AN ASEPTICAL OPERATION.}

Ist. PROCURE THESE SPECIAL SUPPLIES FOR THE OPERATING ROOM.-It is needless to state that the veterinarian cannot afford to use the expensive supplies that adorn the human operating room. In the treatment of a wound for several weeks, the cost of the surgical dressings alone might easily exceed the value of the patient. The following articles are sensible, practical, economical as well as effectual in bringing the best possible results.

(a) Sterilized bandages are made directly from a bolt of unbleached muslin, by tearing them into strips five yards long and four inches wide, and then keeping them stored in a large, salt-mouth, glass-stoppered bottle, filled with mercuric chloride solution I-IOOO. A bottle with a capacity of two gallons is quite appropriate.

(b) Sterilized wound packing is made from common cheese cloth, torn into strips, rolled into loose bandages and stored in the same manner as the muslin bandages.

(c) Sterilized sponges for bailing and wiping the blood from wounds are obtained by keeping a liberal supply of them immersed in a salt-mouth bottle containing either mercuric chloride I-I000 or alcohol.

(d) Sterilized sutures are obtained by keeping a liberal supply of saddler's linen thread or silk thread immersed in a jar of alcohol. The thread is unraveled through a perforation in the lid. In addition, sterilized silk and sterilized catgut purchased in hermetically sealed tubes may be kept on hand for special operations.

(e) Sterilized instruments are always available in a few minutes by immersing them in a 95 per cent solution of carbolic acid, a quart jar of which is included among the operating room supplies. This jar is carpeted at the bottom 
with cork, rubber, felt or cotton to prevent the knife points from blunting against the glass.

(f) Absorbent cotton in the original package.

2nd. Use No Less Than Five White, Clean, Porcelain Pans For Each Operation. A veterinary surgical operation cannot be a clean one if only one or two containers are used for the antiseptics, because different solutions are required for different purposes, and because the use of a certain solution for one purpose will render it harmful for another.

(a) Pan No. I contains soap, hot water, a sponge or pledgets of cotton, scissors, razor and clippers. An ordinary metal pail would answer for this purpose.. This pan is used to carry out the first step of disinfecting the operating field, and since its contents become soiled with dirt and hairs it is no longer safe to use for the subsequent steps of the operation. When the hair is shaved and the field washed it may be emptied and used thereafter for the slopjar, for dissected tissues, soiled sponges, pledgets of soiled cotton, etc.

(b) Pan No. 2 contains mercuric chloride I-500, or even 1-200, and several sponges or a number of pledgets of cotton. It is used to wash the operating field and to rinse the hands, which of course were previously washed. The pledgets of cotton or sponges are taken from the solution one after another to wash the field, but are never carried back into it. When soiled they are cast into pan No. I,-the slop-jar,and not rinsed out in this pan, because carrying a sponge back and forth from pan to patient will pollute the solution. When the field is disinfected it is set aside for rinsing the surgeon's hands, which often become soiled during the operation.

(c) Pan No. 3 is the assistant's bailing pan. It contains sterilized water of a weak antiseptic solution,- - three per cent carbolic and I-2000 mercuric chloride,--several 
sponges or pledgets of cotton. The sponges or cotton wads are used by the assistant to bail or wipe the blood from the wound as the dissection proceeds. When soiled or overfilled with blood they are cast into the slop-jar and are never carried back and forth from wound to pan, because if rinsed in the solution it would soon be bloody, probably septic and always unfit for the intended purpose.

(d) Pan No. 4 is the instrument tray. It contains a three to five per cent solution of carbolic acid and all of the instruments required for the operation. A clean instrument table may be used as a substitute for this pan, but the latter is preferable because its contents prevent the instruments from becoming too badly infected from septic tissues and from the air that has not been entirely cleared of its dust.

(e) Pan No. 5 is the needle pan. It contains a three to five per cent solution of carbolic acid, as many threaded needles as there will be stitches required to close the wound, a needle holder, and a dissecting forcep. The use of this pan prevents the sutures from being contaminated during the operation, and besides if they are kept among the instruments the threads readily become tangled.

3rd. Avoid the Use of the Bare Hands as Much as Possible. The fact that the veterinarian's hands are always nore or less infested with pyogenic microbes, together with the impossibility of keeping them from coming into contact with dirty objects as the operation proceeds, renders this precaution specially important.

In sewing wounds the needles are picked up with the dissecting forceps and placed in the jaws of the needleholder. The lip of the wound is lifted with the forceps and the needle inserted with the needle holder. This manipulation eliminates the direct use of the fingers, which are never safe enough to handle sutures. All of the other 
aseptic precautions are useless if the wound is "pawed over" with the hands.

4th. Avoid Operating in a Dusty Environment, especially in the dust of a stable or veterinary hospital.

5th. Secure the Patient so that the seat of operation is under perfect control. Dust, flying hairs, flying litter, and the dirty hands are certain to contaminate the wound if the patient is permitted to thrash about. General anæsthesia is commendable.

6th. Use Only Sterilized Sutures, and thread the needles before the operation begins and thread them with just enough thread for a single stitch. Long threads are dragged over dirty parts of the patient and operating table. Insert them only with the needle holder, and use absorbable thread for buried sutures.

7th. Use Only Sterilized Dressings.

8th. Provide Effectual and Adequate Drainage. A poorly drained wound on an animal will seldom remain nonpurulent until it is healed. Remember that dryness is the ideal condition to prevent microbian growth.

9th. Avoid Unnecessary After-Care. Leave a welldrained wound much to itself, rather than submit it to the mercies of the unskilled attendant. 


\section{PART II}

\section{PATHOLOGIE CHIRURGICALE GENERALE}

$B Y$

P. LeBLANC, C. CADEAC and C. CAROUGEAU

TRANSLATED, EDITED AND ENLARGED 



\section{CHAPTER XII.}

\section{TRAUMATISMS.}

The word "traumatism" is applied to local affections produced in an abrupt manner by physical, chemical or mechanical agents.

ETIOLOGY.-The physical agents, heat and cold, cause special lesions, designated as "burns" and "chills," and more rarely the chemical agents, acids, and caustics, which produce injuries to the integument. It is the mechanical agent that occasions the greatest number of traumatisms. The use of domestic animals as motors exposes them to various mechanical injuries.

The bodies which inflict wounds are sticks, knives, hooks, nails, whips, goads, pitchforks, and now and then the horns of ruminants and the iron shoes of a horse. And the surgeon himself by his justifiable intervention produces traumatisms. The ablation of a tumor, operations with the hand, castration, or cauterization with the actual cautery, constitute so many designed and necessary traumata, for the purpose of gaining an economic result or fixed therapeutic object.

PATHOLOGICAL ANATOMY.-The nature of injuries to the tissues is essentially variable. The injured point,the traumatic center, - is designated as an open or exposed wound when the skin is broken, and a closed or subcutaneous wound when the skin remains intact. When the body that inflicts the wound penetrates deeply and opens a cavity, the wound is called a cavernous wound. Every wound, from the standpoint of anatomical alteration, generally presents zones that are easily recognized:-The central zone is the 
region of a wound in which anatomical elements are destroyed and are no longer living tissues. The middle zone is formed by tissues upon which the action of the trauma has not been sufficiently severe to destroy them. The cellular elements are only "benumbed," in which state they become easy prey for micro-organisms.

In the peripheral zone the tissues have preserved their integrity but the general physiognomy of the region is changed by vascular modifications due to the shock.

Whatever may be its form the traumatic center presents the contents, and a wall or boundary. The contents are composed of a cellular waste, extravasated blood and lymph, with which is sometimes mingled a substance from a neighboring cavity. The wall is linear, sinuous or anfractuous. In wounds made by cutting instruments it may be regular, the anatomical elements having only been separated, but in those made by contusions, the tissues constituting the wall, are bruised and soaked with blood and serosity.

SYMPTOMS.-The symptoms of traumatisms are both local and general. The local symptoms manifest themselves at the injured point, while the general are felt over the entire system. Verneuil has also distinguished remote symptoms which appear in organs more or less remote from the wound, that have not been injured by the initial violence.

The local symptoms are separation of the wall of the traumatic center, pain, and the hæmorrhage. The separation is more or less evident at all times, but is especially well marked in wounds made with sharp instruments, and in regions where the skin is tight, e. g. the abdomen and limbs. It is, however, non-existent in wounds from contusion, in which instance the tramatic center may be sunken and forced into neighboring regions. The pain varies in intensity with the character of the traumatized center. It is not so acute in clean-cut wounds, but is very excessive when the 
tissues are bruised. It does not vary in proportion to the richness of the nerve supply, but according to the nature of the trauma. Regions rich in nerve endings, other things being equal, are no more painful when wounded than those poorly supplied with nerves.

The hæmorrhage varies with the vascularity of the wouncled region, the extent of the wound and the nature of the body inflicting the injury. [Wounds inflicted with sharp instruments bleed proportionately more than those which are lacerated or contused. L. A. M.] In certain animals hæmorrhage may become alarming, even after insignificant lesions. It is not rare, for example, for a donkey to bleed abundantly from small wounds.

The general symptoms are not intense, as a rule, so long as the wound remains free from microbian invasion: The general reaction, aside from microbian infection, is confined to nervous disturbances which vary in intensity with the temperament of the animal. Formerly; it was supposed that the fever was the result of shock and that every trauma was accompanied with a more or less manifest febrile state. Fever was even considered necessary and useful to the patient. These ideas have not been entertained since the discovery of wound nicro-organisms and their secretions. The remote symptoms are rare in domestic animals, but have nevertheless been noticel. A kick on the abdomen of a horse has been found to cause polyuria, and nephritis has been known to develop from violence to the dorso-lumbar region. Emboli proceeding from traumatic foci, provoked by inflamnation of the vessels traversing the region, should also be included among the remote symptoms.

The local symptoms previously mentioned are, so to speak, immediate symptoms. In time the tranmatic focus changes its physiognomy and the local secondary symptoms begin to appear. These secondary signs are the work of 
reparation. The tissues endeavor to repair the breach made at their expense, and the lesion proceeds towards cicatrization, which may be accomplished by:-( I) Immediate union, (2) First intention, (3) Second intention, (4) Retarded cicatrization.

Immediate union takes place in a few hours. It is rare and is possible only in small, aseptic, incised wounds that have bled but little. It is recognized by reunion of the walls of the trauma. The serum coming from the wound is interposed between the lips of the lesion and agglutinates them. The repair is, however, not complete. If the phenomena of repair are followed closely from this point it will be found that the clot between the edges of the wound now constitutes a framework or support for the connective tissue elements, which hasten to proliferate (first intention). These elements shoot forth extensions which cross each other, and ere long form a rather substantial network. In the days which follow, the connecting fibres make their appearance and the regeneration is complete. The white and red blood cells, mixed in the fibrinous coagulum, become the prey of the connective tissue elements and are starved in the activity of the process.

Secondary or mediate cicatrization, or healing with suppuration, is a much longer process, and is the usual mode by which extensive wounds heal when the tissues are bruised. It is also observed in simple wounds that are soiled, infected, or improperly treated. Here the healing process is more complex. The tissue, having to reconstruct or reunite its injiry, must eliminate its disaggregated or dead elements. Cure is effected by self-amputation. A line of demarcation is established between the elements capable of living and those which are irreparably injured. As in primary cicatrization the embryonic cells are multiplied as the fine capillaries which bring them the elements needed for their 
activity are formed. The proud-flesh-(granulation tissue)appears in the wound, forming a rose-colored mass which bleeds at the slightest contact. This is the germinative tissue of Ziegler. From this moment the integrity of the tissue is accomplished. The new products form a barrier through which the microbian agents cannot traverse. In a comparatively short time the granulations fill up the cavity of the wound, its essential role being to bring the adjacent structures to a level. At this moment there appears at the periphery a whitish border of epidermic cells which gradually covers the granulated surface.

When the breach has been completely filled up with the granulating tissue, sclerogenesis begins. The fibroblasts become active and construct connective and elastic fibrillary frameworks. This evolution occurs rapidly; the new cells very speedily acquire adult life with "all its burdens and fatigues." Promptly they become flattened, raveled, and transformed into ordinary connective tissue cells, which are not slow in exhibiting all the signs of a premature senility. This cellular retrogression is accompanied by a. resolution of new tissue. The capillaries flatten, atrophy, contract, and disappear, leaving behind only traces of some cells.

The cicatrix, thus completed, then changes its character. Rose-colored at first, it later becomes white. It shrinks more and more, becomes depressed, and on account of the absence of hair follicles on its surface it leaves behind an indelible blemish (retarded cicatrization).

"Thus the scar tissue is produced. It is a tissue as dried up as its generator-the granulating tissue-was succulent; anæmic as much as the other was sanguineous; dense and firm, thanks to its fibrous bundles, as much as its predecessor was loose and incapable of much resistance; and poor in cells that are flat and even atrophied, as much as the granulating tissue was living and richly cellular. In a word, the young, 
vascular, connective tissue has been replaced by a tissue of sclerosis." (Letulle)

PROGNOSIS.-The seriousness of a wound depends upon different factors. When the wounded tissue is a healthy one and that of a sound animal, the cicatrization is generally rapid and the termination is simple. Matters are, however, different when the injured animal is diseased or placed in bad hygienic conditions, or when the injured tissues were previously impaired. The prognosis is then dependent on a number of factors, viz.: the animal wounded, the wound, the environment and the part wounded.

The environment in which a wounded patient is placed is significant so far as the progress of the disease is concerned. Formerly, when rules of hygiene were little known, animals kept in infected places frequently died. Complications were the inevitable result. The animals wounded or operated upon in the presence of spectators in the hospitals of Alfort, from the time of Renault, almost invariably succumbed to purtulent infection. With improvement in the surroundings this sequel disappeared. These complications were then attributed to changes in the surrounding atmosphere, but as the researches of chemists did not furnish the necessary explanation, search elsewhere became necessary. In 1860 the great controversy about spontaneous generation occurred. After impassionate conflicts the victory remained with Pasteur, who cleared up points that had heretofore remained obscure. It is easy to understand the successes achieved by Lister and J. Guerin who, placing the wounds out of the reach of air, thus preserved them from any infection from without. It is, however, established today that, since the beginning, too much importance has been attached to dust and aerial germs. Nearly always the complications are traceable to the operator or his instruments. It is by their agency that micro-organisms are carried to the wouncls. 
Kept for several weeks without careful disinfection. instruments and dressings, become genuine vehicles. They become re-infected from each subject operated upon, thus becoming conveyors of exceedingly virulent germs which show an increased virulence with each intervention. Today it has been shown that operations can be performed with impunity in a septic environment. The contrary condition is, of course, preferable, but "in emergency, let the surgeon learn to create for himself an aseptic operating field in a septic environment." (Reclus)

The gravity of the disease is equally subordinate to the nature, seat and extent of the wound. Other things being equal, wounds which are deep, anfractuous, or the result of crushing, are infinitely more serious than those made with sharp instruments. Then, again, wounds affecting important organs or ducts may be exceptionally serious. Wounds of the urethra are often fatal; those of Steno's duct may cause death from loss of saliva, and lacerations of the udder may provoke a lacteal fistula of prolonged duration.

The character of the instrument inflicting the wound needs aiso to be taken into account. If it is aseptic or only the conveyor of benign germs the wound will behave as an aseptic trauma. Formidable complications may, on the contrary, supervene if the instrument inflicting the wound, having been previously soiled, deposits pyogenic or other septic organisms into ${ }^{\circ}$ it.

The physiognomy of a wound may also be modified in a very perceptible manner hy the state of resistance or enfeeblement offered by the individual. The condition of the wounded animal has a manjfest influence on the evolution of the lesion of which it is the bearer. This important point which was ail but known fifty years ago, has been elucidated only by scientists of the French school. Not only does the state of the wounded animal direct the trauma along a 
special course of action, but the trauma may in turn reveal the general condition of the injured subject. A long time has elapsed since Mae Schiller insisted on the importance of traumatism in tuberculosis localization. Although this action may today be challenged, it seems that good reasons are on the side of the German experimenters. Has not Peuch succeeded in disclosing the presence of the bacillus of Koch in the pus collected in the passage of a seton in a tuberculous cow? The introduction of pneumococcus into the trachea of sheep leaves the animal unaffected so long as the trachea has not been wounded. The bacillus of symptomatic anthrax, when introduced into the blood, produces no injurious effect, but let any part of the bocly be wounded and an anthracoid tumor will develop.

Without insisting on these examples, which belong to the domain of general pathology, there can be no doubt that every injured tissue, whatever may be the nature of the initial lesion, is placed at a manifest disadvantage from the point of view of protection and repair. Disease imprints on the tissue an altered vital action and modifies the energies of the cellular interchanges. The organic elements, rendered anæmic, protect themselves badly, and become impaired by morbid absorption for want of sufficient destruction of poisonous substances, and the stagnation of the products of disassimilation. The influence of diabetes on the evolution of wounds is common knowledge, and the effect of albuminuria and of cancerous diatheses, although less known among domestic animals than among human beings, nevertheless exists. Cadiot and Almy report a case of a dog affected with sarcoma at the head of the femur, which, when irritated by cauterization, soon became general.

The age of the wounded animal can no longer be ignored in estimating the gravity of traumatisms. The young resist them better than old animals which have impaired nutrition, 
fatigued tissues, enfeebled important organs. In the pregnant ferrale a wound may become the origin of complications. Occasionally it provokes abortion, and if this is escaped. the wonnd may prove serious by compelling a permanent state of decubitus; without taking into account the fact that cicatrization is hindered in consequence of the weakened state of the organism during the development of the fœtus.

CLASSIFICATION.-Trammatisns are divided into two great groups:--Wounds and Contusions. The latter liffer from the fnrmer in that the wound remains protected by the common integument. Although every classification of wounds is open to criticism, it is nevertheless indispensable to classify them in order to make their description more clear. The simplest classification is that of our predecessors, and is as follows:

( I) Wounds by sharp instruments. (Incised wounds)

(2) Wounds by penetrating instruments. (Punctured wounds)

(3) Wounds by tearing. (Lacerated wounds)

(4) Wounds by teeth-bites.

(5) Poisonous and venomous wounds.

(6) Contusions and contused wounds.

\section{WOUNDS BY SHARP INSTRUMENTS.}

(Incised wounds)

Incised wounds are made with a sharp blade,- - knife or scythe,-which cuts the integument by sliding. on the surface. They are called superficial when only the skin is divicled, and deep when the subcuten and adjacent tissues are severed. These wounds are generally symmetrical. Measured with the long axis of the body or the region affected, they are distinguished as horizontal, vertical, or oblique. Most frequently they present two sharp angles or commissures, a long axis and a short axis. Incised wounds 
are the most numerous. "They form the basis of every surgical operation."

SYMPTOMS.-Incised wounds present three primitive signs: pain, separation of the lips of the wound and the hæmorrhage. The pain, as is manifested by violent efforts of self-defense. It varies in intensity with the richness of the nerve supp.y of the region concerned. It is always accentuated in the skin and musc?es, and is less acute when the incision is made with a sharp instrument than with a notched or dull blade that will saw or tear the anatomical elements. The separation of the lips of the wound is implediate. but varies in extent according to the tension of the part incised. It is very marked on the skin covering the abdomen, in arteries, and in muscles having prononnced retractility. Separation is generally more accentuated at the surface than in the deep layers, which circumstance favors the outward flow of morbicl products cleposited on or formed in the wound.

The hæmorrhage is very abunclant in regions where superficial vessels are numerous or when the instrument has severed and artery. The skin bleeds but little, while muscle, on the contrary, bleeds profusely owing to the development of its blood-vessels.

As asepsis is rarely perfect in clomestic animals, immecliate union, even in the small animals, is quite exceptional. Paul Bert's experiments of reuniting the amputated tail of a rat, and of repairing the severed spur of a cock have not materially simplified the question from a veterinary standpoint.

TREATMENT.-When the accident is recent, the wound clean and aseptic, immedinte uninn should be attempted. The suturing thread should be absorbable, and as in the case of all instruments used, it should be aseptic. The interrupted stitch should be preferred, as it assures a 
more perfect coaptation of the edges. The sutures draw together the separated elements, which have only been benumbed, and play the part that fibrin does in wounds where the separation is insignificant. If the wound is deep and different kinds of tissues are divided, the sutures must be made to join similar tissue as muscle to muscle, etc. When the wound is closed it should be coated with iodoform collodion and covered with an aseptic dressing. Cicatrization will then take place very rapidly. If the wound is deep, anfractuous and soiled, abandon the idea of suture, even after disinfection. Leave it exposed to the air; irrigate it copiously, so as to cleanse even the underlying parts, and then sprinkle with an absorbent antiseptic substance. It is not necessary in any case to cover over these deep wounds. In sheltering them from the air, the swarming of certain microorganisms, notably the septic vibrio, is facilitated and the outward flow of microbian secretions is prevented. To use an old term "We have shut up the wolf in the sheepfold." The bacterial poisons absorb, kindle fever, impair the cellular elements and thus form an obstacle to reparation.

\section{WOUNDS BY PENETRATING INSTRUMENTS.}

\section{(Punctured Wounds)}

Wounds by penetrating instruments-punctured wounds - may be described as solutions of continuity made by fine instruments-pointed and regular, such as a needle, a trocar, a bayonet, a nail, a pitchfork, the goad or the trident of an ox-driver; or by irregular objects, such as thorns, fragments of wood, and pieces of glass. In the former the penetrating body does not generally remain in the wound, while in the latter the irregularity of its surface is an obstacle to its exit. It may remain hidden in its entirety in the tissues, or may be broken to pieces in the interior of the wound.

SYMPTOMS.-The pain varies with the volume and 
regularity of the penetrating body, with the sensitiveness of the wounded animal and with the region affected. Generally it is so slight as to totally escape notice. The hæmorrhage is also insignificant. It is often rendered impossible by the smallness of the cutaneous orifice, which is concealed by the hair. When the penetrating body is aseptic or clean, and is not left in the wound, there will be no appreciable sign of the accident, but if the puncture is infected the physiognomy of the lesion will be quite different, and disorders of exceptional seriousness will supervene, if important organs are affected. Bones, tendons, aponeuroses, and serous membranes punctured, may be injured to such an extent as to endanger the subject's life. A street nail penetration may prove grave by terminating in suppurative arthritis or tetanus. The condition is less alarming when the infected body is implanted in the connective layers, subcutem or muscles. In this event, if gaseous gangrene does not result, an infectious nodule is created and an abscess formed. The region becomes swollen, hot, painful, edematous and gradually fluctuating. The pus evacuating brings with it the remains of the foreign body left in the tissue, and the morbid process comes to an end. It is now and then necessary to nuake an incision to facilitate the evacuation of the foreign bociy.

Melany reports the case of a horse in whose shoulder a rivet head was lodged as the consequence of a boiler explosion. At first the accident passed unnoticed, but subsequently developed into a fistula, which, when opened, was promptly cured.

TREATMENT.-In simple cases, punctures are of little consequence. The anatomical elements are more separated than destroyed, and the wound has the physiognomy of a small cut, and if not infected it is repaired as such. When an aseptic penetrating instrument has opened a serous membrane, the opening must be quickly obturated. To probe 
the depths of any puncture of this character is not necessary. In fact, probing, which was once general, is now condemned by every surgeon. If a foreign body is aseptic it becomes encysted, if infected it is eliminated by suppuration.

When an abscess formation becomes evident, it is not often that the process can be aborted by antiphlogistics, cold baths or continuous irrigations. In most cases this result can only be attained by an incision through the traumatic focus. In punctures of the foot, baths of copper sulphate, preceded by jarring of the sole, are often sufficient to combat the complications. The infection is generally light, as the micro-organisms are brushed from the nail on its passage through the hoof.

\section{WOUNDS BY TEARING.}

\section{(Lacerated Wounds)}

Wounds by tearing-lacerated wounds-are solutions of continuity caused by a violent traction which is either direct or combined with torsion.

ETIOLOGY.-Wounds by traction are rare among domestic animals. In veterinary pathology, the pulling off of the horse's hoof, or the tearing off of the ox's horn. claws or hoofs, constitute the best examples of these injuries. Furlanetto reports four cases of the wrenching: off of the hind hoof and in each case the accident was caused by a cart wheel. In the ox he reports cases of the wrenching off of the dew-claws of the fore fetlocks. Such accidents are observed on animals employed about railway stations, in consequence of having the horseshoe caught about the rails. The violent movements made by the animals to extriçate the foot wrench off the hoof. Lacerations of the nostril, eyelids and lips are encountered occasionally. Ray reports a horse whose inferior maxillary was completely torn away. "Bites of animals may also result in the instantaneous laceration of an ear, eyelid, scrap of skin or flesh." 
Tearing is sometimes used as an expedient for exeresis. It is also a common recourse in obstetrics when embryotomy is necessary. Pedunculated cutaneous tumors of small dimensions are not infrequently torn off by the surgeon. Certain forms of warts in the ox are removed in this manner.

SYMPTOMS.-The solution of continuity is irregular, and the hemorrhage is slight, in consequence of the vascular obliteration produced by stretching of the vessels. The pain does not seem as excessive as that produced by similar injuries in the human being. The wrenching occurs most frequently at the articulations. The skin is found mangled and drawn over the wound, in which are found fragments of muscle, tendons, or ligaments and sometimes portions of bone, and extremities of vessels and nerves.

It is evident that during the traction, the skin offers the greatest resistance, for when it has once yielded the other structures separate with much more facility. A knowledge of the resistance and peculiar elasticity of the skin is important in obstetrics. The removal of a limb from the fotis must always be preceded by incising the skin and cutting it, as far as possible, from the subjacent tissues.

TREATMENT.-Death may result from these grave traumatisms. When the accident is irremediable. it is advisable to carefully trim the wound, to disinfect it and to cover it with an absorbent antiseptic powder. The tissues injured beyond the capabilities of regeneration will be eliminated by suppuration, making the anfractuous wound assume a more regular physiognomy.

\section{WOUNDS BY TEETH-BITES.}

Bites are solutions of continuity which combine together the features of punctures, lacerations and contusions. They vary with the species of the animal which inflicts them.'

ETIOLOGY.-Bites are very frequently observed in 
veterinary practice. Horses that bite are not rare. They attack their fellow animal in the neck and withers. Except when afficted with rabies or psendo-rabies ruminants do not attack with the teeth. The bites of shepherd dogs having hard teetl, wound sheep, oxen and even horses. Dogs bite horses in the scrotum, nose and ears. Bites of the wild carnivora and wounds of birds are only in rare instances inflicted upon domestic animals.

SYMPTOMS.-The physiognomy of the bite depends upon the biting animal. The horse crushes the tissues. His bite is rather characteristic-the incisors seize the tissues and crush them without chewing. There is no laceration except when the bitten animal makes a vigorous effort to escape. The teeth leave an elliptical, cutaneous lesion that becomes tumefied, and defined by a furrow of more or less depth where the tissues are crushed. The bite of a dog is very different; it combines the punctured with the lacerated wound. When the dog bites he imberls the tẹeth into the tissues and drags them toward him. by abrupt side-movements of the head. The nature of the lesion is sometimes difficult to determine.

The ox bites only in very rare instances, and in consequence of the absence of unner incisors and the mobility of the inferior ones his bite lacks the gravity of that of the preceding animals.

When there has been no laceration the region bitten, if observed a short time after, is tumefied, hot and very painful. The skin may be mortified and the connective tissue may be the seat of abundant hæmorrhage.

Bites are always serious. When the teeth have not inoculated a specific pathogenic micro-organism as in rabies they frequently leave other virulent germs in the tissues. The inoculated focus is bruised, composed of traumatized elements, is soaked with serum and blood, and has become an excel- 
lent environment for the growth of the micro-organisms it may contain. Before the application of antiseptic methods suppuration and gangrene were not exceptional terminations. When important organs are injured the prognosis is still more grave. Letulle almost lost a horse on which a bite had opened the carotid artery and the jugular vein. Cavalin and Bournay report the loss of a horse from a bite in the trachea. Ismert reports an inflammation of the withers following the bite of a horse. He found it was necessary to kill a magnificent Barbary steed the withers of which had been so badly mutilated that the apophyses of three vertebra were denuded; two were literally ground to pieces, and bony fragments were found in the bedding.

\section{POISONED WOUNDS.}

Poisoned wounds generally have the same physiognomy as punctures, as it is nearly always after penetration with a sharp point that the poison is introduced into the tissues. The nature of the active substance changes the appearance of the local lesion and sometimes stamps a speçial cast on the general symptoms. The substance which soils the wound may be a mineral poison, a venomous element, or a virus.

Whatever may be the active substances, they provoke various modifications at the point of inoculation. They may kill the anatomical elements which they contact, they may make an impression on the neighboring elements, or act on the entire system, by penetrating into the vessels which supply the wounded tissue. They are rarely ever totally absorbed. A certain portion of the poison remains at the point of inoculation for a considerable period after the wound is inflicted. This must not be overlooked, as it indicates the necessity of cleansing the poisoned wound even though it is not one of recent date. 


\section{VIRULENT WOUNDS.}

Virulent wounds do not cliffer from poisonous wounds, except in the fact that in the latter the poison deposited is not renewed. Virulent wounds, on the contrary, are genuine laboratories where active substances are manufactured, and act upon the system after entering the blood, which they corrupt. In the poisoned wound only the soluble substances are absorbed and drawn into general circulation, while in the viru?ent wound, both micro-organisms and their poisons pass through the absorbing surface. The micro-organisms colonize at distant points, create foci of secondary infection, and by cultivating themselves in the wound inject the system with fatal poisons. The domain of virulent afflictions, formerly restricted, is now considerably enlarged, and the study of microbian complications of wounds is now relegated to the description of the diseases themselves.

The greater portion of these afflictions-tetanus, septicæmia, gaseous gangrene, pyæmia-are considered in succeeding chapters.

Envenomed wounds are characterized by the inoculation of a poison secreted by a venomous animal or insect. The poison is known as "venom."

Serpents are the most formidable of venomous animals. In France the viper is not rare. In the year $1896,16,000$ of them were killed in the department of Doubs. It bites horses under the belly, on the limbs, and on the neck and shoulders; cows on the udder and dogs in the throat, the lips and the paws. Being abundant in tropical countries, venomous serpents frequently cause fatal accidents. They inoculate the product of the venomous glands, which are analogous to the saliva of mammals, into the animal bitten by means of their fangs. The most deadly are the naja, the cerastes, the bothryps, the bomgarus ceruleus and the cobra. 
In Firench country districts insects may also cause grave injuries. Though not possessing a venom of such pronounced activity as that of serpents, they may, by their numbers, cause formidable cutaneous lesions which may prove fatal. They operate by their sting, with which they penetrate the tissues. Bees, wasps and hornets have a hollow sting, out of which flows the product of a contractile vesicle situated at its base. This product is a very active, deleterious liquid that produces an intense burning. sensation. Gadflies are equally dreadful insects, and certain other flies are known to produce fatalities by their sting. Robellet reports serious injuries to a cow from flies that were engaged in despoiling the carcasses of putrefying moles. Gnats should also be classed in the category of dangerous insects. In I863 Tisserand was called upon to report upon the ravages among cattle made by the Simulium maculatum. This insect, very abundant in the neighborhood of Lyons at that period, atacked solipeds and bovines. The small ruminants were less affected. The animals were stung where the skin is thin-the udder, the belly, etc. Independently of serpents and insects, there is observed in France, notably among dogs, injuries due to the sting of the scorpion. The black scorpion of the Indies, a giant species, is so very dangerous that its bite is always invariably fatal to human beings, and the domestic animals seldom survive its sting. In the Danubian countries, especially in Hungary, Servia and Roumania, the gnat of Kolumbaty made veritable ravages. It was looked upon as a scourge. In spring, about the end of April and in May, it descends into the valleys in such great numbers as to form thick clouds, which rush upon the herd of buffalo kept upon the banks of the Danube. It attacks the buffalo, the ox and the horse, which it stings in the nostrils and the insides of the thigh with such fury that every animal stung is doomed. Cases of recovery are exception- 
al. During the year 1892 , in the district of Vidin alone, there died from the sting of gnats:

Large ruminants...........534

Buffaloes ...............220

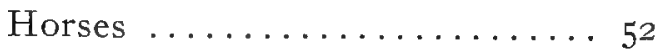

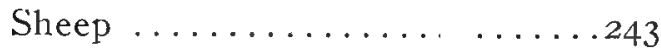

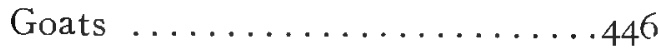

Hogs ..................

These numbers represent a mortality of from I3 to I4 per cent of all the ruminants of the district. With the exception of scab-rot, no contagious disease had previously produced such ravages.

SYMPTOMS.-The aspic viper is more dangerous than the pelias. The former causes very grave injuries, while the bite of the latter is more superficial and its venom less active. The prick does not appear to be very painful, but is followed immediately by a hæmorrhagic tumefaction that rapidly extends over the entire surroundings. At the same time general symptoms,-stupor, profuse perspiration, colics and diarrhoa,-are observed. The gravity of these bites is real as it is sometimes impossible, in spite of every attention, to save the injured animal. Kretschmar saw a bitch die after having been bitten on the lip by a viper; Chanel, a brood mare bitten on the udder; and Roche, I4 sheep out of a flock of 60 .

The serpents of tropical countries inoculate a venom of an intensely poisonous character. Cogan Haaris saw a twoyear-old black deer die in two hours after having been bitten by a cobra. Half an hour after the bite the injured animal had lost all power of co-ordination, kept its eyes fixed, fell repeatedly, and rapidly died. The bite of the black naja (a spitting serpent) causes faintness, convulsions and enormous swelling of the puncture and its surroundings. Dr. Boye reports a cow that was bitten in the udder by a ceras- 
tes, as follows: The region of the udder was extremely bulky; œdema extended over the entire lower part of the body; the animal lay upon its sides, panting, and with its limbs in complete disuse; a thick foam was issuing from the mouth and asphyxia was imminent.

When insects (bees, wasps, etc.) light upon animals in great numbers they produce multiple stings. They attack the head and acnestis in preference to other parts. In a few seconds edema appears on all the spots which have been stung. The skin is hot, painful and tumefied, and the frightened animals manifest signs of very acute pain. They often run away with their vehicles and roll themselves to escape - the enemy. These accidents are rather frequent in districts where bee culture is extensively followed. Horses employed in field labor in the neighborhood of beehives are particularly exposed. In years when wasps are numerous, the destruction of their nests in plowing, is often the cause of a sinilar acciclent.

Lucet has seen oxflies attack sheep and sting them deeply in the ipper portions of the body, - the withers, loins and buttocks,- and a few days after five of the animals exhibited abscesses, surrounded by œdema, at the punctured points. One of the patients died of purulent infection and the other four were killed. When stung upon certain parts of the body, - the head for example,- the horse may present the physiognomy of animals afflicted with purpura hamorrhagica. In this event respiration may be menaced by obstruction of the nares and tracheotomy may become necessary. Fatal cases have been reported by Gueron and Henon, in which death supervened in a few hours. Guilleville reported five horses which succumbed in one hour; Clichy saw five horses die in two, three, four and five hours; Funfstuck has seen an animal die in six hours; and in a case reported by Albrecht death supervened after ten hours. The dog is 
not attacked as often as the horse. When stung, he barks plaintively, turns the head to the side stung, raises the aching limb, refuses to move, and if numerously stung emesis may be provoked. D'Arboval and Crepin report having seen dogs die after bee stings, and Lange described a curious case of a flock of seven geese attacked by a swarm of bees, of which six died and one survived but remained blind.

TREATMENT.-For the sting of bees or wasps, the first requisite, if one is on the scene at the time of the accident, is to wash off the insects by ablutions. When the injury is done local applications of moist earth are of great assistance. Lotions of diluted vinegar and diluted ammonia may also be advantageously employed. When systemic disturbances result, it is clesirable to administer stimulants, heart tonics and evacuants. Coffee is very suitable under these circumstances.

For bites of serpents the following course is recommended:

(I) To oppose the absorption of the poison.

(2) To facilitate its elimination.

(3) To inject the therapentical remedies.

I. When present at the moment the animal is bitten, the immediate indication is the application of a tight ligature above the bitten spot.

2. The elimination of the poison is facilitated, when the ligature is attached, by incising the wound so as to make it bleed. The flow of blood will carry away a part of the poison and a copious irrigation will remove what remains upon the surface of the wound.

Injections in loco of permanganate of potash, one per cent, and applications of chloride of lime, I-I2, are recommended by Calmette. The latter should be applied to the zone surrounding the spot stung. Recent researches by 
Phizalix and Bertrand have demonstrated that chloride of lime effects a cure by its local action.

3. The injection of antitoxic serums, which have been employed since the researches of Calmette. The serums possess both preventive and therapeutic antitoxic properties, which are all the more effectual when promptly applied. If the bite is recent ten cubic centimeters of the serum are injected, and if systemic disturbances already exist the dose is doubled. When the symptoms are alarming the serum may be injected intravenously. In the dog this can easily be accomplished through the saphenic vein. The immunity is rapidly established, but as promptly disappears.

\section{CONTUSED WOUNDS.}

Contused wounds are produced by external violence. In these wounds the integument is pressed in and the subcutaneous structures crushed. When the outer skin remains intact, the traumatic focus is a simple contusion proper. Contused wounds are generally produced by blunt instruments which divide the skin only when the violence is excessive.

ETIOLOGY.-Contused wounds are most frequently caused by stakes, the shafts of vehicles, the end of a pole, the friction of a rope or the application of a ligature that is too tight or is left in place too long. Dollar reports the history of a horse in which case the end of a pole entered the right side of the chest, pentrating upward, lacerating the trachea and making an opening at a higher point on the opposite side. We have ourselves observed an analogous instance, of a horse under whose shoulder a shaft penetrated and caused an actual dislocation of the limb. Colin refers to a contused wound in a dog following the attachment of an elastic band around the paw.

SYMPTOMS.-Contused wounds exhibit numerous 
forms. They include simple abrasions in which only the epidermis is affected, and erosions frequently seen in horses that fall and drag a portion of the body along the ground for a variable distance. Since the body inflicting the injury is blunt it is essential that it take effect on an anatomical region with a resisting or bony base. Thus, in wounds of the orbit caused by falls, the skin is crushed and divided from within outward. Contused wounds may also be caused by blows with a stick or whip handle, or from prolonged decubitus. The physiognomy of these wounds varies with their nature. In slight ones there is generally no hæmorrhage, but the pain may be rather intense, or even acute. When the traumatism has been very violent the disturbance produced may be considerable. The skin may be loosened over a large surface, the tissues may be crushed and torn and important organs may be exposed. But in spite of these serious disturbances the hæmorrhage is nearly always insignificant, not only because of the crushing of the blood vessels but also on account of the traumatic shock which is always accompanied by a fall in the arterial pressure due to enfeeblement of the cardiac impulse.

Contused wounds were formerly of a very formidable character. Most of the infective morbid processes were ascribed to them. Their irregularity, their extent, their deepseated nature, the variety and number of tissues affected, the abundance of coagulated blood, and of accumulated serum, and the difficulty often experienced in cleansing them, made them veritable nests of infection. Gangrenous septicæmia, pyæmia and tetanus so frequently resulted that they might have been referred to as "classic soils for complications."

PATHOLOGICAL ANATOMY.-Their anatomo-pathological characteristic is the absence of precise limits. The contused wound is bounded by bruised, mangled and irreg- 
ular lips. The skin may be torn loose over a wide surface, and by displacing it so as to expose the subjacent tissues, they are found shapeless, composed of remains of muscles, the ends of blood-vessels, torn tendons and nerves, at which surface are collected coagulated blood and serum. The periphery of the wound is also more or less bruised, infiltrated and dissected by the blood, and also, in itself: constitutes an excellent environment for the culture of micro-organisms.

TREATMENT.-The general directions are the same as in the preceding instances. Usually, the spacious wound must be left uncovered. Shower-baths, and continual irrigations of cold water, constitute excellent therapentic expedients. When the region permits, antiseptic baths give satisfactory results. Powdered carbolic preparations find their greatest value in these wounds. When these directions are properly followed and rigoronsly applied it is sometimes astonishing what success is obtained. A cure is often effected in cases that appear very grave. Dollar's horse recovered in sixteen days and our case was perfectly well in three weeks; and Cadiot and Almy report a no less remarkable cure due to antiseptic baths.

\section{GUNSHOT WOUNDS.}

On account of their peculiar etiology these wounds are studied with special interest everywhere.

ETIOLOGY.-Wounds by firearms are not rare among hounds. Except in war they are not encountered in the horse, unless through some accidental cause. They result from discharges of grains of shot, from balls of revolvers and guns, or from bullets. Guns loaded with shot, at short range, cause accidents similar to that of balls. When fired at long range the shot scatter and riddle the skin without penetrating more deeply. 
SYMPTOMS.-The physiognomy of gunshot wounds varies with the kind of projectiles and the distance at which they are fired. The balls of the Chassepot, the Gras gun and the Lebel gun have no different effect, except in the power of penetration.

When important viscera are affected, animals rapidly succumb, sometimes instantaneously, and at other times after walking but a few steps. The hamorrhage is usually insignificant and if no important organ is injured the animal exhibits no particular symptom. Salle relates the case of a mule that was accidentally wounded by a ball from a Gras musket. Upon being struck the animal stumbled, and then resumed its journey. Donough cites the curious case of a red setter that received a ball in the sub-lumbar region without appearing seriously indisposed, and yet the omentum protruded and was torn into shreds.

Projectiles may pass through the bodies of animals or remain in the tissues. In the former case they produce two perceptibly different wounds. The entrance wound appears to have been made with a punch. It is generally small and hollow in the infundibulum. The exit wound is always more extensive. The dimensions are in proportion to the distance. The greater the distance the more extensive is the exit wound, whatever may be the pattern of the gun. It is often very large, with wide open and projecting edges. With the Gras and Lebel rifles at short range the wound of exit and that of entrance do not differ much in size. Grapeshots, small cannon balls, shells and splinters of shells cause frightful lesions.

PATHOLOGICAL ANATOMY.-Whether the projectile passes through the body or stops in the tissues it excavates'a passage of variable extent and regularity. The projectile may remain just beneath the skin, penetrate into a large cavity or traverse the entire body. It may excavate 
a veritable gallery through the organs from 0.40 to 0.60 meter long.

The tract is either rectilinear or tortuous. It is tortuous when the ball has spent its force and is unable to penetrate directly through the bones. When the projectile makes its exit without penetrating into the muscles, it is similar to that of a seton. When the passage is blind the ball is left in the tissues, and yet a wound of exit is no assurance that a fragment of the ball, split by striking a bone, is not left behind, as is frequently the case with the dum-dum bullet.

The tract in the muscles is surrounded "by a hemorrhagic muff," and is carpeted with a friable detritus. When the ball has penetrated in the usual manner the muscular fibres are only separated. The bones are either penetrated or scattered and the organs seem reduced to a pulp. It is not rare for the ball to stop beneath the skin after passing through the body where it is found beneath a large ecchymosis. The dum-dum bullet used by the English troops in India produces lesions of exceptional gravity. It splits upon striking and ploughs up the tissues in its course, tearing the parenchymatous organs, pulverizing the bones and reducing everything it meets to a veritable pap composed of bony splinters, blood and muscle fibres. It causes the formation of extensive traumatic foci, irregular and without any definite character, in which it is difficult to recognize the physiognomy of the mutilated organs.

Projectiles, or shells, take off limbs by cutting or shattering them, producing a huge, gaping wound containing mangled bones, muscles, etc., which mingle confusedly, and cause the limb to dangle by shreds of skin and muscular debris.

TREATMENT.-Formerly, great stress was placed tupon immediate intervention; the extirpation of the bullet was advised in nearly every case. Surgeons of the old school 
regarded a bullet in the tissues as a diabolical surgical arsenal. The practice has been abandoned for several reasons. The ball remaining in the tissues may prove quite tolerable, may cause no trouble and mà become encysted. Examples of complications occurring as a consequence of a bullet remaining in the tissues are rare. The observations of Carnet are exceptional. It is not common to let animals die from purulent infection without trying to evacuate the abscess producing it. Muller has seen a horse that retained a ball, which had slowly descended from the lumbar region to the umbillicus, for ten years without causing any complications. The Journal of Comparative Pathology records the case of a cat whose eye was pierced by the shot of an air pistol. The shot lodged in the retina, where it became encysted by a thick sac. As analogous instances are numerous, it is eviclent that bullets when antiseptic and when the skin at the point of entrance is clean, may be perfectly tolerated by the tissues.

If the bullet does not encyst it causes suppuration and produces an abscess. The seat of the abscess indicates the seat of the bullet, which could not have been located when the course is tortuous. The abscess, when lanced, will eject the bullet as soon as the pus is evacuated. It is bad practice to act differently, as the search for the bullet is always painful; it may aggravate the injury the organs have already sustained, reopen obturated vessels and obstruct possible prompt cicatrization of the tract, without taking into account that the search is so often without results. This is especially the case when the tract is tortuous and an aponeurosis has been pierced. Aponeurotic structures immediately reclose after being divided by a bullet. Do not, therefore, search for foreign bodies in gunshot wounds unless they can be c'early felt through the integument, but leave them 
to the tissues where they will either be tolerated or expelled by suppuration.

Having adopted these directions, treat gunshot wounds as any other. Carefully disinfect the wounded spot, apply tepid antiseptic baths. if possible, and redouble the effort if the wounded patient is liable to become infected. Gaseous gangrene and tetanus are not exceptional complications of gunshot wounds. They are seen most frequently from hunting accidents sustained at the end of a day's hunt, when the clothes are covered with dust and the ball has carried dust containing tetanus germs into the wound.

In the domestic animals intervention is useless where a wound is inflicted to an important organ, and as the consequence of such wounds is fatal, the only sensible advice is to slaughter the animal.

\section{CONTUSIONS.}

A traumatism may injure the tissues, more or less deeply, and leave the skin intact, in which event the lesion is designated as a contusion. A contusion, then, does not differ from a contused wound except that it is sheltered, and the traumatic focus protected against infection from without by the integument.

ETrOLOGY.-On account of their economic destination, contusions are frequent in the domestic anima!s. In the horse the kick of an attendant, or vicious animal, blows from whip-handles, pressures of the collar, friction of the harness, an abrupt dismount from a horse that has carried a rider a long time, etc, are the most frequent causes of contusions. The ox resists them better on account of the thickness of the skin, yet, horn-thrusts may crush the tissues without penetrating the skin, and bruises may lue sustained from the shoes worn by working oxen, and finally friction from the girth may cause a serous or sanguineous 
effusion. The dog and the other small animals are less exposed to such injuries.

The physiognomy is variable. The symptoms by which they are recognized may be either grave or trivial, according to the region attacked and the importance of the organs affected. Human surgeons, first, Boyer, and then Dupuytren, have designated different degrees of contusion. from which the following, purely conventional classification emanates: (I) A first degree contusion, or simple bruise of the skin consisting of ecchymosis due to the rupture of its capillaries; (2) a second degree contusion in which large blood vessels have been ruptured and a sanguinary tumor has resulted; (3) a third degree contusion in which there is crushing of the subcutaneous tissues, disturbances and mortification of the anatomical elements, fatal gangrene of the affected structures, and frequently synovial and serous effusions.

Briefly considered these three varieties of contusions demand the following description:

\section{Contusions of the First Degree.-Ecchymosis of the} skin.-The character of first degree contusions depends much upon the preservative vitality of the part afflicted. The anatomical elements have only been disorganized,--separated. The traumatism has not been forcible enough to cause mortification of the entire focus; only a few cellular elements are destroyed, and these become isolated by the infiltration of blood or serum. The ecchymosis is manifested by a diffused. reddened area easily seen on an unpigmented skin. It is accompanied by a stinging and benumbing pain When the blow is violent and affects a limb, the pain may be sufficient to cause lameness, and a pronounced odema may soon appear as a consequence of the resulting vaso-motor paralysis. In the domestic animals, with the exception of the hog, cedema is often the only indication that a contusion exists. 
In time the tint of the ecchymotic skin changes to green or black. But this transformation, though very important in human medicine, is too trifling an issue in veterinary pathology to warrant the discussion of the theories advanced in explanation of the cause of these color changes.

2. Contusions of the Second Degree.-Blood tumors.A more violent blow causes an ecchymosis, as in the preceding variety, but in addition there is a cavity filled with bruised tissue, serosity and blood. The skin is raised by the accumulated liquid in the injured focus and a swelling results. When the blood accumulates on a resisting surface, as bone, the swelling is designated as a sanguineous tumor, (blood tumor) and when formed in the soft tissues it is called a sanguineous sac (blood sac). A distinction may, therefore, be made between the two expressions.

The sanguineous tumor may be very large, and yet produce no lameness. The rapidity of its formation and the sensation of crepitation felt on palpation are sufficiently characteristic. The crepitation is distinguished from that of fracture by the harshness of the latter. It is soft and reappears with each formation of the coagula.

The sanguineous accumulations may become complicated with suppuration and gangrene. The former is not rare among debilitated animals or those under the bane of a microbian affection. A sanguineous sac nearly always suppurates in the horse afflicted with strangles, or in a dog suffering from distemper. The injured point is weakened and the micro-organisms circulating in the blood find an excellent environment for growth.

Pathological Anatomy.-The blood that flows after the blow pushes the tissues apart and thus creates a cavity for itself, which becomes lined with fibrinous deposits, and soon a genuine sac is found to be established. These immediate phenomena are accompanied by the work of or- 
ganic repair and cicatrization. The fixed cells attached to the surface of the fibrinous layer proliferate simultaneously with the accumulation of leucocytes at the periphery, thus constituting a sac. The accumulated blood is rapidly modified. The serum absorbs and the coagulum remains clinging to the walls of the sac, and dries up, or else the corpuscles become dissolved, and leave a sac that fluctuates indefinitely. In time their coloring matter becomes dissolved and destroyed, leaving the liquid contents brown, then yellow, and finally amber colored, in which event, the case being old, the exact origin of the lesion will be difficult to determine.

3. Contusions of the Third Degree.-In contusions of the third degree the tissues are cleeply impaired. The muscles are crushed and torn, infiltrated or separated by hæmorrhage, and the skin covering the injured area readily becomes necrotic. Tendons are ruptured, aponeuroses torn, nerves crushed, vessels ground, and the whole area transformed into an irregular focus impregnated with a liquid that exudes at the surface, and if infection intervenes death is quite certain.

Contusions of the third degree include primary serous effusions. This lesion consists of an ovoid tumor of limited height, but extending over a considerable surface, and is frequently encountered on a level with and slightly behind the coxo-femoral articulation. It is cold, indolent and very fluctuant, and any force applied to its surface produces an undulating movement which spreads over its entire area. The skin is markedly detached and the contents are easily displaced. Primary serous effusions are rather common in the horse in places where the muscles are enveloped in a strong aponeurosis such as exists in the region of the buttocks. They are not entirely absent in the ox, in which animals they are encountered on the other side of the stifle. 
They vary from the size of a hen's egg to that of an infant's head. They have a very thick wall and cause no elevation of temperature. These tumefactions appear suddenly, and in some cases without the possibility of determining their cause.

From falls, when the animal drags along the ground, and from bruises of carriage wheels the skin is loosened, and a copious accumulation of serum in the space is the result. Whence comes this serum? Different views have been advanced on the subject. Morel-Lavalle thought it flowed out of the vessels that were drawn out, like the heated glass tube or the Bunsen burner. Granfelt opined that it had its origin in the hypersecretion of the areolar tissue, and finally Verneuil advanced the hypothesis of an interstitial lymphangitis. Whatever is the origin, the accumulated liquid is always of an amber color and faintly tinted with red by the blood.

The collection of serum is rapid. It increases rapidly for a few days and then retains almost the same dimensions indefinitely. The lesion is easily recognized. The location, the rapidity of formation, the clearly perceptible fluctuations, are its typical symptoms. It is scarcely possible to confound serous effusions with sanguineous sacs, owing to the characteristic crepitation of the latter, and which does not exist in the former.

The oleaginous effusions, described by Violet and Gavard, result from a fall, the bite of a horse or a kick. They are seen especially in the region of the stifle. Out of 34 cases observed by Gavard Io were in the region of the patella, I6 on the carpus and 8 on the fetlock. Their formation is analogous to that of serous effusions. "They are either entirely dormant, without any lesion of the skin, or else more or less sensitive and accompanied with an inflammatory obstruction which causes a lameness of variable intensity." They 
contain an amber-colored liquid of oily appearance whicl presents the characteristics of synovia, but which does not, like it, coagulate in the air, but concretes slightly. It has been found that in man this liquid would produce an oil stain on paper, and that it contains margarin. In the course of time and after the first evacuation it becomes turbid, owing, probably, to the fat it contains. These accidents, which are very uncommon, do not exhibit any grave features. They ustually disappear after appropriate treatment.

TREATMENT.-Contusions in the first degree,-ecchymosis,-constitutes an insignificant lesion in the domestic animals, and is only treated in subjects kept as luxuries. Resolvents, camphorated alcohol and Goulard's extract are the preparations to recommend in these cases. When the pain is acute, preparations of cocaine are specially indicated, and in the dog prolonged baths at a high temperature (40 C.) (II2 Fahr.) afford immediate relief.

The sanguineous tumor yields to compression, which hastens absorption by spreading out the mass. Friction, massage and the application of an elastic band bring effectual results.

When the inner face of the sanguineous sac is organized, tapping, combined with aspiration and breaking down of the coagulum is advised. The tapping may be profitably followed by the injection of an antiseptic liquid, carbolic solution, creosol, tincture of iodine or sulphate of copper. When the walls are indurated excision or enucleation may become necessary, in which event the sanguineous tumor is disposed of in the same manner as a solid tumor.

Serous effusions do not always promptly disappear after tapping with the trocar combined with energetic compression; in nearly every case the sac will refill. In that event the operation is repeated and followed by injections of iodine, the concentration of which is gradually increased. Cradu- 
ally the collection becomes more limited and finally disappears. Incision, followed by careful disinfection, is much recommended by all practitioners. Blisters and setons should be absolutely abandoned, in spite of some good results which have been claimed.

For oleaginous effusions, Violet and Gavard have used irritant preparations, liquid caustics and actual cauterization, and claim they have good results.

In the ox Furlanetto obtained a complete cure by passing a ribbon seton through a tumor and smearing it every four or five hours with an ointment of bichromate of potash, I to 8 .

\section{BURNS.}

Burns are lesions produced by the action of heat. They may be caused by solicl bodies, by gas raised to a high temperature or by chemical caustics.

ETIOLOGY.-Of all the clomestic animals the horse is most exposed to burns. In the ox they are more rare. The cat and the dog, being in constant close relation with man, are, like him, exposed to burns by oil or by boiling water. Independent of these conditions, which refer to the mode of utilization of animals, it is indispensable to add that death by burns or by asphyxia frequently occurs in burning buildings. In these events the animals seldom succumb to actual burning. In nearly every instance they are asphyxiated by gases before the flame has reached them. The irritating fumes also cause coryza, laryngitis and even bronchitis and pneumonia, which the subjects find difficulty in resisting.

Solid bodies do not inflict very extensive burns, as their effect is confined to the parts they touch. Shoes applied too hot to the foot often cause burns of the sensitive sole, and even the cautery when used for curative purposes may cause serious accidents.

Liquids by spreading over the skin produce large burns, 
for which reason oil is still more dangerous than water. Rainard reports the curious case of a horse that sustained a serious burn by plunging the nose into a bucket of boiling water that was intended for fumigating purposes.

Gases produce large burns in oil mines : the horse is sometimes the victim of explosions which cause large and fatal burns, and fumigations which are too hot may cause dreadiul accidents.

The flame of fire is capable of burning deeply and may cause extensive damages. Gohier reports the death of a horse whose genital organs had been burned by teamsters in their effort to make it draw a heary load.

Many chemical agents are capable of producing grave burns. Rey has often observed burns caused by sulphuric acid on horses and (logs belonging to druggists, and points a1so to the frequency of burns in the extremities of horses by quicklime.

Burns from lightning do not present any special features; except in the arrangement of the designs it forms. Radiating heat produces only superficial burns, and solar heat may produce erythema ancl sometimes serious conditions designated as "insolation" or "heat-stroke," but these should be studied in internal pathology.

SYMPTOMS.-Since Dupuytren's time six degrees of burns have been distinguished in human surgery, but as this is an entirely arbitrary division that is more anatomo-pathological than clinical there is no reason, although Rey has preserved it, for applying it in veterinary surgery. Cognizance of but two burns is sufficient,-circumscribed burns and diffused burns,- both of which may be deep. This division is more simple than that of Bouley, which distinguished between simple burns and disorganizing ones, and more simp'e than that of Hertwig who distinguished: (I) 
Erythema, (2) Simple inflammation, (3) Inflammation with secretion or suppuration, and (4) Necrosis of the tissues.

Local Symptoms.-The physiognomy of the lesion varies markedly with the nature of the body that has cauterized it. Burns with boiling water may be diffused, but are always superficial, and those with solid bodies are deep but linited. Burns from sulphuric acid are generally diffused and reddish and are covered with a reddish-brown scab.

Circumscribed burns manifest themselves, when produced by a solid body, by disappearance of the hairs, which are redrened at the point touched. The skin hecomes dark red and erythematous, and the pain is brief but acute. If the artion of the heat has been intense, the skin becomes tumefied and blistered, or else shrivels up and sloughs away. When the surface is extensive there is erythema around the periphery, blisters in the middle zone and necrosis in the center. The skin may be destroyed and the subjacent tissue partly carbonized. Slight burns produce no systemic disturbances.

Diffused burns, although superficial, will give rise to serious general phenomena, which are-still more accentuated if the burn is cleep. They may affect the muscles, the bloodvesse!s, the nerves and even the bones. In these cases the periphery of the burned area exhibits every degree of burns and the central zone contains a scab which emits the odor of scorched flesh. Cicatrization is only possible when the damage is limited. Slight burns last but a short time, although those exhibiting a scal, may require a considerable time to heal.

General Symptoms.-Diffused burns are followed hy general complications. Animals suffer from nervous shock and pass into a comatose state as the result of the intense irritation of the scnsitive nerves. The pulse is feeble, the respiration irregular, the temperature lowered and hamoglobinuria appears. Horses eject an intensely dark red urine, 
The mouth is hot and discharges a ropy saliva, and the loins are stiff. The dog exhibits general weakness, somnolence, deliriun, cramps and even general convulsions. The digestive functions are disturbed by emesis and diarrhoea.

How is the appearance of these symptoms to be explained? This question has interested scientists at different periods, and although various hypotheses have been advanced the problem is not definitely solved. The interpretation of these complications is grouped into five theories: That of irritation was one of the first, and was defended by Petit, Remy, etc. : that of shock, advanced by Dupuytren, attributing every complication to the shock of the nervous system or to its exhaustion; a third, that of purely mechanical blocking of the circulation, sustained by Dupuytren, Couston and Morton. These different hypotheses do not appear to have long enjoyed the favor of the medical profession. They have been abandoned and other theories have taken their place.

For a long while nearly all physicians attributed all the results they observed to disturbance of the function of the skin, - the skin being destroyed the body is deprived of one of its excretory channels and the blood will become corrupted by the accumulation of waste matter. The disturbance of the cutaneous function is therefore closely connected with corruption of the blood, which is increased by absorption of products from the burned area. This point of view is upheld by Reiss, Kijanitzin, Boyer and Guinarrl. The burnt tissue is the starting point of the formation of toxins, (Reiss) which arise from the action of organic ferments and the dead elements. Reiss classes these toxic substances with pyridine and quinoline, while Kijanitzin compares them to the peptotoxins of Brieger.

Boyer and Guinard have attempted to demonstrate that the body of the burned subject absorbs poisons of consid- 
erable potency in the production of general symptoms. This theory is incontestable, as proofs are found of the symptoms and in the lesions, as well as in the particular toxicity the urine acquires.

COMPLICATIONS.-When burns are infected, lymphangitis, abscess, gaseous gangrene, pyæmia and tetanus may result. Complications of œdema of the glottis, bronchitis and pneumonia occur among animals that inhale smoke.

PATHOLOGICAL ANATOMY.-The local lesions have already been described. In the horse when death supervenes the subcutaneous tissue is thickened, hard, infiltrated with serum, the lungs congested, the pericardium filled with reddish serum, the blood in the heart, reddish, and the brain, injected. When complications of bronchitis have occurred the bronchi, trachea and larynx are greatly inflamed and contain false membranes. In the dog, the blood is dark, thick, coagulated and scantily supplied with water. The red blood corpuscles are deeply altered and the gases diminished-a diminution which affects the conveyance of both oxygen and carbonic acid, but especially the latter.

PROGNOSIS.-The prognosis varies with the extent and depth of the wounds, with the importance of the organs attacked and the nature of the cauterant. It is graver in young than adult animals in consequence of the great anæmia resulting from the vitiation of the blood. Burns of the sole may prove fatal, and those which affect the walls of the great splanchnic cavities may become complicated with pleurisy or peritonitis.

TREATMENT.-For a long time burns have been treated without taking new data into account. Most surgeons used substances that had only a nominal microbicide value, but today nearly all have adopted antiseptic treatment. Burns, in fact, should not be treated differently from other wounds, and as wounds of domestic animals are never 
aseptic, it is indispensable to render them so. Tepid antiseptic baths, when practical, and carbolized powders, are said to give good results. In the dog; if the pain is acute, preparations of cocaine should be used in the dressings and baths.

When general symptoms are inevitable asthenia must be combated. Revive the heart's action with caffein and avoid chilling of the patient that is already showing signs of hypothermia. If the theory of morbid absorption is admitted, it must be combated as far as possible, by promoting the elimination of poisons and by the use of artificial serums, which will free the blood of toxic products it contains and augment the action of the heart and urinary function.

Thierry insists on the good effects obtained from picric acid in superficial burns. The solution he used has the following composition:

Picric acid ........... 22 grams

Boiled water ............. I litre

In times past a whole series of formulae were recommended, all of which contained fat substances as excipients. Capitan recommended the following ointment:

Vaseline ........... 30 grams

Salol .............4 grams

Cocaine hydrochlorate ....25 centig

Reclus has advantageously employed the following formula :

Vaseline ...........40 grams

Boric acid ............ grams

Antipyrin ........... grams

Iodoform ........ 0.5 to I gram

These can be greatly varied.

On a horse burned in different degrees by an explosion of gas, Porteus made use of compresses soaked with a mix- 
ture of three ounces of tincture of iodine and one pint of water. Over this he spread a coating of starch. This treatment, repeated each day, brought about a cure in three weeks.

Haas has obtained remarkable results from the following prescription :

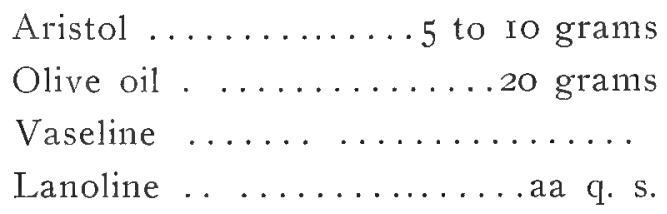

The aristol should be dissolved in the oil, and the vaseline and lanoline added.

Graze employs the following mixture for extensive burns:

$$
\begin{aligned}
& \text { Powd. gall-nut } \ldots \ldots \ldots \ldots \ldots \text { I gram } \\
& \text { Boric vaseline } \ldots \ldots \ldots \ldots \ldots 8 \text { gram }
\end{aligned}
$$

This mixture attenuates the secretions, is deoderant and prevents the exuberant growth of proud flesh.

\section{CHILBLAINS AND FROST-BITES.}

The word "chill" is used to describe the local or general action of cold: on the organism. "Freezing" and "congelation" are also employed as almost synonymous terms.

ETIOLOGY_Frost-bites are extremely rare among clomestic animals in the climate of France, owing to the thickness of their skin and the abundance of hair with which they are provided at the approach of winter. Except in isolated cases solipeds and ruminants resist the effects of cold remarkably well. The resistance is especially great when the organism is not debilitated by previous diseases or privations. It is especially interesting to consult the memoirs of soldiers in the Russian campaign on this point. We gather from them the fact that men and beasts died chiefly from lack of nourishment, and that the only horses that resisted 
temperatures of 27 degrees $\mathrm{C}$ were those whose riders were as much concerned about their animals as about themselves; they rifled the thatched roofs of cottages in orcler to get them a little coarse mouldy straw.

As a general rule the southern breeds resist cold better than the horses from the north. In the Crimea, cluring the Franco-English expedition, the Barbary and Arab horses resisted better than the English horses, and the winter of I870-I87 I killed more English than African horses. The ox sometimes exhibits frost-bites on the extremities, on the scrotum and on the udder. Robeis observed an epiclemic gangrene of the nipples of a cow that he connects" with the effect of cold. The dog is not exempt from the same accident. Hunting dogs, from running on the ice, sonmetimes exhibit frost-bites on the paws and ears. In birds frost-bites of the feet and comb often occur simultaneously.

Every species of animals do not offer the same resistance to cold. The-rabbit endures temperatures of IO, I 5 and 25 degrees $\mathrm{C}$, without undergoing any apparent diminution of animal heat. The sheep and the hog also possess great powers of resistance, while the dog and the horse show far more sensibility to cold.

PATHOGENESIS.-By what mechanism does cold cause organic troubles? It provokes a backward flow of blood toward the large veins and the heart. The cutaneous vaso-constriction which is nature's self-defense against cold, is a remedy that is often more terrible than the injury it aims to combat-coldness. The blood flows from the periphery and flows toward the center of the body and ascuminlates in the parenchymatous organs and clogs them. The blood corpuscles are piled up and crammed into the lungs, which then become the seat of a congestion so intense as to prevent normal hæmatosis. The respiration is accelerated and painful and asphyxia may supervene. 
Cold has a very perceptible action on the brain. Does it act abruptly?. It stimulates cerebral anæmia. Is its action gradual and progressive? It ends in, cerebral congestion, which is absolutely opposed to the former. These different manifestations may be combined in different ways and give rise to a complex and variable symptomology.

Locally cold causes different lesions, according to whether the part is frozen or threatened with freezing. It provokes a vaso-motor constriction,- - a real anæmia, which deprives the tissues of their nutritive elements and causes gangrene if the action has sufficient duration. Generally matters do not reach this point, for cold, unlike heat, has a slow progressive action, and has not the same disorganizing effect. It predisposes to, rather than causes gangrene.

SYMPTOMS.-As in the case of heat, degrees of cold have been designated in human surgery, but it does not seem necessary to apply them to veterinary surgery. Freezing caused by snow, exhibits itself in the horse, by gangrene of the skin on the fetlock and pastern, and sometimes on the velvety tissue of the foot that is insufficiently protected; and exceedingly tenacious ulcerations often appear on the flexion surface of the pastern. Real incrustations may be produced and the skin may be eliminated in shreds. Stottmeister refers to the loss of the skin of the four extremities of a cow, and Kekeler to an analogous injury in a colt.

PATHOLOGICAL ANATOMY - Slight and transient cold leads to rubification of the skin, and more severe cold to blisters and gangrene, but these lesions are not immediately produced; they are secondary, since the primary phenomenon is a marked vaso-constriction-a stage of anamia. If the refrigerating action yields before there has been any vascular impairment resolution will be quite prompt, but when the latter occurs morbid processes are positive. 
Thromboses obstruct the frozen vessels, then the skin and subjacent tisues, being deprived of nourishment, become necrotic. Utchinsky's experimental studies of lesions in the tissues caused by cold, show that ether, in only four minutes, caused contraction of the blood vessels and heaping up of the red blood corpuscles, and after twelve hours accumulation of leucocytes with the formation of intra-epidermic vacuoles, while a more intense refrigeration provokes hæmorrhages in the papillæ.

That frozen organs can be restored to their normal condition has been shown experimentally by Hunter, who froze the ear of a rabbit until an incision with a bistoury brought no blood, and then warming the organ to its original temperature, found that the circulation had resumed. Frozen organs regain the normal temperature by degrees, and then become the seat of a rather acute inflammation, which gradually subsicles.

TREATMENT.-When animals have been exposed to intense cold they should not be immediately placed in a hot stable. Dogs should be kept away from stoves. If this rule is ignored, simple accidents may terminate in gangrene. Gentle rubbing with snow or ice is very efficacions against frost-bites. When ulcers or scabs have formed the lesion should be treated with respect to antisepsis. They should be thoroughly cleansed and coated with a protecting substance. Iodized glycerine or an iodoform ointment is very suitable for frost-bites in the extremities, and a clean, dry litter is indispensable. In dogs Moller successfully made use of warm baths from I6 to 18 degrees $C$, of which the temperature was gradually raised. Occasionally amputation of a frozen extremity may become necessary in the dog or cat.

Systemic disturbances resulting from exposure to cold 
are combated by friction, stimulants of hot wine, light alcohol and coffee.

\section{TRAUMATIC HÆMORRHAGE AND ANÆMIA.}

Traumatic hæemorrhage may be clefined as an escape of blood, beyond ordinary limits, from an accidental or surgical wound. Hremorthage may be either primary or secondary: -it is primary when occurring immediately after a trauma, and secondary when some time elapses between the infliction of the wound and the occurrence of the hamorrhage. In contused wounds it is often seconclary as the blood vessels are bruised and their lumina closed by the coagula, which circumstance temporarily arrests the flow of blood, but when animals are left to themselves they may bite the wound or rub it against neighboring objects and thus provoke a secondary hamorrhage by breaking up and detaching the clots.

Hamorrhage may be especially abundant in certain subjects. Donkeys and mules are normally hæmophilic, as a trifling wound, the excision of proud flesh or the cutting of a shallow fistula are often sufficient, in these animals, to provoke hremorrhages that show no tendency to arrest spontaneously, as in the other species. Whether the hæmorrhage diathesis is a vascular defect, a disturbance of the vaso-motor nerves or a chemical modification of the blood is difficult to determine. It may be either of these factors or an associated action of all.

The amount of hæmorrhage equally clepencls upon the richness of the blood supply of the injured region. It is always abundant in the tongue, foot, anus, walls of abscess, fistulie, etc.

The nature of the trauma, the character of the wounded vessels and the constitution of the tissues in which the wound occurs, are so many, more or less, important factors in the production of hæmorrhage. The state of the blood 
vessels concerned is an item of utmost importance. When they are hard and fibrous, or their walls are in a state of chronic inflammation, they do not retract into the tissues and their lumina remain wide open, and the flow of blood, as a consequence, shows no tendency to stop.

Late secondary hrmorrhages are often the result of phlebitis. The coagulum closing the vessel becoming infected, disintegrates, liquefies and flows out, and thus reopening of the opening is effected.

Hremorrhages are more serious in the young and the aged animal. Arterial hæmorrhage is recognized by the flow of a jet of blood of a very bright red color, that stops by compression between the wound and the heart. If the wound is deep or the orifice small, it flows out in a sheet over the skin, or else dissects its way beneath the skin and collects in the connective tissue spaces forming a sanguineous tumor upon which pulsations can be felt. The result is a diffuse transitory aneurism. Venous hæmorrhage is recognized by the dark color of the blood, by the stopping of the flow when compression is applied between the wound and the capillaries, and by the copious flow when an obstacle is applied between the wound and the heart. When the wound in the vessel and that of the skin do not coincide the accumulation of blood beneath the skin produces a thrombus. In capillary hæmorrhage the flow is sheet-like, its color is intermediate between arterial and venous blood; and the flow is not generally abundant.

Copious hemorrhages are followed by weakness, rigors, enfeeblement of the pulse, acceleration of the respirations, paleness of the mucous membranes and coldness of the extremities. In slow and repeated hemorrhages there is languor and weakness, and the slightest effort causes shortness of breath, fatigue and disturbance of the circulation. There is intense thirst but no appetite for food. 
TREATMENT-Capillary hæmorrhages are often arrested by baths of cold or hot water at 50 to 60 degrees $C$. It is not necessary to resort to perchloride of iron or actual cautery except when other remedies have failed. Compression with absorbent cotton soaked in an astringent solution is generally effectual.

Large vessels, arteries or veins are seized and twisted with the hæmostatic forceps, and their complete occlusion secured with a ligature. When, in consequence of the depth or irregularity of a wound it is impossible to adjust the ligature, the forceps may be left tightly fixed to the vessel and removed only after the coagulum is sufficiently consistent to resist the blood pressure. When the hrmorrhage has been sufficient to enclanger life, subcutaneous or intravenous injections of artificial serum (salt solution) are indicated.

\section{TRAUMATIC EMPHYSEMA.}

Traumatic emphysema is an accident caused by the passage of certain quantities of air beneath the skin, or of gas proceeding from the digestive tract. It is frequently noticed in the horse following wounds of the face and trachea, or after solutions of continuity occurring where the subcutaneous connective tissue is abundant and loosely arranged. In the ox it occurs under the same circumstances, but is also seen as the sequel of pulmonary emphysema, after tapping the rumen, and in certain cases not well determined it is generalized without any certainty as to its origin. In the sheep it is frequent after the bites of dogs. When the wound is inflicted on the neck the trachea is perforated and the air dissects its way over the neck, shoulders and front of the chest. In the dog traumatic emphysema is rare.

SYMPTOMS.-Bouret reported a subject that, in consequence of a wound at the elbow, became absolutely monstrous. The limb, neck, trunk, and head were transformed 
into shapeless objects, and completely obstructed the outlines of the skeleton. A similar case in a mile is reported by Pellegrin, from a wound in the same region, in which the emphysema extended over the lower third of the neck, the lower part of the shoulder and breast and backward over the lower third of the ribs as far as the twelfth. The same writer reports another case in a "heavy" mare, in which the obstruction covered the neck, the two shoulders, and the ribs, restlting from a fracture of a rib and a wound of the lung.

PROGNOSIS and TREATMENT-Traumatic emphysema is not generally serious, as air may remain in the sulbcutaneous connective tissue without any serious detriment to the organism, and besides, unless generalized, it yielcls to appropriate treatment.

Scarifications must be avoided as far as possible. Efforts must be made to remove the air through the original opening by pressure and massage. If the entrance wound is small it may be enlarged. When, with gas, there are irritating substances, such as alimentary matter for example, scarification and disinfection of the cavities so formed, may be resorted to to complete the procedure.

\section{CICA TRICES OF THE SKIN-CUTANEOUS SCARS.}

The word "cicatrix" or "scar" applies to the new formation that succeeds a loss of substance or a breach of continuity. All of the tissues, muscles, tendons, bones, etc., are capable of forming scars, but in this article only cutaneous scars will be considered.

SYMPTOMS.-As soon as the tissues are divided by a traumatism the organism becomes occupied with the repair of the breach. In order to fill the gap caused by the loss of substance the affected tissues build up flesh vegetations 
called "granulations," whose development is either normal or abnormal. In normal granulations the wound is of a good character and of good aspect, and the granulations of which it is composed are regular, rosy, and firm. In the abnormal granulations the cicatrization is slower, more irregular and the breach assumes variable characters. The granulations may be exuberant when they pass considerably beyond the level of the surface to be repaired, fungous when they lie outside of the limits of the wound and erethic when the sensibility is excessive. And finally, they may become the seat of infectious complications called diphtheritic granulations which are coated with a pultaceous, yellowish substance that hinders disinfection. The surface is smooth and is only disinfected with the greatest difficulty. These mouldy sores, well known and described by military veterinarians, are especially frequent at the close of summer, in animals exposed to water or treated continually by showering. Uider the outer pultaceous coating the granulations are reddish and flabby, bleed from, the slightest contact, and show no tendency to complete their organization. The product that covers the granulations is composed of cellular waste, colonies of streptococci and most frequently mucus. They are frequently complicated with lymphangitis.

Lastly there are cases where the scar develops in the regular manner but sustains injury during the process of healing. This injury is frequent in animals that again fall upun their incompletely healed knees and tear open the scar that was closing around the breach. The new lesion is often deep and extensive, but heals by a perfectly normal process.

\section{KELOIDS.}

DEFINITION.-In both immediate and secondary cicatrization the growth of granulations first brings the scar to the level of the wound and thus fills the trammatic cavity; 
the granulations then become covered with epidermic patches, from periphery to center, which serve as the definite covering. Rather frequently the hyperplastic reparative process continues, and the connective-vascular tissue becomes an abnormal, exuberant vegetation that results in the formation of hypertrophic sclerosis. The totality of the process is the formation of a cicatricial keloid-"a sort of subacute fibroma of inflammatory origin."

ETIOLOGY.-Among the domestic animals the horse is a frequent sufferer from keloids. In this species they result from the actual cautery, contusions, blows, vesicants, and even simple irritants. They are encountered most frequently where the skin is not firmly attached to the subjacent tissues. The anterior face of the hock, the tendons and the fetlock, the flexion surface of the pastern, and the coronet are their locations by predilection. They frequently originate from a prominent cicatrix, linear or in buttons, according to the form of the lesion, which is generally considered to be a fibroma and which is extirpated or caurerized. These manipulations arouse the dormant cellular activity, and the insignificant lesion of the beginning becomes a. voluminous tumor that is extremely vascular and capable of rendering the animal useless. Young weak subjects, reared under bad hygienic conditions, are predisposed to the lesion.

Among the bovines these accidents are rare. Moreau has observed some on the rump of a cow, that traced a long cicatrix in the form of a scar, circumscribing the hip and rumning along the entire extent of the left flank. They are exceptional in the dog and small animals of the farm. Labat refers to one in the ram.

SYMPTOMS.-Mediate abnormal cicatrization may give rise to projecting scars, sensitive scars and finally keloids. The first two states are often the starting-point of 
the last. Projecting scars are of but little importance, as they are no obstacle to the usefulness of the animals affected, and they do not disfigure unless very extensive. Sensitive scars are found in the regions where the skin is displaced during locomotion. Those located on the neck or shoulder often become so painful as to seriously interfere with the adjustment of the collar. Carougeau reports a lameness due to a sensitive scar resulting from the extirpa-

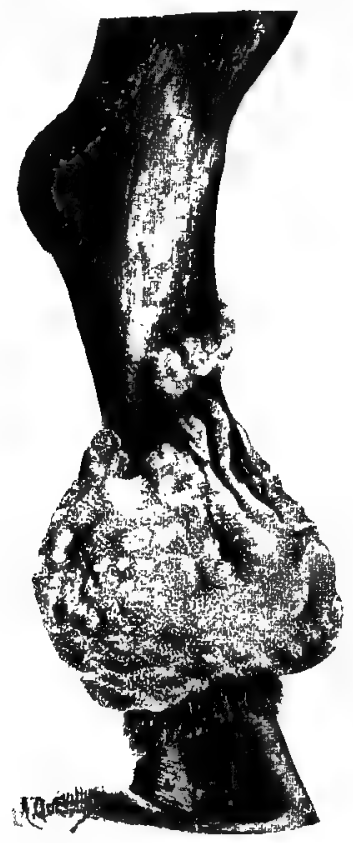

FIG. 31.

Monstrous Keloid Caused by Accidental Cauterization with Sulphuric Acid.

tion of a cold abscess of the mastoido-humeralis, in which the removal of the scar tissue rendered the animal useful. We have observed sensitive scars on the tendons of a horse, following the actual cautery, so painful that the animal was useless until median neurectomy was performed. The operation of neurectomy, badly performed, often is followed by 
sensitive scars that provoke a continuation of the lameness, in spite of the division of the nerve.

Keloids are real fibrous tumors generated on irritable, projecting scars. They are the expression of a too active. cicatricial process, and of an over-abundant cellular hyperplasia. They may develop in different ways after insignifi. cant wounds.

They reappear and extend after ablation. Their origin has not been accurately determined. They may follow the most trifling lesion and clevelop in animals in perfect health. Slight but continuous irritations of wounds in the course of cicatrization seem to be the cause in many cases. They may acquire considerable dimensions and weigh many kilo. grams. They occur in the form of large, reddish, oozing tumors that bleed easily. They, are complicated with lym phangitis and often render the subject useless.

The observations published on this subject by Labat are very interesting.

PATHOLOGICAL ANATOMY.-Cicatrix. Tissue in - the course of cicatrization, besicles very numerous vascular plexi and different connective elements, contains speciai cells termed fibroblasts containing a highly colored nucleus and a granular protoplasm provided with extensions which meet progressively in connective fasciculi. The development of the connective tissue takes place rapidly. By degrees the connective cells are flattened and smoothed out, and then assume the physiognomy of ordinary connective tissue. Simultaneously the rest of the tissue is modified. The newly formed blood-vessels sink, retract, atrophy and disappear without leaving any traces of their existence except some anæmiated cells and some canaliculated capillaries.

Thus the scar tissue is produced. It is a tissue that is desiccated as much as the granulation, its generator, was succulent; ischemic as much as the other was largely irri- 
gated; dense and firm owing to its fibrous fasciculi as much as its predecessor was loose and unresisting; and lastly, poor in flat cells, and even in atrophied ones, as much as the granulation tissue was living and richly cellular. In short to sum up, in a word, the young and connective-vascular tissue has been replaced by the tissue of sclerosis.

Keloids.-From the microscopic point of view the keloid is similar to the cicatrix, with the difference that its blood-

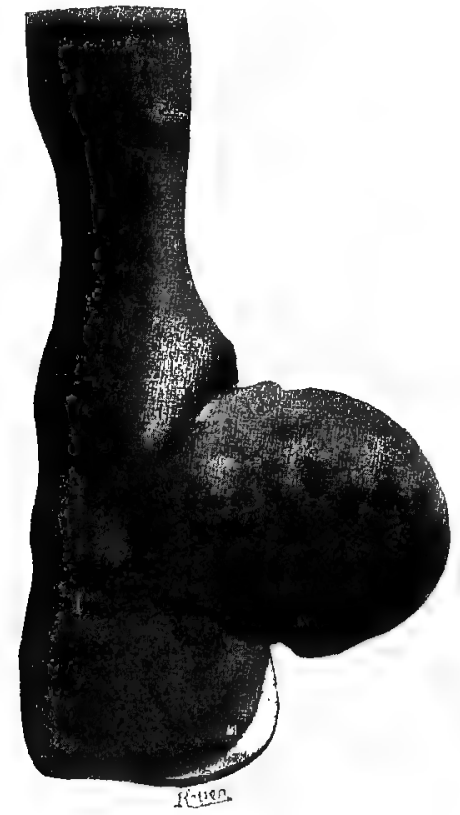

FIG. 32.

Keloid Resulting from Some Form of Firing.

vessels are numerous. They are accompanied with embryonic cells which are very probably the centers from which the tumor extends. When they have a wide base, the blood-vessels, the nerves and the tendons of the region are clrowned in a fibrous tissue which is the zone of attachment of the neoplasm. This arrangement is well marked when they are located in the flexion of the hock. It is evident that their extirpation is not only inadvisable but actually impos- 
sible. They may be covered with horny epidermis and composed of dense, hard and fibrous tumors. This phenomenon is not rare in connection with the keloids of the horse and the ox.

TREATMENT.-Some cicatrices, those of the knee of the horse for example, materially diminish the value. They may be removed, if not too extensive, by resection of the bare spot. The operation is known as "operation for broken knees." The projecting cicatrix of limited size, that causes no obstruction to locomotion, should be left alone. Sensitive scars that prevent the use of the animal require surgical intervention. If a scar in the skin of the shoulder, the inodular tissue should be extirpated, if the collar can not be advantageously adjusted. If the scar of neurectomy is sensitive it may be necessary to make a new incision and resect the proximal end of the nerve.

Keloids can not be successfully managed by any sort of surgical intervention. They must be left untouched. In every case in veterinary practice the extirpation that is not complete has been followed by a recrudescence of the phenomena of proliferation and by the re-formation of a tumor more voluminous than the preceding one.

This re-formation has been known for many years in human surgery, and as a consequence those neoplasms are not molested. It is actually hazardous to remove all the tissues of the scar, and even if the operation is performed under the strict rules of asepsis, the results are not often favorable.

The reappearance of these neoplasms is due, in all probability, to the absence of immediate cicatrization, or else to the regeneration that occurs from some embryonic islets that the surgeon has spared or has been unable to remove. Ries advises excision, followed by a compressive dressing, astringents and actual cauterization. The recent observa- 
tions of Professor Labat show the excellent results it is possible to obtain by the application of the elastic band. The latter is placed around the base of the tumor, in a groove made with the bistoury. The growth is carefully disinfected and showered each day. Labat attributes the wonderful effects to the showering and the protective dressings which are applied to the tumor.

\section{FOREIGN BODIES.}

The accidents caused by foreign bodies are frequently observed in veterinary pathology. In every species foreign bodies, entering from without, may cause a variety of different lesions. The gluttonish habits and the rapid first mastication of bovicle renders the acciclent conspicuously frequent among them. Ordinarily the foreign body is ingested, as the keeper, perceiving possible danger, rushes upon the animal to snatch it away.

Independently of the ingested foreign body, all animals sustain variable accidents by the penetration of different objects under the skin and into the muscles. Sprigs of wild grass may penetrate the dog's paws and cause fistula, while pieces of iron, splinters, needles and nails may pierce into the body and break off, leaving a fragment and some mutilated tissue nore or less deeply imbedded. When of small dimensions they may pass through the skin, and at first not betray their presence by any perceptible symptom. If aseptic they may remain in the tissues indefinitely without causing any inconvenience, and if absorbable may break up and disappear little by little. (Fig. 33.) The organism possesses a remarkable toleration in this connection. Bullets and shot received by dogs while hunting may remain in the tissues without harm for years.

SYMPTOMS.-If a foreign body is septic, its presence soon provokes a reaction manifested by inflammatory phe- 
nomena and the appearance of an abscess at the affected point. The collection points at the surface and discharges an unhealthy liquid that is generally fetid, and if the body is not removed the suppurating process will continue, the tract will become fistulous and the wound will not cicatrize. Incision and exploration of the fistulous tract then become necessary to reach and remove the irritant body that perpetuates the purulent discharge. Cadeac thus cured a pleural fistula in a horse by incising the external orifice and searching for a foreign body in a partitioned pouch of the pleura, which proved to contain a glass tube. It would require sev-

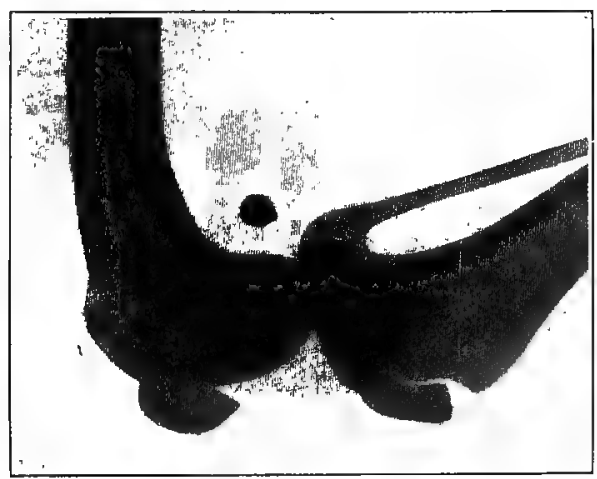

FIG. 33.

Revolver Bullet in a Dog's Stifle (Radiograph).

eral chapters to complete the studies of the various accidents in animals caused by foreign bodies. Only the most frequent ones can be considered, and those only in a general way.

In the dog and cat foreign bodies rather frequently lodge in the mouth. Twine, thread or rubber bands sometimes become knotted on the tongue and cause tumefaction. Savigny reports such a case as occurring in the hen. In the horse and the ox they may lodge between the teeth, in the tongue, in the internal face of the cheek and in the pharnyx, provoking stomatitis, pharyngitis and more or less marked dys- 
phagia. When the sharp bodies once pass the pharynx they generally descend into the digestive receptacles and there cause varied manifestations. Blunt bodies of considerable dimensions may lodge in the pharynx and cause signs of asphyxia or else descend into the osophagus and obstruct it. And, again, such bodies may descend into the stomach and accumulate there in considerable quantities. They may remain in the rumen of an ox for an indefinite period without clisclosing their presence by any morbid phenomena, if composed of inert substances (lead, paper-covered plaster, etc.)

The operations of sharp bodies are varied. They may impede the movements of the rumen by their bulk.

Mathis reports the interesting history of a cow that swallowed the stiletto of a probang, which, being of considerable size, could be made to project by depressing a point in the lejt flank, from whence it was surgically removed.

Sharp bodies are tusually small and may remain in the digestive organs for some time without causing any trouble. Most frequently they become fixed in the reticulum, a contractile organ with an areolar arrangement that is well adapted to admit of implantation and fixation of foreign bodies. If they take a forward course they cause pericarditis, purulent pleurisy, or pulmonary abscess. When directed upward and to the left they cause abscess of the spleen, and when downward and to the right an abscess of the liver. If they remain in the rumen they may become fixed to the walls and cause local peritonitis and at the same time excavate a more or less extensive passage through the abdominal muscles, at which point a phlegmon appears and then terminates in an abscess that points and discharges pus containing dregs of alimentary matter from the rumen. This termination constitutes the well-known fistula of the rumen. In other cases the rupture of the abdominal wall may cause hernia of the rumen. And lastly there are exceptional in- 
stances in which the bodies travel a considerable distance before effecting an exit. If they meet an important organ on their passage they deposit the seeds of abscess in them. If they encounter an important artery an aneurism is formed or fatal internal hæmorrhage results.

The other open cavities, besides the digestive, do not frequently receive foreign bodies. However, Guillebeau, in a report on a series of cases, refers to one in which the vaginal mucosa was injured by the maneuvers of a psychopath. In the respiratory organs, bodies that accidentally fall into the trachea may cause broncho-pneumonia. In certain diseases of the ox fragments of hay and bits of chaff may aspirate into the bronchial tract. This accident is often compatible with life, attacking but a single lobe, which is generaliy the anterior. The conjunctiva does not endure a foreign body well. In the large and small ruminants seeds, chaff. etc., may lodge under the eyelids and provoke a serious sup purative process if ignored.

TREATMENT.-The mode of intervention is necessarily variable. It is well to follow the principle of respecting an aseptic foreign body that causes no disturbance. If infection is suspected,- - and it soon becomes apparent,prompt intervention is necessary. The nail prick or strcetnail wound are grave accidents that require special as well as prompt surgical treatment. Accessible foreign bodies causing fistula should be dislodged and removed. Those fixed in the mouth or pharynx may be pulled out with forceps or hands, and those in the osophagus are carried back into the pharynx or pushed into the stomach; or, in the case of failure, œsophagotomy will become necessary. Foreign bodies in the stomach and intestines may be removed by laparotomy, and gastrotomy or enterotomy. Morez removed a spoon from the stomach of a dog, and a bullet from the intestines of another. The operation performed by 
Mathis to remove a probang stiletto from the rumen has already been mentioned.

When abscesses form at the surface, they must be opened and appropriately treated. I'or abscess of the pericardium Moussu's operation may be attempted. The operation consists of making an incision into the pericardial sac, through the abdominal wall at the bisection of the angle formed by the xiphoid cartilage and the median line of the body.

Foreign bodies in the eye are removed by cocainization, with the forceps, folded carcl, or the index finger.

1 further consicleration of this subject would be out of place here, as these several accidents, their development and the details of their treatment, are inc! tuded in surgery of the different regions. 


\section{CHAPTER XIII.}

\section{ABSCESS.}

DEFINITION.-An abscess is a collection of pus that hollows out a cavity in the tissues by destroying and expanding them. The word "abscess" may also be appliecl to depos. its of pus in the natural cavities, i.e., in the sinuses, in the guttural pouches, etc. The term "collection" is, however, a mire suitable designation for such accumulations.

CLASSIFICATION.-The study of abscesses requires 11 umerous classifications. We distinguish hot (or acute) abscesses when the evolution is rapid, and cold (or chronic) abscesses when the progress is slow. These are again subclivided into superficial, deep, idiopathic and symptomatic. Multiple suppurating foci following a fixed collection of pus are called metastatic abscesses.' Critical abscesses are those which supervene as the sequel of a grave malady. Their appearance is followed by a marked improvement in the patient's condition. Closely related to this variety are sudden abscesses, which occur chiefly among clebilitated subjects and appear without any signs of inflammation. Pneumonia in the horse, terminating unfavorably, is often marked by an outburst of abscesses of this character. As to their contents, abscesses may be classified according to the special products they contain, into biliary, stercoraceous, urinary, milky, etc.

ETIOLOGY.-The dominating causes of abscesses are either predisposing or exciting:

I. Predisposing causes.-(a) Species.-All writers concu1 in admitting that certain species suppurate more readily than others. The horse, for example, is usually regarded 
as having the greatest pyogenic aptitude among the domestic animals, but this is not entirely correct, because horses as beasts of burden are more exposed to conditions which favor abscess formation than the other species. In spite of the impression that solipeds are more susceptible to abscesses, it seems that collections of pus are not more frequent than in the other species, proof of which is shown by the following statistics:

In 438 dogs observed at the Lyons Hospital in the course of several we ks nine abscesses were found, being a proportion of 2.05 per cent.

In 590 horses, only 20 cases, or $3.3^{8}$ per cent.

In $26 \mathrm{I}$ oxen, only Io cases, or 3.83 per cent.

In 70 hogs, only 3 cases, or 3.94 per cent.

In 144 cats, only 6 cases, or 4 .I 6 per cent.

In 5 I goats, only 5 cases, or Io.Io per cent.

These statistics demonstrate very clearly that abscesses occur in the following scale of ascending frequency: Dog, horse, ox, hog, cat and dog.

(b) Race.-Horses of coarse breed-lymphatic animals -are more subject to abscesses than those of the finer breed. [The greater liability of the coarser breed to injury must be taken into account.-L. A. M.]

(c) Age, diet, mode of utilization, and the hygienic conditions in which animals are kept, are also well-known factors in the etiology of abscesses.

2. Exciting causes.-In the horse contusion, friction, pressure, or abrasion is usually the primary cause of an abscess.

Pre-existing diseases-endocarditis-may frequently prove a dominating factor. Cadeac has seen an abscess suddenly attack the anterior limb of a horse recovering from endocarditis. The sane author has seen an ileo-spinal abscess as the result of a nail wound in the foot. Operations 
upon animals threatened with strangles may be followed by an abscess formation. Firing, for example, may result in cutaneous suppurations.

In the horse abscesses make their appearance in regions most susceptible to injury, - the poll, the withers, the acnestis, the buttocks, and the lower extremities of the limbs.

In the ox, horn-thrusts, blows, and bruises of the teats are the most common effective causes. Among milk cows abscess occurs in the udder, three times in ten cases; and in the parotid region twice out of ten cases. Collections of pus are also found with considerable frequency in the flanks, the thigh, the shoulder, the costal region and the ischial region

In the goat abscesses are most frequent in the region of the horns; in the hog, about the toe-nails, the lesions of thrush being often their starting point.

In the canine species collections of pus are encountered more especially in the eyelids, the ears and the tail. In the cat they are found in nearly every part of the body.

Role of Micro-organisms. - The causes above enumerated in the different species operate by bruising the tissues and by provoking the formation of "points of least resistance," but they also introduce at the same time the indispensable element in the formation of every non-experimental abscess -namely, the infection.

The introduction of pyogenic micro-organisms into the subcutaneous connective tissue, under usual conditions, is promptly followed by a collection of pus at the seat of inoculation. The role played by the organisms in the genesis of the abscess is therefore easily shown. Aseptic traumatism brings negative results. Introduce aseptically a foreign aseptic body beneath the skin and no results will follow, but soil it and abscess will be promptly evolved at the seat of the inflammation. Research, especially cn the human body, has shown that every idiopathic abscess contains a more or less 
pure culture of a certain micro-organism. Ogston, since I88I, has found micro-organisms in all of 74 abscesses examined.

In abscesses of the domestic animals, the same pyogenic micro-organisms are found as in the human species. These are:

Staphylococcus pyogenes albus

Staphylococcus pyogenes aureus

Staphylococcus pyogenes citreus

Staphylococcus pyogenes flavus

Streptococcus pyogenes

Streptococcus of strangles

Bacillus Pyogenes Fetidus (Coli Communis).

The microbian flora of abscess of the horse has been studied by Nocard, Schultz and Lucet. Lucet examined 93 abscesses and found the white staphylococcus, pure or mixed, 47 times, - three of which were pure cultures, and the citrine variety 28 times, out of 75 cases examined.

Among the animals of the bovine species the pyogenic micro-organisms are very probably the same as those found in the other species. The differences in the cultures on microscopic examination must be attributed to the modifications of the environments. Lucet, in studying the microbian flora of abscesses in the ox, has met chiefly staphylococci and bacilli. In this species must be included the bacillus of Hofflich and Enderlen, which generates pyelonephritis with abscess formation in the kidneys. In the hog the microbe of Charon is frequently associated with the ordinary microorganisms of suppuration. (Cadeac)

In birds fetid abscesses are sometimes observed in the neck and head. (Jacquot and Legrain). They are noted for the offensive odor of the pus they contain. Jacquot and Legrain have found this pus to contain a thick, short, motile bacillus I to I. 2 microns thick, and I. 8 to 2 microns in length. 
They have cultivated it on gelatine, gelose, potato and in bouillon. The pure cultures have produced typical abscess in hens. A young pigeon has developed chronic septicæmia, and several mice have died in from fifteen to twenty days foilowing an inoculation of these cultures. The authors are of the opinion that the bacillus in question belongs to the species bacillus pyogenes fetidus, and that it exists abundantly in dung.

Among the various pyogenic micro-organisms the staphylococcus pyogenes aureus is the most frequently encountered. Karlinski found it 82 times, the albus 55 times, streptococci 45 times, and the other different organisms only I I times in 193 cases examined.

Pyogenesis is not a specific infection and is therefore not the work of these microbes alone. Suppuration is, in fact, a general mode of reaction of the tissues against microbian infection.

Without wishing to rewrite a history of suppuration, it is desirable to point out the elements found in pus and the mode in which it has been possible to produce an abscess with the micrococcus tenuis, the micrococcus prodigious, the proteus vulgas, the bacillus anthracis, the fetid bacillus, etc.

In the horse and ox botryomyces and actinomyces may be the starting point of pus collections; and, besides, certain microbian toxins possess marked pyogenic properties, and some inert powders and some chemical substances have an analogous action. But the pus found in these cases differs from ordinary pus in that it is reabsorbable and inoculable.

These data clearly show the important part played by micro-organisms in the formation of abscesses.

PATHOGENESIS.-We shall study what takes place in the subcutaneous connective tissue-the areolar system being the system in which abscesses develop or toward which they all tend. When the infection has 
taken place, it creates a toxi-infectious nodule in the connective tissue; that is to say,-a point at which the first morbid phenomenon is marked by the death of a certain number of cellular elements. The moment it is attacked the tissues react; the phenomena of vaso-dilatation make their appearance. The blood carried in large quantities to the exact spot affected, throws into the abscess in miniature a considerable amount of lymphatic elements, which, being added to the fixed cells originally attacked, constitutes, with the serum exuded, the first evidence of pus. The latter, increasing in quantity through the incessant addition of new elements and the dissolving action of microbian diastases, hollows out a cavity and presses upon the surrounding tissues. The tissues build protecting materials,-the pyogenic membrane,-around its mass. The pyogenic membrane of the author's is the patent manifestation of these protective elaborations. When the abscess has been established, its formation, which finally results in the encystment of pus, may be regarded as a victory for the tissues, as against the micro-organisms. The formation of pus is, in the absolute sense of the word, a curative effort, a step in the process of recovery.

\section{HOT, OR ACUTE, ABSCESSES.}

I. SUPERFICIAL ABSCESSES.-In every species, hot superficial abscesses are announced by well known local and general symptoms.

(a) Local Symptoms.-At a given point of the surface of the body there is noticed, in addition to a tumefaction, all the signs of inflammation. The tumefaction is hot, painful, diffuse and œdematous, and the skin, when not masked by hair or pigment, is reddened. After a time, varying with the character of the abscess, the original tumor becomes wontracted, localized, diminished in size and more projecting, 
and then generally at this period the central part becomes softened and the phenomenon of fluctuation comes into evidence.

The word "fluctuation" is applied to a combination of sensations determined by the sense of touch, disclosing the presence of a liquid collection in the tissues. Fluctuation is recognized by placing two or three fingers of the same hand on the tumefaction, while, with the fingers of the other hand, pressure is cautiously exerted at another point of the tumor. The liquid displaced by the active hand lifts the fingers of the hand that remains passive. Several repetitions of the manipulation are essential to make a successful deduction. Fluctuation associated with inflammatory manifestation is a positive indication of abscess.

(b) General Symptoms.-The establishment of an abscess is usually accompanied by general systemic disturbances which are often scarcely perceptible, but may in other cases be rather intense. The symptoms marked during the early stage of the process become modified as the tumor defines its limits, and disappear entirely when the abscess formation is complete.

2. DEEP ABSCESSES,-The first evidence of deep abscess is announced by a train of very grave symptoms without any indication of the abnormality on the surface of the body. Pyrexia, acceleration of the respirations and pulse rate, injection of the mucous membranes, and anorexia are notably in evidence. This first period, exclusively characterized by general symptoms, is of short duration. After forty-eight hours to three days the temperature lowers, and a "revealing œdema" makes its appearance at some point on the surface of the body. Shortly the œdema defines its limits, and having partially disappeared, the fluctuation becomes perceptible. During the progress of development abscesses of this variety are usually accompanied.by special symptoms 
-lameness, roaring, etc.-according to the organs affected.

Preceding the clinical study of deep abscesses it may be advantageous to consider pus collections which gather in the extremities, the rectum and the pharynx with reference to their frequency and their gravity, making of them a group of abscesses of which an accurate knowledge is essential.

(a) Abscesses of the Limbs.-Abscesses at the lower extremities of the limbs announce their presence by intense lameness and the appearance of an œdematous swelling. The thickness of the skin on the limbs, and the existence of an unyielding aponeurosis, make these abscesses behave much the same as the genuine deep variety, and, if ignored, the pus spreads along the tendons, leads to considerable detachment, and sometimes to irreparable accidents.

(b) Abscesses of the Rectum.-Pelvic Abscesses. In this form of abscess the mischief is often first revealed by a lameness in one of the hind legs, followed at about the same time by a hot, painful obstruction, especially pronounced about the pudendum. The anus is more or less displaced, defecation difficult or impossible, and the general symptoms always very grave. They are frequent among colts suffering from strangles.

(c) Abscesses of the mouth and pharynx are the consequence of the insertion of foreign bodies in the buccal mucosa, or the remains of former sore throats. The movements of the head, neck and shoulders are limited and painful, and the muscles of the neck and shoulders are contracted. The parotid region and throat are the seat of a very accentuated obstruction. The patients are subject to roaring, deglutition is painful or impossible, and the pyrexia and other general symptoms are pronounced.

PROGRESS. - The progress of abscesses is variable. Some of them develop in eight days, while others delay several weeks before maturing. Gienerally speaking; the 
rapidity of their development depends upon the resistance of the tissues of the region where the abscess is growing. Pus collections have a tendency to burst through the skin, or just at its junction with mucous membranes. Explanation of this phenomenon has been sought and the most improbable hypotheses have been advanced. Hunter has compared the tendency of pus to direct itself towards the external surface of the body with the unknown force that guides the feather towards a wave of atmosphere. The pulsating force of the arteries has been relierl upon, and researches have been made which tend to demonstrate the part played by the blood current in the movement of pus. In reality the exact canse of this phenomenon has yet to be discovered. In certain organs the path taken by the pus can easily be explained. In the cornea, for example, the lamina have a disposition which in a very manifest fashion favors the movement of pus towards the surface. In other organs pus obeys the laws of gravity. Its tendency is downward when its course is not governed by muscular movements which can compel it to spread in one direction or another. It is, however, necessary to bear in mind that the different muscles are joined together by connective tissue,_-"ce tissu d'union,"as Bichat called it, and that pus has quite a natural tendency to $s^{1} i p$ into the interstices at points where there is the least resistance. These connective tissue spaces are in more or less close relations with the dense areolar tissue beneath the skir and this favors the collection of pus at that point. The micro-organisms and their diastases then tulcerate an orifice through the skin and the collection empties itself externally. It is not rare to have purulent eruptions of the withers forming abscesses in the chest. Diseases of the foot are often starting points of abscesses in the pastern, and abcesses of a scirrhous cord may burst in the upper inguinal region, etc. 
When an abscess is near a mucous cavity in the interior of the body it progresses downward, and frequently bursts within the cavity. It is usual for pus to burst into the mouth, into the pharynx, into the œsophagus, and into the rectum or vagina. On the other hand it is exceptional to see it penetrating into the peritoneum.

TERMINATION.-Whether the abscess bursts spon. taneously or in consequence of a liberating incision, the flow of pus is established, and the purulent secretion continues for several days, if the interior of the pus cavity is not modified.

PATHOLOGICAL ANATOMY.-The pathological anatomy of an abscess comprises the study of two essential parts: (r) The container, the sac previously described as the "pyogenic membrane," and (2) the contents, the pus.

I. Study of the Container-the Sac.-Since Delpech's work the internal face of the pus sac has been described by the term "pyogenic membrane." The cavity is sometimes traversed by vessels and nerves that have resisted the disso!ving action of the pus. The walls of the cavity which the pyogenic membrane confines are tomentous and draped with connective tissue cells in the course of proliferation. According to Le Tulle the pyogenic membrane resembles a serous membrane in the state of irritation. It differs in no way from inflammation of the peritoneum. It leads to an excessive diapedesis. The pyogenic membrane is constituted at the expense of the connective tissue in which the abscess is developed. Sometimes it leans against a muscle, a tendon, an aponeurosis, a bone, a blood-vessel or a nerve, which react more or less to the inflammation.

The muscles offer little resistance to the dissolving action of the microbian products, especially when they are not protected by a thick fibrous membrane. On the other hand the aponeurosis and the tendons are not easily encroached upon. Their close texture protects them against destruction 
unless the phlegmon is sufficiently intense to provoke exceptional disorders. The fibrous tissue then changes its character; it becomes leaden, and assumes a characteristic gray or greenish color. The periosteum and the serous membranes offer marked resistance and become thickened. Blood vessels may remain in the midst of a purulent center without appreciable alteration for a rather long period, but will eventually yield to the dissolving process, and when suppuration persists, their coats may become corroded, completely perforated, and death may supervene from hæmorrhage.

2. Study of Pus.-The study of pus belongs more particularly to the pathological anatomy of inflammation in general.

Pus collected in a vessel after opening of an abscess is a creamy, opaque liquid, varying slightly in color and consistency, according to the kind and nature of the suppurating organ. The pus known as "laudable" is a liquid of animal odor, sweetish taste, and of alkaline reaction. That of the mouth and rectum is fetid; that of the parotid region has a repulsive odor; that of the urinary tract has the smell of mice; and that of the mamma the smell of milk.

In the horse pus is generally yellowish and of a semiliyuid consistency, while in the bovine species it is thicker. In certain cases it may acquire the consistency of putty, for which reason it may require more time to perforate the skin. In consequence of the thickness of the integument, the serum may be absorbed by degrees and leave in the cavity only a thick, doughy product adhering to the pyogenic membrane. In the hog, pus is sometimes colored green, owing to the presence of the bacillus pyocyanus. In the dog, pus collections are frequently mingled with more or less blood, and hence are of a chocolate color.

In every species pus collected is evidently composed of two parts, like blood and lymph: One part, solid, consisting 
of purulent globules, and the other liquid, which is simply serum. Serum and leucocytes are not in unchangeable proportions: The first is in proportions of 7 to IO, 8 to ro, or 9 to IO; and the second of 4 to IO, 3 to IO, or 2 to IO.

The solid part of pus contains leucocytes belonging to different varieties. There are also modified fixed cells, connective tissue fibers, elastic fibers, hematic fibers or fibrin, and crystals of various kinds. Different parasites,-echinococci, botryomyces,-may also be found. These different elements sooner or later, undergo fatty degeneration. In other cases the serum being absorbed the pus is transformed into a cheesy mass which becomes harder and harder until it resembles chalk, and which is infiltrated with calcareous deposits. The serum separated by filtration is a clear liquid of a yellowish hue, which becomes coagulated from the effects of heat and of nitric acid. It contains lecithin, cholesterin, fatty bodies, chloride of sodium, carbonates and phosphates, to which it owes its alkalinity.

DIAGNOSIS.-The diagnosis of superficial abscesses is always easy, but deep collections of pus may present serious difficulties. Blood tumors, cysts and hernias are the ailments which may be confounded with abscesses. Blood tumors are rapidly formed, are immediately fluctuating, and they become crepitant when coagulation has taken place. A cyst is uniformly fluctuating, without œdema and without any sign of inflammation. Hernias, especially in the bovine species, may Le confounded with abscess, which they often accompany, and, like them, may on their first appearance be accompanied with œdema. When the latter disappears the proof of the hernial orifice removes every doubt. When the abscess is deep there must be no hesitation about resorting to exploratory puncture, which is always free from danger. This recourse removes every doubt and may furnish a basis for therapeutic purposes. 
Diagnosis of Suppuration by Blood Cultures. (See Chapter on Hæmatology).

PROGNOSIS.-The seriousness of abscess depends upon its location. Collections of pus are always grave when located in the neck, shoulders, withers, poll or limbs, and the gravity is still greater when the abscess is deep-seated. Abscesses of the pharynx may lead to asphyxia; those of the pelvis may obstruct the expulsion of excrement; those of the sheath may prevent the emission of urine, etc., without taking into acount the numerous complications which they may provoke. Idiopathic abscesses are less grave than those which are the manifestations of a general malady,strangles, glanders, tuberculosis, etc.

TREATMENT.-Abcesses being so frequently the result of a summary infection of wounds, the first requisite is to prevent them by keeping wounds aseptic. When a phlegmon is terminating in the formation of an abscess, and attempts at aborting it have failed, it is necessary to wait until it is "ripe" before opening it. Formerly, especially in human medicine, efforts were made to abort the process by medical means, or rather by premature tapping, but this mode of treatment has had its day. Incisions into undeveloped abscesses may be followed by relapses. Today the "ripening" of the phlegmon is hastened. Hot baths are certainly the best preparatory remedy for abscesses in the limbs. In other regions blisters are often used in veterinary practice. This expedient hastens the progress by augmenting the inflammatory phenomena at the affected point. Emollients such as poppy ointment, camphorated pomade and preparations of vaseline, without having the properties of a blister possess an unquestionable alleviating action. When fluctuation appears, the abscess should be tapped. The opening should be made with the bistoury, the cautery or the trocar. The operation is much more urgent when the pus collection, 
while developing, threatens to effect an entrance into the neighboring muscular plane, the synovial membranes or into the large splanchnic serous membranes.

The incision is made in the most dependent part in order to facilitate the flow of pus. If sinuses, anfractuosities or diverticula exist, a counter opening is made. For superficial abscesses simple tapping may be sufficient, but when the cavity is one of any considerable size a liberal incision is es. sential. For deep abscesses the incision must be made successively through the different layers.

Veterinarians frequently use the cautery instead of the straight bistoury for this purpose. The cautery must be brought to a white heat, and must be inserted into the fluctuating part by pressure and semi-rotation. By this method the hæmorrhage that follows is considerably less than with the bistoury, and the cutaneous wound has not the same tendency to close too rapidly. The trocar is specially used in tapping abscesses that threaten to burst in the region of a mucous membrane, but it offers no special advantages.

Whell an abscess is opened should it be left to itself? In the last stages it has been advised to currette the fleshy tomenta from the pyogenic membrane in order to remove the microbian cultures with which they are impregnated, but this is a useless precaution. This does not mean, however, that nature must be left alone, as repair will be more speedy, and the cure more rapid if the sac is well washed out with a solution of carbolic acid to draw out the remainder of the pus and disinfect the pyogenic membrane and thus facilitate the constriction and reunion of the fleshy tomenta. Under these conditions cicatrization rapidly supervenes.

\section{COLD ABSCESSES.}

It is difficult to draw a well-defined line of demarcation between hot (acute) abscesses and cold (chronic) abscesses, as between these two are all the intermediate varieties. 
Chronic abscesses are characterized by the slowness of their development and the mildness of the inflammatory phenomena of which they are the seat. They are divded, like het abscesses. into idiopathic and symptomatic. In the latter category are included local symptomatic abscesses which develop in the neighborhood of the affection by which they are generated, and of which suppurative phlebitis is an example. In this group is likewise included abscess from congestion, an examp.e of which are the abscesses that occur in the shoulder as a sequel to an affection of the withers; lymphatic. abscesses that have their seat in a gland; and finally abscesses (the term beino descriptive of a general malady) of strangles, g.anders, tuberculosis, etc.

From a clinical point of view these distinctions are not important, cold abscesses being classified for this purpose, according to their consistency, into hard and soft abscesses.

FREQUENCY.-Chronic abscesses are frequent in the horse, the ox and the dog.

ETIOLOGY.-Hard chronic abscesses occur in the hurse as the sequel of repeated contusions. Contact of the harness in certain regions, at the neck, withers and the point of the shoulder is the principal contributing cause. They are a!so produced in the limbs by "interfering" and on the projecting points of the body from the decumbency of diseases. Frequently they result in necrosis or caries, and are often observed among debilitated animals exhausted by hard work or by a previous pathological state.

In the ox the greatest number of cold abscesses arise from the passage of a foreign body through the tissues, after an acute abscess has inoculated the neighboring regions.

PATHOGENESIS.-Not long ago it was supposed that all cold abscesses in man were of tuberculous origin, but the recent researches of Mauclaire, Bertoye, Roger, Arloing, Achalne and Conamin have demonstrated that these pus 
collections may be the work of the pyogenic micro-organisms,-staphylococcus, pyogenes alluws and aureus, streptococcus pyogenes, pneumococcus, etc. In domestic animals chronic abscesses are likewise provoked by the inoculation of the micro-organisms of suppuration. Bossi has stated that in abscesses of the shoulders there are special nicro-organisms which may exhibit particular qualities, but wich may have only a moderate degree of virulence. In reality, however, these agents belong to the pyogenic micro-organisms.

In our bacteriological examination of the microbian flora of cold abscesses we have found that in every case the organisms were those of suppuration, notably streptococci and rarely staphylococci.

Their introduction into the subcutem occurs through excoriation of the skin, or through the medium of the cutaneous glands or hair follicles. Their propagation through the blood channel is exceptional.

SYMPTOMS.-Hard Abscesses.-These pus collections are characterized by a more or less voluminous and diffused tumefaction, without any clearly marked acute features. Sometimes they are accompanied by a light "edematous cloud." They are not hot, slightly or not at all painful, and the skin is only feebly attached to their surface. Finally they develop the characteristics of genuine fibrous tumors, and fluctuation is scarcely ever a perceptible feature. When located in the mastoido-humeralis, at the base of the neck and shumldcrs, they rencler the animal unfit for service by the permanent pain produced by the pressure of the collar.

Soft Abscesses.-Soft abscesses present the physiognomy of cysts, and are uniformly fuctuating without inflammatory characteristics. They may, however, display a slight peripheric cdema, and may be the seat of a rather keen sensibility. They are nearly always abscesses from congestion. 
PROGRESS.-As a rule chronic abscesses have a tenclency to remain stationary. In exceptional cases inflammation ma be aroused and the abscess transformed into a hot abscess.

DIAGNOSIS.-Chronic abscess of the neck and shoulcler nay be confounded with fibroma or melanosis. The seat may, however, lead to a diagnosis. The existence of a tumor more or less fibrous in front of the shoulder, for exaniple, would at once suggest itself as a cold abscess. The differentiation is based upon the perfect delimitation and the absolute mobility of the skin if the case is one of neoplasm. If. on the contrary, the case is one of cold abscess, the region will not be absolutely free from swelling and the skin will be more or less adherent and thickened. Fibrous tumors associated with botryomyces always expose to view a fistula which discharges a purulent product rich in parasites.

PROGNOSIS.-Chronic abscesses are always graver than the hot ones on account of the slowness of their evolution, the nature of the causes which govern their development, and the difficulty which is often found to make them disappear. When they make their appearance after the necrosis or caries of a cartilage or ligament (fistula of the withers, poll-evil, quittor, etc.) they still further complicate matters.

PATHOLOGICAL ANATOMY.-The hard variety are composed of a fibrous wall, sometimes very thick and adherent to the skin, which is generally thickened, infiltrated and hardened over a considerable area. It is unusual to see the wall of the abscess adhere to or become mixed up with muscular tissue, as the latter will be found to have undergone fibrous degeneration. On being cut the hard abscess reveals one or more cavities of very limited dimensions, containing a thick, greasy pus that is subject to calcareous infiltration when the lesion is of long standing. 
The soft variety, on the contrary, have a thin wall and the pyogenic cavity is very large and filled with a serous pus that is deficient in formative elements.

TREATMENT.-Here, as in the hot abscess, it is indispensable to provoke the elimination of the pus. This is sometines obtained by reviving the inflammatory phenomena in the hard abscess by the irritating effects of a b!ister of biniodicle of mercury. Usually these expedients end in nothing and the case will require lancing with the bistoury or cantery.

Tapping with the bistoury is often without resu ts in cases where the purulent center, very slightly developed, is composed of cheesy pus. In this event it is necessary to plunge the bistoury several times into the fibrous tumor. The inflammation resulting from this operation may break up the purulent center and liquefy it.

Tapping with the cautery is more frequently resorted to than the preceding method; it may, in fact, while provoking the elimination of the pus, hasten the disappearance of the inflammatory tumor.

In some rare cases these two methods of intervention are of no avail. Bayer and Langzillotti recommend that under such circumstances three deep incisions should be made in the wall of the tumor. In each of these incisions a drain is inserted and kept in place by a suture. The cavity of the abscess is each day cleansed with a solution of mercuric chloride. At the end of two weeks the drains are removed, and the cure is generally complete in from twenty-five to thirty days. Finally there are some cases in which the extirpation of the abscess is the only proper means of intervention. This operation, which was greatly in vogue some years ago in Germany, does not always yield the desired results. The part operated upon often remains painful and the op- 
eration is often dangerous on account of the proximity of the jugular and carotid artery.

Chronic, soft abscesses should be opened, like hot ones, in the most dependent point, and the cavity disinfected by some antiseptic solution.

\section{ANNOTATION.}

Special Treatment.-Abcesses in any part of the muscular system. accompanied with a thick wall of degenerated muscular tissue (muscle sclerosis), but especially the common example, developing in the mastoidohumeralis at the point of the shoulder, very often prove obstinately persistent and refractory. Extirpation of the entire tumefied area is the most effectual cure, but it often necessitates almost impossible transgression into structures supplied with large vessels which render the operation rather hazardous. This is particularly the case with the cold abscess of the shoulder that is closely related to the carotid artery and jugular vein. And besides, being a tumefaction without any well defined limits, and deeply rooted into the muscle tissue, it is absolutely impossible to locate its nutrient vessels until they have been cut in the course of the dissection. Then, often the flow of blood is so copious and the location of the vessel so hidden, that its arrest is extremely difficult. Ligation is usually impossible and grasping with the hæmostatic forcep is seldom successful. Compression by packing the wound taut with wadding and then suturing it is the only method remaining that will save the patient's life. This emergency recourse is not without danger. The blood may still flow inwardly and dissect its way upward along the loose areolar tissue of the jugular groove, or else into the thiorax. One case operated upon by Dr. (—) died six hours after operation from hrmorrhage into the thoracic cavity after the wound had been hurriedly packed to save the patient's life. Kragness reports two similar cases answering the same description, in which the life was saved only by the timely administration of salt solution.

Now, in view of these possible accidents, it is evident that total ablation of all of these tumors is not advisable. Only those having more or less well defined limits and located at a safe distance from the jugular groove should be submitted to the radical operation of total extirpation. The others, which are in the majority, may be successfully eliminated by combined incision, enucleation and cauterization. After the tumor is shaved and disinfected It is divided in two equal halves by a vertical incision through its sclerotic walls. The incision, which in addition liberates the pus, is made across the entire tumor and into its pus cavity, but no farther. A considerable portion of the sclerotic wall, between the skin and the pus sac, is then cut away by sectional enucleation on each side of the incision, so as to hollow out a cavity of considerable size. This done, a hot iron, (or a number of them consecutively) is plunged into the hollow cavity until the remaining part of the tumor (its periphery) is literally cooked. In a few days the, eschar sloughs out and cicatrization promptly terminates the condition. This operation may be performed without any hæmorrhage at all if the hot iron is applied to the wound as fast as the incision and the subsequent enucleation 
are made, but this precaution is hardly necessary in view of the extremely short duration of the operation. Special irons are required to obtain the best results. They should be shaped like a drum-stick, with a head about one inch to one and a half inches in diameter. Only the head is heated, and the handle, before plunging the head into the wound, should be cooled completely with cold water, to prevent burning the skin and superficial tissies. With this latter precaution it is indeed remarkable how painless the oferation really is. Only the first plunge causes a slight resistance. The virtue of this technique depends, not alone upon the sloughing of the tumor. It seems that the intense heat to which the surrounding parts are submitted terminates the degenerative process in the tissues, and at least thoroughly disinfects them.-L. A. M.

\section{ANNOTATION.}

\section{Thecal Abscess of the Horse.}

The term "thecal abscess" is the appropriate appellation for a number of special lesions of the horse, consisting of accumulations of purulent products in great tendon sheaths. They are found chiefly in the navicular sheath, the sesamoidean sheath, the tarsal sheath, and occasionally in the carpal sheath. Other sheaths (those of the extensors) are also frequently affected, but only the larger ones are of any serious consequence in this connection. These suppurations are of sufficient frequency and seriousness to require special mention in the study of abscesses. In the sheaths of tendons concerned in supporting weight, (the sheaths of the flexors) abscesses are attended with grave consequences, that either prove fatal, produce permanent blemishes and lameness or at least run a troublesome course of long duration.

Etiology.-Sheath abscesses are always traumatic. They are due to the introduction of infective matter into the synovial membrane through abrasions, punctures or contusions. They are rarely ever secondary abscesses. In the colt, secondary articular abscesses occur from navel infection, but in these cases the sheaths are never implicated except through their contiguity to the purulent articulations. Sheaths may, however, become infected from one another by the spread of an infective inflammation from below upward in the affected limb. Abscess of the navicular sheath due to a nail prick, may thus, in rapid succession, attack the sesamoidean and the tarsal sheaths, by encroaching upwards along the tendons. This spread of the disease is, however, limited to very acute infections. The usual thecal abscess remains quite tenaciously isolated within the limits of the synovial membrane that sustained the initial injury.

Abscess of the navicular sheath is generally caused by the nail wound that penetrates into its synovial membrane. In some instances it follows the apparently trivial nail puncture, owing to the encroaching tendency of the infective inflammation, so thoroughly confined beneath the hoof. These abscesses also follow gangrenous dermatitis (furunculus) in the region of the heels. The sloughing of patches of skin often opens channels for the borrowing of purulent products downward into the synovial membranes in the region, and thus creates a serious synovitis with all its attending results. In this manner the sesamoid sheaths are frequently infected. These structures are, however, most frequently injured from interfering. The contused 
skin produced by constantly striking the fetlock with the opposite foot or shoe becomes the seat of an infective inflammation which, by continuity of tissue, extends into the synovials beneath. Accidental punctures with stable forks are also often the starting point of these serious abscesses, in the region of the fetlock. The tarsal sheath is generally infected from-kicks, fork-punctures, etc. The aspirating needle inserted to evacuate the contents of a thoroughpin has bèen known to carry infectious matter into the synovial membrane, and thus set up a serious infective inflammation of the entire sheath, that terminated by producing a prolonged suppurative process, an acute obstinate lameness and a permanent blemish of the region. Tarsal abscess may also occur from somewhat mysterious causes and in total absence of any perceptible breach in the skin. Severe straining of the part, followed by the localization of pyogenic microörganisms in the injured focus, offers the only rational explanation of some of the suppurative tarsal inftammations. In many instances the articular synovials are implicated either primarily or secondarily.

Symptoms.-The first evidence of the trouble is a more or less marked lameness, tenderness of the region and a hot odematous tumefaction spreading in every direction from the trauma. Shortly the symptoms accentuate. The lameness increases, the tenderness becomes more acute and the limb no longer supports weight. If in a posterior extremity, the foot is held some distance from the floor in a forward direction and is kept in constant motion from the pain. When forced to move the patient will hop with difficulty on the sound limb. In the anterior limb, the carpus is flexed, the elbow dropped and the foot rests almost on its anterior face. When the morbid process is located in the sesamoid, carpal or tarsal sheath, the œdema becomes more circumscribed, and the tenderness diminishes simultaneously with the appearance of one or more fluctuating points somewhere along the outlines of the sheath. When lanced, or allowed to burst spontaneously a combination of liquid and coagulated synovia is discharged. This event, contrary to the usual expectation, is not followed by any amelioration in the lameness. The inability to support weight persists stubbornly and the abscess openings continue to discharge the purulent synovia in somewhat limited quantities. The openings dry up only to point elsewhere, until finally the entire synovial membrane is destroyed by the inflammatory process. Then, and then alone, will the period of improvement commence. As long as there is a vestige of the infected, secreting membrane left, the abscesses will continue to form.

Gradually the patient begins to place the foot, heel first, to the floor, and to show some slight inclination to bear weight when forced to walk. For some weeks the leg is pushed well forward in walking. The stride is exclusively anterior. The lameness becomes less and less during two or three months, and finally ceases entirely, but the region remains hard, indurated and tumefied. In some cases the lameness never entirely disappears and the patient remains a more or less decrepit subject thereafter. These symptoms apply equally to the abscess of the sesamoid, tarsal and carpal apparatus, as the navicular sheath, owing to its confined position within the hoof, presents a somewhat different aspect. The first evidence of the real nature of the trouble in this sheath is severe lameness accompanied with synovial discharge from the nail puncture. At first the patient walks upon the toe, but soon no weight is supported whatever, and ere long a marked 
swelling appears above the coronet between the bulbs of the heels. The abscess may or may not burst at this point. If the inferior opening freely pcrmits the outward flow of the secretion, the tumefaction above the hoof may not point. In very acute infections the inflammatory process advances to the.articulation or progresses upwards along the tendons and proves fatal from septicæmia and exhaustion or loss of the hoof. The recovery is slow and often incomplete, as in the case of the other varieties.

General Symptoms.-Aside from these serious local manifestations grave systemic disturbances always accompany the thecal abscess. The appetite is impaired, the temperature is elevated, often to Io6 degrees F., the pulse is fast, the respirations accelerated and frequently local areas of perspiration appear at different parts of the body. The patient will either remain permanently recumbent or stubbornly persist in remaining in the standing posture until the opposite limb becomes swollen and painfully exhausted from supporting the added weight. If the patient is not placed in exceptionally appropriate surroundings and given the best of general attention, septicæmia, exhaustion and decubitus soon terminate its existence.

Treatment.-The thecal abscess necessitates more general management of the patient than local treatment of the affected part. The subject must be placed in a large, well bedded place and induced,-even by force,--to lie down to protect the sound leg from the injury that may be sustained by supporting the entire weight of the affected end of the body: It is essential to roll the subject over several times during each day and to occasionally induce it to return to the standing position from time to time. In fact, the usual precautions to protect the patient against the inevitable results of decumbency. must be carefully executed. The internal administration of febrifuges, stimulants, and laxatives may be of service. The diet should consist of mashes, boiled feeds and the best quality of hay. Succulent feeds, in season, should not be omitted.

The infected region must be submitted to a frequent or even constant immersion with antiseptic lotions. Thick woolen wraps, repeatedly soaked in hot solutions of carbolic acid, creolin or mercuric chloride, ate highly recommended, on account of the practicability of their application. When the abscesses point they are lanced and irrigated moderately with antiseptics. After the abscess formation has entirely ceased. feather firing or blistering may be resorted to, in hopes of hastening the termination of the lameness. Shoeing with exceptionally high heel calks will also assist in hastening the healing process by encouraging the patient to walk about.-L. A. M.

\section{ULCERS.}

DEFINITION.-An ulcer is a suppurating wound that shows no tendency toward cicatrization. The process that terminates in an ulcer is called "ulceration." The word "ulceration" should therefore not be employed as a diminutive of "ulcer."

Ulcers are either symptomatic or idiopathic. Sympto- 
matic ulcers are those which are associated with a special disease, such as glanders, tuberculosis, carcinosis, etc., whose numbers seem to diminish from day to day, while idiopathic ulcers are still said to be results of unknown causes. Ulcers are classified acording to their special characteristics, into atonic, callous, fungous, inflammatory and phagadenic.

FREQUENCY.-Ulcers are rare in the domestic ani. mals except in the specific maladies. They are seen in the horse after carcinosis (and are commonly associated with glanders.-L. A. M.). On the extremities they are sometimes the result of blows, and now and then they can be traced to protracted decumbency. In the cat and dog they are found on the surface of certain tumors and on the paws and ears. The auricular canker of the $\operatorname{dog}$ is but an ulcer. Among bovine animals working in the marshes or rice fields or among those living in damp or dirty stalls, ulceration of the heels and extremity of the tail, is frequently observed. In the same species carcinosis may be the starting point of an ulcer.

PATHOGENESIS.-Ulcers are the result of a special diathesis, vascular changes, either arterial or venous, or they may be connected with nerve lesions. Brian has shown experimentally that perforating wounds in the paws of dogs follow section of the posterior (superior) roots of the lumbar and sacral nerves. Their frequency in the extremities has led to the supposition that they may be the result of an impediment of the return circulation, the tissues soaked with serum and placed in conditions of impaired vitality not being able to react against external morbific influences. Primary lesions of the veins, arteries, nerves and sometimes, the bones, are predisposing factors. The tissues supplied by these organs are themselves, more or less, seriously impaired. To use the expression of Reclus, they are "infirm tissues" If a solution of continuity is created in an insig- 
nificant trauma, a slight inflammation, or fugacious dermatitis, an ulcer may be promptly evolved. The anatomical elements, already diseased, having become deeply injured, are threatened with impending death. The trophic lesions on the extremities of neurotomized horses give a very clear idea of the phenomena presenting themselves in the evolution of an ulcer. Previous pathological conditions which diminish the vitality of the tissues, lessen the nutritive phe-

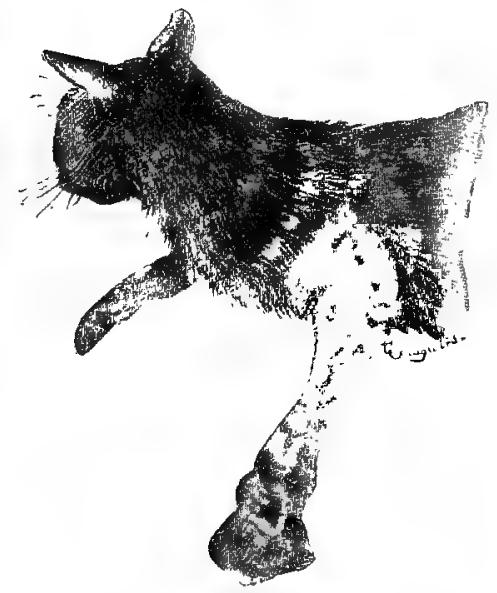

FIG. 34.

Ulcer in a Cat's Paw. (Cadeac.)

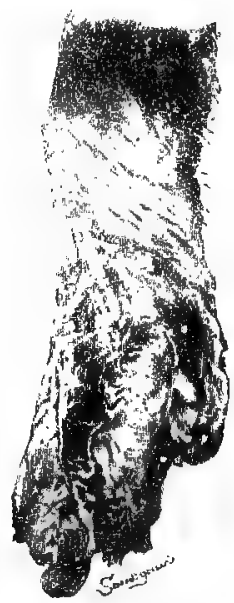

FIG. 35.

Ulcer in a Dog's Paw, (Cadeac.)

nomena or impair them, may be, for the same reasons as the preceding lesions, effective causes in the development of ulcers. The latter may in fact be met in cardiac and albuminuric patients. A peripheric neuritis may favor the appearance of sores which have no tendency to heal, but which, on the contrary, become aggravated upon the slightest friction or contact.

It may therefore be said, in a general way, that every influence that weakens the resistance of the tissues, places them at the mercy of every attack. The cellular elements of which they are composed no longer possess sufficient vitality to repair injury, and the lost substance can not be reproduced by the process of cicatrization. 
Under these conditions, it is easily conceived how trivial may be the causes responsible for the starting of ulcerating sores.

SYMPTOMS.-An ulcer may start with a cutaneous excoriation, an abscess, or an eruption. Its aspect is essentially variable according to the age of the lesion and the amount of celema of the region. When the part is œdematous the ulcer is depressed and spongy in appearance, and the sound skin shows a striking tendency to expand over it. The bottom is indented, greyish, and covered with little hremorrhagic spots. The granulations on the surface are soft, translucent and verging on necrosis.

When the lesion is old, the ulcer becomes inactive and is surrounded by a fibrous tissue that is very hard, very consistent, sclerotic and lardaceous, constituting the lesion known as callous ulcer. Its edges are cut perpendicularly, are hardened and devoid of all mobility.

When certain phenomena of gangrene appear at the level of the ulcer and the process spreads so as to destroy every tissue it encounters the lesion is known a phagadenic ulcer. (Fig. 34). Ulcerous sores with a pale, smooth, pultaceous, greyish back-ground and without granulating elevations are known as atonic ulcers. Those which bleed easily are designated as hæmorrhagic ulcers, and those which have been keenly sensitive from the beginning are called irritable ulcers. In all varieties the surrounding skin is hard and smooth. (Fig. 35). Their evolution is extremely slow and irregular, and they usually develop without pain, but may impede locomotion, and the ability to support weight, and they generally present symptoms of improvement and aggravation alternately. Spontaneous recovery is raré.

DIAGNOSIS AND PROGNOSIS.-The determination of the disease is simple enough, but the estimation of its exact proportions is often surrounded with real difficulties. 
The prognosis is always grave. Large animals may exhibit indelible blemishes after recovery, and the dog and cat have often to be destroyed on account of the expense entailed by protracted treatment.

PATHOLOGICAL ANATOMY.-In the environs of an ulcer the horny layer of the epidermis thickens while the deep layers become weaker; the papillz of the skin become enlarged and the blood-vessels increase in diameter. The skin becomes sclerotic and the ulcer itself is constituted of embryonic tissue resting upon a clense fibrous bed.

Ulceration is essentially characterized by the phenomenon of molecular necrosis accompanied with an inflammatory reaction. The adult elements of the tissues are replaced by embryonic elements, but the inflammation which is responsible for these transformations is not a typical one; it is always spurious and incapable of repairing the lesion. The newly-formed elements, being subjected to the same depressing influences as the pre-existing elements, degenerate and retrograde, before reaching a state of complete development. The phenomenon of mortification usually prevails over the process of repair. There is a conflict between two absolutely different states, and as one or the other predominates, the tulcer either becomes larger or is transformed into a benign sore.

TREATMENT.-The treatment must vary according to the nature of the ulcer. When it is atonic, essence of turpentine, or cauterization with silver nitrate is the most frequent recourse. If, on the contrary, it is fibrous and lardaceous, the thermo-cautery lightly applied has the most successful effect. Proud flesh, exuberant and colorless, which has no tendency to organize, should be destroyed with permanganate of potash, copper sulphate, or chromic acid. Painful ulcers may be treated with hot antiseptic baths and soothing ointments, and those having a tendency to trangress on- 
ward, should be combated with strong antiseptics in lotions or ointments.

The treatment of symptomatic ulcers varies according to the nature of the disease upon which their existence depends.

\section{FISTULA.}

The word "fistula" is applied to abnormal ducts (or channels) either congenital or acquired.

CLASSIFICATION.-Fistulæe are divided into two categories,-congenital fistulæ and pathological fistulæ. The former, which will not be discussed in this work, have their origin in an opening or embryonic cleft that fails to close. Of the latter,-pathological fistulæ,- there are several varieties. They are called blind or non-communicating when they terminate with a cul-de-sac in the tissues; complete or communicating when they have two openings; idiopathic when they follow an abscess; and symptomatic when they are connected with a deep lesion. When they burst in a mucous membrane they are called mucous fistulae, and if they open on the skin they are cutaneous fistulæ. They may be mixed, cutaneous, mucous, bi-cutaneous, or bi-mucous. German writers divide fistulæ into two groups:

Ist. Those which discharge pus.

2nd. Those which secrete or excrete the product of an internal organ.

FREQUENCY.-Fistulæ are of frequent occurrence in the horse, the ox, the sheep, the goat, and the dog. They are especially common among the horse and ox.

ETIOLOGY.-In the domestic animals hot abscesses are often the origin of fistulæ, especially when their evacuation has been delayed too long, or when the incision has not been made at the most pendent point. Lesions of the poll and withers of the horse, are for this reason so frequently complicated with fistula. Hot abscesses in contact with a 
duct, in a like manner, produce fistulous tracts, by dissolving its continuity. Peuch has reported the history of an csophageal fistula in a horse, following an abscess in the lower part of the neck. Ries mentioned a cutaneo-mucous, buccal fistula succeeding án abscess of strangles. Necrosis of the lateral cartilage is always complicated with a cutaneous fistula (quittor). Dental decay is a frequent cause of bimucous fistula in solipeds (dental fistula). In solipeds, foreign bodies are often the origin of inexhaustible suppuration. Cadeac observed a fistula that resulted from the retention of a glass tube in the pleura. Certain parasitic tumors of botryomycostic origin are nominally fistulous in character. In bovines the two sole causes are hot abscesses and foreign bodies. Abscesses of the abdominal wall which have been inauspiciously opened often leave fistulæ as a sequela. Abscesses of the udder may cause perforation of the galactophorous sinus or of the teat (lacteal fistula). Gastrotomy, rumenotomy, or urethrotomy, are nearly always followed by incurable fistulæ. Actinomycostic tumors are noted for their fistulous tracts after having attained a certain development.

In the ox, foreign bodies are the chief factors in the production of fistulæ. As foreign bodies are capable of penetrating everywhere and of ulcerating the skin at any point, they can thus excavate fistulous tracts in any part of the body. Berger has drawn a pair of scissors from a fistula in a cow. Observations of this kind are too numerous to mention. In the goat, fistulæ following abscess of the udder are very frequent. Coronary phlegmon or whitlow often leave very extensive fistulous tracts behind them.

In the dog dental fistulæ are not uncommon. Certain ulcerated tumors may cause complete perforation of the lips and finally it is not uncommon to find fistula following punctures of the plantar pad or interdigital spaces. Ducournean 
has reported cases of fistula in the dog due to punctures of the toes either from thorns or spikelets of grass.

SYMPTOMS.-The symptoms of fistula vary according to the character of the integuments or organs affected. Salivary, lachrymal, anal, and rectovaginal fistulæ borrow a particular physiognomy from the products to which they give issue. Some fistulæ discharge pus or mucous, while others emit milk, urine or stercoral matter. All, however, exhibit an orifice and a tract answering the following description:

The ofifice is either projecting or depressed, and may by virtue of its appearance be called "cul de poule." When there are a number of openings it may be designated as a "watering-pot" fistula.

The tract may be simple or bifurcated, rectangular or sinuous, of small or great dimensions. The tissue surrounding the tract is generally fibrous, lardaceous and resisting. The discharges irritate the integument and cause a superficial inflammation of the skin and loss of its hair.

There is also more or less emaciation, due either to the loss of some indispensable product,-saliva, gastric juice, intestinal juice, etc.-or to the inexhaustible suppuration.

PROGRESS.-As a general rule fistulæ show no tendency towards spontaneous recovery. Those which discharge a glandular or organic product remain stationary for an indefinite period. The persistence of fistulæ is due in some cases to infection; in others, the continuous flow of the liẹuid secreted has a permanent irritating action, which is further reinforced by a mechanical action resulting from the shrinkage or closure of the tract and the formation of new abscesses. Finally, others keep constantly extending until some effective treatment is given to oppose the effect of the pus in producing necrosis.

PATHOLOGICAL ANATOMY.-Idiopathic fistulæthose which follow abscesses, are nothing more than can- 
aliculate abscesses. Their tract is covered with a velvety membrane having the appearance of a mucous membrane, having a base of thick fibrous tissue. The surface of this pseudo-mucous membrane is constituted of proud flesh, which tends to become covered with an epidermic layer in the region of the orifice. The tracts which close up, cause a chronic inflammation in the surrounding tissues. The connective tissue is odematous and becomes thick and sclerotic, the vessels obliterate and the muscles degenerate, giving to the fistula the quality of piercing through a fibrous mass that obstructs cicatrization.

DIAGNOSIS.-Fistulæ may be readily recognized from the preceding symptoms. The discovery of an orifice that will admit a probe to a considerable depth, together with the continuous flow of a purulent product or liquid secretion, is sufficient evidence upon which to base a diagnosis. Injections with a liquid will determine whether the fistula is blind or complete, and will furnish more or less accurate information as to the capacity and extent of the tracts. And finally, an acute and persistent pain in a region where a sore of small dimensions is situated, e. $\mathrm{g}$. in the digital region of the horse testifies as to the existence of fistula.

PROGNOSIS.-The gravity of the disease varies according to the nature of the fistula. Secreting or excreting fistuia are always more serious than those which result from the non-cicatrization of an abscess. Their seriousness is also subordinate to the anatomical constitution of the region they traverse, and the nature of the lesion that perpetuates the suppuration. Every veterinarian is familiar with the gravity of fistula of the poll, of the withers and of the region of the lateral cartilages.

TREATMENT.-Having stated above that fistulae are nothing more than canaliculate ulcers, it is evident that their therapeutics must have some analogies with that of ulcers. 
The inflammation in fistulous tracts is always spurious and must therefore be transformed into an acute, free process. For this purpose three principal expedients are employed, viz., caustics, actual cautery, and setons. Among the caustics preference is given to sulphate of copper, sulphate of zinc, solutions of the three sulphates, nitrate of silver, chloride of zinc, permanganate of potash, resinate of copper, tincture of iodine and five per cent solution of carbolic acid.

When the fistulous tracts are very large and have a pendent cul-de-sac, the seton allows the pus to flow outward and by irritating the tract transforms the chronic inflammation into an acute process. It is sometime necessary to augment this effect by applying some irritating substance to the seton. For this purpose blisters, oil of turpentine, etc., are frequently employed. When the tract is limited in extent the cautery gives positive results.

These directions apply only to idiopathic fistula. For symptomatic fistula the therapy is quite different. Symptomatic fistula are manifestations of a deep lesion (necrosis or caries) and as a consequence the tract will only dry up after the purulent center has been removed. The preceding directions are therefore insufficient as a guide for the cure of the symptomatic variety. Recourse must be had to surgical intervention. The necrotic center must be extirpated, and provisions must be made to prevent the pus from lodging in the tissues during the healing process.

These general data require completion by the study of special methods of treatment employed for the various fistulæ (See Vol. III).

\section{ERYSIPELAS.}

During the last few years the word "erysipelas" has been employed by veterinarians to describe a number of surgical complications which have more or less remote analogies to 
the disease that occurs in man. Human erysipelas is caused by the introduction of the streptococcus, isolated and studied by Fehleisen, into a cutaneous wound or abrasion.

Whether the disease affects animals or not is a debatable question. It seems that certain observations warrant an affirmative answer, while others on the contrary, appear to establish the fact that so called, erysipelatous conditions in animals are the work of entirely different agents than the streptococcus. However in veterinary medicine the name "erysipelas" is applied to describe a series of affictions that have a doubtful relation to each other, and some recent observations seem to connect them with the same pathological stite. These observations try to connect erysipelas with a special disease characterized by rapid swelling of the head, whether accompanied with phlegmonous and gangrenous swellings or not.

SUSCEPTIBLE ANIMALS.-Moler, Hoffman, Lucet, Cadiot and Semmer, all have either observed the above disease in the horse or have published reports on it. In the ox, erysipelas has been described by Rychner and Forassassi, and Nuvoletti, Jucet, Cadiot and Fröhner have seen it in the dog. According to Fröhner it is no longer unknown in the hog.

ETIOLOGY.-As in man the clisease should be a complication of cutaneous abrasions, through which the microorganism should penetrate. Semmer has observed it in the horse after flexion crevices produced by severe cold. Lucet. Malzew, and Semmer have made a bacterial study of the disease in the horse with the following results: Lucet isolated a micro-organism having the characteristics of Fehleisen's streptococcus, that existed in small numbers in the blood, sp.enic and ganglionic pulp, and in large numbers in serum from the seat of inoculation. His microbe took the Gram and the Weigert stain, was cultivated in broth, on gelose and 
gelatine, and proved virulent to the rabbit on intraveneous injection. In three horses apparently afflicted with erysipelas, Malzew also found a streptococcus, which, inoculated into the horse, produced an injurious effect analogous to the original disease. Semmer obtained different results from the preceding writers. The entire three horses that came under his observation rapidly succumbed, and with the serum extracted from the swellings he inocuated a rabbit, a cavy, a cat and two mice, with negative results. On postmortem he found some staphylococci in the subcutem, and streptococci and bacilli in the effusion of the great cavities. Negative results likewise resulted from the inoculation of a horse with these effusions as well as with cultures of the organisms on agar-agar and gelatine. An erysipelatous swelling was, however, produced in a horse by an inoculation of a culture of the staphylococci on potatoes, and a cavy was killed by the same culture. In the cow Lucet found microorganisms in the serum of an erysipelatous phlegmon, in the form of diplococci and streptococci which took the Gram and Weigert stains. The micro-organism was easily cultivated in broth, on gelose, on gelatine and on potato, but inoculation of a rabbit produced no visible effect. Nuvoletti collected a bacterium (diplococcus or micrococcus) from the subcutem, from the pus of the abscesses, and from the excretions of affected bovine animals, which was well cultivated without losing form, on gelatine or on potato. The germ colored well, like the micro-organism of human erysipelas, with all the aniline colors, and it yielded to Gram's method. When inoculated into a rabbit it generated a disease similar to that in the ox from which the cultures were obtained. These bacteriological researches, which are the only ones known to the writer, do not entirely harmonize.

SYMPTOMS.-In the horse the disease presents the following manifestations: There are general symptoms 
of variable intensity preceding the appearance of any local disturbance. The patients lose their appetite, and now and then show signs of slight colic. They are melancholy; feeble, dejected and the thermometer testifies to an increased temperature. On the next day there appears a doughy and painful tumefaction, that rapidly increases in size. In Lucet's observation the tumefaction has developed on the head to the extent of disfiguring the animal. The head resembled an irregular, swollen mass which concealed all the prominences. In Malzew's horses, the tumefaction which first appeared on the lips extended rapidly to the different parts of the face, neck and chest, and resulted fatally in a very short time. Cases observed by Semmer had an absolutely analogous aspect. The patients presented tumefaction of the lips which rapidly reachéd the cheeks, throat and lower parts of the neck.

In the ox and in all bovines identical manifestations occur. Lincet reports that he saw the characteristic tumefaction commence at the left maxillary spine, spread rapidly over the same side of the face, throat dewlap and breast. These symptoms were accompanied with intense fever, suppression of the lacteal secretion and suspension of the appetite and rumination. During the days following, the general symptoms become more aggravated, the temperature rises to $40,40.5$, or 4 I.2 degrees $C$., the tumefaction spreads to various points of the body and the animal succumbs. According to Nuvoletti the bovine variety of the disease presents much more analogy to human erysipelas than that of the other clomestic animals. The infection generally proceeds from a lesion of the mouth or nasal mucosa. The tumefaction is hot, sensitive, œdematous, but not crepitant. It starts at the lower extremity of the head and reaches the neck and shoulders and sometimes displays small, purulent foci. 
Moller has observed a typical case in the dog. Fröhner, out of 70,000 sick dogs, has met the clisease four times.

In the hog, erysipelas exists in a popular form, and the disease can be transmitted to other animals of the same species. (Fröhner).

PATHOLOGICAL ANATOMY.-In the regions invaded, the skin is thick with infiltration, and the subcutaneous connective tissue contains a coagulum that creeps between the subjacent muscles. The mucous membranes are cadaverous and covered with hæmorrhagic exudation, the lymphatic glands are hypertrophied and dark, and the parenchymatous organs are engorged and hæmorrhagic. The pleura, peritoneum and pericardium contain a muddy red serum and are strewn with reddish stria and partially covered with petechial spots, which likewise affect the mesentery and mesenteric glands. The lungs and intestines are normal, the liver is attacked with fatty degeneration and the spleen is engorged with blood. The pulp of the spleen and liver and the blood contain streptococci in variable numbers. Microscopic examination of the diseased integument shows the connective tissue fibers dissociated by infiltration and the lymphatic vessels distended and filled with lencocytes. The specimens sometimes contain streptococci in considerable quantities. These descriptions of these lesions will also apply to the ox.

DIAGNOSIS.-The recognition of the disease seems always easy. The location of the swelling, its character and its rapid development are the important items, which with the general symptoms and the proof of the existence of the streptococcus (?) make the diagnosis certain.

PROGNOSIS.-If the published observations are true the disease is a serious one in the horse. Lucet's case died in forty-eight hours, Malzew's succumbed in a short time and none of Semmer's three survived. Death may also sup- 
ervene in the ox and dog. Oxen brought to the abattoir for slaughter are condemned.

TREATMENT.-The best method of combating erysipelas is to prevent it. As in all surgical complications, prophylaxis is the essential part of the therapeutics. Complete and methodical disinfection of the wounds and the preservation of the breach from every infecting object are the important directions. When infection has taken place and the disease has appeared antisepsis in all of its forms is the surgeon's recourse. The prolonged employment of tepid antiseptic lotions, subcutaneous and intradermic injections of disinfecting substances-powders and solutions-are generally sufficient to stop the extension of the tumefaction. Carbolic acid and mercuric chloride solutions give excellent results. When complicated with abscess formation, lancing and disinfection or cauterizations is essential.

\section{SURGICAL SEPTICÆMIA.}

The expression, "surgical septicæmia" is used to describe a group of pathological conditions that are less clearly defined in veterinary science than in human pathology. The group includes traumatic fever, acute septicæmia and putrid infection or hectic fever. These different morbid manifestations should be regarded as different phases of the same infection. Traumatic fever represents the initial stage-the most benign stage. It is a slight surgical septicæmia. If the symptoms by which it is revealed become more aggravated and persistent, the traumatic fever becomes acute septicæmia. The distinction between these two conditions is therefore not well defined; they blend into each other with all the intermediate stages between a passing traumatic fever and a very acute septicæmia which rapidly terminates fatally. Chronic septicæmia is the condition designated as putrid in- 
fection or hectic fever by old authors. In this condition the symptoms are less intense and more prolonged.

These conditions are the real septicæmias in the proper sense of the term. They are characterized by a poisoning of the blood rather than by microbian infection of that liquid. These surgical complications are, therefore, very different from pyæmia, which is itself an embolic septicæmia, as Vermulil correctly called it. Purulent-infection pyæmia is, in fact, a distinct colonization of micro-organisms, while surgical septicæmia is a poisoning of the anatomical elements by the microbian products elaborated in the wound and carried into the system by the blood. Before the time of Pasteur it was thought to be the absorption of certain particular substances, one of which-sepsine-seemed to produce all the trouble. In I 868 Bergmann claimed to have isolated this poison. Gradually the microbian doctrine, becoming better understood and better interpreted, supplanted the former theory.

The discovery of microbian toxins, and the numerous experimental researches in that connection, cast a new light on the origin of these affections. It was then admitted by all that fever following surgical operations is due to the entrance of toxins into the blood, that were elaborated by infectious agents in the wounded tissues. The studies of Bouchard and his school brought into full view the function of microbian toxins, their action on the nerve centers and particularly on the thermic centers. The experiments of Claude Bernard arrested the evolution of the doctrine of Pasteur and a long controversy resulted. The learned physiologist, Claude; maintaining that no fever resulted by implanting a nail in a horse's foot, the nerves of which had been previously cut, drew the conclusion that the fever should be attributed exclusively to the influence of the nervous system, and Vulpian shared in this opinion. But is not the absence of fever af- 
ter perfect antisepsis of the wound sufficient proof of the preceding doctrine? This does not, however, signify that there is no non-infectious fever. Bouchard has, in fact, demonstrated that nervous excitement, muscular fatigue and certain sensations, peculiar to hysterical subjects, may provoke a rise of temperature. But these are exceptional cases on which there is no occasion to dwell. In surgery fever should always be regarded as of an infectious nature. This is so absolute that fever is the precious symptom that informs the surgeon in the most perfect manner of the state of infection or asepsis of the part operated upon. If the temperature rises a day or two after the application of a bandage over a fracture there should be no hesitancy in uncovering the diseased region; suppuration has resulted; the asepsis has not been sufficient. If the temperature curve is normal after operation upon a nail wound in a horse there should be no fear in allowing the dressings to remain in place as long as the normal temperature exists. The revelations of the thermometer are therefore the most valuable to the surgeon.

The established fact that septicrmias are the result of poisonings of the blood with toxins does not mean that micro-organisms do not gain entrance to the "inner environment." In fact, bacteria are frequently carried along by the blood, but they are never numerous and never remain long in the system. They are either destroyed by the resources of the body or else expelled by the emunctories which constitute the excretory channels for waste products. Monod and Macaigne have met with streptococci in the blood-vessels of man, and Enriquez has shown that some are eliminated by the urine.

Together with, properly called, traumatic fever, there exists a febrile state designated as epitraumatic fever, in which the traumatism is not the direct cause of the pyrexia. In this event the fever supervenes because the injured point 
becomes the seat of a toxic elaboration recalled by a previous febrile state, as for example paludal fever.

In view of the preceding considerations a brief reference to the pathological anatomy, symptoms and therapy will be sufficient.

I. Traumatic fever appears after the absorption of toxins that are secreted in the wound. Certain conditions, more particularly the application of compressive dressings, which thus permit of accumulations of toxins, are favoring causes of the first order. The purulent product of an abscess cavity is in excellent condition to be reabsorbed unless it is allowed to flow out freely, in which event there will be no traumatic fever or septicæmia. The surgeon may make interesting demonstrations of this fact every day. If, in performing castrations, the incision is small, infection is facilitated and the microbian products not flowing out, accumulate in the scrotum and provoke fever. If the incision is enlarged and the sac drained and irrigated, the temperature retu. 'ns to normal. More than this, horses suffering from nail wounds frequently exhibit intense fever with the lomeness, which promply subsides after the operation of removing the infected tissues. What, then, becomes of the febrile action of the traumatism?

SYMPTOMS.-On the second or third day following the operation the general state of the disease is aggravated. The temperature, previously normal, rises to 39.5 degrees $C$. to 40 degrees $C$. The heart beat and the pulse rate are accelerated and the respirations become more frequent. Sometimes digestive and nervous disorders ensue. If matters are not remedied two issues are possible. Either the fever subsides and does not reappear, or else it rises again the next day and following days with exacerbations in the evenings. The former instance is one of simple traumatic fever. The temperature curve coincides with the absorption of toxins, 
which do not again appear, no doubt, because the micro-organisms which have penetrated into the blood have been destroyed by the phagocytes. In the latter instance there exists a condition intermediate between traumatic fever and acute septicæmia.

SYMPTOMS OF ACUTE SEPTICAMMIA.-Th e symptoms are graver and more persistent than in the preceding form. In the horse and dog the temperature rises from 40.5 degrees to $4 \mathrm{I} .5$ degrees $\dot{C}$. It lowers in the morning to gradually rise again in the afternoon, reaching its maximum at night. For several days the same condition continues. The animals are clejected and refuse all nourishment. The pulse and respirations are accelerated. If the lesion is on a limb there will be intense lameness, and unless relieved by liberal drainage of the poison the patient dies of exhaustion.

\section{SYMPTOMS OF CHRONIC SEPTICAMIA.-This} pathological state is described by the expression "putrid infection" or "putrid intoxication." It is characterized clinically by a slow febrile state of intermittent type. It is the result of the repeated absorption of microbian toxins in small doses. It was once described as hectic fever. The condition is seen in the course of chronic suppurations, as a sequel of certain extensive chronic abscesses,-fistulæ of the withers, poll-evils, quittors, etc. It also occurs in animals suffering from diseases which necessitate prolonged decumbency, especially when insufficient bedding is allowed. The numerous cutaneous wounds thus produced become the seat of a cupious absorption of toxic products which provoke fever and assist in exhausting the patient. Horses suffering from diseases of the extremities and cows after difficult parturition, presenting lesions of the bones and muscles, and animals affected with acute arthritis sonetimes die of putrid infection from 
this canse. In constant clecubitus, animals emaciate rapidly. They take but little food and the bruised skin becomes dry and adheres to the adjacent tissues. The hair is staring, dull and brittle, abundant dirt adheres to the integument, and all signs indicating a thorough impairment of the cutaneous functions follow. Gradually the eyes become sunken in the orbits, the extremities swell and the patient becomes marasmic.

The fever is never high, but it is constant and prevents the patient from eating and regaining its strength. It cundemns them to permanent decubitus, in which state they die after several weeks.

Colin has succeeded in realizing this pathological condition experimentally. The fever is due to products of putrefaction. When it supervenes fractures or contusions without wounds it should be regarded as the result of absorption of liquids and dead cells. Fever may also be produced by injections of sterilized putrid liquids of hay, mygdalein emulsion, myrosin, chymosin, stale beer, decomposed meat and stagnant water, even if it has been freed from microbes by filtration. Under usual conditions, however, there is no doubt that the intoxication is the work of numerous microbian colonies, combined secretions of which are absorbed in the region of the wound.

TREATMENT.-With the preceding data as a basis, a rational treatment is easily established. Prevention is par excellence the best method in combating these diseaces. Prophylaxis plays an important part in the treatment. That is to say, the surgeon, assistants, instruments, and surgical dressings should be clean, and the asepsis of the part operated upon ought to be as complete as possible. But when, in spite of everything, the disease has followed, the surgeon's attention must be constantly fixed upon the temperature. If the temperature rises it is of the utmost importance to reopen the 
wound and disinfect it. This is a law. For example, it is a matter of current observation to see firing with an infected cautery cause fever. The removal of the dressing and the thorough disinfection of the cauterized parts is rapidly followed by a fall of the temperature. Examples of this kind are of such frequent occurrence that it is unnecessary to dwell upon them at length.

In cases of acute septicamia, when the fever is high and persistent and the life of the patient is threatened, "washing the blood" is considered excellent treatment. The treatment causes expulsions of the poisons carried along with the blood, by rendering the secretions more active. The urine, copiously excreted, is the chief vehicle. These injections are made with sodium chloride solution, 7 to $\mathrm{I}, \mathrm{OOO}$, injected subcútaneously at a temperature of 38 degrees $\mathrm{C}$. It should be administered in large doses and frequently. A transient discomfort soon gives way to a marked improvement of the general condition. The fever abates, the animal becomes brighter, the mouth cool, the skin moist and all the chief functions are normally performed. At the same time, rich and easily digested food should be given to strengthen the patient. Even in the horse, milk is highly recommended. To combat the fever, if it persists, sulphate of quinine is the best remedy that could be prescribed. The administration of internal antiseptics is not to be recommended, as most of them give only meagre results. The effects of carbolic acid are more debatable. Alcohol and coffee are excellent aids in combating weakness and debility. They relieve the patients by stimulating them and producing diuresis.

\section{MALIGNANT EDEMA-GANGRENOUS SEP- TICÆMIA.}

DEFINITION.-Gangrenous Septicæmia or malignant cedema is an inoculable disease caused by a specific micro-organism swarming in the tissues. The 
specific agent responsible for this affection is the septic vibrio of Pasteur. [Koch designates it as the "bacillus of malignant œdema." (I. A. M.)]. The disease usually originates from an anfractuous wound, and it manifests itself by necrosis of the affected tissues, by the production of gas in the areolar tissue and by general symptoms of exceptional gravity. Its progress is rapid and termination usually fatal. It is a grave disease, and, like tetanus, it constitutes one of the most fearful complications of wounds. "It is," says Pasteur, "putrefaction of the living subject."

It was for a long time confounded, in veterinary practice, with purulent infection or putrid infection. It is now known to differ from these pathological states and to have nothing in common with them, as Colin states, "except septicity."

SYNONYMS.-The disease is known by several different names: (I) Traumatic gangrene. (Renault). (2) Acute purulent œdema. (Pigoraff). (3) Thundering, encroaching and instantaneous gangrene, and gangrenous septicæmia. (Chaveau and Arloing). (4) Very acute septicæmia, gaseous septicæmia, traumatic emphysema, gangrene with emphysema, malignant œdema (Koch), and bronzed erysipelas.

HISTORY.-Malignant œdema was well known to the old veterinarians. It was one of the formidable complications of "ante-Lister" surgery. At Alfort, Bartholemy and Dupuy attempted to reproduce it by placing putrefactive matter into the tissues of a horse. Vatel pointed it out as a sequel of setons. "In the course of the year I825," says Renault, "malignant œdema was so frequent after the application of setons that many veterinarians discontinued their use." In 183 I many horses of the eleventh artillery corps that were sent to Alfort were attacked with pulmonary diseases, and many of them died after the application of the seton as a counter-irritant. The frequency of these cases enabled Renault to study the disease carefully from the 
standpoint of its symptoms and pathological anatomy. To him the vitiation of the exuded blood exposed to the air wis the origin of the disease. He repeated the experiments of Bartholemy and Dupuy and reproduced the affection with its essential features. Renault was a precursor, as his idea of the disease has proven very accurate. "It is the entire economy," he says, "that is penetrated with poison, which exhibits its presence and effects by the following symptoms, etc."

For a long time the etiology of the disease remained hidden in profound obscurity. Different hypotheses as to its nature were advanced, but all were insufficient until the bacteriological era at last revealed the secret of the affliction. Some physicians,-Nepveu, Bottini and Samue1,- - found microbian agents in the blood and in the liquid of the lesions. In 1877 Pasteur brought to light the role of the septic vibrio in a case of animal septicæmia, and Morand attributed gaseous septicæmia of man to this agent. Davaine, Coze, Feltz, Chauveat and Arloing, above all, proved experimentally the identity of the septic vibrio of Pasteur with the microbe of gaseous septicæmia. Henceforth, gaseous septicæmia constitutes a well-defined morbid entity and numerous recent researches have confirmed the previous deductions. Nearly everyone recognizes the action of Pasteur's microbe. The German school alone, with E. Franckel, disputes the identity of the vibrio and the microbe of gangrenous septicamia, but up to the present time, this school has not advanced any fact that absolutely proves the doctrine it defends.

SUSCEPTIBLE ANIMALS.-Most all of the domestic species are liable to succumb from the inoculation of the septic vibrio. Cornevin, who has studied the receptivity of the different domestic species, has classed them according to their susceptibility in the following manner:

In the first rank-Horse; donkey. 
In the second rank-Sheep; pigeon.

In the third rank-Rabbit; cock.

In the fourth rank-White rat.

In the fifth rank-Dog; cat; duck.

During the period of lactation the receptivity is nearly equal in every species, the inoculations being always positive.

BACTERIOLOGY.-The septic vibrio discovered by Pasteur in 1875 has been called the bacillus of malignant œdema by the Germans,-Hoch and Gaffky. It is found in the blood, the peritoneal fluid, and muscular juices.

When examined immediately after death in blood serum, without staining, it is found in abundance. In this event it presents itself with the peculiar aspect of excessive length, which is often longer than the entire field of the microscope, and so translucent as to escape observation. However, when finally perceived it is found rampant, flexuous and creeping among the blood cells, as a snake moves aside the blades of grass in the bush.

On the borders of the preparation, where the air penetrates, it does not exhibit any movements, as oxygen is a veritable poison to it.

After staining with the various aniline dyes, but by preference with Loeffler's or Kuhn's blue, it appears clearly in the field of the microscope. It shows itself to be composed of unequal segments, placed end to end, flexuous, and crossing each other in different directions. Examined in the peritoneal fluid collected, for example, on the surface of the liver, it is found in considerable quantities. Its segments are short and isolated, united in pairs, flexuous, or joined in parallel series. Muscular juice, also very rich in the vibrio, shows ovoid bacilli swollen to the form of a club or bell hammer. Their peculiarities resemble those of black-leg, 
but contrary to the latter it does not take the Gram stain.

CULTURE.-Pasteur has described the method of culture in bouillon, and Liborius and Roux in solid media. It may also be grown in blood and in peritoneal fluid. When the first liquid (blood) is selected it is necessary that it be collected and brought to the incubator in a pipette, if taken immediately after death. The vibrio then grows exuberantly, producing numerous bubbles, and disorganizing the blood column. In bouillon it grows slowly at ordinary tempera-

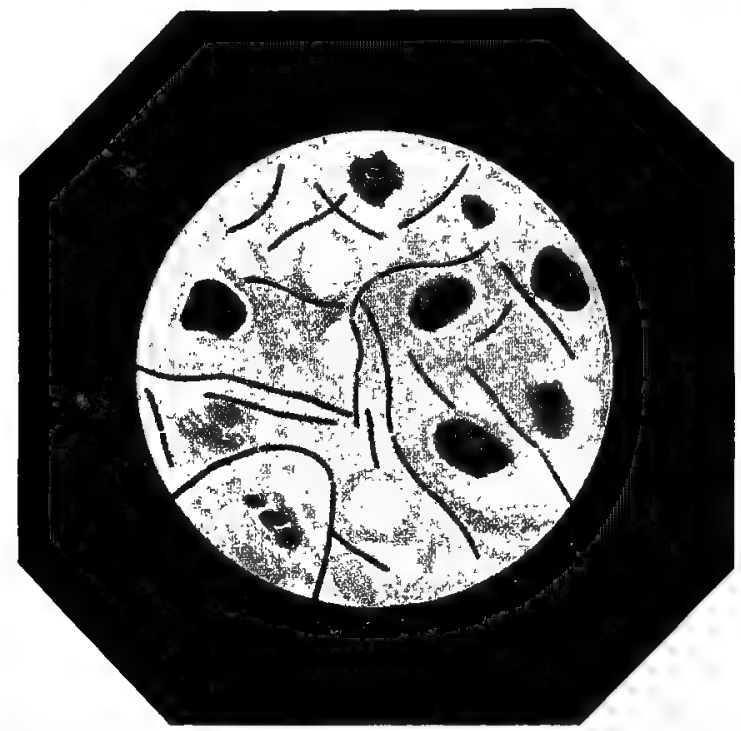

FIG. 36.

Blood of a Cavy, Containing the Septic Vibrio in Short Rods and Long Filaments.

tures. On the contrary, it grows rapidly at 37 degrees $\mathrm{C}$. It does not grow except in a vacuum or in an inert gas, hydrogen, nitrogen or carbonic acid.

The septic vibrio-bacillus septicus is, in fact, strictly anaerobic. In bouillon at 37 degrees $C$, the medium becomes cloudy, and after twenty-four hours a trifling quantity of carbonic acid and hydrogen is set free. Gradually the me- 
dium gets c'earer and a precipitate falls to the bottom of the container. On gelose the vibrio forms little whitish spots, which when examined with the microscope show themselves to be composed of tree-like filaments. In gelatine the colonies cause local liquefaction, which manifests itself by forming small liquid spheres. This liquid, clear in the beginning, becomes muddy and shows fine radial striations at the periphery.

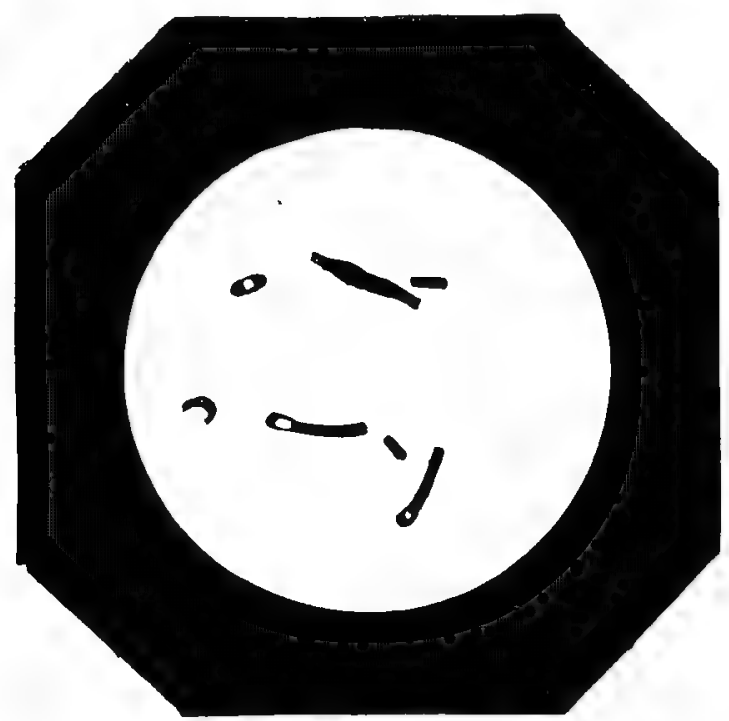

FIG. 37.

Spore Formation of the Septic Vibrio. (From Roux and Mace.)

On potato, the colonies develop deeply and form a closemeshed net-work. On solidified serum, liquefaction occurs rapidly.

EXPERIMENTAL STUDY.-It was Bottini, of Novar, who first tried the inoculation of the septic vibrio in the rabbit and cavy. He has exactly described the resulting lesions. In 1879, Tedenat, in Lyons, successfully inoculated the serum of blisters. Chauveau and Arloing submitted the knowledge of that day to the control of an extended exper- 
imentation that was methodical and rational. They classed domestic species in order of receptivity:
I. Man
7. Cat
2. Horse
8. Cavy
3. Donkey
9. White rat
4. Sheep
Io. Rabbit (average receptivity)
5. $\mathrm{Hog}$
נI. Chicken
6. Dog
I 2 . Duck

The inoculation of the frog by introducing the virus into the dorsal lymphatic sac is followed by no noticeable results, but if the aquarium is heated to 26 to 28 degrees C. the frog dies in two or three days, and comes floating to the surface all bloated with gas.

When inoculated in the connective tissue of the skin, the

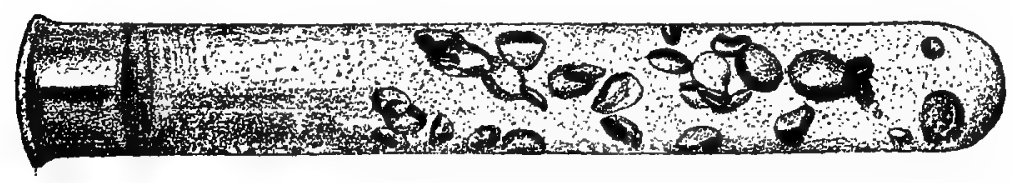

FIG. 38.

Culture of Septic Vibrio in Gelose after 24 hours at $34^{\circ} \mathrm{C}$. (Frankel.)

sheep becomes lame the succeeding day, and on the days following the inoculated limb is hot and tumefied; then the cedema spreads and the animal dies. In solipeds the progress of the disease is about the same. In birds there is an extremely abundant cedema rather than gaseous infiltration. From injections in the areolar tissue the cavy survives twenty-four to twenty-six hours; the dog four to six days; the ass two to three days; and the sheep two to eight days, and often succumbs earlier. Intravenous injections in doses of two to forty drops in whatever species or condition are never fatal. A dog injected with sixteen cubic centimeters of a virulent pure culture was not incommoded, but appeared more refractory from a subcutaneous inoculation. From intra-arterial injections the results are the same. Through the 
respiratory tract the results are negative, and the digestive tract is an equally unfavorable entrance channel in the reproduction of the disease.

Gangrenous septicæmia is transmissible from mother to fœtus.

The effects of the toxic products of the septic vibrio have been studied by Roux and Chamberland with intraperitoneal injections and by Rodet and Courmont with intravenous injections. Intravenous injections always provoke trouble and may cause death. The respiration is always markedly affected and the action on the heart is announced by diminution in the pulse rate and a brisk drop in the ar-

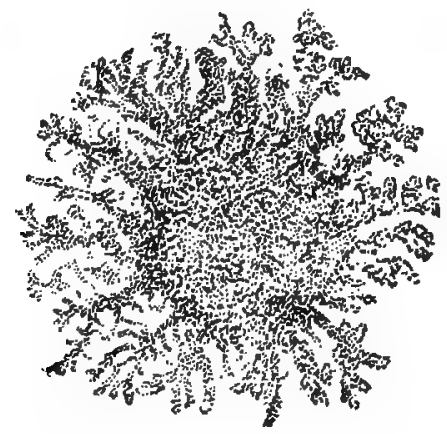

FIG. 39.

Bacillus Septicus. Colony Isolated in Gelose.

terial pressure. The temperature lowers and may fall to 35 degrees C.

RESISTANCE OF THE VIRUS.-The researches of Arloing have clearly placed in evidence the considereble resistance of the septic vibrio to the various antiseptic agents. Niter twenty-fours of contact, the mixture of equal parts with the following substances was not followed by any sensible modification in the properties of the virus:

Carbolic acid at 3 and 5 per cent

Boric acid at 2 per cent

Permanganate of potash at $\mathrm{I}$ and 2 per cent

Iodoform 
Oxygenated water at ro volumes of oxygen

Salicylic acid, I to 20

Solution of thymol

Solution of eucalyptol, I to ro

Corrosive sublimate, I to 200

Chloral, I to 5

The virus Toses its pathogenic properties after twentyfour hours contact with sulphuric acid, and only after fortyeight hours with the following substances:

Permaganate of potash, I to 50

Nitrate of silver, I to 500

Bromine vapor

Desiccation, and its abode in water, or soil, do not alter its virulence. The fresh virus is killed after several minutes exposure to a temperature of 100 degrees $C_{\text {., }}$ and the spores taken from the gelatine or agar cuitures are killed in five to fifteen minutes at Ioo degrees C.; in thirty to thirty-five minutes at 90 degrees, or in eleven to twelve hours at 80 degrees. The spores are not destroyed from prolonged desiccation of several months, and they equally resist the action of sunlight for fifty hours. Heating the dried virus at 120 degrees $C$ does not destroy its virulence when the temperature is only maintained five minutes, but the pathogenic activity is abolished if the heat is continued for ten to twelve minutes. Since I869, Semmer has established the fact that boiling water is not sufficient to sterilize a septic liçuid.

ATTENUATION OF THE VIRUS.-In I888, Cornevin clemonstrated that the septic vibrion was enfeebled by the action of several substances belonging to the phenol group in a temperature of 38 to 40 degrees C. He experimented with tannic, pyrogallic, gallic, phenic and salicylic acids; with quinine, hydrochlorate of quinine, sulphate of quinine, hydroquinone, resorcine, hydrochlorate of morphine, pyrocatechine and tonka bean. He thus pre- 
pared a weak vaccine with coumarrine and a strong one with gallic acid, and communicated immunity with this attenuated virus, especially to dogs and fowls, but the immunity never lasted more than twenty-four hours. $\mathrm{He}$ also demonstrated that the virus became only slightly attenuated after several passages through the white rat, and that the seventh generation thus produced killed cavies in the usual manner, and a part of the rabbits and pigeons inoculated, but respected the duck, the dog, and the cat, at least it was not effectual in the young subject. On the contrary, virus taken from the chicken and transmitted to the rabbit showed an augmented activity for the latter.

STUDY OF THE TOXIN.-Rosenberger was the first to study the septic poison. He endeavored to infect animals with small quantities of serum or blood taken from subjects that were killed with boiled septic liquids. Boiled putrefied blood should kill by simple intoxication and the liquids from animals dying from its action should not possess infectious properties. He proved that the boiled blood killed like ordinary blood and that the organic liquids of the experimental animals contained the same proto-organisms. He remarked, however, that the dose necessary to kill was greater than with the non-boiled liquid. In order to kill all the micro-organisms he carried the temperatures of the virulent liquids to I 40 degrees and even then produced death the same as with blood charged with the septic agents. From these results he concludes that the vibrion owes its action to the poison it elaborates,-sepsine.

Besson, after Arloing, Roux and Chamberland, Rodet and Courmont, also studied the septic poison, and proved that the toxicity of cultures attains its maximum on the sixth day; that in order to kill the cavy it is necessary to inject five to ten cubic centimeters of the culture into the peritoneum; and that the products secreted by the vibrion pos- 
sess a negative chemiotactic action. If heated to 85 degrees the chemiotactic properties of the poison become positive. He also studied the action of the pure spores on the organism in the liberation of their toxin by prolonged immersion or by allowing them to remain in the incubator for several months. The spores thus obtained could be injected in large quantities without causing death. The inoculation, at the point injected, was followed by abundant phagocytosis; the leucocytes seized and digested the spores. The best proof of the exactness of this deduction is found in the following example: If in some manner phagocytosis is arrested, septicæmia evolves. The spores treated with several drops of lactic acid,- - a substance having negative chemiotactic action,--produces the disease, as the chemical agent arrests phagocytosis. In the same manner, the addition of a small quantity of the toxin to the spores is followed by the appearance of phenomena that are rapidly fatal.

ETIOLOGY.-PATHOGENESIS.-Renault was one of the first in the veterinary profession to insist on the danger in exposing the tissues and the possible complications of wounds covered with blood-clots and mortified tissues. The contused foci sheltered from contact with the air constitutes a very favorable soil for the development of the disease, although it sometimes follows a small solution of continuity. Delamotte has seen it follow a subcutaneous injection with an improper syringe, and it is not exceptional to see it follow castration, strangles, setons, and compound fractures.

It was quite frequent at one certain epoch and is not absolutely rare today. It depends upon the abundance of the bacillus septicus (septic vibrion) in their exogenous habitat. Pasteur found it in cultivated earth where he was searching for anthrax bacteria, and Cornevin affirms that it is exceedingly abundant in the soil. He also found it in the 
water from the Saone, when, in winter, it was muddy and swollen. Before the work of Chauveau and Arloing, Professor Nocard, who has found the bacillus septicus in the tissues of animals that have died of gaseous gangrene, admitted that the disease is caused by Pasteur's microbe. Although the experimenters of Lyons affirmed that it was im. possible to differentiate between the septic vibrion and the microbe of gangrene, their ideas were not admitted without contest. In Germany, Vicklein wrote that gangrenous septicæmia can be caused by bacteria analogous to, but not identical with the bacillus septicus. Frankel is said to have found an agent very different from the vibrion of Pasteur in the lesions of malignant œdema, and to have reproduced a grave inflammation with gas and without pus, by subcutaneous inoculation of a cavy. Whatever he thought of these divergent results, the whole world admits the identity of the septic vibrio and the microbe of gangrenous septicæmia as settled.

What is the behavior of the vibrion of Pasteur in the tissues that it should cause death? This question, although very simple to solve or decide today in certain infectious diseases, is not completely brought to light in this disease. It is based upon the researches that have established the properties of microbian secretions. Some have thought its action is due to a veritable intoxication, but the researches of Roux and Chamberland have demonstrated that such is not the case. These authors have in fact tried to kill cavies with filtered serum of animals that died of the disease, and they did not obtain positive results from injections of considerable doses. Before the failure of these experiments one was obliged to admit that microbian intoxications were almost exclùsively attributed to the bacteria, and what gives this conclusion more force is the fact that injection of pure cultures into the veins confers immunity to, instead of kill- 
ing, the animals. It was also concluded to attribute the poison to the young virus which produced gangrene by capillary obstruction with microscopic thrombosis. In reality, nothing is more poorly demonstrated; today one is leit to believe that the vibrion does not act alone, and that associated micro-organism play a predominant role. It has also been said that the disease is the result of two bacilli; whereof one causes the septicæmia, while the other produces the gangrene. The fact is, streptococci and various varieties of staphylococci are always found. Besson has shown that the role of associated micro-organism and traumatism has been considerably represented.

Earth containing the vibrion without being submitted to any special preparation, inoculated under the skin of a cavy, rapidly causes death, but when it is freed of its accessory germs by a temperature of 80 degrees $C$., it does not, under the same conditions, cause a single morbid phenomenon. The bacteria which are particularly favorable are the staphylococcus, the micrococcus prodigiosus, etc.

The traumatism has an equally important role, which is well demonstrated by the conditions under which gaseous gangrene is evolved. The experience of Chauveau on the ram, castrated by torsion of the testicles, and the proof of Besson, confirm the matter from observation. Necrosis of the tissues, fractures and foreign bodies, by provoking local or diffused destruction of the tissues, are favoring causes of the first order.

IMMUNIZATION.-Since I88I, Semmer and Visjewski have proven that rabbits become refractory to injections of septic blood when they have been previously inoculated with the same septic blood exposed for six minutes to a temperature of 55 degrees $\mathrm{C}$. He obtained the same immunity in rabbits with bouillon cultures of septic bacteria made at a temperature of 35 to 40 degrees $C$, and then 
exposed six minutes to 55 degrees $C$. A small portion of exudate taken from a phlegmon that killed a cat was exposed to a temperature of 55 degrees $C$, and then inoculated into another cat. The latter became refractory to the inoculation of blood from a dead horse. From these facts, Semmer concludes that the bacteria of septicamia introduced into the organism opposes the multiplication of bacteria of the same genus in the organism previously impregnated.

From numerous researches that have followed those of Semmer it results that immunity can be conferred by three different methods:

I. By intraveneous inoculation.

2. By inoculation with attenuated virus.

3. By inoculation with toxins.

I. By Intraveneous Inoculation (Chauveau and Airloing).- The professors from Lyons obtained, in the ass, the most beautiful examples of immunity by introducing strong doses of the virus into the veins at different periods. If a subject thus vaccinated, is inoculated subcutaneously with one-half to one cubic centimeter of virulent serum, ai: alsscess will form at the seat of inoculation, but gaseo:is gangrene will not supervene. In the dog and sheep intmunization by this procedure is more difficult to obtain.

2. By Inoculation with Attenuated Virus.-This method, reported by Cornevin, is not susceptible of giving sensible results beyond an immunity that disappears in twenty-four hours.

3. By Inoculations with Toxins.-In I887, Chamberland and Roux succeeded in assuring immunity by intraperitoneal injections of perfectly pure cultures of the septic vibrion exposed to a temperature of IO4 to IIO degrees C. In eight days each cavy received in three times, one dose of ninety cubic centimeters. The injection was followed 
by no appreciable trouble, and the subcutaneous inoculation of the virus was without results in the immunized subjects. The immunity was still real after thirty days.

SYMPTOMS.-The Horse.-The disease begins two or three days after an accident or operation, without a single special sign to announce its approach. It is manifested by local and general symptoms of exceptional gravity.

I. Local Symptoms.-A hard and advancing œdematous engorgment makes its appearance at the periphery of the wound responsible for the infection. Very painful at the beginning, it becomes less and less as it gradually extends. It radiates in every direction, up, down, right and left, and it remains sensitive only in the peripheric regions which constitutes its zone of extension. The central part becomes cold and painless, and can be attacked with a bistoury or cautery without provoking the least movement of defense on the part of the animals. An incision into the skin is followed by a flow of pus that dispels a stinking odor. The muscles laid bare reflect tints of brown, violet and black and have lost their tenacity and tear without resistance. The areolar tissue is infiltrated with a frothy lemon-colored serum. The blood vessels are either empty or else filled with a species of black, fetid pap.

In the majority of cases nothing can stop the extension of the engorgement. When it begins at the shoulder it advances on both sides of the neck, head, chest, breast and girth; and in the pendent parts it assumes frightful dimensions. It gives the patient the appearance of an attack of purpura hamorrhagica. The wound from which the disease originated is black and blue; suppuration has ceased if the wound is old, and the products discharging are stringy, yellowish or bloody, bubbling and fetid.

2. General Symptoms.-The preceding local modifications are accompanied by exceptionally grave general 
symptoms. The patient is anxious, a prey to restlessness or fright, and the countenance expresses intense pain. In a short time the nostrils contract, the eyes sink, the respiraations accelerate, it is absolutely unconscious of its surroundings, will not respond to a call, and moves with difficulty when forced to do so. The pulse becomes weaker and weaker, and at the same time the heart beats become accentuated, loud, resounding and perceptible at a distance. The timber is dry and metallic as if the first bruit is fused with the second. Chills run over the body and are especially intense at the olecranon and thigh, and abundant perspiration appears. When the end is approaching the patient is much excited and agitated but soon shows all the signs of a serious affliction. It remains motionless in the middle of the stall with the head down and then falls to the floor to die after a few seconds of agony. Other times it lies down and rises several times and then falls as an inert mass to die immediately.

The fever is not always manifested by an elevation of the temperature; generally when there is purulent infection at the commencement it attains 40 degrees $\mathrm{C}$. or more. In some cases it is below the normal and reaches 38.8 to 38.9 degrees with difficulty.

PROGRESS.-Gaseous gangrene has a galloping progress, its evolution is sometimes exceptionally rapid. Death may supervene in twelve hours, in twenty-four hours, in three days and in five days. Its duration is seldom longer.

The Ox.-Actually there is still some doubt whether buvine animals die of gaseous gangrene. Renault reported, nevertheless, that in I786 Desplas wrote with much care about an epizootic that sapped the oxen of the province of Quercy, as follows: A large number of animals in which setons were inserted developed tumors, thought to be anthrax, at the point of insertion four, six, eight, ten or 
twelve hours after. While it is not absolutely certain that Desplas's cases were gangrenous septicæmia, the report is no less interesting, in that it should have been left ignored during these numerous years. It is not until 1884 that Biot, of Pont-sur-Yonne again attracted attention to the possibility of cattle dying from gangrenous septicæmia, affirming what is now a contradiction of the researches of Chauveau and Airloing.

He reported the history of a bovine animal that succumbed to septic complications, resulting from the lancing of a large sanguineous sac. Unfortunately Biot's observation lacks a bacteriological confirmation. In I889, $\mathrm{M}$. Nocard, in a communication to the Societe Centrale, affirms having found the bacillus septicus in muscular tumors of cattle. During the same period the Germans were more advanced than us, on the subject. Since I884, Kitt conveyed gaseous gangrene to the various domestic species and has observed it in the horse and ox. Attinger reported that he had seen eleven cases of malignant œema during the years I889-189o. The disease appeared at all seasons of the year and struck all of the animals during a year and a half. In one case the infection resulted from an abscess of the pharyngeal lymphatics. During the following year, Reuter, (1896) wrote of a case of malignant oedema in the ox, that recovered spontaneously. $\mathrm{He}$ insisted upon the similarity of gaseous gangrene and symptomatic authrax. Horne published, some time later, an observation on four cases that were sustained by bacteriological examination and inoculation. In his statistics of diseases of large animals, Eberhardt, gave the following repetition of the cases of malignant œdema he was able to observe:

In the year $1888 \ldots \ldots \ldots \ldots 2$ cases

In the year $\mathrm{I} 889 \ldots \ldots \ldots$. . . case

In the year $1890 \ldots \ldots \ldots \ldots$. case 
In the year $1891 \ldots \ldots \ldots \ldots$.

In the year $1892 \ldots \ldots \ldots \ldots$ case

In the year $1893 \ldots \ldots \ldots$..... case

In the year $1894 \ldots \ldots \ldots \ldots$.... case

In the year $1895 \ldots \ldots \ldots$. . . a c

In the year $1896 \ldots \ldots \ldots \ldots 3$ cases

These sundry observations attest, in a convincing manner, that the German veterinarians do not consider the bovine family refractory to gaseous gangrene. Just recently, in fact, Koninski drew the following conclusions from similar observations:

I. The spontaneous evolution of malignant œdema in bovida can be considered as well proven.

2. In certain cases malignant odema can become enzootic and may present forcible resemblance to symptomatic authrax.

3. As is the case with anthrax, enzootic malignant œdema appears to reign above all in low lands.

4. The clinical symptoms, the same as in the enzootic character, do not permit of a positive differential diagnosis from anthrax. In either case it is necessary to make a microscopic examination of serum taken from the cedema, in lieu of a recourse to experimental inoculations.

5. If one considers the endemic faculty of malignant œdema, the clinical characteristics common to this affection and symptomatic anthrax and that the micro-organisms of both diseases resemble each other, it is easy to conclude that both germs have a common origin, or at least a close relation. Aside from the preceding observations which compel the deduction that gangrenous septicæmia is an allied disease, note the observations of Strebel, who saw a cow die following blood-letting from the coccygeal vessels. The lower part of the tail was gangrenous and infiltrated with gas. The tail was amputated, but in spite of complete 
disinfection of the caudal stump, the infection reached the croup and the skin became under-run with crepitant infiltration. Further surgical treatment proved futile and the animal died.

I wish to report briefly that while in the service of the college clinic, M. Mathis had occasion to observe the disease twice. One was the sequel of an abortion, and the other of castration of a bull. In both of these cases bacteriological examination and inoculation gave positive results.

The disease may result from the puncture of a sanguineous sac. It may complicate abortion, castration and penetrations of the heart by foreign bodies. It may follow parturient metritis, may supervene the lancing of an abscess, and finally it may result as a complication of mammitis.

SYMPTOMS IN THE OX.-It nearly always appears four or five days after parturition. It begins by the appearance of a rapidly advancing œdema that surrounds the vulva, and then descends into the limbs and surmounts the pelvis and back. The oedema is crepitant, and painful at the start but becomes insensitive after several hours. It gives to the patient the special physiognomy of being insufflated with air. Later the vulva presents a shapeless tumefaction of variable volume. The skin of the vulva-lips is tense, shiny and purple, and the lumbar region is insensitive to pressure. During the time these local symptoms evolve, general symptoms of real gravity also appear. The patient trembles, staggers, hangs the head, arches the back and presents an anxious expression. Frequently the animals are down and refuse to rise except by repeated urging. The ears are cold, the muzzle dry and the eyes sunken. The respirations are accelerated to 50 or 60 , and the pulse to IOo or IIO. When the infection is not a mixed one the dis- 
ease progresses without fever, but when mixed, pyrexia of $4 \mathrm{I}$ degrees C. may appear. The condition of the patient rapidly grows worse and he will have to be killed or meet an early death from the disease.

PROGRESS. - The manifestations develop very rapidly. Animals die in twenty-four hours or in two days or three days after the first symptoms appear. Recoveries are exceptional.

Sheep and Goat.-The disease has been observed in the sheep by Kitt, and was experimentally reproduced in a ram by Chauveau. In several instances it has been known to occur as a complication of scabies. The traumatic gangrene that was referred to by Girard in 1818 has been frequently observed since.

Symptoms in Swine.-In the porcine species malignant cdema occurs in both males and females as the result of castration. In the female the disease begins at the seat of the surgical wound by the appearance of an odematous and crepitant engorgement that often disappears after having acquired the size of an egg. When the animal does succumb the swelling spreads in every direction and loosens the skin from the subjacent tissues. The patient remains in continual decubitus and will not rise in spite of any excitement to which he may be subjected, and it is not rare to observe symptoms of paralysis, which prevent it from maintaining the standing posture. The snout is dry, the ears cold, the tail unrolled, the eyes retracted, the respirations snoring and dyspnœic, and the abdomen is sunken on the opposite side and insensitive. Death rapidly follows.

Symptoms in the Dog.-Malignant odema is rare in the dog. Renault reported two cases brought to the clinic of Professor Vatel, who also observed four cases, all of which resulted from the lancing of sanguineous tumors. Soula 
observed a case that followed parturition and Nocard one in a young beagle hound that was fed with raw meat. Fröhner insists that there is equal occasion to observe it in the dog, although the clinical symptoms may be wanting. The symptoms are the same as in the other species. According to the observations of Soula the lesions remain localized in the breast and show no tendency to spread. Recovery is rapid.

PATHOLOGICAL ANATOMY.-(a)-The Horse. The carcass of an animal that has died from malignant œdema emits an odor that is repulsive and fetid. The original focus of the gangrene is the seat of a very pronounced lesion. The surrounding muscles are reduced to a putrid mass and are macerated into a sanguineous, infected serum. The gas and the liquids separate and infiltrate the subcutaneous and intermuscular connective tissues to a considerable extent. Analysis of the gas shows it to consist of a mixture of hydrogen, atmospheric air and carburetted hydrogen. The muscles are discolored, whitish as if cooked, and present numerous ecchymoses of variable dimensions. In a short time even in winter they take on a yellowishgreen color that is especially pronounced in the injured muscles.

In the gangrenous parts the septic vibrion are found in abundance, associated with other micro-organisms, micrococci and streptococci that have complicated the primary infection. The systemic lesions are equally well marked. If the carcass is examined immediately after death the specific micro-organism is not found in abundance in the blood, but is found to be multiplied if the autopsy is made some hours after death. The blood is tarry, dark and greasy. If examined microscopically it is found rich in fat, and is seen to contain various mobile bacteria and sometimes micrococci. The endocardium is red, infiltrated and col- 
ored with the hæmoglobin. The heart is cooked, discolored, friable and ecchymosed. The pericardium is full of blood serum, and the pleura is injected, ecchymosed, and contains a variable quantity of fluid similar to that of the pericardium. The parenchymatous organs-the lungs, liver and kidneysare obstructed, the spleen is hypertrophied and the divers organs are friable and infiltrated. The peritoneum is roughened, slate-colored and covered with soft adhesions, and it contains a blood serum like that of the other serous membranes.

(b). The Ox.-In the ox, the lesions are similar to those of the horse. The subcutaneous tissue and the muscles around the affected area are found to be the seat of a sero-hamorrhagic and emphysematous infiltration. The affected region presents characteristics slightly different from those of symptomatic anthrax. In the latter disease, gas is found in large quantities, but it is rather spare in malignant œdema, and the liquid that impregnates the tissues is always of limited quantity.

Section of the muscles shows them to be black in symptomatic anthrax while, on the contrary, in malignant œedema they are livid and blanched. The changes in the serous membranes and the parenchymatous organs are the same as in the horse.

(c). Swine.-The lesions in porcine species are always very intense. There is a marked gelatinous infiltration of the subcutaneous connective tissue and a considerable thickening of the abdominal wall around the seat of infection. At the seat of castration the surface is purple and the deeper parts are yellowish-green. The intestines are the seat of a violent inflammation and the peritoneum is eroded and overrun with numerous capillaries. The peritoneal cavity contains a highly colored liquid. In all the lesions the septic vibrion is easily found and it is often associated with a staphylococcus. 
DIAGNOSIS.-The determination of the exact nature of the disease is usually easy. The existence of a sheltered anfractuous wound in a horse, that is followed by the appearance of an odematous, crepitant engorgement. with local mortification of the tissues, leaves but little doubt as to what the symptoms reveal. In the ox it may be confounded with symptomatic anthrax, but the history generally makes it possible to exclude the latter disease. The non-existence of symptomatic anthrax in certain localities, and the development of an advancing odema from the periphery of a wound or sanguineous sac, or following parturition, would constitute symptoms of real importance. In the other species, the hog for example, the diagnosis is of little importance. The disease is only verified when it is too late for intervention.

\section{ANNOTATION.}

Malignant oedema is a much more frequent disease in America than was once supposed. Heretofore the real nature of the affliction has not always been recognized. Its galloping course often terminates the patient's life before expert advice is procurable. The disease has been so little studied in the American schools that the cases encountered seldom arouse suspicion as to their true nature. Inquiry among veterinarians proves that some have never thought of the disease, others have recognized a few cases, while still others claim to meet it quite frequently.

Malignant odema is, in fact, quite a common disease of citv horses. It supervenes nail punctures of the feet, surgical operations, setons, and various accidental punctured wouncls. Among the latter, the wounds about the shoulders seem particularly prone to favor its evolution. Operations upon fistula of the withers, poll-evils and shoulder tumors are especially dangerous. The malignant oedema cases following nail punctures are probably of very common occurrence. The cases from this cause are, however, subjects for future study, because the clinical aspect is not always typical enough to make an absolutely positive diagnosis. Acute blood-poisonings, terminating fatally a few days after the nail wound is sustained, are at least sometimes, typical cases of the disease. About twenty-four hours after the nail is "picked up" the patient begins to manifest unusual symptoms of distress which seem entirely out of proportion to the apparent character of the wound. The temperature is elevated, the appetite nil, the respirations grcally accelerated. The foot is found hot, the lameness intense, and the wound absolutely free from suppuration. At the end of forty-eight hours the symptoms are accentuated and the wound, when reopened, will bubble as if gas is escaping. Within a few days the patient dies rather suddenly. In some cases the hoof falls off shortly before death. The post-mortem exam- 
ination reveals the typical lesions of malignant-œdema cases occurring from wounds in other parts of the body. and bacteriological investigations prove the presence of the vibrio septic of Pasteur.

The cases following wounds about the body are so absolutely characteristic, so absolutely unlike any other disease of the horse, that a clinical dingnosis is always sufficient, but the nail-puncture odema, having its seat between two unyielding walls that always mask the true character of the affection, often remains incognito.-L. A. M.

In all the animals it is possible to complete the clinical diagnosis by bacteriological examination and inoculation. The experimental indication we have described will nearly always lead to a correct diagnosis. It is nevertheless well to state, as shown by Nocard and Leclainche, that there seem to exist in domestic animals, as in man, crepitant tumors of microbian origin that have nothing in common with malignant odema. Airlong, et al, have studied various cases in man that furnish evident clemonstration. In veterinary subjects it is possible that certain cases might be traced to other bacilli. The cases of limited gaseous gangrene mentioned by Violet probably belong to this category. In Germany Kitt seems to have the credit of commencing the study of this question. In a work published in 1897 he speaks of the bacillus of malignant cedema and of a bacillus of pseudo-malignant œdema.

Storch describes four cases of gangrene of the vulva of cows that have considerable analogy with malignant cdema. All four of the animals died. It is probable that they were not cases of gaseous gangrene because the clinical characteristics of the cedema were different from those of gangrenous septicæmia. Section through the affected tissues showed them to be relatively dry, red, strewn with petechia and ecchymoses, and without gaseous infiltration. A bacteriological examination revealed, in the lesions, cocci and bacilli that were different from those of malignant odema in their pathological reactions.

PROGNOSIS.-Malignant oedema is an exceptionally 
grave disease. It is rare to see it terminate in recovery, and in that event it is perhaps permissible to surmise that the case was one of pseudo-gaseous gangrene caused by a bacillus other than the vibrion septic of Pasteur. Violet has seen a horse recover, and Ehrhardt had made a similar observation in the cow. Recovery is certainly more rare in the bovine species, as their feeble receptivity cannot be doubted. Swine nearly always die, while in the dog, on the contrary, the lesion remains local and recovery is the rule.

TREATMENT.-Prevention here, as in all surgical complications, is the important role. The surgeon should employ only sterilized instruments. Wounds soiled with earth, dung or excrements of whatever nature should be thoroughly disinfected. The researches of Besson have shown that the disease will not develop when only the spores of the vibrion septic are brought into contact with the wound. The presence of accessory microbian colonies seems indispensable to the evolution of the disease. If the methodical disinfection of the surface of the wound rids it of the accessory parasites, the wound, no matter how badly infected with the spores of the vibrion, will not cause the gangrenous process. Anfractuous wounds covered with blood clots should receive particular attention. Setons must be banished, or else their tracts must be perfectly irrigated. The accidents of parturition should never be neglected, especially when the labor was attended by incompetent hands and infectious matter from the earth or excrements may have been carried into the vaginal canal. The disinfection of the generative tract with a solution of mercuric chloride I to 4000 is generally sufficient in the cow.

When the disease has once started, prompt and energetic intervention is necessary. The understanding of the gravity of the diseases should not discourage the surgeon. Open the gangrenous tissues with multiple incisions. Em- 
ploy by preference the cautery. Remove all of the gangrenous tissue and even invade the healthy tissue beneath. If it occurs in the limb of a small animal, amputation is indicated. Completely destroy the tract of the seton, or lay bare the fistula of the withers or poll-evil and then energetically irrigate the wound with antiseptics. Bouley recommends sodium chloride-Labarraque's liquor; Trasbot employs tincture of iodine injected throughout the entire engorgement, and Viand used intermuscular injections of cresol. Antiseptics capable of yielding oxygen to the tissues-oxygen water, permanganate of potash-and from another idea-sulphate of sodium, on account of its sulphuric acid-constitute so many agents of real value.

The general treatment should be considered of secondary importance, but should not be neglected. The patient should be supported with stimulants-hay-tea, milk, bouillon, alcohol-and when needed nutritive solutions may be administered per rectum. In the majority of cases, in spite of prompt intervention all of the means employed are impotent.

\section{ANNOTATION.}

The following line of treatment has been successful in three cases. The disease was recognized early. The emphysema and odema were still confined to a small area. In one case the entire swelling was no larger than a man's hand, while in the other two it covered a space of about one foot in diameter. All three of the cases were located in the region of the shoulders and were typical examples of the affection.

The swelling of each case was surrounded with a series of incisions through the skin, two inches long and two inches apart. The skin was then freely loosened from the subjacent tissues, some distance in every direction from each incision so as to freely admit air to the whole environs. Two or three liberal gashes were also cut into the infected center. All of these openings were injected with hydrogen peroxide night and morning during the next few days. In one of the cases the advancing swelling escaped beyond the line of incisions at two or three different points, and as a consequence a second series of incisions were made some eight inches from the first series, and the same treatment accorded to them. The febrile state, dejection, and in fact all the general symptoms. lasted some ten days with decreasing intensity. At the end of that time a second febrile state supervened from secondary infection of the numerous openings with pyogenic 
microörganisms, which in each case was even more stubborn than the original disease. No internal treatment was administered.-L. A. M.

\section{PURULENT INFECTION-PYEMIA.}

DEFINITION.-Purulent infection is a disease determined by the penetration of pus, micro-organisms and their toxins into the blood vessels. It is characterized by the formation of multiple abscesses in the organs, and is accompanied with a chain of symptoms that vary in gravity with the species ancl nearly always terminate fatally.

The disease designated as "purulent infection" by Velpeau has been given various names that describe accurately enough the interpretation of what was known of this pathological state at the different epochs. "Purulent resorption," "purulent fever," "purulent diathesis," "traumatic embolic fever," and "suppurative, metastatic phlebitis" are the expressions that have been most frequently employed. Nowadays most of these names are forgotten and the process is seldom referred to except by the name of "pyæmia" or "purulent infection."

SUSCEPTIBLE SPECIES.-Purulent infection in veterinary practice as in human medicine, has become a rare disease. All of the species are not similarly exposed. Certain ones suppurate more readily than others and from Bouley's point of view the domestic species are classed in the following order: Ist, fowl; 2nd, oxen; 3 rd, dogs; 4 th, swine; 5 th, sheep; 6 th, rabbits; 7 th, horses, starting with the animals in which suppuration is rare and ending with the horse, in which the simplest trauma becomes purulent. Bouley's scale, although exact on large lines is not infallible, because in admitting the incontestable pyogenic aptitucle of the horse it is not denied that the ox suppurates frequently enough. Lucet and Bourney have already observed that suppuration in bovine animals is not rare, and the opinion is shared by all 
country practitioners. It is much more rare, for example in the groat, the hog and the dog.

ETIOLOGY.-Strangles and glanders are frequently complicated by general suppuration. Besides these two special pathological states, the evolution of purulent infection is subordinate to the environment and the condition of the individual.

The Environment.-Before Pasteur discovered the lower forms of life it was difficult to explain why all subjects operated upon, both large and small, would die from purulent infection if placed in the certain habitats. The observations of Renault at Alfort showed that matters were the same in veterinary as in human pathology. The renovation of the Alfort hospital and the observance of the rules of hygiene was followed by the complete disappearance of purulent infection.

The Individual.- - If the environment plays an important role in the evolution of the disease, the role of the individual is equally evident. Although infected with agents of suppuration of considerable virulence all animals are not necessarily condemned to die of purulent infection. Some are easy prey for microbian infection. The debilitated, the overworked and the anæmic belong to that category. And besides, the young--the new-born-in which infection through the umbilicus causes a fatal pyæmia, must be taken into account.

Except in the predisposed, purulent infection will not evolve as the result of any wound whatever. Certain accidents are more prone to cause the disease than others. Anfractuous wounds and fistula from crushing may cause pyamia, while plain wounds, although extensive, are generally without danger.

In the horse fistula of the withers, poll-evil, quittor, arthritis, wounds of castrations, acute mastitis, and purulent 
metritis are nearly always the origin of purulent infection. In the ox the disease has been actually observed as the result of compound fractures, accidents of parturition, metritis, abscesses from foreign bodies and from traumatic pericarditis. In the hog the disease occurs from castration and in the newborn from infection of the umbilicus. Valley reported a veritable enzootic of pyæmia in new-born lambs. In the dog complicated fractures and crushing of the limbs are the usual causes.

PATHOGENESIS.-It would be tedious to unfold at length the history of the various conceptions of the subject of pyæmia. We shall only mention those which seem to have enjoyed special favor. Most of the theories aim to explain the formation of metastatic abscesses. Before Boerhaave, Ambrose, Pare, and Morgagni have held to the theory of purulent resorption. Boerhave in I 720 entertained the theory of penetration of pus into the blood vessels, but his hypothesis was not universally accepted. One hundred years later Breshet and Littre held that pus found its way into the blood by simple osmosis. The theory of phlebitis that succeeds the preceding was generally accepted until recently. It was supposed that the inflammation of the veins caused coagulation of the blood on their interior, and that suppuration supervened in the clotted blood, and that the blood thus became mixed with pus. The studies of Virchow on emboli (r856) partially explained the formation of abscesses. From I 869 to I87I, Verneuil engaged in demonstrating the common origin of septicæmia and purulent infection. He proved that intravenous injections of filtered pus-serum caused septicæmia and that inoculations of the solid parts of pus caused pyæmia. To him purulent infection was embolic septicæmia.

The recent studies of infectious micro-organisms made Vernetil's theory fruitful and explained the obscure points. The microbian theory has supplanted all the others. Al- 
phonse Guerin, in the treatment of surgical accidents by sheltering them from the air, neatly demonstrated the miasmatic nature of purulent infection. The study of anthrax singularly facilitated the study of this disease. Various micro-organisms were found in pus, and the explanation of their action soon followed. The septic agents penetrate into the blood vessels through abrasions in the capillaries of the granulation tissue, or through larger vessels, or possibly gain the endovein by causing inflammation of the vessel.

A broken vessel is rarely ever the entrance point of purulent infection. Generally pyæmia results because the agents cause local, multiple phlebitis. In veterinary pathology purulent infection is at other times frequently the result of phlebitis of the jugular vein. Irritated by the septic agents, the walls of the veins become successively inflamed; the vascular endothelium becomes wrinkled and gathers an infected thrombus little by little. From this clot emboli break away and are carried into the right side of the heart, and then immediately to the capillaries of the lungs, where their considerable dimension opposes their onward course. Arrested at this point they provoke obstruction and local mortification that becomes the center for the formation of an abscess.

Wher the emboli are of small dimensions, or when the septic agents are launched into the general circulation through the medium of the leucocytes, the small dimension of these elements permits them to pass through the network of pulmonary capillaries to colonize elsewhere,--in the parenchymatous organs, the kidneys, the brain, the muscles, etc. It causes alterations that are more particularly formidable in organs that are already diseased or have been previously injured. The firing of horses affected with or approaching an attack of strangles is nearly always followed by the appearance of an abscess at the seat of the cauterization. The 
microbes that produce pyamia are not specific; they are the ordinary agents of suppuration; the streptococcus and various varieties of staphylococci.

It is nevertheless necessary that they possess special virulence in order to cause the general symptoms. In most cases in the horse the streptococcus is found alone. Arloing and Chantre found a pure culture of it in a horse that died from purulent infection following castration. Cadiot on two occasions found the streptococcus alone, and once he found it associated with staphylococci.

In the ox purulent infection can result from the streptococcus, from the bacillus of pyelo-nephritis, and from divers cocci and bacilli. In the hog the usual agents of pyremia are the staphylococcus, pure or associated with bacilli.

If the preceding lesions permit of easy explanation of the formation of metastatic abscesses, the suppurations of synovial membranes seem more difficult to solve. They are not very vascular but it is probable that microbian agents arriving in contact with them find an excellent environment for growth where they can develop in abundance. The microbian theory, therefore, constitutes the synthesis of the preceding theories, enriched with that of Pasteur.

SYMPTOMS.-Nowadays purulent infection. is a rare clinic. The following manifestations are eviclent in all the species. It is announced in the wounded subject by a brisk aggravation of the general condition and by a brisk elevation of the temperature. The patient, previously well in every respect, suddenly changes its physiognomy. It is depressed, anxious, pays no attention to its surroundings, disregards the food given and is attacked with general trembling and chills. The respirations are accelerated and plaintive, the heart beats violent, the pulse small, the mucous membranes injected, and the temperature may be elevated to 40 degrees C. This chain of symptoms is accompanied by a notable 
change in the character of the wound from which the disease resulted. Formerly red and covered with exuberant granulations that are discharging an abundance of pus of usual character, it is seen to modify as the disease begins. The granulations become sunken, tarnished, livid, gray, leadcolored, and flabby, and discharge a bloody pus, tainted with a stringy serum of a reddish-yellow color.

After a variable time the general symptoms amend and the patient seems to recuperate its health. The temperature falls and the principal functions regain their normal condition. But suclenly another chill supervenes, more intense than the first, accompanied with profuse perspiration, staring coat and elevation of the temperature. These ațtacks succed one another in an irregutar fashion every half hour, every hour, or at longer intervals, and each one leaves the patient more depressed. They correspond to successive infections, which appear to be formed in large doses. Symptoms which vary according to the organ in which the abscess is located then supervene. If located in an articulation there will be an inability to support weight ancl very acute pain over the joint, and the purulent collection will clevelop rapidly and will always contain a large quantity of pus. If the abscess is in the lungs, attention is drawn to the respiratory symptoms. There will be an icterus if the liver is the seat, and purulent nephritis if located in the kidneys.

The urine is albuminous and contains biood, pus cells and micro-organisms. The patient becomes weaker and weaker, emaciates rapidly and may die of cachexia or adynamia. These symptoms, although general, apply particularly to solipeds. In the cow the clinical picture is not very different. There appears suddenly a formidable chill, staring coat, tremor over the entire body, sunken eyes, dry muzzle and pale mucous membranes. The skin, horns, ears, forehead, and extremities are cold, and the patient pays no 
attention to what occurs around her. Rumination is suspended and she will occasionally switch the tail and lightly rest one posterior limb. The respirations are accelerated and heart beats violent, the pulse fast, small and difficult to perceive. The temperature is 40 degrees $C$. The hog will hide away in the litter or ground and will not move when presented with food. The skin is either discolored or becomes red or purple. The disease may develop tardily and may not kill the animals for several weeks after castration.

PROGRESS.-The course of the disease, although irregular, is generally slow, unless complicated with septicremia. Death occurs after eight days, twelve days or several weeks. The rapidity of its evolution is subordinate to the virulence of the micro-organisms, and the resistance of the patient.

PATHOLOGICAL ANATOMY.-Metastatic abscess is the "signature" of pyæmia. It can be encountered in all the organs, the lungs, kiclneys, heart, liver, spleen, brain, musc'es or articuiations. Often it exists only in one of them. The lungs are seldom idemnified. They present changes that vary with the, rapidity of the process from so-called infarction to well developed pus collections. As the infarct results from vascular obstruction, its extent will depend upon the size of the obstructed vessels. It appears macroscopically as a red and brown patch that breaks up in cones in the pulmonary parenchyma. The infarets are fragments of the mortified and inoculated lung, and if the animal lives they are transformed into abscesses, the volumes of which are variable. They are found in all states of development, the size of a millet seed, hazelnut, walnut or the fist. They contain purulent products that are yellow and quite liquid. Their internal faces are not different from those of idiopathic abscesses. They usually occupy the 
periphery of the pulmonary lobes. Next to the Jung the liver is the organ most frequently affected. It is found enlarged and presenting abscesses in a circumscribed zone, and with the biliary canals containing pus that is yellow and clotted. The lesions of the kidneys are usually less developed. The affected kidney presents whitish, rounded spots on its surface that penetrate into the parenchyma in the form of white tracts as far as the vascular arch. They lose these characteristics when the infarct has become an abscess. The brain, the spleen, the heart, the muscles, the testicles, the bones, the articulations, and the walls of intestines and blood vessels, can become the seat of these purulent collections. The blood does not present any marked changes, but is nevertheless tarry, sticky and changes rapidly.

The pus found in metastatic abscesses of the horse rarely concretes because their development is usually rapid. $M$. Laulane reported an interesting observation on this subject: -pus of the abscesses of a horse affected with pyæmia of slow progress became caseous. In the ox affected with pyamia Morot found abscess in the muscles of the croup, pectoral region, cervical region, the longissimus dorsi, the anteaspinatus, and in the extensors of the forearm. They also exist in the kidneys, the heart and the spleen. In the observations of Bournay, the spleen, the liver and the lungs contained pus collections. In Mathis' report a pulmonary abscess is described as having united the right pulmonary veins together and partially obstructed them with a purulent clot. The right femoro-tibial articulation contained pus and there was a vast abscess in the posterior crural muscles. Ellerman, who observed pyæmia in a cow following a fracture of the sternum, found the following lesions: sere-purulent accumulation at the seat of the fracture; degeneration of the heart; congestion of the lungs and infiltration of the lymphatics; diversfied lesions of the parenchy- 
matous organs, and redness and liquefaction of the marrow.

The observations on pyxmia in the hog reveal the same lesions. In one case Cadeac found abscesses in the lungs, the heart, the temple, and acute inflammation in the meninges. In another case milliary abscesses were found in the peritoneum from a purulent focus in the tibia.

DIAGNOSIS.-The determination of the exact nature of the disease is not generally difficult. The sudden aggravation of the condition of the patient; the consultation of the temperature curve; the modifications occurring in the wound; the digestive and urinary troubles and the sounds revealed by ausculation can leave but little doubt as to the nature of the disease.

PROGNOSIS.-The disease is excessively grave-nearly. always fatal. In exceptional cases, when the patient succeeds in overcoming the condition, it is left in a state of decrepitude and misery that will render it useless for a long time.

TREATMENT.-The complete and methodical disinfection of bad wounds; the cleansing of obstinate fistule, and the free opening of deep, suppurating foci will prevent the disease in the majority of cases. When the disease is once started the surgeon's efforts should be directed toward the complete disinfection of the wound causing the trouble. Antiseptic powders of high potency, applied freely, is the best method of treatment. When the wound is anfractuous and irregular, and presents excavations, there should be no fear to cauterize the wound and make it smooth. The general symptoms must be attacked in every manner possible. Sulphate of quinine, alcohol, coffee and salicylate of soda can render some service. When the patient refuses nourishment it must be fed with bouillon, milk and hay tea, administered per os or per rectum. In the great majority of cases when the disease is confirmed all the medication will prove insuf- 
ficient. The clinician is absolutely disarmed and must stand without being able to relieve the affliction of his patient.

\section{TETANUS.}

DEFINITION.-Tetanus is a local infectious disease caused by an anaerobic micro-organism-the bacillus of Nicolaier-which generates a poison to the nervous system that provokes spasmodic contraction of the whole or a part of the striated muscles.

Tetanus attacks man and the domestic animals. It is particularly common in the horse, the donkey and the mule, and is also observed in a decreasing ratio of frequency in the small ruminants, the hog, the ox and the carnivora. The disease has been reproduced in all of these animals by inoculation by cultures or soluble products of the bacillus. It has also been thus produced in the mouse, the cavy, the chicken and the frog.

HISTORY.-Tetanus has been observed in man and the domestic animals since the most remote antiquity. The works of Hippocrates describe it, and it also attracted the attention of Hippiaters. Although closely studied in the horse from the symptomatic point of view by Solleysel, Vatel, Gohier, Trasbot, etc., and in the ox by Gelle, RocheLubin and Cruzel tetanus has, until late years, remained a mysterious disease the pathogenesis of which has been the subject of most conflicting theories. The numerous relations, published up to $\mathrm{I} 884$, are confined to a discussion of the gravity of the disease and its symptomatic features, but taught nothing as to the nature of its lesions; and the varieties of treatment recommended serve only to clemonstrate the futility of therapeutics. Tetanus was then regarded as a neurosis. They recognized an essential or rheumatic tetanus due to the action of cold, and a traumatic tetanus due to accidental wounds or surgical operations. It was thought 
to develop from nerve irritation caused by abrupt impressions of cold or traumatism and that it was only a reflex having for its origin a peripheric irritation, for its condition a functional over-activity of the nerve centers, and for its effect muscular contraction.

With B. Travers, Simpson (1854), Rose, Billroth, Panum, etc, a new opinion came into existence. They regarded tetanus as the consequence of a specific intoxication formed in the region of the wound that, when absorbed, took effect on the nerve centers. Under the influence of the microbian doctrine its possible infectious character was speculated upon. Already real epidemics of tetanus had been observed among human beings and animals. In man great numbers of cases of tetanus-veritable epidemics-were noticed following great battles. After the battle of Prague (Seven Years' War) 4,000 wounded men were attacked with tetanus in a single night, and Larrey has testified to an epidemic in Egypt and in Saxony. It was also seen in the Crimean War, at Metz, etc. Surgeons refer to similar facts in wounded soldiers in a single hospital ward.

Veterinarians have furnished important information which tends to prove the infectiousness of tetanus. Lacoste reports striking examples of it in a report on castration, in which Cagnet is said to have castrated six horses with the same ecraseur and all died of tetanus. After the ecraseur was disinfected no further deaths occurred. Similar examples have been published in, great numbers. Lister had no more cases of tetanus following his operations after he introduced antisepsis.

Experimenters finally reached the last step of these investigations. Carle and Rattone (I884) succeeded in inoculating tetanus. Nicolaier discovered the microbe and obtained pure cultures of a virulent character that produced tetanus fatal to animals. These results were con- 
firmed by Rosenbach in I886, completed by Kitasato who isolated the specific agent in 1889 , by Sanchez Toledo and Veillon (1889) and by Rouget, whose elegant works threw a vivid light upon the pathogenesis of the disease. In I 899 , Knud Faber began the study of the soluble product contained in the cultures, and Vaillard, Behring and Kitasato subsequently vaccinated animals and demonstrated the preventive properties of their blood serum.

Thus the nature of this disease. which had remained a mystery for centuries, was elucidated. It is today a much studied disease, as effective treatment remains to be discovered.

Tetanus exists everywhere-in every latitude. It seems to be frequent in equatorial regions. In the Antilles-in Havana-it causes a third of the mortality among the newborn. In equatorial Africa, as well as in Ireland, it is found. In France it was very frequent in certain districts during the past century. Among animals it is relatively rare.

\section{ANNOTATION.}

In America tetanus is a common disease of the horses working in the larger cities. While it has been observed in all parts of the United States the cases occurring in the country districts are comparatively few as compared with city animais. In the routine of a city practice it is encountered only in the horse. The other animals seem to be possessed of a pronounced immunity against accidental infection of tetanus. In eighteen years' practice in the City of Chicago, where equine tetanus is ever present, we have never met the disease in either the dog or ox. White saw two cases in the dog in seventeen years of continuous dog practice.* In the American cities the disease is much more common among animals than in the human being. In the latter it is comparatively rare, so rare that even the wide-awake American surgeon does not often regard it necessary to administer preventive treatment to patients suffering from wounds suitable for the propagation of tetanus. The greater number of cases are seen following the Fourth of July, on which day many boys sustain wounds from toy pistols and fire crackers, which are precisely the kind of lesion that favors the disease. With the exception of this incident, - which often assumes the proportion of a veritable epidemic,-tetanus cases in the human being are mighty rare in the country districts and of only nominal frequency in the cities.-L. A. M.

* The June number of the Bulletin of the Chicago Veterinary College reports another case of tetanus in a dog from Dr. White's hospital. 
BACTERIOLOGY.-The infectious nature of tetanus was suspected long before the discovery of the specific micro-organism. The discovery was assisted by work that is interesting to recall: B. Travers, Rose, Bantum and Billroth considered tetanus a special intoxication. The first experimenters tried to transmit tetanus but failed. Arloing and Tripier failed to transmit tetanus to the dog, the rabbit and the horse by venous inoculations of blood and pus taken from a tetanus patient twenty-fours after cleath, and by the injection of 200 grams of blood of a horse affected with the disease into the jugular vein of a healthy horse. Billroth and Anthona failed to transmit the disease by injecting the blood of tetanic subjects into healthy animals, and Nocard likewise obtained negative results from.intraarachnoidean, peritoneal, venous and subcutaneous inoculations of cephalo-arachnoidean liquid or bulbar substance taken from a horse that had clied from tetanus.

These experiments dicl not demonstrate, as some writers thought, "that tetanus is not an infectious diserse," but that the virus is not in the products used for inoculation. Carle and Rattone in I 884 were the first to transmit tetanus by inoculation. An animal having died from tetanus caused by scraping a pustule of acne, the pustule and its surrounding tissues were excised and made into an emulsion, which was injected into the sheaths of the sciatic nerres of rabbits. Out of a-clozen rabbits inoculated eleven presented symptoms of tetanus. Fragments of tissue taken from the inoculated places on the rabbits that died were inoculated into other rabbits, with fatal results from tetanus. The infectious nature of the clisease was thus demonstrated, but the specific micro-organism remained to be founcl. This discovery was made by Nicolaier in 1884 . I'hile studying the pathogenic micro-organisms of the soil at Gottingen he inoculated white mice, caries, and rabbits 
with the earth of the streets and fields, and found that most of them exhibited symptoms of tetanus and perished. The dogs he inoculated proved refractory. At the autopsies a little pus was always found at the seat of inoculation. This pus, inoculated into rabbits, caused tetanus in numbers, while the inoculation of fragments of liver, spleen, spinal cord, etc., gave no results. The microscopic examination of the pus found at the point of inoculation revealed different micrococci and a specific bacillus in the form of long, lank, slender filaments, a little longer but less thick than the bacillus of Koch's mouse septicamia. Nicolaier implanted the pus deeply into a media of solidified sheep serum and obtained pure cultures of the bacillus, which, when inoculated into animals, produced tetanus.

Rosenbach in r 886 found the bacillus of Nicolaier in the wound of a man that had died from tetanus. The tissues of the wound inoculated into animals caused tetanus in series. Cultures were obtained by punctures in solidified merlia, and Rosenbach demonstrated that the bacillus is anaerobic, and formed spores which develop into a_bacillus having the aspect of a drum-stick or of a pin. He did not, howerer, succeed in obtaining pure cultures.

The discovery of Nicolaier was confirmed at every turn. A great number of observers transmitted tetanus by inoculations from the wounds of tetanic animals. Nocard in 1887 produced the disease in rabbits, cavies and a donkey by inoculating them with fragments of caseous matter obtained from horses that died from tetanus following castration. Since then the bacillus of Nicolaier has been regarded as the specific micro-organism of tetanus. Some writers contested this action and claim to have isolated different tetanigenic germs, but their work fell flat before that of Kitasato, who obtained pure cultures of the bacillus of Nicolaier, and thus established its specificity in the year I88g. 


\section{ISOLATION OF THE MICRO-ORGANISM.-In or-} der to isolate the microbe of tetanus Kitasato implanted tetanic pus in serum. At a temperature of 36 to $3^{8}$ degrees $C$. he obtained impure cu1'tures at the end of forty-eight hours which contained numerous baci!li and foreign micro-organisms. He submitted them to a temperature of 80 degrees C. for three-quarters of an hour to one hour, and found that Nicolaier's bacillus a'one resisted. Cultures made in vacuum or in an atmosphere of hydrogen are the on'.y ones that developed to show the agent of tetanus in pure co?onies. These colonies cu!tivated in serum or agar-agar and incubated in a vacuum or in hydrogen show growth constituted only of the tetanus microbe, which, inoculated into mice, produced the characteristic tetanus.

By this process it is possible to isolate Nicolaier's bacillus because it gives spores more rapidly than the other bacteria which accompany it. The latter are killed at the temperature of 80 to 90 clegrees, while the tetanus spores resist. It is owing to the great resistance of the spores that the isolation occurs. Vaillard and Vincent cultivated tetanus pus in beef tea at 38 to 39 clegrees, sheltered from the air, for five to six days, and obtained growths of different varieties of micro-organisms. With a sand bath they then heated the culture to Ioo degrees for one to two minutes in a closed vessel. The spores of Nicolaier's bacillus resisted and the other microbes were killed, and when cultivated on gelatine or hot bouillon pure cultures were obtained. Sometimes a single heating did not destroy every impurity, in which event a second or third operation was necessary. The vibrio septic and the hay bacillus can resist this temperature and remain mixed with the tetanus bacillus. In order to isolate the latter recourse must then be had to Roux's process of cultivation on gelatine or of Vignal's method of separating anaerobic micro-organisms. 
MORPHOLOGY OF NICOLAIER'S BACILLUS.The microbe of tetanus is polymorphic. Microscopic examination of pus from a tetanus wound reveals fine, slender, rectilinear bacilli, slightly longer than those of Koch's mouse septicamia, and with longer forms that are almost filamentous. The bacilli are motile and their movements are slow. The motility is due to the abundance of flagella, with which it is provided. It is the most bristly of all microbes.

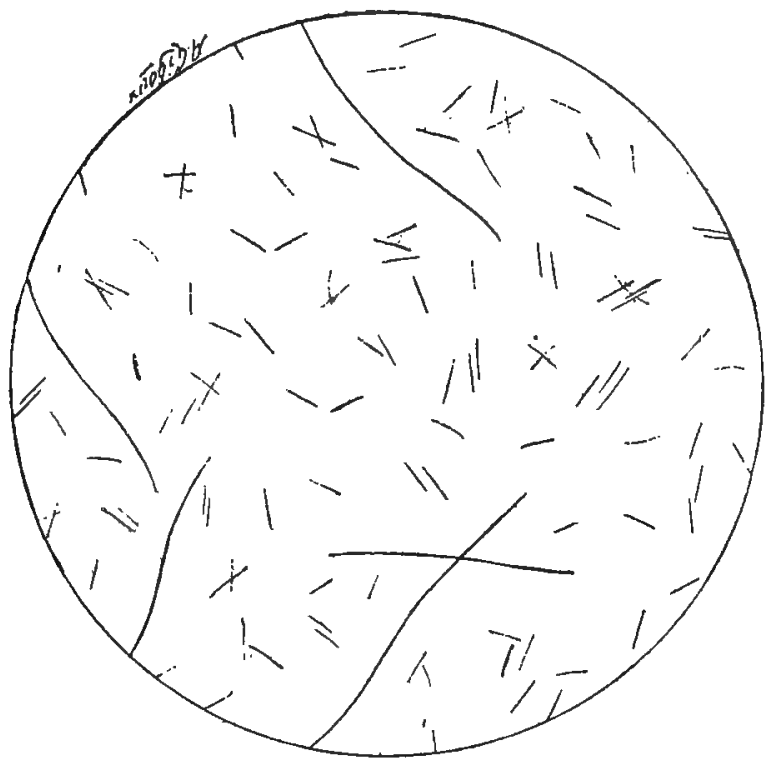

FIG. 40.

Bacillus of Nicolaier. Fifth day.

In the bouillon cultures the bacilli are at once linear, from 5 to 8 microns long and 0.3 to 0.5 thick, and sometimes filamentous and motile in the absence of air. In the sport1lated form they are homogeneous and regular.

The spores develop from the cultures in eight to ten days at the heat of the room, and in two to three days in the incubator at 35 to 38 degrees. One of the ends of the bacillus swells and it assumes the typical pin or drum-stick form. The brilliant spherical spore is usually at one end, 
but may sometimes be found in the middle of the bacillus, or even at the two extremities. It is always thicker than the rod and may reach a width of $\mathrm{I.5}$ microns. In very old cultures they are only spores,- the rod has disappeared.

STAINING.-The bacillus of Nicolaier is easily stained with any of the aniline colors, gentian violet, etc, and by Ziehl's or Gram's method. The spores are well stained by

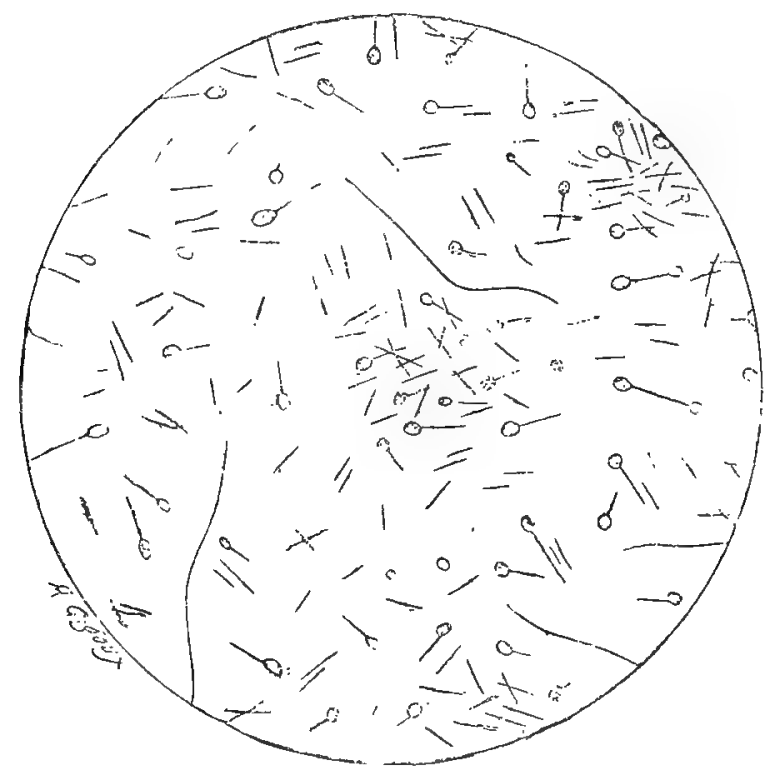

FIG. 41.

Bacillus of Nicolaier. Twelfth day.

Ziehl's or Ehrlich's stain, and the flagella by Van Ermenghen's process.

CULTURES.-The bacillus of tetanus is anaerobic. It my be cultivated in slightly aerated environments. but beatutiful colonies are obtained only in a vacuum or in hydrogen. Any of the media may be used. The temperature which favors its growth varies between $\mathrm{I}_{4}$ and 43 degrees. The most favorable temperature is 38 to 39 degrees.

The aspect of the cultures varies with the nuedia. In the alkaline bouillons, containing 2 per cent of glucose, and 
in fresh bouillons of beef, veal and chicken the bacilli grow quickly. In twenty-fours the media are uniformly cloudy and fine bubbles of gas are set free by agitation. About the fifteenth day germination stops. The culture has the special odor of burning horn or hair, or, more exactly, of ripe cheese. The bouillon always exhibits an alkaline reaction. In the bouillons more than eight days old the spores do not germinate. In five to six days the spores are formed

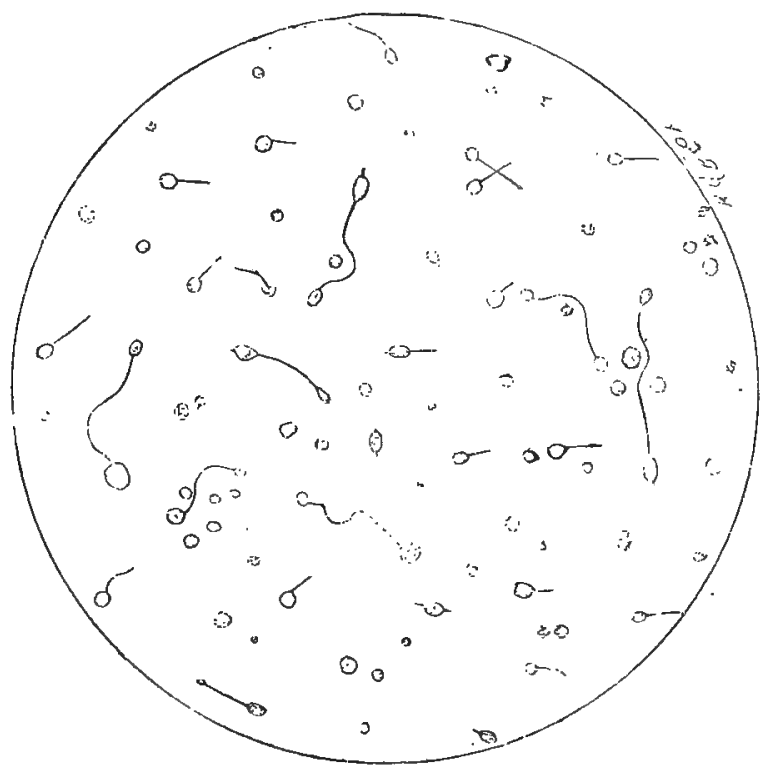

Fíg. 42.

Nicolaier's Bacillus. Old Culture.

and the bacillus loses its cilia and precipitates to the bottom of the vessel.

A deep stab in a gelatine tube to the depth of ten to twelve centimeters will show nebulous streaks at the encl of four to six days in a temperature of 18 to 20 degrees. The streaks, which might be compared with very fine needles entangled at the center, radiate in every direction toward the circumference of the tube. By degrees the gelatine is liquefied and the colony appears in the form of a 
whitish, creany flake, surrounded by a zone of solid gelatine. On gelatine plates at the temperature of 20 to $22 \mathrm{de}-$ grees the cultures also have the racliating character. Gelose is not liquefied, but, like gelatine, liberates gas. The colonies are less flaky and the streaks coarser. Serum, when coagulated, is a good medium. It is inoculated by puncturing, and then covered over with hot gelose. After fortyeight hours in the incubator at 37 degrees the colonies are alreacly apparent. The serum is not liquefied.

The bacillus of tetanus grows poorly on potato. It forms

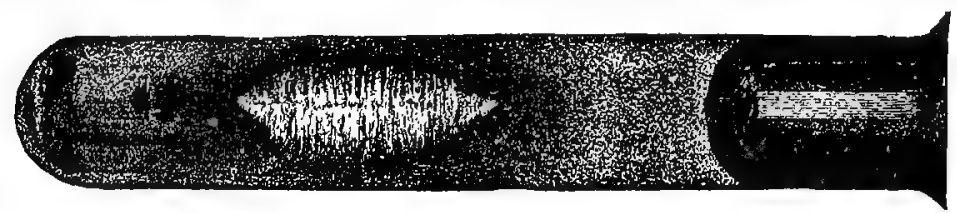

FIG. 43.

Culture of the Tetanus Bacillus in Glucose Gelatine, Inoculated by a Deep Stab. Six Days Old.

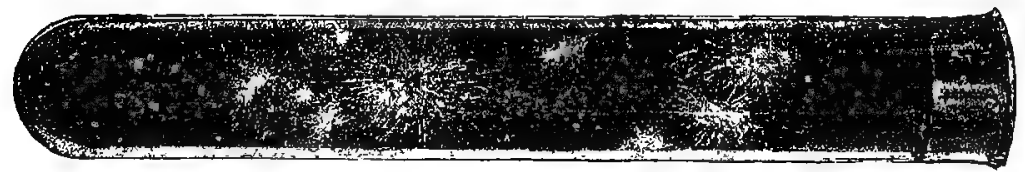

FIG. 44.

Culture of the Tetanus Bacillus in Glucose Gelatine, after Separation of the Inoculated Substance. (Frankel and Pfeiffer.)

but fine, slight beds, keeps in the filamentous form, and cloes not form spores, even after months. Egg albumen, fresh serum and aqueous humor are not favorable media. Cultivation is favored when reducing substances (glucose. 2 per cent; blue litmus tincture, 5 per cent, or sulpho-indigotate of sodium, o. Io per cent) are added to the media.

RESISTANCE.-Cultures of Nicolaier's bacillus preserve their vitality for a long time in the incubator, or at ordinary temperatures when air is excluded. The spores are very resistant to the action of heat. They are not killed when exposed to a temperature of 80 degrees for six hours. 
In a closed vessel and humid environment they are destroyed in fifteen minutes at roo degrees and in five minutes at II 5 to r2o degrees. When dried up in the atmosphere away from sunlight they remain virulent for years. They are, however, very sensitive to light, especially sunlight, which promotes oxidation, and will perish after about a month's exposure. Before they die they progressively lose their virulence and become incapable of forming spores. In artificial cultures, the action of oxygen will also arrest sporulation.

Kitasato has shown that tetanus bacilli will resist carbolic acid, five per cent, for more than ten hours; that it requires more than three hours for mercuric chloricle, I to $\mathrm{r}, \mathrm{O00}$, to kill them, and that chloroform destroys them only after two days. Nitrate of silver, I per cent, destroys them in one minute; iodine solution, 50 per cent, carbolic acid, 50 per cent, and permanganate of potash, I per cent, will destroy the activity of the spores after some minutes' contact. In the soil the spores of tetanus may find conditions which will preserve their virulence for a long time. They are especially well preserved when in an albuminous environment. Eiselsberg has proven that a splinter taken from the hand of a man suffering from tetanus was still virulent at the end of two years and a half. Soil impregnated with spores, kept in a diffused light, was shown to be very virulent after several months. Putrefaction does not alter the spores.

HABITAT OF THE BACILLUS.-Nicolaier's bacillus is very widely disseminated. It is found in the soil. It was in earth that Nicolaier discovered it. It exists in the dust of streets and dwellings, in cultivated fields and on the surface of vegetables. The dust of hay is tetanic.

The spores are much more abundant at the surface of the earth than underneath it. Below the depth of a milli- 
meter they become rare. They have been found in marshes, in the slime of the Dead Sea, in the bogs of New Hebricles, Santa Cruz, etc. In the Solomon Islands it is found in a sort of eartl which the natives use to poison their arrows. La Danzea has shown that the wounds caused by these arrows are followed by tetanus.

Tetanus spores are preserved while being carried along by streams of water, creeks, rivers, etc. The bacilli abound in dung, especially that of the horse, and are found preserved in the intestines of many animals. Animals fed with earth-soiled substances, or tetanus cultures, have rejected active bacilli without having clied of the clisease. The excrement of the horse and cow inoculated into rabbits produces fatal tetanus in five to six days, showing that the spores resist the action of the gastric juices. In animals that have died from tetanus, the bacillus is always found at the point of inoculation. They remain localized during the entire progress of the disease and are not founcl either in the blood or parenchymatous organs. Sanchez. Toledo and Veillon have shown that they sometimes pass into the general circulation a few lours before cleath.

INOCULATION AND RECEPTIVITY.-The inoculation of finished cultures gives tetanus to susceptible animals. The white mouse is highly susceptible. Cultures forty-eight hours old, in closes of I-500 of a cubic centimeter, kill them after a short period of incubation. Eight hours after inoculation in the paw tetanus appears, and at the end of fourteen hours it becomes generalized. The cavy is also very susceptible. Tetanus appears eighteen to twenty hours after inoculation of $\mathrm{I}-500$ of a cubic centimeter. In the order of susceptibility man comes after the cavy. The horse-is killed with from one-half to one cubic centimeter after an incubation of seven days. The mule is similar to the horse. The rabbit offers niore resistance, but, like the dog, is killed 
by a close of one cubic centimeter. The frog can be tetanixed, especially at the temperature of $30^{\circ}$ to $35^{\circ} \mathrm{C}$. Marie claims to have caused tetanus at a temperature of $\mathrm{I} 2^{\circ}$ to $\mathrm{I} 3^{\circ} \mathrm{C}$. after a long period of incubation, and chiefly with the grey frog, but the experiments of the Italians, of Ehrlich and of Morgenroth fully confirm the previous cleduction as to the surrounding temperature. The sheep and the hog are highly susceptible.

The question as to what becomes of the bacilli reveals the fact that they remain at the point of inoculation, and that they become fewer and fewer, and finally leave only their spores behind. The disease is therefore only local. If the tails of twelve mice are inoculated with fatal closes of the culture and then cut off five, ten, fifteen and thirty minutes after, only the last one will die from already having absorbed sufficient poison to cause cleath, although all of the micro-organisms have been removed by the amputation. It is the tetanus poison that kills.

PATHOGENESIS.-The bacillus of tetanus inoculated alone, that is to say without toxin, is not pathogenic. Thus, cultures in gelatine at 20 degrees have no toxin until four or five days. They may be inoculated with impunity on the third clay. The spores can also be separated from the poisons elaborated by the bacilli by washing or by heat without impairing its potency. The poison is sufficiently deteriorated by a temperature of $80^{\circ}$ for three hours to rencler possible the inoculation of considerable numbers of spores without producing serious intoxication. Cultures thus purified when inoculated into very sensitive animals. like the cavy, in closes of one-half or one-third of a cubic centimeter, give negative results. Rabbits have been given 30,40 or even 65 cubic centimeters of purified spores contained in broth cultures without exhibiting any tetanic symptoms. These facts demonstrate clearly that the spores are 
incapable of germinating when they enter the sound tissue of an animal in good health and in a state of purity. This is due to the fact that when they are introduced under the skin or into the peritoneum they are surrounded, immobilized and destroyed by the polynuclear leucocytes. This prophylactic role of the leucocytes is established by examination of the part affected. After inoculation of a heated culture there is a thickening of the connective tissue-a nodosity due to the influx of the polynuclear leucocytes. By excising the tumefaction and examining the contents, it is easy, by staining with fuscin or methylene blue, to follow the fate of the spores and to understand their relationship with the cellular elements. It is proved that lencocytes abound at infected points. At the end of twenty-four hours there were thirty leucocytes to every spore. Gradually the exudate becomes richer in leucocytes as the free spores diminish, and at the end of two or three days all are incorporated. It is established that they diminish little by little, undergo degeneration and after five or six days are destroyed.

But whether the resistance of the germs varies, or whether the cells are not always equally capable of destroying them, some spores may remain intact and virulent in the cellular protoplasm for three months or more. This view is important in explaining the origin of cases of tetanus whose cause remains obscure.

There is a direct relation between phagocytosis and the preservation of the animal. All the influences that lessen or hincler the work of the leucocytes likewise permit the growth of the spores and thus favor the development of the disease, and substances having negative chemiotactic properties, added to spores deprived of toxins, favor the development of fatal generalized tetanus. The rabbit which resists an injection of 55 to 65 cubic centimeters of the cul- 
ture becomes susceptible to one-eighth to one-third of a cubic centimeter of the same culture to which lactic acid or trimethylamine has been added. These chemical agents have a repelling effect on the leucocytes. At the autopsy they-the leucocytes-are not found at the point of inoculation.

The spores may also be protected against phagocytic action by chemical means, for example, by shutting them up in an envelop of filter paper which is afterwards introduced aseptically under the skin or into the peritoneum of a rabbit. The paper sheath does not obstruct the passage of serum to the spores to form a favorable medium for their growth, but, on the other hand, on account of its thickness, it retarcls the inward migration of the leucocytes. Under this condition the spores grow, the bacilli elaborate their toxins, and when the leucocytes finally reach the interior of the paper, the time for effective struggle is over. The toxins then spread through the surroundings and drive away the leucocytes.

Again, if the leucocytes are paralyzed by intravenous injections of finely pulverized regetable carbon, tetanus may be produced by the injection of spores deprived of their toxins. In this experiment, as well as in the preceding one, the spores are protected against leucocytes-the latter being occupied with the carbon are motionless and incapable of coping with the micro-organisms.

Traumatism and foreign bodies play the same role. The association of various micro-organisms with the spores of the tetanus bacillus favors infection. In the natural infection numerous micro-organisms that live in the soil are inoculated with the specific germ, and they generate the impurities that make a favorable field for the growth of the tetanus bacillus. These facts, which Verhoogen and Baerten anticipated in I890, were demonstrated by Vaillard Vincent and Rouget by means of the following experiments: 
When a portion of tetagenic earth is inoculated into animals it always causes tetanus. If it is heated to a temperature that will destroy the other micro-organisms without lessening the vitality of the tetanus spores, the earth loses its virulence. But if the other microbian species are restored to this same earth its virulence is likewise restored. If the earth heated to 85 degrees ceases to be virulent, it is not the result of vitiation or destruction of the tetanus spores, because the viru'ence can again be restored under certain conditions. The expianation is found in the fact that the heat, while respecting the specific germs, has eliminated the greater part of the other micro-organisms and has suppressed the pathogenic puperties of those that have resisted. The cultivation shows that the proportion of viable germs is very small in this heated earth. It is incapable of producing either a local lesion or tetanus. but if this inactive earth is again supplied with the adventitions germs the original virulence at once returns, hence the following conclusion: In tetanus following an earth inoculation, the bacteria, other than the specific germ, play an important role in the pathogenesis of the disease. These bacteria produce lesions in which the tetanus spores may grow. Their co-operation is indispensable and when they are not present the spores do not germinate and tetanus is not produced.

Among the bacteria that favor tetanic action, the bacillus prodigiosus deserves notice. The mixture of $I_{-1} 5$ of a cubic centimeter of a culture containing spores without toxins with 0.5 cubic centimeters of a culture of bacillus prodigiosus, one month old, inoculated into cavies, produces tetanus in thirty-two hours that becomes general and proves fatal fifty hours after inoculation.

111 micro-organisms do not facilitate the evolution of tetanus spores to the same extent. The staphylococcus pyogenes aureus, the streptococcus, the bacillus subtilis and 
the bacillus of Friedlander do not exert any favoring influence. All of these details apply exactly to the pus co'lected from the wound of a tetanic man or animal that has died from the inoculation of earth. Deposited under the skin of a cavy this pus rapidly produced fatal tetanus. But after heating ten to fifteen minutes at 68 to 70 clegrees $C$. it becomes inactive, even with a clouble dose. Here, again, it is easy to establish what is the indispensable element in the pathogenesis of tetanus, which heat caused to disappear. It is not the specific bacillus; the cultivation of a quantity of this pus not heated, equal to that which has been inoculated, gives a very toxic culture. It is the bacteria that vegetate with it that have been eliminated, and which thrive in the tetanic wound of both man and anima!s. Tf, by cultures in gelatin, in the air, and in a vacuum, attempt is made to determine the quantity of other microbes contained in this pus, before and after heating, striking results are obtained. The quantity sown remaining identical, the pus before heating always gives a consiclerable number of various micro-organisms. After heating only colonies of tetanus bacilli are formed. The heated pus contains only the tetanus bacillus that is not virulent and does not cause local lesions. The pus that is not heated is tetanigenic. Besides the tetantis bacillus it contains a number of different micro-organisms. It is because the latter have disappeared that the heated pus does not cause local lesions, and that in consequence of the fact that the specific germs cannot grow.

\section{ANNOTATION.}

The following, then, is the summary concerning the action of $B$. tetani and its terms as confirmed by the foregoing experirnents:-

I. B. Tetani is the specific capse of tetanus.

2. Other bacteria, such as B. prodigiosus, are indispensable to the germination and growth of the tetanus bacillus.

3. The toxin acts in special thermic conditions and always in every animal after a period of incubation, which does not vary as to the dose given. 
4. The toxin cannot be found in either the tissues or liquids of the body during the entire course of the disease.

5. During the disease it is temporarily fixed by the nerve cells and in some way a new substance is produced which does not diffuse through the body but remains intracellular.

6. The nerve fibers conduct the toxin. It does not reach the nerve centers through the circulation; as it does not kill by intravenous injection of the toxin.-L. A. M.

Microscopic examinations of cultures give a definite idea of the variety of bacterian species found in tetanic wounds. The isolation of these species is possible, but they do not all act as favoring agents. This property belongs only to some of them. There is 'found constantly in the wounds of tetanic subjects this or that bacterium, which, combined with a small dose of tetanus spores, will probably give tetanus to the cavy. Inoculated alone this bacterium does not cause the disease, and acting alone the tetants spores will give negative results, but when associated tetanus is produced. The associated bacterium taken at the autopsy on the dead tetanic subjects can be recognized as the one that was introduced beneath the skin to produce the disease.

By studying these lesions the mechanism by which the micro-organisms facilitate the generation of tetanic bacilli is made apparent. In spite of their different physiognomies these alterations are always characterized by leucocytic influx ending either in a pus-like collection or in the formation of a pseudo-membrane. In both cases most of the leucocytes are dead, while the others are filled with the micro-organisms they have englobed. The "favoring" bacteria have the property of actively attracting. the leucocytes and also of killing them. The filtered cultures act in the same manner. They seem to supply the tetanus spores with the support they require by destroying the activity of the leucocytes, which obey their dominant chemiotactic solicitations and then strike them dead. These experimental and significant facts interpret the 
mode of development of tetanus under many circumstances. They cast a vivid light upon the etiology of tetanus, and show all of its factors. The spores existing everywhere in nature are frequently inoculated, but pure. Tetanus requires the foreign body that carries also pyogenic microorganisms into the wounds. The traumatism plays the part of lactic acid. It is thus that contused wounds soiled with earth or dung are most liable to be followed with tetanus.

Dung contains many spores. The bacillus of Nicolaier finds in the intestines, the conditions favorable to anaerobic life, and multiplies. In spite of the wide diffusion of the tetanus germ in the soil, in the dust of dwellings, dungpiles, stable-floors, etc., and in spite of the numerous circumstances which faror the contamination of wounds by these germs, tetanus is comparatively rare. According to the statistics of Hering there might be one case of tetanus among three thousand patients. This comparative rarity of tetanus is due to the fact that the bacillus of Nicolaier cannot exert its pathogenic action except in special complex, multiplied conditions that are not often combined. Viallard, Rouget and Vincent have experimentally determined the combination of conditions required for the propagation of tetanus spores in a nervous organism. They have shown that tetanus spores exist in nature in a different form from that observed in the laboratory. Instead of being impregnated with toxins, like the laboratory cultures, they are destitute of them. When introduced into the tissues they must first propagate before secreting the poison that produces the disease In order to study how the spores act on the living organism, under natural conditions, it is necessary to operate with spores deprived of their toxins.

ETIOLOGY.-Tetanus is always due to the introduction of the bacillus of Nicolaier into the body. Spontaneous teta- 
nus is constantly cleveloped in consequence of the contamination of a wound with products containing the spores of the tetanus bacillus. Any acciclental or operative trammatism may be followed by tetanus.

Horse.-In the horse tetanus has been noticed to follow a great variety of traumatisms. Their seat has a considerable influence. Wouncls of the extremities are in the first rank.Quittors, wire cuts, street nails, nail punctures, corns, kicks. etc., are often complicated with tetanus. Wounds of the trunk and head do not frequently exhibit this complication. The most superficial woundis may be followed by tetanus. However, contused wounds, deep and anfractuous, broken knees, wounds by crushing, tearing or puncturing, irritating wounds caused by foreign bodies, splinters, iron snags, nails, etc., all have a specially predisposing tendency. Wounds caused by the harness also give rise to visitations of tetanus. and the harness may serve as the agent of direct infection. Oliver saw three donkeys successively inoculated with tetanus by use of the same halter. The surgical wounds most frequently followed with tetanus are: Castration when performed by clamping, amputation of the tail, application of setons, foot operations, caudal myotomy, operation for umbilical hernia by clamping, or by cauterization with nitric acid, the ablation of tumors, puncture-firing, blood-letting in the jugular, blood-letting in the palate when performed with the chamois' horn, amputation of the penis and hypodermic injections.

In every case the infection is caused by the operator. The instruments used serve as carriers of the contagion, or the operating field may become soiled with earth, dirt, dung, etc. when the animal is lying clown. It is especially the clamps, ecraseur, and emasculator that infect the wound of castration, and the catheter may serve to inoculate the disease into a susceptible subject. 
Certain empirical practices are the cause of tetanic complications. The use of spider's web as a hæmostatic is dangerous because it is always filled with dust. Burns may be infected when clressed with scrapings of raw potatoes, which vegetable is never entirely free from earthy particles. Poultices of cow's clung. frequently used for foot diseases, are also a cause of infection.

Wounds of the Mucous Membranes.-The buccal mucous membrane by clental irregularities, intestinal mucosa by foreign bodies, conjunctiva, vagina, etc., may serve as passages for the micro-organism of tetanus. Among colts, tetanus may be the consequence of infection through the umbilicus. Hartman observed it as an enzootic outbreak.

Ox.-In the ox all kinds of wounds (horn-thrusts; wounds of the neck from the tie-rope; the application of trochisci of hellebore root; dystocia; metritis; wounds at the base of the horn from the tie strap; abortion) may be followed by tetanus. It is more specially observed after parturition. This is puerperal tetanus; the bacillus is inoculated in wounds of the vagina or uterus. Its evolution is favored by the state, of putrefaction existing from retained placenta.

Among calves and lambs omphalophlebitis is often complicated with tetanus that occurs as an enzootic disease. Umbilical infection often follows the custom of dressing the navel with potter's clay. The castration of lambs with clamps or by extraction, or the amputation of their tails, is often followed by enzootic tetanus. The inoculation of sheep-pox has also caused an outbreak of tetanus in the epizootic form. In the hog tetanus has occurred after castration and after different wounds. In the dog it is especially seen after crushing of the toes or extremities of the limbs.

TETANUS TOXIN. TETANIC POISON.-Nicolaier's bacillus, remaining at the point of inoculation, causes the symptoms of the disease because of the poison it secretes. 
Nicolaier was first to suspect its existence. Kitasato soight to isolate it. He, too, held the view that the poison was an alkaloid acting like strychnin.

Since $1886-87$ Brieger has found evidence of the presence of poison in impure cultures made in beef, and in the brain of sheep. He extracted from them certain venomous bases:

Tetanin $\left(\mathrm{C}^{13} \mathrm{H}^{30} \mathrm{O}^{4}\right)$ which in very small doses causes prostration, lethargy, the classical symptoms of tetanus and cleath, in small animals;

Tetanotoxin $\left(\mathrm{C}^{5} \mathrm{H}^{11} \mathrm{Az} z^{6}\right)$ a liquid that boils at $100^{0}$ with a disagreeable smell : a rather large dose of it causes restlessness, tremors, and violent convulsions;

Spasmatoxin similar to cadaverin: it is very active and tetanizing;

Hydrotetanin which is convtlsant and sialagogic.

Brieger and Fraenkel have also separated a toxalbumin from cultures of the tetanus and thought it was the principal poison of the bacillus of Nicolaier.

\section{ANNOTATION.}

Recently (1899) in the Annals of Institut Pasteur, Madsen has described a toxin of tetanus which Ehrlich had previously shown to be hæmolytic. This is tetano lysin. It is destroyed by $15 \mathrm{~min}$. exposure to $60^{\circ} \mathrm{C}$. and has a constitution similar to toxin. Normal horse serum possesses a marked antihæmolytic action to tetants.-L. A. M.

It is demonstrated that the above bases and toxalbumin of Brieger compete in causing the symptoms of tetanus, but are only satellite products, that is, assistants to what is called tetanus toxin. The latter, easily revealed in cultures, was first obtained by Knud Faber, a student of Salomonsen of Copenhagen, in 1890 . He separated it by following the process employed in diphtheria. He filtered a bouillon culture of the bacillus of Nicolaier through porcelain and thus obtained a liquid free from its germs, and which proved to be very toxic when injected under the skin of animals (rabbits, cavies, mice, etc.). It causes, after a period of incubation of 
twenty-four to thirty-six hours, stiffness of the inoculated member that spreads over the entire body and causes a typical tetanus. Knud Faber demonstrated that the tetanic poison is very active, that intraveneous injections lead to immediate generalized tetanus, that its ingestion is harmless and that it is destroyed by heating at a temperature of $55^{\circ} . \mathrm{He}$ advances the opinion that the toxin is a soluble product that closely approaches diastases, an albuminoid substance resembling diphtheritic poison,- - a venom and not an alkaloid. Tizzoni and Cattani, Villard and Vincent and Rouget in I89 I and I892 continued and completed this study.

PREPARATION OF TETANUS TOXIN.-To prepare the toxin a medium is made of neutral or alkaline, peptonized, salted, fresh beef bouillon to which a small amount of gelatine is added. Considerable quantities,-flasks of two litres,-are used, and the cultures are left in the incubator for eighteen to twenty days. The poison (toxin) is dissolved in the bouillon. By filtration through porcelain, the dissolved toxin is separated from the bacilli. In this manner a very toxic liquid, free from germs, is obtained. The liquid must not be exposed to air, light nor heat. Its activity is then increased by inoculating it with a new tetanic colony which will develop well in the environment where the first generation has already lived. A dose of I-I 50 of a cubic centimeter of the liquid of the first generation will kill a cavy. The liquid of the second generation after having been in the incubator another eighteen days, kills by a dose of $\mathrm{I}-500$ of a cubic centimeter.

The third generation is only possible by adding a small quantity of new bouillon (20 c.c. per 350 c.c.). If filtered after sixteen days in the incubator a dose of I-IOOO of a c.c. will kill a cavy, and I-IOO,OOO c. c. will kill a mouse. The quantity of real toxin contained in this small amount of liquid is trifling. A cubic centimeter of the liquid evaporated in a vacuum 
leaves 0.040 grams of a dry residum containing 0.025 grams of organic matter. Even if this last were pure toxin the conclusion would be that this amount is sufficient to kill a thousand cavies or a hundred thousand mice. The fatal dose for a cavy is therefore 0.000025 grams, and for a mouse 0.00000025 grams. And besides, this slight quantity of organic matter is not all toxin; it also contains foreign substances, showing the extraordinary activity of the poisons liberated by the micro-organisms.

What is the nature of this poison? It is a diastatic substance presenting in all of its biological properties, great analogies to jequiritine, zymasis or soluble ferments, and to the poisons of diphtheria. Knud Faber, Tizzoni and Cattani and Vaillard and Vincent have shown the close relationship of these poisons.

The activity of the tetanus poison is markedly modifier! by heating to the temperature of $60^{\circ}$ for forty minutes. 1 heating of thirty minutes at $65^{\circ}$ in a closed vessel partly kills it, although this same temperature will not kill the microorganisms. The temperature never completely suppresses its action even at $80^{\circ}$ or $90^{\circ}$. The toxin preserves its activity for years if kept in a closed vessel and out of the reach of light. On the contrary, in the air and light it become rapidly inactive in consequence of the phenomenon of oxydation. It is precipitated by alcohol, is soluble in water, is not modified by tartaric acid and it is not easily dialyzed. Like diphtheria poison and cliastases, it possesses the property of adhering to certain precipitates produced in its solution. The alumina, and above all the dibasic and tribasic phosphates of lime, attract it, and when these precipitates are carefully washed and inserted under the skin they produce fatal tetanus.

Its properties are utilized to obtain solid toxins. They are precipitated with five to six volumes of alcohol and then 
clesiccated, but the toxins are not pure and may be heated to $\mathbf{I O O}^{\circ}$ without a!teration. Tannin and magnesia sulphate also precipitate them. With the latter the precipitate rises to the surface and can be easily skimmed. Successive precipitations bring out all of the toxins. The dry precipitates keep for an indefinite period, and their activity is formidable.

The bacillus of tetanus elaborates a diastatic substance in the cultures that acts much like digestive ferments. It liquefies gelatin. It is perhaps mixed with tetanic toxins, as the cultures attenuated by heat, light and air, lose not only their toxicity but also their property of liquefying gelatin.

\section{EXPERIMENTAL STUDY OF TETANUS TOXIN.}

-The toxin, when injected into the body of an animal, produces symptoms iclentical with those of spontaneous tetanus. The sensibility of animals to tetanus toxin is parallel to their receptivity to the complete culture. In order of a decreasing sensibility we may successively enumerate man, white mouse, cary, rabbit, solipeds, dog, pigeon and the hen.

I-I,O00,000 cc will kill a mouse

I-IO,OOO cc will kill a cavy

I-4 cc will kill a rabbit

I-2 cc will kill a frog

$2 \mathrm{cc}$ will.kill a horse

4 cc will kill a dog

Io cc will kill a hen.

The hen is not naturally immune, as was once supposed. Courmont and Doyon have shown that fatal tetanus may be easily produced in this animal with moderate doses. The pigeon is more sensitive than the hen to tetanic toxin. The frog has been used in the laboratories since the researches of Courmont. Metchnikoff has demonstrated that the tortoise, the alligator and the scorpion are refractory to tetanus and will resist even large closes of the toxin, either hot or colcl. In order to give tetanus to a frog it is necessary to work 
in a sufficient temperature: in summer at a temperature of $28^{\circ}$ to $3 \mathrm{I}^{\circ} \mathrm{C}$. the frog is easily rendered tetanic; in winter at $5^{\circ}$ to $8^{\circ} \mathrm{C}$. the frog is refractory, and in order to produce the disease the temperature must be artificially raised to $30^{\circ}$ to $39^{\circ} \mathrm{C}$. The order of sensibility to tetanus toxin is as follows, if we base our conclusions on the dose which kills a gram of animal weight. By starting with the dose that kills one gram of horse: To kill a gram of cavy requires twice as much; of dog four times as much; of mouse twelve times as much; of rabbit two thousand times as much; and of hen two hundred thousand times as much.

Mode of Action of Tetanus Toxin.-The writers who first devoted themselves to the study of the poisons of Nicolaier's bacillus thought that these soluble products acted directly upon the organism like other well known toxins or alkaloicls, e. g. strychnia. Tetanus toxin, although exactly similar to diphtheric toxin in its essential properties, differs from it in its action on animals. Diphtheria toxin has both immediate and remote effects, while tetanus toxin never acts immediately. An uncertain period of time (several hours or several days) always elapses between the injection of the toxin and the appearance of the first tetanic symptom. It is a real period of incubation during which there is no noticeable disturbance to the health. This incubation has been observed since the first studies on the subject, even by Knud Faber. Courmont and Doyon have shown that this period of incubation is constant in all animals, whatever may be the dose or the channel of entrance. The close has but a slight influence on the duration of the period of incubation, which varies with the different aninals. From the subcutaneous injection of an average close it is of eight hours chration in the white mouse, thirteen to eighteen hours in the cavy, eighteen to thirty-six hours in the rabbit, thirty-six to forty hours in the dog, four days in man, four to nine days in the 
chicken, five to seven days in the horse, and four to six days in the frog. In the moule, rat and cary it also varies with the point of entrance; for example, an injection into the brain is followed by a longer period than an injection beneath the skin. The close has a little influence on the period of incubation.

By beginning with a given dose that gives the minimum period of incubation, colossal doses of the toxin can be injected, but the duration is not modified. The time intervening between the appearance of the first symptoms of tetanus and the death of the animal, however, varies with the dosage. It is therefore evident that the period of incubation is unobservable. There are neither respiratory nor circulatory disturbances, but the period is nevertheless noted for organic modifications of a chemical character, as the following statements seem to indicate: Brunner in 1898 proved that gaseous changes are notably active from the very beginning of the period of incubation. D'Arsonval and Charrin in 1898 , by placing the same animal in a D'Arsonval calorimeter, proved the existence of a disturbance in the temperature curve; the dissipation of heat was lessened.

From these facts it follows that tetanic toxin belongs to a rather peculiar class of toxins. It acts in special thermic conditions and after a period of incubation. The toxin is spread through the organism by degrees, and its ultimate behavior and disposition is interesting. In most infectious diseases the toxins become more and more abundant as fast as the micro-organisms become more and more numerous, and they are found in the liquids and tissues of the patients. But this is not the case with tetanic toxin. - It beconres modified in the organism and cannot be found in either the tissues or liquids of the body during the entire course of the disease. Arloing and Tripier have already attempted in vain to reproduce tetanic spasms by the transfusion of blood from ani- 
mals suffering from tetanus. Pestana in i89 I inoculated cavies with tetanus toxin and sacrificed them by hamorrhage after the first appearance of the first symptoms. He gave tetanus to mice by injections of fifteen clrops of the blood or emulsion of the muscles of the injected point, but extracts from the kidneys, spleen, liver, lungs, medulla oblongata and other muscles proved innocuous. He proved that if the cavy is not killed until after generalization of the disease the quantity of the toxin is diminished. It is never found in the nerve center, medulla or urine. Similar results have been obtained by other experimenters. Kartulis and Blumenthal in I896, Knorr, Marie and Jacobs in I 897 , and Courmont and Doyon in 1898 , could not prove that the toxin was eliminated by the kiclneys. Marie, in 1897 , systematically investigated from hour to hour the behavior of the toxin in a rabbit that had received ten culbic centimeters of toxin into its blood. He has established, in the mouse, that the bloud is no longer poisonous after the seventh hour, although contractions clo not appear until the forty-eighth hour. The nerve centers, muscles, kiclneys, liver, testicles, ovaries, spleen ancl marrow, ground and emulsified and injected into mice proved to be harmless. The toxin has clisappeared and is not found in the secretions. Marie supposes that it has combined with the cellular protoplasm.

These experiments have been confirmed by Blumenthal. He has found it easy to find toxin in the tetanized cavy. while in the rabbit the poison clisappeared about the thirtieth hour, that is, during the period of incubation. It was always absent when the symptoms have appearecl. He assumes that the toxin has combined with the cellular substance. In coldblooded creatures, such as the carp, the frog and the axoloth, which do not become tetanized at a low temperature, as well as in the turtle, Metchnikoff has found the toxin to be preserved for months. In the frog heated and tctanized 
there is always toxin in the blood at the time of the appearance of the first symptoms, but this toxin always clisappears after some days of tetanus. The frog still lives, suffering from intense tetanus after the disappearance of the toxin, and its nerve centers are always scantily supplied with the poison. In the hen tetanic toxin subsists for a long time without modifications. It is not eliminated but transformed. From these experimental investigations it is eviclent that tetanus does not act alike on all animals. It is important to remember that in the rabbit the toxin has clisappeared from the blood and emulsions of the organs, a long time before the disease begins; that certain refractory or slightly sensitive animals like the frog, turtle and chicken may preserve the toxin intact for a long time, and that warm frog may become tetanized without having any toxin in the nerve centers.

The mode of action of tetanic toxin has been very clifferently interpreted. Some have claimed that it does not unclergo any modifications in the organism, but that it cliffuses by degrees and becomes fixed in the cells of the nerve centers. This hypothesis rests upon the following fundamental experiments of Wasserman and Takaki. The normal brain of a cavy is pulverized in ten cubic centimeters of salt water. A cubic centimeter of this emulsion mixed with tetanus toxin neutralized ten fatal closes, and attenuated sixty fatal doses for mice. It is therefore evident that the brain substance has rendered the toxin harmless. The brain of the rabbit. the pigeon, the horse and man, exhibit the same properties. The brain medulla, or other parts of the body, in lieu of brain substances for this experiment, do not attenuate the toxin.

These statements have been verified by different authors. Metchnikoff and Courmont have established that the antitetanic property is the privilege of the nerve centers in mam- 
mals. In the cold-blooded animal, the frog, turtle, etc., no neutralizing action occurs. These writers have demonstrated in opposition to Wasserman, that the nerve substance is neither vaccinating nor curative. Other substances, nervine, and hydrochlorate of betaine also neutralize the toxin. By basing conclusions on these experiments it has been supposed that the toxin localizes in the nerve cells of the brain and causes alterations which are responsible for the tetanic spasms. But in reality the toxin cloes not combine with the nerve-cell protoplasm. It is simply fixed, and the fixation is not permanent. If an emulsion of brain charged with toxins is macerated it will diffuse through the menstruum, and will prove to be active in proportion to the amount of toxin contained. The experiment of Wasserman is therefore artificial and insufficient to explain the development of tetanus. There is nothing to prove the affinity of nerve protoplasm, so clear in vitro and also recognized in vivo. The diffusion of the toxin is not regular. As has been already shown animals may remain tetanic when their nervous systems no longer contain toxins. In order to clearly demonstrate the affinity of the cerebral cells to tetanic toxin, Roux and Borrel have produced "cerebral tetanus." They injected tetanus toxin directly into the cerebral substance of the rabbit, the cary and the rat, and thus obtained a localized disease of the brain, characterized by symptoms of cerebral origin:-hallucinations, epileptic crises and cerebral ex+ citement, without medullary symptoms or spasms. In the rat, the phenomena were most startling. It became mad and the victim of hallucinations. The poison was immediately fixed by the brain and was not cliffused as far as the spinal cord. It seems then, that the cerebral cells fix the toxin in the living animal as well as in the crucible,- - "in vivo as in vitro." Courmont and Doyon have endeavored to prove that fixation has really occurred. The fact that the toxin 
does not diffuse is not sufficient proof. They injected the toxin into a part so poorly supplied with blood that the puncture could not be found at the autopsy, and found that it diffused with great difficulty. This is so true that if toxin is injected into the brains of animals rendered immune by antitetanic serum it will be found that those that accidentally bleed at the point of inoculation will not contract the clisease because of the flow of anti-toxic blood into the toxic focus. It is as difficult: for the toxin to diffuse as it is for the antitoxic blood to come into contact with it. Finally, and this is particularly important, the injection into the brain shortens the incubation but does not eliminate it. How can we explain the incubation period if the tetanic symptoms are due only to the fixing of poison by the cerebral cells? And even in the rat it requires more toxin to kill by cerebral than by subcutaneous injections, and the period of incubation is rather prolonged. All of these arguments clemonstrate that the theory that aims to explain the pathology of tetanus, by simple physical phenomena--the fixing of toxin in the cerebral cells-is insufficient, as it can not explain all the peculiarities of the morle of action of tetanus toxin, notably the period of incubation. The others,- the chemical theories,admit a transformation and combination of the tetanic toxin in the organism. Courmont and Doyon thought that the toxin acts similarly to a soluble ferment. When introduced into the organism it was supposed to form a new tetanizing substance, at the expense of the organism, that caused the spasms. The period of incubation would then be explained by the influence of the temperature, the disappearance of the toxin during the period of incubation. the preventive and non-curative action of the serum, etc. This would also harmonize with the chemical characters of the toxin. Courmont and Doyon have found the strychnizing substances in the blood, the organs, the urine, etc. They have shown that 
the transfusion of the blood of a tetanic log to a healthy one may now and then cause slight tetanic spasms, that the watery extract of tetanic muscles injected into the frop strychnizes it, and that the urine of tetanic animals is very productive of convulsions. This substance has been found by Buschke and Ergel, and by Blumenthal and Tanher, but has been sought for in vain by Brunner, Ouchinsky and Marie. It is therefore inconstant, and cannot be taken into account in the general pathogenesis of tetanus.

The German theories sustained by Ehrlich, Wasserman and Blumenthal are also "chemical theories" closely approaching the preceling. They hold that the toxin is combined with the nerve-cell protoplasm to produce a new substance that does not diffuse through the liquids of the body, but remains intracellular. It is therefore useless to search for it in the liquids of the body or in emulsions of organs. According to some authors it might diffuse at times and lodge elsewhere in the cells of diseased organs. Wasserman's experiment is the basis for all of these theories. They vary more or less according to the authors who have upheld them. It is held that the cells of the nerve centers of normal animals contain an antitetanic substance,-a preformed antitoxin, - that is similar to the blood of animals vaccinated against tetanus. Then, in the cases of tetanus there would be a saturation of the cellular antitoxin by the toxin, and the production of new substances; but notice, in orcler. the difference in the mode of action of brain emulsion and the antitetanic serum. The second is preventive, while the first requires mixture "in vitro." And besides, there is no destruction of the toxin, since Danysz separated it from the emulsion by maceration.

According to certain authors, the new substance remains intracellular, while others hold that it may diffuse and be again found in the organs and the liquids of the body. Ac- 
cording to Behring an animal has a given amount of natural antitoxin capable of neutralizing a given dose of toxin. If only a certain close is injected the animal does not take tetanus. When the close is large, there is not sufficient antitoxin to neutralize it and tetanus results. Death supervenes through lack of antitoxin. It might be said in reply to Behring's view that the animal clead from tetanus still has a rich stock of antitoxin, as its brain is still capable of neutralizing many toxins in vitro, and that the antitoxic supply from the brain and medulla is not measured by the progress of the tetanus.

Another argument in favor of the "chemical theory" has been invoked; - the antitetanic serum is preventive and not curative, because it can only neutralize the toxin itself. It is without effect on the new substance, which in reality causes the tetanus.

These different theories are rather obscure. They are mere hypotheses, and are all open to criticism. They are insufficient to explain the pathogenesis of the tetanus spasms.

\section{ANNOTATION.}

It has aiso recently been shown by Hektoen that some of the lower animals are immune to tetanus because other cells such as liver cells combine with tetanus toxin and thus prevent its action upon the nervous system.L. A. M.

It remains to be explained in what part of the nervous system the tetanic toxin, or the new substance,-the formation of which has been admitted,-acts. The investigations of Courmont have demonstrated that the tetanic poison has no effect on the muscles. It is not a muscle poison, but acts exclusively on the nervous system. By submitting tetanized animals to the action of curare, which paralyzes the motor nerves and thus separates the muscular system from the motor nervous system, the spasms immediately and entirely disappear. Tetanic toxin is therefore not a muscle poison. Section of motor nerves, or of their medullary roots, renclers 
impossible the contraction of the muscles they supply, and destruction of the medullary centers renders impossible the contraction in the corresponding region. Chloroform considerably diminishes the contractions, or makes them disappear entirely. When the motor nerve of a muscle is intact, it can no longer undergo contraction from the infuence of tetanic toxin, if not supplied with sensitive fibers.

The study of the comparative excitability of the different parts of the nervous system elucidates the mode of action of the toxin. The tetanic poison cloes not modify the excitability of the motor nerves; but acts as if it was concerned with the sensitive system. It acts on other nerve centers, arriving at them by way of the nerves, after more or less time. The symptoms of tetanus vary according to the point of inoculation and the part of the nerve centers first attacked. It seems that the nerve fibres act as conductors of the toxin. Thus, M. Binot has shown that it is possible to produce varieties of tetanus in the cary, according to the point of inoculation. Subcutaneous or intramuscular inoculation gives generalized motor tetanus. Inoculation in the testicle or uterus gives visceral tetanus with a long period of inculbation and without spasms. The cary, thus inoculated, quivers at the least touch, walks upon the tips of its toes as if on needles, and dies usually from asphyxia. The toxin was conducted by the sympathetic nerves. By inoculating under the skin, but in a tuberculous gland innervated by the sympathetic system, there will be visceral tetanus but no contractions.

The nerve fibers therefore conduct the toxin. It does not attack the nerve centers through the circulation, as it does not kill by the intravenous inoculation of a dose seven times larger than a fatal subcutaneous dose. This fact is demonstrated by the following experiment:-Two rabbits are each inoculated in the sciatic nerve with a fatal dose of the toxin; 
the nerve is divided in one of them,-it resists, while the other dies.

IMMUNIZATION.-Tetanus is not a self-limiting disease. One attack roes not secure the patient against a second. Every animal is immune against the microorganism deprived of its toxin, but none is ever im. mune to the toxin. Tetanus is a toxic disease. To immunize against it is to immunize against the tetanic poison. N11merous attempts have been made to give animals complete immunity. By repeated injections of small doses of culture Tizzoni and Cattani in I89 immunized a dog and two pigeons. Instead of inoculating with pure cultures, Behring and Kitasato, in I89o. sought to modify the culture by adding certain substances. They inject a virulent culture into a rabbit, then, immediately, three cubic centimeters of a one per cent solution of trichloricle of iodine. In twenty-four hours four similar injections are given. Rabbits thus acquire a clegree of immunity that permits them to receive with inpunity from two to three cubic centimeters of a virulent culture at the end of fifteen to twenty-five days. Their resistance increases, and soon they are able to resist five cubic centimeters of very active culture. The method is, however, not perfect, as a great number of the animals succumb. -probably 6o per cent. And, besides, it is not a general process; it is applicable only to the rabbit; it fails in the cavy and mouse.

Brieger, Kitasato and 17 asserman have sought for a more perfect method. They have obtained better results by using cultures of tetanus bacilli in extract of thymus glands. The micro-organisms thus cultivated formed neither spores nor toxins. The inoculation of these cultures, which are vaccinative and not toxic, immunized the rabbit and the mouse. Vaillard recommended a very differant method, which consists of utilizing the micro-organism without attenuation or 
modification. He determined the refractory point of the rabbit "by repeated inoculations into the connective tissue of the tail, beginning with small quantities, to which lactic acid had been added." This method is not general. It succeeds with the rabbit, but is more risky in the cavy and rat. Cavies have, however, been rendered refractory in this manner.

Instead of using complete cultures for vaccination. one can use cultures filtered of the micro-organisms, and that possess vaccinating properties. This method is more certain and more practical than Behring's and Kitasato's. The animal is first accustomed to the tetanic poison by the inocilation of small closes of filtered cultures whose toxic activity has not been modified. It is necessary to proceed cautiously, as inoculation with one dose that is a little too strong will kill the subject. The process proceeds well with the rabbit, but is less certain with the cavy, because of a greater sensibility. By an artifice, Vaillard diminished the activity of the toxin before injecting, and was thus able to operate with greater certainty. Filtered cultures are partly deprived of their toxicity by heating, and are introduced into the blood in large doses, heated at gradually decreasing temperatures$60^{\circ}, 55^{\circ}, 50^{\circ} \mathrm{C}$. These are followed by gradually increasing quantities of filtered cultures that are not diminished in toxicity. The application of this method is easy in the rabbit. At intervals of three days two doses of ten cubic centimeters of a filtered culture, heated to $60^{\circ}$, are injected into a vein of the ear of a rabbit. Five days after, ten cubic centimeters of cultures, heated to $50^{\circ}$, are injected. This already gives immunity to the animals and confers antitoxic properties to the blood. The state is afterwards reinforced by injections of cultures having their full toxicity. For this purpose "increasing doses, 5, I0, I 5, 20 and 30 cubic centimeters, are injected every eight to ten days. In all the animals so treated 
a lasting immunity is obtained, and the blood of the animals so vaccinated preserves a great antitoxic power for eight months or more. By using doses infinitely smaller, but more frequently repeated, identical results are obtained on the cavy. The immunity is also lasting. It persists for at least one year, and in the female it is transmitted to the offspring.

Other means may be applied in the rabbit, or even more sensitive animals, like the cavy and rat. One of them consists of using toxin modified by iodine instead of by heat. Iodine, in small doses, instantaneously neutralizes tetanic toxin. If to five cubic centimeters of a filtered culture, of which $I-6,000$ of a cubic centimeter is sufficient to kill an adiult cavy, an equal part of iodine water,- - I to 500,-is added, the entire mixture may be injected into a cavy without producing the least tetanic symptom. The injection of comparatively large closes of this mixture into the veins of a rabbit gives negative results. After injecting increasing doses of this kind animals acquire immunity. The process is applicable to the cavy and the rat and is absolutely free from all danger. The same method rapidly immunized the sheep, the calf and the horse. The immunity thus conferred on the horse may be very considerable. Its degree is subordinate to the amount of toxin injected into the vaccinated animal. Nocard immunized a horse that was capable of receiving 250 to 300 cubic centimeters of tetanic toxin in the jugular with impunity, that is to say, sufficient to kill 2.500 horses. Other substances will attenuate or neutralize the tetanic toxin and replace iodine. These are trichloride of iodine, used by Behring and Kitasato, extract of thymus, by Brieger, Kitasato and Wasserman, chlorine solution, 5 per cent, and carbolic acid solution, 5 per cent, by Tizzoni and Cattani. The hypochlorites, permanganate of potash and carbonic acid 
have also similar properties, but are not as easily managed as iodine, nor do they give any better results.

SEROTHERAPY.-Animals immunized against tetanus may receive large doses of tetanic toxin. They are refractory to the poison. At first it was thought that the property of destroying the effects of the poison was due to a substance existing in the tissues of the vaccinated animal (antitoxin) that possessed the power of annihilating the toxin when brought into contact with it. The hypothesis was advanced by Behring and Kitasato, who based their conclusions on the following experiment:

If the blood serum of an animal raccinated against tetanus is mixed with toxin, the mixture may be safely injected into the most sensitive animals. The serum of vaccinated animals seems to neutralize the tetanic toxin as an acid nettralizes an alkali, and may be injected into different regions with impunity when mixed with fatal doses of the pure toxin. Furthermore, the injection of toxin is without effect if preceded a few hours by an injection of the antitoxic serum. It will also prevent effects from inoculations of tetanus spores impregnated with toxins.

These effects are the basis of serotherapy. The blood serum of vaccinated animals is immunizing. It may be used as a vaccine and as such will immediately confer immunity. The discovery of the vaccinating properties of the vaccinated blood was confirmed by Tizzoni and Cattani in $189 \mathrm{I}$, but they state that the serum cannot cure tetanus once developed. In I892, Vaillard showed that the blood of a hen, an animal slow in contracting tetanus, does not neutralize the toxin, but becomes antitoxic about twenty days after an injection of filtered culture into the peritoneum. He also established that the bacillus may grow and secrete toxin in the serum of refractory animals; that it multiplied in the living tissues of vaccinated animals and that it is not attenuated by the pro- 
longed action of the liquids of a refractory animal. The serum is therefore not a bactericide. It is only antitoxic and its preventive action is clue, not to the destruction of the bacillus, but to the neutralization of its poison. Roux, Vaillard and Nocard, in 1893 , had completed the study of antitetanic serum, and had indicated the mode of preparation and direction for its use in practice.

\section{PREPARATION OF ANTITETANIC SERUM.-The} horse is used for the preparation of antitetanic serum because it is capable of supplying a consiclerable quantity of blood; because of the limited natural toxicity of its serum, and because of the facility with which it can be injected and bled.

The animal is first raccinated by subcutaneous injections of an eighth or a quarter of a cubic centimeter of toxin mixed with a few drops of solution of iodine. An injection is marle every four or five days, and the dose is gradually increased. The injections are made subcutaneously. They cause a little local swelling, but not general disturbances. Later. when the blood has become antitoxic, the injections may be begun with pure toxin, but in small doses, and at intervals of nine days. Again, these doses are gradually increased until the horse is able to withstand 30 to 40 cubic centimeters of pure toxin. Henceforth the dose is more rapidly increased until 200 to 300 cubic centimeters of very active toxin is injected in a single dose.

At the end of some months the horse is sufficiently immunized to warrant a trial of its serum. If the blood is found insufficiently antitoxic the injections are continued after an interval of ten days rest. When the desired antitoxic potency has been obtained, from five to six litres of blood are drawn from the jugular vein. The blood-letting is done with a sterilized trocar inserted into the vein from below upwards, and attached to a rubber tube connected with a flask in which the separation of the serum is to be effected. 
The antitoxic effect of the serum is preserved for a long time, even more than a year, and its strength is measured according to the recommendations of Behring: The quantity of serum required to immunize a gram of white mouse against a fatal dose of toxin is taken as a unit. A gram of young cavy will answer for a gram of white mouse. When antitoxic serum is said to possess an activity of a million units, that means that a cubic centimeter of the serum is sufficient to immunize 1,000 kilograms ( $1,000,000$ grams) of mouse; or, again, that one mouse of 20 grams is rendered immune by two hundred thousandths of a cubic centimeter.

In practice antitoxic activity is measured in the following manner: Mice are injected with gradually increasing doses of the serum, starting with a dose supposed to be ineffectual, then from ten to twelve hours later, each mouse is given a fatal dose of the toxin. Thus the extent to which the mouse has been vaccinated is determined, that is to say, the amount of resistance to the poison is made known.

Serums of exceptional activity may be obtained. They may be represented by figures o.00000000oI or even 0.0000000002 according to Nocard. At each bleeding the activity of the serum should be tested as to its antitoxic potency. The antitoxic potency of blood of the horse used will be found to diminish regularly from the time the vaccinating injections cease. Although the immunity lasts for a long time, often for two years, the clecrease in the antitoxic strength of the serum is already manifest 15 to 20 days after the last vaccinating injection. In order to maintain an active serum it is necessary to regularly inject toxin into the animals which supply it. This does not only prevent the enfeeblement of the toxic and immunizing products, but also increases them incessantly in proportion to the quantity of toxin injected.

The antitoxic substance exists only in the serum. It is 
not found in the coagulum, but is met with in the transudate of œdemas and in large quantities in the milk, to which Ehr. lich attributed the hereditary transmission of the immunity. The saliva, the aqueous humor and the urine may exhibit feeble antitoxic activity.

PROPERTIES OF ANTITOXIC SERUM.-The. principal properties of antitetanic serum are already knowr. Mixed in vitro, with the toxin, it neutralizes the latter, and the action is instantaneous. As soon as the mixture is effected it is harmless to animals. When gradually increasing quantities of toxin are poured into a given quantity of antitoxic serum there arrives a time when the antitoxic activity of the latter is destroyed, and the liquid will give tetanus to animals that receive it.

It has been thought that the toxin and the antitoxin were capable of uniting into a definite unpathogenic combination, which is, however, not the case, as Roux has shown that the toxin is not clestroyed, and that both the toxin ancl the antitoxin preserve their specific properties. The injection of the mixture is harmless in the most sensitive animals. only when they have never been subjected to the action of micro-organisms or microbian products. But if the mixture is injected under the skin of cavies that have been previously subjected to the action of cultures of the bacillus prodigiosus, the streptococcus, the bacillus coli communis, etc., although having all the appearance of health, they die of tetanus.

The resistance of cavies to a mixture of toxin and antitoxin serum is not due to the destruction of the toxin, but to the serum exerting a peculiar stimulating influence over the cells to such an extent as to render them capable of resisting the action of the poison. Whatever may be the mechanism of the action of the antitoxic serum, it is none the less true that it is preventive. If injected into an animal at the same time, before or immediately after the toxin, tetanus does not 
develop. Antitoxic injection made before the tetanic infection enables animals to resist the disease with absolute certainty. The serum confers the genuine immunity, and the immunity is acquired immediately after the introduction of the serum into the animal. It is proportionate to the dose. and is very different from the slow, lasting immunity acquired through iodated or heated toxins.

Antitoxic Immunity is Immediate but Transient.-It is incapable of curing or attenuating tetanus that has once developerl. It may interfere with free toxin in the organism or with that produced at the tetanic focus, but is ineffectual aganst the toxin that has been absorbed and fixed by the nerve cells.

Tetanic antitoxin is precipitated from the serum by alcohol, magnesia sulphate and tannin. It is altered by heat. passes readily into the dialyzer, and can be preserved in the dry state.

Whether the antitoxin is the. result of transformation of the toxin or is elaborated by the cells is a question. It seems possible that it is the product of cellular secretion, probably of the leucocytes. Vaillard bled an immunized rablit. white, again and again, and found its serum was always antitoxic. We must needs conclude that the antitoxin carried off with each bleeding was replaced.

The antitoxic activity of the serum and the immunity do not go hand in hand. Vaillard succeeded in immunizing a ralshit without its serum becoming antitoxic. He inoculated the animal in the tail with spores and lactic acid two or three times, in small doses. The rabbit afterwarrl resisted toxin and bacilli mixed with favoring micro-organisms and yet its serum was not antitoxic. Furthermore, anima!s that have been immunized and kept for a long time lose their antitoxic power. If at a given moment they are again injected with toxin they will be found to resist it quite as well as 
before, and yet their serum is not possessed of antitoxic potency. Therefore, there is a cellular immunity besides the antitoxic immunization which follows it. Behring first thought that cellular immunity did not exist, but finally admitted the fact.

Antitetanic serum is strongly agglutinant for bouillon cultures of Nicolaier's bacillus. It agglutinates at I per 2,000, or even at I per I 50,000, while the serum of a normal horse does not agglutinate above I per $5^{\circ}$.

SYMPTOMS.-It is desirable to study the symptoms in every species, beginning with man.

Man.-In man tetanus makes its appearance after a period of incubation of at least four days. It averages between six and fifteen days, and may be prolonged to three weeks. The clinical types of the disease vary some, according to the point of inoculation, that is, the point of entrance of the micro-organism. Following a wound of the extremities, hands, feet or trunk, tetanus usually manifests itself at the end of eight to fifteen days by the appearance of contractions of the facial muscles (trismus), which afterwards advances to the neck and becomes generalized over the trunk. At the same time there are circulatory and respiratory disturbances. The pulse reaches Ioo to 115 , and the respirations 30 to 40 . The acceleration of the pulse and respiration are grave symptoms.

During the entire continuance of the disease the temperature does not rise over 37 to $38.8^{\circ} \mathrm{C}$., but between twelve to six hours before death, it may rise to $39,40,41,42$ or even $43^{\circ} \mathrm{C}$. at the moment of death. The rise is rapid. The disease lasts four to five days and then passes into the chronic stage, and on rare occasions is cured. The mortality is from 85 to 90 per cent.

When the virus is introduced into a region innervated by the sympathetic system, tetanus is always serious and 
the clinical aspects are different. (Splanchnic tetanus). Puerperal tetanus, that which occurs as a consequence of ruptured uterus is always fatal. Binot has experimentally reproduced these forms in the cavy. The incubation is as long as from nine to twelve days, and from the beginning the trismus is very intense and uncontrollable, and deglutition is impossible. The patient raises his hand to the heart and experiences a sensation of torment. Spasms of the tongue lasting three to four minutes then follow. and produce almost complete asphyxia. The patient faints as successive spasms occur. Death is inevitable and supervenes at the end of forty-eight hours. In splanchnic tetanus the mortality is roo per cent.

If the inoculation is in the face the disease occurs in another form. (Cephalic tetanus). The period of incubation in this event is very short. It is constantly four days.

There is trismus and facial paralysis in the side inoculated that do not arouse the suspicion of tetanus until the ninth or tenth day after the wound is inflicted. At this time a slight constriction of the throat is observed, a fleeting spasm, that reappears with a period of asphyxia. Death comes with the twentieth or thirtieth spasm, at the end of twenty-four to thirty-six hours.

The last form is tetanus a frigore, which occurs after a prolonged chill. The trismus first makes its appearance, and in twenty-four to forty-eight hours the tetanus is complete. The duration is always long, and the mortality relatively slight, 25 to 30 per cent. This tetanus, which comes so quickly, is not attributed to tetanus toxin, because the latter requires at least four days of incubation, and, besides, in this form the contractions are not permanent. There is a general stiffness, but the contraction can be overcome. The patients can be made to walk and to sit down, and, moreover, no wound is found. It is always febrile, $3^{\circ}$ to $38.5^{\circ} \mathrm{C}$. It 
must be due to a micro-organism other than the bacillus of Nicolaier, perhaps the toxin of the pneumococcus. It is, perhaps, a toxic form of pneumonia. Marcheux has shown that the pneumococcus has a powerful action on the nervous system. He has found that epidemics of cerebro-spina! meningitis have been confounded with epidemics of tetanus. These studies lead to the conclusion that the pneumococcus might be taken into account in the epidemics of tetanus, following great battles.

Symptoms in the Horse.-In the horse a rariable period: of incubation elapses between the introduction of the fetanic germs and the appearance of the first symptoms. The average duration of the period of incubation is two weeks, but it is, however, subject to remarkable variations. Certain exceptional events have shown that tetanus may appear a few hours after the accident. Hoffman observed it six hours after the prick of a nail. It usually develops between the twelfth to the thirtieth day.

\section{ANNOTATION.}

The supposed short periods of incubation so frequently mentioned in connection with tetanus probably never occur. Tetanic contractions following shortly after the infliction of a wound are no doubt due in every case, to some previous trauma that has escaped notice, or that cannot be found at the time of the appearance of the disease. In all of these cases the second trauma is a mere coincidence. Numerous incidents of this kind have been observed. A large draft horse sustained a frightful injury to the fetlock by wedging a heel calk into the slot of a cable car track. In falling to the ground while making desperate efforts to release the fixed limb, the fetlock was dislocated. The subject was carted to the stable with an ambulance, being unable to support weight on the injured leg. The pain was extreme. Six hours later an examination revealed the subject afflicted with acute tetanus, which the owner, of course, attributed to the dislocated joint. A careful examination of the feet, however, disclosed a forgotten nail puncture that had been sustained some ten days earlier. Another interesting case followed two tail operations, - pricking and docking,--performed by two different veterinarians. On a certain date one of the veterinarians performed caudal myotomy on a valuable coach horse. Ten days tater a second surgeon amputated the tail. Three days after the second operation the patient fell sick with sub-acute tetanus, and died two weeks later. The intensity of the disease pointed to the myotomy as the cause, while the relative rarity of tetanus from caudal myotomy as compared with amputation, was pointed out as an 
argument against the last operation. The records of similar (supposed) short periods of incubation were also advanced as against ampufation. While the merits of this case were never decided, it is very evident that the first operation did the mischief. The intensity (subacute) pointed to a much longer period of incubation than three days. Other cases of this character are too numerous to mention. It is indeed very frequent that veterinarians must investigate two wounds, inflicted at different times, to determine which one is tetanogenic. When the character of the wound is not convincing, the period of incubation, compared with the intensity of the tetanic contractions, will always assist in correctly accusing the guilty trauma.

The great frequency of these coincidences would indicate that the tetanus cases reported as occurring one or two hours after the wound has been inflicted, and Hoffman's cases of six hours, were all caused by wounds previously sustained. The discovery of tetanus bacilli in the last wound would be no proof that it is tetanogenic, as these microörganisms are indeed very liable to find their way into many wounds that prove innocuous, and besides the mere discovery of virulent bacilli in a wound is by no means a proof that they have exerted their pathogenicity on the organism.-L. A. M.

The first appearance of tetanus may be preceded by some premonitory symptoms: uneasiness; difficulty in walking, masticating and swallowing; rigidity, and phenomena that rarely attract attention. Most frequently it manifests itself in the rery beginning by pronounced contractions. The neck becomes stiff, and movements of the lower jaw and of the head are painful. The stiffness usually begins at the head, but may also commence at the posterior extremities or where the virus has entered the body. At the start there is. little or no fever. W'hatever may be the starting point of the contractions, they become accentuated and generalized in from two to ten days, at which time all of the muscles are tense, the tetanus is general, and spasmodic attacks become manifest.

The general aspect of the patient is now more characteristic. The attitude is quite peculiar. The head is stretched forward from the neck, which is forcibly elevated. The ears are erect, the vertebral column bent downward, the tail is either horizontal or elevated, and the limbs are stiff and spread apart. The limbs diverge and scarcely bend in walking, which is impossible or very difficult. The movements are made in a single stride. The animal remains motionless 
and uneasy. The muscles are hard and projecting. He seems mummified. The contractions give a very special physiognomy to each region.

In the head, the alae nasi are turned up, the nostrils dilated, the lips hard, rigid and tense, the eyes fixed, glittering and retracted to the depth of their sockets, so as to force the membrana nictitans over the eyeball and the ears

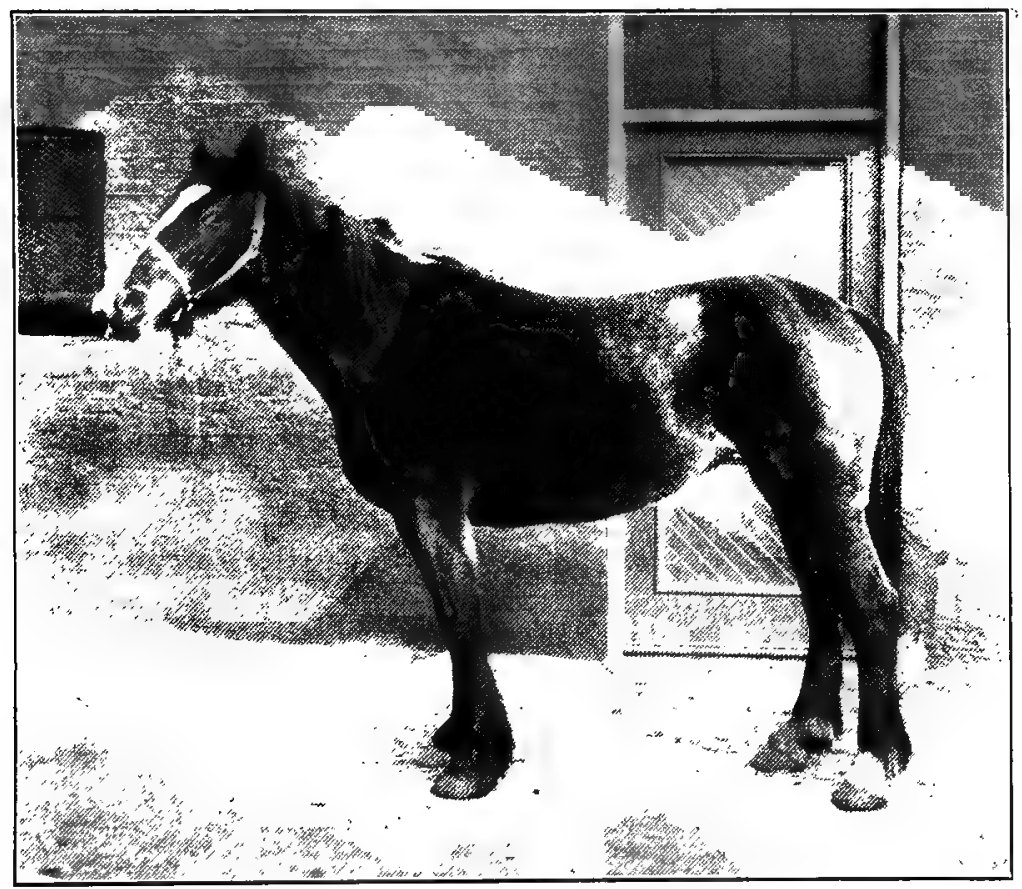

FIG. 45.

Incipient Subacute Tetanus.

upright and drawn together. The masseters compress the dental arcades: this is lockjaw. The jaws cannot be separated, and mastication, at first difficult, becomes impossible.

The contractions of the muscles of the pharynx render deglutition difficult or impossible, and those of the thorax and abdominal wall render respiration difficult and compress the stomach. 
The attitude of the animal varies with the severity of the contractions. It is according to the attitude that the varieties of the disease have been established. If the contractions are in opposite groups of muscles, the pátient is rigid; the head, neck and trunk are on a horizontal line: this is orthotonos. Generally the head is elevated and thrown backward, the dorso-lumbar column is concave and the tail is raised: this is opisthotonos, the most frequent attitude. It occurs in various degrees of severity, and may be limited to the cervical or to the dorsal region.

\section{ANNOTATION.}

There is no good argument in favor of preserving the words "emprosthotonos" and "pleurosthotonos" in the nomenclature of tetanic attitudes of veterinary patients. These conditions are remarkable chiefly for their scarcity in the tetanus of domestic animals. The fact is, they never occur, in infectious animal tetanus. Lateral and inferior incurvations are quite impossible from generalized muscular contractions such as occur from the poison of Nicolaier's bacillus. When the typical attitude of any given infection manifests itself the contractions are already well distributed over the entire muscular system. Although some particular part of the muscles may be more rigid than others, it cannot be disputed that in animals tetanus is always pretty well generalized in the strictest sense of the term. This being the case, opisthotonos is the only tetanus possible. The attitude is due to the great strength of the dorsal muscles as compared with the ventral ones. The jaw is locked shut because the muscles that control the mouth are much more forcible than their opponents, the head is extended because of the greater power of the superior cervical muscles, and the spinal column is depressed because of the enormous size of the longissimus dorsi and its assistants. The hind legs are drawn backward owing to the strength of the gluteal group, and outward on account of that of the abductor muscles of the thigh, as compared with that of the muscles that draw the limb toward the medium line.

Furthermore, impressions that tetanic contractions vary in strict obedience to the location of the initial trauma, cannot be strengthened by observation. That the point of infection has any influence whatever on the degree of contraction in any part of the body would not be seriously entertained by any experienced veterinarian. The tetanus cases following nail punctures in the hind feet, for example, are not uniformly severe in the posterior groups of muscles. Neither can it be claimed that these muscles are always the first to become contracted when the "entrance point" has been the hind foot. Tetanic contractions are not always uniformly severe over the whole body, however. A slight trismus may accompany severe contraction of the posterior groups and vice versa, but the difference is seldom traceable to the location of the trauma.-L. A. M. 
An opposite incurvation is rarely observed; the head is lowered by the muscles of the neck, and drawn downward between the forelegs, and the vertebral column is curved upward: this is emprosthotonos. Reference has been made to lateral curvature- pleurosthotonos-but this is a rare entity. The cause of these various attitudes cannot be explained, unless they be attributed to the seat of the original traumatism.

Whatever may be the form of tetanus and the extent of the contractions, the latter are not permanent. From time to time they lessen for a time and then are followed by

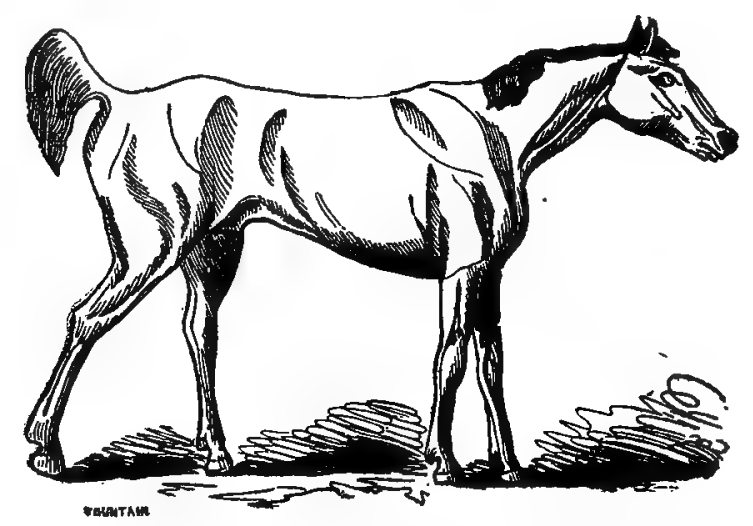

FIG. 46.

Acute Tetanus. (Youatt.)

spasms, These crises or paroxysmal seizures supervene on the least excitement. The patient is remarkably susceptible to the least noise, a vivid light, the slightest contact, the administration of medicines, the taking of the temperature, etc., all of which ape equally liable to provoke paroxysmal fits, convulsions or general spasms of variable violence. Spasms of sufficient piolence to cause rupture of certain muscles haye been observed. Several vertebræ may even be crushed, and the patient nay be thrown to the fleor dur= jng the seizure:

The temperaturure of tetanic subjects is ușually above nor= 
mal. It rises gradually and its increase seems to be proportionate to the gravity of the disease. There are, however, cases of tetanus in which the temperature does not rise above $39^{\circ}$ Generally speaking the high temperature is characteristic of grave tetanus in which the rise is rapid. It may reach $42^{\circ}, 44^{\circ}$ and even $45^{\circ}$, continue until death, and still accentuate in the corpse. Bayer has referred to an instance of a temperature of $45.5^{\circ}$ in fifty minutes after death in a horse having a temperature of $44^{\circ}$ at the moment of death. The thermic curve of tetanus presents no regularity. The

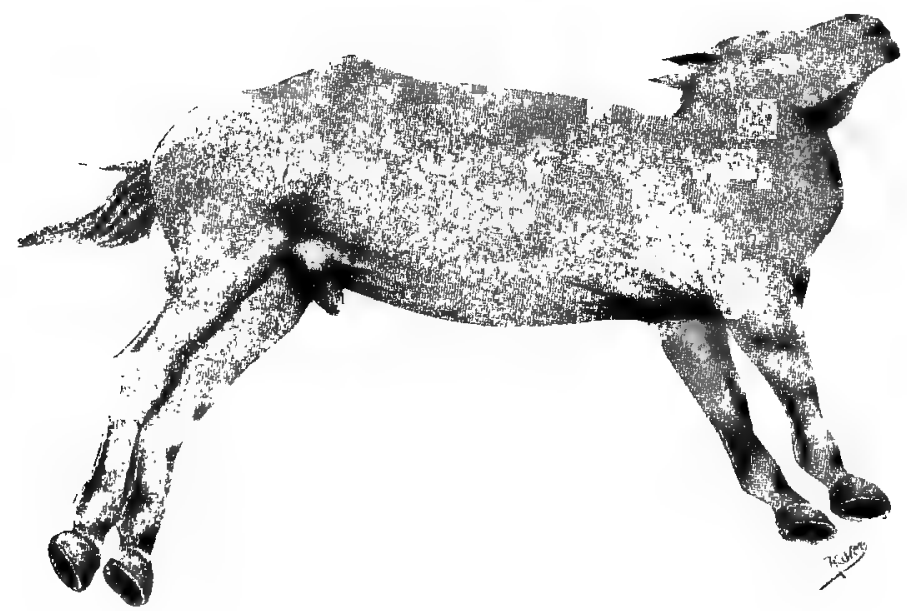

FIG. 47.

Acute Tetanus; recumbent position. (Cacleac.)

temperature rises during the convulsions. (Fig. 48). The hyperthermia depends upon the muscular contractions and the toxic products secreted in the wound by Nicolaier's bacillus and the associated micro-organisms. It also depends upon the disturbance to the thermic centers and upon the complications: broncho-pneumonia, etc.

In the greater number of cases the intellect remains clear and the appetite is preserved. In the beginning mastication is possible, but difficult, and in exceptional cases it continues throughout the entire course of the disease. As a rule the 
constriction of the masseters renclers mastication impossible. Solid food cannot be ingested; liquids are most easily swallowed, but when contractions affect the pharynx and œsophagus, even these are rejected. The saliva itself escapes through the commissures of the mouth. The intestines are contracted, their movements are suspended and constipation results.

The respiration is normal in the beginning, but becones disturbed when the spasmodic contractions invade the muscles of the trunk and diaphragm. They become rapid, short. jerky, painful, panting, and more and more difficult and dyspnœic as the movements of the ribs and diaphragm become more limited. It is during the paroxysmal seizures that the respiratory movements reach the highest mark. They may count 60 to 90 per minute, in which case asphyxia is imminent.

The pulse harmonizes with the contractions. It is normal at the commencement, but becomes accelerated, especially during the paroxysms. It may reach 90 to roo or more, ancl is then feeble, small and hard.

Uncler the influence of contraction of the sphincter of the blarlder the urine is retained. The micturitions are scanty and there is evidence of albuminuria.

From the standpoint of its progress tetanus exhibits two forms: an acute tetanus and a chronic tetanus.

Acute tetanus has a very short period of incubation. It becomes generalized very rapidly and is ushered in by violent contractions, complete trismus and a very high temperature. Deglutition is soon impossible, the respirations are accelerated, the paroxysms are frequent and the animal is covered with perspiration and threatened with speedy exhaustion. The patient soon falls in complete lateral decumbency, and the limbs are so rigid that they are carried some distance from the floor. The agitation is permanent. It 
takes possession of the entire body and death rapidly supervenes. The duration is from one to ten days. In some instances tetanus may develop with frightful rapidity and prove fatal in a few hours.

Chronic tetanus has a much slower evolution. Its period of incubation is long. Localized at first, it is characterized by an incomplete trismus which admits of slow mastication and easy deglutition for a few days, and sometimes for several weeks. The extension of the contractions is slow and the temperature does not rise much above normal. The

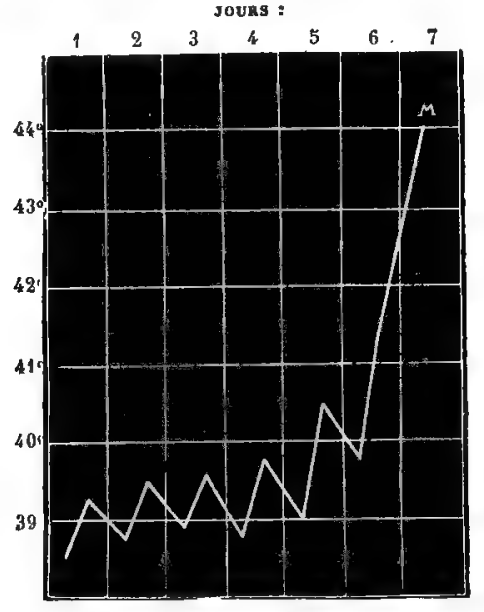

FIG. 48.

Thermic \$ketch of a Fatal Case of Tetanus.

dyspnoea does not become intense and the paroxysms are rare. There may be periods of abatement and return to the normal state. At other times the contractions generalize, the convulsive fits become more frequent and exhaustion and death supervene. There may be a transformation of the chronic into the acute form, these two varieties not being clearly separated.

When patients have resisted for several weeks a cure may be expected. The cure is manifested by a progressive diminution of the rigidity, chiefly in the region of the jaws, 
the pharynx, the neck and the limbs. The temperature falls, mastication and deglutition are easy and paroxysms disappear.

Resolution is slow. The convalescence is always long, from three to four weeks. Certain regions may remain contracted for a long time. The movements are rather easy, but limited, and relapses are possible during the entire period.

The cause of death from tetanus is not satisfactorily explained. It may result from asphyxia, syncope, exhaustion of the nervous system, and from inanition. Poisoning from the toxin seems to have a predominating influence. It may also result from a complication. Pulmonary complications, -pneumonia,-from false deglutition is of common occurrence. It occurs at the beginning of or during convalescence, and death results four or five days after.

Ruminants.-The symptoms of tetanus in ruminants are similar to those of the horse. The rigidity and the contractions attract the attention to it. It first manifests itself by difficulty in locomotion and mastication. The contractions rapidly become apparent. The head is bent, the neck and shoulders are rectilinear and erect, the ears are turned ontward and fixed, and the eyes, drawn deeply into their orbits, are covered with the membrana nictitans. The lips are eon tracted and shriveled, the jaws are set, and the tongue is hard. The entire body is rigid, the limbs are bent with diff:eulty and the tail is moje or less raised. Walking is almost impossible. The contractions of the muscles of the trunk render the respirations painful, dyspncic and accelerated, and tucks the abdomen.

Deglutition and mastication being impossible the patient becomes emaciated, and rumination being suppressed leads to bloating and constipation. The paroxysms are wanting. The rise of the temperature varies from $40^{\circ}$ to $42^{\circ} \mathrm{C}$. The 
evolution of the disease is variable. Death may supervene in from two to ten days and sometimes at the end of two weeks. Among the bovines the mortality is about 50 per cent, while among lambs it reaches 90 to Ioo per cent.

The Hog.-The clisease is rather rare in the hog. It is characterized by contractions that are localized before they become general. They begin in the region of the jaws and trunk. The subject stands upright and its rigid body moves with difficulty. The head is bent over the neck and stands stiffly upwards. When the tetanus has become generalized the patient remains in lateral clecubitus, and cannot return to the standing posture. The muscles are excessively contracted and possess the hardness of stone. The limbs are in an extreme state of extension. It is impossible to bend them. The jaws are locked and the membrana nictitans almost cover the eyeballs. The contraction of the muscles of the trunk curves the dorso-lumbar column upwards. Sendrail has seen a tetanic hog with the incurvation of the trunk forming the letter "S." In the region of the kidneys the body is arched upward, while the cervical region is arched downward. The head is inclined backward. The respirations are accelerated, dyspncic and rattling. He emits plaintive grunts. External excitement, blows, noise, etc., cause the patient to squeal and aggravate the symptoms. The disease ends favorably in two to three weeks or else the patient dies in a few days.

Dog.-Generalized tetanus is by no means frequent in the dog. Cacleac has seen only one case in twenty years' clinical experience. Labet has observed it in shepherd clogs. Moller refers to one case out of 50,000 sick dogs, and Freiclberger and Fröhner one case out of 25,000 patients. White (Chicago) has seen two cases in twenty years.

The symptoms are similar to those of other animals. The contractions first localize at the point of infection and after- 
ward become general. The patient then exhibits general rigidity. He walks with difficulty. 111 of the muscles are strained, projecting and hard, and display convulsive tremors. The ears are straight and the eyes wide open and drawn toward the temporal canthi. The pupils are dilated, the eyebrows knitted, and the forehead crimped. The neck is stretched out, straight and rigid, the back is straight or curved and the tail projects backward. The four legs are stiff and carried in abduction. The flanks are tucked, the hypochondria prominent and the abdomen taut and hard. There is dysphagia, dyspnoea and a hard, irregular pulse. The patient moves with difficulty and falls to the floor.

The progress of the disease is variable. It may exhibit paroxysms that simulate rabies. In fact, the spasms of the pharynx render deglutition difficult and the voice hoarse. Death occurs during a fit. Recoveries are frequent. Warnesson cured 59 per cent. Convalescence lasts about one month.

LESIONS.-Tetanus has been the subject of numerous anatomo-pathological investigations, but no constant, specific or absolutely characteristic lesions have been found. Most of the lesions found in post-mortem are commonplace or secondary, dependent on complications resulting from tetanic infection. Therefore, very different lesions are found in every organ and in every portion of the body.

The initial wound cannot always be found. When the period of incubation has been long, cicatrization may be complete. Often, however, a deep, anfractuous wound, containing a hamorrhagic exudation, is found in the region of the trauma. Neuritis has been found extending a short distance from the wound.

The subcutem may be the seat of ecchymoses and effusions incident to the falling or struggling of the patient on the floor. Fractures of the cranium and abrasions of the 
limbs may also be observed. The muscles may be the seat of traumatic alterations, ruptures of the fibres, ecchymoses, hæmorrhagic infarcts and degenerative changes caused by the contractions. They are deep red, blackish, infiltrated and full of numerous ecchymoses. They are baked, so to speak. Microscopic examination shows them to be granular. The striations cannot be seen and they are tumefied and implicated in fatty or vitreous degeneration. The lungs show lesions of asphyxia. They exhibit congestion, superficial ecchymoses, hamorrhagic infarcts and finally pneu-

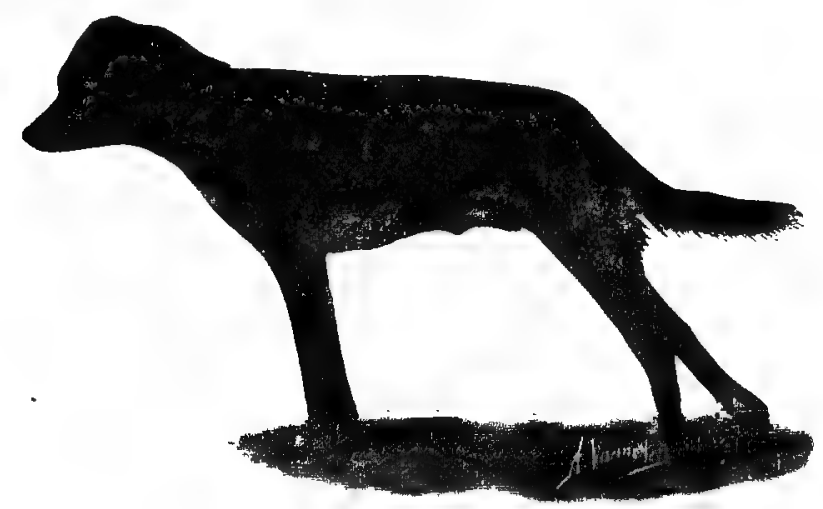

FIG. 49.

monia from foreign bodies, the consequence of false deglutition, which is so frequent in tetanus.

The mucous membrane of the respiratory tract exhibits inflammatory lesions of variable intensity. Ecchymoses and hæmorrhagic foci are found in the pericardium, endocardium and myocardium. The spleen is congested and the kidneys are the seat of hremorrhagic foci and of a more or less violent inflammation. Every mucous membrane may exhibit ecchymotic spots and hæmorrhages.

These lesions are the result of secondary infections. Most of the investigations have been directed to the nervous system with the aim of ascertaining the specific lesions. In the case of both spontaneous and experimental tetanus, micro- 
scopic examinations show absolute integrity of all the nerve tissues in one instance and various lesions in others.

The meninges may be hyperamic and ecchymosed and the medullary substance sometimes presents congestions and hremorrhages. The endyma is thickened with a cellular infiltration. Rokitansky and Demme have described changes that have no pathological significance (proliferation of neuroglia). Bonhomme mentioned degenerative changes in the white substance of the spinal cord and in the roots of the nerves. Achard has seen degenerated nerve fibres situated in the region of the wound. Nerlich found degeneration in the motor nuclei of the trigemini, the facial and the hypo$\mathrm{glossal}$. The method of Nissl ( 1895 ), so precious in the study of minute lesions of the nervous system, has recently been applied to investigating the lesions of tetanus, and the most varied results have been obtained. He refers, without describing them, to lesions of the brain cells and their nuclei in tetanized cavies and rabbits.

Marinesco found lesions in the spinal cords of tetanized cavies which depend upon the activity of the virus and the duration of the intoxication. The gray matter showed diffused hremorrhages that are especially numerous in the anterior (inferior) columns. He observed cellular lesions consisting of apparent modifications in the form and volume of the chromatophilous elements. They are smaller and sometimes reduced to granulation of irregular form and disseminated through the body of the cell in the appearance of a network. In some of the cells they may disappear entirely. The protoplasmic prolongations as well as their chronuatophilous elements have an increased volume and their edges are irregular and granular. In some cases the cell retains its external form and is transformed into a homogeneous mass of high color which renders difficult the perception of the chromatophilous elements. Those observed are profoundly 
modified in volume and structure. It is probable that these instances are cases of coagulation-necrosis. The nuclei present ill-defined outlines and the nuclear plexus is wanting. The neurogliac cells, augmented in volume, transgress upon the nerve cells. If these lesions would be absolutely constant and special in tetants they might be attributed to the combination of tetanic toxin with the protoplasm of the neuron.

When tetanus passes to the chronic form degenerative changes are found in the white matter. Nissl (1897) described different lesions and admitted that they are similar to those observed from ligation of the aorta and from different poisons. Courmont, Doyon and Paviot continued the investigations of Marinesco in an endeavor to explain the period of incubation of tetanic intoxication. They utilized the cavy and the dog. They found alterations of the cells of the anterior (inferior) columns of the cord, but subsequently found them also in the medullary substance of sound cavies. The only alterations of at least some of the medullary cells is therefore an action of Nissl's method rather than that of the tetanic toxin. In a cavy, killed when the contractions were still local (the posterior paw) the same alterations were bilateral and occupied the entire medulla. Their topography therefore did not correspond to the contractions. The relations could not be the relations of cause and effect. In the tetanized dogs hæmorrhages were never found and the medullary cells were absolutely sound.

Nissl's method is accordingly incapable of revealing characteristic lesions of tetanus. While this opinion is diametrically opposed to that of Marinesco it has been confirmed ly all subsequent investigations.

Goldschreider and Flatau have studied nerve cell alterations in a large number of poisonings, by Nissl's method, and have concluded that similar clinical phenomena may be observed from various lesions and vice versa. The same le- 
sions may exist without any etiological analogy. Strychnized rabbits killed three minutes after injection exhibit lesions of the motor cells as in tetanus

These lesions are neither characteristic of tetanus nor productive of it.

Blumenthal expresses the opinion that the lesions observed on animals suffering from tetanus are neither specific nor constant. Tetanus must be consiclered as a consequence of anatomical alterations of the nerve cells.

Nageotte and Ettlinger studied the medullas of caries without finding the lesions described by Marinesco. They found chromatolysis, racuoles and fissures. The lesions occupied the entire medulla and therefore have not the topography that corresponds to the contractions. They may exist in cavies not tetanized, for example in poisoning from potassitum iodicle. Writers consequently deny that there is any connection between the lesions observed in animals affected with tetanus and the contractions which characterize the intoxication. In recent works Courmont, Doyon and Paviot have shown that the medullary lesions seen in the cavy may exist without tetanus and that tetanus may exist without them. They are not pathognomonic. From all of these investigations the conclusion must be drawn that the actual nerve lesions of tetanus are not known.

DIAGNOSIS.-The diagnosis of complete tetanus is extremely easy. The symptoms are typical. There are few diseases so well characterized. General rigiclity, difficulty of movement, trismus, displacement of the membrana nictitans and the position of the head, ears and tail are pathognomonic symptoms in the horse. In the beginning, when the tetanus is incomplete and local, indecision is permissible, but this phase of the clisease is of short duration. It rapidly becomes general. Tetanus is easily differentiated in the horse, from laminitis, rabies and cerebro-spinal meningitis, 
which diseases may exhibit some analogy with it. Laminitis has a single common symptom with tetanus: difficulty of movement. This is differentiated by the absence of trismus, rigidity of the neck and trunk, etc., and by symptoms peculiar to it: heat and sensibility of the hoofs, etc. In rabies there is rigidity instead of contractions, fits of rage, tendency to bite, etc., which must not be confounded with the paroxysms of tetanus. And besides the jaw is paralyzed instead of locked. Cerebro-spinal meningitis which manifests itself by trismus, grinding of the teeth, tetanic contractions of the masseter muscles, etc., is distinguished from tetanus by the succession of the periods of excitement, depression, vertigo and finally coma.

In ruminants cerebro-spinal meningitis is somewhat similar; but the spasmodic contractions differ by remaining local and of short duration. The existence of cerebral symptoms, and the appearance of progressive paralysis also assist in making the differentiation. In the dog, tetanus must be differentiated from rabies and strychnin poisoning. Rabies: especially dumb rabies, exhibits several points in common with tetanus, but the paralysis of the inferior jaw and the absence of permanent general contractions are sufficient to prevent all errors. Strychnin poisoning exhibits paroxysms of but a few minutes duration. The progress and the reflex excitability are sufficient to make a differential diagnosis from tetanus.

During the period of incubation tetanus .cannot be diagnosed. It can not be recognized until the contractions appear. If such a diagnosis were possible preventive inoculation of antitetanic serum could be successfully applied in every case, as it certainly hinders the appearance of the contractions if used before incubation. It may therefore be understood that means have been, sought to make a hurried diagnosis of tetanus Courant wondered whether the phe- 
nomenon of agglutination would not apply to tetanus and constitute a practical expedient of making a diagnosis during the silent period of incubation. Sabrazes and Riviere have declared that the blood of tetanized animals agglutinates Nicolaier's bacillus.

Courmont and Julien have studied the question systemat= ically and have concluded that no dependence should be

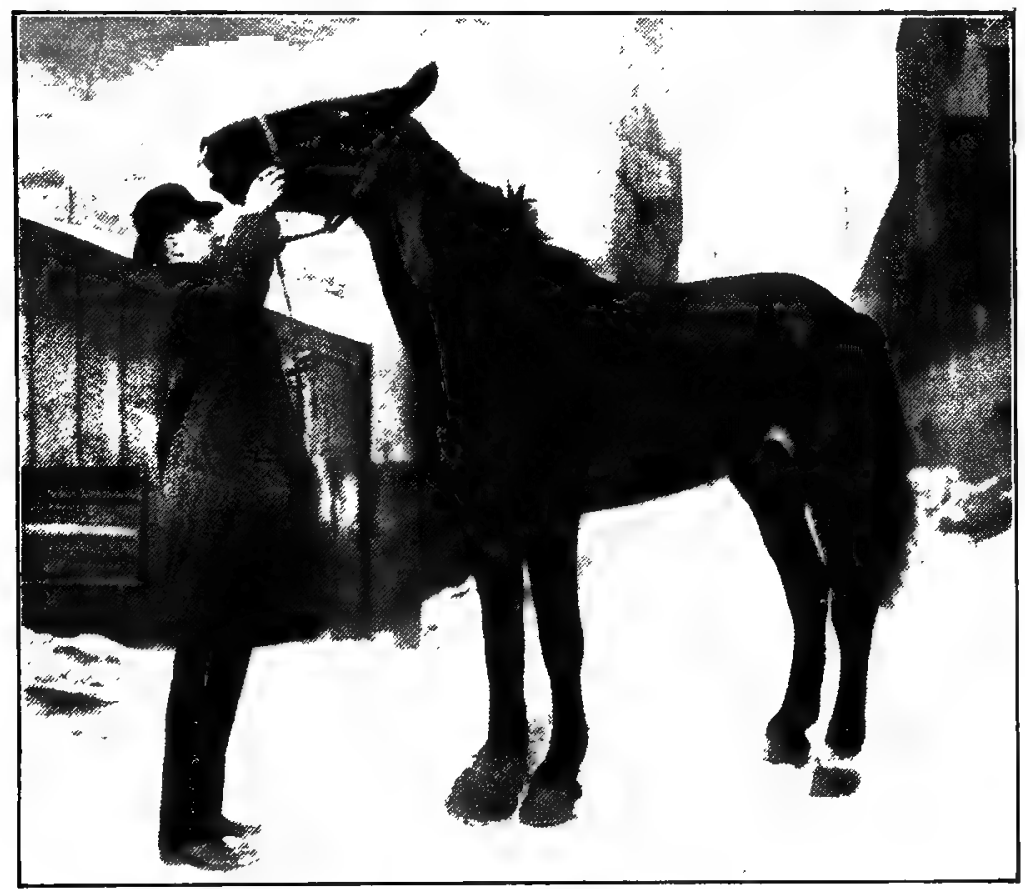

FIG, 50 .

Method of Diagnosing Incipient Tetauus.

placed upon the agglutination of Nicolaier's bacillus by the blood of tetanized animals, either in making an early diagnosis or in confirming a clinical diagnosis. The blood of man, the mouse, the cavy, the rabbit, and the dog suffering from tetanus has never exhibited agglutinating properties. The blood or serum of man, the cavy, the mouse, the hen, the dog, the frog and the turtle is not agglutinant to tha tetanus 
bacillus, but the blood or serum of the horse and donkey exhibit agglutinative properties. This normal agglutinant power of the horse's blood is found in animals strongly immunized against tetanus. Thus, in relation to the horse Julien has stated that, after immunization, the normal agglutinant property has increased a thousand fold. Immunization also develops agglutinant properties in an animal that does not exhibit them before,- the rabbit. The results of these investigations slow that agglutination cannot assist in the diagnosis of tetanus.

PROGNOSIS.-The prognosis of tetanus is always grave. It is frequently fatal. Its gravity is demonstrated by all the various statistics. The average mortality in the horse is about 70 to 75 per cent, in the ox from 50 to 60 per cent, in the small ruminants and in the hog 90 per cent and in the dog about one-half of the cases recover. The maximum gravity is exhibited in the young, particularly the new born. In the case of lambs it is invariably fatal.

The prognosis of tetanus depends upon the duration of the period of incubation. According to the duration of the period of incubation the distinction is made between the acute and the chronic form. The first, which is characterized by the rapid appearance of the symptoms, is always fatal. In the second, on the contrary, the contractions which make their appearance after a long period of incubation, do not generalize. This form is capable of being cured, and that without medicine. The prognosis should be based upon the rapidity of the invasion of the contractions, on their localization, on the intensity of the trismus and paroxysms, on the acceleration of the respirations, and on the elevation of the temperature.

TREATMENT.-The treatment of tetanus is prophylactic and curative.

Prophylactic Treatment.-Knowing that tetanus is al- 
ways a complication of wounds, efforts should always be made to prevent the infection of traumatisms by the bacillus of Nicolaier. The antiseptic precautions render the execution of this recommendation possible and reduce the chances of infection. As tetanus spores strongly resist antiseptics they are hard to destroy, but Vaillard's experiment has shown that the spores are harmless without being associated with other bacteria, and as these are more vulnerable, they can be destroyed by antiseptics. The tetanus spores thus deprived of the aid of the associated bacterian species become the prey of the leucocytes.

Accidental wounds soiled with earth, straw or dung, so often tetanigenic, should be cleaned, irrigated carefully, purified, rid of foreign bodies and freely submitted to the action of antiseptics. Anfractuous wounds with bloody infiltration and necrosis of the tissues should be surgically renovated and then appropriately protected against subsequent infection with surgical dressings. Operating tetanus is prevented by using only sterilized instruments and dressings. Cases of enzootic tetanus following castration are thus prevented.

Accidental or surgical wounds that cannot be properly disinfected must be prevented from causing tetanus by the use of antitetanic serum, which will positively prevent the evolution of the disease.

Nocard has experimented with the serum, and he has published results obtained by a number of veterinarians, which confirm his experimental data. He relates the treatment of 2,705 animals, of which 2,373 were horses, donkeys or mules, 44 bulls, 82 rams or lambs, and 206 hogs. Fach of these animals received two injections of the serum at twelve days interva1, 20 c.c. for big animals and 6 to ro c.c. for the sheep and hogs. The inoculations all followed either an accidental or surgical wound. Among these 2,705 animals, 2,300 of them received the first inoculation immediately after 
the operation to which they were subjected (castration, amputation of the tail, umbilical hernia, cryptorchidectomy, etc.), and not a single one contracted the disease. The second group comprises 400 subjects which received the serum one, two, or four days after the accidental traumatism of which they had been victims (street nails, nail punctures, quittors, bites, etc.), soiled by earth or dung, and not one succumbed from tetanus. Only one horse, treated five days after the accident (pricked by a horse-shoer) presented tetanic symptoms, but the disease was the most benign imaginable. Injected too late to prevent the disease, the serum considerably reduced its gravity.

The results published by Nocard are of special importance from the fact that the preventive treatment is now in use in localities where the disease was so frequent that proprietors refused to submit their animals to the usual operations,-castration, caudal amputation, etc., in the farm stables where tetanus had found victims before. Finally, during the time occupied by the experiment, the veterinarians who lost none of the 2,705 animals preventively treated, observed 259 cases of tetanus not treated. Of these I.9 I were horses, 57 rams, and I r bovines. All of these points demonstrate that antitetanic serum is absolutely effectual as a preventive. Among the cases which prove its efficacy the following is the most striking. A veterinary hospital became infected to the extent that five out of eight surgical subjects contracted tetanus. From the very moment that every animal operated upon received the serum, and out of ${ }^{6} 63$ castrations of horses placed in the same premises, not a single case of tetanus occurred.

The technique of preventive inoculation is simple. It consists of the hypodermic injection of the serum in any part of the body, preferably the region of the neck or shoulders. The guantity used is Io c.c. for the horse and bovines and 3 
to 5 c.c. for the small animals-sheep, goats and hogs. Two injections are made at intervals of twelve days. The immunity, although transient-two to six weeks duration-is sufficient to carry the subject safely over the period of cicatrization to the infected wound. For wounds of a longer duration, a third or fourth injection must be made. The serum causes no inconvenience. It is as harmless as it is effectual.

It is supplied by the Pasteur Institute of Paris in bottles containing Io to 20 c.c. The firm of Meister, Lucius \& Bruning, of Hochst-on-the-Main, furnishes desiccated serum that is used in 5-gram doses dissolved in 45 c.c. of distilled water.

\section{ANNOTATION.}

Besides the Pasteur Company, which has distributing offices in all the principal American cities, the serum can also be obtained in America from Mumford \& Co., of Philadelphia, and from Parke, Davis \& Co., of Detroit, Mich.-L. A. M.

Curative Treatment.-The most varied treatments have been recommended against tetanus. Their number and diversity shows their inefficiency. The fact is, in spite of innumerable attempts, no agent has been found capable of hindering the progress of confirmed tetanus. There is nothing more uncertain than the treatment of tetanus. It is useless to recall all the expedients that have been successively extolled. Recoveries have occurred under the infuence of a variety of different medicines: in reality it cures itself. The medicines have counted for nothing in the matter.

The most rational treatment based on the theoretical knowledge acquired during the last few years as to its etiology and pathogenesis, is both local and general.

Local treatment is directed toward the suppression of the bacterian hot-bed. Its object is to destroy the microorganisms that produce the toxin. This recommendation may be effected by different surgical expedients: cauterization, ablation, amputation, disinfection, etc. The ablation 
is easy when the wound is conveniently located, for example, the end of the tail. If from a seton the entire tract is laid bare by incision. Such operations must be performed early to be of service. They must be performed on the very first appearance of the symptoms in order to remove the micro-organisms before they have injected a fatal dose of the toxin into the organism. Anæsthesia is essential to prevent excessive excitement. Such operations are only advisable when the wound is located in structures of secondary importance (skin, muscles, etc.). Simple disinfection alone is possible when important organs are involved, or where the operation would necessitate extensive mutilation. Besides, the effects are very uncertain. Vaillard had shown experimentally that the extirpation of the muscle into which a few drops of toxin have been injected does not prevent the development of tetanus.

\section{ANNOTATION.}

The results obtained in practice from extirpation, ablation, re-amputation, disinfection, etc., of the tetanigenic wound is never followed by any immediate change in the progress of the disease. Alone it will accomplish nothing, but when applied as one of the various efforts, there is but little doubt that scme benefit is actually derived. In the very early stage, while poison is still being elaborated in the wound, and in view of what is now known of the pathogenesis of tetanic intoxications, the treatment of the wound, by either mechanical or medicinal disinfection. is a palpable duty of the surgeon.L. A. M.

General treatment aims at the toxin already in the organism and the arrest or diminution of its effects on the nervous system. The first recommendation is the injection of antitetanic serum. Contrary to the opinion once held by Behring and Kitasato, antitoxic serum is not curative. Its employment is, however, useful in neutralizing the poisons not yet fixed in the nerve cells and which continue to be formed in the wound. The antitetanic serum will give variable results. If the animal has absorbed a fatal dose of the toxin, no benefit will be derived, while in the opposite case its action is effectual. It prevents further intoxication 
and enables the animal to eliminate and destroy the poison already combined. In cases of chronic tetanus, progressing slowly, the serum has produced good results in the hands of Dieudonne, Chennot, Rohr and Lang. In acute cases it was always a failure.

The investigations of Nocard show that injections of the serum are the best treatment for confirmed tetanus. The crises are less intense, less numerous, and when recovery begins the period of convalescence is shortened.

Statistics support this opinion. In 28 olservations by Dieckerhoff the mortality fell from 80 and 90 per cent to 50 per cent. The injections should be given in the jugular in doses of $20 c c$ every two or three days. According to Nocard the results should be better among cattle and sheep.

Treatment by Intracerebral Injections of Antitetanic Serum.-Roux and Barrel have shown that in animals injected for preventive purposes the antitoxin remains in the blood and does not come into contact with the nerve centers. If tetanic toxin is injected into the brain of the subjects thus immunized they will exhibit tetanic manifestations (cerebral tetanus). It is therefore clear that the serum treatment is ineffectual after the disease has once cleveloped, because at that time a part of the toxin is alreadly adhering to the nerve cells. The antitoxin neutralizes the toxin that still circulates, but it does not attack that part already settled in the nerve centers. These authenticated facts have suggested the idea of directly introducing the antitoxin into the nerve centers. The antitoxin introduced into the brain protects it, if the medulla is not already affected. This method has given good results against experimental tetanus of rabbits and cavies, and it has been applied to man, but the results up to the present time are not conclusive. Out of 24 cases in the human being treated in this manner 6 recovered and 18 died, a mortality of 75 per cent, which is higher than 
the average mortality in man. Most of the failures, however, have been in cases of grave prognosis and rapid development. There are no publications obtainable which relate to the treatment of tetanus by intracerebral injections in the lorse. The experiment is yet untried. In every instance they should be resorted to as early as possible.

The tolerance of the brain to antitetanic serum is interesting. The practice of intracerebral injections is simple, althougl the most absolute asepsis is essential. In the horse it would first be necessary to resort to complete anresthesia by chloroformization, and then incise the skin parallel to the median line about two centimeters outside of the parietal crest. After clissecting the subcutaneous connective tissue and the insertion of the temporalis muscle, a small trepanation is made about 3 centimeters above the union of the parietal crests. The needle of Pravaz's syringe, five to six centimeters long, is then passed through the white substance into the lateral ventricles. The injection of about ten cubic centimeters is made, clrop by drop, into each hemisphere. The operation is followed by consecutive psychical disturbances, which are, however, of short duration. At the autopsy no trace of the needle puncture in the cereliral substance is found.

Medical Treatment.-The medical treatment aims to modify the excitability of the nerve centers caused by the poison. Seclatives and hypnotics are used. Chloral is the proper drug for the horse, and it should be administered in enemata in doses of about Ioo grams per day. It hild sometimes been administered intravenously. Essence of turpentine in large doses, subcutaneous injections of carbolic acid solution, oxygenated water, quinine, antipyrin, frictions with carbolic acid solution, intratracheal injections of potassium bromide, potassium iodide, atropine, curare, eserine, and pilocarpine have been successively recommended in 
the treatment of tetanus. J. Courmont and Doyon have shown that the subcutaneous injections of carbolic acid completely fail against experimental tetanus.

All of these medical expedients are credited with successes, but none are obligatory. The results obtained depend upon the form of the disease rather than upon the medicines administered. When used, they may as well be forgotten.

The therapeutics of tetanus is completed by special hygienic precautions which are applicable to every case. These are,-rest and absolute isolation of the suffering animal. The patient must be kept in a stable that is shaded, quiet, silent and rather warm, with a uniform temperature, with the purpose of avoiding any excitement that may lead to convulsive fits. The food should consist of liquid nourishment that is light and easily digested. If the trismus is intense, alimentary enemata are recommended. Light purges should be given to prevent constipation. This course of treatment limits the contractions and limits the paroxysms and general convulsions. It prolongs the patient's life.

It must be remembered that the lingering cases are the curable ones.

\section{ANNOTATION.}

In acute tetanus treatment is futile while in the extremely chronic vanety it is unnecessary. Chronic tetanus will recover spontaneously under sensible stable attention as regards proper dieting, sanitation, etc. It is the sub-acute variety that calls for special therapeutic, hygienic, antitoxic, and surgical intervention, which, although not always successful, will often guide a seemingly fatal case toward recovery. A course of treatment, based upon what is now known about the pathological state of a tetanic subject, and upon the good results obtained from its application to a large number of cases, is as follows:

Ist. At the very onset of the symptoms, or as soon as the subject is submitted for treatment, unless the symptoms are well advanced, the patient is placed in an appropriate position for the disinfection of the wound. The disinfection is either mechanical or medicinal, according to the location and character of the trauma. If the operation will be long and painful, the patient is placed on the operating table and anæsthetized with chloroform. The wound is opened, drained, irrigated and curetted, laid bare, extirpated 
or amputated according to its position and condition. In the after care only nominal effort will be made to prevent subsequent infection with pus germs. The free admission of air is preferable to the applicationi of an impervious covering which will again shelter the anaerobic microörganisms it may still contain. Packing with loose gauze soaked with potent antiseptic solutions is ample treatment for the wound thus renewed. The aim here is to prevent further absorption of tetanic toxin.

2 d. At the completion of the foregoing step 20 c.c. of antitetanic serum are injected into the jugular vein. These doses are repeated every second or third day during the first twelve days. The aim is the neutralization of the free toxin in the organism that has not been appropriated by the cells of the nerve centers, and which are still capable of yielding to the antitoxic action.

3d. The subject is placed in as quiet a place as possible, where the light is neither too vivid nor entirely absent, and then put into a sling that is carefully adjusted to the body. As the tetanus patient does not survive the recumbent position and can only be returned to the standing position with the greatest difficulty, slinging is always a wise precaution if not an absolute necessity in the treatment of sub-acute tetanus.

4th. The character of the food will depend upon the intensity of the trismus. Hay, oats, corn, mashes or any of usual feeds are admissible if the patient is capable of masticating them; that is, if the trismus admits of mastication. When the motion of he jaw is more limited liquid diets consisting of oatmeal gruel, linseed tea, hay tea, bran gruel, cornmeal gruel, etc., are the only nourishments the patient is capable of ingesting. In these liquids liberal amounts of alcohol may be added to augment their nutritive value. Feed stuffs administered per rectum will prove a disappointment. By restricting the diet and by always giving it fresh and clean, various useful medicaments can be administered with it. In this manner two to three grams of calomel can be administered per day to prevent constipation and colics.

5th. Potassium bromide, chloral hydrate and carbolic acid seem to give the best results. These are named here in the order of their practical applicability if not in that of their therapeutic value. Potassium bromide is tasteless and soluble in the liquid food and can therefore be administered in any reasonable quantity without exciting the patient. Chloral hydrate cannot be administered per os on account of its irritating properties. If diluted sufficiently the quantity precludes easy administration. Per rectum, if continued during several days, even highly diluted it causes irritation of the rectum that is often serious. Carbolic acid administered subcutaneously is objectionable because the operations excite the patient and thus provoke harmful paroxysms. Too much importance must not be placed upon the internal medication. At best it does but little permanent good.-L. A. M.

\section{ACTINOMYCOSIS.}

DEFINITION.-Actinomycosis is a parasitic disease caused by the vegetation, in the tissues, of a specific parasite belonging to the genus Actinomyces, and described under the name of Actinomyces Bovis. 
SUSCEPTIBLE SPECIES.-Animals of the bovine species are very often affected, but the disease is also observed in the hog, the horse and the sheep. It was seen once in the dog by Heim. Schreiber has pointed it out in the cat, and Montfallet has mentioned cases in the llama and the guanaco. It has been reported as having been seen in the elephant. In man it was noticed for the first time by Lucet in 1888 . Since that time it has been the subject of numerous investigations, especially by the practitioners of Lyons.

HISTORY.-The clinical aspects of the disease have been recognized for a long time in veterinary medicine. In the beginning of the nineteenth century, a disease which in most cases was actinomycosis was described by veterinarians under the names of "osteosarcoma," "osteoporosis," "spina ventosa," "cancer," and "farcy of the bones." About the middle of the century Langenback (1845) and Leber (1857) found radiating bodies in osseous tumors of man, which they reyarded as crystals and to which they attached no real importance. Davaine (I850) and Laboulene (I853) studied osseous tumors which today seem to have been actinomycostic. Up to that time nobody had any suspicion that these tumors were of a parasitic nature. Rivolta, who in I868 found "elements in the form of little rods". in the tumors of bovine animals, looked upon them as crystals. In I875, Perroncito again found the crystals of Rivolta, but he was of the opinion that they were cryptogamic productions. Rivolta, returning then to his previous studies, confirmed Perroncito's views and gave complementary inclications.

The disease was studied in Germany. In 1870 , Hahn found an ox attacked with "wooden tongue," an analogous growth to those pointed out by Rivolta. In 1877 , Bollinger" established the constant presence of the radiating parasite in the tumors of the maxilla. He made a botanical study of the parasite and named it "actinomyces" and the disease "actinomycosis." 
These investigations were rapidly followed by many others. In the course of a few years the parasite was discovered, studied, and described in nearly every country of Europe. Johne tried to cultivate it in 1885 , as did other numerous experimentalists.

In France, the disease was recognized by Nocard in 1885 . The following year Professor Thomassen reported the successful results of treatment with iodine. Since that time publications on the subject have been very numerous, and they have dealt with every point. It may be safely said that
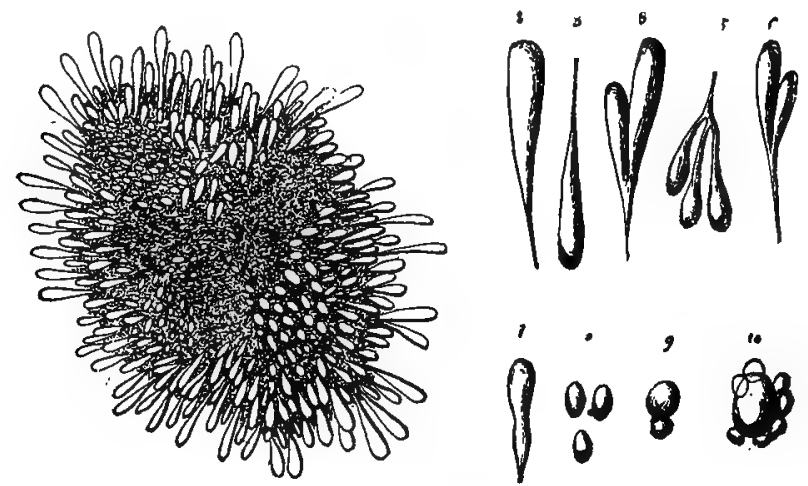

FIG. 51.

Actinomyces from Tumor of the Inferior Maxillary of an Ox. 1. One Granulation Entire. 2, 3, 4, 5, 6, 7, Different Club-Shaped Forms ; 8, 9, 10, Rounded Elements.

actinomycosis has been more closely studied than most any other disease.

Character of the Parasite in the Tissues.-When a section is made through an actinonycostic lesion, or when the pus discharging from the fistula of the tumors is examined, "yellow grains" are seen at the surface of the section or incorporated with the pus. These grains, when calcified, feel like fine sand, and the proof of their presence reveals the exact nature of the morbid process. They vary in size; some are microscopic, while others, on the contrary, are the size of a pin-head. They are coated with gelatinous substance that is difficult to remove completely. 
MICROSCOPIC EXAMINATION, even without staining, permits the determination of their nature witl great accuracy. Staining with picro-carmin gives the most beautiful preparations. The yellow stain becomes fixed on the center of the parasite, while the carmine stains the periphery, the gelatinous covering and the lymphatic elements, which the latter contains. To simplify the description, two zones are distinguished in the tufts of the parasite. The central zone is composed of fibrillary matting whose filaments are spiral and ramified and they radiate toward the periphery. (Fig. $\left.5^{1}\right)$. The peripheric zone is constituted of club-shaped elements. These pear-shaped en!argements are characteristic; they are yellow, very refractive, homogeneous, four to twelve microns long and four microns wide. They are simple or bifurcated and sometimes vary in length. Some of them extend perceptibly beyond the limits of the tuft. Numerous hypotheses have been advanced on the constitution, the nature and the function of these enlargements. Bostrom thought he had found a filament in their axis that is continuots with the filaments of the central zone. Harz, Carmel and Babes at one time maintained that they were organs of generation. It is now established that they are naught but involute arrangements.

METHOD OF STAINING.-Staining is necessary in order to properly study the details of the structure of the parasite. The actinomyces either does not take Gram's stain well or else is badly stained by that method. Picro-carmine, on the contrary, gives a good preparation. Lemiere and Beene approve the following method: Place a little of the pus in a watch glass and wash well with ether and allow it to bathe for some time in a recently prepared concentrated solution of caustic soda or potash, and then from ten to fifteen minutes in a 5 per cent aqueous solution of eosin. The specimen is then washed in a concentrated solution of sodium or potassium acetate, mounted in the same solution and imbedded 
with paraffin. This process, which gives satisfactory results, is also recommended in the notes of Baranski, Petrow, Babes and Flormann.

CULTIVATION.-The parasite is facultative. It can be cultivated on potato, especially in the absence of air and with the aicl of pyrogallic acid, at a temperature of $22^{\circ}$ to $24^{\circ} \mathrm{C}$. The culture is thick, wrinkled and of a sulphur yellow color. Its characters are rather quickly modified. It becomes gray, then white, and finally a yellowish green. The latter color is developed from the exposure of the culture to light. In serum the surface of the culture becomes covered with a dirty, white coating. The parasite also grows on agar at $35^{\circ}$ to $37^{\circ} \mathrm{C}$, and on glycerinated agar. The cultures may remain for months in the form of grey tubercles, consisting only of ramified filaments which do not give spores. In glycerinated peptonized bouillon the ray-fungus forms white, round masses that do not cloud the media. It may also be cultivated on the grains of cereals. Berard and Nichols have made cultures on grains of oats and wheat, which they were able to preserve for four years. At that time they presented the form of a yellowish powder, consisting of spores that weire isolated or collected into chains. These elements exceeded the different cocci in size.

The parasite thus preserved, when re-cultivated and then inoculated into the rabbit, produced lesions in which the typical forms were again founcl.

The character of the ray-fungus in the tissues and in the cultures has enabled naturalists to classify it, although there is still some difference of opinion on that score. Some hold that it belongs to the oöspora, while others oppose this view and maintain that the oöspora and the actinomyces are distinct types. Gasperini recognized four species of the rayfungus, but Neumann considers that all forms of actinomyces belong to one species,--the actinomyces bovis. 
INOCULATION.-The ray-fungus is difficult to inoculate. Wolf and Israel nevertheless succeeded in communicating the disease to the rabbit by introducing parasitic tufts into the peritoneum. Johne made use of the subcutaneous inoculation and with Gutmann succeeded in reproducing the affection in the cow, the horse and the bull. Caufit obtained results in the ox by introducing the parasite into the peritoneal cavity. At the autopsy he found the lymphatic glands oi the meso-colon congested and covered with tubercles. The heart was adhered to the pericardium and on both were numerous yellow tubercles containing the fungus. Ponfick has been able to infect the calf; Mosselman and Lienaux, the rabbit; and Mandereaut, the goat, the rat and the sheep. The cat, the log, and the cavy are refractory. The inoculation of pure cultures has been successful in the hands of Mosselman, Lienaux, Wolf, Israel and Mandereau.

ETIOLOGY.-It is now known that the actinomyces is, above all, a vegetable parasite that exists only accidentally on animals and that the tissues of animals do not form a very favorable medium for its development. It is through the medium of vegetables, on which it lives, that man and arimals are infected. The latter are infectel as a consequence of dental irregularities or abrasions of the mucous membranes caused by the mastication of stalks. Dry fodder is a natural source of infection. The chaff and the stalks of gramineæ inoculate the mucous membrane or tear it for subsequent infection, or they may become wedged between the teeth and deposit the parasites which they carry upon the tissue into which they are lodged. The hordeum murinum (wall-barley, mouse-barley, wild-barley, so-called) is particularly dangerous. Observations by Jensen and Neuwirth establish the fact that straws are not highly infective. Krantz and Tribout found actinomyces on thorns implanted in the buccal mucous membrane of African oxen. Johne found the 
fungus on a barley-beard implanted in the velum of a hog, and Korsak on a beard of grain inserted in the tonsil. Analogous facts are reported by Neuwirth, Kouritzine and Piana.

In man, the part played by cereals is likewise clearly shown by exact observations. In five cases of human actinomycosis, Bostrom found fragments of barley in the tissues invaded. The vegetal, saprophytic life of the parasite also explains the cutaneous inoculations. The fungus fixes itself to the surface of the skin after an excoriation or superficial wound. Actinomycosis of the knee, of the interdigital space and of the external side of the limb, are developed from this circumstance.

In the hog and ox infections have been known to take place on the spermatic cord, and in the flank wound of the sow, following castrations. Mammary localizations in the sow and cow result, beyond all doubt, from an infection ascending up the galactophorous canal, and in a like manner the hen becomes infected through the oviduct. Actinomyces has been found in the eggs of hens.

Actinomycostic infection is rendered more frequent by the great resistance of the parasite. Its spores will resist boiling for fourteen minutes, a dry heat of $145^{\circ} \mathrm{C}$., five minutes contact with corrosive sublimate, $\mathrm{I}$ to $\mathrm{r} 000$, and a 5 per cent solution of carbolic acid.

It is very improbable that the affected animals communicate the disease, except indirectly, by throwing off the parasite into external environments. This is largely on account of the fact that the parasite discharged from the fistula are degenerates which cannot cope with the organic forces of the tissues it infects. Its inoculation is always fruitless. If positive results have been obtained by Johne, Max Wolf, Israel, and others, they must be considered as exceptional and attributed to the fact that the parasite was placed directly into 
recent lesions and at a time when it was still in the filamentolis form. Liebmann has demonstrated that the pathogenicity of the parasite emanating from an actinomycostic fistula can only be restored by passing it through a plant cultivation.

Therefore the instances of enzootic outbreaks must not be regarded as the result of contagion. Actinomycosis is no more contagious than anthrax, for example: the cause of outbreaks in a large number of animals in one region, or on the same farm, is the ingestion of the same food.

These considerations demonstrate that the danger of infection from meat of actinomycostic animals is nil. The parasite in meat is already almost deprived of its pathogenicity, and cooking, even though slight, removes all grounds for apprehension. It is therefore absolutely illogical to attribute the cases of intestinal actinomycosis to the ingestion of meat taken from infected animals.

Is the actinomyces pyogenic? This question seems perfectly useless since the parasite discloses its presence in the tissues by suppuration. This point is, however, disputed. Netter holds that the parasite is clearly pyogenic, while others hold that the pyogenic power is the work of parasitic bacteria of the fungus. In fact, cases of mixed infection have been pointed. The investigations of Israel, Babes, Gottotein, and Roussel demonstrate the constant association of parasites with different microbian forms. The part assigned to these different agents in pyogenesis is still undecided. Some writers are inclined to believe that the cases of spontaneous recovery are due to a vital combat between the bacteria and the fungi.

PATHOGENESIS.-How does the parasite behave in the tissues? Its penetration provokes an organic reaction which ends in the construction of a nodule called an actinomycoma, which in its histological constitution resem- 
bles the tubercle or the follicle of Koster. "The histological lesions of the actinomycostic nodule and the tuberculous tubercle are alike. The invading parasite alone is different."

The actinomycostic nodule is primarily composed of parasites surrounded by giant cells around which are found accumulations of epithelioidal cells. When the process is very rapid the nodule has all the characters of Virchow's lymphoid tubercle, but it does not undergo caseous degeneration like the so-called tubercle. In time it undergoes fatty or puriform degeneration, or else is transformed into cicatricial tissue.

The first sign of degeneration is marked by leucocytic infiltration of the epithelioidal cells. The white cells dislocate the parasites of the neoplasm and cause degeneration or purulent exudation. Degenerated epithelial cells are therefore found in actinomycostic pus, in addition to capitula of dead actinomyces, hyaline bodies resulting from breaking up of the clubs, pus cells and free grains of chromatin. The infiltration of the actinomycostic neoplasm by polynuclear leucocytes is therefore the first step in the degeneration of the nodule, and is not a part of its normal constitution. These data on the constitution of the actinomycostic neoplasm have been elucidated by the investigations of Johne and Moorbrugger, and were completed by the studies of Pawlowsky, Maksutoff and Hoche. These writers have, in fact, demonstrated that the penetration of the parasite into the tissues is immediately followed by an organic reaction that aims to surround and destroy it. The phagocytes, by reason of their chemiotactic property, surround the fungtus and undertake its dislodgment. They carry off the filaments which develop slowly from within the parasite when it is not destroyed.

If the tissues weaken, the fungus destroys the cells that surround it, and gradually transgresses farther. If, on the contrary, the tissues resist, the phagocytes grasp the para- 
sitic extensions and endeavor to break them up and destroy them, and the fungus thus takes the form in which it is found: the form of degeneration or involution. The hyaline globules are the remains of the shattered club-forms.

Generalization of the disease is very rare and is explained by the parasite's behavior. The disease is diffused through the medium of cells laden with living filaments, perhaps, also, but more rarely, through the lymphatics and finally sometimes through the blood vessels. Its localization has been observed in the blood vessels. It must not, however, be supposed that the actinomyces behaves like a microbe. Its behavior is entirely different and its generalization should be regarded as exceptional.

GEOGRAPHICAL DISTRIBUTION.-The disease is found in nearly every country of Europe. In France the maxillary form is especially noticed. The lingual form is rare. Nocard and Leclainche show by statistics collected at the market of Villette the proportion, in round numbers, of 0.72 per rooo: the stock of Maine (2.94 per Iooo), of Holland ( $1.5^{2}$ per IOOO), and of Normandy ( $1.5^{2}$ per Iooo) furnished the highest figures.

At Saint Etienne, out of I3,905 cattle slaughtered in I894, only one case of maxillary actinomycosis was found, as was also the case in I895 out of II,I56. In I896 and I897 none were found. In 1898 , only one case was seen out of an analogous number of slaughtered animals. All of the cases noticed were in animals coming from one district. The disease is rare in the cattle supplying the market of Amiens. In the course of one year,- - 898 , - the abattoir inspection revealed but four cases. The statistics for the several years is sufficient to show the rarity of the disease in the cattle of Flanders, Normandy and Picardy. In Dijon the proportion is perceptibly higher than in the above mentioned towns. 
The following figures are taken from statistics furnished by M. Carreau:

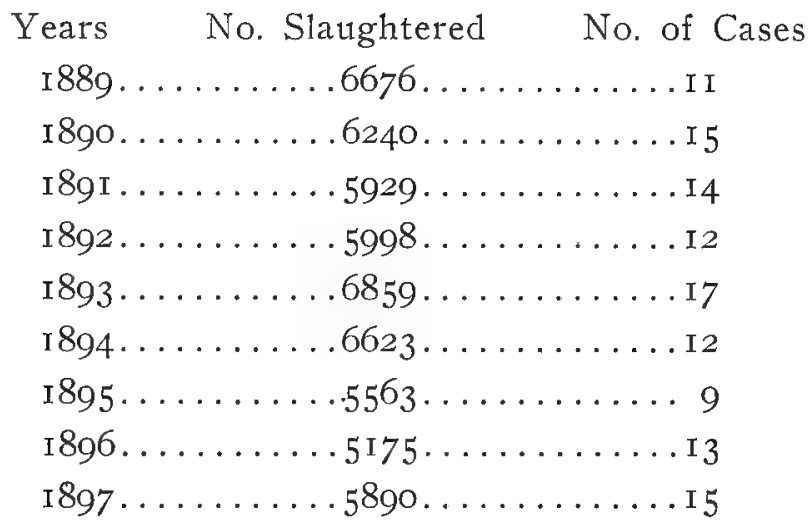

In Lyons out of an average of 36,000 animals slaughtered annually, the number of cases of actinomycosis wás 69 in I895, and 63 in 1896 . Lingual localization was rare. In the abattoir of Troy, Morot observed I9 cases out of 4,I 85 slaughtered cattle, and from January, i898, to September of the same year, ten cases, of which one was of the lungs and nine of the head.

In Germany, maxillary actinomycosis is common in Silesia, and maxillary and lingual localizations are seen in Franconia, Palatinate and Bavaria. Degenerate changes of the pharynx and parotids are found in 5 per cent of the cattle of the districts at the mouth of the Elbe. The disease is frequent in Denmark, Sweden, Italy, Russia and England. Canada and Chili are also considerably infected.

\section{ANNOTATION.}

Frequency of Actinomycosis in the United States.-Actinomycosis is a fairly common disease throughout the Mississippi Valley, with the exception of the Upper Ohio Valley, where it is seldom encountered. It is found in the Mountain states, on the Pacific Coast and throughout the South. The Eastern states have fewer cases than any other part of the continent. In 1903 the Bureau of Animal Industry condemned 824 carcasses and 837 parts of carcasses, affected with actinomycosis, out of a total of 6,134,4IO cattle inspected in stock yards and abattoirs. During the same year 32 carcasses and 44 parts were condemned out of a total of $21,793,738$ hogs inspected.-L. A. M. 


\section{BOVINE ACTINOMYCOSIS.}

The disease may be enzootic. Steinon found it raging on one farm. Preusse observed it in the low districts of Prussia after floods lasting two months. Twenty per cent of the cattle fed with the hay gathered in the inundated regions contracted the disease, and all of the various localizations were observed. Villemin also saw the disease attack a number of animals in a single cow-house. The problem here is not that of contagion, as has been claimed by some writers. The disease takes the enzootic form because the cattle attacked were exposed to the same source of infection. If the fodder of a district is infected with the actinomyces all of the animals that consume it are liable to contract the disease. It is therefore not a question of transmission from animal to animal.

SYMPTOMS.-As far back as I 884 Bang distinguished four clinical forms of actinomycosis:

Ist. Actinomycosis of the jaws.

2nd. Actinomycosis of the tongue.

3rd. Actinomycosis of the soft structures of the face and of the neck.

4th. Actinomycosis of the pharynx.

Generally speaking the lesions caused by the ray-fungus are the same. The peculiar physiognomy of the different forms is due to the region in which the disease develops. The parasite will construct tumors in any tissue.

Actinomycosis of the Jaws.-These localizations were known to the old veterinarians under the names of "osteosarcoma," "wen," "cancer of the cheek," etc. It is the most common kind encountered in France. It is usually located on the inferior maxillary; sometimes, but more rarely, on the superior.

Generally, the commencement of the tumor escapes no- 
tice. It is, at least, only brought to the notice of the practitioner when already well developed. The following symptoms are usually observed: One of the branches of the jaw is deformed, bent round; sometimes the deformity is diffuse; at other times it has clearly defined limits. When the lesion begins it is nearly always diffuse, that is to say, concealed by a more or less pronounced œdema that occupies the periphery and fills the cavity between the jaws. In time, the cdema is reabsorbed as the tumor becomes more pronounced. Its relief is exposed more and more by the sinking of the peripheric zone. The tumor, which varies greatly in

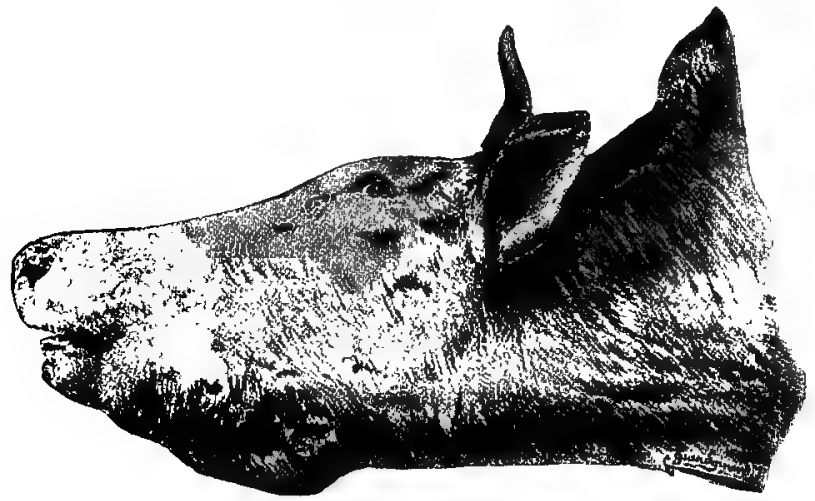

FIG. 52.

Antinomycostic Tumor of the Inferior Maxillary (Le Blanc).

size, is now found to be hard and of a fibrous or cartilaginous consistency. It is very adhesive to the deep tissues and is covered over with skin that is nearly always intact at the beginning but clings strongly to the neoplasm.

If this tumor is allowed to run its course it generally in-s vades more surface, sometimes it increases in thickness. It undergoes an important phenomenon: it is softened at many localized points and exhibits fluctuating zones corresponding to small abscesses which end by bursting at the surface and leaving fistula behind them. The osseous neoplasm covered over with a normal skin at the beginning now becomes a fistulous tumor. 
The fistulae are seldom very deep, but they have a sinuous tract. On digital exploration the fistula appears to be carpeted with a lining analogous to the pyogenic membrane of an abscess. They discharge a purulent product containing the "yellow grains" in suspension, which are characteristic of the disease.

The local symptoms for a time co-exist with a satisfactory state of health. For months the tumor may grow without causing any perceptible inconvenience to the animal. It is only when the neoplasm has attained a considerable volume, when it has distorted the buccal cavity and loosened the teeth, and when it has ulcerated the mouth, that it becomes troublesome. The movement of the jaws is then limited. The prehension of food is difficult and mastication is painful and insufficient. In this condition of inconvenience and pain the subject grows lean and becomes wretched.

Spontaneous recovery is exceptional, and it is rarely ever cured by the different methods of treatment recommended. In 1884 , Johne distinguished two varieties of the disease affecting the jaw: one which originates in the bone tissue, or myelogenic actinomycosis, and a second which has its origin in the periosteum, or periosteal actinomycosis. In the first case, and according to Johne's conception, the tumor is exclusively osseous, while in the second it is formed at the expense of the periosteum and the subcutaneous connective tissue. The latter is then especially fibrous and hence less grave than the first.

Medical men acquainted with the disease accept Johne's classification. It might perhaps explain the difference of the results obtained with iodine treatment, which may temporarily cure the fibrous form, but has no effect on the myelogenic variety.

Actinomycosis of the Tongue.-This form is rare in France. It has been observed by Godbille and by Rigal. 
It is occasionally encountered in the large abattoirs, but is incomparably less frequent than the maxillary localization. In England (and America) it is known by the name of "wooden tongue." In Germany it is called "holzzunge," and in Italy "mal di rospo."

The disease manifests itself in the beginning by copious salivation, difficult prehension and painful deglutition. These signs are accompanied by an odema that fills the cavity between the rami of the lower jaw. The patient resents being handled and examination of the mouth provokes resistance. The buccal cavity presents an abnormal redness

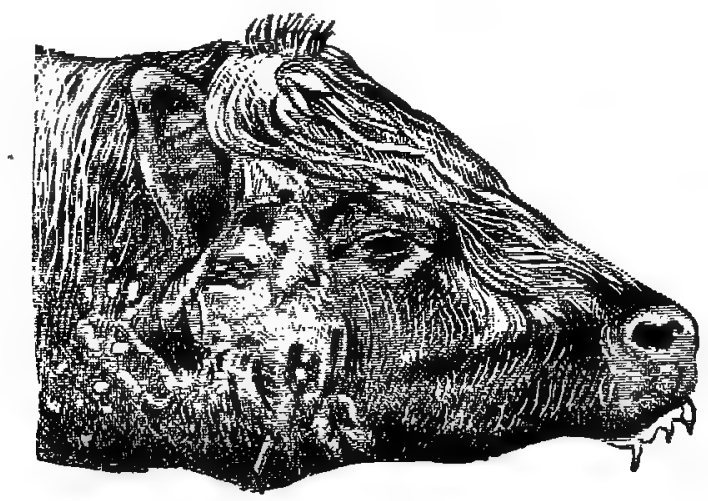

FIG. 53.

Pharyngeal Actinomycosis.

of the mucous membrane and a painful tumefaction of the tongue. The appetite is capricious, the thirst intense and the ptyalism more and more profuse. From this period, there are usually yellowish spots on the tip and lateral faces of the tongue which succeed superficial ulcerations scattered all over the organ. The tongue is hypertrophied and is retained in the buccal cavity with difficulty. It hangs from the mouth and the patient experiences a great deal of trouble in returning it. By degrees it assumes a woody consistency and hangs out to the right or left, according to the side most affected. It is cut and lacerated by the molar 
arcades and the patient ends by refusing all nourishment. There is rapid emaciation and death from cachexia. Spontaneous cure does not occur.

Actinomycosis of the Soft Parts of the Face and of the Neck.-This localization has been especially observed in Denmark by Jansen and Bang, and in Germany by Preusse. It has been found in France.

It is marked by the formation of tumors, which appear to be developed after inoculation through cutaneous wounds. They make their appearance on the cheek, on the surface of the parotid, in the retro-pharyngeal and sub-parotid lymphatic glands, and on the lateral faces of the neck and shoulders. They are hard at first, but soon become ulcerated and discharge pus containing the typical "yellow grains." Two tumors are sometimes found in the same region, which instance a subcutaneous, fistulous communication between them. The muscles adjacent to the tumor became fibrous, hard and incompressible. In favorable cases they become isolated and encysted.

Actinomycosis of the Pharynx.-The invasion of the pharynx is accompanied by symptoms of sore throat. Johne refers to a cow that exhibited sore throat and dyspnoea, and the autopsy proved it to be a case of actinomycosis. When the pharynx is affected the region under the jaw is œdematous and sensitive.

The lesions may be diffused or in the form of tumors. When isolated, the lesion is usually situated supero-posteriorly on the sphenoid-(posterior pharyngeal actinomycoma)-or immediately anterior-(anterior laryngeal actinomycoma). They extend over the mucous membrane, become ulcerated like the cutaneous neoplasm, and discharge a clotted and fetid pus. When voluminous they become an obstacle to deglutition and cause roaring. The subject becomes emaciated very rapidly, and may have to be killed, 
even before the nature of the disease is known to a certainty.

Other Localizations.-Actinomycosis of the lips has been frequently observed by Mari in the abattoirs of Moscow. Ignatieff has found lesions on the lower lip. Oscholkow says that 5 per cent of all animals slaughtered are affected with it. The infected lips are covered with small tubercles, hard and capsulated, and varying from the size of a pin-head to that of a hazelnut. They are of a yellowish gray color and contain a creamy or caseous pus.

In the œsophagus the lesions may exhibit either the granular or tumor form. In the latter event they may cause osophageal obstruction, as in volume they may acquire the dimensions of the fist. This form of the disease is seen most frequently in adults, but may also affect calves.

Neoplasms are found in the skin, the reticulum, the abomasum, the intestines, and the liver. The latter organ may become the seat of either neoplasms or abscesses. The kidneys and the spleen are rarely ever affected. .

The respiratory passages are no more spared than the digestive tract. Preusse has found the lesions in the nostrils on several occasions, and Roder cliscovered an actinomycoma in the nasal cavities. Jansen refers to the invasion of the Schneiderian membrane with small muriform tumors that showed a tendency to become ulcerated. With the nasal mucosa the turbinated bones may be attacked. Jansen has noticed twenty cases of actinomycosis of the larynx, and Claus six cases. The trachea and the lungs may also show lesions. Pulmonary lesions are no longer regarded as exceptional. Jansen and Claus have reported observations made on the subject, in which they state that the lesions were not of the large variety, but of scattered hot-beds of variable dimensions. Pleural actinomycosis often coexists with that of the lungs. Hink found the right pleura adhered to the lung through the medium of yellowish ac- 
tinomycostic tubercles the size of beans. With the exception of the lesions affecting a large portion of the perenchyma, grave pulmonary lesions are exceptional. The udder may be attacked, in which case the infection occurs through the milk channels. It is manifested by the development of neoplasms of a variable volume in the parenchyma, or by a glandular sclerosis that is not unlike tuberculosis. The tumors form abscesses that become ulcerated at the surface of the skin. Bang, Harms, Jensen, Johne, Erhardt, Williamson and Korevaar have observed actinomycosis of the udder. Tumors may grow under the skin in different parts of the body, but especially in regions exposed to wounds of the integument. Jensen noticed an actinomycosis of the knee; Lupke in the interdigital space; Nystrom in the region of the phalanges; Le Blanc a neoplasm on the outside of the leg; and Preisse a tumor on the inner aspect of the hind leg.

The parasite may affect the generative organs and the spermatic cord after castration. The muscles may be encroached upon, but this localization is exceptional. The bones, notably the vertebræ, and the bones of the cranium, are sometimes attacked. MacGillivray reports a cow that exhibited symptoms of general paralysis, and at the autopsy he found a tumor in the lungs and an actinomycoma of the first dorsal vertebra. Jensen found nests of actinomyces in the sternum, and Bang in the metatarsus. Pieroni refers to an ox presenting the symptoms of turnsickness that proved on post-mortem to have suffered from the effect of an irregular actinomycoma in the internal surface of the cranium.

PATHOLOGICAL ANATOMY.-(a) Lesions of the Maxilla.-The tumors developed in superficial structures are composed of a hard, lardaceous tissue, riddled with abscesses of small dimensions, containing purulent product 
rich in actinomyces. The underlying periosteum is attacked. When the parasite is deeply implanted, when it has attacked the jaw by way of the mouth, it produces an irritation that acts chiefly on the maxilla. Nests are formed in the cancellated tissue of the bone. It swells the osseous plates, separates them, weakens the compact tissue and raises a tuberous mass that becomes soft and often ulcerated on the surface, either in the buccal cavity or externally. When located in the superior maxilla, even the nasal cavities and their appendages become involved.

There is a manifest deformity of the jaw, consisting of

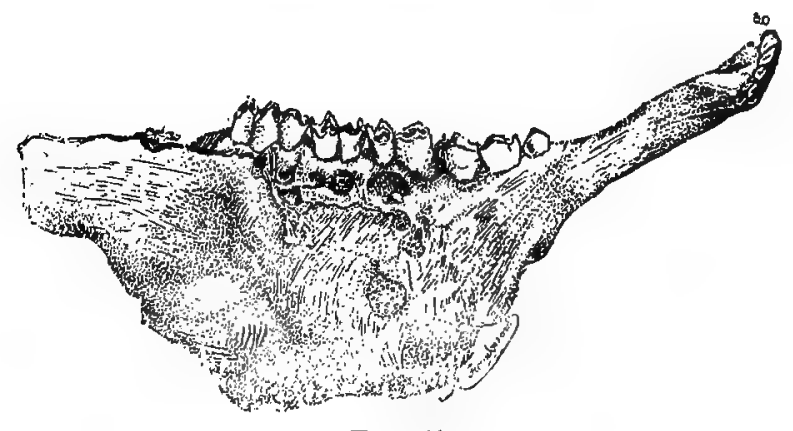

FIG. 54.

Actinomycostic Tumor of the Inferior Maxilla, Showing Ulcerations and Granulations.

an apparent separation of the osseous plates. When the lesion is much developed the molar teeth loosen and fall out. It appears as if an "osseous sponge" had been interposed between the sound bone plates. The tissues are corroded and hollowed out, with irregular cavities united by bridges of bone. In the recesses of these cavities the bone tissue is hard, but perforated with an indefinite number of hol'es that give the appearance of a sponge. The swollen osseous mass contains, here and there, very thin translucent lamellæ that resemble fine lace.

At the other limits of the lesion the periosteum has lost its toughness and is pierced with innumerable openings. 
When the lesion is located on the superior maxilla, the cranial bones participate in the morbid process, which transforms them into papyraceous lamellæ. (Fig. 57).

(b) Lesions of the Tongue.-Very frequently the tongue is hypertrophied and deformed. The tip is drawn down or up, to the right or left, according to the seat of the lesion. It is hard, fibrous and woody. These alterations, scarcely noticed when the disease is new, are all the more manifest when it is of longer standing. The lingual mucosa is excavated with ulcerations or embossed by tumors under the

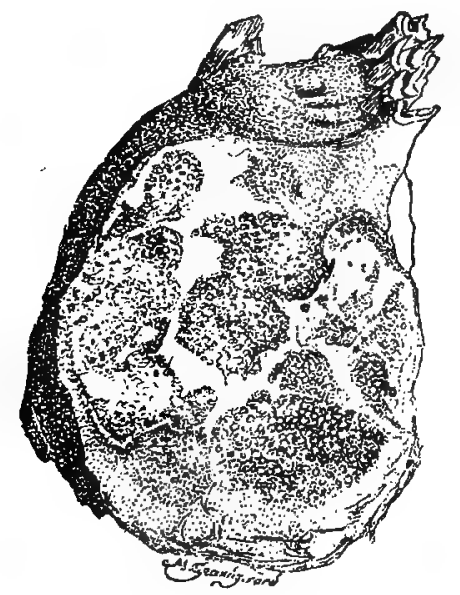

FIG, 55.

Transverse Section of the Preceding

mucous membrane or in the muscle. When incised the tongue gives the impression of a sarcomatous mass. A microscopic examination shows very marked lesions of interstitial myositis. At a higher power the sections show invasion by connective tissue proliferation. The muscular fibres are atrophied, choked up, disjointed and shattered. and the parasites are lodged in the fibrous islets. With a still higher power, the center of these fibrous zones show the existence of nodules already in the course of degeneration.

The actinomycoma in miniature gives rather the impres- 
sion of a tubercle. The parasitic tuft is in fact surrounded by giant cells, which are drowned in their circumferences with masses of lymphoid elements. The organism in reacting against the actinomyces constructs a lesion similar to a tubercle. Only the foreign body differs.

(c) Lesions of the Integument.-The skin may show lesions of chronic inflammation, sometimes to a considerable extent. It is hardened, pasted to the subjacent tissues, cracked and overrun with furrows, which penetrate into the muscular masses, transforming them into fibrous zones. Circumscribed tumors, surrounded with a fibrous capsule, are also found on the surface of the skin.

(d) Lesions of the Lungs.-In the lungs the tumors are

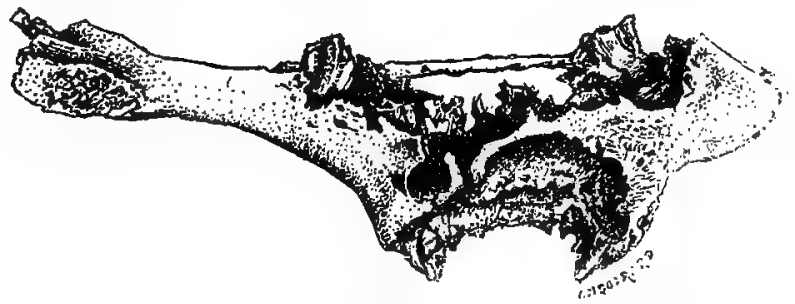

FIG. 56.

Inferior Maxilla of an Ox Attacked with Actinomycosis.

of variable size, have well defined limits and are surrounded by a thick, fibrous capsule. They contain a product that readily undergoes purulent transformation and fatty degeneration. At other times the lesions are composed of little nodules, found only by palpating the organ, but in which the parasite is easily found. On microscopic examination these nodules prove to be constituted of small fibrous masses containing the parasite in their centers, surrounded with giant cells and lymphoid elements. The neighboring air cells are compressed and sometimes filled with red corpuscles.

In the liver, the udder, the peritoneum and the pleura the lesions exist in the form of tumors. 
DIAGNOSIS.- (a) Actinomycosis of the Maxilla.-Generally speaking, the diagnosis may be said to be easy. It is sufficient to have seen a case or a photograph of the disease to recognize it. There are few lesions of the maxilla similar to actinomycosis. When the tumor is excavated, with a number of fistulæ discharging a pus that contains "yellow grains," an opinion may be given without recourse to the microscope. When the tumor is not ulcerated, and when it is of long standing, localized lesions having the appearance of actinomycosis are nearly always found in the mouth. Bang refers to the possibility of confou1ding the lesion at the beginning with an ordinary osteo-sarcoma, and

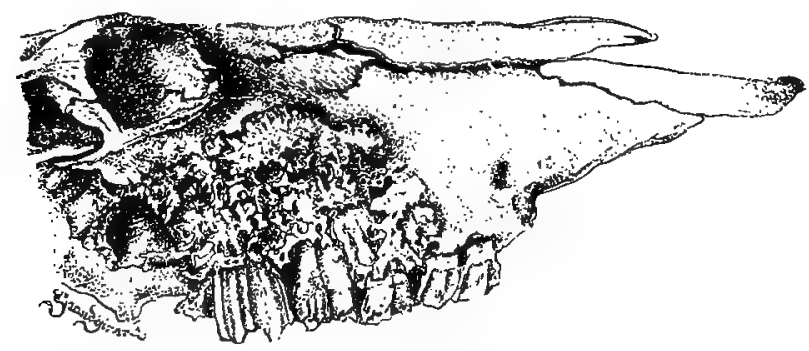

FIG. 57.

Superior Maxila of an Ox Attacked with Actinomycosis.

Morot reports the case of an ox exhibiting an osteo-sarcoma of the maxilla that was first thought to be actinomycosis. Mathis refers to a similar case in which an ulcerated sarcoma of the extremity of the jaw bore a fairly exact resemblance to the actinomycostic lesion. In the bovine pathological department of the school at Lyons there is a specimen of dental abscess at the extremity of the jaw that more or less resembles actinomycosis.

With the exception of these cases there is no confusion possible, especially when the "yellow grains" are found in the pus.

(b) Actinomycosis of the Tongue.-When the lesion of the tongue co-exists with a maxillary neoplasm the diagno- 
sis is simple. When the tongue alone is affected, its increase in size, the yellow ulcerations of the surface, the tumors, and the impossibility to trace the trouble to trauma are leading symptoms. The discovery of the parasite removes all doubts. Tuberculosis of the tongue may present these changes, but tuberculous lesions nearly always present ulcerations with festooned borders, and the contractility and motility of the organ are preserved. Bacteriological examination and tuberculin will render the differentiation possible.

Pflug refers to a glossitis not actinomycostic, that is refractory to iodine treatment. This lesion must be rare and a mere knowledge of its existence is sufficient to prevent errors.

PROGNOSIS.-Actinomycosis is always a grave malady. It is not always fatal, however. The lesion may be a cutaneous tumor. In any event the cure is always difficult and its existence is always to be dreaded. The maxillary localization is always very grave and often incurable, because it is often accompanied with buccal lesions, on which it is difficult to operate, and because the iodine treatment on which so many hopes have been based is so often disappointing. Lingual actinomycosis appears to be less grave. The majority of observers agree that it yields to the use of iodine, even when the lesions are accentuated to the point of almost preventing deglutition.

TREATMENT.-The methods of treatment vary according to the cases. The cure should be advised and undertaken only when it is economical. And in the majority of cases, unless the subject is a working animal or a valuable sire, it must be acknowledged that it is not. With these reservations the practitioner might follow the following directions :

(a) Lingual Actinomycosis.-Internal actinomycosis, when recognized, and the lingual form are amenable to the 
iodine treatment. Experience seems to have demonstrated that lesions of the soft tissues yield rather readily to treatment with the iodide of potassium. This medicament is administered internally at the rate of eight to fifteen grams (two to four drams) per day for some days. There is nothing to fear from symptoms of iodine intoxication, except when the animals become indisposed and cease to eat. In these cases the treatment is discontinued for five to six days, and then resumed when the symptoms of iodism disappear. When the tongue is tumefied to the extent of preventing deglutition, the medicament is administered per rectum, dissolved in half a liter of water.

(b) Maxillary Actinomycosis.-For maxillary actinomycosis the most favorable results are obtained from the continued use of the iodine treatment and surgical intervention. It is indispensable, however, to anticipate a successful result, that the tumor should have well defined limits, and not involve the important vessels and ducts of the region.

The operation consists, first, of the excision of the exuberant actinomycoma, and, secondly, the curettage of the maxillary bone. By using internal treatment with iodine bases, succeeding this intervention, a cure may be obtained in four to six weeks that could not have been hoped for by any other form of treatment. Imminger considers that the extraction of the molar teeth implanted in the affected point is necessary. The extraction should be made with care not to disturb the neighboring teeth. After the teeth are extracted, the tumor is incised at the most prominent point to effect an opening for curettage and admission of the iodine dressings.

Although these different methods of treatment may be the only logical ones, they must not be considered as absolutely effective. There are many cases in which they will only effect a brief amelioration.

(c) Cutaneous Actinomycosis.-For therapeutic purposes 
these lesions must be classed under two heads: Ist. When the tumor is slightly or not at all adherent to the deep tissues on which it is fixed with a narrow base, the method to recommend is total extirpation. The neoplasm is detached from the structures on which it rests by dissection. If nests of actinomyces exist on the outside of the capstule, the fibrous zone on which they are lodged must be extirpated and the wound cauterized with tincture of iodine. The edges of the wound may then be reunited with sutures. 2nd. When the tumor rests in deep tissues, on a large and adherent base, its total extirpation might create a considerable wound, and it would be difficult to define the limits between the healthy and the infected zones. In this case, it is advisable to make an incision into the tumor, curette the interior, stuff it full of cotton soaked in tincture of iodine, and then suture the wound. In forty-eight hours the plug is renewed. After eight days cicatrization is well on its way and the cure is complete in three to four weeks. In no case is it necessary to curette beyond the limits of the fibrous capsule which constitutes an isolating envelope.

It is interesting to note in conclusion that iodide of potassium has no direct action on the actinomyces. In fact, Nocard, Dor, Dubreuilh and Berard have been able to cultivate it in a nutritive media containing one per cent of iodide of potassium. This medicament, therfore, acts by modifying the nutrition of the cellular elements and transforming the chemical phenomena. The iodide imparts a fresh, vital energy to the tissues, which enables them to struggle victoriously against the parasite.

\section{PORCINE ACTINOMYCOSIS.}

After the ox, the hog is the animal most frequently attacked. The udder, the tonsils and the muscles are the organs in which the fungus settles by preference. 
(a) The Udder.-Bang found 52 cases of actinomycosis of the udder of sows. Rasmussen reports several cases and Hertwig saw all the mammæ and the lumbar vertebræ invaded in a sow. The mammary tumors may at first be mistaken for adeno-fibromata or sarcomata. Their extirpation is followed by relapse. Microscopic examination reveals the nature of the lesion. The middle udders are the usual locations of the tumors. They start by an induration of the base of the teat that ends by becoming ulcerated. The inguinal lymphatics are not usually infected.

(b) Muscles.-Virchow in I865, and Duncker in 1884 , found actinomycostic tubercles in the muscles of the hog, the nature of which was determined by the latter. Duncker's opinion, ratified by Virchow, Israel, Shultz and Plant, was contested in later years by Zuiniscemann, Lemke and Olt. Muscular actinomycosis does not appear to have any influence on the general health of the hog. It has been observed only in Germany, where it is common in the abattoirs of Berlin, and in Italy.

(d) Tonsils.-The disease manifests itself by the formation of tumors that disturb deglutition and respiration. A number of cases of chronic sore throat in the hog might lead to the suspicion of actinomycosis.

(e) Other Localizations.-The maxilla may be attacked, and not infrequently the scars of castration in both males and females are the starting points of infection.

The lungs, the pharynx, the kidneys, omentum, the bones and the skin are sometimes attacked, but actinomycosis is seldom generalized in the hog. Carl found all of the left side of the abdominal cavity of a hog invaded, and all of the lymphatic glands of the posterior part of the body showed lesions. There were abscesses in the spleen, liver, lungs, stomach, and the intestines. The initial point of infection was the wound of castration. 
PATHOLOGICAL ANATOMY.-The physiognomy of the mammary lesions or other localizations is the same as in the bovine animals. The tumors of the udder are composed of fibrous tissue, white, lardaceous, sprinkled with greyish, yellow, cellular islets, regularly rounded with well-defined limits, projecting above the general level of the section. Their dimensions vary from the size of a hemp seed to that of a walnut or larger. Now and then we find soft ones representing so many cysts with purulent contents. The pus is greyish and contains a great abundance of grains of the color of sulphur, characteristic of actinomycostic pus.

Muscular actinomycosis chiefly affects the abdominal muscles, the intercostals and the diaphragm. The histological study of the diseased muscles shows fragmentation of the fibers, clisappearance of the striations and vitreous degeneration. The mycostic tufts are englobed in a connective tissue production constituted at the expense of the sarcolemma, and the granulations often undergo calcareous degeneration.

DIAGNOSIS.-In communities where the disease is frequent localizations in the pharynx and udder should always be looked upon with suspicion. Neoplasms of the udder can nardly be confounded with other lesions. They are hard, ulcerated tumors that discharge a purulent product containing the actinomyces. The neighboring lymphatic glands are scarcely ever invaded.

PROGNOSIS.-The gravity of the disease is subordinate to the extent and duration of the lesions.

TREATMENT.-As in the ox, iodide treatment is advisable. For manmary tumors surgical intervention may be necessary. The udders affected are extirpated, as in the bitch, and the two contiguous ones are also sacrificed to avoid relapse. The surgical wound is packed with iodine-soaked cotton held in place with sutures which also serve the purpose of diminishing the dimensions of the wound. These dress- 
ings are removed after forty-eight hours and the wound is disinfected every day with an antiseptic solution.

\section{Equine Actinomycosis.}

Actinomycosis, rare in the horse, affects the jaw and the tongue.

(a) Maxillary Actinomycosis.-The tumor is formed at the expense of one of the jaws, and the symptoms are similar to those in the ox.

(b) Lingual Actinomycosis has been observed by Truelsen, Eberhardt, and Struve. As in the ox the tongue is hypertrophied, painful, red, uneven and immobile. Mastication is difficult, the mouth is hot and ptyalism is abundant. Examination of the mucous membrane reveals the existence of tubercles or yellowish nodules containing caseous matter.

(c). Other Localizations.-Actinomycosis has also been seen in the maxillary lymphatics, in the subcutaneous tissue of the hip, in the region of the stifle, on the withers, in the spermatic cord after castration, over the larynx, on the tibial region, on the costal surface, the thigh, the udder and the muscles.

\section{Ovine Actinomycosis.}

Actinomycosis is very rare in the sheep. Pulmonary localizations have been pointed out by Grips and by Stettin. Hamond reported a case of the lingual form and Berg two of the lingual and one of the maxillary variety. Muscular actinomycoses have been observed in the abattoirs of Berlin and Copenhagen. Hertwig also has met with the disease in the sheep.

\section{ANNOTATION.}

Copper Salts in the Treatment of Actinomycosis.-The destructive effects of copper salts, especially of the sulphate. in destroying vegetable parasites have been amply enough demonstrated by scientific agriculturists to warrant their application to the destruction of the actinomyces in animal tissues. Copper sulphate in weak solutions is now widely and successfully em- 
ployed to kill the parasites of vegetables. Its potency against algæ and the low forms of vegetable life is no longer a matter of doubt. With this fact as a basis Bevan (Chicago) employed it on a number of cases of "internal actinomycosis" that had proven refractory to the combined use of iodide of potassium and the X-ray, and the results obtained seem to correspond with the high potency of the chemical against exogenous parasites. The drug is used both internally and as an irrigation of the fistulae of the neoplasms, and may be used in addition to the iodide treatment. The fact that the iodide treatment is most effectual when used at regular intervals"leaves room for the administration of the copper salt between these intervals. The administration of liberal doses of potassium for three or four days, followed during the next three or four days by doses of copper sulphate, should, from this reasoning, constitute the appropriate internal medication for the non-operable actinomycostic lesions. In the superficial localizations the copper salt in solution is applied alike in the surgical wound following ablation or incision of the tumor as in the fistulæ of the non-operable variety.-L. A. M.

\section{BOTRYOMYCOSIS.}

DEFINITION.-Botryomycosis is a parasitic disease caused by a fungus belonging to the genus botryomyces, and described by the name "botryomyces equi." It resembles actinomycosis in its clinical aspect and in the character of the parasite.

HISTORY.-The knowledge of the disease is of recent date and the credit of having determined its nature is due to German and Italian authors. I879, Rivolta discovered the presence of the fungus in the spermatic cord. The observations of Johne, Semmer, and Rabe directed attention to this new mycosis. In I884, Rivolta, while pursuing his investigations, gave a complete description of the fungus, under the name of dyscomyces. Rabe, in I886, called it the micrococcus botryogenus, and Johne, the micrococcus ascoformans. The latter named the tumor in which the fungus is found, mycofibroma equi. Bollinger named the disease botryomycosis.

Although observed for a long time in Germany and Italy alone, botryomycosis was noticed for the first time in France by Soula, in I 887 . Since that time a considerable number of publications have mentioned its existence in our country.

SUSCEPTIBLE ANIMALS.-Although chiefly ob- 
served in the horse, the disease has nevertheless been seen in the ox, the hog, and even in man.

ETIOLOGY-AND PATHOGENESIS.-The disease is caused by a special parasite, which, when examined by a low power, appears in the form of colonies of micrococci in rounded muriform masses of variable dimensions, each of which seems to be surrounded by a gelatinous capsule. It is easily stained with picro-carmin, and sometimes it presents the aspect of grantular discs. Rabe cultivated the fungus on potato and gelatinized peptone. On gelatine the botryomyces give white, round colonies which in the course of time become grey. On glycerinated gelose the colonies are orangecolored and on potato they are yellowish and emit an odor of ripe strawberries.

On microscopic examination of the cultures, the parasite is seen in the form of micrococci measuring about I micron, collected in heaps. It is easily stained by all aniline dyes, but especially by Loeffler's methylene blue.

Experimental inoculation has been successful in the horse. Rabe has successfully produced lesions analogous to those developing spontaneously. In other species,-cavies, sheep and goats, - the experimental inoculations have only caused necrotic inflammatory processes. Under natural conditions it is not doubted that inoculations occur through wounds of castration, or cutaneous abrasions caused by defective harness, collars or halters. The tumors are frequent in the spermatic cord, on the shoulder, in the parotid region, at the elbow in animals that lie cow-fashion, on the lips, on the tail, between the thighs, in the pasterns, in the udder, and on the mucous membranes. Wester reports the case of a horse that very clearly demonstrates the possibility of infection by the harness. The collar of a horse affected with botryomycosis, when placed on another horse, inoculated the latter in the same part of the body. 
During the past few years there has been considerable discussion as to the identity of the botryococcus. Some regard it as a special parasite quite distinguishable from the rest, while others, on the contrary, consider it a variety of the staphylococcus. $U_{p}$ to the present time there is nothing to demonstrate the exactness of the latter hypothesis. De Jong tried in vain to make botryococci from staphylococci. By injecting botryomyces subcutaneously into the horse a mycofibroma is produced, while the staphylococci produce only a transient inflammation, or an abscess.

Kitt has established that the pigment of the staphylococcus aureus is stable, while that of the botryomyces is, on the contrary, absolutely variable.

At $18^{\circ} \mathrm{C}$. on agar the botryomyces gives orange colored colonies. If this very culture is resown and the temperature is raised to $37^{\circ} \mathrm{C}$, it becomes white in twenty-four hours. When brought back to $18^{\circ}$ it becomes yellow and finally orange yellow.

In 1897 Poncet and Dor found the botryomyces in some pediculated, frambosioid tumors of the human fingers and hand, and they tried to identify them with scirrhous cords of animals. The identity, however, has yet to be demonstrated, in spite of the opinions of some writers. The scirrhous cords of animals and the tumors of man are two different lesions, even though the same parasite is found in both. Poncet and Dor state that they found muriform bodies in recent tumors, which is again incorrect, as botryomyces are found only in the old lesions that have already been hollowed-out by fistulæ. This fact leads to the inference that the tumor is not primarily due to the botryococcus, but that it appears on the scene later. They have also advanced the hypothesis that the botryococcus is the product of cellular degeneration, that makes its appearance in the nucleus and grows larger by degrees as the protoplasm disappears; but this reasoning 
has no better foundation than the preceding hypothesis. How can it be conceived that cellular degeneration takes the same form even in different tissues? How is the specificity of a product of cellular degeneration to be explained?

\section{BOTRYOMYCOSIS OF THE HORSE.}

In the soliped, botryomycosis is manifested by the appearance of tumors on the skin, the spermatic cord, the udder and the viscera.

(a) The Skin.-Certain regions are more particularly attacked. The disease begins in the form of chronic inflammatory neoplasms, which become fistulous and discharge a purulent product containing the botryococcus. The neoformations have a tendency to invade the surrounding tissues. They gradually enlarge by their contiguity to the adjacent tissues. The neighboring lymphatic glands are seldom attacked. According to Cadiot and Almy "most all fibrous tumors excavated with fistulous tracts are connected with botryomycosis."

The neoformations may acquire a considerable size. Soula observed a botryomycostic tumor on the forearm that measured 32 to 35 centimeters in diameter. Thomassen refers to a voluminous growth, of this nature, on the breast, and Fröhner to one on the elbow, three times the size of a man's head, and measuring 86 centimeters through its long axis. Fröhner also observed botryomycomas of different sizes in the leg, the thigh, the scrotum, the inferior maxillary, the fetlock and the parotid. Perroncito reports a case in the horse that exhibited a botryomycostic growth on the stifle caused from a kick. Johne found the parasite in fistulous tumors on the back, the chest, and the nostrils. Jensen found one on the fetlock and Kitt one on the tail.

The disease appears to be very frequent in Germany, as Fröhner met $5^{2}$ cases in two years. In France, although 
the disease may not be recognized as such, it is certainly tolerably rare.

(b) Spermatic Cord.-After castration a tumor is found growing at the end of the spermatic cord, to which the name "champignon" (or "scirrhous cord") has been applied. Some writers claim that this lesion is always botryomycostic, and the opinion is probably accurate, yet it should not be admitted without restriction. The recent cases never contain the parasite, or if it does exist, it occurs in some unknown form. Consequently, unless a larval form is discovered, it must be admitted that its role in the scirrhous cord is that of secondary infection. Kitt and Hofflich found the bacteria of hæmorrhagic septicæmia in the spermatic cord. The lesion seldom becomes general, although Semmer observed a case involving the inguinal glands, the liver, the diaphragm and the lung, and Fröhner refers to a case that involved the surrounding skin, abdominal muscles and lymphatic glands. Pedrozzi and Bosso mention a case of chronic funiculitis with an encroaching tendency, and Thomassen the development of a tumor within the abdomen that grew from the spermatic cord.

(c) The Udder.-Botryomycosis of the mammary glands has been observed in the mare. In I890, Neilson and Sands reported such a case, and since that time Sands and Muller have published other observations on the subject. Fröhner reports a case in the mare in which the right gland was absent and the left had become as large as a child's head. Its surface was irregular and mammillated, and a microscopic examination disclosed the presence of botryomycosis in the pus. We have ourselves observed a case of botryomycosis of the udder, in which the two lobes were voluminous and fibrous, and each presented a fistula that discharged pus containing the parasite.

(d) Other Localizations.-Fröhner observed a botryo- 
mycoma in the left nostril. The tumor was fungoid, soft, multilobulate, polypoid and as large as a walnut. A section through it disclosed numerous purulent foci, rich in botryomyces. Bollinger, Fröhner, Steiner and Thomassen have pointed out two cases of pulmonary botryomycosis, either primary or secondary. When the parasite attacks the abdominal viscera, it is generally by continuity that the disease advances to them. Semmer refers to a case in which the inguinal glands, the liver, the diaphragm and the cæcum were invaded through the existence of a tumor of the abdominal wall.

Thomassen observed a case of chronic peritonitis with thickening and adhesions of the omentum, and Reich noticed the generalization of the disease in the uterus, the ovaries, the mesenteric glands, the spleen, the liver and the diaphragm. Boulin reports a horse affected with botryomycosis of the periosteum of the tibia.

DIAGNOSIS.-The diagnosis of the disease cannot be made with certainty except by proofs of the existence of the botryococcus in the pus of the lesions. Nevertheless, the existence of fibrous and fistulous tumors at different parts of the body, especially the shoulders, should lead to the suspicion that the disease is possibly botryomycostic.

PROGNOSIS.-The gravity of the disease is subordinate to the extent of the lesion.

TREATMENT.-When the lesions are accessible surgical treatment is most appropriate. The internal administration of potassium iodide need not be expected to cure the disease, in fact, this is a medicine to which we are too ready to attribute special potency.

\section{BOTRYOMYCOSIS IN THE OX.}

Botryomycosis is rare in the ox. Csoko and Immelmann reported two cases affecting the udder, and Gunther found botryomyces in tumors of the liver. They were formed of 
a connective-tissue stroma, in which was enclosed a brownishyellow mass containing the parasite. Reali also found botryomyces in fistulous tumors of the shoulders" and buttocks. After failing to modify them by actual cauterization and iodine, recourse was had to extirpation. The two scapular tumors weighed 1.72 kilograms, and although the surgical wound was a large one, the cure was complete. The wound cicatrized in a comparatively short time.

\section{BOTRYOMYCOSIS IN THE HOG:}

The only observation we possess as to the disease in the porcine species comes from Wilbrandt, who reports the infection developing at the extremity of the spermatic cord after castration. The infection spread to the muscles of the thigh, white and fibrous, and contained purulent foci rich in parasites.

\section{ANNOTATION.}

All of the scirrhous cords ablated at the clinics of the Chicago Veterinary College during the past four years contained the botryomyces in the fistulous tracts. The microörganisms were, however, wanting in all cases of short development, which circumstance leads to the conclusion that botryomycosis is a secondary infection that has found a favorable field for development in the exposed, inflamed tissues of the scrotum after improper methods of castration. The funiculitis caused by septic hands or instruments or the protrusion of the spermatic cord into or through the incision, and exposed to the filthy stable, are undoubtedly the primary lesions of these common tumors.-L. A. M. 


\section{CHAPTER XIV.}

\section{MELANOSIS.}

\section{SOLIPEDS.}

The name "melanosis" is applied to a morbid condition characterized essentially by the overproduction of pigment, with or without neoplasms. The term signifies abnormal pigmentation of the tissues by a substance known as melanin. This substance may accumulate in any part of the connective tissues of the body, - - the derma, the intermuscular, interfascicular, subcutaneous, or the interstitial connective tissue of any of the organs. It may infiltrate organs and form into more or less voluminous masses known as melanoses, or form into neoplasms called melanotic tumors. In some cases it forms little projecting grains around the anus, while in others it forms into tubercles which are found in the lungs, the lymphatic glands, the liver, the spleen, etc. Still others develop into voluminous tumors. The tumors are usually simple, that is to say, they are deprived of all neoplastic tissue, the melanin deposited in the pre-existing tissue, forming the entire tumor.

Simple melanosis and melanotic tumors are found in the horse, the mule, the ox, the sheep, the hog, and the dog.

HISTORY.-The word "melanosis" was not used before the beginning of the present century. Laennec, in I806, regarded melanosis as a variety of cancer, which opinion was held by 'Trousseau, Le Blanc and Cruveilhier. In I826, Breschet attributed the origin to the effusion of blood into the tissues. Brugnone, in I87I, and Gohier, in I8I3, etc., drew attention to its frequency among white horses, thus leading veterinarians to look upon it as a manifestation of an aberration of the pigment, which, not being util- 
ized in the skin and hairs, is deposited in the tissues. But this theory does not explain the existence of melanotic tumors in animals with pigmented skins,-bay horses, black horses, etc., -and their absence in albinotic animals.

Melanosis has been the subject of numerous anatomopathological and chemical investigations. Lebert, Robin and Broca have described numerous melanotic tumors in man; Virchow and Kindfleisch fibromatous, sarcomatous and carcinomatous melanotic tumors. Carnil and Trasbot, in 1867 , published an important note on melanosis in the horse.

Robin's chemical study of melanosis, pursued with such success, has recently been completed by investigators who determined its composition.

MELANIN.-Physico-chemical Character.-Melanin is an organic substance, semi-solid, pultaceous, dense or concrete, brittle, easily pulverized after desiccation, and found in normal organs (choroid, etc.) in the form of very small. rounded granules ( $\mathrm{I}$ to 9 microns) and in pathological states, in the form of irregular bodies, consisting of an accumulation of the granules. Its color varies from black to reddish or deep brown. It has neither taste nor smell, and putrefies slowly and with difficulty when exposed to the air. When burned it emits an empyreumatic odor and is converted into carbon.

Melanin is chiefly characterized by its fixity and insolubility in most of the reagents, cold or hot water, alcohol, ether, dilute mineral acids and concentrated acetic acid. It is feebly soluble in certain weak organic acids, such as uric acid. It is soluble in cold ammonia, potassium, sodium and the alkaline carbonates. Hippomelanin is insoluble in these same reagents, but is dissolved by boiling alkalines. $\mathrm{Hy}$ drochloric acid precipitates the melanin and hippomelanin .in these alkaline solutions. Chlorine decolorizes melanin 
and partly dissolves it. Nitric acid reddens the granules and to some extent dissolves them.

Melanin is found in the cells of the choroid, the ciliary processes and the iris, in the form of fine pigmentary granules. It is found, more or less abundant, according to the region of the body and the species of animal, on the surface of the derma (under the epidermis) in the epithelial cells, and rarely in the form of free granules. Numerous investigations, which cannot be reiterated here, have been made by Thenard, Clarion, Lassaigne, et. al. Some have found iron and sulphur, while others found only sulphur as the mineral constituent.

The fact is there are infinite varieties of melanin as there are infinite varieties of egg albumen or coloring of grapes. a fact found in history of many complex substances. Its component parts are albuminoids, sulphur and sometimes iron. Brandt and Pfeiffer found 0.424 to 0.625 per cent, and Morner 0.075 to 0.2 per cent of iron in their analyses, which shows that composition is rather variable. Bard and Hugouneng found:

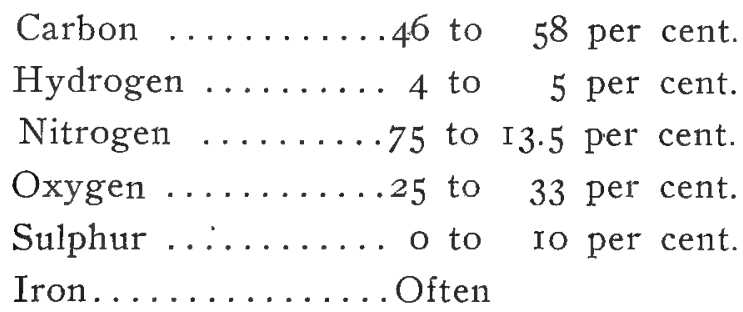

The melanin of the choroid contains neither iron nor sulphur. Melano-sarcoma of the horse contains a pigment called hippomelanin, which contains no iron and has the following composition:

Carbon ........53 to 55.5 per cent.

Hydrogen ...... 3.7 to 3.9 per cent.

Nitrogen . ...... IO.5 to Io per cent.

Oxygen .......28 to $3^{2}$ per cent.

Sulphur ......2.7 to 3 per cent. 
In a melano-sarcoma of man, an analogous substance richer in sulphur, and called phymatorusine is found.

Knowing its physico-chemic characters and origin, the localizations may then be considered.

ORIGIN.-The origin of a melanotic pigmentation has been the subject of considerable discussion. According to Breschet, it is of a hamorrhagic nature; the blood poured out becomes decomposed and provokes pigmentation by the transformation of hrematin in melanin. According to other writers, melanin results from alterations in the coloring matter of the blood, while still others opine that the pathological black pigment is elaborated by the cell, because it may appear at some distance from the blood vessels.

Heurtaux, endeavoring to reconcile the various theories, says: "If the hæmatin furnishes the elements of the pigment, it is certainly not owing to an infiltration of a sanguineous nature, and an alteration caused by extravasation of the corpuscles among the elements of the tissue. The authors who believe in the hamatic origin of the pigment have not maintained that the blood poured out is directly converted into melanin. They simply intended to say that its materials have been borrowed from the blood, and especially from its coloring matter." In favor of this theory it is pointed out that in melanæmia a great number of sanguineous elements contain particles of pigment, especially hxmatin, which leads to the formation of a pigmentary emboli. On the other hand, it has been discovered by Bruckmuller that in two horses suffering from melano-sarcomatosis, there were similar lesions which imparted a deep brown color to the blood. A like phenomenon in a calf was observed by Bollinger. It might, of course, be contended that the melanotic granules might have been carried into the blood by the leucocytes.

The process of pigmentation is the same in the 
pathological as in the normal state. The former is but an excessive activity of the hyperplasia of the pigmentary cells. But it is not always easy to distinguish the normal state, because some animals possess a special aptitude for the accumulation of pigments in the connective tissue cells. The calf, in particular, sometimes exhibits a niarked pigmentation of the connective tissue cells of the endothelium of the peritoneum, the meninges, and the connective tissue surrounding the nerves, etc. In the ox there is frequently found a slate colored pia mater over the frontal lobes, which on microscopic examination proves to be a simple superpigmentation. analogous to that seen in toads and fishes, with the excepticn that there is no cellular proliferation. The slight difference between the physiological and pathological states at ccmmencement is clifficult to comprehend.

It has, therefore, been wrongly claimed that the melanotic pigment is formed in the blood. The blood, and especially its coloring, must furnish the cells with materials for their elaboration. Ehrman has seen the pigment form at the expense of extravasated blood, and in following the development of amphibians with unpigmented eggs (salaman(lers) he has clearly seen the pigment develop, but only after the development of the blood vessels. Other facts support this view. Pouchet has demonstrated that in pouring blood into an alcoholic solution of bichloride, a precipitate of black pigment is obtained that has all the appearances and properties of the choroid pigments, the melanotic pigments, etc. This artificial pigment occurs in the form of lozenge-shaped bodies of crystalline aspect, or rather of spherical or irregular masses ( $\mathrm{I}$ to 40 microns). It has the chemical reaction of melanin. This experiment demonstrates the possible transformation of the coloring matter of the blood into melanin. Brandt and Pfeiffer have always claimed that there is an impoverishment of the blood in hamoglobin in melan- 
otic animals, as the number of red blood cells diminish by one-fourth. The presence of melanotic granules in the blood of melanic horses does not prove that the pigment is formed there and afterwards deposits itself in the tissues. The granules are rather carried into the blood by the leucocytes, which have brought them from without.

It is known today that the pigment is contained in the proper cells of the tissues, that the melanotic granules have no proper organization, and lastly that the blood that has flowed out undergoes different transformations. It must logically be admitted that melanin is produced like all other pigments, in a word, that it is the result of cellular activity. The data we possess on the general physiology of pigments shows that they are elaborated by special cells of mesoblastic origin, that the pigments are formed by the cellular protoplasm, and that they accumulate in the latter without encroaching on the nucleus. The behavior of these pigmentary cells on the lower vertebrata (toads) has been closely studied. The studies have shown that they are cells of mesoblastic origin, ramifying and migratory, which form pigmentary granules and which perform the function of transporting them into the midst of the epidermic cells, which in turn incorporate the pigment. In the case of highly pigmented mammals, for example, the gorilla, it has been proven that there are ramifying pigmentary cells in the superficial cells of the derma, which send extensions among the elements of the malpighian bodies to which they give up their granules. The same phenomena occurs in the negro. A layer of starry pigmentary cells, forming plexuses between the dermis and epidermis, is found in the horse and the ass, proving that there are cells the function of which is to elaborate the pigment. In short, melanin is elaborated by certain cells and conveyed into the tissues by them or by the blood. It remains to determine the mechanism that gov- 
erns the transformation of coloring matter into melanin, and how the multiplication and functional superactivity of the pigmentary cells occur. It is well established that the chromatoblasts of certain inferior animals, - the lizard and the frog,- - are directly dependent upon the nervous system, and that those of mammals are also governed by the nerves. It is, therefore, easy to conceive that the trophic influence of a lesion of a nerve center can provoke general hypertrophy of the pigmentary cells. Pathological pigmentation is in fact only an exaggerated and perverted normal pigmentation.

It has been established that the white color of hair constitutes a predisposition to melanosis. From an etiological

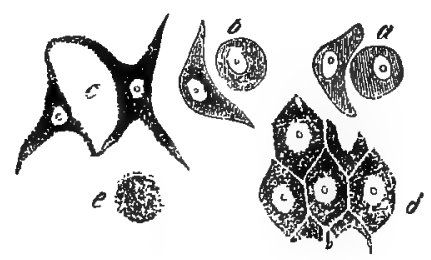

FIG. 58.

Cells in the Different Phases of Pigmentation.

$a, b, c, e$, Cells of a Pigmented Cancer.

d, Pigmented Epithelium of a Blood Vessel.

point of view it should be known that melanic products are generally found in animals having coats more or less depigmented, and, according to observations of Arabs, in animals that have wavy and woolly manes and tails. But on this point it might be asked whether the tumor led to curliness or whether it is a state directly connected with melanic alterations. In order to explain the formation of melanotic tumors in animals with coats of light color, Trasbot resorts to the melanogenic (?) power, which, not being spent, is kept in reserve, and at a given moment begins to accumulate. But knowing the rapidity with which unused elements are eliminated from the body, the theory cannot be defended. Besides, although it is true that the white horse is chiefly 
affected, it would be an error to erect this fact as an absolute law, as observations of melanosis among animals with dark coats are not rare. They have been observed in roans, blacks, dark bays and chestnuts. Although the common breeds are most susceptible, the disease has been seen in thoroughbreds.

The heredity of melanosis is certain. Gohier reports the following facts: A stallion with white hair was used from two to four years, and then invalided on account of melanosis. He had begotten a great number of both males and females, which all inherited his coat and developed the disease in aclult life. Even those born while their sire was still a colt, and before the disease had appeared, were not exempt. Schults and Raabe are also of the opinion that melanosis is congenital.

Age has an unquestionable influence on the appearance of the tumors. True, they have been seen in young horses but only in a few cases and always in small forms. They are generally found in animals from six to twelve years old and chiefly after the age of ten. From the twelfth to the fifteenth years, generalization takes place. Sex has no influence. It has been even sought to show that contagion plays a role in the matter, but Gohier, and others failed to reproduce it by inoctilation. Virchow had only negative results in introducing human melanosis into dogs and rabbits. Intravenous injections produced pulmonary emboli, in the center of which were found melanic substance without modification. However, according to Baillere, Goujon and Klenke, melanosis may be transferred to the horse and dog by inoculation. According to Bard, melanosis must be a parasitic disease with the role of the microbe to be determined. He based his conclusions on extended arguments that deserve no consideration, and finally abandoned the hypothesis.

DEPOSIT OF MELANOTIC SUBSTANCE.-A search 
for the points of predilection, where melanic pigment is usually deposited reveals the fact that the connective tissue is a favorite location, but that certain organs, like the liver, spleen, etc., may be affected, and, finally, that any of the tissues or organs may at times harbor melanism.

The connective tissue is the seat of predilection for melanic deposits. It has shown elsewhere that its formative elements accumulate more particularly in tissues that have the same blastodermic origin, and, since the connective tissue is distributed over the entire body, the varied locations of melanism are clearly explained. The pigment may be conveyed by the blood or by the elements which have formed it. The claim that it always locates at the seat of neoplasms is not true, for, in fact, the pigment is most frequently deposited in the absence of new formations. Investigations and observations, based on the dissection of old animals in veterinary schools during a long period, emphatically establish this fact. Indeed the frequency of melanosis in the horse does not correspond with the rareness of true tumors. Out of I,700 horses slaughtered, Bard found but three real tumors, while melanosis was found in every tenth horse. And, finally, melanosis does not cause cachexia in the horse.

Moreover, microscopic examinations have shown that melanic tumors often contain no other organization except the connective tissue in which the pigment is deposited. Most frequently there is no trace of new formation. This statement does not, however, imply that fibromata, sarcomata, carcinomata, etc., are not sometimes impregnated with melanosis. The truth is that these tumors may, like any other tissues, be impregnated with melanin, yet pigment masses of the tail, anus and perineum may at a late period be found associated with a fibroma that contains no pigment. With these facts as a basis, two varieties may be distinguished: 
Ist. Melanotic tumors or melanotic infiltration of tumors.

2nd. Melanosis, properly so called, simple melanosis, or melanotic infiltration of the tissues, with or without subsequent neoplasia.

Melanotic Tumors.-The history of this form of melanosis is confused with that of tumors in general. There is but one additional element, the pigment. In some of these tumors is found a structure analogous to the melanotic nodt1le, which is subsequently described as a manifestation of simple melanosis. The fundamental difference lies in the preponderance of cellular proliferation, and the embryonic character of the proliferated cells belonging to the melanotic neoplasm. In other words, the melanotic tumors have the evolution and the characters of special tumors infiltrated with melanotic substance. The fundamental cells are very variable. Sometimes they are embryonic connective tissue cells, sarcomatous, fibromatous or myxomatous, while at other times they are epithelial cells of different forms distributed through the alveoli (melano-carcinomata and melano-epitheliomata). The pigment is found distributed in abundance in the connective stroma and new-formed cellular masses, where it assumes the form of round spots or disseminated islets similar to those of the melanotic nodules already described. Their growth is rapid, and they generalize rapidly through the medium of the lymphatics. Cachexia may supervene from the neophasia, but not from the melanism.

Dexter found a melano-sarcoma of the cardiac lymph glands of the horse, but they may be located in the cardiac septum. The tumors may generalize. Mawri reports a small tumor on the tail that recurred twice after ablation, and that was eventually followed by manifestations of paraplegia. The patient died and the autopsy revealed a tumor in the neural canal at the third lumbar vertebra. The cord 
was compressed, softened and reduced to two-fifths of its normal size. Similar tumors have been found in the sacral region, in the liver and in the endocardium. It has been shown in a number of cases that the animals may die of cachexia in consequence of the generalization of these tumors. This form of melanosis is of the rarest kind. It is as rare as the tumors which serve as the substratum for the deposit of the melanic substance.

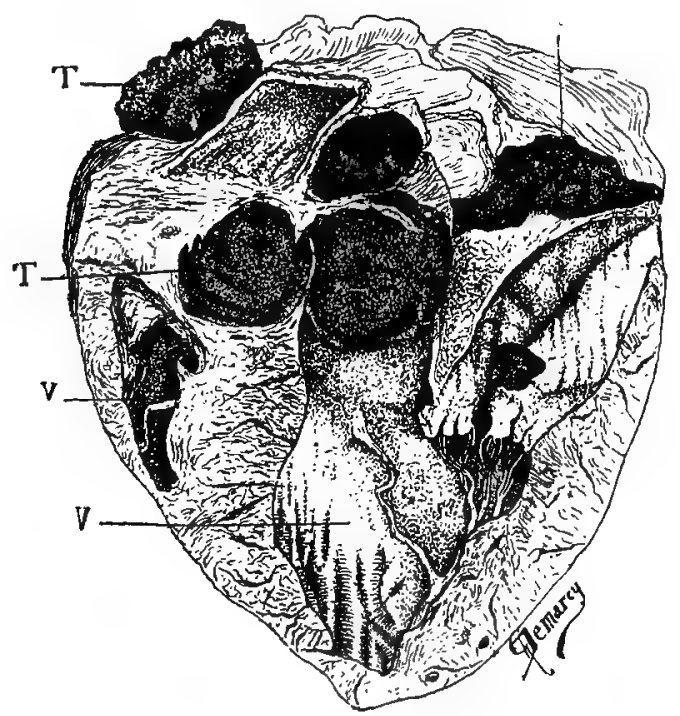

FIG. 59.

Melano-Sarcomata of the Heart. (M. Blanc.)

$\mathrm{V}, \mathrm{V}$, Ventricles.

T, T, T, Melanotic Tumors.

Simple Melanosis.-In simple melanosis the accumulation occurs without presenting any of the characters of true tumors. The accumulation of the pigment is the essential process. The masses have variable dimensions, from those of microscopic particles to volime of the fist, the head, etc.

The action of the melanic substance on the tissue varies: Ist. The pigmentary granules accumulate in the pre-existing tissues, and infiltrate and bury their cells, without des. troying them. The process is one of simple melanic ob- 
struction. The tissues, at first, exhibit no trace of reaction. A microscopic study of the growth shows a connective tissue, with cells hypertrophied and laden with pigment, without any evidence of cellular multiplication or alteration of its fibres. This is the maculous melanosis of Gurth. 2nd. When the masses become more voluminous, the infiltrated parts cause an inflammatory reaction of slow development that provokes the formation of a nodule of fuso-cellular, sarcomatous appearance, in which the pigment continues to accumulate. (Fig. 59). In the course of time the melanic neoplasm is established, the normal structure disappears, and the melanic mass exhibits, on section, the aspect of car-

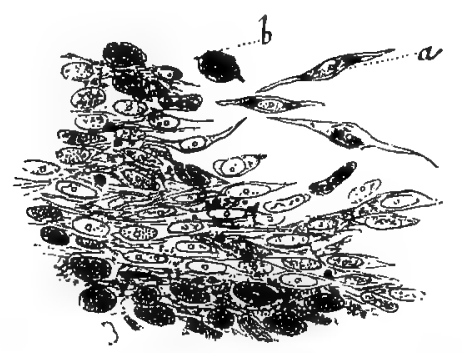

FIG. 60 .

Fibro-plastic Melanotic 'Tumor (200 diameters). a. Fusiform Cells Containing Black Pigment. b. An Element Completely Infiltrated with

Pigment.

bon in which no detail can be distinguished. Washing of the section with a brush removes the infiltrating granules with difficulty. To this form the name simple melanotic tumor may be applied. It is a circumscribed mass in the form of a tumor, the tuberculous tumor of Gurlt. It is the most frequent form in the horse. When the masses become too voluminous the pigmentary granules cause destruction of the cells in which they were accumulated, and the lesion then presents the form of a cyst filled with black granules and vestiges of the original tissues.

The third form more nearly simulates tumors, although it is still simple melanosis. It is habitually found in the 
foci of recent formation, and deserves the name of melanotic nodule. The nodules, enclosed in the cellular tissue, are of globular aspect, and divided into round secondary lobes of fibrous septa harboring black foci of the disease. The blood vessels are but few and slightly developed, and the cells of which the tumor is composed all belong to the connective types. These cells are found in every stage of development, from the young cell to the fibrillary adult tissue. The small round embryonic cell of true sarcoma is never encountered. These nodules are constituted by the reaction of the tissues and the more or less abundant proliferation of the connective tissue cells, but the process is rather more exuberant than destructive. It simulates the fibrous tubercle more than the sarcoma or fibroma. The elongated and spindle form of the elements of the nodule has caused the majority of writers to confound them with sarcomata or fibromata, so that they are almost always described under the name of melanotic-fasciculated sarcomata.

The three forms are seldom pure and isolated. Even in the same tumor a cyst may be found associated with the nodules. The organs in which melanism may be observed are:

I. Connective Tissue. Every portion of the connective tissue may be invaded. The connective tissue constituting the cutaneous derma (true skin) usually presents the first perceptible alterations, but since the epithelial covering has a share in the process it deserves separate consideration. The subcutaneous intraglandular and intramuscular connective tissues exhibit every variety of melanosis, but it seems that they are invaded later than the skin. The loose connective tissue at the entrance of the thoracic and pelvic cavities is frequently invaded. Tumors of considerable size have been found around the bladder and the rectum. In the pelvic cavity the masses may surround the kidneys, the ureters, the 
posterior aorta and completely obstruct the rectum. In the thorax, tumors have been found in the mediastinum, around the trachea and along the œsophagus. The submucous tis-

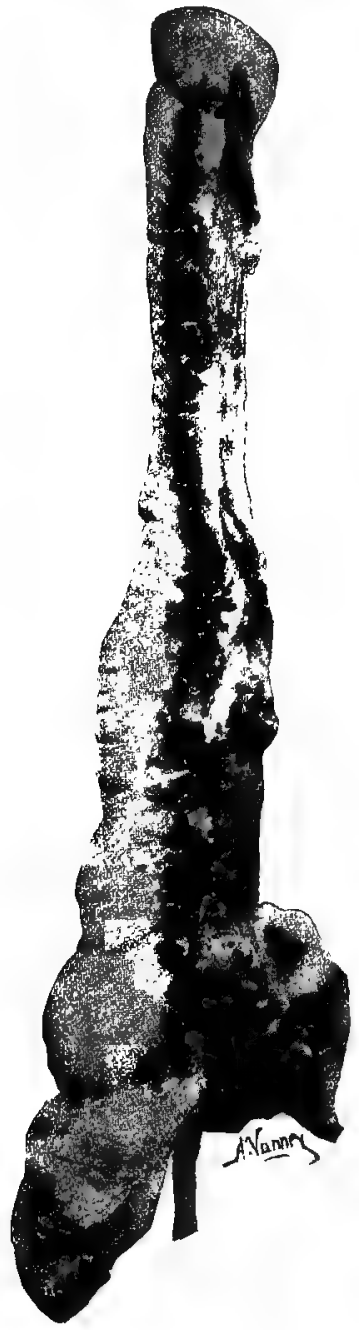

FIG. 61.

Peritoneal Melanosis Localized on the Large Intestines.

sue of the larynx and the soft palate may be invaded. The serous membranes, - the pleura, the peritoneum and the pericardium,-show round or irregular black patches, more or less thick, more or less numerous, and scarcely larger than 
a hazelnut. The generalized form is often accompanied by abundant infiltration of the connective tissue of the peritoneum, in which are found enormous black masses involving the mesentery and the intestinal walls. (Fig. 60).

The melanic pigment may infiltrate the bones, even those of the head, especially the tempora1. It invades the osseous medulla and thus provokes fractures of such bones as the pelvic arch, the dorsal vertebre and the ribs. Nibbert has observed cystic-melano-sarcomata develop originally on the ribs.

2. The Muscles.-Black tumors have frequently been found in the different muscles under the shoulder; in the rhomboideus and upon its surface; in front of the shoulder, under the mastoido-humeralis; in the longissimus dorsi; under the tensor fascia latæ; in the region of the scapulohumeral and coxo-femoral articulations; near the pterygoids, etc. The diaphragm is not always spared. It may be invaded with oval, flattened tumors that simulate the cotyledons of the uterus of large ruminants, or pigmentary infiltration similar to that of the peritoneum.

3. Cartilage.-The lateral cartilages, the scutiform cartilage, the membrana nictitans and the caruncula lachrymalis are the cartilaginous structures occasionally affected.

The eyes, atrophied from periodic ophthalmia, are sometimes invaded with pigmentary deposits that replace the vitreous humor.

4. Lymphatic Glands.-When lymphatic glands are encroached upon by pigment they become hypertrophied, hard and fibrous, and on section exhibit the characters already described. Bissauge observed melanosis of the sub-glossal gland, simulating the tumefied gland of glanders. It presented the form of a hard, lumpy, adherent, and insensitive body the size of a walnut. The nostrils discharged a suspicious mucus that gave negative results when inoculated 
into cavies. Ablation proved it to be a gland infiltrated with pigment. Dexter observed melanosis in the cardiac glands. The bronchial, the mediastinal and the mesenteric glands may also be invaded and transformed into enormous masses.

5. Viscera.-The viscera are seldom spared. The spleen

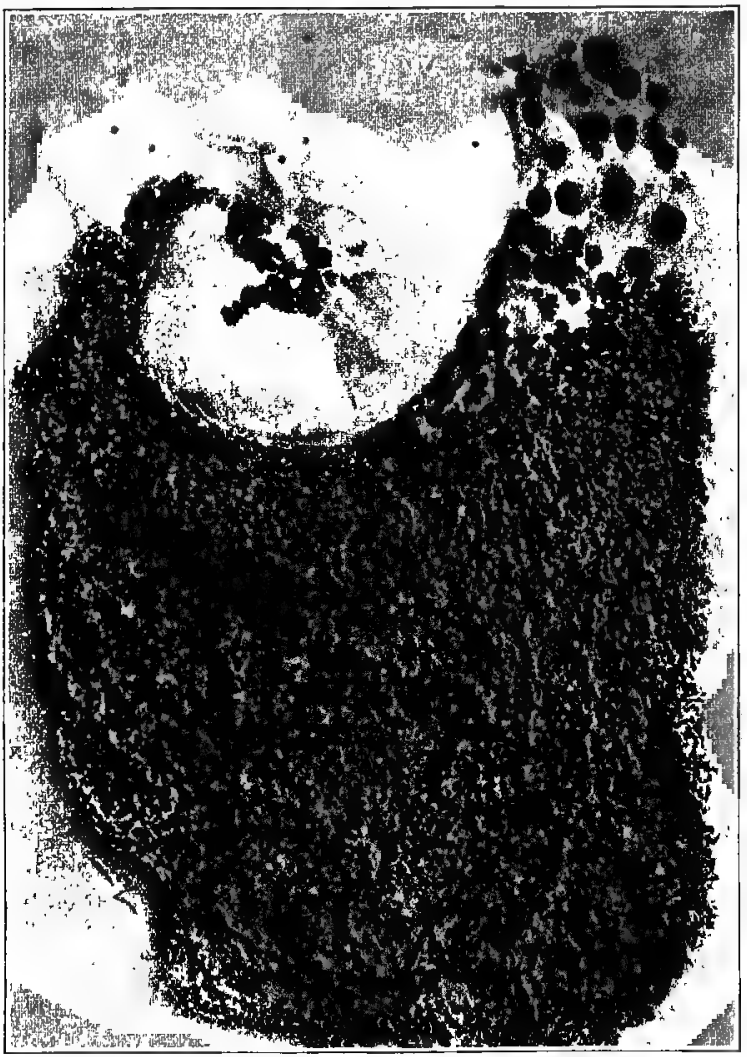

FIG. 62

Melanosis of the Omentum.

is often encroached upon with melanosis, either simple melanosis or melanotic tumors. The former are of the typical form already described. (Fig. 63). Melanotic tumors, and particularly the sarcomatous variety, have been observed. Their weight may reach 25 kilograms (Gregory) or even 33 kilograms (Nibbert). The surface and the edges are strewn 
with secondary nodules the size of a gall-nut, a hen's egg. or even larger. The growths considerably enlarge the dimensions of the organ. It may be 90 centimeters to I meter long, and instead of being smooth, it exhibits ovoid, spheroidal or tuberous protuberances, and a brown color with violet or bluish streaks. Sections show variegated colors, deep red, inky and black. The tissue proper of the organ may have entirely disappeared. Its consistency is increased and scrapings stain paper like India ink. The serons membrane and the fibrous stroma are thickened to several millimeters, forming a very solid framework. The tumor may be ruptured by external violence and disseminate its blackish, manure-like product throughout the abdominal cavity.

They are also found around the heart, in the coronary furrows. Reboul found a veritable melanotic bandage extending around the heart between the auricles and ventricles, and Vianet a melanotic growth of 25 kilograms investing the arterial trunks that caused acute cardiac disorders. Sometimes these tumors invade the interior of the ventricles and auricles or develop over the valves. The cardiac wall may itself be invaded by nodules that develop in its own depth.

The liver and the spleen are the most frequently affected. Melano-sarcomata, or simple melanosis, from slight pigmentation of the elements to pronounced overloading obstructions are encountered in these organs. The word "sarcoma" has frequently been applied to these simple, nodular melanoses. The pigmentation of the hepatic cells is observed in cases of disseminated melanosis in the different parts of the body. When the generalization is complete the pigmentation is well marked. The liver is wrinkled, very dark and hard, and has a uniform*or marbling black tint.

The section is dry and as tough as leather. It presents the same aspect as the external surface. Scrapings show little hepatic cells that are surcharged with accumulated pig- 
mentary granules, especially around the nuclei. Sometimes the protoplasm is so loaded with pigment that the nuclei are imperceptible. The black granules are spread throughout an entire lobule, but are more abundant in the center. The periphery is somewhat more clear, and thus gives the section the appearance of cardiac liver. At this phase the connective tissue of the organ is but little modified.

The melanotic sarcomata and the melanotic nodules are sometimes so numerous that the organ is destroyed. The surface of the liver is covered with a thick incrustation deposited on Glisson's capsule. The alterations vary accord-

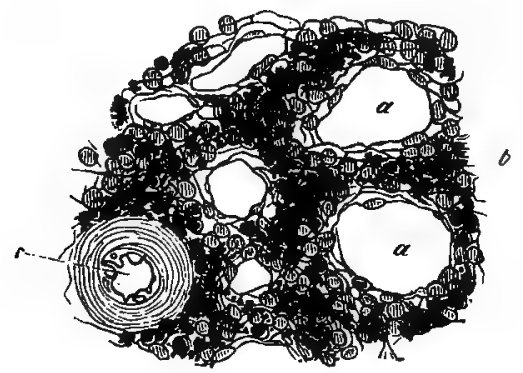

FIG. 63.

Melanosis of the Spleen. Section through the Middle of the Organ.

a. Cavernous Veins. b. Intervascular Bands with Their Fragments. c. Branch of Artery. (300 diameters.)

ing to the gravity and duration of the lesion. In every case the organ has lost its smoothness. Its surface has become irregular, mammillated or riddled with nodules, dependent upon the number and size of the tumors, which are diffused or confluent. In some cases they are chiefly found on one lobe, while in others the whole parenchyma is affected. They are separated by healthy tissue, which is slate colored, and which encroaches on the prominent black portions. The melano-sarcomata are of variable sizes. They become as large as the fist, or even larger. Bouley mentions one that weighed 24 kilograms. To the touch they are firm, elastic, resistant and hard to dent with the finger. A re- 
markable feature is the fact that they are always globular. The outer color is brown with violet or bluish streaks. The tissue, when cut, is marbled, and resembles a Strasburg pie well stuffed with truffles. The tissues hiss when cut with a sharp instrument, and a deep black blood escapes, but no central softening nor suppuration can be found. Intermediate phases of the disease may, of course, be enconntered.

The kidneys, suprarenal capsules, and the ovaries may also be invaded with simple melanosis or melanotic tumors. Cunningham drew attention to a melanotic tumor weighing ten pounds attached to the left kidney, and Nibbert found a kidney enclosed in a sort of melanotic sack.

The parotid gland and the udder are frequently invaded in aged animals. When the mammæ are invaded with melanotic masses the skin is tightly drawn and glistening. The thyroid body is sometimes the seat of a pigmentary infiltration, and at others is invaded with melanotic sarcoma. And lastly, horses attacked by generalized melanosis exhibit pigment in the blood, but only in agglomerated masses all ready to form tumors.

The pancreas is occasionally invaded. Bruckmuller saw melanosis of the connective tissue of the pancreas in the form of tubercles ranging from the size of a pea to that of : a walnut, with pigmented deposits in the neighboring connective tissues.

The Lungs.-Simple melanosis and melanotic tumors are found in the lungs. The former sometimes imparts a siaty gray color to the organ analogous to that of pronounced anthracosis, but the chemical characters distinguish the anthracotic particles from pigmentary granules, Under the microscope, fibrous neoplasms and pigmentary infiltration of the different cells is seen. The melanotic tumors of the lungs usually correspond to the sarcomatous type and are of variable numbers and dimensions. When 
the infiltration. is well advanced the structure of the lungs disappears. The bronchial cartilages resist the action of melano-sarcomata found distributed among the masses. Their cells are of a violet tint, shown for the first time by Cornil and Trasbot.

The Nervous System.-Melanosis is found in the men-

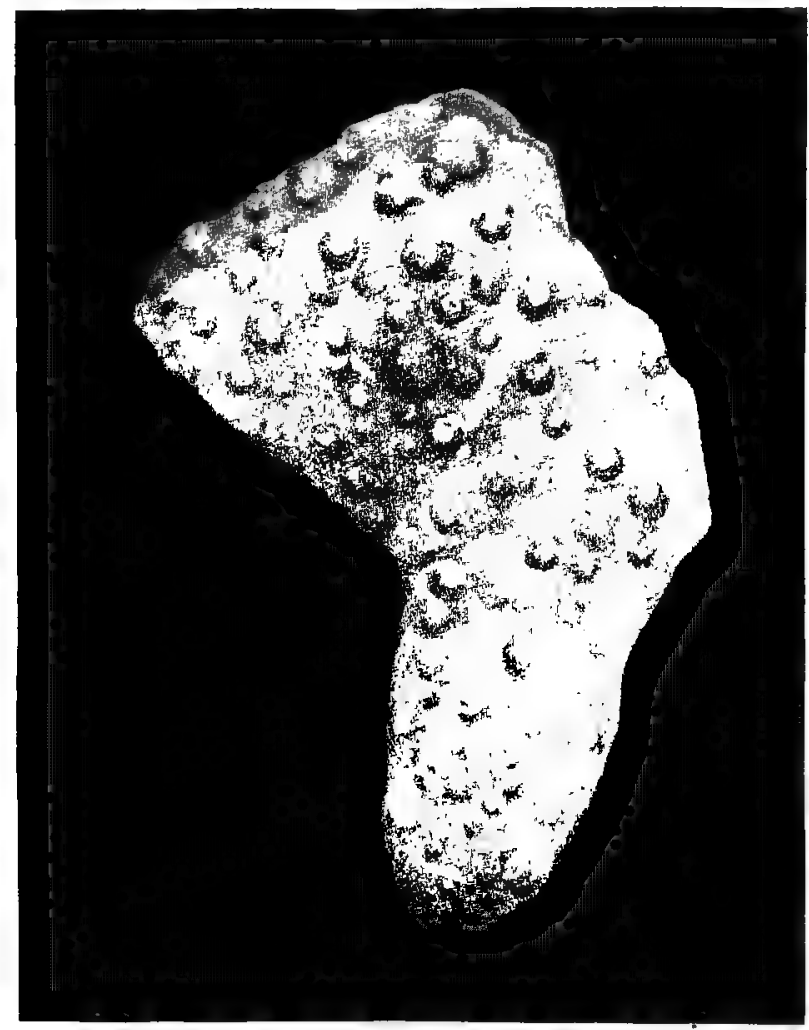

FIG. 64. Melanosis of a Spleen, Weighing 7 Kilograms.

inges of the brain, the cranium or in the neural canal (Fig. 65). The pigment is accumulated in the connective tissue or the bones surrounding the meninges. They may penetrate through the communicating foramina at the roots of the nerves. The masses grow regularly, atrophy the cerebral substance and may attain the size of an egg. Jessen 
found a melanotic tumor two centimeters in diameter in the left temporal lobe, and Nibbert one the size of a pea in the right cerebral hemisphere, and another as large as a goose egg in the orbital fossa. In the brain they have been found in the pituitary gland, the optic commissure, the lateral ventricles, and Bouley refers to one that started from the right petrous temporal and penetrated through the suprasphenoidal duct, englobing the seventh and fifth nerves. Blanc reports an analogous case.

The Skin.-The skin, associated with the epidermis and its glands, presents more complicated lesions. According to an unpublished publication of Blanc, the melanotic infil-

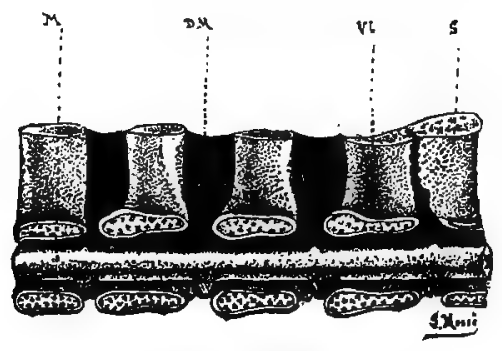

FIG. 65.

Melanosis of the Lumbar Region of the Horse. M. Spinal Cord. D. M. Melanotic Deposit. V. Vertebrae. S. Sacrum.

tration begins in the epithelial cells of the sudoriferous glands, which become tumefied as the result of a reaction of the periglandular connective tissue, which in turn becomes pigmented, and thus forms a nodule of one millimeter at the maximum. In the course of time the nodule swells through the periphery while its central cells,- - sudoriferous glands,-die, and resolve into a mass of granules. The process occurs simultaneously at different points, which often results in very remarkable accumulations in the perineum of the horse. In cutaneous melanosis the skin is thickened, slate-colored, filled with black blotches, tumefied and riddled with black nodules that raise the epi- 
dermis, depress the subcutem, and crush the glands and neighboring follicles.

The melanotic infiltration extends to the subcutaneous connective tissue, and forms masses, more or less regullarly hemispheric or ovoid, with clear, firm outlines, unyielding to the touch and very dense (Fig. 67). At the commencement of the disease they usually occur in th:

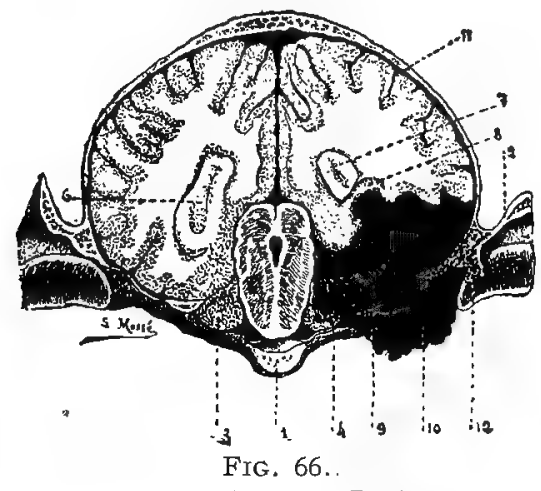

Melanosis of the Brain.

1. Body of the Sphenoid Cut through the Sella Tursica.

2. Zygomatic Process of the Temporal.

3. Left Gasserian Ganglion.

4. Right Gasserian Ganglion Invaded with Melanosis.

5. Section of the Aqueduct of Sylvius and Cerebral Peduncles.

6. Section of Left Hippocampus.

7. Section of the Right Hippocampus Pressed Back by the Tumor.

8. Sphenoidal Lobe Pressed Back.

9. Melanotic Tumor (Intracranial Portion).

10. Melanotic Tumor (Extracranial Portion).

11. Pia Mater Infiltrated with Pigment.

12. Internal Ear Infiltrated with Pigment.

form of little grains projecting from the inferior surface of the tail, around the anus, vulva, udder, or prepuce. The tail and perineum is the specially selected place, but they may be found in the subcutaneous tissue of any part of the body. In the stifle, on the elbow, the shoulder, the coronet, in the tracheal region, on the parotids, between the jaws, etc.

The masses are varied in form and size. At first they are little granular nodes in the skin, perceptible to the 
touch, colored black, but not prominent. They are associated in variable numbers and have well defined limits. The size increases by degrees, until they attain the dimen-

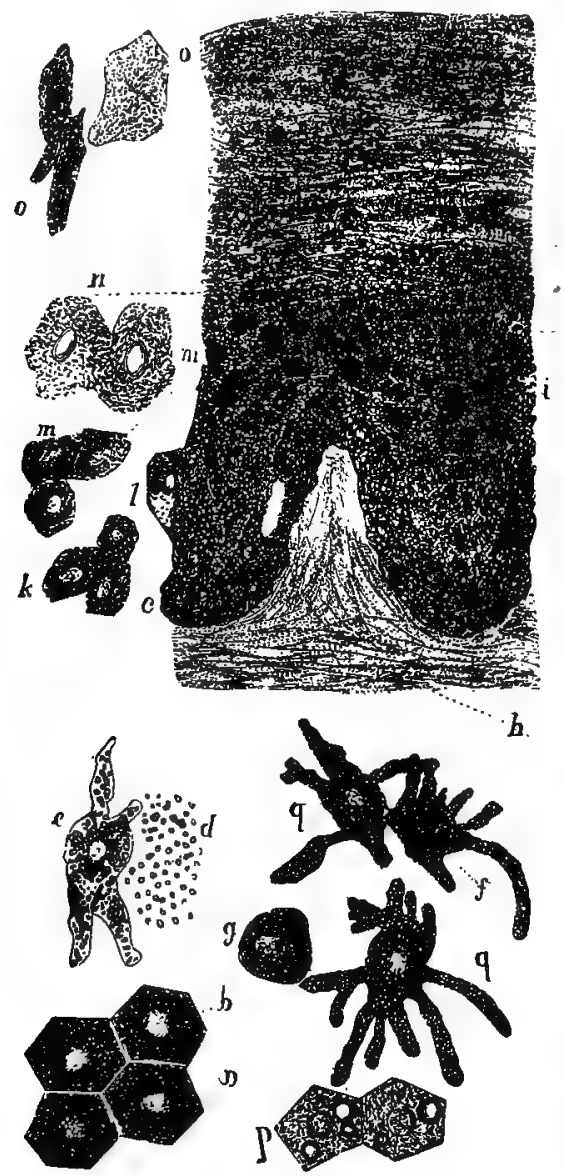

FIG. 67. Pigment.

d. Free, Pigmentary Granules. c, n. Pigment Granules of the Cells of the Malpighian Layer of the Skin. 1, m. Masses of Pigment in the Cells.

b. Polyhedric, Pigment Cells of the Choroid. q. Irregular

Pigmentary Cells. g. Pigment Cells with Drops of Oil in Their Midst.

sions of a hazel-nut, a walnut, an egg, the fist, the head, or even enormous masses with irregular, tuberous, mammillated, hard, unyielding and elastic surfaces. The masses are dark and destitute of hair. [The cutaneous melanotic 
tumor retains the hair, and is only destitute of it in the perineum, inferior surface of the tail, etc.-L. A. M.] They may become soft, fluctuating or ulcerated, in which case they discharge a thick, dirty liquid of the color of blacking.

SYMPTOMS.-There is no symptomatic tableau more varied than that of melanosis. Every structure, every organ in the system, may be invaded with melanotic infiltration. Every function may be disturbed, mechanically impeded, or suppressed by deposits of pigmentary tumors. Only the principal localizations can, therefore, be reviewed (Fig. 68). The skin and the subcutaneous connective tissue are sometimes the seat of semi-lunar or ovoid tumors of variable sizes on the lateral surfaces of the neck and shoulders, at the collar, seat, in the costal region, etc. Generally they accumulate in the region of the tail, the rectum, vulva, or prepuce. Isolated in groups, they have their limits well defined. They are hard, elastic, sluggish and without any peripheric inflammatory infiltration, characteristic of phlegmons in the course of development.

When numerous around the anus, they first present rounded outlines, but sooner or later fuse together, acquire considerable dimensions, and become the seat of inflarima. tions occasioned by the crupper (Fig. 68a). When thus injured, they may soften, fluctuate and even ulcerate, sut the discharge of black substance, the existence of other tumors and the pigmentary infiltration of the surrounding parts disclose the origin of these pseudo-purulent foci. Cicatrization is slow; the anatomical elements, incumbered with pigment, find difficulty in regenerating. The external melanotic infiltrations are accompanied, also, by numerous functional disturbances. Melanosis of the rectum and vagina, although compatible with health for a long time, generally ends by deforming these orifices and impedes the exit of excrements. The walls of the vagina and rectum 
may become indurated and an obstinate constipation re. sults that will provoke colic every day, and necessitates the extraction of the fecal matter. When the coprostasis is neglected the rectum dilates, finally ruptures and causes death (Fig. 69). The female cannot fecunclate and the passage of urine is more or less obstructed. The melanotic tumor may invade the foreskin and prevent protraction of the penis, cause retention of urine, incontinence and intense

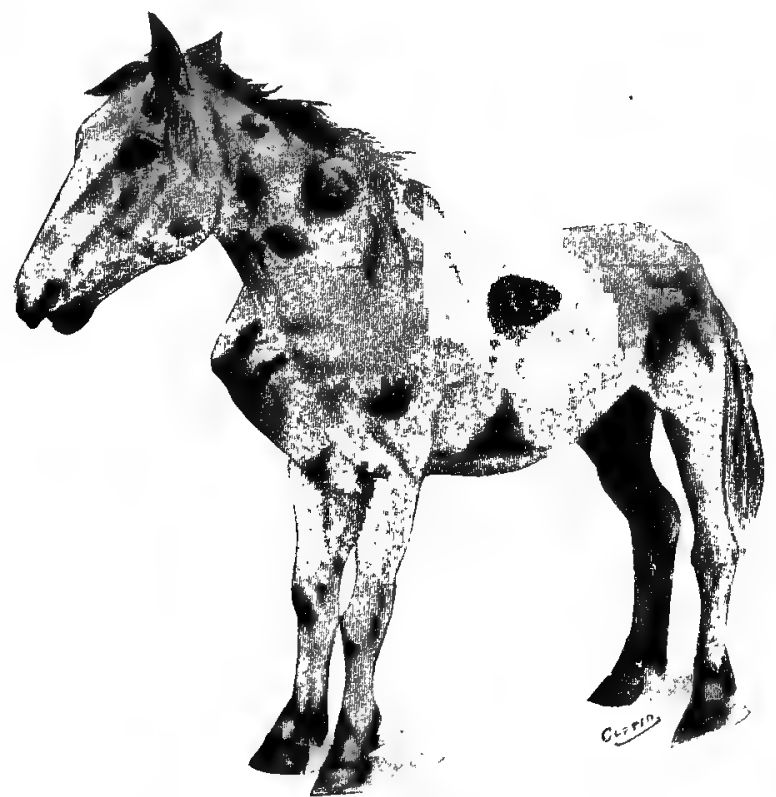

FIG. 68.

Cutaneous Melanosis (Cadeac).

irritation of the prepuce. Those at the extremity of the tail constantly become bruised, mutilated and ulcerated, and become the seat of a blackish fetid suppuration. They may, on the other hand, encroach on the sacrum, and the other bones of the pelvis, or to the terminal extremity of the spinal cord or the region of the coxo-femoral articulation, and provoke lameness.

In the parotid region they fill up the parotid cavity, emboss the region and may even cause roaring. When the 
thyroid gland is affected it exhibits all the characteristics of cancerous goitre, from which it can be differentiated only by section. Melanosis of the eye generally affects.the menbrana nictitans, carunula lachrymalis and the upper eyelid.

Melanotic tumors of the limbs are rarer. They may counterfeit side-bones. They sometimes develop under the mastiodo-humeralis and present the appearance of the cold abscess so common in that region.

They may compress the pneumogastric nerve and cause tumultuous and sonorous beatings of the heart. In the lymphatic glands they are often the cause of grave functional derangements. In the subglossal gland it may be mistaken for glanders (See Bissange's case page 539). The glands of the groin and of the mesentery are frequently attacked. The former may impede the movements of the limbs, those of the lungs may reproduce all the phenomena of bronchial adenopathy, etc. The nerve tissues sometimes become compressed with melanotic infiltration. We have reported cases of the brain, the spinal cord, the lumbosacral plexus, etc. King found a large one in the subsacral region that extended into the neural canal and compressed and atrophied the spinal cord. Similar tumors are occasionally encountered. Mawry observed a compression of the radial nerve from a melanotic tumor situated in the forearm. They have also been observed to envelop the cervical ganglia of the sympathetic system and the pneumogastric nerve. In this localization they cause paresis, paralysis and paraplegia.

Independent of digestive disorders succeeding obstruction and contraction of the rectum and anus, disorders of deglutition may result from invasion of the retropharyngeal gland, compression of the pharynx, trachea, œsophagus, bloodvessels and nerves. The tumors developed in the abdominal cavity (peritoneum, diaphragm, intestines, 
etc,) are seldom large enough to provoke grave digestive disorders, but they may impede the return circulation or compress the aorta, and produce odema and ascites. They may predispose to attacks of colic or rupture of the intestines, by traction on the mesentery.

Melanosis of the liver may disturb its function and cause degeneration and rupture. This termination is especially frequent in melano-sarcoma. The splenic form generally passes unnoticed, and is found only at the autopsy.

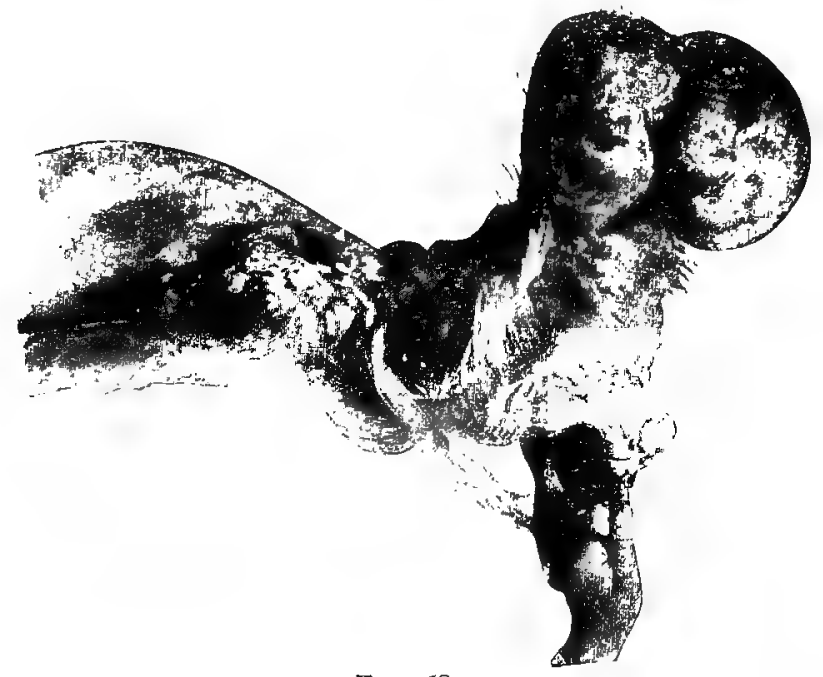

FIG. 68A.

Melanosis of the Extremity of the Tail (Tail Elevated).

The respiratory form characterized by infiltration of the lungs, the pleura and the periphery of the heart manifests itself by dyspnceic symptoms of more or less intensity. The respirations are alternately calm and accelerated. Sometimes the subject is seized with general tremors and exhibits asphytic symptoms. Then again they may cause a fatal hæmoptosis. In some cases they cause circumscribed sudation by compressing the sympathetics. Bouley reports a case in the horse that sweated copiously over the left side of the head, neck and shoulder. Sometimes the melanotic 
infiltration of the trachea, bronchial tubes and lungs is so intense that the jugular, glossofacial and other superficial veins are extremely distended. The pneumogastric the recurrent laryngeal and the phrenic nerves may be compressed by melanotic masses. The trachea may be flattened and almost obliterated and thus cause roaring that is audible even in sleep, and even death from asphyxia.

PROGRESS, DURATION, TERMINATION.-Melanosis is compatible with health as long as it does not obstruct or suppress the functions. It is a benign affection with a slow and chronic course and without any reverberation on the health.

The acute forms [rare-L. A. M.] causing emaciation and cachexia, with generalization of the tumors, have an undeniable cancerous character and do not belong to the same category as simple melanosis.

DIAGNOSIS.-The advanced age of the animal and the coat [white-L. A. M.] facilitate the diagnosis. It does not generally appear before the age of eight or ten years, and simple melanosis is scarcely ever observed except in white horses. Most of the melanoses observed in animals of dark coats are fibromata, myxomata, carcinomata, or sarcomata that are incidentally infiltrated with pigment.

The absence of any inflammatory tumefaction and the slowness of the development signalizes the nature of most of the subcutaneous or intermuscular melanotic masses. Besides, recourse may be had to puncture or incision of the tumor to unmask its nature. The internal forms are revealed by the functional disturbances accompanying them, by the absence of fever, and by the presence of melanotic nodules in the tail.

PROGNOSIS.-The prognosis is comparatively grave, by reason of the heredity of the disease, of the fact that generalization is always possible, and of the numerous and 
variable functions it is capable of provoking. Besides, flesh of melanotic horses is condemned as food, and as the majority of white horses are affected at an advanced age, the white horses cannot be consigned to the butcher. The gravity also depends upon their nature. Simple melanosis, or melanosis by infiltration, is always less grave than mel-

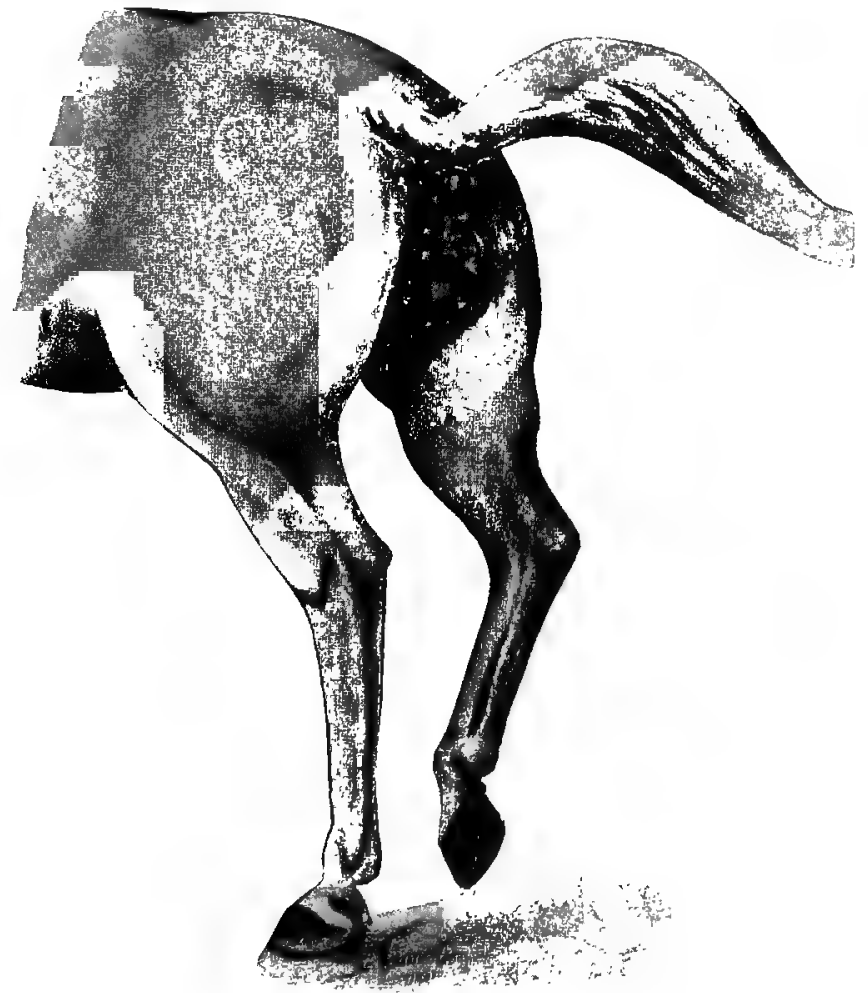

FIG. 69.

Melanosis Surrounding the Rectum.

anosis with tumors. In this form the fibromata and myxomata are benign. They never become generalized, as different observations testify.

Mawry reports that on two occasions he removed a melanotic tumor from the extremity of the tail of a bay horse. In this case, after the second reappearance of the tumor, symptoms of paralysis appeared and the autopsy disclosed 
the existence of a tumor of the same nature in the neural canal at the third lumbar vertebra that compressed the spinal cord to $2-5$ of its normal size and softened it. Melanotic tumors were also found in the liver, under the sacrum, in the spleen and on the endocardium.

TREATMENT.-The deposit of melanotic pigment cannot be prevented, nor can it be made to disappear. Once commenced the process continues through the entire life of the animal. External tumors that obstruct certain functions,-micturition, defecation, respiration, etc.,--or those which prevent the animals from being untilized, can be removed. The extirpation should be complete, as the tissues impregnated with melanin cicatrize badly.

\section{MELANOSIS IN RUMINANTS.}

DEFINITION.-The melanosis of ruminants, as in the horse, consists essentially in pigmentary aberrations. In exceptional cases, the pigment is deposited in the elements of fibromata and myxomata.

ETIOLOGY.-The physico-chemical properties of melanin are the same as in the horse, and the deposits, too, are similar. The melanosis of calves and that of adults should, however, be distinguished.

Calf.-More or less complete researches on melanosis of the calf have been recently made. Wehenkel gives the first account of the subject. Trasbot, in the article on melanosis in the "New Dictionary," mentions that Goubaux found an authenticated case of melanotic infiltration in a calf. In 1885 , DeGive found melanotic infiltration of the meninges, lungs, liver, skin and the heart in a calf five days old. In 1887, Morot brought several cases of melanic pigmentation among calves to the notice of the Societe Centrale. The lungs are the organs most frequently attacked. Also reports, with the observation of Albert, that he discov- 
ered an enormous melanotic tumor on the cheek of a calf six weeks old. Its histological nature was not determined. In I896, he cited the case of a calf afflicted with melanic infiltration of the cerebrum and cerebellum. Spots also appeared in the mouth, cheeks, perilaryngeal region, œsophagus, trachea, parietal pleura, and the investing membrane of the thymus gland. There were also spots on the surface and in the depths of the lungs, the heart, the liver and the spleen.

Dr. Fiorentini has seen numerous cases of melanosis in the calf, most of which showed black spots on the lungs with clear outlines. The spots penetrated to varied depths into the parenchyma, and microscopic examinations proved that the function of the lungs were not altered in the diseased portions. In the connective tissue melanosis of the calf assumes a special form.

In the calf, the etiology is distinguished from that of the ox. The nutritive anomaly of the subcutaneous connective tissue manifests itself by deep spots of a black hue, which are described under the name of "macular melanosis." When skinned there are found in the under layer of the skin, brilliant, black, clearly circumscribed spots two centimeters in diameter, shaped like feathers, and giving the impression that black feathers had been pasted under the skin. Only the loose cellular tissue shows this coloring, and they can be removed with forceps and deposited in a steel blade. The microscope reveals fibrillary fasciculi, crossed with voluminous fusiform cells of granular nature with colorless nuclei. These cells are analogous to the lamina fusca of the eye, ramified in stars and incurved. The entire connective tissue is strewn with free melanic granules.

This affection, is a congenital, heterotopic anomaly of the mesodermic pigment, which disappears at a later period. It has never been found in the adult bovida. According to 
Fiorentini it is due to hamorrhages accompanying painful delivery, the calf being more or less compressed in the passages and the extravasated blood undergoes a melanic transformation. The spots thus observed are destined to disappear. But this theory of Fiorentini on the formation of pigment is not based on probability. Indeed melanotic tumors that are not traumatic are found in calves. Besides. if the origin which Fiorentini attributes to it were correct, the melanic infiltration would disappear with age, but it has often been found in adult animals. Moreover, how could the frequency of melanosis in the protected places like the brain and the spinal cord be explained? The formations of melanotic alteration in the calf must be explained otherwise.

The first theory held by Breschet, et al, acknowledged that melanosis is an alteration of the coloring matter of the blood,- the hamatin,- - and there is some reason in explanation, as Heurtaux says: "If the hrematin supplies the elements of the pigment it certainly is not the consequence of sanguineous infiltration, and of an alteration resulting from the red corpuscles extravasated among the elements of the tissues. The authors who believe in the hæmatin origin of pigment have not claimed that the blood outponred is directly converted into melanin. They simply meant to say that its materials have been borrowed from the blood, and particularly from the coloring matter."

The second theory, defended by Lebert, et al, attributes the fabrication of melanin to the cell, and Schultz to the connective tissue cell, without the intervention of the red blood corpuscles. Of these theories the first is unacceptable and the two others lead to the conclusion that among six weeks' old calves intended for the butcher melanosis is congenital. It may be acquired in older animals and both origins may be found in the same animal. The color of the animal's coat would not, any more than among soli- 
peds, enter into the explanation of the melanic pathogenesis. In fact, kidneys as black as ebony have been found in dark colored animals.

Adults.-As in the case of solipeds, there are two modes of melanotic deposits, - the neoplastic and the simple deposit. There are numerous examples of the one and of the other form, which facts demonstrate that it is not enough for a tumor to exist in order that melanin be deposited. Dupont and Denuce point out that the melanic pigmentation of the peritoneum rendered the abdominal serous membrane perfectly black, and Morot draws attention to a similar infiltration of the parietal pleura. Gurlt has also found melanosis in the intestinal mucous membrane of a cow, a somewhat unusual case, according to Zundel, who says that melanoses are only observed in the subcutaneous tissue. Perroncito has described melanosis in the ox simul. taneously affected with tuberculosis. Baillet has reported a case of melanic infiltration of the lumbar division of the neural canal, and of a portion of the pancreas. Browier and Megnin have observed a melanic infiltration of the long bones and the flat bones, and Mosselmann et al have published two interesting cases concerning abnormal coloring of the skeletons of animals intended for the shambles. Nothing characteristic was found in the viscera, but the entire osseous system exhibited a characteristic red-brown tint. The osseous tissue appeared uniformly colored in its different layers. Chemical analysis demonstrated that the coloring matter was represented by nitrogenous and ferruginous organic products, while according to Borow the organic pigment would contain 0.25 per cent of iron. Microscopic examination reveals that the coloring matter is not uniformly distributed in the osseous substance, but, on the contrary, is found settled in the osseous cells or exclusively located in the fundamental substance 
of bone, sparing the bone cells. Attention has been drawn to melanosis of the skin and the meninges, which are occasionally as black as ink, or melanosis of the viscera. Muscular pigmentation has been observed by Gotz, in the heart, the muscles of mastication and the tongue, which exhibited a deep brown color. This black matter in the flesh would proceed, according to Rabe and Schultz, from a pigmentary infiltration in the connective tissue. The same authors have also found similar alterations in the liver of an ox, that presented lesions of chronic hepatitis.

Cases of generalized melanosis are not extremely rare. Albert observed a case in which the muscles, the parenchymatous organs and the dorsal and lumbar vertebra had undergone melanotic pigmentation, and Nuvoletti refers to a case of generalized melanosis in a cow exhibiting numerous tumors, first in the subcutem, then in the muscles of the thigh, shoulders and back; in the intermuscular connective tissue and in the peritoneum and walls of the intestines. The spleen contained a melanotic tumor as large as the fist, and the diaphragm and pleura were studded with them. Analogous tumors have likewise been observed in the region of the elbow joint, and in various other regions. Nuvoletti found a three-year-old ox that exhibited blackish stains of the viscera, pleura, Schniederian membrane and subcutem. In I885, Cadeac published the history of a case of melanotic fibroma in the depths of the right buttocks, and similar observation were reported by Cancel, who found melanotic tumors in the pelvis of a cow. Hamberger describes a tumor on the pleura of an ox that presented the structure of a chondro-fibro-melano-sarcoma.

Melanomata are frequently nothing more than melanotic myxomata, as was shown by LaBlanc in the case of a four-year-old cow, exhibiting a tumor having the consistency of a myxoma, in the abdominal wall, which, when but 
slightly magnified, revealed a clear, attenuated stroma filled with black detritus. When magnified 330 diameters, it presented a clear stroma having clear spaces as its limits, that contained pigment deposits. The pigment was found in the connective tissue cells, in which the nuclei were intact. The infiltration, which consisted of brown granules, was more copious around the blood vessels, where it was so in-

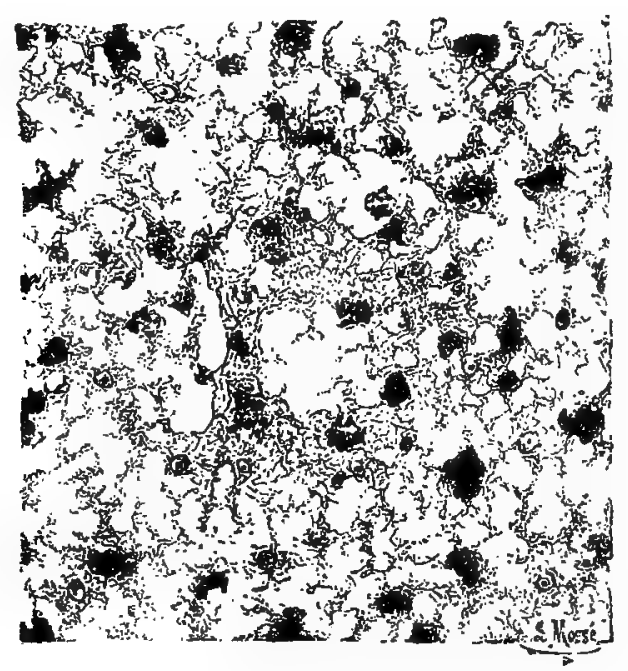

FIG. 70.

In the Middle of the Stroma, in an Irregular Manner, the Pigment is Seen to Invade the Cellular Elements, Some of which Remain Intact (330 Diameters).

tense as to reach the muco-connective fibrilla constituting the network of the growth.

It will be observed that in the ruminant, melanotic deposits do not have the same well-determined places of localization as in the soliped. The infiltration affects all the organs.

\section{Melanosis in Sheep.}

Melanosis is comparatively rare in the ovine species, but is occasionally encountered. Worsley observed nervous disorders traceable to melanotic deposits in the medulla of 
the nerve centers, and others have shown the existence of similar deposits in the lungs alone or in the lungs, liver and some of the muscles. Morot refers to several cases of melanosis in the sheep. He even found melanotic infiltration of the bones the color of waxed walnut in a four or fiveyear-old sheep. LaBlanc found accentuated pigmentation of the anterior portion of the cerebrum of a sheep. Under the name of Black Liver of Russian Sheep, Barrier describes melanotic alterations occupying the periphery of the lobules. The same changes were pointed out by Siedamgrotzky.

\section{Melanosis in the Hog.}

Of all the domestic animals, the hog is the least stbject to melanotic alterations, because usually sacrificed for meat before melanin is deposited. Melanotic deposits occur chiefly in the adult. The pigment has never been observed in the adipose tissue cells of this animal. The few observations on the subject show that the infiltration occurs in the interlobular connective tissue tracts. In 1878 , Saake observed pigmentation of ventral lard, and De Jong, continuing the study, referred, to an alteration of the bones in a hog, that appeared to be of a melanotic nature. All of the bones, without exception, exhibited a deep black tint. The marrow of the long bones was brown, the color of chocolate. Ostertag, in his manual refers to black pigmentation of the skin and of the panniculosis adipostus of sows. De Jong concludes that pigmentation occurs chiefly in black and piebald pigs.

The pigments are found in the kidneys and in the liver and according to the observations of Forreggiani it is 10cated in the capillaries of the kidneys and in the glomeruli, while in the liver it is found in the hepatic cells. 


\section{Melanosis in the Dog.}

Simple melanosis is very rare in the dog. Nevertheless, Kolesnikoff observed six cases and claims to have found striated muscular fibers in them. Kitt found them in the neck, and melanotic sarcomata were observed in the region of the incisor teeth by Cadeac. According to Bayer, et al, melanosis can be transferred from the horse to the dog by inoculation. 


\section{CHAPTER XV.}

\section{TUMORS.}

DEFINITION.-Formerly the word "tumor" was used as the universal designation for all enlargements and projections on the surface of the skin or subcutaneous connective tissue, which circumstance accounts for the application of the word to abscesses, phlegmons, hernias, cysts, hæmatomata, aneurisms, etc. Gradually, with the progress of medicine and surgery, the different abnormal tumefactions were properly distinguished from one another, and as a result the sense of the word was restricted to enlargments accompanied by the formation of new tissue. This conception, although more appropriate than that which had prevailed before, was not entirely satisfactory because certain inflammatory pathological processes combine in the formation of new tissue that is absolutely different from the genuine neoplasm. Nowadays, only masses of new formation that have a tendency to increase or persist independently of all inflammatory processes, are regarded as tumors. This view, although criticised and open to criticism, is, as a matter of fact, quite generally admitted as logical, and may as well be maintained until new investigations,-some of which have already shed new light on the subject,-remove the majority of tumors considered as pure neoplasms from this category. [For an anatomo-pathological and historical study of tumors the reader is referred to works on General Pathology, as only their clinical aspects will be considered here. 1

FREQUENCY.-Tumors are not rare among domestic animals. Named in a scale of decreasing frequency the do- 
mestic animals are classified as follows: Dog, cat, horse, ox, small ruminants, and hog. All of the tissues, but especially the mammæ, the liver, the kidneys, the ovaries, the testicles, the thyroid body and the lungs, are susceptible to neoplasms. Their importance varies with the nature and degree of development. From the clinical point of view and preceding the advent of the miscroscope, neoplasms were divided into two great groups: Ist. Benign tumors or those which are compatible with life and comparatively good health, and 2nd. malignant tumors, or those which provoke disorders necessitating intervention. Tumors possessing the following characteristics should be regarded as malignant :

Ist. All tumors having a deep, diffuse implantation, and which propagate by continuity of tissue.

2nd. All tumors susceptible to generalization.

3rd. All tumors that recur after ablation.

4th. All tumors having cellular colonies that impregnate the organism with products capable of provoking cachexia.

When extirpated, the malignant tumor is usually found clevoid of any membranous envelop or isolated capsule. They are quite generally known by the name of "cancer," and may attack every organ-the mucous membranes, the liver, the pancreas, the kidneys, the prostate, the testicles, the thyroid, the mammæ, the ovaries, the parotids, etc. Out of 57 cases of malignant tumors Semmer found 32 sarcomata and 25 carcinomata, and in further observations found more cases of sarcomata. The following statistics furnish a fair example of the relative frequency of the two principal varieties of malignant growth in the different animals : 


\begin{tabular}{|c|c|c|c|c|c|c|}
\hline \multirow[b]{2}{*}{ ANIMAL SPECIES } & \multicolumn{3}{|c|}{ Semmer's Statistics } & \multicolumn{3}{|c|}{ CAdiot's Statistics } \\
\hline & $\begin{array}{c}\text { Carcino- } \\
\text { ma }\end{array}$ & & & $\underset{\text { oma }}{\text { Epitheli- }}$ & Sarcoma & $\begin{array}{l}\text { Indeter- } \\
\text { minate }\end{array}$ \\
\hline \multirow{7}{*}{$\begin{array}{l}\text { Dog } \\
\text { Horse } \\
\text { Ox } \\
\text { Hog } \\
\text { Cat } \\
\text { Birds } \\
\text { Fishes }\end{array}$} & 7 & 17 & 30 & 27 & 11 & ............. \\
\hline & 14 & 7 & 12 & 4 & 1 & 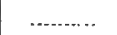 \\
\hline & 4 & 2 & 4 & -......... & …..... & …...... \\
\hline & $\cdots \cdots \cdots \cdot$ & 1 & 2 & $\ldots$ & ............ & …....... \\
\hline & (n........ & 4 & 6 & $\begin{array}{c}1 \\
\ldots \ldots \ldots\end{array}$ & (n........... & 3 \\
\hline & …….... & 1 & 2 & & & $\ldots$ \\
\hline & 25 & 32 & 56 & 32 & 12 & 3 \\
\hline
\end{tabular}

In the schools of Berlin, Munich and Dresden during seven years, there were found out of:

86 , I 3 horses I, II 3 cases of tumors.

85,337 dogs 4,020 cases of tumors.

4,972 bovids 1,020 cases of tumors.

Out of 643 tumors in the dog, Fröhner found 47 per cent of them malignant; out of 47 tumors of the horse 34 per cent; and out of 75 cases in the ox 29 per cent were malignant. The same writer publishes more recent statistics as follows:

Sarcomata ...........28 per cent.

Fibromata ............. I5 per cent.

Carcinomata ............. Io per cent.

Adenofibromata .........4 per cent.

Lipomata ............ 2 per cent.

Osteomata ............. 2 per cent.

In Fröhner's list, the botryomycostic tumors, keloids, papillomata, and keratophyllocele which he places in the category of neoplasms have been omitted.

\section{CLASSIFICATION.}

I. Tumors of the epithelial type.
(a) Adenoma.
(b) Epithelioma.
(c) Carcinoma. 
2. Tumors of the vasculo-connective tissue type.
(a) Sarcoma.
(b) Fibroma.
(c) Lipoma.
(d) Myxoma.
(e) Chondroma.
(f) Osteoma.
(g) Lymphadenoma.
(h) Angioma.

3. Tumors of the nerve type.
(a) Glioma.
(b) Neuroma.

4. Tumors of the muscular type.
(a) Myoma of striated muscles.
(b) Myoma of non-striated muscles.

5. Complex tumors.
(a) Dermoid cysts.
(b) Cystic tumors.
(c) Solid tumors.

TREATMENT. General Indications.-The past as well as the present methods of treatment, which still enjoy some favor, should indicate that medical treatment be not insisted upon. The poor success obtained from medical applications has led to their almost total abandonment. In veterinary medicine, the medical methods of intervention are rather numerous. Professor Trasbot recommends compression, refrigeration, and scarification, with the addition of electricity, but these processes cannot be utilized in animals, and besides they have not given sufficiently satisfactory results in human practice to justify their adoption in the treatment of domestic animals.

Cauterization is a much more practical method. It may be accomplished with chemicals or with the actual cautery, but no matter what agent is employed, there is never any 
certainty that the entire growth is destroyed, owing to the impossibility of appreciating its exact extent. When neoplasms are pedunculated they may be extirpated with the ecraseur or elastic ligature. The latter has the advantage of not producing hremorrhage and the disadvantage of being rather slow and of leaving a more or less voluminous, mortifying mass in contact with the body for some days, and besides, when dealing with a house dog, the offensive smell is of no small significance. As a general rule, ablation should be the chosen method. The operation should be preceded by all the precautions recommended for the management of hamorrhage. In every case the large blood vessels should not to be divided until they are ligated. The ligation of both the nutrient artery and its co-lateral vein is necessary. Copious hemorrhages may also be controlled with the actual cautery.

Some tumors may be easily enucleated with the fingers after the skin is incised, while in others it may be necessary to dissect away the capsule that surrounds them, and even encroach upon the healthy tissues. When all suspected parts have been extirpated, the wound should be smoothed up and cleansed. The coagulated blood should be removed, but if the operation has been properly performed, antiseptic irrigation will be unnecessary. When there is reason to fear infection, a drainage opening is provided at a dependent part for the discharge of pathological products. A protecting dressing is then applied and the temperature and general physiognomy should be watched for possible evilence of suppuration, in which event renewal of the dressings and antiseptic treatment will be essential.

\section{EPITHELIAL TUMORS.}

I. ADENOMA.-The adenoma is a benign tumor constructed in the form of a gland. They are rare in the domestic animals. They consist of new formations, intermixed 
with the glandular tissue upon which they develop. These growths are sometimes soft and but slightly vascularized.

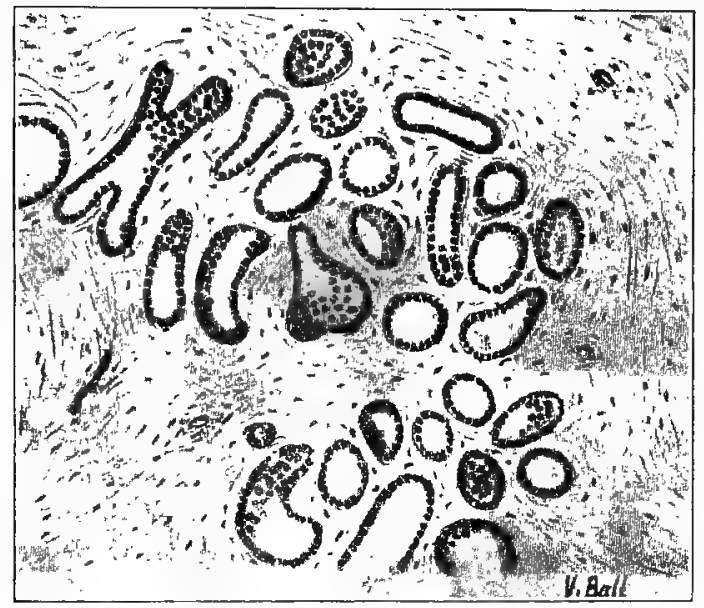

FIG. 71.

Adenotma of the Uterus.

Leisering found an example of them in a cow (Fig. 7I); Lienaux published an account of one in the cutaneous

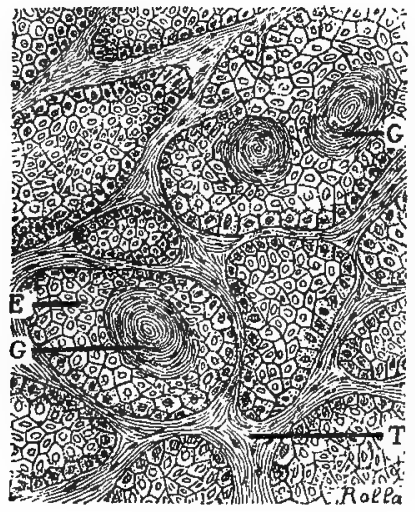

Fig. 72.

Lobulated Epithelioma of the Penis of a Horse. GG. Horny Globes.

E. Epidermic Cells. T. Stroma.

glands; and Stockman of one in the udder of a goat that generalized in the lungs.

2: EPITHELIOMA.-The epithelioma constitutes, with 
carcinoma, the properly called cancer. These neoplasms are not rare in the bitch and the mare, in which animals they occur in variable sizes. They consist of a flesh-like, regular growth, sometimes hard and protuberant and other times fluctuating at one or more points. (Fig. 72). The skin may be either intact or ulcerated. In the latter event they present crater-form openings that discharge a purosanious product. (Fig. 73). According to the kind of epithelial cells constituting them, they are either pavemental or cylindrical epitheliomata. They are found in the mouth, the anus,

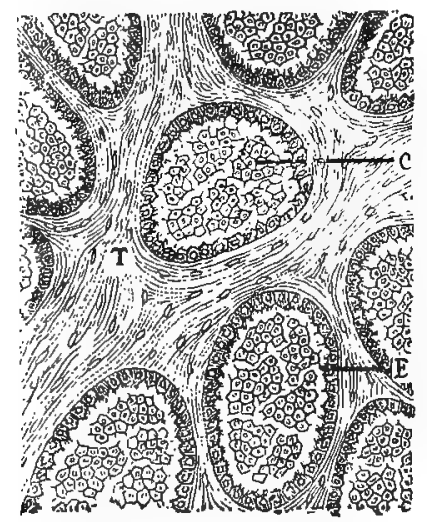

FIG. 73.

Lobulated Epithelioma from the Peritoneum of a Dog. C. Central Epithelial Cells. E. Epithelial Lining Cells. T. Stroma.

the penis, the prepuce, the udder, the testicles and the liver. (Fig. 74).

When located in positions that can be explored with the hands they are found to present a wide, diffused base, and an engorgement of the neighboring lymphatic glands. They are always incurable, and cause death by generalization or cachexia.

A few years ago the treatment was both medical and surgical, but today it cannot be too strongly emphasized that only the latter is of value. (Fig. 75). For years alka- 
lines, mercury, arsenic, iodides, and certain tinctures,-thuja, condurago and hydrastis,--were used internally. At different times, as a balm to our conscience, we have tried tincture of thuja, of which we had many favorable reports, but in all of our experiments the effort was fruitless.

The only effectual intervention is surgical ablation, resorted to as early as possible, in fact, as soon as the nature of the growth has been determined. In the dissection, the scund tissue at some distance from the tumor should be

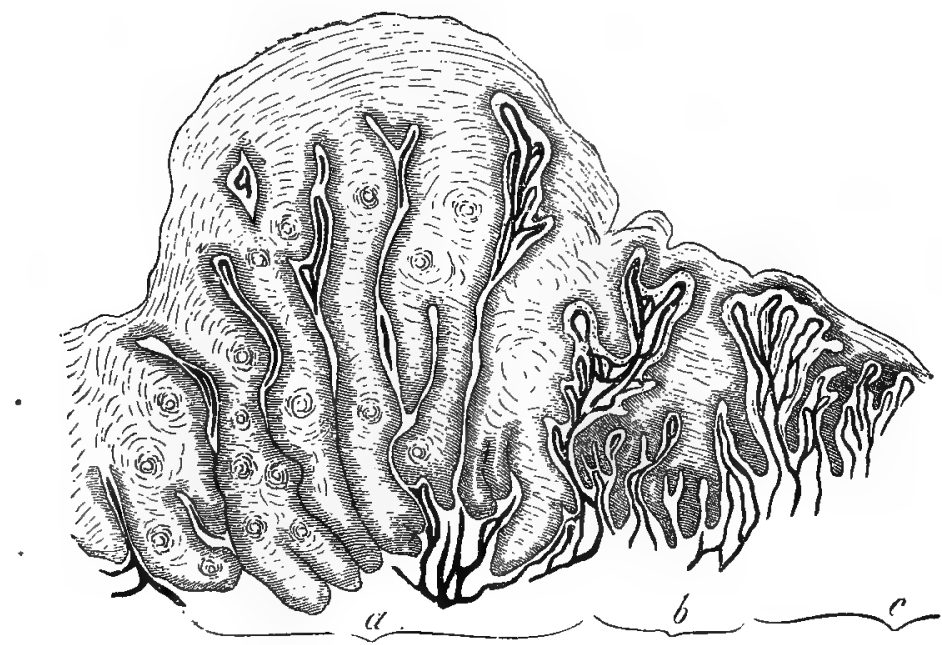

FIG. 74.

Limited Zone of a Cutaneous Epithelioma. a. Tumor Perfectly Developed.

Cylindrical Epithelium Traversed with Epidermic Globules. b. Se-

baceous Follicle Enlarged. Epidermic Cones Extending into the Depths (150 Diameters).

included, and if the growth is located in a mamma, the entire gland, and even its contiguous glands, should be ablated. They sometimes recur, which event will necessitate a second operation. Surgical treatment is never contraindicated (in animals) except when generalized, or when important organs would be sacrificed.

3. CARCINOMA.-The considerations mentioned with regard to epithelioma may, from a clinical standpoint, be ap- 
plied to carcinoma, which is closely related to it. Some authors do not even regard it as a variety of the latter; but simply designate it as an infiltrated epithelioma. (Fig. 77). It is less frequent in the $\operatorname{dog}$ and horse than epithelioma, but is emphatically the malignant neoplasm. Its generalization has been shown by Morot, Cadeac, et al. Carcinoma justifies prompt surgical intervention.

\section{CONNECTIVE TISSUE TUMORS.}

r. SARCOMA.-Sarcoma is a tumor constituted by young connective tissue cells. It is often voluminous, globu-

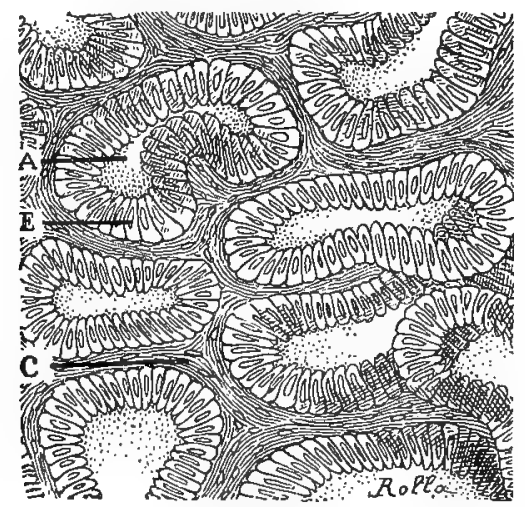

FIG. 75.

Cylindrical Epithelioma of the Intestines of a Dog. A. Follicular Cavity. E. Epithelial Lining. C. Stroma.

lar, and presents an adhesive base that is drowned in the surrounding tissues. The surface is often the seat of numerous ulcerations, through which suppurating granulations protrude. (Fig. 79). In certain cases they are protuberant, movable and have a clear boundary. Their consistency varies with their nature. The embryonic sarcoma is soft; the sarcoma with fusiform cells is firmer; while those containing cartilaginous or osseous productions are excessively hard. 
Sarcomata are not rare in domestic animals; Cadeac found six sarcomata out of a total of nineteen tumors. They are found in the udder, skin, bones, kidney, uterus, muscles, etc. Melanotic-sarcoma is very frequent in the horse, in which event it usually begins in the skin. Trasbot and Cornil found forty-seven that started as cutaneous tumors.

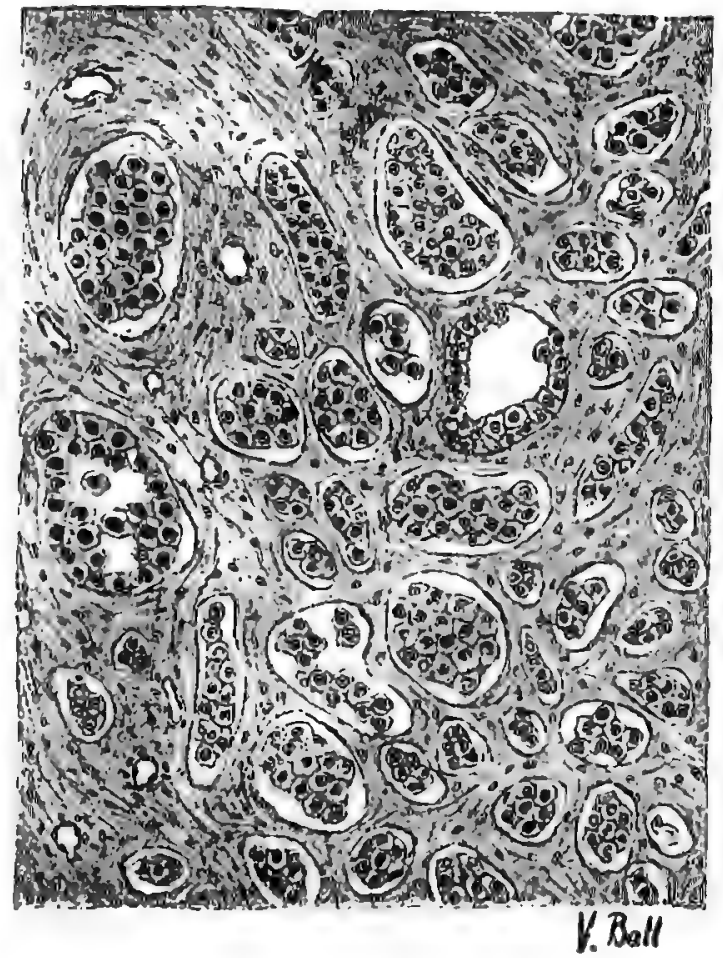

FIG. 76.

Epithelioma of the Udder of the Bitch.

fifty in the eye and seventeen in the internal organs. The death from sarcoma is either from generalization or cachexia. It has characteristics by which it can be distinguished from epithelioma. It is more movable, less ad. hesive in its deeper parts, and is not accompanied with engorgement of the neighboring lymphatic glands, its generalization being effected through the venous system. 
TREATMENT.-The only appropriate treatment is surgical intervention. The sooner the tumor is removed the

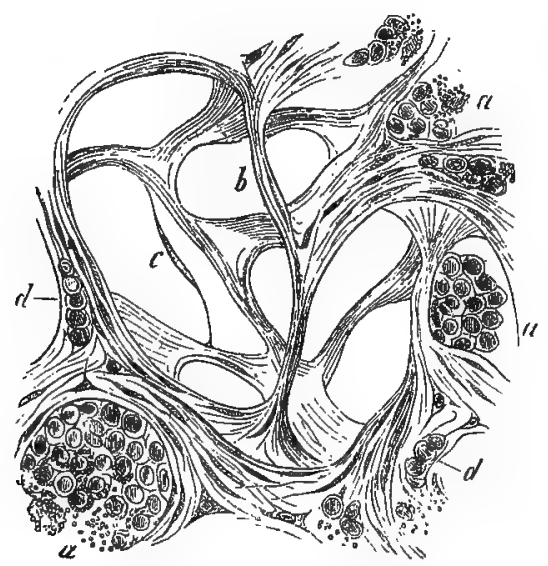

FIG. 77.

Stroma of a Soft Glandular Carcinoma. a. Section of Cellulo-Cancerous

Cylinders. b. Fasciculi of the Stroma. c. Fusiform Cells Extending

Transversly from One Fasciculus to Another, along which is

Deposited the Fundamental Substance Serving for the Forma-

tion of a New Fasciculus. d. Infiltration of Round Cells

Within the Stroma (300 Diameters).

greater will be the chances of effecting a complete cure. In the beginning sarcoma develops slowly. It is essential, not

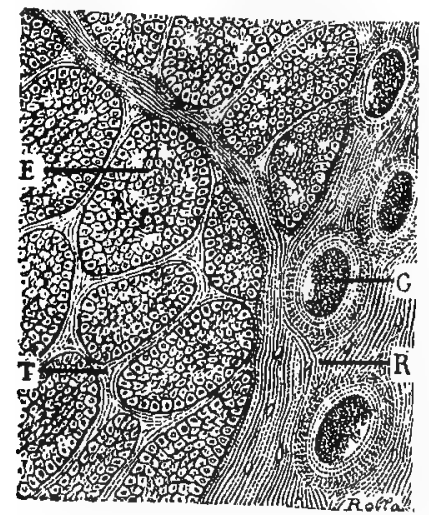

Frg. 78.

Carcinoma of the Kidneys of a Dog.

only to enucleate the growth, but also to completely extirpate its capsule when one exists. Enucleation is always an imperfect operation and may be followed by recurrence. 
2. FIBROMA.-The fibroma is a neoplasm constituted of adult connective tissue. It is hard, dry, and unyielding, and is found in the skin, subcutem, glands, uterus, etc. A number may develop in the same region (multiple fibromata). The evolution is slow and prognosis is favorable, and they only discommode the subject when located in an important organ or its vicinity. Unless they attain a size sufficient

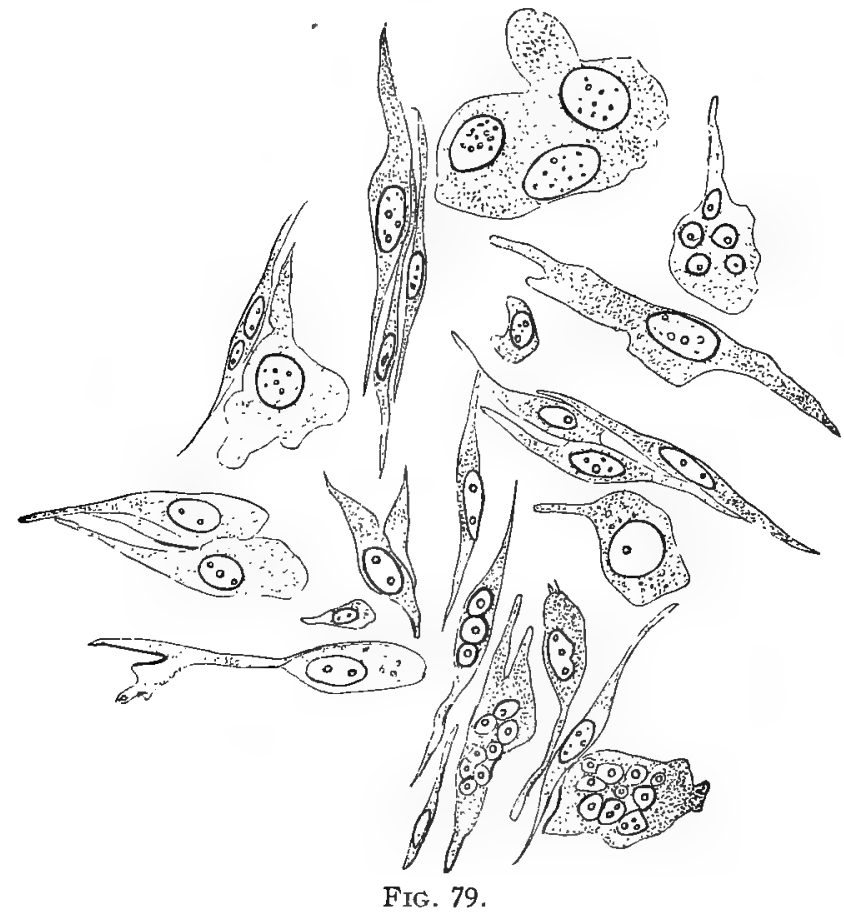

Fusiform Cells of a Sarcoma.

to hinder locomotion or interfere with the harness, they may be safely left unmolested. Surgical intervention is, therefore, but a matter of propriety.

3. LIPOMA.-The lipoma is a tumor constituted of adipose tissue. It is rather frequent in the dog, the horse and the ox. Its dimensions are variable; its shape round or oval; its limits easily defined, and finally it may exhibit sensations of pseudo-fluctuation so pronounced as to give 
the impression of cyst or cold abscess, from which it can only be differentiated by tapping with a trocar.

The lipoma is ustally found in the subcutem, subserous or submucous tissue. In the large serous membranes, it may be pedunculated. As these tumors are emphatically

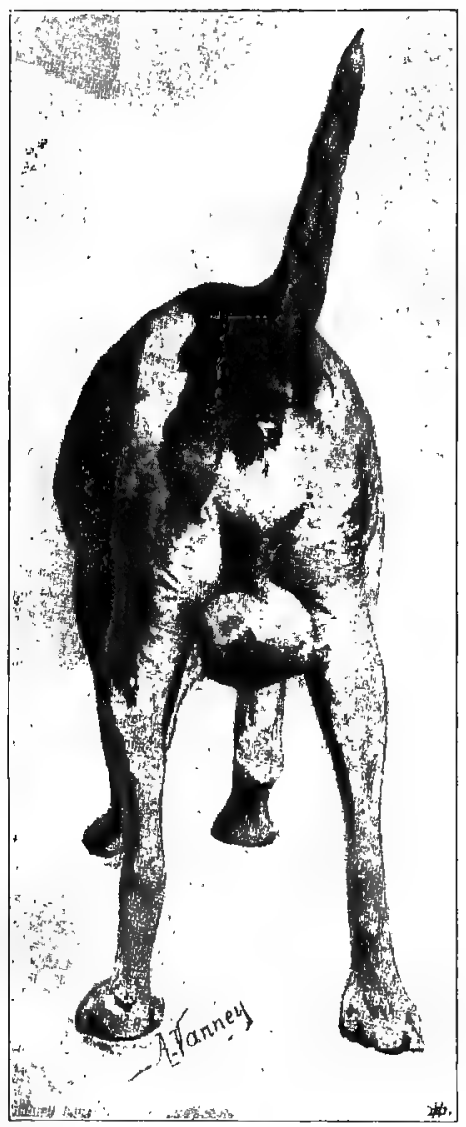

FIG. 80.

Sarcoma of the Testicle.

benign they seldom warrant extirpation. In the bitch, when located on the teats or groin, it is sufficient to incise the skin and enucleate them. If the skin is much distended a cutaneous flap may be resected.

4. MYXOMA.-The myxoma is constituted of mucous 
tissue. They are soft, gelatinous and rather rare among domestic animals. Lucel observed a case in the third right teat of a six-year-old bitch. It consisted of an enormous trailing, piriform mass. (Fig. 83). Wre have observed two cases of melanotic myxoma in cows, both of which were as large as a child's head, clearly pedunculated and located in the umbilical region. They are benign tumors and easily ablated when not diffuse.

5. CHONDROMA.-The chondroma is constituted of

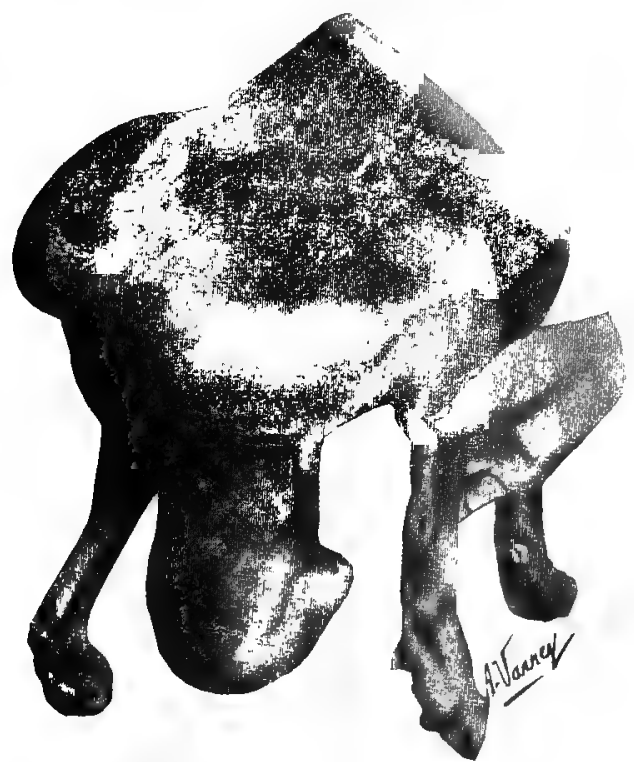

FIG. 81

Telangiastic Sarcoma of the Mammæ (Photo).

cartilaginous tissue. They are hard, unyielding, irregular and sometimes elastic. (Fig. 85). They develop slowly and rarely generalize. They are found in glands and bones. (Fig. 86). The fact that their generalization is possible must not be ignored. Nocard et al. observed such cases. A case reported by Generali generalized in the lungs, kidneys and nerve centers after having been extirpated from the teat. The enchondroma, when recognized, should be surgically removed. 
6. OSTEOMA.-The osteomata are constituted of definitely organized osseous tissue, and are distinguished from osteoid growths by the fact that in the latter the osteoblasts are not transformed into osteoplasts. Osteomata are frequent in the horse and the dog. Le Blanc et al. have reported cases which they have observed. They are found in the nasal cavities or their appendages, and have only indirect relations with the bones. They are covered with the mucous

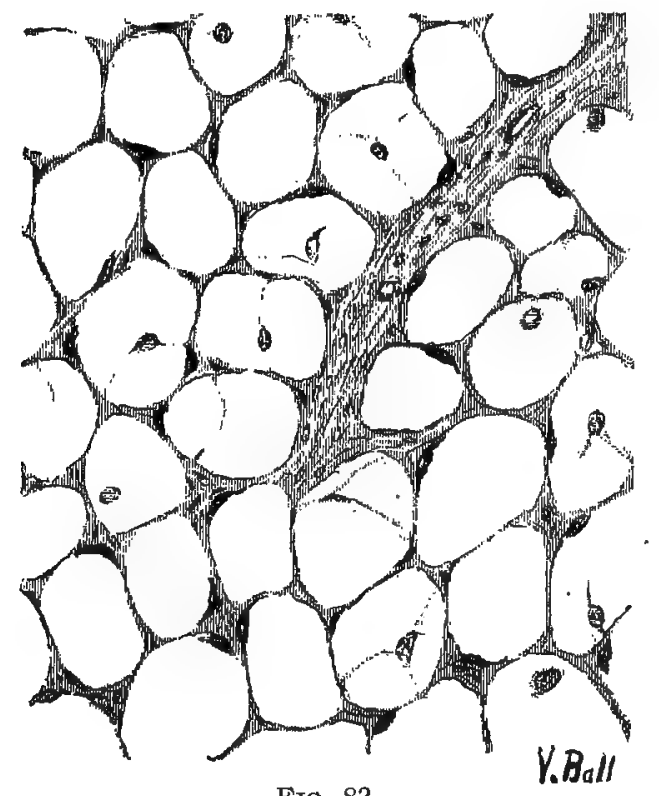

FIG. 82.

Lipoma of the Mammæ of a Dog (Ball and P. LeBlanc).

membrane and are constituted of a hard or cancellated osseous tissue.

7. LYMPHADENOMA.-The structure of the lympha. denoma is similar to that of a lymphatic gland. These t11mors are rather frequently observed in veterinary practice, according to the observations of Jobelot, Peuch et al., whose descriptions would indicate that they are connected with leucocythæmia or some other microbian affection. In the ox, for example, cases of chronic pneumo-enteritis may be 
due to tumors having the characters of lymphadenomata that develop under the irritating influence of the microbian agents. (Fig. 87). These tumors develop in the lymphatic glands, the spleen, in closed follicles, the liver, the testicles,

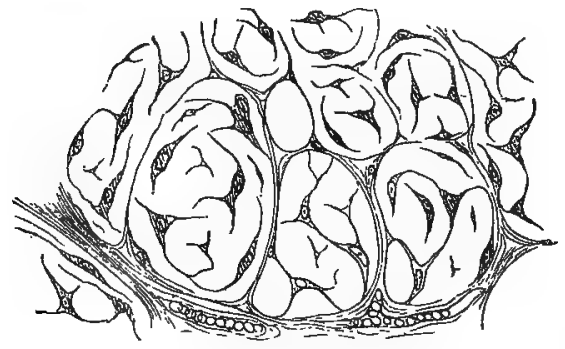

Frg. 83.

Hyaline Myxoma Developed in the Subcutem near the Angle of the Jaw (300 Diameters).

the ovaries, the pancreas, etc. They are difficult to diagnose because of the impossibility of distinguishing them from other lymphatic hypertrophies caused from a general disease (peritonitis, tuberculosis, etc.)

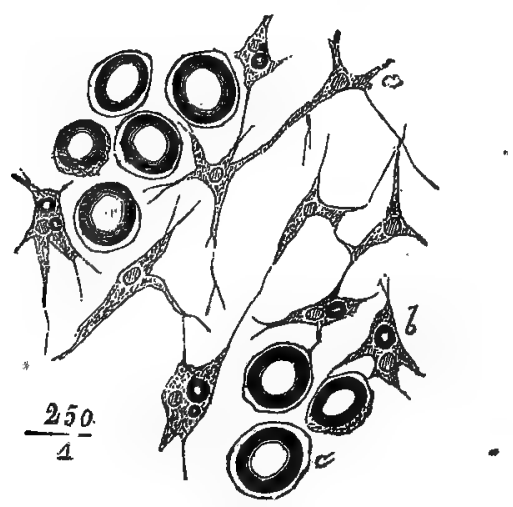

FIG. 84.

Section of a Lipomatous-Myxoma. The Cells of the Mucous Tissue are

Filled with Fat. a. Normal Cell. b. Cell with Fat Globule.

c. Cell Completely Filled (250 Diameters).

Iodides, iron and Fowler's solution have been given hypodermically for these growths, with no appreciable results. Surgical intervention is likewise useless, owing to the liability of recurrence. 
8. ANGIOMA.-The angiomata may be divided into two groups: sanguineous angiomata or hæmangiomata and lymphatic angiomata or lymphangiomata.

Hæmangioma.- This tumor is due to dilatation and neoformation of the capillaries. Virchow describes two varie-. ties, simple and cavernous. Although rare in domestic animals, some cases have been observed in the horse. Trasbot and Montane have each observed one case, and Lucet and Bournay each describe a case in the dog. (Fig. 89). They consist of small, soft tumors that have no tendency to generalize, but which are prone to rupture and cause death from hæmorrhage.
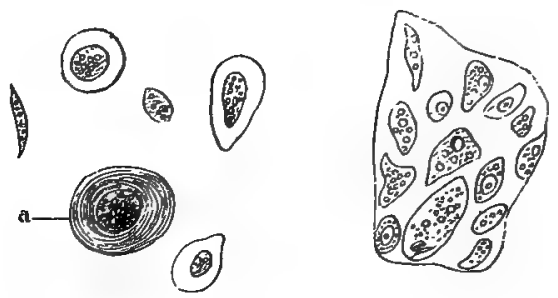

FrG. 85.

Cartilaginous Cells that Have Become Free in an Enchondroma after Having. Undergone Mucoid Metamor phosis. Section of Hyalin Cartilage.

Lymphyangioma.- These are formed by the anastomosis of lymphatic vessels. They are exceedingly rare among domestic animals and consist of flabby, succulent neoplasms.

\section{NERVE TUMORS.}

I. NEUROMA.-Under the term "neuromata" must be included the tumors formed entirely of new nerve tissue. They have been found in the plantar nerves, the femoral, the sub-zygomatic, the cranial nerves and in the brain. 'They are divided into two groups: ganglionic and fasciculated. Blanc has demonstrated that neoformations on the nerves of ruminants should not be regarded as neuromata, but as 
myxo-sarcomata. Neither should the mixed tumor developed at the extremity of divided nerves be regarded as neuromata, as these cases are inflammatory growths that are extremely sensitive, which should be extirpated. When neurotomy has been performed under aseptic conditions these growths do not develop.

\section{ANNOTATION.}

The so-called neuroma following plantar neurotomy is not always the result of delayed healing of the wound from infective inflammation. These growths are met in two distinct forms. One is always due to, or at least al-

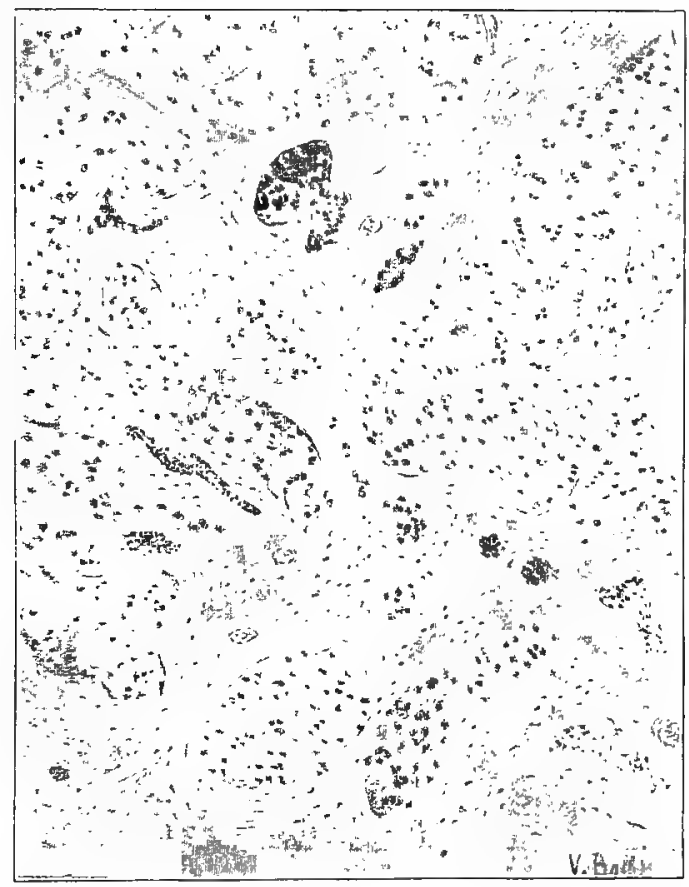

FrG. 86 .

Chondroma of the Mammæ of a Dog.

ways follows the slow-healing, suppurating, granulating wound, and manifests itself in the form of a sensitive cicatrix as soon as the wound eventually heals. Histologically, it is scar tissue infiltrated with nerve elements that emanate from the axis cylinders of the proximal end of the divided nerve. It is, in fact, an effort of the organism to reunite the breach. This form of the growth always shows at least some tendency to diminish in sensibility with time. The second variety presents itself in the form of a small nodule 
- that is movable and non-adherent to the adjacent tissues. It is less sensitive than the first named variety, but shows a manifest inclination to increase slightly in size and to grow more sensitive, probably owing to the constant friction caused by movements of the parts. This variety has an obscure origin and will often develop in spite of asepsis. The fact is, they occur most frequently after the operation that was followed with absolutely prompt urion of the cutaneous incision.-L. A. M.

\section{TUMORS OF MUSCULAR TYPE.}

I. MYOMA.-The myoma is a tumor formed by the muscular tissue. There are myomata of non-striated muscle and those of striated muscle. (Fig. 82). The former

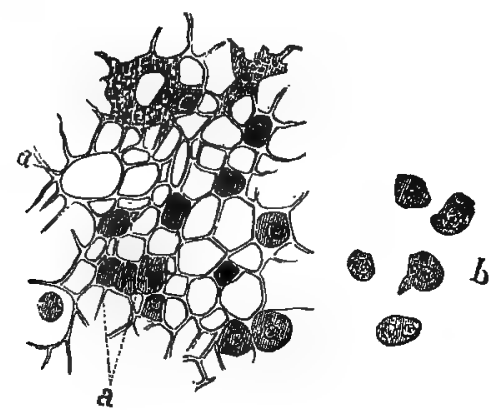

FIG. 87.

Lymphadenoid Tumor, ss. Fine Reticulum Circumscribing Little Alveoli

Some of which Still Enclose Their Cell While Others are Empty, the

Cells Having Been Dislodged by Filaments. b. Isolated Cells.

may be found in the uterus, œsophagus, the stomach, the intestines, and, in short, in any part having non-striated muscular tissue. They are benign tumors that provoke disturbance only by mechanical obstruction. They rarely suppurate or mortify.

\section{COMPLEX TUMORS.}

Complex tumors are those formed of different tissues, without evidence of the tissue primarily concerned in the elaboration of the tumor. They are known by the names of teratomata, mixed tumors, congenital tumors and tumors with numerous tissues. Most of them are cystic. 
I. CYSTS.-Cysts are closed cavities having connective tissue sacs lined within by epithelium. The cavity contains products of different naturre and aspect, distinguished as serous cyst, mucoid cyst, sebaceous cyst and dermatoid cyst. The latter refers to the position, while others indicate the contents.

Serous cysts have whitish contents that are slightly viscous. They are single or multilocular, and are found in the subcutem and in the walls of the tendonous sheaths.

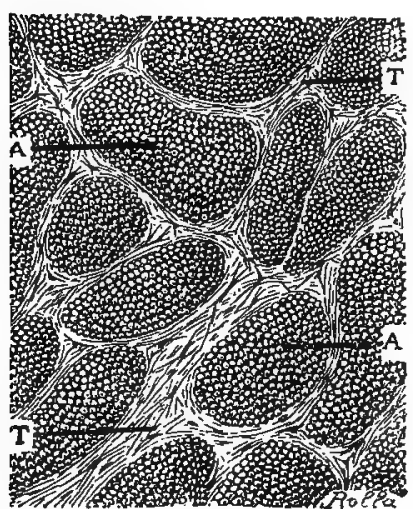

FIG. 88.

Cavernous Angioma in the Neck of a

Dog. A. Cavernous Spaces Filled with Blood. T. Fibrous Tracts Bounding the Spaces.

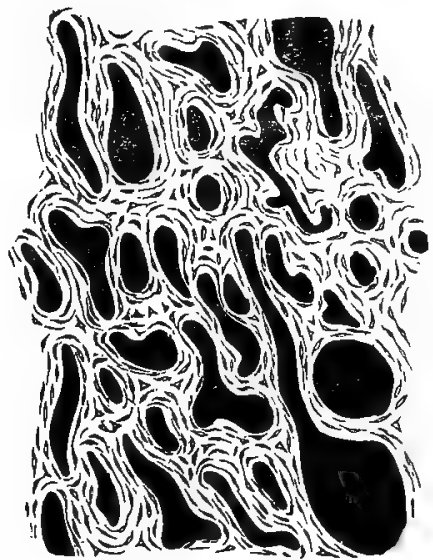

FIG. 89.

Section of Cavernous Angioma.

The mucous cysts have a dermo-epithelial wall and are found in the ovary, kidney, udder, testicle, liver, etc. The sebaceous cyst contains epidermic growths and hair follicles. It is doughy, fat and chocolate colored. Dermatoid cysts are very frequent in the horse and ox. They are particularly noticed in the orbit, surface of the cranium, on the neck, in the thoracic walls, about the maxillaries, and in the ovaries. The contents vary. Sometimes they contain a clear liquid and at other times horn, cartilage, bones, teeth, etc. 
Generally, their contents are expelled at the end of a certain time, in consequence of ulceration of the skin covering them. It is probable that these cysts are the result of an enclavement of the branchial fissures. Quenu believes that in addition to the theory supported by Verneuil there is an epithelial involution. But these theories do not explain the dermatoid cyst of the ovary.

TREATMENT.-The treatment is purely surgical. Extirpation, when possible, and at other times tapping, followed by injections of irritating substances into the cavity with the purpose of modifying the wall and promoting their adhesion. This last process is usually employed. The tap-

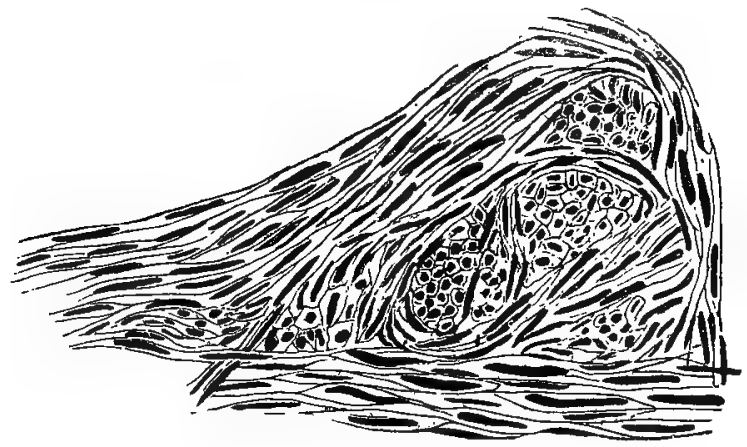

FIG. 90.

Non-Striated Myoma. Nuclei Stained with Carmin. The Round Forms are Transverse Section through the Long Elements.

ping is performed with a small trocar. The skin is shaved and washed, the trocar singed and then inserted into the cyst. When the contents flow out the solution selected is injected slowly, as the cyst is gently massaged with the fingers to bring the liquid into contact with every part of the sac. The excess of the solution may be drawn away, and then a protective dressing is applied to the surface. After a few days the sac begins to fill with granulations and the different parts adhere as the tumor disappears. 


\section{OSSEOUS TISSUES : GENERAL AFFECTIONS.}

\section{RACHITIS.}

Rachitis and Osteomalacia are general diseases of the osseous system due to disturbances of the nutritive function. The two processes are very grave. They have often been confounded, but are, however, clearly separate conditions. Rachitis is a disease of the young, occurring during the period of growth, while osteomalacia is an abnormality of the adult.

DEFINITION.-Rachitis is a disease of young animals, characterized by aberrations of physiological ossification, which produce transient or lasting deformities of the skeleton. It is constitutional dystrophia of the bones which seemingly depends upon, in general, disturbances of nutrition reflected over the entire anatomical apparatus. It is observed in man and in most of the domestic animals. It especially attacks pups and pigs, and is somewhat rare in the colt, the calf and the lamb. It is also quite frequent in poultry and in wild animals kept in captivity, - the lion the tiger, the ape, etc.

ETIOLOGY AND PATHOLOGY.-The causes of rachitis have remained obscure, in spite of the numerous studies undertaken to determine them. Its study must, therefore, revert to an investigation of the influence of age, species, race, heredity, hygienic conditions and alimentation; an examination of the different opinions advanced in its pathogenesis, and the researches made with the view of reproducing it.

The influence of age is certain. The young alone are attacked. It sometimes begins in the intra-uterine life,congenital rachitis, - but more frequently after birth, acquired rachitis,-during the first year, before the epiphy- 
ses unite. Some species,--pups and pigs,-—are specially predisposed to the affection. It is more frequent among the highly developed breeds. The Newfoundland and Great Dane dogs and the English breeds of hogs are particularly susceptible.

Heredity, by transmitting a special mode of nutrition, predisposes toward insufficient calcification of the osseous tissue. In the hog the entire litter is usually attacked, leaving the impression that the process of ossification is vitiated because of defective hygiene and alimentation. Lafosse attributes its frequency in the hog to the permanent housing of sows in unhealthy, cold, moist, dark and badly ventilated places, and to insufficient and deficient nourishment. which produces a dearth of phosphates in the milk supply. Captivity seems to provoke rachitis in the offspring of animals kept in menageries-lion's whelps, young tigers, panthers, apes, etc.

The relations existing between alimentation and rachitis have frequently been mentioned. Premature weaning, uniform and exclusive diet, dearth of mineral substances in the food, and acid food are supposed to be potent factors, and facts of observation and experience have been advanced in support of this opinion. The import of this cause is emphasized by all observers. The disease has been found to develop in pigs fed exclusively on raw potatoes, in calves nourished by mothers suffering from osteomalacia, and in young lions and panthers fed only upon meat. Roloff noticed it in lambs and pigs suckling mothers nourished with food containing a dearth of lime salts. J. Guerin produced the disease in two pups, one month old, by feeding them only with meat, but the experiment is not cunvincing, because it is not accompanied with data as to the lesions presented at the autopsy.

The disease was experimentally produced by Letellier, 
Bibra and Milne--Edwards, and Heitzmann claims to have obtained similar results by subjecting pups and kittens to a diet destitute of calcareous salts, but rich in lactic acid. The experimental animals, from the second week, exhibited a swelling of the epiphyses, which accentuated up to the fifth week, together with a bronchial and intestinal catarrh, and incurvation of the bones of the limbs. After five or six months the diaphyses were flexible, and when slaughtered at the end of eleven months the autopsies disclosed lesions analogous to those of osteomalacia. Rabbits subjected to the same dietary did not develop the osseous degeneration.

These experiments are not decisive. There was softening of the bones, but no formation of the spongoid or chondroid tissue, characteristic of rachitis. The continuation of Heitzmann's experiments by Tripier and Toussaint shows that young cats cannot be made rachitic at will by feeding exclusively on meat, even by exposing the animals to wet and cold. But in spite of this, it seems that the dearth of lime salts and phosphates in the food, or the insufficiency of their absorption governs the etiology of rachitis.

Recently Pornmay produced lesions of the trunk, limbs and head, and spontaneous fractures in birds, that appeared analogous to rachitis, by feeding them exclusively on bread crumbs, yellow of egg and meat deprived of its juices. But opposite results were obtained by Chossat and Friedleben, who showed that the privation of calcareous salts caused a rarefaction of these salts without producing histological alterations similar to those of rachitis. More recently Delcourt sought to produce rachitis experimentally in young animals by means of lactic acid, the lactic ferment of Heuppe and phosphate of potash, and came to the conclusion that lime salts play but a secondary role in the pathogenesis of 
the disease, and that the augmented elimination of calcareous salts is a symptom and not a cause.

He proves that lactic acid, while increasing the elimination of alkaline salts, does not produce rickets; that the lactic ferment, while making the calcareous salts of the food hard to absorb, does not cause rickets; and, finally that the potassium salts have a marked action on the cartilages in the stage of growth, and that they produce lesions characteristic of rickets, probably by removing salts of sodium from the organism that are necessary for the ossification of the primitive cartilages. These etiological factors may be summed up as an insufficiency of phosphates, which may be due to a diminution of the acidity in the intestinal secretions. Bouchard states that phosphates are not absorbed, but that phosphoric acid is assimilated as an alkaline phosphate, especially of phospho-glyceric acid, and calcium in the form of an alkaline or organic salt. Phospho-glyceric acid is produced in the duodenum. Phosphoric acid is generated by the action of the hydrochloric of the gastric juice, and is combined with the nascent glycerin resulting from the breaking up of the fats by the pancreatic juice. This phospho-glyceric acid is afterwards combined with the calcium in the tissues on the way to ossification. These successive reactions could not take place in every instance of alterations in the gastro-intestinal apparatus. That is to say, that the principal cause of rachitis would depend on a vicious or defective operation of the functions of the stomach and pancreas. Kassowitz regards this affection as the result of an inflammation of the osseous tissue, -an osteitis. He claims that the exaggerated vascularization of the periosteum prevents the deposition of calcium carbonate, but these lesions are found in normal bones. Ossification in young animals is accompanied by intense vascularization. Rachitis has also been looked upon as an infectious dis- 
ease, but the theory does not rest upon any authenticated fact.

None of these theories must be accepted as final, except that the etiology is governed by insufficiency of mineral salts and defective alimentation.

SYMPTOMS.-The symptoms are general and local. The latter consist of deformities of the skeleton, which appear about a month after birth. The disease develops slowly and its commencement passes unnoticed. The first symptom is disturbance of the general nutrition, which cannot at first be connected with this disease. There is diminution and irregularity of the appetite, hide-bound, staring coat, bad appearance, feebleness, more or less accentuated emaciation, digestive troubles, diarrhœa and a general, wretched aspect of the anima1. Bronchial and nasal catarrh may also be observed. The animals are weak and sluggish. They remain constantly lying down, and rise with difficulty. Every part of the body is sensitive to pain. This general hyperæsthesia is specially frequent among pigs, which at the slightest touch utter piercing and continuous shrieks. After this period, the general symptoms become more accentuated and more clear, and finally the local signs (the deformities of the bones) make their appearance. They exhibit themselves in the vertebral columns and the limbs. 'The deformations of the long bones are the consequence of alterations in the cartilage of conjugation and consist of rickets at the épiphyses and incurvation of the diaphyses. The rachitic bone has all its diameters augmented, especially at the epiphyses. The result is that the shafts are twisted. The articulations become swollen, deformed and rickety, and the nodosities are hard and painful. The deformations are easily perceived on palpation. They are found in the humero-radial and the femoro-tibial articulations, and on the carpal, tarsal and metatarsal bones. The curvatures and deformities are 
the result of muscular action, the weight of the body, walking, and the feeble resistance of the bones, all of which prevent the animal from standing upright. From a front view the legs may form a parenthesis "(.)", or at another time an "X", "K", "D" or "B", according to the deflections of the axes of the limbs. In the hind legs different abnormal curvatures may be found. The pelvis may be deformed, flattened or curved in the cotyloid cavities. The latter state may contract the pelvic passage and cause dystocia in the adult. It is difficult for the animals to keep in the standing position

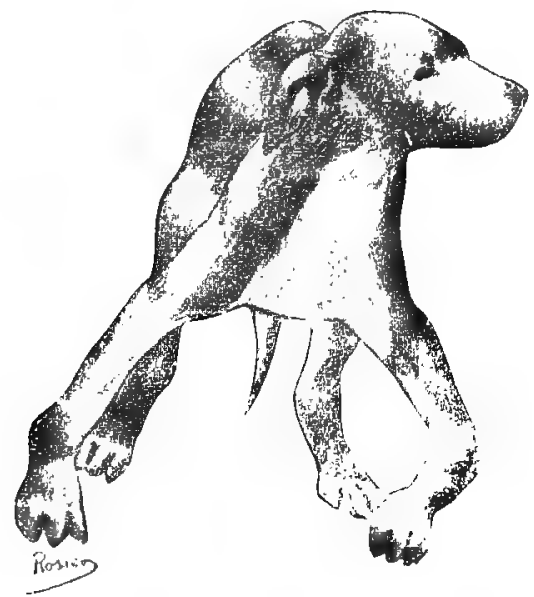

FIG. 91.

Rachitis in the Dog (Photo. Cadeac.)

or to walk. The femurs are bent and rickety. The deformations of the vertebral column are varied, presenting different abnormal incurvations-arch-backed or kyphosis; saddle-backed or lordosis; right or left inclination or scoliosis. These deviations have been observed in colts, and when consolidation of the bones occurs later in life the subject often becomes useful, even for fast work. The ribs may be knotty at each cartilage and thus produce a deformity of the thoracic cage. The head is not exempt from rachitic manifestations, which cause an increase in the volume of 
the cranium and a swelling of the maxillary bones. This deformity is especially observed in the hog.

Rickets has been frequently associated or confounded with schnuffelkrankheit (sniffling disease), an entirely different condition, described in subsequent paragraphs.

The local manifestations are reflected on all the functions, besides giving the animals a special physiognomy, rendering the gait abnormal and producing complications, such as fractures, which consolidate later in life. The concomitant systemic troubles are those of development ending in general atrophy.

The deformities of the limbs are never as pronounced in the colt as in the dog. Dogs and hogs have been seen to crawl upon their carpi or tarsi in a kneeling or squatting posture.

Lafosse refers to a case in the colt, presenting a double inflection of the vertebral column,-lateral and vertical. There was a concavity behind the shoulder and one in front of the sacrum, and a pronounced convexity at the junction of the dorsal and lumbar regions.

The disease may last for months, during which time the patients' condition becomes worse and worse. They are lean, unthrifty and poorly nourished. The feebleness finally becomes extreme, and a majority of them exhibit accentuated digestive disorders (continued diarrhoea) in addition to the elimination of considerable quantities of lime through the urinary channel. Death occurs from marasmus.

In some cases, however, the bones may consolidate and the nutrition return. But the deformities persist and the animals remain small (below the average size) and deformed through life. The limbs are shortened and the attitude is abnormal.

The course of the disease is slow; it lasts from two to four months, or even more. In some cases the bones re- 
turn to their normal condition and in others the reaction makes the epiphyses harder. The disease has been observad at birth, that is to say, rachitis may be congenital, and characterized by voluminous articular tumefactions which cause dystocia. This special feature is, however, rare. In addition to the special lesions of the skeleton, there is general atrophy of all the tissues and various secondary lesions occurring toward the termination of the disease.

DIAGNOSIS.-At the beginning, the disease presents no special characteristics and could not be recognized except by the existence of other cases in animals exposed to the same influences. But when the disease has reached its crisis, it cannot be confounded with any other disease. Infectious polyarthritis is easily differentiated; its onset is sudden and it is accompanied with intense pyrexia and swellings of the articulations, which sometimes suppurate. The differentiations between rachitis and articular rheumatism is equally simple; the latter has a sudden beginning and is accompanied by a violent reaction that is entirely absent in the former. In colts the disease must not be confounded with osseous or synovial inflammations, nor congenital deformities. Here a differentiation is made by the shortening of the bones, their incurvations and the enlargement of the epiphyses.

PROGNOSIS.-The prognosis is always grave. Nevertheless, in the beginning, an appropriate course of treatment may ameliorate the condition of the affected subject. When the deformed bones have consolidated the defective position of the limbs cannot be remedied, and as a result the value of the animal is greatly diminished. The prognosis is, therefore, particularly grave from an economical paint of view.

PATHOLOGICAL ANATOMY.-The degenerative changes of rachitis are essentially osseous lesions. They 
consist of defective development and defective classification. The rickety bone is but slightly resistant. It is easily bent, deformed, fractured or severed. The deformities are varied. The long bones exhibit incurvations of the diaphyses and rickets of the epiphyses. They are swollen at the cartilage of conjugation. On section they appear very red and very vascular. The cartilage of conjugation is large, thick, hypertrophied, bluish or violet colored, and in its surrounding tissues are seen small cartilaginous nodules, scattered promiscuously and enclosed in osseous tissue. From the cartilage irregular spaces project into the diaphysis. All these structures are very vascular, non-resistant and areolar, and if scraped they are found to be encrusted with calcareous salts. Under the periosteum the tissue is reddish and areolar, very non-resistant, pits on pressure with the fingers, and cuts easily with a knife. The periosteum is thickened and very vascular. When removed it leaves the surface of the bone knotty and porous. The medullary canal is larger than normal and the marrow is rich in blood-vessels. In the flat and long bones there is marked vascularity and rarefaction of the cancellated tissue. All of the bones are affected, but in unequal proportions. Everywhere the inorganic substance has diminished, especially in its phosphate of lime. The organic substance and the adipose tissue are augmented.

The lesions have been but little studied, microscopically, in animals. In man Carnil distinguishes three periods: (I) period of commencement; (2) period of deformity; and (3) period of repair. The first period is characterized by alterations of the epiphyseal cartilages. They are thickened and the zone of ossification exhibits an increase in all its diameters. Instead of possessing its normal thickness of one millimeter it may reach a centimeter or more, and its edges are crumpled and irregular. The cartilaginous projections 
penetrate into the diaphyseal bone. In order to understand thoroughly the histological lesions of rickets the mechanism of normal ossification must be understood. Bones grow from the simultaneous operations of the cartilage of conjugation, the periosteum and the marrow. A section through the cartilage shows that near the line of ossification, the cartilaginous cells are arranged in a series of lines, separated by zones of hyaline substance. The cells multiply, new elements appear with separate capsules and enveloped in groups

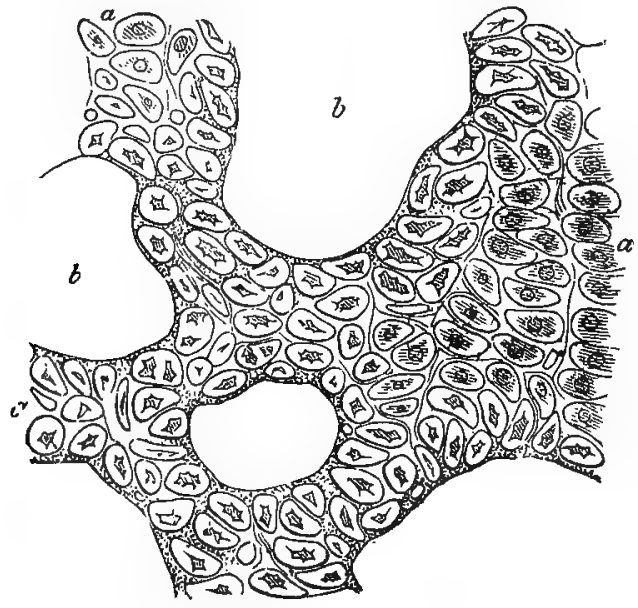

Frg. 92.

Zone of Proliferation of a Rachitic Epiphyseal Cartilage (500 Diameters).

(a) Unossified Cellular Columns. (b) Medullary Spaces of Cartilaginous Tissue in Course of Ossification.

with a large common capsule. These capsules are then dissolved and the spaces are filled with round cells without capsules. These are medullary spaces. Vessels penetrate to them and some of the round cells are transformed into osteoblasts, which secrete the osseous lamella from the circumferences to the centers of the medullary spaces, and then send out canaliculate and anastomotic extensions to become osteoplasts.

On section of the rachitic bone this progressive evolution, which ends in substitution of the cartilage, is markedly 
modified. After the zone of hyaline cartilage the cartilaginous cells are found in very irregular series, and the arrangement in series is even much less clear in the large spaces. The parent capsules do not dissolve and their cells within also remain surrounded by their capsules. The structureless, cartilaginous substance is strewn with numerous capsules containing a single cell with a more or less condensed protoplasm; or with parent capsules containing minor capsules.

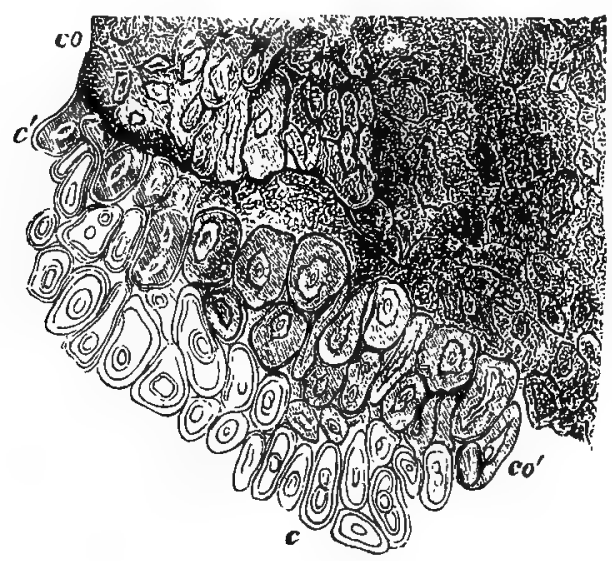

FIG. 93.

Zone of Ossification in a Diaphyseal Cartilage Attacked with Rachitis (300 Diameters).

cc. Ordinary Cartilage Developing. The Capsules are Thickening and Have Formed Cavities Containing Stars (Cartilaginous Cells that Have Become Bone). co. Calcareous Infiltration of the Bone Cells of the Cartilage. co. Capsules of the Cells, Invaded with Lime Commencing to Mingle Together. o. Osseous Substance.

Around the capsules may be seen undissolved granulations of calcareous salts, and lastly, at the same point, large vessels are found in the cartilage. This cartilaginous tissue sends irregular prolongations into the diaphysis, which may even become isolater by forming cartilaginous zones in the midst of the bone. In the medullary cavities, which form a continuation of the rachitic cartilaginous tissue or which are, as it often happens, contained in the midst of this tis- 
sue, is found fibrous tissue overrun by dilated blood-vessels. This fibrous tissue contains some round embryonic cells, but they are not arranged along the medullary wall in the form of epithelial osteoplasts, such as occurs in physiological ossification. These cavities are not bounded by genuine osseous tissue, but by gaps filled up by shrunken

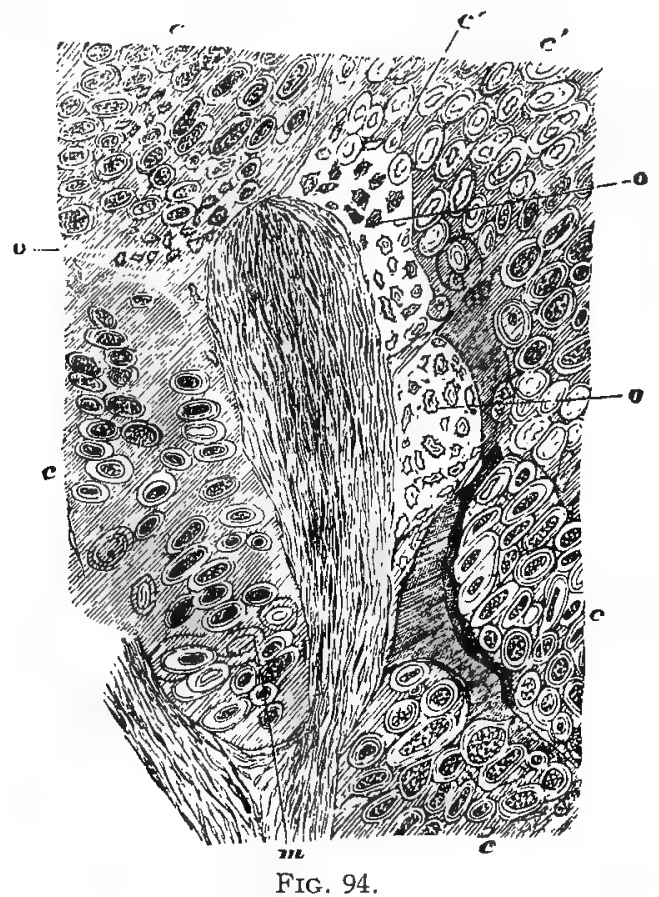

Vertical Section of a Diaphyseal Cartilage of a Rachitic Tibia, in Course of Development. A Large Medullary Cone Sends a Lateral Branch to the Left ( $\mathrm{m}$ ) and is Extended into the Cartilage. ccc. Represent the Periphery of the Cone, the Cartilage of which is Undergoing Proliferation and is Exhibiting Cells and Groups of Cells. c'c'. Represents the Capsule of the Cartilage, Thickened and Crumpled in the Interior. At oo They Meet and Form an Ossified Tissue.

cartilaginous cells and connective tissue cells within the cavities which are lined with complete calcareous incrustation. The cells and their nuclei are small; the tracts containing them are softer than the normal osseous tracts, and microscopically they resemble bone partially decalcified by acids. 
The rachitic osseous tissue is not arranged in concentric lamelle. It does not contain osteoplasts. The cellular bodies enclosed in the calcified fibrous tissue have no anastomotic branches characteristic of normal bone cells. These modifications are observed over the entire diaphysis. The Haversian canals are dilated, filled with a cellular marrow and lined with a fibrous zone. The medullary vessels are numerous; their walls are thin; they bleed easily, and the fibrous stroma is strewn with yellowish pigments, accumulated in heaps. Besides this rachitic tissue (the osseous tissue of Virchow and the spongy tissue of Guerin) can be distinguished old osseous lamellae, arranged concentrically and exhibiting osteoplasts. They are made thinner and are partly re-absorbed by the mechanism of Howship's lacunæ.

The modifications of the periosteum and of the medullary canal are also interesting. The periosteum is thickened and very vascular, and between it and the bone are elongated connective tissue cells instead of medullary cells, and a zone of rachitic tissue in which the tracts, instead of being parallel to the long axis of the bone, are promiscuously arranged, perpendicularly, oblique, etc. The rachitic tissue developed by the periosteum is similar in structure to that of the diaphysis. In the medullary canal analogous modifications are observed. The marrow becomes fibrillary and even fibrous, as if beneath the periosteum, and it is the seat of a special calcification, which is only incomplete ossification. In the midst of all this portions of normal bone, recognizable as lamella and osteoplasts, are always found. These parts are always softened by a process analogous to that of a rarefying osteitis.

The rachitic bones are easily fractured, but owing to the thickness of the periosteum there is usually no displacement (sub-periosteal fracture). They are repaired by a layer of rachitic osseous tissue formed by the periosteum. 
The crisis of rickets has a variable duration, followed by the period of reparation. The repair is characterized by the substitution of normal osseous tissue for the rachitic tissue. The cure results from the introduction of calcareous salts and the calcification of the rachitic bone, or of the appearance of the normal osseous marrow in the rachitic lacunæ, which renders physiological ossification possible. The osseous tissue thus formed, instead of being rarefied, is more dense than bone in the normal state. In addition to lesions specially connected with the skeleton there is general atrophy of all the tissues and secondary lesions resulting from digestive and pulmonary disorders, which are observed at the termination of the disease.

TREATMENT.-The treatment should be immediate and based upon the etiology. Prophylaxis is especially important. The mothers must be attended to before parturition by good hygiene and an excellent system of alimentation. The young should be placed in good hygienic surroundings and if the mother's nourishment is insufficient other diet must be provided. It is through the medium of the mother that the suckling young are acted upon in the endeavor to increase the absorption of mineral salts.

From the curative point of view, the animal should be made to absorb substances rich in calcium carbonate. Herbivora should have a grain diet of oats, corn, barley and farinaceous food generally, and carnivora must be given a diet of meat. The administration of lime salts in the natural state-bone powder, calcium phosphate, etc.- has been recommended, but as these substances are not assimilable, the chloro-phosphate of lime should be substituted. Oleum phosphoratum, I per cent, in Io to 20 gram doses, is advisable, but it must be stopped at the first sign of intolerance. Powdered meat and cod liver oil are very easily absorbed and 
are excellent remedies. Recently, animaline has been highly recommended.

\section{OSSEOUS CACHEXIA.}

SYNONYMS.-Osteomalacia, Osteoclasis, Osteoporosis.

DEFINITION.-Osseous cachexia is a disease of adult bovids, characterized chiefly by an altered nutrition that reflects on the osseous tissue and ends in the resorption of calcareous salts and dissolution of the osseous reticula. It is cachexia accompanied with softening and fragility of the bones, resulting in deformities and multiple fractures. It is entirely different from rachitis, which disease, although characterized by modifications of the osseous system, attacks only the young during the development of the skeleton.

Osseous cachexia is a disease that has received various names. Its numerous synonyms are sufficient to show that the opinions relative to its nature have been varied. The term "osteomalacia," frequently applied, is hardly suitable, because the softening of the bones is but the ultimate phase of the disease. The word "osteoclasis," suggested by Zundel in I87o, only applies to a series of symptoms-the fractures. The Germans have named it "osteoporosis" which refers to an important character of the lesions-rarefying osteitis; but this alteration is also observed in other diseases. It has also been described by the name of "enzootic osteitis" because it has been observed to affect a number of animals simultaneously; by that of "milk-cow disease," although it also attacks males, and by that of "gout" and "muscular rheumatism" when complicated with synovitis and osteitis. These improper designations must be rejected for the better term "osseous cachexia" suggested by Cantiget. This term indicates the general characteristics 
of the disease, the progressive cachexia of all the organs re. flected chiefly upon the skeleton.

ANIMALS ATTACKED.-Osseous cachexia is frequent among bovidæ, especially the cow. It is rare in the horse, the sheep, the goat, the hog, and the dog. Animals kept in captivity in menageries and zoological gardensgiraffes, rabbits and birds-may also be attacked.

HISTORY.-It has been observed since ancient times, when Vegetius clescribed it under the name of "syntexis," and was noticed at different epochs in every part of Europe. In Norway, about I650, it was attributed to the ingestion of a plant called "sterregrass," and it was treated by the administration of bone dust. It often appeared in Germany, where Hinke, in 1870 , was first to observe it. A good description was given of the disease by Gleditsche in 1787 , in Brandebourg and Magdebourg. It was afterward studied by Bohlmann in 18.23 in the Duchy of Anhalt, by Hertwig and Gurth in $1859^{\circ}$ and later by Zundel in Alsace. It was also observed in Belgium for the first time by Dale in 1836 , in the neighborhood of Antwerp, then by Maris in $185^{2}$ in the agricultural colony of Laumel, and more recently by Duvieusart in 1875 and by Mosselmann and Hebrant in I895, who made a thorough scientific study of the disease.

Osseous cachexia has a wide geographical distribution. It has been found in Italy, Switzerland and in the region of the Great Lakes in America. In France it was noticed by Roux in 1825, and by Dupont, of Bordeaux. The German investigations were brought to the attention of veterinarians by Neyen in $186_{3}$, and later by Zundel, who observed the disease in Alsace, where it was the subject of an interesting discussion at the Veterinary Society in 1870 . It is from this time that our knowledge of the disease became definite, thanks to the writings of Bouley, DeTherry, Leclainche, Collard, Tapon and Cantiget, whose recent works 
are accurate and have thrown some light on the pathogenesis of the affection.

The disease is known to rage in various localities. In 1892 it ravaged Le Berry, La Sologne, La Brie and all of the Eastern region of France. It is constant in some districts, and the intensity varies with different years. In some years the cases are isolated, while in others they are so numerous that it assumes the character of an epizootic disease.

ETIOLOGY AND PATHOGENESIS.-The conditions under which the disease appears are numerous. In spite of our knowledge of them the pathogeny has not been settled. The following is an enumeration of the etiological conditions and the pathogenic theories:

It must first be remembered that osseous cachexia is an affection of nutrition reflected chiefly on the osseous tissue which becomes demineralized. Every influence that is capable of causing vitiated and imperfect nutrition has been dwelt upon as productive of the disease. These are: Bad hygiene, defective alimentation and over-work. Females during the period of gestation and lactation, are particularly predisposed. This fact is acknowledged by all writers, and many show that the disease often appears soon after parturition. The different opinions advanced as to the mechanism of osseous cachexia are as follows:

Ist. It is the consequence of the ingestion of materials insufficient for the nutrition of the skeleton, or the excessive elimination of the elements indispensable to it. This is the theory of inanition, gestation, lactation, etc.

2nd. It is due to a dissolvment of the calcareous salts,the lactic acid theory.

3 rd. It is due to a microbian agent which causes inflammatory disturbances, - the microbian theory.

The preponderating part played by alimentation and in- 
directly by the geological constitution of the soil, is based upon numerous facts, and seems to have been presented by the old writers. It was thus that Dupont attributed the disease to a special anther (antheriod ossifragum). Thumbler, a physician of Bergen, refers to the habit of Norwegian peasants of feeding their cattle with crushed bones. In I780, Hinter accepted Linnæus's idea, Haubner and Kurst attributed it to another plant, the molinia caerula, and Roloff thought the equisetum played an important part. But these ideas are insufficient to explain the appearance of the disease in regions where these plants are absolutely unknown.

More recently the chemical theory of the insufficiency of the food, based on very complete investigations as to the composition of the alimentary substances and the constitution of the soil, has been accepted. The disease prevails chiefly on lands under cultivation where the soil is bad and anything but productive, and where the system of culture is not properly understood. A soil poor in calcic phosphates necessarily produces poor forage. In every locality where the disease is prevalent the poverty of the food in phosphates has been proven. Hering, Spinola, Roloff and Zundel support this theory. Cantiget, who has studied osseous cachexia very closely and observed it for ten to fifteen years on badly cultivated farms, has shown how the disease has disappeared by the improvement of the land and its fodder. It retained its hold, however, in localities where the soil was not improved. The works of Schloesing and Joulic have demonstrated, nevertheless, that the ratio of phosphoric acid in good lands is 4000 kilograms to the hectare (about $2 \frac{1}{2}$ acres). They found that the disease occurred on lands which had.less than 2000 kilograms per hectare. Cantiget analyzed soils from lands where there was little or no osseous cachexia and found 
that the proportion of phosphates increased as they entered lands where the disease did not exist at all.

The constitution of the forage depends entirely upon that of the land. Analogous statements on the subject, with analyses to support them, have been made by the Veterinary Society of L'Aube in I893. This theory is, however, only that of inanition of the German authors, based chiefly on the experiments of Roloff and Voit, who were able to reproduce osseous cachexia experimentally by diminishing the proportions of lime salts in the aliment. The part played by alimentation in the development of the disease has also been proven by Germain in Cochin China and by Theiler of the Cape country. The experiments of Roloff and Voit are, however, subject to criticism, because they used only young animals for their experimental subjects.

Other opinions confirm the theory of alimentation. Zundel attributes the disease to soils abounding with silicates, which as a consequence, do not retain water, and Hertwig, on the contrary, claimed that clay sub-soils play an important part. It is, however, important to note that the disease may occur where the land is rich in calcium carbonate and poor in phosphoric acid. Yagow has attributed the disease to a dearth of calcareous salts in the water supply, and Rossignol, who observed it in animals fed on the by-products of distilleries and sugar-refineries, blamed the poverty of these feeds in phosphates.

All of these influences are indirectly associated with alimentation, as the character of the soil affects the nutritive value of its flora. The influence of drouth on vegetation has often been held responsible for the disease, as it leads to the growth of feeds having a dearth of nutritive elements, especially of salts, which, lacking water as a conveyance, remain incorporated in the soil. This theory is held by 
many writers. Aside from these influences which depend upon the external environment, gestation and lactation play an important predisposing role. They modify and depress the nutritive movements of the body, and from the standpoint of osseous cachexia they play the part that traumatisms do in certain infections. During gestation the female should supply the needs of the fotus, and convey to it the salts required to construct the skeleton, and as growth occurs, both before and after parturition, in spite of the mother's deficiencies in this connection, the needs of the skeleton are borrowed from all of the organs containing them. The mother supplies the new-born an average of 6.64 grams of salts per day, which she draws not only from her blood but also from any of the organs capable of furnishing them, and even from the nervous system itself. If these elements are not furnished in the food materials transferred to the young, the mother's tissues are impoverished, resulting in an abnormal construction of all new tissues formed in both the fœtus and the mother. The cessation of lactation is nearly always followed by a spontaneous amelioration; but the disease resumes its course at the next period of gestation. All of these influences tend to produce the same pathological results, -an incomplete nutrition of the skeleton, which is so vitiated as to destroy the equilibrium between assimilation and disassimilation.

The theory of acids and that of inflammation complete the subject of its etiology. According to the first, lactic acid by dissolving the salts, in excess, would cause osseous cachexia. This theory is supported by the experiments of Heitzmann, and is defended by Bouchard (see rachitis). It is very debatable, as the experiments and the resulting facts in its favor are by no means convincing. The latter theory is that of inflammation, said to be caused by an irritant circulating in the blood-phosphorus, or some infectious mat- 
ter, as Zurn found a micrococcus in the marrow that he considered pathogenic, Leclainche, Tapon and Butel admitted the infectious nature of the disease. Padrone maintains that the agent is the germ of nitrification. He was able to reproduce the disease by infecting with this microorganism and clains that he found nitric acid in the urine of animals suffering from osseous cachexia. Tschissowitshe failed to reproduce it and showed that nitric acid is also present in the urine of animals suffering from other diseases.

"Chemical Theories."-Haller, and after him, Navier admitted the presence of an acid in the blood, and according to Renard it is phosphoric acid. According to Stansky and Proisseau osseous cachexia is a special osteitis. According to Rindfleisch it is a venous sanguineous stasis of the bones, or a superabundance of carbonic acid in the blood and a premature senility of the bones. Ranvier, partially supports this theory and is of the opinion that the formation of carbonic acid dissolves the osseous tissues. Marchand, Schmidt and Weber admit the presence of lactic acid in the bones. According to Volkmann, Ribbet and Lane, it is a demineralization of the bones, an opinion that Kassowitz rejects. According to Trousseau osseous cachexia is rachitis of the adult. According to Bouchard it is an acid dyscrasia quite distinct from rachitis, in which there is an accumulation of lactic acid in the osseous tissue, that causes a dissolution of the calcium phosphate. DeCosali and Durhann, assign the cause to defective alimentation and repeated pregnancy. According to Comby it is caused by digestive troubles due to dilatation of the stomach, and reverberating over the osseous system.

The parasitic theory has been maintained by Zuern who believed he had found the micro-organism in the marrow, but negative bacteriological results are numerous. Arcangeli found nothing in blood cultures. 
The theory of infection-by nitric ferment or the microorganism of nitrification is held by Petrone, but it is hard tc understand what association of ideas led the Italian investigator to this new conception of the disease, knowing on one hand the properties of nitric ferment and on the other hand the powerful microbicide action of chloroform on the micrococcus nitrificans. Was he not led to suspect the pathogenicity of this micro-organism by this very knowledge and to seek a cure or amelioration so evident after bilateral castration, ovariotomy, etc., in this microbicidal action on the nitric ferment? He has inoculated dogs intravenously with nitrates and with pure cultures of the nitric ferment and produced the disease. In the blood of animals affected with osteomyelitis, he has shown the presence of round cells associated with two specific nitrate micro-organisms. Chloroform and chloral possess a powerful bactericidal action against the nitric ferments. The administration of chloral for three weeks, in doses of two grams per day, would have cured an attack of osteomalacia; but Latzko relying on his own experiments, holds Petrone's statements to be unconfirmed, because he has always observed relapses after brief periods of improvement, and he did not find the micro-organism in the blood of any of ten patients, nor an abundance of nitrates in the viscera. Petrone's theory is certainly astonishing, and with the numerous facts to support it, it is not an exaggeration to join with Schauta in designating it as a romance. Positive facts must always be taken into account and hence it must be admitted that there are many varieties of the disease, some being curable with chloroform and others not.

Utero-ovarian Theory.-According to Fehling, osseous cachexia is the consequence of a pathological hyperactivity of the ovaries. From this fact the ovaries would be the starting point of reflexes which create an activity of the 
vaso-dilator nerves of the blood vessels in the bones, ending in passive hyperæmia, and consequently in energetic reabsorption of the elements of the osseous tissue. Castration, by suppressing the seat of these reflexes, would again contract these vessels and cure the disease. This theory is opposed by certain facts. Cures have been observed after Casarian section and from medical treatment, and besides, man is not immune. More recently Fehling declared his opinion anew, saying that osseous cachexia is a trophoneurosis of the osseous system dependent on a disease of the ovaries - a trophoneurosis, which on account of the important part played by the marrow in the composition of the blood, usually leads to a general vitiation of the blood. It must be also remembered that when Porro's operation is performed the annexes seem sound, and that the micrococcus nitrificans is a saprophyte of the uterus.

The direct or indirect role of the nervous system has been postulated as a cause of the disease by Ogle, and Virchow, in his classification of osseous cachexia, mentions that there is a form of the disease dependent upon nervous affections. Moses, on the other hand, has reported cases of osseous cachexia in animals affected with syringomyelia.

On the whole, all of these theories are absolutely insufficient to explain the appearance, development and lesions of the disease. They do not supply demonstrative facts confirming the theory of alimentary insufficiency which is defended in the opening paragraphs of this chapter.

SYMPTOMS.-Osseous cachexia always makes its appearance insidiously and without fever. The first symptoms pass unnoticed. During the first few weeks they are too insignificant to be connected with osseous cachexia, except where the disease is common or when it is enzootic. The first manifestations are systemic disturbances. The patients lose their spirits and display feebleness and lassi- 
tude by prolonged decubitus of twelve to twenty-four hours. There are then noticed slight digestive disturbances, consisting chiefly of perversion of taste, and pica; but the principal functions are not disturbed. The respiration and circulation are normal, the temperature is but slightly raised, but the patients thrive poorly.

The disease develops slowly. The lesions of the bones reflect more and more upon the general system and the organs of locomotion. The suffering becomes more intense and renders every movement painful, whether walking or rising. After having stood up with difficulty, or often after having fallen down again, the animal remains motionless. When standing the back is arched and the legs parted like females. They will lift first one leg then another, as if suffering a dull pain. The loins are sensitive to pressure. When moving about they may moan. The walk is stiff, painful, hesitating and irregular.

Lameness appears abruptly in one or several legs, without apparent cause and without a fixed location; or after having walked a hundred meters. At this period of the disease osteoperiostitis and synovitis makes its appearance, as a rule in the sesamoid sheaths, producing permanent decubitus or very acute pain. Articular cracking can be heard and enlargements appear at the various articulations. The nutritive disturbances lead to emaciation accompanied with perversion of the appetite. The pica is more or less pronounced according to the subjects. They lick everything. Licking becomes the sole occupation of the patient. They attack the walls, the mangers and the hay-racks, and search for all kinds of solid bodies to devour-rubbish of plaster, ashes, sand, soil saturated with urine, bitters, old leather, rags, etc. The appetite is very capricious. Fodder of the best quality is refused. These symptoms are accompanied with profuse salivation, intermittent meteorism and 
emaciation. The hair is prickly, discolored, and bristly; the skin is dry, hard and bound; and the eye is sunken.

The permanent decumbency for whole days causes dermal sores which become infected and produce febrile complications. The milk secretion lessens and then becomes entirely exhausted, and is at all times watery. In the advanced stages of pregnancy, miscarriages sometimes occur. The general condition becomes worse and worse. So great is the emaciation and anæmia that the patients are unable to rise; they are almost paralyzed. When urged they rise on the fore-legs, the posterior part of the body remaining upon the floor, and it is only after several efforts that they are able to regain the standing position.

The affection becomes aggravated by degrees and the osseous lesions become progressively accentuated. The bones become less resistant, and the decalcification ends in deformities, but more frequently in fractures, which nay occur in any part of the skeleton from such slight causes as a fall.when attempting to rise, a slip, a delivery, etc. The fractures are located in the diaphyses of the long bones. They may be found in the posterior limbs and in the pelvis, which causes dropping of the hip and depression of the coxo-femoral articulation. The seat of the fracture may be difficult to locate and they are liable to occur at any pointthe ribs from decubitus, the vertebral column from slipping, or in any part of the extremities. The fractures are always numerous, but are not accompanied with hæmorrhagic effusions and show little tendency to reunite. The osseous ends become smooth instead of forming a callus, showing clearly the poverty of calcium salts and the feeble reactive property of the tissues. The ends of the fracture may penetrate the skin, and sometimes the tendons tear away from their attachments.

Deformities without fractures are rarer and are only 
seen at the final stage when the bones have become softened and deformed. The bones may be so soft as to yield to the pressure of the finger. The deformations take place in the articulations, the epiphyses and in the diaphyses. The pelvis may become so deformed as to render parturition impossible, and the vertebral column may become curved and otherwise deformed. A sufficient analysis of the urine has not been made, but it is cloudy and contains a large quantity of phosphate of lime.

COURSE, DURATION, TERMINATION.-The disease develops slowly but progressively. The duration is from three to four months, or even more. Its evolution is more rapid in pregnant cows, and unless prompt treatment is administered it terminates fatally, from cachexia, general enfeeblement and infections resulting from decubitus.

Sheep.-The disease is rather frequent in the sheep, and is characterized by osseous pains and lameness. The patients may walk on their knees, but owing to the fact that they are generally slaughtered they seldom reach an advanced stage.

Swine.-In the hog the disease appears early, and may as a consequence be confounded with rachitis.

Goat.-In the goat, it attacks chiefly the inferior maxilla. The rami are thickened, painful to the touch, and sometimes softened to such an extent that they can be depressed with the fingers. The teeth sink in the alveoli as if implanted into India-rubber, and render mastication impossible. The jaw hangs as if paralyzed, in consequence of arthritis of the temporo-mandibular articulation, which frequently complicates the condition. The animals scarcely grow at all, and move about on their knees. Lesions of the joints are wanting, but there is often incurvation of the bones of the extremities. The principal lesions are located in the head, where they reach considerable proportions. 
HISTOLOGY.-The osseous tissue is found to have almost disappeared. Instead of 50 per cent of phosphates there is but 2 to 3 per cent. The Haversian canals, which can scarcely be found, are surrounded with fibrous tissue, indicating not only an inflammation, but also a perversion of nutrition, bearing a close resemblance to a fasciculated sarcoma, and forming wound-up-elements that are but slightly vascular.

LESIONS.-The principal lesions are those of the bones. Every portion of the skeleton is attacked. Their essential character is the rarefaction of the osseous tissue by the disappearance of the osseous elements. The alterations are visible to the naked eye, and they vary according to the extent of the disease. They are found to attack the flat bones, the bones of the head, the ribs, the posterior extremities, the pelvis, etc., but to an unequal extent. At the early stages the bones are congested and ecchymosed. The medullary canal is dilated and the marrow is hyperæmic. In the more advanced stage these characteristics are accentuated. The bones become more fragile and friable, and may fracture without producing a sound. They are easily cut with a knife; diminish in density; and lose Io to I 5 per cent in weight. The periosteum has various aspects. It is slightly changed, bluish or reddish color, and is very vascular, thick, pulpy and easily removed. The articular surfaces are violet colored, ulcerated and exhibit necrosis of the articular cartilage. There is arthritis, abundant secretion of synovia and tumefaction of the articulations, of the binding ligaments and of the tendinous attachments.

The bone, when stripped of its periosteum, presents a porous surface resembling a skimmer or sieve. When squeezed, an oily liquid mixed with blood is pressed out. They are not resistant. The vertebra and the flat bones are particularly rarefied. Numerous fractures are observed 
but without any tendency toward the formation of a callus. The ends are worn and inflamed, and may be the seat of an imperfect fibrous callus. As many as thirty fractures have been observed in the ribs, some of which were in the course of cartilaginous consolidation. In the pelvis fractures may involve the acetabulum. In the long bones the medullary canal is found to be enlarged and to contain an increased amount of marrow presenting hæmorrhagic foci. The compact tissue becomes spongy and presents areolæ much larger than in the normal state, and the lamellæ may be detached. When the disease has reached the last phase, the lesions are still more accentuated. The compact tissue assumes the character of cancellated tissue, and is reduced to thin lamella at the surface. The marrow assumes the aspect of splenic pulp, then becomes yellow, gelatinous and sometimes as liquid as oil. The histological study of osseous cachexia should be directed to both the marrow and the osseous tissue:

The marrow is congested, is rich in blood vessels and exhibits interstitial hæmorrhages. The fat cells are diminished and the whole mass is transformed into a gelatinous substance of a more or less reddish color. The osseous tissue exhibits modifications similar to that of a normai bone decalcified by an acid,-in fact the essential process is a decalcification of the osseous elements. The fundamental substance, deprived of its mineral matter, becomes finely striated, and is differentiated from the part remaining normal, by the manner it fixes the stain (carmin). In the fundamental substances thus modified are included osseous cells which, deprived of anastomotic prolongations, display small, dark spots, linear or rounded, and frequently full of fat.

The lesions move from the center to the circumference. They are well marked around the Haversian canals, which are enlarged and exhibit an irregular outline, with points 
similar to the lesion of rarefying osteitis. New canals and cavities are formed in the fundamental substance. In the first period of' osseous cachexia there is, therefore, a dissolution of lime salts, and later the tissue thus deprived of its mineral substance undergoes farther ill-defined modifications, consisting of a transformation into a mucous substance. The degenerative changes are more intense in the cancellated tissue, but they always reach the compact tis-

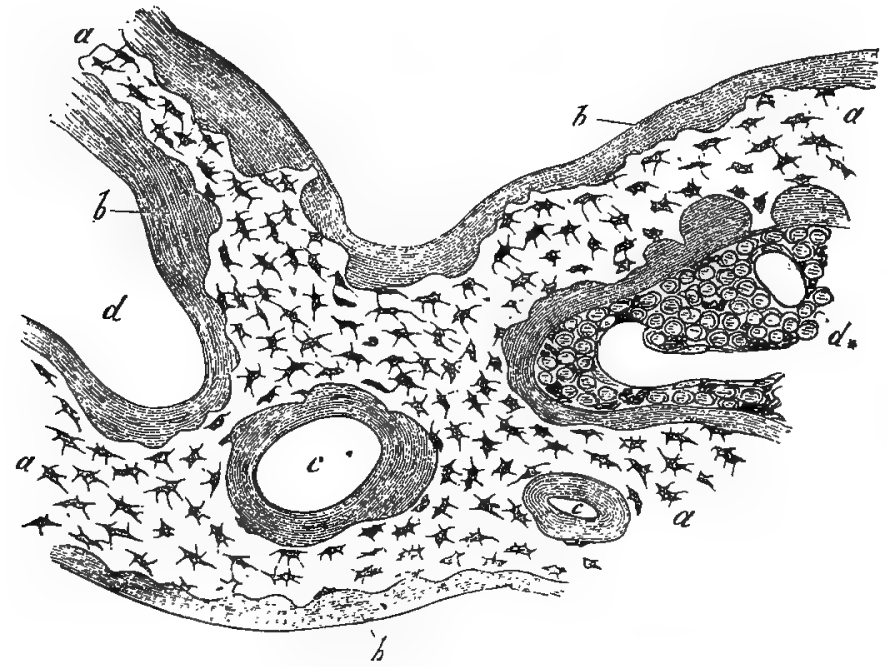

Fig. 95.

Osseous Cachexia. Fragment of the Cancellated Tissue of an Osteomalactic

Rib. (a) Normal Osseous Tissue. (b) Osseous Tissue Deprived of

Calcareous Matter. (c) Haversian canals. (d) Medullary Space Filled with Red Medulla; the Capillaries are Open.

sue, which finally disappears from center to circumference. This explains the enlargements of the Haversian canals and the medullary canal of the long bones. The alterations form cavities in bones having none with the result of diminishing both the density and the weight. In view of the extensive deteriorations of the osseous tissue, the chemical constitution of diseased bone, compared with that of normal bone, is an interesting study.

Numerous analyses, all of which vary somewhat, have 
been made, and the following table is the average results obtained.

\section{Analysis of Normal Bone.}

Tribasic phosphate of lime. 85 to 87 per cent.

Tribasic phosphate of magnesia..I.7 per cent.

Carbonate of lime...... 8 to ro per cent.

Fluoride of lime..........35 per cent.

Chloride of sodium...........23 per cent.

Bones contain 60 per cent of calcareous salts and 40 per cent of ossein. In diseased bone, most chemists clain that there is a diminution of phosphate of lime and of mineral substances.

More recently Mosselman and Hebrant made interesting analyses of the bones of a cow of ten years.

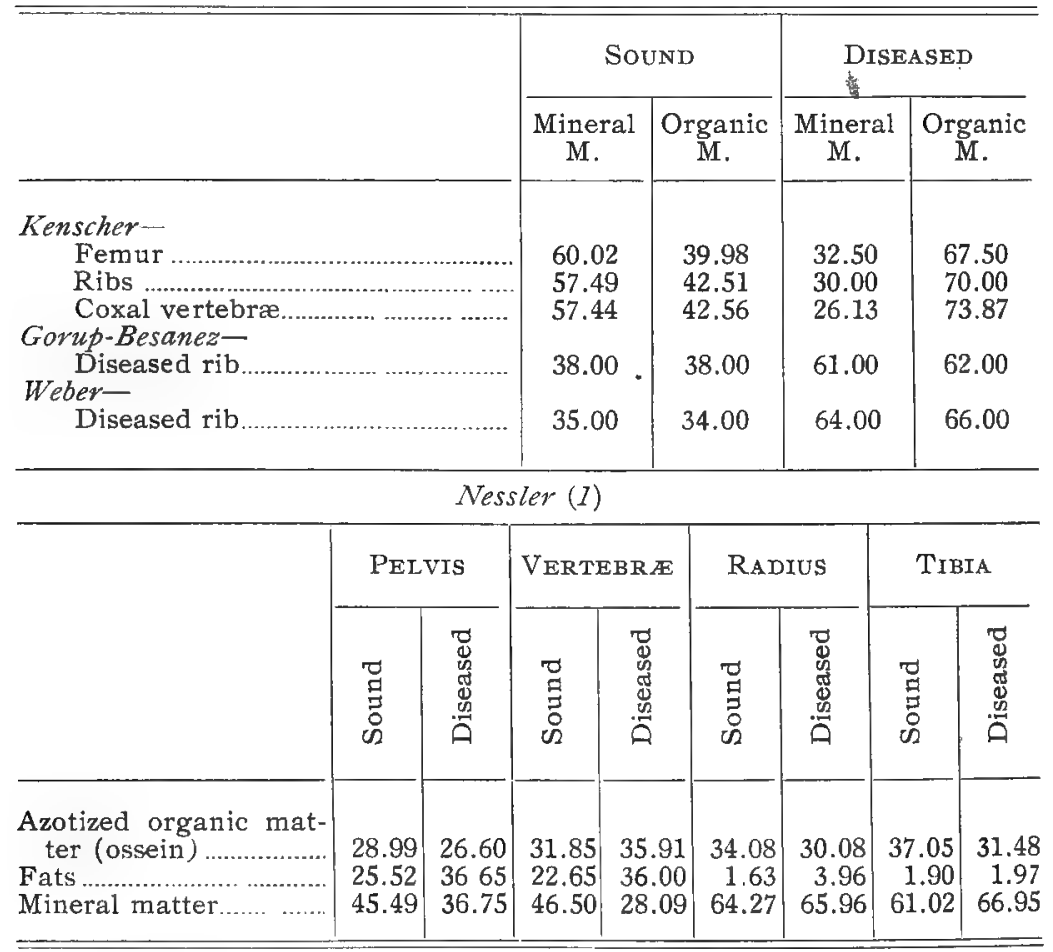

(1) Nessler: Chemical Analysis of the Bones of Animals Attacked with Osteodiastasis (Der Thierartz, 1973). Analysis of Zondel (Col. of Med.Vet. 1874, p. 144.) 


\section{METACARPALS.}

\begin{tabular}{|c|c|c|c|c|}
\hline & \multicolumn{2}{|c|}{ COMPact PART } & \multicolumn{2}{|c|}{ SPONGy PART } \\
\hline & Diseased & Sound & Diseased & Sound \\
\hline $\begin{array}{l}\text { Water } \\
\text { Fat } \\
\text { Ossein } \\
\text { Lime } \\
\text { Phosphoric acid } \\
\text { Magnesia, carbonic acid, etc }\end{array}$ & $\left.\begin{array}{r}10.45 \\
1.10 \\
35.91 \\
25.96 \\
21.32 \\
5.26\end{array}\right\} \mathrm{w}$ & $\left.\begin{array}{r}8.40 \\
2.00 \\
34.50 \\
26.48 \\
22.29 \\
6.33\end{array}\right\}$ 它 & $\left.\begin{array}{l}16.02 \\
26.88 \\
27.80 \\
15.83 \\
11.50 \\
1.92\end{array}\right\}$ & $\left.\begin{array}{l}13.83 \\
13.15 \\
36.79 \\
16.72 \\
15.55 \\
4.06\end{array}\right\}$ ळ \\
\hline
\end{tabular}

According to these results the alterations are most pronounced in the cancellated tissue, the water and the fat. The latter is augmented and the minerals and the ossein are diminished.

These analyses show that the osseous tissue is deprived of its minerals by degrees, and that the fundamental substance is transformed into a sort of mucous substance and then into fat. These alterations do not exist to the same extent in all of the bones, which explains the different results obtained by chemical analysis. Other peculiarities have been brought into prominence by these analyses. Hoffman and Bergmann found the diseased bones richer in phosphates than the sound ones. Marchand and Schmidt have noticed lactic acid in them, and this was at first considered very important. It is, however, only the basis of the theory that aims to explain the disappearance of the calcareous elements by the solvent action of lactic acid. This view is disputed nowadays. Kopp, Lucanus, Hoffman, Nessler and Leclainche found an abundance of fat in their analyses, in fact there is a rarefaction of the compact tissue,-a kind of eccentric rarefying osteitis.

Moreover there are general lesions of cachexia and anæmia, but these are of secondary importance. The muscles are pale, emaciated, infiltrated, degenerated and fatty, 
and the fat has disappeared from the connective tissue. There are also lesions of prolonged decubitus, consisting of œdematous infiltrations in the subcutaneous tissue and serous membranes, ecchymoses, dermal wounds and eschars.

DIAGNOSIS.-The diagnosis is easy, especially by at tentive observation of the patients, and when the disease is enzootic. It may then be recognized on first sight. The age of the patient is sufficient to distinguish it from rachitis. During the painful period it may be confounded with rheum atism, but the persistence of the affection, the etiology and commemoratives facilitates the differentiation.

PROGNOSIS.-The prognosis is all the more grave when the disease rages as an epizootic. The cure is difficult and can only be accomplished in the early stage. The prognosis is therefore always unfavorable. In connection with years of scarcity and droughts it is often impossible to procure the diet necessary to ameliorate the condition. All treatment is useless when the disease has advanced, and especially to the period of fractures. For rich land owners, who can furnish the necessary good nourishment, the prognosis is more favorable, and especially when the animals are only slightly affected. But in bad years, when good nourishment is scarce, even the mild cases cannot be saved. Animals in the middle of an attack may sometimes be saved by an appropriate diet. The fact is that the prognosis depends upon the economical condition of the agriculturists.

TREATMENT.-The treatment must be prophylactic and curative. Prophylaxis, based upon etiological facts, has been earnestly recommended by Cantiget, and it has been accomplished by cultural improvements in the districts where the disease was enzootic. The use of phosphates and superphosphates as manure has given good results. The use of fodder obtained from districts where the disease is unknown is also advisable. Emigration into countries 
rich in carbonate of lime is not practical. Curative treatment should be applied early. It is recommended to place the sick animals in the best hygienic conditions, to change the diet, to give food of good quality, rich in calcareous salts and in phosphates,-cereals, barley, oats, bran, leguminosæ, cotton oil cake, peanuts, - and finally to counteract the progressive enfeeblement by appropriate medication.

Bone-dust has been recommended. Soluble phosphates of lime, oleum phosphorus o.I per cent in milk, at doses of 20 to 30 grams, cod liver oil in doses of 500 grams per day, are all good remedies if not too expensive. Condiments, sea-salt, bitters, iron, nux vomica, hydrochloric acid, or gentian may be added to the food to facilitate absorption. There is no occasion to treat the complications. Roloff advised that the treatment of the fractures and deformities should give way to their destruction as food, unless they are too loathsome for that purpose.

\section{EQUINE OSTEOPOROSIS.}

SYNONYMS.-Osteomalacia of the horse. Bran sickness. Osseous cachexia of the horse. Diffuse rarefying osteitis. Big-head.

DEFINITION.-Osteomalacia of the horse is characterized by an inflammation diffused through the entire skeleton, but especially through the bone of the head. Its course is chronic, its termination is fatal, and its principal features are co-existing rarefaction and hypertrophy of the osseous tissue. Authorities differ both as to its nature and its appropriate denomination. It has been regarded as rachitis, especially when it attacks young animals. At other times it has been attributed to a special or exclusive diet,-bran sickness,-and finally, it has been described as a special form of osteomalacia.

The disease was studied in France by Laquerrier in 
1876 , and by Soula in I888. It has been observed in different countries, - in England, in South Africa, in Germany, in Switzerland, where it is known as "bran sickness," in Cochin China, and in America, where it is known as the "big-head." It has also been described under the name of "osseous cachexia of the horse," "osteomalacia," "generalized ostoeporosis" and "diffuse rarefying osteitis" analogous to osteomalacia of man.

The descriptions given of the disease are incomplete and the "pathologies" are the object of only an insignificant paragraph. It was, however, pretty well described in 1860 by Warnell, who had observed numerous cases while on an exploitation. He gave a complete anatomo-pathological description of the disease, after microscopic investigations by Scharpey. It was observed in Germany by Bagge, Zipperlein and Hering, and has been closely studied in Italy by Marcone.

ETIOLOGY.-The etiological conditions that govern the development of the disease seem always the same. In every case the patients have received a diet of bran or millproducts for some time previous to the appearance of the disease, hence the name "bran sickness." A case reported by Porcher and Carougeau had been submitted to a diet consisting of six kilos of bran of the best quality at each ration, and similar observations have been made by Crowhurst since 1869 .

The disease usually attacks animals between the age of four and eight years, and is very rare in colts. The cases observed in very young animals are undoubtedly rachitis. However, the young horse, according to the observations of the American veterinarians, Morris, Faville and MacLead, are not entirely immune and animals of both sexes are equally susceptible. It may now be safely stated that horses affected with deformities of the bones of the head, 
especially the maxillary bones, have been suffering from the disease for a long time.

The disease often assumes an epizootic form. Theiler, a veterinarian in Johannesburg, has described a veritable epidemic of osteomalacia among tramway horses. The bacteriological investigation of the disease has thus far given only negative results, and its infectiousness therefore remains unproven. It is supposed to be connected with goiter. Dor has compared it with Paget's disease and Marcone with osteomalacia of man. The latter regards it as an inflammatory process beginning in the Haversian canals and ending in rarefaction of the osseous tissue.

SYMPTOMS.-The premonitory symptoms are vague and are usually regarded as due to "muscular rheumatism." Its beginning is obscure and evolution slow. The appetite is preserved and the condition of flesh is well maintained. The general condition is but little altered, but the vigor is considerably depressed. They become weak and easily fatigued without any evidence for attributing the change to any definite disease. Some animals suddenly show signs of either a slight or marked lameness, which is migratory and remittent and often attributed to rheumatic diathesis, or of late years to osteitism, resulting from painful sensations in the bones. These pains are expressions of a diffused rarefying osteitis, attacking the bones successively. alternately or simultaneously. The lameness is accompanied with articular or tendinous tumefactions which usually yield to the application of counter-irritants. Sometimes numerous and successive inflammations of several synovial membranes and a large number of bones are observed. These inflammations evolve without any perceptible rise of the temperature, and without leaving behind any indurations and ankyloses as relics. The dorso-lumbar region is stiff and painful on pressure and some animals show paresis or paraplegia. 
During the development of these sudden and movable pains the bones submitted to the most exertion become areolar, and as the entire skeleton is affected with a rarefying osteitis, numerous accidents, such as detachment of tendons and ligaments, fractures and deformities, may occur. The accident which deserves special description, usually takes place in the anatomical structures supporting the greatest weight. The resistance of the bones being diminished, they become incapable of sustaining the attachments of the ligaments and tendons, or of serving as levers for the supporting organs. Fracture is the inevitable result. These accidents are regarded by Joly, Jacoulet and Vivien, as the consequence of osteitism.

Tumefaction of the bones of the head is the only pathognomonic symptom of the disease, but it is agreed that the nutritive trouble that intensifies the fragility of all the bones, and that which manifests itself by the increase in the size of the bones of the face, are identical. On the other hand, it is certain that many cases of osteitism in young animals are not osteoporosis.

The different manifestations of the alterations of the bones are as follows: Ist. Detachment of ligaments and tendons. 2nd. Fractures. 3rd. Tumefaction of the head. 4th. Urinary disturbances.

I. Detachments of Ligaments and Tendons.-Marcone deserves the credit of having established the role of the osteitic process through which tendons are torn away from their attachments and bones are fractured. These accidents often occur before it is possible to make a clinical diagnosis of osteoporosis. The only fact connecting these accidents to the disease is their occurrence without violence and in animals in apparent health. Veterinary literature abounds with facts in this connection. Marcone reports the following cases: 
In 1884 , Kay reported a horse that fell suddenly lame from detachment of the plantar aponeurosis of three legs. The inferior sesamoidean ligaments were detached and had drawn shreds of bone from their insertions. Schraml related an identical case, and Schellhase one in which the inferior sesamoid ligaments of the right fore-foot were detached and had torn away spicula of bone. Pendry testifies to the detachments of the sesamoid ligaments of the left hind leg: Blumentritt reports the case of a three-year-old colt that suddenly became lame in one fore-leg without any particular cause. The fetlock was swollen, excessively extended, (dorsal flexion), and crepitated at every attempt to support weight. At the autopsy the inferior sesamoidean ligaments, including the middle and lateral ligaments, were found completely and irregularly torn away from their attachments, and holding pieces of bone varying from the size of a millet seed to that of a pea. Degive and Hendricks report some very curious facts which furnish evidence of long standing alterations of the osseous and ligamentous systems. An eight-year-old horse exhibited a lameness from which he apparently recovered, but when started in a short race of two kilometers, he suddenly became lame and broke down with complete detachment of the inferior sesamoidean apparatuses of both hind legs. The phenomenon was accompanied by an enormous oozing, sanguineous engorgement extending upward as far as the hock.

An eight-year-old pony suddenly fell lame with a complete detachment of the perforans tendons at their plantar insertions of all four legs. In the hind feet, the plantar aponeuroses were detached from the os pedis along the entire semilunar ridge, which was partly torn away, and adhered to the end of the tendon in the form of perceptible osseous fragments. The navicular bone, in consequence of the detachment of its binding ligaments, was drawn backward 
and upward. In one of the hind legs the navicular bone was crushed and a narrow and thin layer of its articular cartilage was left attached to the posterior lateral ligaments. In one of the fore-legs, there was a complete detachment of the glenoid fibro-cartilage that unites with the perforatus at the superior extremity of the os corona.

In another horse, ten years old, Degive and Hendricks observed a partial detachment of the plantar aponeurosis of a hind-leg from a violent effort to dislodge the leg from a fixed position, but this accident may be analogous to fracture of the patella from violent muscular contraction, in the human.

The detachment of the great suspensory ligament may result from the same cause, but when four legs are affected simultaneously it is only reasonable to suppose that the accident is the result of a nutritive disorder of the osseous tissue. Among the tendinous and ligamentous detachments noticed in works on surgery there àre many which result from the diffuse rarefying osteitis under consideration. Thus, Bayer and Frohner report that out of 287 cases observed among horses, 8I.I8 per cent were cured, 9.06 per cent were improved, 9.06 per cent were killed, and 0.07 per cent died. It is quite certain that the last three groups contained numerous examples of osteoporosis. The flexor tendons are the most predisposed to these detachments. Out of 2 I 4 cases it has been shown that IIO, or $5 \mathrm{I} .40$ per cent, were complete lacerations of the flexor pedis perforans; 24 , or II.2I per cent, were lacerations of the suspensory ligament, and 8 , or 3.74 per cent, were lacerations of the extensor tendons. Among the cases of ligamentous detachments it was observed that in I 53 subjects 99 , or 84.7 I per cent, were cured; 25 , or 16.34 per cent, were improved; 8 , or 5.23 per cent, died, and $2 \mathrm{I}$, or 35.25 per cent, were killed. 
Of this number 30 per cent were in the fetlock. Detachments in the other articulation is less frequent.

Flatter has clescribed two cases of detachment of the tendo-Achilles, which at the autopsy showed also a partial clisinsertion of the caput muscles at the olecranon.

Marcone gives accounts of some observations of tendinous and ligamentous ruptures in horses apparently sound, but which in reality were affected with a generalized osteitis that was amply proven by minute microscopic examination of the skeleton. A horse six years' old, in good condition and of beautiful appearance, when brought from the stable to be hitched with another horse, exhibited an intense lameness with painful tumefaction of the fetlock. When examined the articulation was found to be the seat of exaggerated movements. The flexors gave way, and as a result, the extension of the articulations became abnormal. In the course of a few days the weight was supported upon the posterior surface of the fetlocks, and the animal died in agony. The autopsy disclosed complete detachment of the inferior ligaments, the free extremities of which were covered with blood and rough from the presence of small, bony splinters. The flexor tendons were studded with hremorrhagic spots. A study of the skeleton demonstrated the existence of a generalized osteoporosis.

In another case studied by Marcone the question had been raised as to whether a certain horse had been suffering with rheumatism. At the autopsy the perforans tendons of the hind legs were found detached from the semi-lunar ridges, riddled with hæmorrhagic dots, and considerably thickened. Their extremities were studded with osseous debris, and the navicular bone was detached as the consequence of laceration of its ligaments, besides being fractured in the antero-posterior direction: In the right foot the navicular bone was fractured in two places. 
Independent of the local lesions found in these two cases there are general lesions characterized by the easy detachment of the periosteum and a dark, reddish-blue color of the bones beneath it. These lesions are the result of a general morbid process that belongs more to clinical pathology than to surgery, although in these cases there is no deformation in the bones of the head.

2. Fractures. Fractures occurring without a perceptible cause, or even those due to accidents, are very frequently due to osteoporosis. Formerly, it was admitted that fracture may result from a previous osseous alteration, but up to the present time it was supposed that the alteration was limited to the bones fractured. The latest ideas on this subject are furnished by Vivien and Marcone, who attest the existence of a general osteitis of a diffuse and rarefying character, of a11 of the bones of the skeleton. When the morbid process in a bone reaches a state in which its resistance is not equal to the required strength it will fracture or its ligaments and tendons will detach. The shape, strength or inclination of a bone may predispose toward fracture, but the osteitis responsible for the accident affects the fractured bone exclusively. The entire skeleton presents the very same alterations in most cases, and the mechanical forces produce the fractures at the predisposed points.

The most susceptible bones are the lumbar vertebræ, the ribs and the metacarpals, but the fragility may manifest itself by numerous fractures. In a case reported by Balts, fractures in the course of repair were found in the eighth and ninth ribs, and new fractures of all the ribs from the eighth to the fourteenth. Marcone observed a fracture of the ninth, tenth and thirteenth ribs on the right side. We have observed analogous cases, and Blanc noticed numerous fractures of most of the ribs resulting from a probable osteoporosis. 
Deformities of the Head.-In spite of the generalization of the process throughout the entire skeleton, it is evident that some bones exhibit preponderating lesions. Some animals may present ligamentous detachments without tumefaction of the skull bones, while others exhibit the tumefaction of the facial bones as the most conspicuous phenomenon during the entire development of the disease. These deformities appear to be chiefly expressions of the old chronic case. The lively petulant animals seem to be most susceptible to detachment of the ligaments and to fractures. The shape of the body and the distribution of strength may have some influence in the causation of the secondary lesions,-fractures, detachments, and deformities of the head. The gravity of the disease and the reparation from the periphery or the center of the bones must also be taken into account.

The participation of the facial bones in the generalized inflammation of the skeleton is proven by the fact that the animals can no longer eat hay without rejecting quids into the manger at each meal, but will easily swallow grass, bran, oats and cooked potatoes.

An examination of the head reveals a striking roundness and enlargement of the face. The depressions on the superior maxillary are filled up, and the head in its entirety looks enormous. On palpation each branch of the inferior maxilla is found hypertrophied to the extent of diminishing the cavity between them into a narrow cleft, through which the fingers can scarcely be inserted. The projection of the maxillaries gives the head the appearance of the muzzle of the ox. Closer examinations will then reveal similar alterations in different parts of the head.

There is slight pain on pressure and an examination of the mouth reveals the teeth regularly implanted in the maxillaries, but considerably encroached upon by the bone, and 
comminuted food is found lodged in the cheeks. The diseased animals become weaker, their general condition grows worse and they become slightly emaciated. As they finally are unable to take nourishment the emaciation becomes accentuated, the gait becomes tottering, and standing erect difficult. Toward the later stages the patients will lie down all day and become perfectly useless. The tumefactions that deform the facial bones become more pronounced and

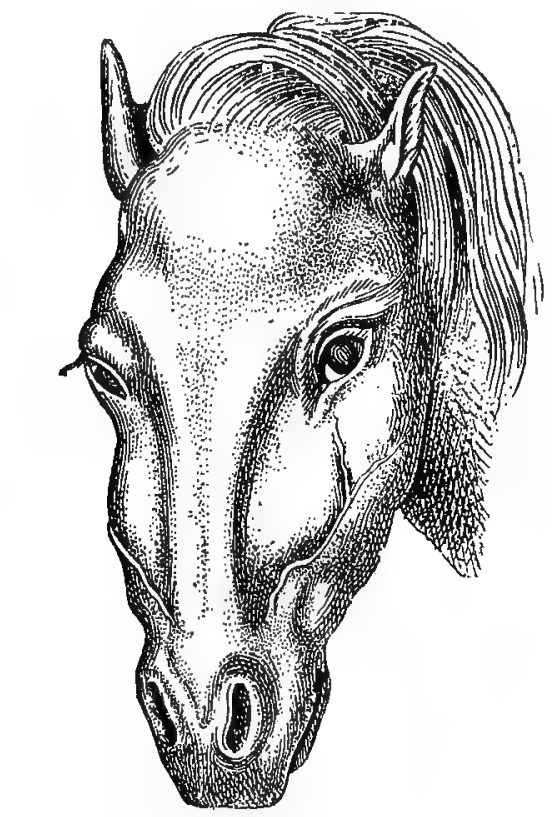

FIG. 96.

Tumefaction of the Facial Bones (Marcone).

extend to the lachrymals, the zygoma, the turbinated and frontals, giving a singular physiognomy. The teeth are surrounded by tumefied, red, and indurated guns, the hard palate and tongue are tumefied, and the teeth loosen in the alveolar cavities. The osseous tumefactions diminish the lumina of the nasal cavities and obstruct the respirations, which become loud and sniffling, and even threaten to cause death from asphyxiation. Finally, the bones having grown more and more tumefied, they lose their solidity and yield 
to pressure with the finger. They become as soft as a pear. The weakness and emaciation increases until the subject remains permanently recumbent.

4. Urinary Disorders.-Alterations in the composition of the urine appear to keep pace with the osseous alterations. Every case of general rarefying osteitis is accompanied with phosphaturia in proportion to its extent and intensity. Normally, the elimination of phosphates varies with the alimentation. From analyses made by Marcone on healthy horses fed with various feeds, the quantity of phosphates (measured as phosphoric acid) eliminated, at no time exceeded 9.60 grams daily, and sometimes it fell as low as 0.40 grams. On the contrary he found that horses suffering from osteoporosis eliminated as high as 14.440 grams, and even 28.80. However, the diseased horse may not always show an increased elimination of phosphoric acid, because there may be momentary arrests of the process and hence arrests of the elimination of phosphates.

TERMINATION.-Death is the rule, but American veterinarians have shown that a cure may be effected in some cases. The evolution of the disease may, however, be interrupted with frequent halts, as is proven by the momentary disappearance of the lameness. And besides, autopsies often reveal points in the osseous tissue where the dilated Haversian canals and enlarged lacunæ have been filled with adipose tissue. The disappearance of the embryonic elements, and their replacement with adipose tissue, testifies to the arrest of the process,

The incurability of the disease is certain when the process has reached its ultimate phase,-tumefaction of the facial bones. All of the horses observed by Varnell died. In spite of these assertions it has been shown that cures,perhaps exceptional,- - have been effected in horses presenting deformities of the head. Marcone cites one instance of 
this kind, and Vende another. Hoskins (Philadelphia) also states that he has seen cures in both young and adult horses having tumefied maxillaries. Faville and Herbang observed similar cures. They are, however, rare. Diecherhoff refers to cases of four-year-old horses with deformed maxillaries that did not afterward exhibit any morbid symptoms. Arrests in the morbid process are, on the contrary, very common. The attack always coincides with the phosphaturia, and arrests with its disappearance.

\section{ANNOTATION.}

Cures, or at least arrests of the morbid evolution of the disease, are not at all uncommon in American horses. When the disease is recognized in its "rheumatic period" or when the tumefaction of the skull is still in its incipiency, osteoporosis is frequently seen to yield to a course of hygiene, dieting and medication that will palliate, arrest or even entirely cure the condition for all practical purposes. Failure to recognize the disease until it has developed to an advanced state will prevent successful intervention. The cases of tumefaction in young horses referred to by Diecherhoff, cannot be claimed in the same category of affections, as osteoporosis. Bulging of the superior maxillaries and sometimes the inferior maxillary in three, four and five-yearold horses, without any diffused morbid process to correspond, is a mighty common disease in all breeding districts. This particular lesion is local; it is dental in origin and presents no phase by which it could be connected with grave constitutional affections of the skeleton. (See Vol. I, Osteoma of the Maxilla.)-L. A. M.

DIAGNOSIS.-The diagnosis of osteoporosis is very difficult at the beginning, at which time it manifests itself exclusively by a migratory and remittent lameness and by a more abundant elimination of phosphates. These symptoms disclose the intra-osseous pain and the destructive process taking place in the bones. At a later stage, fatigue and tumefaction of the facial bones become apparent, but in some cases the detachment of a ligament or tendon, or the fracture of bones, may cause death before the appearance of the facial deformities. The symptoms bear some resemblance to pseudo-leukemia or pernicious anæmia, but are distinguished by the absence of the remittent lameness so characteristic of osteoporosis. It is differentiated from rheumatorn liv phusphaturia. 
PATHOLOGICAL ANATOMY.-The important lesions are localized in the skeleton, and consist, essentially, of a pronounced vascularization which is the expression of a rarefying osteitis. The other systemic alterations are those of inanition.

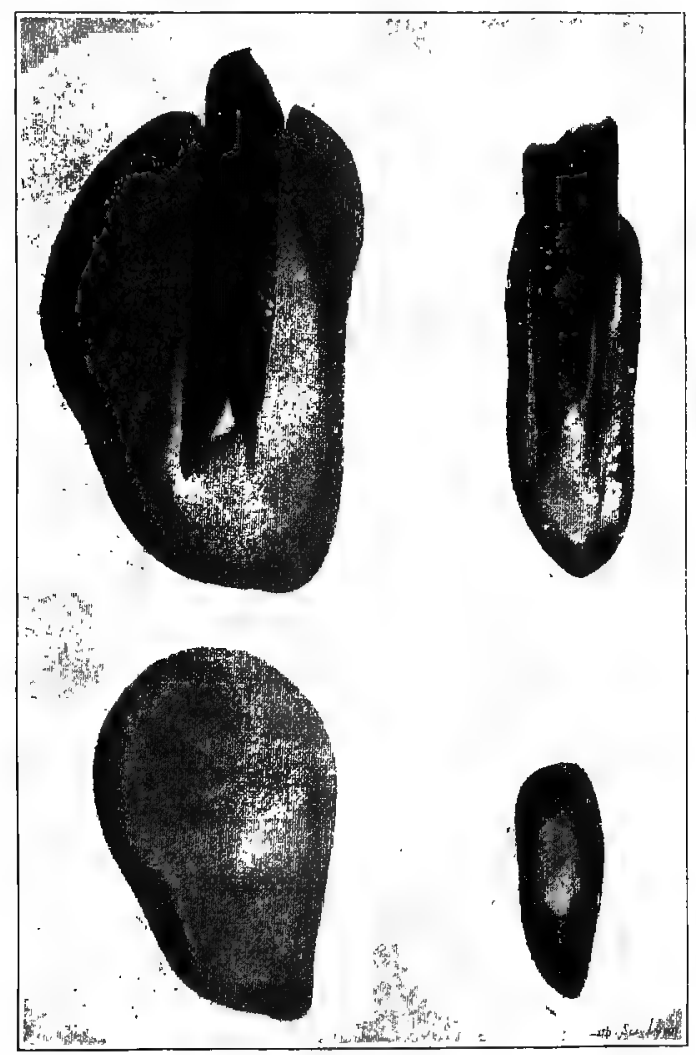

FIG. 97.

Section of the Inferior Maxilla in the Region of the Third Molar. 1. Diseased Jaw. 2. Normal Jaw.

Section through the Inferior Maxilla at the Interdental Space. 1. Diseased Jaw. 2. Normal Jaw.

The bones of the head which have been well studied, are found to be the seat of a pronounced alteration. The periosteum, which is excessively vascularized, is easily torn off and brings with it processes that extend into the osseous tissue. The bone can be easily wounded with the finger 
nail, and is of a dark red or violet color. Its surface is rough and uneven and presents the aspect of a fine sponge. It exhibits a large number of elongated oval openings several millimeters wide, through which the blood vessels enter. The surface of a section through the bone is reddish, violet or yellow. It is soft to the touch, and no distinction can be made between the compact and the cancellated tissue.

The maxillary bones present the most pronounced lesions. They are particularly hypertrophied along the molars, sometimes attaining three times their natural size. The rami of the inferior maxilla are very thick. The teeth are implanted in the thickest portion of the bone, the table surface is almost on a level with the alveolar border, and the roots closely approach the inferior borders of the rami. When a dissection is made of the bone they are encountered at a depth of less than a centimeter. The teeth are, however, easily extracted in spite of their deep implantations. but they never fall out as in other abnormalities of the jaws. As a whole the skull bones are hypertrophied, swollen, and tumefied, especially in the transverse axis.

The osseous tissue has a rosy tint and blood oozes from its surface when the periosteum is torn off. The tissue is non-resistant to the touch, but it has not lost its density to the extent of yielding to pressure of the finger. It is easily cut with the knife.

A transverse section of the maxillaries, made at different points, shows interesting modifications which are rendered particularly conspicuous by the radiograph view of a section four to five millimeters thick. The above cuts show principally the extent and aspect of the lesions. The diseased bones are uniformly gray throughout while the sound bones present the normal reticule of the compact tissue distinctly separated from the cancellated tissue. In this respect a section through the superior maxilla is instructive. On the 
left side the palatine arch is considerably thickened, while in the right it has preserved its normal appearance. The contrast would even be greater in an older horse.

The entire osseous tissue becomes spongy, in fact there is actually re-absorption of the compact tissue until it has become porous. The cancellated tissue is hypertrophied to

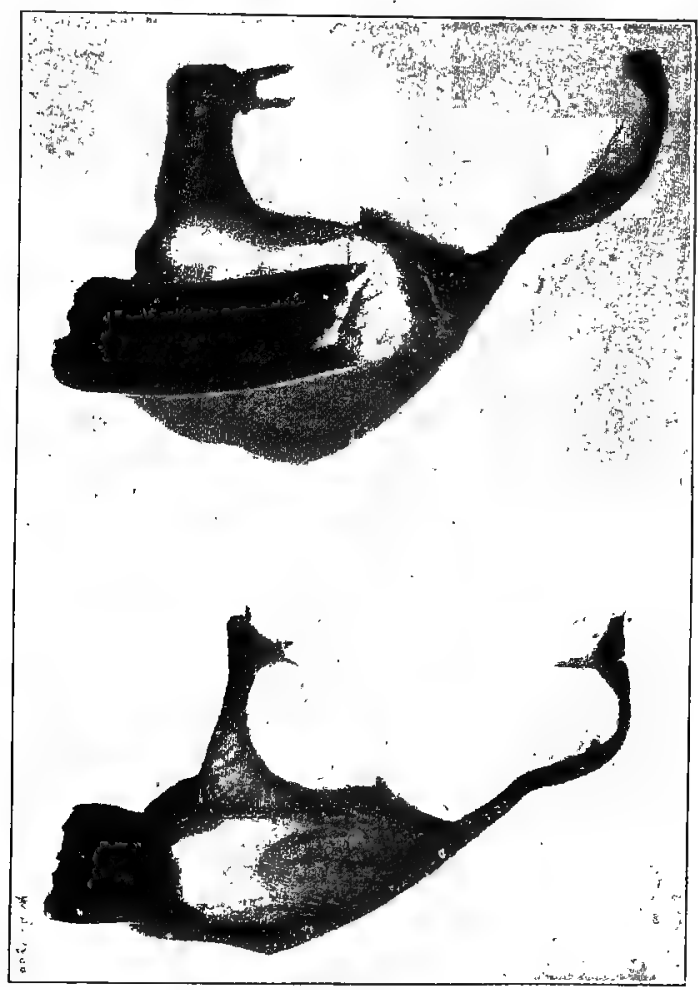

FIG. 98.

Section of the Superior Maxilla in the Region of the Third Molar. 1. Diseased Bone. 2. Normal Bone. (The Nasal Bones are Removed.)

the extent of enlarging the rami of inferior maxilla to the thickness of six centimeters. The compact tissue of the rami has completely disappeared and is replaced with a tissue resembling pumice stone. The tissue has considerably diminished in density, and encloses a reddish yellow and very vascular marrow. 
In the superior maxilla degenerative changes of the same kind are observed. The bones of the palate are also thickened. All of the bones of the head after maceration and exposure in the air are very light. The entire horse's head, excluding the teeth, often weighs but 400 grams. Occasionally it weighs but 600 grams including all the teeth. A very large head including all the teeth and inferior maxilla may not weigh more than I,I60 grams. On the other hand there are cases in which the head when greatly tumefied loses but little weight. Some may weigh four kilograms, others 2,500 grams, and still others as high as 3,300 grams, including all the teeth. The head of a sound horse of the same size would weigh about 4,040 grams. The maxillary sinuses are nearly closed on account of the swelling of the bones forming their boundaries. The openings in the maxillaries are more numerous and nearer to one another than in the other diseased bones. On skulls which exhibit the more acute rarefaction the bones forming the orbital arch, with the exception of the orbital process, have almost normal surfaces. And the same may be said of the basilar process of the occipital and the squamous portion of the temporal. Even in a single maxillary, every degree of the alteration may exist. The symmetrical swelling of the maxillaries is the cause of the facial deformity aiready mentioned. The sutures of the bones are all quite visible, and the external foramina are more numerous.

The other bones of the skeleton are as much deteriorated as those of the head, but they attract little attention because they show no special deformities during life. In all long, short, and flat bones the richness of the blood supply is in evidence. In long bones like the tibia the marrow is excessively red and rich in fat, and the osseous substance of the diaphysis cut transversely sometimes shows a bluish tint in the eccentrical zones. When the process is advanced the 
marrow is red and the medullary canal is enlarged. In some cases the marrow is yellowish. On the surface of the section may be seen reddish or grey dots, more or less large, in which the osseous substance has been displaced by a soft tissue. In the centre of the short bones the osseous. substance has undergone great changes. It is softened and formed into a large cavity filled with a sanguineous pulp, which, pressed between the finger, reveals the presence of bony splinters-the remains of the osseous lamellæ. The areolæ of the epiphyses are considerably enlarged and filled with a sanguinolent marrow. A fresh bone when macerated and exposed to the air loses three-quarters of its weight.

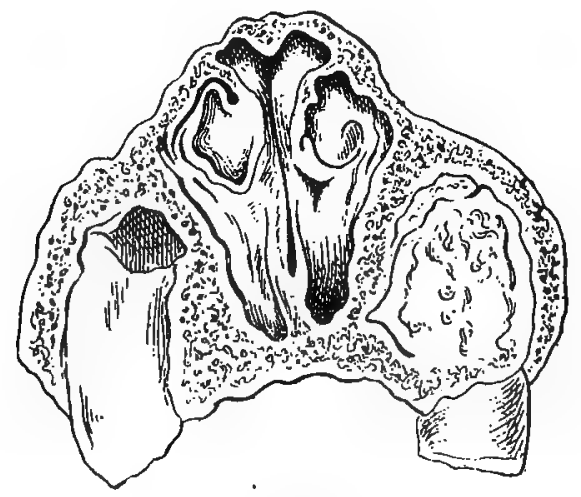

FIG. 99.

Section through the Head at the Fourth Molar Showing the Narrowing of the Cavities and Sinuses.

A fresh tibia that weighed 735 grams, after maceration and desiccation, was reduced to 250 grams. A humerus weighed 270 grams, a scapula $\mathrm{I} 55$ grams and a femur 320 grams. In flat bones like the scapula, a very pronounced hypertrophy may often be observed along the entire acromion spine. Similar alterations are seen in the ischial tuberosity and iliac spine. The ribs of horses that have remained permanently recumbent during the last days of their existence are often found fractured. Sometimes one rib alone is the seat of numerous fractures. The ribs, the vertebra 
and all the flat bones are filled within with a gelatinous substance. The articular surfaces, especially of the limbs, exhibit loss of substance, and the cartilage, which is dark in color, is studded with erosions similar to those of dry - arthritis. The disappearance of the cartilage during life results from the disappearance of the subjacent epiphysial osseous tissue.

Evidence has been found of the disappearance of the intervertebral discs and ankylosis of the vertebra with rarefaction of the bones.

The osseous apophyses are softened and traction upon the ligaments easily detaches them, with small particles of bone.

Analysis of the Bones.-The analyses of the osseous tissues have no absolute value. In rachitis and senile osteoporosis the same changes in the proportions of mineral and organic matter may be found. In I887, Marcone analyzed the tibia and the maxilla of a horse that was attacked with osteoporosis and had fractured the two metacarpal bones of the fore-legs; IOo parts of fresh bone contained:

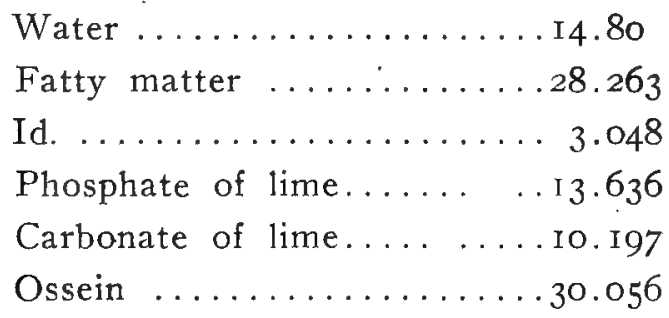

The proportion of mineral matter to organic substances was $6 \mathrm{r} .367$ to 25.833 . The analysis shows that there is a larger quantity of organic matter (more than 50\%) but that the relative proportions of the mineral salts are preserved. Fritz and Abey, on the contrary, found a normal relation, and Germain a relation too exaggerated to be correct. The relation between the organic and inorganic matter was 40.04 to 5.89 . The difference observed varied according to 
the particular bone analyzed. The maceration and desiccation considerably diminished the weight of organic substances. The fat and the osseous tissue that fill up the lácunæ after destruction of the osseous lamellæ disappears. The analysis of the desiccated bones gives only the relations that exist between the mineral substance and the ossein without taking into account the enormous amount of fat accumulated in the bones. On the whole there is always a considerable increase in the organic matter, and a diminution in the inorganic substances.

HISTOLOGICAL LESIONS.-Marcone's histological description of the bones is as follows:

Short Bones.-The rarefaction of the osseous tissue is marked. Enormous lacunæ are developed, especially in the center of the sesamoids. They are round ovoid, or prolonged, and border upon other smaller ones which communicate with them. Osseous lamellæ are found inclosed in the connective tissue that replaces them. Elsewhere the osseous areolæ may be found to have preserved their lamellar structure and to contain zones not colored by safranin, in which the osseous cells appear red. These zones are already decalcified in the living animal. The lacunæ contain big central cavities surrounded by a single row of fusiform cells, and round embryonic cells, together with adipose cells. Usually the latter nearly fill up the entire lacunæ. The large ones are frequently filled with connective tissue. It is important to note that in the sesamoids, navicular and os pedis, these lacunæ are found not only in the center but also throughout the entire bone, including the parts serving for the attachment of the ligaments, tendons, and plantar aponeurosis.

The long bones that are not decalcified, present extensive repletion, increase in volume, and enlargement of the blood vessels. The primitive lamellæ ạre markedly altered 
by the loss of their radiating canaliculate construction due to anastomosis and distention of the osseous reticula. Some of the Haversian canals are so enlarged that only a few eccentric lamellæ remain and the entire canal is filled with blood. Sometimes a large lacuna filled with a red or yellowish substance replaces an entire Haversian system, and communicates with other systems also similarly destroyed. In the intermediate zone of the long bones, the same enormous

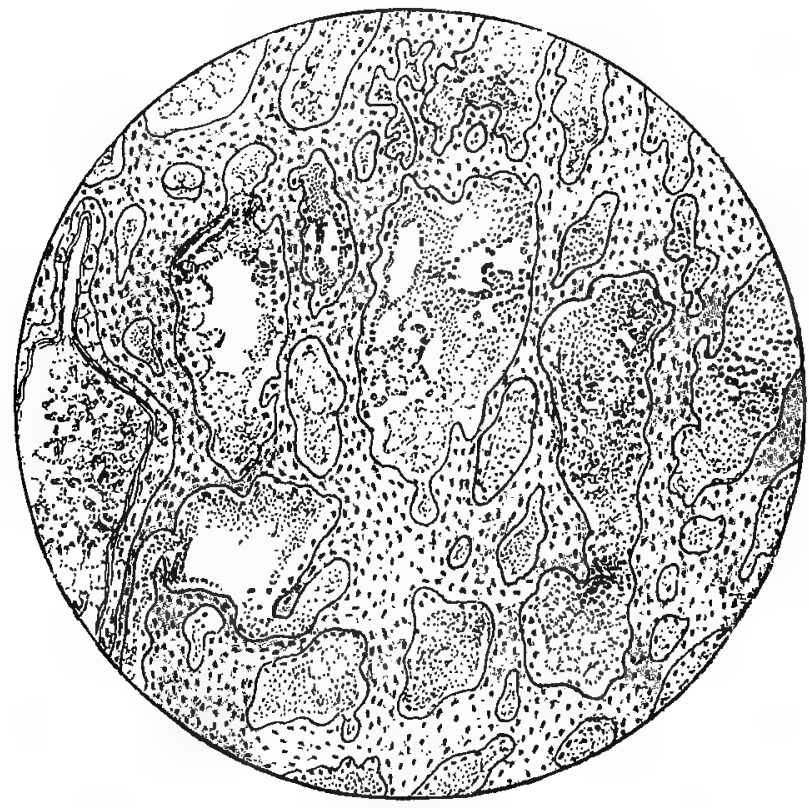

FIG. 100.

Formation of Lacunæ by the Disappearance of the Osseous Trabeculæ.

quantity of blood that fills the enlarged Haversian canals is not found, but instead there is perceived large, irregular lacunæ, filled with a reddish yellow, semi-transparent substance surrounded by primitive or secondary lamellæ in which the osseous cells are replaced by refractive formations. The internal or perimedullary zone presents large, irregular, anastomosing lacunæ, having extensions uniting them to the Haversian canals, and separated with well-preserved osseous tissue. 
The decalcified long bones, on transverse section and stained with safranin (Fleming's Method) do not stain the lamellæe surrounding the lacuna, but the lamella of the eccentric zone stain readily. Sometimes a Haversian system is totally discolored, showing that during life there was a partial decalcification of the osseous lamellae. In the intermediate zone two or three Haversian systems may be fused into one large lacunæ.

The lacunæ contain adipose cells, fibrillary connective tissue, and some embryonic cells which either fill up the cav-

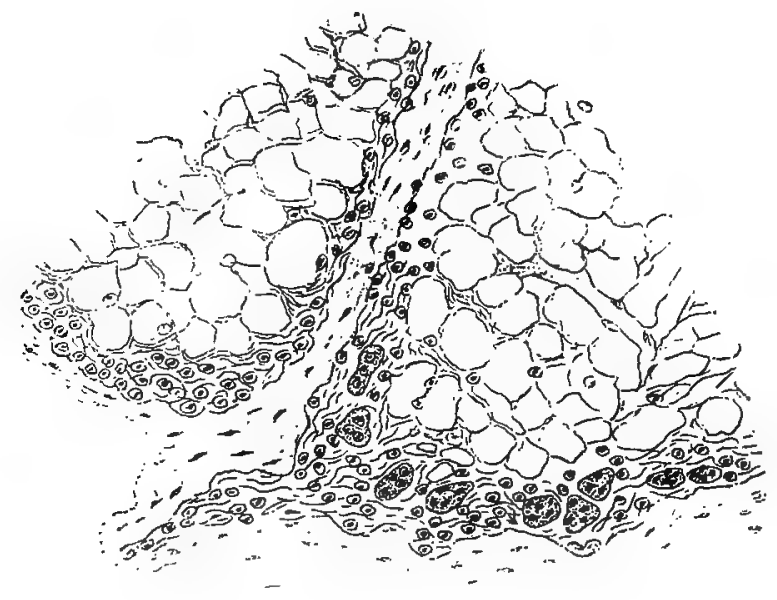

FIG. 101.

Deposits of Adipose Tissue During the Periods of Cessation of the Morbid Process.

ity or accumulate around its circumference. Sometimes the cells consist of a single row in immediate contact with the lamellæ bounding the lacunæ. They are never wanting and are abundant in the lacuna in the course of development. The outlines of the lacunæ are irregular and sinuous. The embryonic cells that fill them increase with each new step of the process. When the process halts they fill with adipose tissue. In this affection the normal osseous tissue forming the Haversian systems is transformed into osteoid tissues, that is to say, the lamellæ are decalcified and exhibit 
imperfect osseous cells. The process is a general rarefying osteitis.

TREATMENT.-There is no known effective treatment. Phosphorus, arsenic and changes of diet have been tried without effect. Dieckerhoff recommends a special diet and intratracheal injections of,

Iodine ................. I part

Potassium Iodide ...........5 parts

Water ................ roo parts.

This mixture is injected in doses of $\mathrm{I} .5$ grams per day for a few days, and then discontinued from time to time. Colts from two to three years old, attacked with tumefaction of the facial bones, have been cured by a regimen of alfalfa and clover. The oats diet is discontinued. At the end of some months, when some improvement is observed, the ra. tion is augmented with small but increasing quantities of barley bran and rice, in the proportion of two-thirds of the former and one-third of the latter.

\section{ANNOTATION.}

Osteoporosis, or Big-head, the names universally applied to the disease by American veterinarians, occurs with remarkable frequency throughout the central, eastern and south-central parts of the United States. It is seen alike in the city and country districts. The facial form of the disease is encountered chiefly in the heavier or medium weight draft horse, while the finer breeds seem to be more susceptible to the form localizing itself in the limbs and resulting in the detachment of ligaments and tendons. This latter form is rare in the heavy breeds but is sometimes seen in the small grade horse such as is used for light delivery purposes. Running horses, trotters, hunters, coach horses, drivers and saddle horses seem to be most susceptible to the disjunction of binding ligaments and supporting tendons, probably owing to the severe strain to which these structures are subjected as the disease slowly weakens the stability of their attachment to the bones.

In both forms of the disease, the premonitory symptoms are alike. There is always the incipient period manifested by mysterious, migratory and remittent lameness which can usually be located in the complicated articulation, such as the coffin joint, the fetlock, the tarsus, the stifle, the hip or the shoulder. It is somewhat more common in the hind-legs than in the fore ones. This lameness is accompanied in every case by evidence of listlessness, fatigue or slight anorexia. None of these general symptoms are very well marked; in fact they frequently do not attract the attention of their keepers until careful inquiry is made. The condition of flesh during 
this period of the disease is but slightly affected. The subjects may even grow fat, or at least retain the flesh remarkably well. In the small animals before any other signs of general osseous lesions appear, they become permanently lame from visible lesions of the affected joints, or else break down by the detachment of the tendons and ligaments of the parts most severely diseased, or fracture the bones near or through the articulations. In many cases, especially when the os pedis and os navicular are intensely involved, the wrenching of the tendons and ligaments may be the very first evidence that any disease has existed, with the exception of the mysterious lamenesses already mentioned. In the larger animals early after the appearance of the lameness and the general symptoms of fatigue, the rami of the inferior maxilla are found to be slightly tumefied along the alveolar margin. When examined by palpation a wide, rounded, somewhat tuberous elevation is felt running along the course of the bone just below the molar arcades, both externally and within the mouth. At this stage the superior maxillary may not be perceptibly involved, but as the disease progresses, it, too, will begin to show a tuberous swelling in the concavity between its spine and the nasal arch. Soon this concavity becomes over-full and gives to the patient the physiognomy which gave birth to the American name "big-head." In the still more advanced stage the respirations become audible and the mastication of food becomes first difficult, then impossible. The patient will also show a tendency to remain recumbent for hours, and will often be unable to rise without assistance. Horses cramped in a small, single stall in the recumbent position for hours are especially liable to refuse to rise until given some assistance, or else dragged out into a more favorable place to make the effort. When this stage is reached they soon become emaciated, suffer from complications, and are finally destroyed to relieve a hopeless condition.

The limb localization usually proves fatal or hopeless much earlier in the course of the process, on account of the greater strain to which the affected structures are subjected. If the bones of the head were subjected to the same strain as the bones and tendons of the legs, they, too, would prove the undoing of the patient much sooner. Their inactive function protects them against dissolution, and permits the process of rarefaction to ccntinue until mastication or respiration or both, are so markedly impaired as to bring about the complications which cause death. Osteoporosis, itself is not a fatal disease in any of its phases until some part of the skeleton breaks down and renders necessary the slaughter of the patient to prevent a lingering death from complications.

The cases of osteoporosis manifested in the better class of horses by the detachment of ligaments and tendons, the fractures of bones, or the compound dislocation of one or more articulations, are too numerous to mention. These patients usually leave the stable in very good spirits, usually after a period of rest made necessary for the treatment of lameness. They travel a mile, two miles or even a much greater distance, suddenly fall severely lame in one foot, perspire quite freely from distress, and finally refuse to be urged farther. Often before the driver leaves his seat to investigate the cause, the patient is walking on the posterior surface of the fetlock, comp'etely broken down. Very frequently, before the animal is unhitched from the vehicle one or even two or more legs, may become similarly afflicted. In one case coming to our notice the coachman while unharnessing his torse to lead him to a convenient stable in the neighborhood, found him 
walking on the stumps of thiee metacarpal bones, which had been completely dislocated from the first phalanx and had perforated through the binding ligaments of the skin. Less than five minutes elapsed between the appearance of the lameness and the complete dissolution of the three articulations.

Analogous instances are not rare. Running horses, laid up for a considerable time for treatment of lameness that is usually diagnosed as a traumatic lesion, but which in reality was osteoporosis from the onset, often break down completely in the first race after the rest. The trainers of such horses, knowing that they are unable to withstand the grief of screre training, usually reserve their efforts for a final grand attempt to win, but to their surprise their horse, before running many furlongs, breaks down with compound dislocations of one, two or even three legs.

When the patient begins to break down on the road, and before there i; any marked alteration in the flexion of the joints, the distress, the difficulty to move, the perspiration, and especially the fact that there has been a period of rest, may lead even an experienced diagnostician to mistake the condition for azoturia. .

Treatment.-It is very evident that Dieckerhoff's cases in young horses that were so promptly cured by alfalfa and clover diet, were not cases of generalized osteoporosis, but local tumefactions of dental origin, so common at that age. These cases usually recover spontaneously in two or three months, and never remain permanent even though they persist a whole year. Is it not possible that the clover diet cured another disease and one that night have cured itself? The genuine case of osteoporosis already presenting deformities of the head (and this is the only symptom by which the disease can with certainty be diagnosed) is not readily cured. We have, however, seen perceptible amelioration by a complete change of diet and protracted rest. Country life, for the city horse. with a nominal grain allowance will often do wonders for even an advanced case. Whatever is done, the city horse must be removed from the foul, ill-ventilated stable, the cheap crushed feed diet, and the hard work, or his usefulness will soon be at an end. Medicines accomplish but little, if anything, toward a cure. Arsenic is probably the most potent remedy, but the benefit derived is too often imperceptible to warrant its recommendation too highly.-L. A. M.

\section{DIFFUSED OSTEOPERIOSTITIS.}

Diffused osteoperiostitis is particularly observed in the dog, but may be encountered in the horse. Wiart and Cadeac refer to periostitis of the knees, the hocks, and the cannon bones from strangles, which seem to be of an infectious origin, and of quite common occurrence.

It is a disease that cannot be easily distinguished from that which attacks the dog. In the dog the words "diffuse osteoperiostitis" are applied to an affection of the osseous system characterized by chronic, hypertrophic lesions and 
deformities, located chiefly in the bones of the extremities. Most frequently it is confounded with rachitis, but this is clearly distinguished from rachitis by absence of lesions in the cartilage of conjugation. It has also been called "osteitis deformans."

ETIOLOGY.-This disease seems to be rather frequent in animals of varied ages. Carougeau observed it in a dog six months old, and also in one two years of age, both of

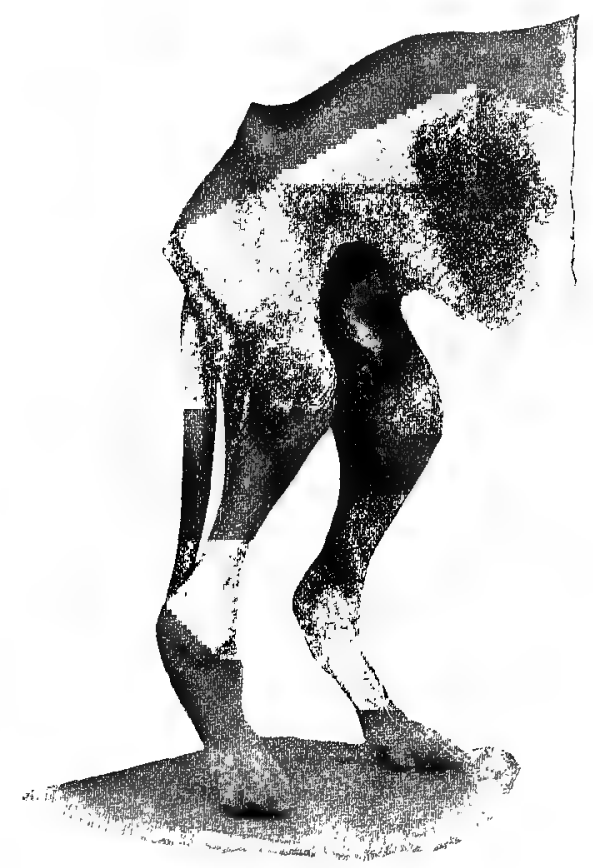

Fig. 102.

Posterior Portion of a Dog Affected with Diffuse Osteoperiostitis.

which were Great Danes. Lienaux observed it in an old hunting dog, and also in a mountain dog ten years old. Identical cases have been reported before the present epoch. Bredin reported a case in the "Annals of French Agriculture for 1809."

It is apparently an infectious disease, and is undoubtedly the relic of some affliction of youth. Moreover, exostoses and hyperostoses can be produced experimentally in rabbits 
by intravenous injections of cultures of micro-organisms found in albuminous periostitis of man. The certain cause of the disease is, however, unknown.

SYMPTOMS.-The patient exhibits a special attitude. The limbs are separated and more or less bent. The vertebral column is arched and the general aspect is that of extreme suffering. The limbs present conspicuous, permanent, deformities, that reach.their maximum at the articular extremities. "The articulations are surrounded by osseous formations which make them large and thick. The. tibio-patellar articulation, the tarsus and the carpus are enlarged, irregular and enormous. The affected area can be easily located by palpation. The surface is hot, but not sensitive. If followed for several months it will be observed to have a slow evolution and an insidious, encroaching mode of progress. It does not seem to shorten life, but make the animal useless.

PATHOLOGICAL ANATOMY.-The macroscopic lesions are located in the osseous system. At the autopsy, the remaining organs seem free from lesions. The skeleton is not all affected. The bones of the limbs are chiefly attacked, but the other parts of the skeleton may also exhibit lesions of osteitis. Lienaux found the vertebræ and the ribs affected. The alterations in the limbs increase progressively and transgress toward the lower part of the extremities. The pelvis and scapulæ show no alterations. The femur and the humerus have exostoses which trail unevenly to connect with the lesions below. The tibia and radius are entirely invaded by exostoses which greatly increase the thickness of the two extremities. The carpus, tarsus, first metacarpals, and first metatarsals are drowned in a veritable osseous gangue of new formation. (Fig. IO4 and Fig. 105).

Generally speaking the longitudinal dimensions of the 
bones are not changed. The modifications affect the transverse diameter. The hyperostoses are exhibited in the form of porous vegetations with uneven surfaces, very irregular, mammillated, granular, and furrowed with narrow fissures. It seems that a great number of bony stalactites may be agglomerated without being soldered together.

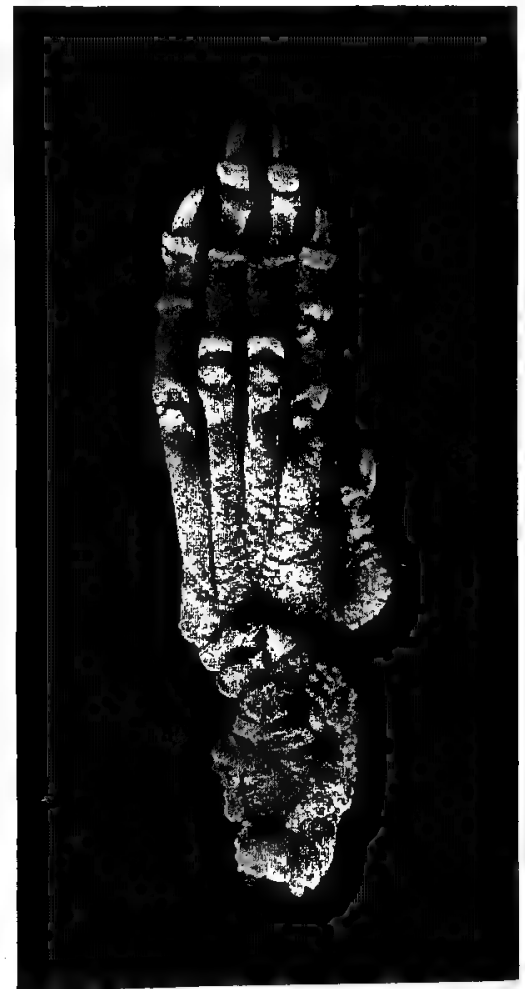

Fig. 103.

Osteoperiostitis of the Tarsus and Metatarsus.

These vegetations are anything but solid. They are easily detached and crushed and thin lamella may be removed from them with a knife. They are spongy. Transverse sections of the tibia, radius, etc., show that the original bone is well formed, and that it preserves its structure, its resistance and its dimensions, but the internal face is incrusted more or less thickly with a spongy bony tissue. 
On the fresh bone the periosteum is thick, resistant and very adhesive. It covers the new formations. The microscopic examination gives but an imperfect account of the manner in which the vegetations are formed. It seems, nevertheless, that they are formed at the expense of the periosteum, which is very thick in the osteogenic layer. Its

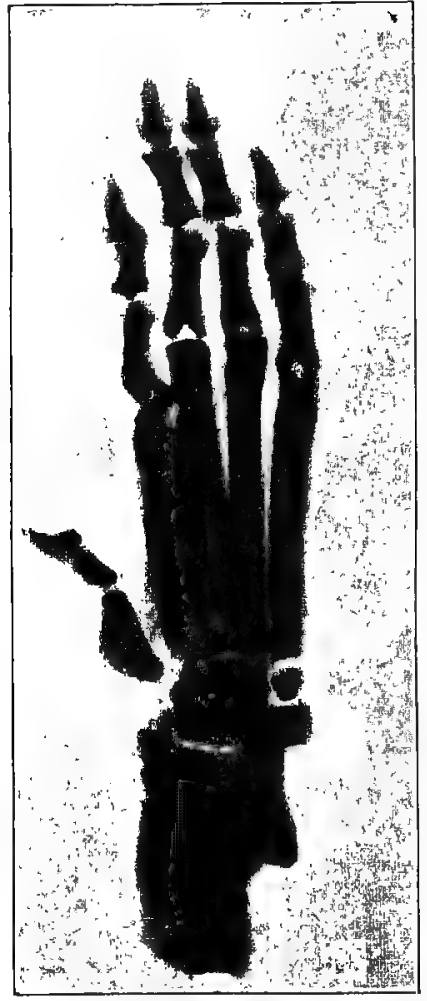

FIG. 104.

Osteoperiostitis of the Tarsus and Metatarsus (Radiograph).

connective fasciculi are set upright,-perpendicular to the long axis of the bone,- - and serve as guiding channels for the deposit of the osseous trabecula. The periosteum is, in fact, composed of osseous stalactites which cling to the surface of the old bone. The radiographs clearly show this arrangement. There is evidence that the bone has preserved its normal characters as regards thickness, direction, 
dimensions of the medullary canal, and that the exostoses are deposited at the surface above the periosteum without apparently sinking below it. The radiograph of a transverse section, three millimeters thick, made at the upper part of the metacarpus is especially demonstrative on this point. These characteristics do-not justify the confusion of this disease with rachitis. In the latter there are deformations of the original bone and grave deteriorations of the cartilage of conjugation, which ultimately substitutes a peculiar osteitic tissue in lieu of bone.

What is the nature of diffuse osteoperiostitis? Only hypotheses can be advanced on the subject. It has been compared to osteitis deformans, or Paget's disease of man. This disease, which has sometimes been regarded as being an appendage to chronic theumatism, and of acid dyscrasias, is today usually regarded as the consequence of trophic disturbances of the nervous system. No investigations have been made in the dog to establish the relations of diffuse osteoperiostitis with a nervous lesion.

TREATMENT.-No effective treatment is known.

\section{ACHONDROPLASIA IN THE CALF.}

In human pathology the word "achondroplasia" is applied to an affection of the fotal osseous tissue, manifesting itself by the arrest of the development of the long bones. The existence of the disease in animals has already been mentioned, but its origin still remains a mystery. It is supposed to be a dystrophia of enchondral ossification, reference to which will be made in the following description.

HISTORY.-Before the present era, achondroplasia was not known by its proper name in veterinary pathology. Animals affected with the disease were described as bulldog calves, or turtle-calves, on account of their rather vague resemblance to these animals. The disease has attracted 
attention since $I 750$, at which time Daubenton referred to it in Buffon's Natural History. After Daubenton, Offa, Owen (1853) and Dareste (1867) referred to the existence of the disease. The latter described it in a course of lectures while connected with the Faculty of Sciences in Lille.

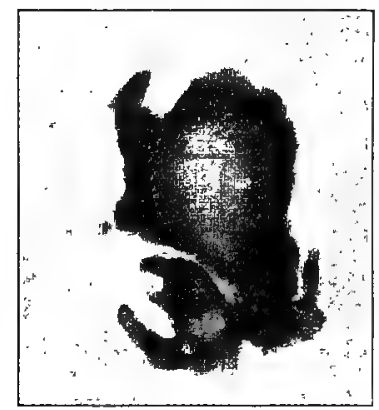

FIG. 105.

Section of the Extreme Upper Portion of the Tibia.

Later, Delchambre, Barrier, Petit and Delplamque published more or less complete accounts of the affection.

DESCRIPTION.-Subjects attacked with achondroplasia are never well developed. They appear to be shortened in the anteroposterior direction, the anterior part of the

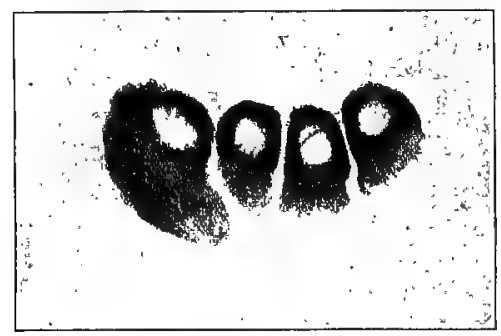

FIG.106.

Section of the Upper Part of the Metacarpus (Radiograph).

body being, as usual, better developed than the posterior. The bones of the limbs are small and their imperfect development consists chiefly of a shortening of the bones radiating directly from the vertebral column. As a general rule achondroplastic subjects are micromeli. The limbs may have their normal direction, but frequently, they are more 
or less out of position and even turned in the opposite direction. The plantar surfaces of the hoofs may be turned forward or attached to the trunk like the paws of a turtle. The head is generally strong, but shortened. The eyes, ears and forehead are normally developed, but the inferior maxillary is curved and projects forward beyond the upper jaw, which is very short. The snout seems to be attached to the frontal bones, the nasal arch being rudimentary. The tongue is held outside the buccal cavity and rises toward the nose. The head resembles a mutilated cone with the basis constituted of the inferior maxillary. It gives the impression of a bulldog's head, [or of a case of exaggerated prognathism. -L. A. M.] The body is short, but normal and plump, with prominent elevations in the region of the shoulders and buttocks. The tail is short and undeveloped. The muscles are slightly modified. The flexors are short and all of the muscles are hard as if in a state of contraction. In reality, there is no contraction, as Petit and Dechambre have shown.* The study of the skeleton shows that only the bones of the head and limbs are involved in the arrest of development. The vertebral column and the ribs are perfect in their conformation. In the head the superior maxillary and the nasal bones are particularly, affected. The teeth are normal and normally inserted on both jaws. They may deviate slightly on the inferior. The most important alterations are located in the bones of the limbs. The scapula contains its complementary cartilage, but is shorter and thicker than in the normal state, and its articular cavity has a diameter almost equal to that of the bone. The humerus is reduced to an osseous disc interposed between two very wide articular surfaces. The transverse diameter is almost equal to the length of the bone. The radius and ulna ex-

*These lesions are frequently associated with an anasarca, probably myxœdema. 
hibit analogous alterations. On reaching the carpus the bones seem to resume their normal physiognomy. The metacarpals and the phalanges do not seem to have been involved in the process of arrested development. The same description answers for the corresponding bones of the pos-

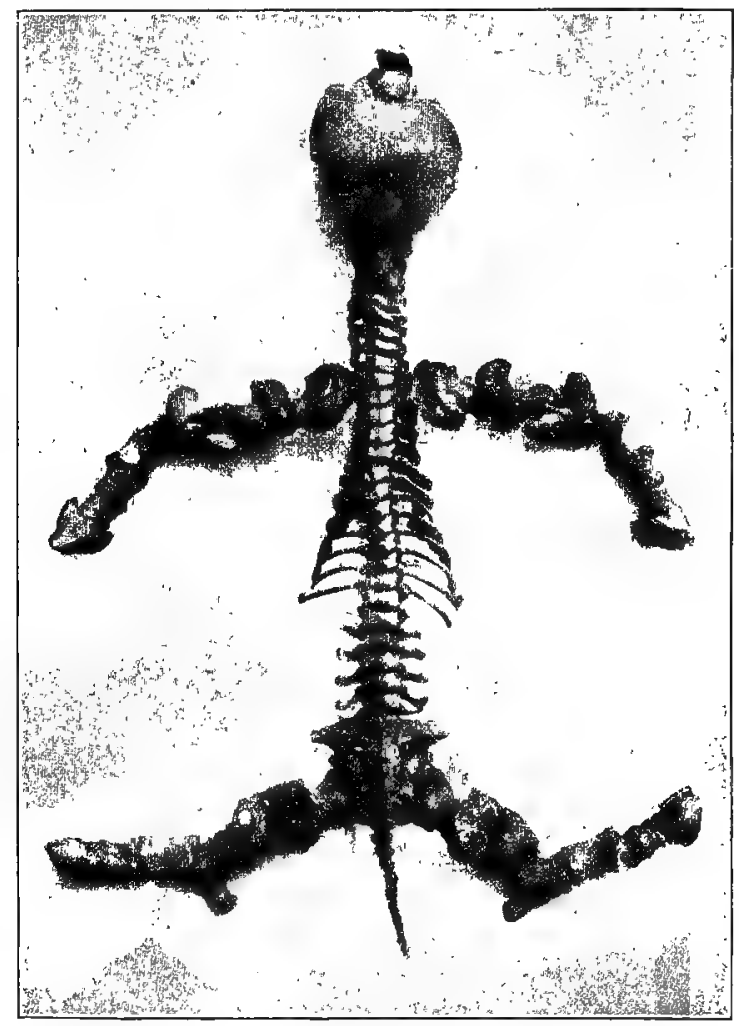

FIG. 107.

Skeleton of a Calf Affected with Achondroplasia.

terior extremities. (See Fig. T05). The dystrophia affects the pelvis as well as the femur and the tibia, and their physiognomy is analogous to that of the fore-limb.

The deformed bones have no epiphyseal center and no cartilage of conjugation, which circumstance prevents their development after birth. These bones are hard, compact and resistant, like well developed ones, showing that the 
case is not one of fotal rachitis. Independently of the fact that the latter is very rare, it is not manifested by analogous lesions, but by osseous incurvations and a special constitution of the bones, which are first fibrous then osseous. Neither is it a case of contracture.

Dareste has shown that the affection is a lesion of a mechanical order caused by amniotic compression. As it is impossible to connect other osseous lesions with those of bulldog calves, further research as to its nature must be made.

PATHOGENESIS.-The cause of achondroplasia is still unknown, although some light has been thrown on the subject recently. It is well known that the long bones, cartilaginous in the fotus, are transformed into osseous tissue by two processes,- - periosteal ossification, which enlarges the transverse diameter, and enchondral ossification, that lengthens them. It is readily seen that the first process is not altered, as the bones have the normal diameters. Accordingly the cause of the alteration must be connected with enchondral ossification. Achondroplasia has been observed chiefly in Normandy calves. Fourreau met with a total of seventeen cases, the mothers of which had all been served with same bull. Parturition is usually easy, only occasionally proving rather annoying to the obstetrician.

\section{SNIFFLING DISEASE OF THE HOG.}

DEFINITION.-Sniffling disease is a microbian affection manifested by fibrous transformation of the bones of the head, especially the maxillaries. It is a chronic affection showing no tendency to recover, and invariably terminating fatally.

HISTORY.-The first account of the disease must be credited to Hurtreld and Arboval, who reported an outbreak in the Duchy of Nassau in 1832. Frodal, the first 
writer to mention it, in a few lines, describes it as a catarrh of the nasal fossa and tissues bounding them. Benion gave an account of some interesting cases, and Lafosse refers to instances in which the nasal cavities were closed to the extent of preventing respiration except through the mouth, which was constantly kept open. Leclainche merely mentions the disease incidentally in his article on rachitis. In I897, M. Petit exhibited the head of an affected hog before the Societe Centrale de Medicine Veterinaire. Finally, in I89o, Struble described a case under the name of "Osteosarcoma of the jaws of a hog" that was an absolutely typical example of sniffling disease. Dor and LeBlanc during I90I several times drew the attention of veterinarians to the disease, and they found a microbe in the lesions that they regarded as the specific agent.

On account of the numerous analogies,-clinical, anatomo-pathological and bacteriological,- - that exist between sniffling disease and rhinoscleroma of man, the two pathological states were compared. Dr. Grenier made this comparison the subject of his thesis at Lyons in Igor. In a review published in 1890 by $M$. Joly, in the Presse Veterinaire, the following information anent the existence of the disease in Germany has been collected. Cases have been observed by Haubner, Haubold and Harms. I878, Schneider saw cases which he regarded as nasal catarrh of traumatic origin. Harthmayer observed it in hogs of English breeds, and Anacker claims that Schnufelkrankheit is but a malignant nasal catarrh that has been erroneously confounded with osteomalacia. Hering advanced the opinion that the disease is a combination of cachexia and scurvy. Freidberger and Fröhner, under the picturesque name of "schnufelkrankheit" suggest in their work that the disease of the hog is a proteiform affection that sometimes resembles rheumatism and sometimes chronic nasal catarrh, either hæmor- 
rhagic or purulent, and finally that it is tuberculous or actinomycostic in character. Only a few years ago, Imminger published a new study of the disease, in which he claims that schnufelkrankheit of the hog is a perfectly characteristic disease, observed in different forms in Bavaria. He regards it as an infectious rhinitis. Under the name of rachitis, Wulf describes lesions absolutely characteristic of this disease.

In France, sniffling disease appears to exist in several parts of the country. Information gathered on the subject

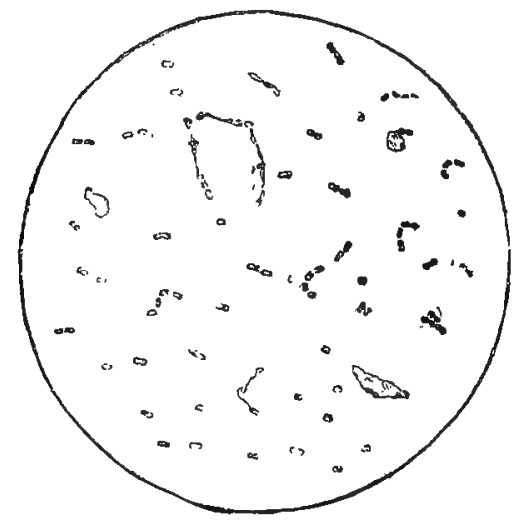

FIG. 108.

Aspect of a Çulture in Bouillon after Forty-Eight Hours (570 Diameters). (LeBlanc).

shows it to be frequent in Ardennes, L'Ain, LaNievre and L'Indre, and we owe our correspondents an argument on its microbian nature. In the Department of La Nievre the disease was rare fifteen years ago, but during the past three or four years the cases have been more numerous, and the mortality is increasing. When the disease first makes its appearance in the pigsty it can frequently be traced to the importation of animals from infected herds.,

EXPERIMENTAL STUDY.-The tissues harbor a thick, short bacillus, found by microscopic examinations of scrapings of the fibrous tumor stained with a hydro-alco- 
holic solution of gentian violet. The preparations thus obtained are usually quite clear, but it may be necessary to prepare several specimens to prove that each one contains the same micro-organism. The microbian agent can be cultivated in bouillon, on gelose, on gelatine, on potato and on carrot. In bouillon the culture is rapid at $38^{\circ} \mathrm{C}$. After sixteen hours a marked disturbance of the media is already apparent, and the turbidity accentuates for forty-eight hours, after which the growth seem to stop. The cultures are deposited in the bottom of the flask in the form of an abundant white coating. At no time does the bouillon give an acid reaction. A study of the micro-organism thus cultivated reveals a diplobacillus, thick, rather large, round at the extremities and reproducing itself by direct division. It

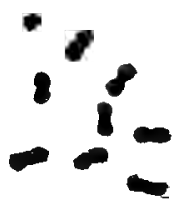

FIG. 109.

The Same Agent Considerably Enlarged (1200 Diameters).

is easily stained with the various aniline stains, especially gentain violet. It is only imperfectly stained by Gram's method. (Fig I09). In the bouillon the micro-organism has but a short life. After three or four days it presents forms of involution somewhat similar to that of the bacillus of leprosy. On gelose the culture is abundant after twentyfour hours. It is irregular, yellowish and oily in appearance. On gelatine the culture is slow and stretches out in the form of greyish white discs with raised edges and projecting centers, and it never liquefies it. On potato the culture is abundant after twenty-four hours and.is composed of a large number of isolated colonies. On carrot the culture is homogeneous, whitish, oily and with a moist surface. 
PATHOLOGICAL REACTION.-The pathogenic agent is non-pathogenic to the cavy, the dog, and the sheep from subcutaneous injections. In the rabbit it is non-pathogenic from subcutaneous or intraperitoneal injections. In the hog it seems to produce the disease spontaneously from subcutaneous inoculations of the face.

SYMPTOMS.-The disease often commences with lameness, the result of multiple arthritis, attacking the complicated articulations having wide articular surfaces, like the femuro-tibio-patellar joint. The articular regions are doughy, enlarged, painful and grow rickety. The articular inflammation may diminish and completely disappear or may accentuate every day until the limbs become deformed. The muscles atrophy from disuse. The patients lie continually and move about only on the knees or haunches to gather their food.

Simultaneously, or after the arthritis has disappeared, the sniffling becomes audible, and an examination reveals lesions of the face, consisting of symmetrical, firm and extended tumefactions along each side of the median line of the head. There is no mucous discharge from the nostrils, but the lesions of the face become accentuated. In some instances the inferior maxillary alone is affected, but usually it assumes dimensions proportionate to those of the superior maxilla. (Fig. I Io.) The sniffling may precede the local lesions by three months. The latter develop imperceptibly and the veterinarian is only consulted when the patient exhibits the following alarming symptoms: The entire maxillary region is swollen, glittering, of a milky whiteness and nearly bereft of hair. The forehead is prominently raised at the frontal arch. The sides of the frontals and the other cranial bones are not implicated. The inferior maxillary is also much tumefied. Its branches are dropped and do not reach the anterior extremity of the premaxilla by several 
centimeters. The superior as well as the inferior incisors are well developed. The superior molars project outward and the inferior arcades are vertical and prominent, and press against the enlarged palate. In profile the palate in its arched condition resembles a prow or the sole of a shoe. The patients find difficulty in breathing. The air can scarcely pass through the nasal cavities and causes the characteristic sniffing sound loud enough to be heard at some

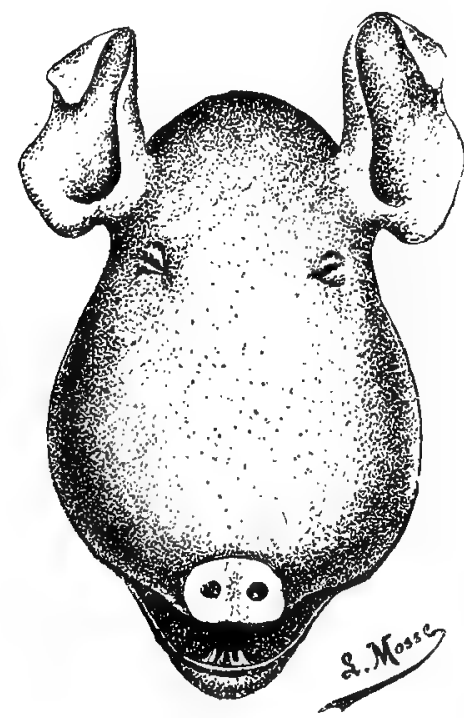

FIG. 110 .

Hog's Head Enlarged Inferiorly (Mathis and LeBlanc).

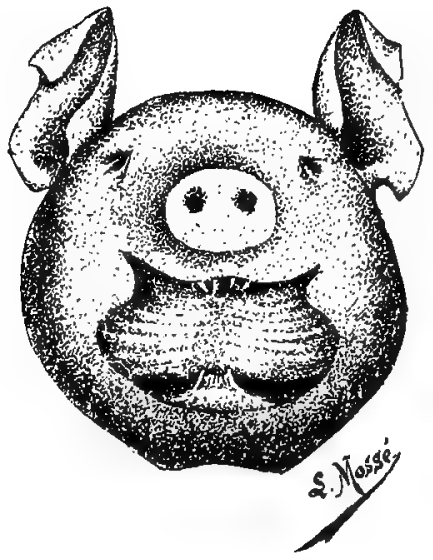

FIG. 111.

Hog's Head Showing Bulging of the Palate.

distance. Expiration occurs through the mouth. The movement of the ribs is difficult, and is out of harmony with that of the flanks. From time to time seizures of suffocation occur, during which the respirations are carried on only through the mouth.

As a consequence of imperfect hæmatosis the skin and mucous membranes become cyanotic, and although death may seem near at hand calmness is restored after a few hours and the skin again resumes its normal color. In many cases the capacity of the mouth is so reduced that the 
patient is unable to keep the tongue within, and as a result it becomes the seat of wounds that show no inclination to heal. Deafness may be observed, but when the patients reach the stage just described they are generally slaughtered, or found dead in their sty.

Previous mention that the disease is preceded by arthritis necessitates the statement that the facial lesions and the arthritis may develop absolutely independently of each other. Whether the joint localization is due to microbian externalization, cannot be affirmed until the provocative micro-organism of the facial lesions is found in the articular cavities.

PATHOLOGICAL ANATOMY.-The only alterations concerned in the evolution of the disease are confined to the head. Besides the articulations, - and they are not always affected,- - the remainder of the skeleton is normal. The spinal column, the ribs, the pelvis and the long bones are not implicated. The physiognomy of the facial tumor resembles the tumor of rhinoscleroma of man as much as two lesions in different species can. The descriptions of Carnil and Alvarez in "Archives of Physiology" apply, without modificatioin, to sniffling disease of the hog.

The nasal cavities, the septum nasi, the turbinated bones, and the ethmoid cells are absolutely free from alterations. There is no lesion in these structures. The mucous membrane is normal, smooth and free from inflammation or coating of any kind. This authenticated fact is not without interest from the standpoint of the seat of inoculation. The new tissue is formed at each side of the nasal fossa and below them, and its extension compresses, contracts, and displaces them from below upwards. They are all the more narrow toward the posterior nares. The transformation of the nasal cavities into narrow cell clefts sufficiently explains the difficult respirations. 
On transverse section of a diseased head the osseous wall is seen to have disappeared and to have been replaced by a circular area of new tissue that forces everything back. (Fig. I I 3). The new tissue is of a lavender shade and studded with a number of hæmorrhagic dots. Its consistency is firm and fibrous, and when pressed a limited quantity of clear serosity exudes. The neoplasm is equally developed on each side and extends from the anterior nares to the pharynx, gradually increasing in size posteriorly. The bony boundary of the nasal cavities has completely disappeared, and the niucous membrane is in close contact with the subjacent neoplasm.

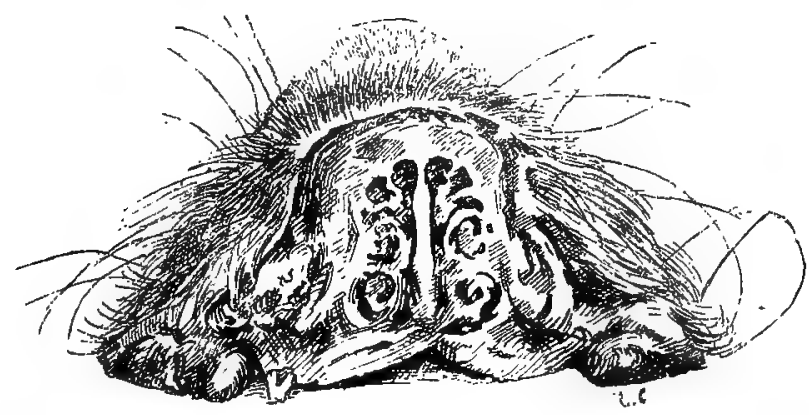

FIG. 112.

Transverse Section Made Just Behind the Snout of a Normal Hog.

The tissue of the tumor appears to be homogeneous through the entire length of the maxillary bones. The teeth are rather solidly implanted in the fibrous bed, but they are generally bent sidewise. The alterations in the superior maxillary are found, also, in the inferior maxillary, which circumstance is important from the standpoint of the nature and origin of the disease, in that it leads to the inference that the inoculation does not take place in the nasal mucous membrane, but probably through erosions of the buccal mucosa. The examination of the inferior maxillary discloses almost complete disappearance of the osseous tissue, separation of the osseous lamellae and the displacement of the cancellated 
tissue by a fibrous neoformation, having absolutely the same microscopic physiognomy as the tumor of the nasal cavities.

A hurried microscopic study of the disease made by Professor Mathis some years ago has shown that the diseased bones are composed chiefly of fibrous tissue harboring in its midst lamella, splinters and tracts of osseous or cartilaginous substances which cross one another. Recent investigations have proven that the lesion from a histological point of view is similar to that of rhinoscleroma of man, with the exception that Mickulicz's cells, which have been regarded as the specific elements, are wanting. As this feature is, however, not absolutely characteristic, it remains to be seen how much importance to attach to it. Mickulicz himself has stated that too much importance must not be attached to the elements bearing his name. The characteristic microbian agents of the disease can not be detected in sections of the tumor, but in view of their form in the lesions this circumstance is not astonishing.

Returning to the original-question it may be said that the tumors are almost always exclusively fibrous, consisting of adult tissue meagerly supplied with cellular elements in the deep layers and new tissue rich in active elements at the periphery, and in certain regions related to fibrous bands that seem to emanate from the remains of the periosteum. The neoplasm is surrounded by a layer of new fibrous tissue in the course of active proliferation, and at certain points on its inner aspect may be found cartilaginous centers the fundamental substance of which does not stain at all, or stains badly, and its cellular elements appear to be surrounded with a rather conspicuous capsule. The peripheric fibrous layer, probably of periosteal origin, sends extensions into the neoplasm that divide it into compartments. These secondary septa again send projections into the depth of the tumor, which are the seat of an active proliferation. 
Macerated fragments very clearly give an idea of the nature of these alterations and their initial seat. The changes are chiefly manifested on the superior maxillary and nasal bones. The other bones of the head, with the exception of the inferior maxilla, remain normal. The diseased part is constituted of osseous interrupted layers, excavated with furrows which in life are filled up with fibrous tissue and layers oi cartilage, a vestige of which still remains. So far as the appearance is concerned the diseased portion may appropri-

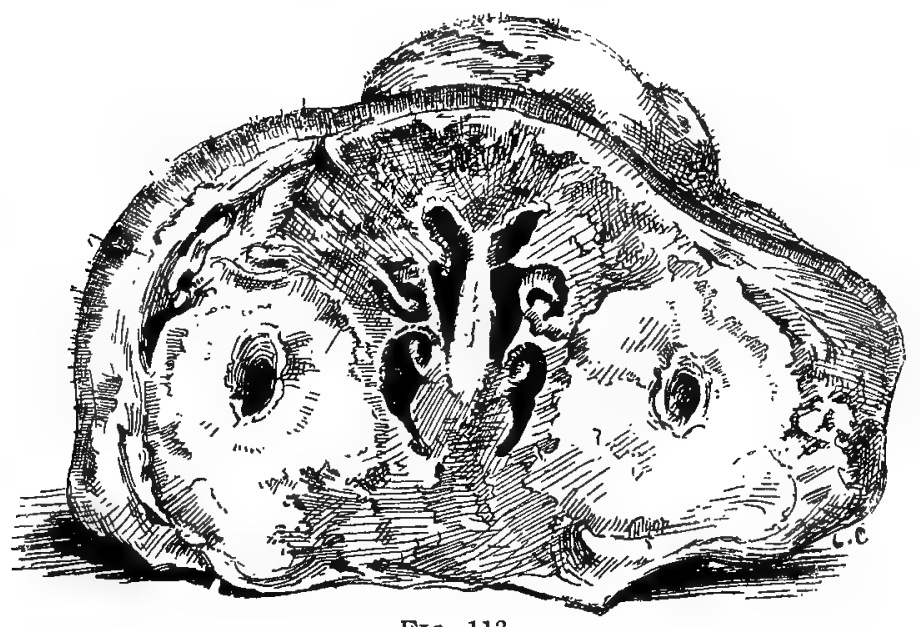

FIG, 113.

Transerve Section Behind the Snout of a Hog Affected with the Disease. A Tumor Containing a Dental Follicle is Shown on Each Side of the Nasal Fossæe.

ately be compared to a sponge. It appears as if a sponge had been pasted on each side of the septum nasi in front of the orbital ridge. The swelling of the bones, which might be described as "blown," causes an almost complete obliteration of the nasal cavities. (Fig. II4). The inferior maxillary exhibits analogous, but less pronounced alterations. The lesions of the joints attack different portions of the articulations, the periarticular tissues, the synovial membranes, the synovia and the articular cartilage.

I. The Periarticular Tissues.-The muscles surround- 
ing or passing over the diseased articulations are degenerated, pale, fibrous, and retracted.

2. Synovial Membranes.-The synovial membrane, instead of being as thin as a cigarette paper, is always considerably thickened. It is fibrous, fibro-cartilaginous, hard, whitish, glittering and poorly supplied with vessels on its inner face.

3. Synovia.-The synovial liquid is neither purulent nor turbid, but is thick, oily and sticky.

4. The Articular Cartilage.-The lesions of the cartilage are the most interesting. They are wrinkled and folded in the direction of their long axes. It seems that their limited

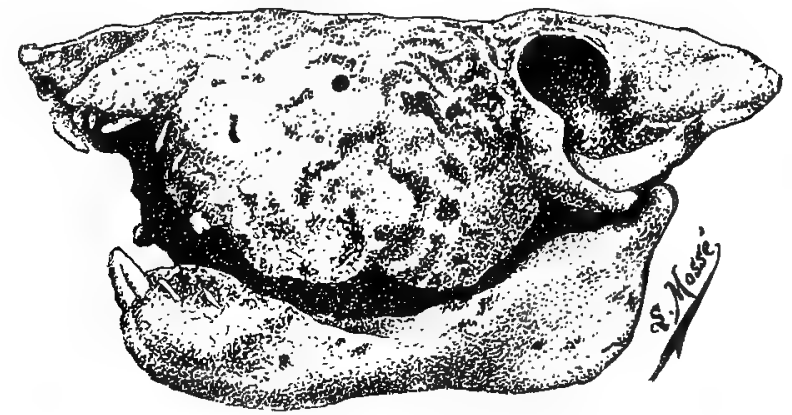

FIG. 114.

Macerated Head of a Hog Attacked with the Disease.

development has not kept pace with length of the subjacent bone. At certain points it is worn or even ulcerated into the osseous tissue. When the lesions are not very old there is no epiphyseal periostosis.

DIAGNOSIS.-The disease may not be recognized for some time after its first appearance. The occurrence of arthritis in communities where the disease exists, and the sniffling, will generally tend to promote a correct diagnosis, and when the convexity of the face appears no confusion is possible.

PROGNOSIS.-The above description justifies a brief consideration of the prognosis. The disease is, in fact, one of $l$ 
slow progress and fatal termination. It leads to death from asphyxia, inanition, or broncho-pneumonia. In other cases various pathological conditions join in precipitating the end.

TREATMENT.-There is actually no known medical treatment. Prophylaxis, alone may be beneficial. Young hogs should not be purchased where the disease exists. Affected hogs should be isolated and the healthy ones kept in the best hygienic conditions. Hygiene seems to play a very conspicuous part in turning the tide of the disease. In fact the disease is only found in communities where hygiene is bad or rudimentary. 


\section{INDEX}

Abnormal Hoof. ........... $\quad 36$

Abscess................ 345

Abscess-Thecal of the Horse.. . 364

Abscesses-Acute.......... 350

Abscesses-Canaliculate. . . . . 374

Abscesses-Chronic.......... 358

Abscesses-Cold............ 358

Abscesses-Deep........ . . 351.

Abscesses-Hard. . . . . . . .359-360

Abscesses-Hot.......... . 350

Abscesses-Soft........... 360

Abscesses-Superficial. ...... 350

Achondroplasia in the calf . . .... 643

Arid Dyscrasia............ 603

Actinomyces Bovis.......... 134

Actinomycosis - Geographical

Distribution............ 491

Actinomycosis-Myelogenic... 503

Actinomycosis-Ovine....... 517

Actinomycosis-Periosteal.... . 503

Actinomycosis-Porcine..... 514

Actinomycosis of the Jaw...... 501

Actinomycosis of the Lips.... $\quad 506$

Actinomycosis of the Pharynx. 505

Actinomycosis of Soft Parts of

Face and Neck......... 505

Actinomycosis of the Tongue... 502

Actinomycosis-Lingulal. . . . . 512

Actinomycosis-Maxillary ..... 513

Actinomycosis-Definition, History, Microscopic Examination, Inoculation, Etiology, Pathogenesis, Symptoms,

Treatment..........490 to 518

Actinomycosis-Bovine...... 501

Actinomycosis-Cutaneous. ... 513

Actinomycosis-F, :ine...... 517

Acute Abscesses. . . . . . . . . 350

Acute Purulent Oedema. ..... 387

Acute Septicæmia,. . . . . . . 380-384

Acute Tetanus......... .469-471

Air-The.............. 25\%

Alcohol, Ether. Chluroform.... 238
Alexin.............. . 45-161

Amboceptor........... 162-167

Ameboid Movement......... 20

Amitosis.............. $\quad 16$

Amputation Neuroma........ $\quad 31$

Analysis of the Bones. . . . . . . 632

Anasarca............... 71

Anæmia................ 330

Anæsthesia.............. 223

Anæsthesia-Contra Indications for ............... 245

Anæsthesia-Duration of ...... 230

Anæsthesia $\leftarrow$ Indications for

General ............... 244

Anæsthesia-Local......... 247

Anæstheria-Partial General... 246

Anæsthesia-Stages of . . . . . . 228

Anæsthetics..............223-237

Anæsthetics-Local......... 247

Anæsthetics-Hypodermic..... 237

Angioma............... 578

Anæsthetics-Mode of Adminis-

tration.............. 232

Anthracite Bacillus... . . . . 141

Anthracis Emphysematosa Bacil-

lus .............. 146

Anthracis-Symptomatici Bacil-

lus ................ 145

Anthrax.............. 143

Anthrax-Bacteridian....... 145

Anthrax-Symptomatic...... 146

Antisepsis............... 254

Antiseptics-Their Containers. , 263

Antitoxin......... 121-156 168

Antitoxin-Preformed,...... 452

Arrest of Hæmorrhage. . . . . . . 192

Arterial Hæmorrhage. . . . . . 188-331

Arterial Sclerosis .. . . . . . . . 66

Arthrosis-Pseudo.......... 28

Arthrospores............. 103

Articular Cracking. . . . . . . $\quad 606$

Ascites,.............. $\quad \mathbf{7 1}$

Ascites of the Dog.......... 74 
Asepsis. . . . . . . . . . . . . . 254-278

Asepsis-Practical. . . . . . . . . 278

Aseptic Fever. 56

Aseptical Operation - Recommendations for an...

281

Asphyxia-Toxic.. . . . . . . 239

Atonic Ulcers, . . . . . . . . . . . . 369

Atrophy............... 82

Atrophy-Circumscribed...... 82

Atrophy-Diffused.......... 82

Atrophy-General. . . . . . . . . 82

Attop'yy-Ischemic..... . . 82

Atrophy-Local........... 82

Atrophy-Nervous......... 82

Atrophy-Pathologic........ 82

Atrophy-Physiologic... . . . 82-84

Atrophy-Pressure. . . . . . . . . 82

Atrophy-Senile........... 82

Auricular Canker of Dog....... 367

Azoturia-Crural Paralysis of. . 87

Bacteria.............. 88

Bacteria-Acid-forming...... 94

Pacteria-Aerobic.......... 100

Bacteria-Aerogenic.......... 94

Bacteria-Alkali-forming. .... 99

Bacteria-Anaerobic.......... 100

Bacteria-Chromogenic....... 95

Bacteria-Facultative. . . . . . . 100

Bacteria-Microscopic Exami-

nation of ............ 110

Bacteria-Non-Pathogenic .... 100

Bacteria-Parasitic......... 101

Bacteria-Pathogenic... . . . 95-100

Bacteria-Photogenic....... 95

Bacteria-Reproduction.... . . 102

Bacteria-Saprogenic........ 94

Bacteria-Saprophytic....... 101

Bacteria-Surgical......... 111

Bacteria-The Cultivation of . . : 103

Bacteria-Zymogenic......... 94

Bacilli................ 93

Bacilli-Tetanus........... . 97

Bacillus Anthracis. . . . . . . 141-143

Bacillus Anthracis Emphysema-

.tosa................... 146

Bacillus Anthracis Symptomatici 145

Bacillus. Edematis Maligni. . . . . 149

Bacillus Chatuvaei, . . . . . . . . . 146

Bacillus Coli Communis. . . . 121-348

Bacillus-Colon.... . . . . . . . 122
Bacillus of Friedlander, . . . . . 437

Bacillus-Hay.............. 426

Bacillus Mallei............ 127

Bacillus Nicolaier. . . . . 134-421-427

Bacillus Pyogenes Fotidus. . 122-348

Bacillus Rauschbrand....... 146

Bacillus Strbtilis . . . . . . . . . . 436

Bacillus Tetani............, 134

Bacillus Tuberculosis........ 135

Bailing. . . . . . . . . . . . . 198

Benign Tumors............. 563

Big Head. . . . . . . . . . 615-636

Bit Gnathitis.... . . . . . . . 64

Black Leg.. . . . . . . . . . . . . . . . 146

Black Liver of Russian Sheep. . 560

Black Quarter............. 146

Blistering of Muzzle... . . . . . . 244

Blood-Coagulability of the... . 193

Blood-Coagulation of ........ 76

Blood Corpuscles - Nucleated

Red.................. 46

Blood-Corruption of the...... 323

Blood-Current-Retardation of

the ................. 18

Blood-Sac............... 316

Blood Tumor.............. 316

Blood-Washing the........ 386

Blood-Vessels.... . . . . . . . . . . 22

Bone ..................24-26

Bone-Necrosis of........... 68

Bone--Spongy ............. 27

Bones-Analysis of the...... 632

Botryomycosis Equi........124-518

Botryomyces-Definition, His-

tory, Etiology, Pathogenesis,

Treatment..........518 to 525

Botryomycosis in the Hog. . . . . 524

Botrymycosis in the Ox...... 523

Botryomycosis of the Horse.... 521

Bovine Actinomycosis........ . 501

Bran Sickness............ 615

Bridge-Temporary......... 26

Brieger-Toxalbumin of....... 442

Bronzed Erysipelas...... . . . , 387

Bulldog Calves............. 643

Burns. . . . . . . . . . . . . . 320

Burns-Circumscribed... . . . . 322

Burns-Diffused . . . . . . . . 322

Btids-Muscle............ 29

Calf-Achondroplasia in. . . . . 643 
:allous Ulcer..... . . . . . . . 369

vallus............... 26

.alves-Bulldog. . . . . . . . . 643

'alves--Turtle............ . 643

¿ancer............. 563-568

Yancer of Cheek........... 501

'anker-Auricular of Dog. . . . 367

'apillary Hæmorrhage. . . . . 190-331

Jarcinoma. . . . . . . . . . . . 569

Jartilage............ 24-26

Jasting Harness-The........ 211

Jauterization............ 198

zells-Epithelioda1......... 21

Yells-Fat............. 25

'ells-Formative. . . . . . . 23-26

Sells-Giant............. 21

Yells-Mast. . . . . . . . . . . 21-46

¿ells of Sheath of Schwann .... 31

Yells-Plasma............ 21

¿entric Paralysis........... 85

¿erebral Tetanus........... 450

thanges in the Fixed Cells or

Tissue. . . . . . . . . . . . . 47

thanges-Senile........... 82

Shanges-Vascular....... . . 43

'hicken-Fat Thrombus. . . . . . 77

'hilblains............... 326

ไhloral Hydrate............ 238

'hloretone.............. 238

'hloroform. ........... 237

לhloroform-Dementia........ 244

'hloroform-Physiological Ac-

tion of . . . . . . . . . . 225

thondroblasts.......... . 24-26

shondroma............ 575

'hronic Abscesses. . . . . . . . . 359

hronic Septicæmia. . . . . . 380-384

'hronic Tetanus. . . . . . . . . . 472

icatrices of the Skin....... . 333

icatricial Scar............ 21

icatrix. . . . . . . . 21-337

icatrix-Horn. . . . . . . . 38

icatrization. . . . . . . . . . 290

ircumscribed Atrophy....... 82

:ircumscribed Burns. . . . . . . 322

laws................ 35

:lot............... 76

Joagulability of the Blood..... 193

'oagtulation of the Blood...... . 76

yoagulum............. 76

jocaine-Action of. . . . . . . 249
Cocainization-Spinal...... 253

Cocci................... 93

Cold Abscesses. . . . . . . . . . 359

Coli Communis-Bacillus.... . . 121

Collection................ 345

Colon Bacillus............. 122

Combustion............. $5_{2}$

Complement...........45-161

Complementophilic Haptophore 170

Complex Tumors........... 580

Compression-Direct....... 195

Compression-Indirect....... 196

Congelation............ 326

Connective Tissue.......... 22

Container-Study of the. . . . . 354

Contractibility of Vessels...... 194

Contra Indications for General

Anæsthesia............. 245

Contused Wounds.......... 308

Contusions............. . 314

Copper Salts in Treating Actinomycosis .......... 517

Coronary Cushion........... 36

Corruption of the Blood ....... 323

Cracking-Articular. ......... 606

Cultivation of Bacteria........ 103

Current Jelly Thrombus....... 77

Cutaneous Actinomycosis. . . . . 513

Cushion-Coronary......... 36

Cutaneous Scars........... 333

Cysts................. 581

Cysts-Dermatcid.......... 581

Cysts-Serous............. 581

Cytase............... .45-161

Cytophilic Haptsphore. . . . . . 170

Decubitus............... 64

Deep Abscesses........... 351

Deformities of the Head...... 623

Degeneration-Senile........ 82

Demarcation-Line of ...... 66

Detachment of Ligaments and

Tendons.............6621

Dermatitis-Gangrenous...... 63

Dermatoid Cysts........... 581

Diabrosis............... 187

Diapedesis. . . . . . . . . . . 44-46

Diaphoresis............. 60

Diffuse Rarefying Osteitis.... 615

Diffused Atrophy. . . . . . . . 82

Diffused Burns............ . 322 
Diffused Osteoperiostitis-Etiology, Symptoms-Pathological Anatomy - Treatment 638 to 643

Direct and Indirect Healing. . . . 17

Direct Syncope. . . . . . . . . . . 242

Disuse................. 84

Dog-Ascites of the........ . 74

Dog-Melanosis in the....... 561

Dropsy............... 71

Dry Gangrene........... $\quad 67$

Dyscomyces.

518

Ecchymosis of the Skin...... 315

Effusions-Oleaginous....... 318

Effusions-Primary Serous.... 317

Ehrlichs Theory ........... 165

Elastic Fibers. . . . . . . . . . 25

Elastic Tissue............. 25

Emaciation.............. 82

Embolic Pneumonia.......... 79

Embolic Septicrmia.......... 381

Embolism................ . 76

Embolus................ $\quad 76$

Emigration............ 19-44

Emphysema-Traumatic...... 332

Emprosthotonos. . . . . . . 468-469

Enchondral Ossification....... 647

Encroaching Gangrene. . . . . . . 387

Endospore............... 103

Endothelial Tubes........... 22

Entrorrhagia.............. 188

Envenomed Wounds.......... 303

Epidermization-Insular...... 34

Epistaxis................ 188

Epithelial Tumors. . . . . . . . . . 566

Epithelioidal Cell. . . . . . . . . . 21

Epithelioma............. 568

Epithelium............ 33

Epitraumatic Fever... . . . . . 382

Equine Actinomycosis. . . . . . 517

Erysipelas.............. 375

Erysipelas-Bronzed. . . . . . 387

Ether................. 238

Ether-Physiological Action of. 225

Ethyl Bromide............ 238

Ethyl Chloride............. 238

Excitement-Stage of . . . . . . 228

Exudates............... 46

Exudation.............. 19

Exudative Inflammation...... 17
Face of Hog-Convexity of.... 657

Fat.................. 25

Fat Cells.............. 25

Fever.............. 52

Fever-Aseptic........... 56

Fever-Aseptic Traumatic.... 57

Fever-Mud.............. 63

Fever-Epitraumatic........ 382

Fever-Purulent........... 412

Fever-Surgical. . . . . . . . . 57

Fever-Traumatic . . . . . . 380-383

Fever-Traumatic Embolic... 412

Fibers-Elastic............ 25

Fibroblasts. . . . . 21-23-24-25-337

Fibroma................ 373

Fibrous Tissue.............. 24

Fission. . . . . . . . . . . . 102

Fistula. . . . . . . . . . . . 371

Fistula of the Rumen...... . 342

Fistula-Watering Pot....... 373

Fistula-Blind............ 371

Fistula-Congenita1........ 371

Fistula-Complete......... 371

Fistula-Dental. . . . . . . . . . 372

Fistula-Idiopathic. . . . . .371-373

Fistula-Pathologica1........ 371

Fistula-Symptomatic....... 371

Fixative............... 162

Fluctuation............. 351

Forcep Torsion. . . . . . . . . 197

Foreign Bodies. . . . . . . . . . . 340

Fractures................ 622

Freezing. ............. 326

Friedlander-Bacillus of. .... 437

Gangrene.............. 62

Gangrene-Dry............ 67

Gangrene-Encroaching. . . . . . 387

Gangrene-Instantaneous. . . . . 387

Gangrene-Moist. . . . . . . . . 67

Gangrene-Mycotic. . . . . . . . 62-63

Gangrene-Operative........ . 64

Gangrene-Pressure......... 64

Gangrene-Primary......... 63

Gangrene-Secondary........ . 65

Gangrene-Spontanesus...... 65

Gangrene-Thundering. . . . . . 387

Gangrene-Traumatic . . . 63-69-387

Gangrene with Emphysema.... 387

Gangrenes-Spreading. ........ 64

Gangrenous Dermatitis. . . . . . 63 
Gangrenous Septicæmia... .386-387

Gaseous Septicæmia......... 387

Gaseous Septicæmia.... . . . . 387

General Atrophy.......... 82

Giant Cells.............. 21

Giant Cells-Multinuclear ... . 28

Glanders. . . . . . . . . . 49-50-128

Glanders-Immunity from. . . . 130

Glanders-Diagnosis of ...... 131

Globules of Fat............ 25

Gout... . . . . . . . . . . . . . 597

Granulation Tissue. . . . . . . . 20-291

Granulations. . . . . . . . . . . . . 334

Granulomata - Infectious . . . .43-49

Granulomata-Specific....... 43

Gunshot Wounds.......... 310

Hæmatoma. . . . . . . . . . . 188

Hæmatomesis. . . . . . . . . . . 188

Hæmaturia. . . . . . . . . . . . 188

Hæmitis................ . 55

Hæmophilia.... . . . . . . . . 188

Hæmoptosis... . . . . . . . . . 188

Hæmorrhage. . . . . . . . . . 187

Hæmorrhage-Arrest of.... . . . 192

Hæmorrhage-Arterial . . . . 188-331

Hæmorrhage by Diapedesis. . . . 187

Hæmorrhage by Rhexis . . . . . . 187

Hæmorrhage-Capillary. . . 190-331

Hæmorrhage-Results of . ..... 191

Hæmorrhage-Traumatic... . . 330

Hæmorrhage-Venous... . . 190-331

Hæmorrhagic Exudation. .... 187

Hæmorrhagic Ulcer . . . . . . . . 369

Hæmostasis... . . . . . . . . . 192

Hremostasis-Artificial . . . . . . 195

Hæmostasis-Natural. . . . . . . 193

Hanging Drop ............ 111

Gard Abscesses............. 359

Haptophore-Complementophi-

lic.

. 170

Haptophore-Cytophilic. . . . . 170

Jay Bacillus.............. 426

Jealing by First Intention. . . . . 17

fealing by Third Intention. .... . 20

Healing-Direct and Indirect... 17

fealing of an Incised Wound. . 17

Iealing Process-Pure. . . . . . 17

leart-beats - Diminution in

force............... 195

Ieat Dissipation.
Heat Generation. . . . . . . . . . 52

Heat Stroke............. 60

High Temperature. . . . . . . . . 54

Hog-Actinomycosis in the... 514

Hog-Botryomycosis in the... 526

Hog-Melanosis in the....... 560

Hog-Osteosarcoma of Jaws ... 648

Hog-Sniffling Disease of . . . . 647

Hoodwink . . . . . . . . . . . 221

Hoof................. 35

Hoof-Abnormal.......... 36

Horn................ 35

Horn Cicatrix........... 38

Horse-Actinomycosis....... 517

Horse-Botryomycosis of..... 521

Horse-Hydrocephalus of the. . 74

Horse-Osseus Cachexia..... . . 615

Horse-Osteomalacia of the. . . 615

Horse-Osteoporosis . . . . . . . 615

Hot Abscesses. . . . . . . . . . . 350

Hydrocele............... 71

Hydrocephalus........... 71

Hydrocephalus of the Horse. . . 74

Hydrotetanin............. 442

Hyperæmia-Passive........ 72

Hypertrophic Sclerosis........ 335

Hypertrophy............ 30

Hypodermic Anæsthetics. . . . . 237

Idiopathic Ulcers . . . . . . . . 366

Immunization . . . . . . . . . . 398

Immunity. . . . . . . . . . . . 150-168

Immunity-Acquired . . . . . 154-164

Immunity-Active. . . . . . . . . 154

Immunity - Natural. . . . . . 153-164

Immunity-Passive. . . . . . . . . 154

Immunity-Theories of. . . . . . 158

Immunity-Types of . . . . . . 152

Inactivity............ 84

Incised Wounds-Healing of. . 20

Incised Wounds............. 295

Indications for General Anæsthesia................ 244

Infection-Purulent. . . . . . . . 412

Infectious Granulomata. . . . . . 43-49

Infectious Polyarthritis. . . . . . . 590

Infectious Venereal Tumor. . . . 50

Inflammation............ 39

Inflammation-Acute....... 48

Inflammation-Anatomical Processes of ............. 43 
Inflammation--Blood . . . . . . 55

Intlammation-Catarrha1. . . . 47

Inflammation-Chronic...... 48

Inflammation-Croupous.... . . . 42

Inflammation-Degenerative . . 42

Inflammation-Desquamated... 42

Inflammation-Diphtheritic .... 42

Inflammation-Emigrative ... 42

Inflammation-Exudative . . . 17-42

Inflammation-Gangrenous... . 42

Inflammation-Hemorrhagic ... 42

Inflammation-Herpetic...... 42

Inflammation-Interstitial. . . . 41

Inflammation-Papular...... 42

Inflammation-Parenchymatous....

$41-42$

Inflammation-Phlegmonous. . 17

Inflammation-Productive.... . 43

Inflammation-Purulent. . . . . . 42

Inflammation-Pustular...... 42

Inflammation-Specific. . . . . . 42

Inflammation--Suppurative. . . 42

Inflammation--Ulcerative..... 42

Inflammation-Vesicular..... . 42

Injury-Physical. . . . . . . . . . 180

Instantaneous Gangrene. . . . . . 387

Instruments--Surgical. . . . . . . 259

Insular Epidermization....... . 34

Internal Governor. . . . . . . . . . 54

Irritable U1cers. . . . . . . . . . . . 369

Ischemic Atrophy. . . . . . . . 82

Karyolinesis............. 16

Keloid-Cicatricial........... 335

Keloids. . . . . . . . . . . . 335-338

Keratogenous Membrane...... 35

Lacerated Wounds. . . . . . . . . 299

Laminae-Sensitive......... . 36

Laudable Pus............. . 355

Leucocytes............. 21-45

Leucocytes-Polymorphonuclear 45

Ligation................ 197

Line of Demarcation. ......... 66

Lingual Actinomycosis....... . 512

Lipoma. . . . . . . . . . . . . . 574

Local Atrophy. . . . . . . . . . . 82

Lockjaw.............. 467

Lopsided................ 85

Lymph............... 72

Lymphangioma........... 578
Lymphadenoma. . . . . . . . . 576

Lymphocytes........... . . 45-46

Lysin-Tetano . . . . . . . . . . 442

Lysis. . . . . . . . . . . . . . . 169

Maculous Melanosis......... 536

Macrocytase............ 45

Macrophages.............23-45

Malignant Oedema......... 386

Malignant Tumors ....... . . . 563

Mallei-Bacillus............ 127

Mallein. . . . . . . . . . . . . . 130-132

Mallein Test. . . . . . . . . . . 131-176

Mast Cells. . . . . . . . . . . . . . 21-46

Matrix............... 35

Maxillary Actinomycosis...... . 513

Measurement of Microörganisms 102

Median Nerve. . , . . . . . . . . . 33

Melanin............ 525-526

Melanoses............... 525

Melanosis-Definition, History, Origin, Localization, Symptoms, Progress, Duration, Termination, Diagnosis, Prognosis, Treatment. . . ..525 to 562

Melanosis in Ruminants. . . . . . . 554

Melanosis in the Dog. ....... . 561

Melanosis in the Hog. . . . . . . . 560

Melanosis-Macular......... 555

Melanosis in Sheep. . . . . . . . 559

Melanosis-Maculous......... 536

Melanosis-Simple.......... 534

Melanotic Fasciculated Sarcomata............... 537

Melanotic Infiltration... . . . . . 534

Melanotic Tumors....... . 526-534

Membrane-Keratogenous.... . 35

Membrane-Pyogenic........ 354

Menorrhagia.............. 188

Metacarpals............. 613

Metaplasia............. 25

Metchnikoff Theory......... 159

Micrococcus Ascoformans. . . . . 518

Micrococcus Botryogenus..... . 518

Micron................. 102

Microörganisms - Measurement of ............... 102

Microörganisms--Pyogenic.... 112

Microphages............23-45

Microscopic Examination of Bacteria............... 110 
Microscopical Picture of Acute and Chronic Inflammation... 48

Milk Cow Disease. . . . . . . . . . 597

Moist Gangrene........... 67

Morphia... . . . . . . . . . 238

Morphology of Nicolaier Bacil-

lus. . . . . . . . . . . . . . 427

Mortification............. 62

Mud Fever.............. 63

Multinuclear Giant Cells...... 28

Vuscle................... 29

Muscle Buds................ 29

Myelocytes............... 46

Mycotic Gangrene........... 63

Myco-Fibroma Equi......... 518

Myelogenic Actinomycosis.... 503

Myoma................ 580

Myxoma............... 574

. Varcosis-Stage of. . . . . . . 229

Natural Hæmostasis. . . . . . . 193

Necrosis.............. 62

Necrosis-Bandage......... 64

Necrosis of Bone ......... 68

Vecrosis-Pressure.......... 64

Necrosis-Stitch........... 6 t

Nerve Tumors. . . . . . . . . . . 578

Verve-Median ............ 33

Vervous Atrophy. . . . . . . . 82

Vervous Tissue............ 30

Veuroma . . . . . . . . . . .31-578

Veuroma-Amputation...... 31

Vicolaier-Bacillus of. . . . 134-421

Vicolaier - Bacillus - Morpho-

logy of. . . . . . . . . 427

Jedema............... 71

Jedema-Acute Purulent. . . . . 387

Jedema-General........... 73

Jedema-Local............. 73

Jedema-Malignant........386-387

Jedema of the Abdomen...... 71

Jedema of the Extremities..... 74

Jedema of the Sheath and Prepuce

Jedema-Simple.......... 71

Jedematis-Maligni-Bacillus.. 149

Jedematous-Swelling....... 71

Jleaginous Effusions........ 318

Jperating Table, the ........ 204

Jperating Table for Small Animals
Operation-Painful and Bloody 183

Operative Gangrene. . . . . . . . . . 64

Opisthotonos.............. 468

Opsonins................. 174

Orthotonos................ 468

Oscillation.............. 19

Osseus Cachexia-Synonyms, Definition, Symptoms, History, Etiology, Pathogenesis, Course, Duration, Histology. Lesions, Treatment . . . . . . . . . 597 to 638

Osseus Sponge............ 508

Ossification.............. 28

Osteitis-Enzootic.......... 597

Osteitis-Diffuse Rarifying.... 611

Osteoblasts. . . . . . . . . . . . . 24-26

Osteoclasis............... . 597

Osteoma............... 576

Osteomalacia....... 583-597-615

Osteoperiostitis-Diffused..... 638

Osteoporosis . . . . . . . . . . 597-636

Osteoporosis-Equine...... 615

Osteosarcoma............. 501

Osteoid Tissue............. 27

Ovine Actinomycosis . . . . . . . 517

Ovoid Tumor............. 317

Ox-Botryomycosis......... 523

Pain-Prolonged or Suffering. . 181

Painful and Bloody Operations. 183

Paralysis of Azoturia.......... 86

Paralysis-Centric.......... 85

Paralysis-Periphera1........ . 83

Partial General Anæsthesia. . . . . 246

Passive Hyperæmia. . . . . . . . 72

Pathologic Atrophy. . . . . . . . 82

Patients' Habitat. . . . . . . . . . 266

Pathological Repair.......... 17

Periosteal Actinomycosis...... . 503

Periosteal Ossification. . . . . . . 647

Perspiration.............. 60

Petechia............... 187

Phagadenic Ulcer. . . . . . . . . . . 369

Phagocytes. . . . . . . . . . . 23-45

Phagocytosis... . . . . . . . 45-159

Phagolysis.............. 162

Pharynx-Actinomycosis of the 505

Phlebitis-Suppurative Metastatic. . . . . . . . . . . . . 412

Peripheral Paralysis......... 83

Physical Injury........... 180 
Physiologic Atrophy, . . . . . . . 82-84

Physiological Action of Chloroform and Ether........... 225

Plasma Cells................. 21

Pleurosthotonos. . . . . . . . . 468-469

Pneumonia-Embolic....... 79

Poison-Tetanic............ 441

Poisoned Wounds.... . . . . . . 302

Poll Evil. . . . . . . . . . . . 25

Polyarthritis-Infectious...... 590

Polymorphonuclear-Leucocytes 45

Polyvalent Serum......... 177

Porcine Actinomycosis........ 514

Post Anæsthesia Complications. 243

Practical Asepsis............ 278

Practical Technique......... 279

Precipitins. . . . . . . . . . 170-173

Preformed Antitoxin. . . . . . . 452

Preparation of Antitetanic Serum .............. 459

Pressure............... 85

Pressure Atrophy. . . . . . . . . 82

Pressure Necrosis. . . . . . . . . . . 64

Primary Gangrene. . . . . . . . . 63

Primary Serous Effusions...... 317

Prodigiosus Bacillus......... 436

Prolonged Pain or Suffering. . . . 181

Pseudo Arthrosis . . . . . . . . . . . . 28

Ptomains............... 94

Punctured Wounds.......... 297

Puerperal Tetanus. . . . . . . . . . 441

Pure Healing Process. . . . . . . . 17

Purulent Diathesis... . . . . . . . 412

Purulent Fever. . . . . . . . . . . . 412

Purulent Infection. . . . . . . . . . 412

Purulent Inflammation. . . . . . 17

Purulent Resorption. . . . . . . . 412

Pus-Laudable.... . . . . . . 355

Pus-Study of . . . . . . . . . 355

Pyaemia............ . 381-412

Pyogenes Fœtidus-Bacillus. . 122

Pyogenic Membrane......... 354

Pyogenic Microörganisms. . . . . 112

Pyrexia................ 52

Quarter Evil............ 146

Rachitis-Definition, Etiology, and Pathology, Symptoms, Diagnosis, Prognosis, Pathological Anatomy, Treatment ................583 to 5 .
Rachitis-Acquired....... 583

Rachitis-Congenital........ 583

Rauschbrand Bacillus........ 146

Ray Fungus............... 134

Receptor.............. 166

Recommendations for an Asep-

tical Operation.......... 281

Reflex Syncope........... 241

Regeneration............ 15

Regeneration of Individual Tissues .................. 22

Regeneration-Pathological... . 16

Repair-Pathological........ 17

Reparative Processes-Simple. . 17

Replacement of Lost Tissue.... 15

Reproduction of Bacteria....... 102

Resorption-Purulent. . . . . . . 412

Restraint............. 200

Results of Hæmorrhage. . . . . . 191

Retardation of the Blood Current ............... 18

Rheumatism-Articular..... 590

Sac-Blood................ 316

Sac-Sanguineous.......... 316

Sac-Study of the ......... 354

Sanguineous Sac.... . . . . . . 316

Sanguineous Tumor......... 316

Sarcina.................. 93

Sarcoma.............. . 570

Scar............ . . . . 17-21

Scar-Cicatricial........... 21

Scars-Cutaneous........... 333

Schnuffelkrankheit.... . . . . . 589

Schwann-Cells of Sheath of.... 31

Sclerogenesis. . . . . . . . . . 291

Sclerosis-Arterial. . . . . . . . . 66

Sclerosis-Hypertrophic. . . . . 335

Seedy Toe.............. 37

Senile Atrophy. . . . . . . . . . 82

Senile Changes............. 82

Senile Degeneration.......... 82

Sensitive Laminæ. . . . . . . . . . 36

Sepsine. . . . . . . . . . . . . . 395

Septic Poison. . . . . . . . . . . . 395

Septic Vibrio. . . . . . . . . . . . . 389

Septicæmia-Actute . . . . . . 380-384

Septicæmia-Chronic... . . . 380-384

Septicrmia-Embolic.... . . . 381

Septicæmia-Gangrenous. . 386-387

Septicæmia-Gaseous........ 387

Septicæmia-Surgical. . . . . 57-380 
Septicæmia-Very Acute.... . . 387

Serotherapy of Tetanus. . . . . . 453

Serous Cyst.............. 581

Serum-Preparation of Antitetanic 459

Serums.............. 176

Serums-Manufacture, Standardization, and Practical Value of ............ 176-177

Serum-Polyvalent.......... 177

Sheep-Actinomycosis....... 517

Sheep-Melanosis in the...... . 559

Shoulder Slip.............. . 30

Shock-Surgical........... 179

Side Chain Theory .......... 165

Side Line-Single. . . . . . . . . . 218

Simple Melanosis........... 534

Simple Oedema............ . 71

Simple Reparative Processes.... 17

Sit-Fast............... 64

Skin-Cicatrices of the....... 333

Skin-Ecchymosis of the. . . . 315

Sniffling Disease. . . . . . . . . . 589

Sniffling Disease of Hog-Definition, Experimental. Study, Pathology, Symptoms, Pathological Anatomy, Diagnosis, Treatment. . . . . . . . .647 to 658

Soft Abscesses.... . . . . . . . 350

Spasmatoxin. . . . . . . . . 442

Specific Granulomata......... 43

Specific Surgical Microörganisms 126

Sphacelus.............. 62

Spinal Cocainization......... 253

Spirilla................ 94

Spongy Bone............ 27

Sporogenic Bacterium. . . . . . . 103

Sporulation. . . . . . . . . . . 103

Spreading Gangrene.......... 64

Stage of Anæsthesia. . . . . . . . 230

Stage of Excitement. . . . . . . 228

Stage of Narcosis. . . . . . . . . . 229

Staining. . . . . . . . . . 105

Staphylococcus............ 93

Staphylococcus-Epidermis Albus. . . . . . . . . . . . . 117

Staphylococcus-Pyogenes A1bus. . . . . . . . . . . 116-348

Staphylococcus-Pyogenes Autreus . . . . . . . 112-348-436
Staphylococcus-Pyogenes Citreus ........... 117-348

Staphylococcus-Pyogenes Flavus. . . . . . . . . . . . . . 348

Stasis.................. 19

Stocks-The . . . . . . . . . 216

Straus Test.............. 131

Streptococcus............. 93

Streptococcus of Strangles. . . . . 348

Streptococcus-Pyogenes . . 117-348

Study of Pus . . . . . . . . . . . 355

Study of the Container. . . . . . . 354

Study of the Sac. . . . . . . . . . 354

Styptics-Application of...... 198

Superficial Abscesses. . . . . . . 350

Suppurative Metastatic Phlebitis 412

Surgeon's Hands.......... 260

Surgical Dressings. . . . . . . . 265

Surgical Fever............. 57

Surgical Field. . . . . . . . . . 261

Surgical Instruments. . . . . . . 259

Surgical Septicæmia... . . . 57-380

Surgical Shock. . . . . . . . . . . 179

Sutures-The............ 263

Swelling-Oedematous....... 71

Symptomatic Anthrax........ 146

Symptomatic Ulcers........ . 366

Syncope-Direct........... . 242

Syncope-Reflex........... 241

Syntexis............... 598

Temperature-High........ 54

Teratomata............ 580

Temporary Bridge......... 26

Tetanic Poison............ . 441

Tetanic Toxin. . . . . . . . . . . 441

Tetanin............... 442

Tetanolysin............. 442

Tetanotoxin............. 442

Tetanustoxin.... . . . . . .442-443

Tetanus-Definition, History, Pathogenesis, Immunization, Serumtherapy. Symptoms, Lesions, Diagnosis, Treatment, etc............ 421 to 490

Tetanus a Frigore. . . . . . . . . . 464

Tetanus-Acute. . . . . . . .469-471

Tetanus-Bacilli..... . 97-134-421

Tetanus-Chronic.......... 472

Tetanus-Cephalic......... 464 
Tetanus-Cerebral........ 450

Tetanus-Puerperal. . . . . . . 441-464

Tetanus-Rheumatic........ 421

Tetanus-Serotherapy........ 458

Tetanus-Traumatic . . . . . . 421

Tetanus-Splanchnic........ 464

Thecal Abscess... . . . . . . . . 364

Theories of Immunity . . . . . . . 158

Thermotaxis............. 52

Thrombosis. . . . . . . . . . . . 66-76

Thundering Gangrene....... . 387

Tissue-Changes in the. ...... 47

Tissue-Connective......... 22

Tissue-Dead and Dying. ...... 66

Tissue-Fibrous.............. 24

Tissue-Granulation......... 20

Tisstue-Nervous........... . 30

Tissue-Osteoid . . . . . . . . . 27

Tissue-Velvety. . . . . . . . . 36

Tissue-Viable. . . . . . . . . . 66

Toe-Seedy............... 37

Tongue-Actinomycosis of. . . . 503

Tongue-Wooden. . . . . . . . . . 504

Torsion-Forcep............ 197

Toxalbumins. . . . . . . . 40-96-442

Toxalbumin of Brieger. . . . . . . 442

Toxic Asphyxia.............. 239

Toxins.............. 96-395

Toxin-Tetanus... . . . . . . 441

Toxins in Diagnosis. . . . . . . 176-395

Tourniquet............. 196

Trabeculæ............... 27

Transmigration............ 44

Traumatic Embolic Fever. . . . . 412

Traumatic Emphysema. . . . 332-387

Traumatic Fever-Aseptic.... 57

Traumatic Fever. . . . . . . . . 380-383

Traumatic Gangrene. . . . . 63-69-387

Traumatic Hæmorrhage...... 330

Traumatisms............ 287

Trismus . . . . . . . . . . . . . 463

Tuberculin Test. ......... 138-176

Tuberculin. . . . . . . . . . . 139-176

Tuberculosis. . . 49-50-135-136-137

Tuberculosis-Bacillus....... 135

Tuberculous Tumor.......... 536

Tubes-Endothelial. . . . . . . . 22

Tumefaction of Bones of Head . . 618

Tumor-Blood............ 316

Tumor-Venereal Infection.... 50

Tumor-Ovoid............ 317
Tumor-Sanguineous . . . . . . 316

Tumor-Tuberculous........ . 536

Tumors-Definition, Frequency, Classification, Treatment....

$\ldots \ldots \ldots \ldots \ldots 562$ to 583

Tumors-Benign.......... 563

Tumors-Complex......... 580

Tumors-Connective Tissue. . . 570

Tumors-Epithelial........ 566

Tumors-Malignant........ 563

Tumors of Muscular Type. . . . . 580

Tumors-Melanotic. . . . . . . . 526

Turtle-Calves. . . . . . . . . . . . 643

Twitch-the............... 220

Ulceration. . . . . . . . . . 62-366

U1cers. . . . . . . . . . . . . . . . 366

Ulcers-Atonic . . . . . . . . . . 369

Ulcers-Callous. . . . . . . . . . . . 369

Ulcers-Hæmorrhagic. . . . . . . 369

Ulcers-Idiopathic. . . . . . . . . 366

Ulcers-Irritable . . . . . . . . . . . 369

Ulcers-Phagadenic... . . . . . . . 369

U1cers-Symptomatic. . . . . . 366

Uniceptor............... 167

Urinary Disorders. . . . . . . . . 625

Vaccination........... 155-177

Vaccines................ 176

Vascular Changes............ 43

Velvety Tissue............. . 36

Venereal Tumor-Infectious.... 50

Venom................ 303

Venous Hæmorrhage. . . . . . 190-331

Vessels-Contractility of. . . . . 194

Vessels-Dilation of the...... 18

Vibrio-Septic.......... 389

Virulent Wounds........... 303

Washing the Blood.......... 386

Wen................... 501

Wound-Cavernous... . . . . . . 287

Wound-Closed............ 287

Wound-Closure and Drainage of,$\ldots \ldots \ldots \ldots \ldots \ldots \ldots 273$

Wounds-Contused ......... 308

Wound-Environs of....... 261

Wound-Examination of...... 268

Wound-Exposed........... 287

Wound-Open............ 287

Wound-Protection and After care................. 274

Wound-Subcutaneous....... 287 
Wound-Surgical Treatment of. 270 Wounds by Tearing..... . . . 299

Wound Treatment-Routine of. 267

Wound Treatment-Routine of

Wounds by Teeth Bites....... 300

Wounds-Gunshot......... 310

Closure and Drainage.... . 273 Wounds-Incised.......... 295

Wound Treatment - Routine Wounds-Envenomed........ 303

Examination........... 268 Wounds-Lacerated......... 299

Wound Treatment - Routine

Examination, Protection and after care.............. 275

Wound Treatment-Proper... . 270

Wounds by Firearms........ 310

Wounds by Penetratine: Instru-

Wounds-Poisoned......... 302

Wounds-Punctured......... 297

Wounds-Virulent......... 303

Yellow Grains. . . . . . 492-503-511

ments. . . . . . . . . . . 297 Zone-Middle............ 288

Wounds by Sharp Instruments . 295 Zone-Peripheral.......... 288 








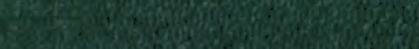

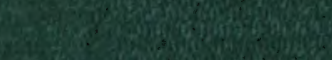

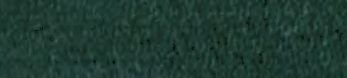

2.

(1)

8.

(4)

(10)

5

8.6.5.

+3.

Q7.

H.t.

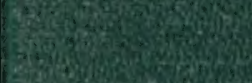

